\title{
KENO-VI Primer: Performing Calculations Using SCALE's Criticality Safety Analysis Sequence (CSAS6) with Fulcrum
}

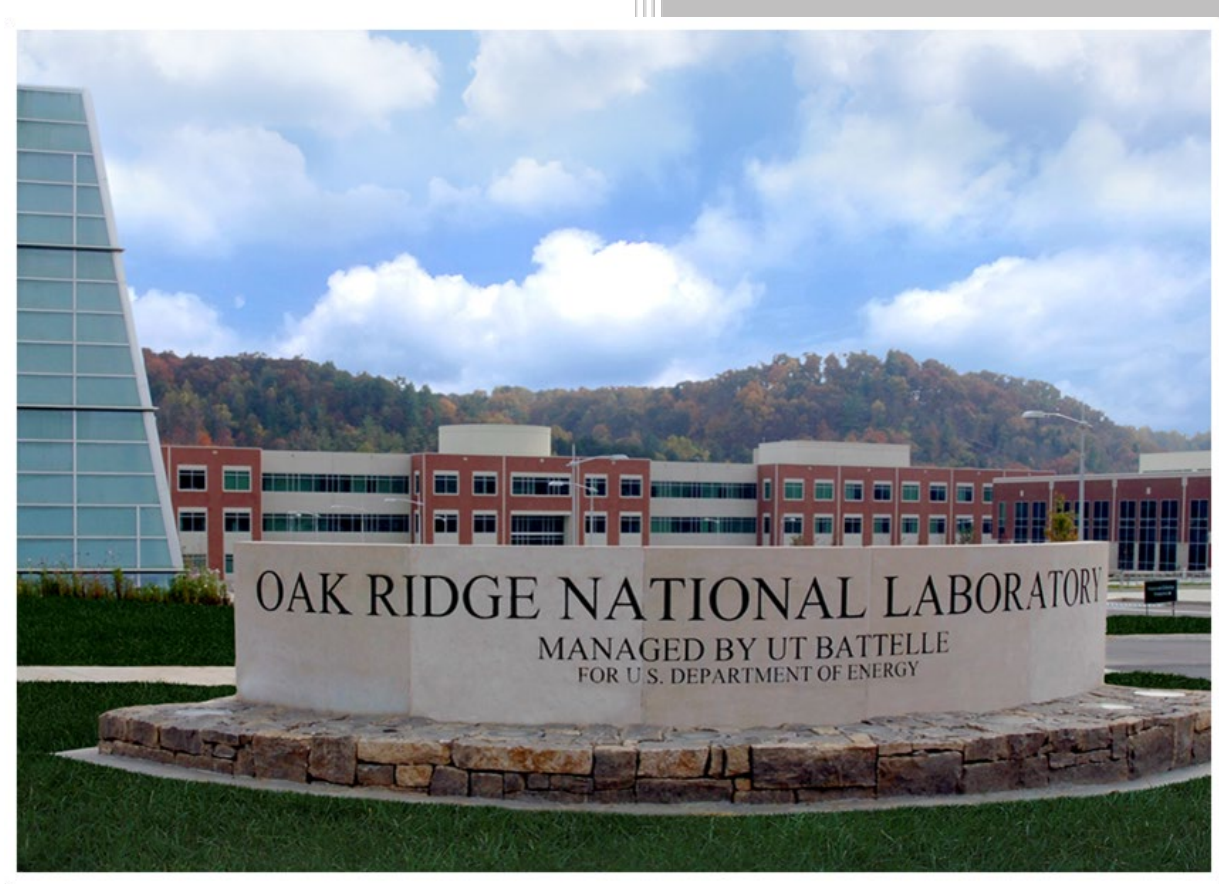

Approved for public release. Distribution is unlimited.
Kursat Bekar Justin Clarity Mathieu Dupont Robert Lefebvre William Marshall Ellen Saylor

December 2020 


\section{DOCUMENT AVAILABILITY}

Reports produced after January 1, 1996, are generally available free via US Department of Energy (DOE) SciTech Connect.

Website www.osti.gov

Reports produced before January 1, 1996, may be purchased by members of the public from the following source:

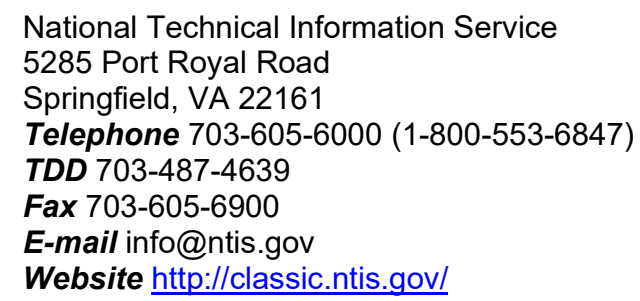

Reports are available to DOE employees, DOE contractors, Energy Technology Data Exchange representatives, and International Nuclear Information System representatives from the following source:

Office of Scientific and Technical Information

PO Box 62

Oak Ridge, TN 37831

Telephone 865-576-8401

Fax 865-576-5728

E-mail reports@osti.gov

Website http://www.osti.gov/contact.html

This report was prepared as an account of work sponsored by an agency of the United States Government. Neither the United States Government nor any agency thereof, nor any of their employees, makes any warranty, express or implied, or assumes any legal liability or responsibility for the accuracy, completeness, or usefulness of any information, apparatus, product, or process disclosed, or represents that its use would not infringe privately owned rights. Reference herein to any specific commercial product, process, or service by trade name, trademark, manufacturer, or otherwise, does not necessarily constitute or imply its endorsement, recommendation, or favoring by the United States Government or any agency thereof. The views and opinions of authors expressed herein do not necessarily state or reflect those of the United States Government or any agency thereof. 
Nuclear Energy and Fuel Cycle Division

\title{
KENO-VI PRIMER: PERFORMING CALCULATIONS USING SCALE'S CRITICALITY SAFETY ANALYSIS SEQUENCE (CSAS6) WITH FULCRUM
}

\author{
Kursat Bekar \\ Justin Clarity \\ Mathieu Dupont \\ Robert Lefebvre \\ William Marshall \\ Ellen Saylor
}

Date Published: December 2020

Prepared by

OAK RIDGE NATIONAL LABORATORY

Oak Ridge, TN 37831-6283

managed by

UT-BATTELLE, LLC

for the

US DEPARTMENT OF ENERGY

under contract DE-AC05-00OR22725 



\section{CONTENTS}

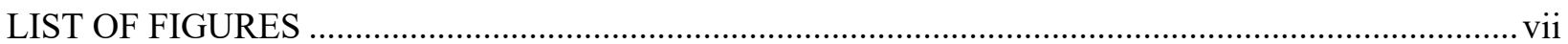

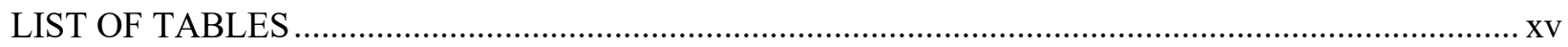

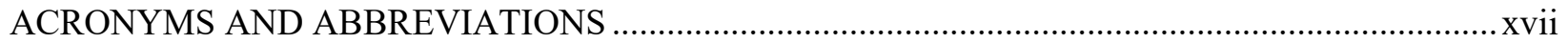

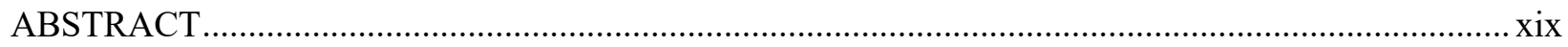

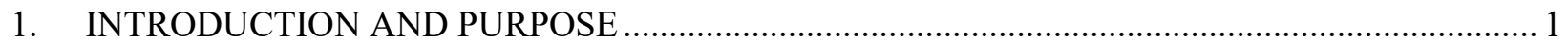

1.1 INTENDED AUDIENCE AND AVAILABLE RESOURCES ............................................. 1

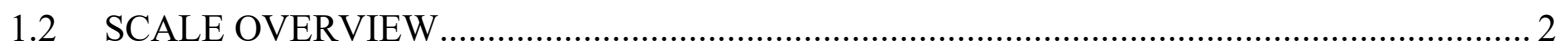

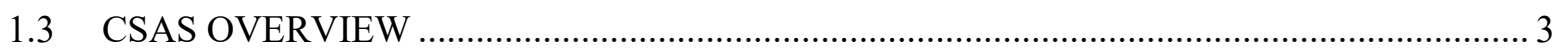

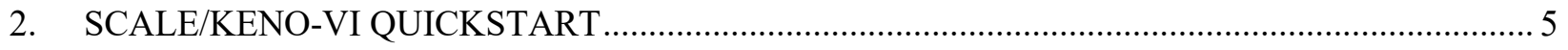

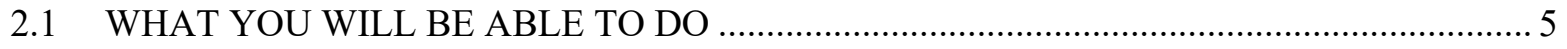

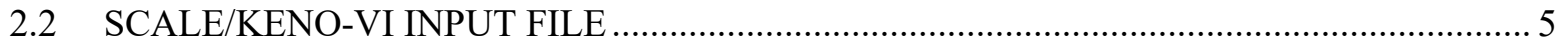

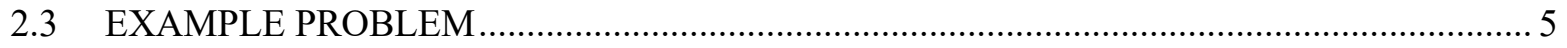

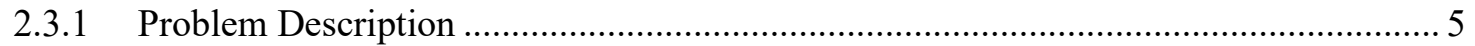

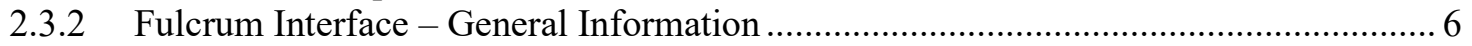

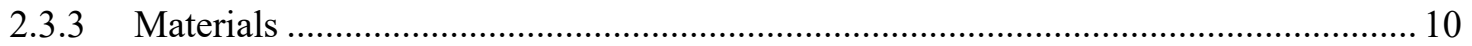

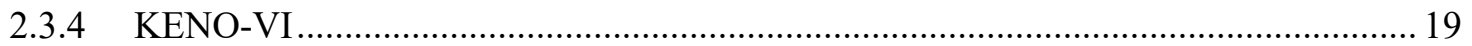

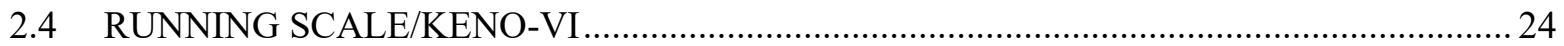

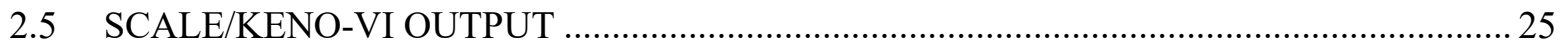

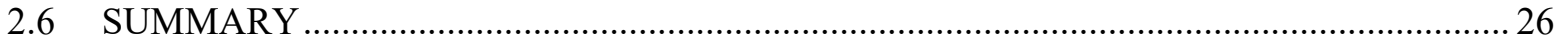

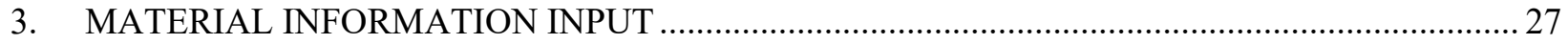

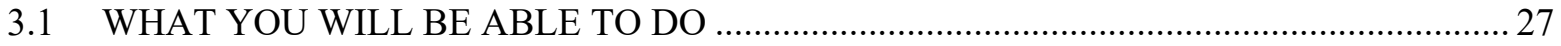

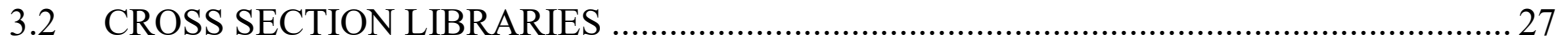

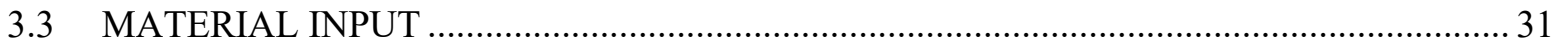

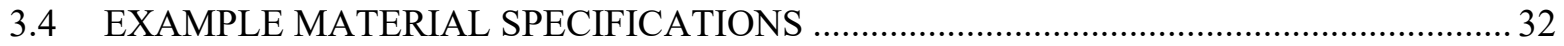

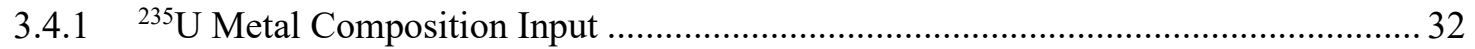

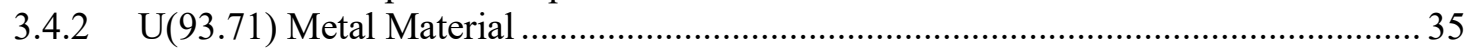

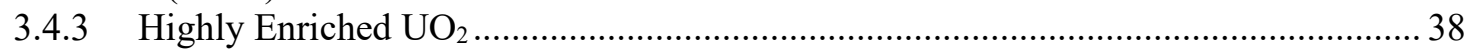

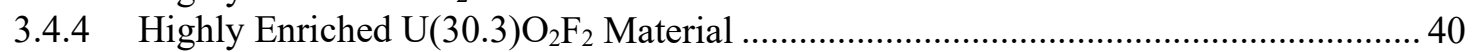

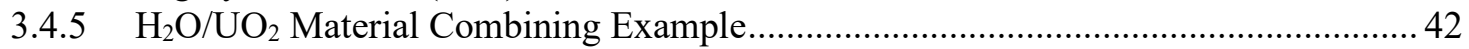

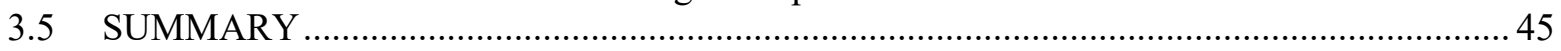

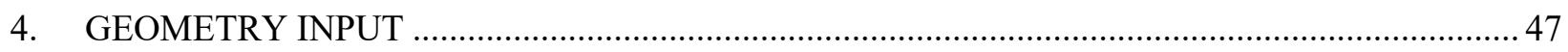

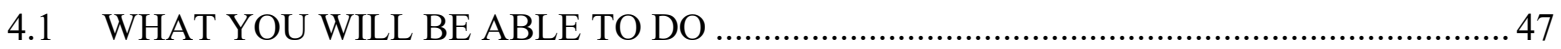

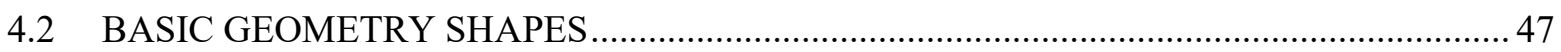

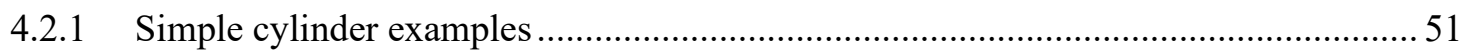

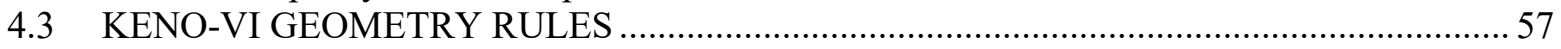

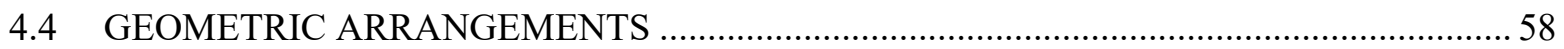

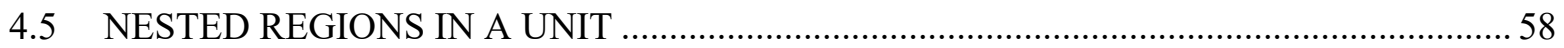

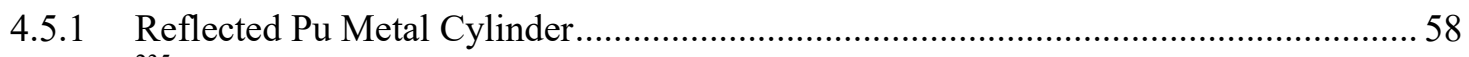

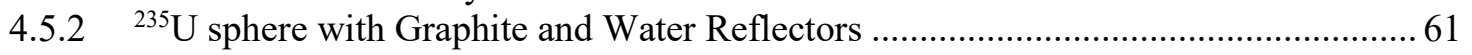

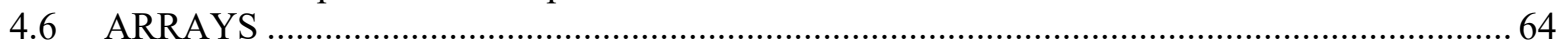

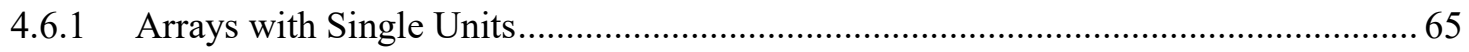

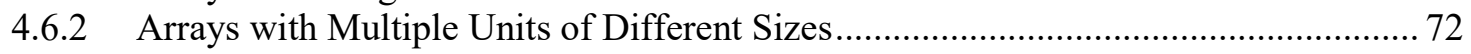

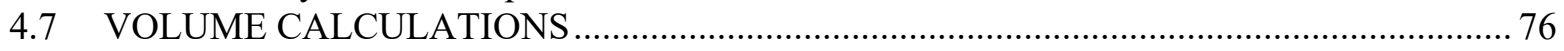

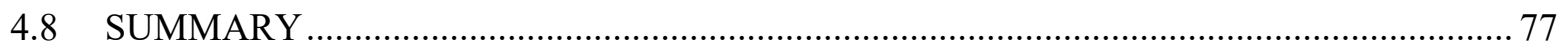

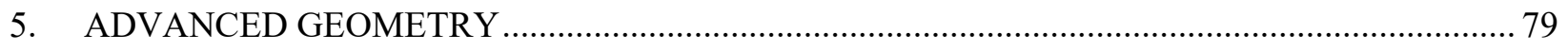

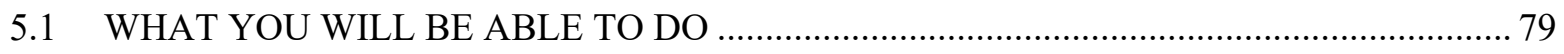

5.2 USING THE CHORD KEYWORD TO TRUNCATE A BODY …....................................... 79

5.3 USING THE ORIGIN KEYWORD TO TRANSLATE A BODY …..................................... 80 
5.3.1 Example Using the ORIGIN Keyword to Create Three Intersecting Cylinders .......... 81

5.4 USING THE ROTATE KEYWORD TO ROTATE A BODY …........................................... 84

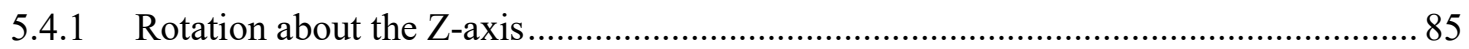

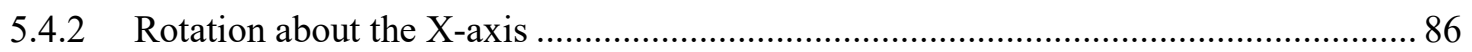

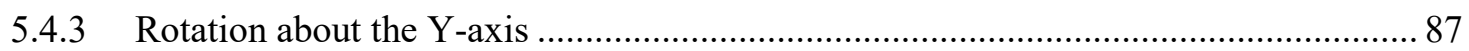

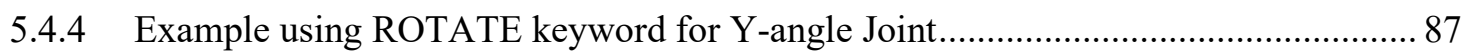

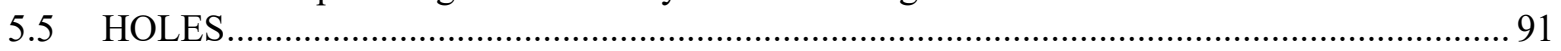

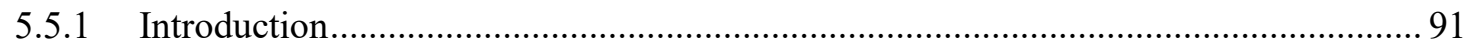

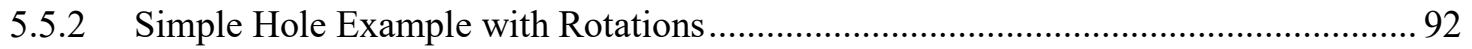

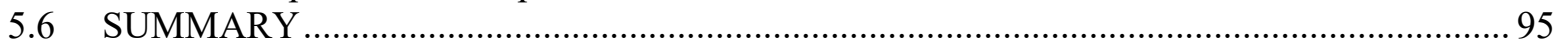

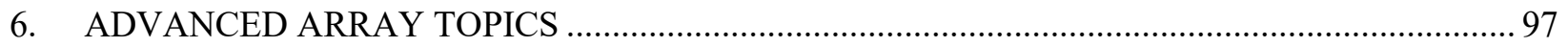

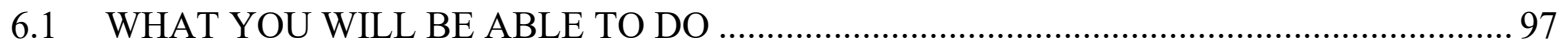

6.2 SHIPPING CONTAINER WITH FIVE CYLINDERS OF CRUCIFORM U(93.2)C

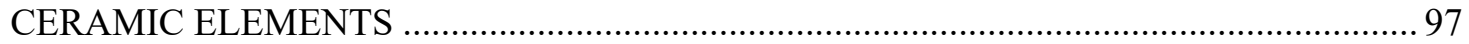

6.3 HEXAGONAL ARRAY WITH INNER AND OUTER BOUNDARIES …........................ 101

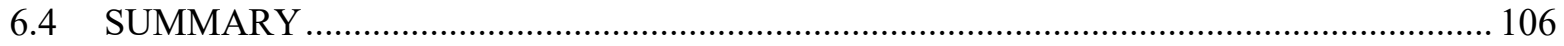

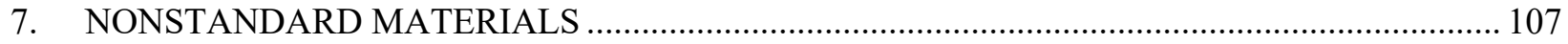

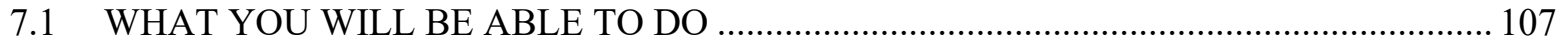

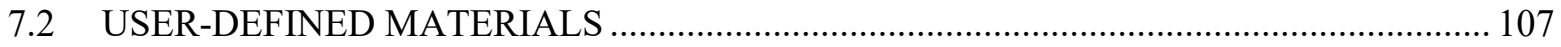

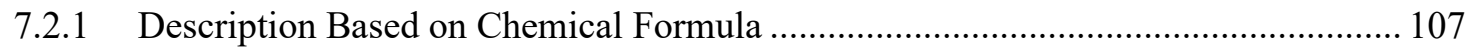

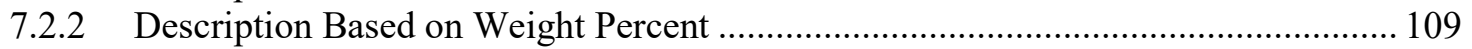

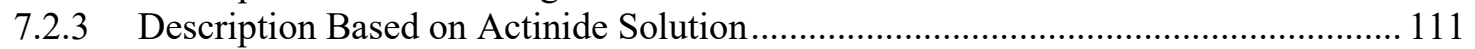

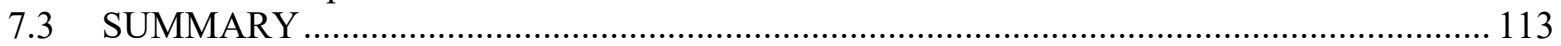

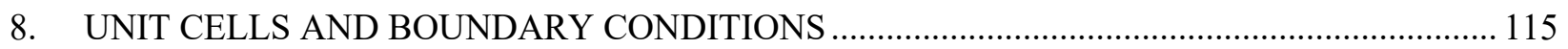

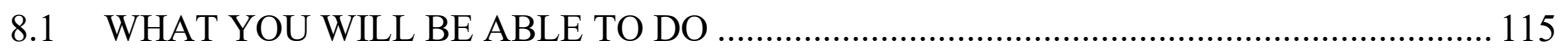

8.2 CALCULATION TYPES FOR PROBLEM-DEPENDENT CROSS SECTIONS ................. 115

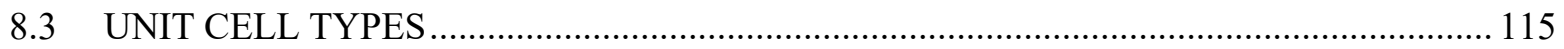

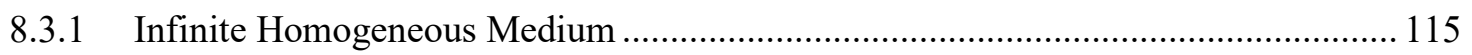

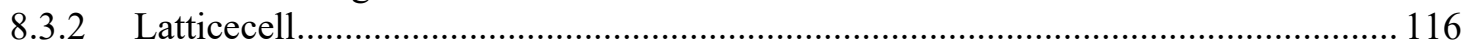

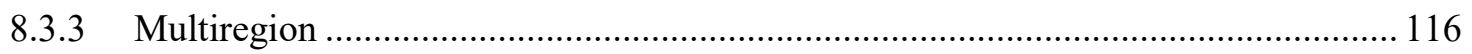

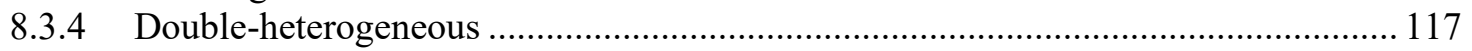

8.3.5 Unit Cell Boundary Conditions....................................................................... 118

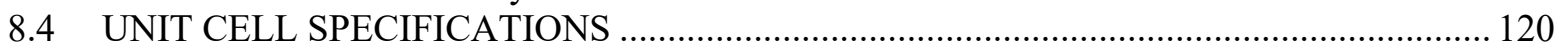

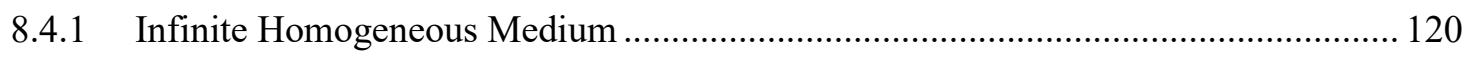

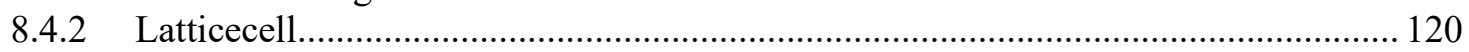

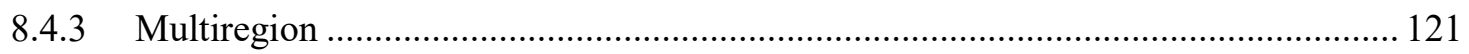

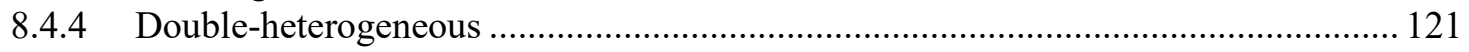

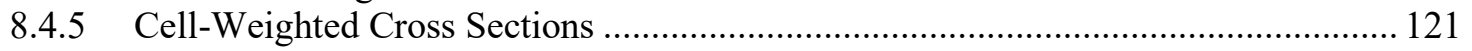

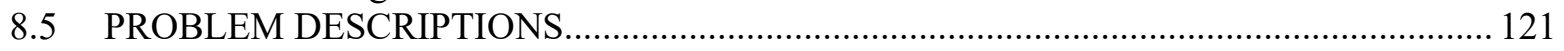

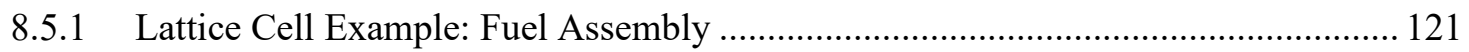

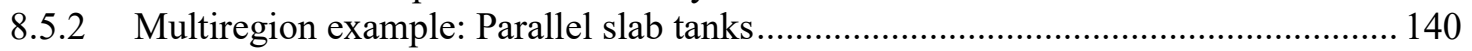

8.5.3 DOUBLEHET Example: Infinite, Square-pitched Array of Pebbles ....................... 149

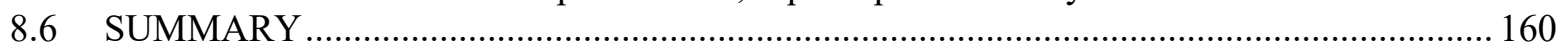

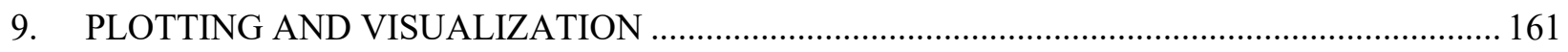

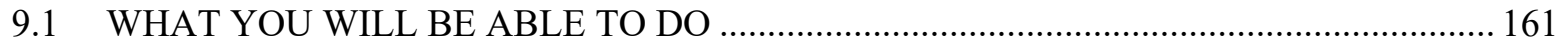

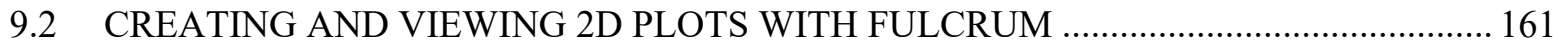

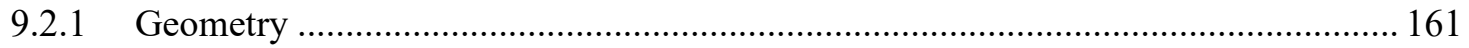

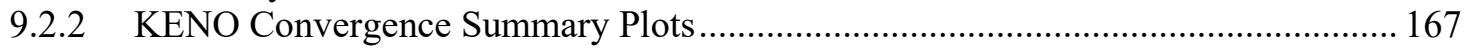

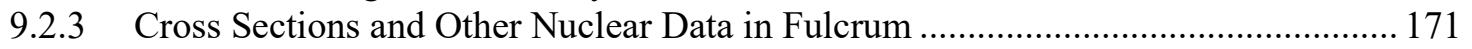

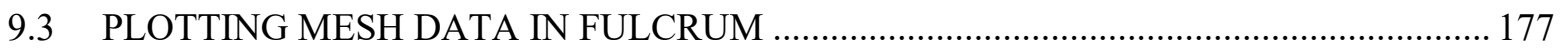

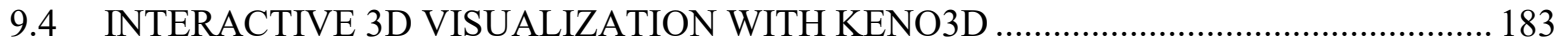


9.5 PLOTTING CALCULATED RESULTS WITH KMART AND KENO3D ....................... 188

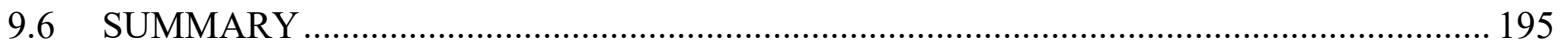

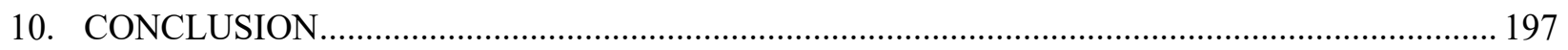

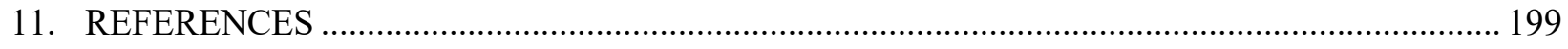

APPENDIX A. INPUT FILES FOR EXAMPLE PROBLEMS …............................................... A-1

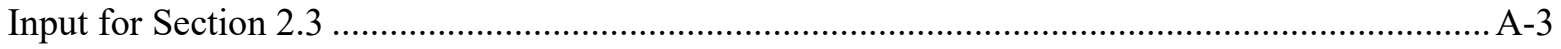

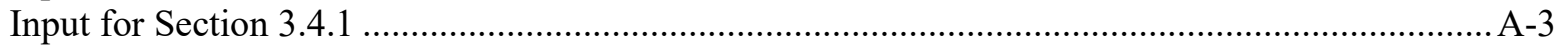

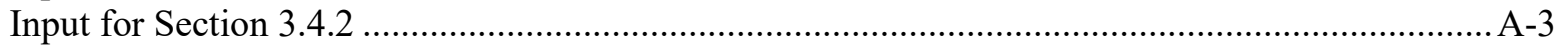

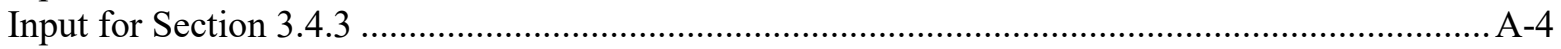

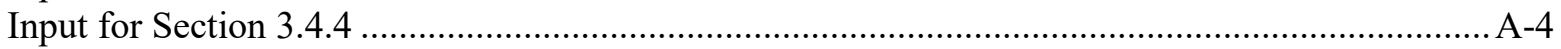

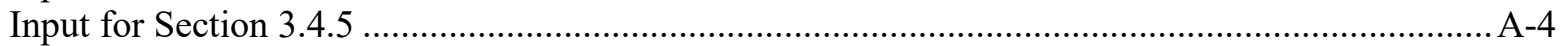

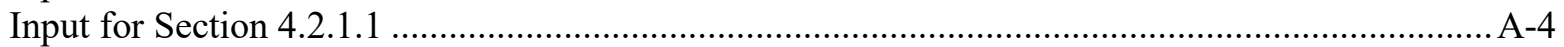

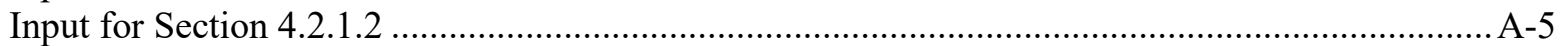

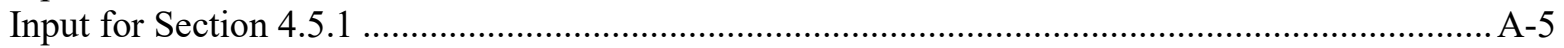

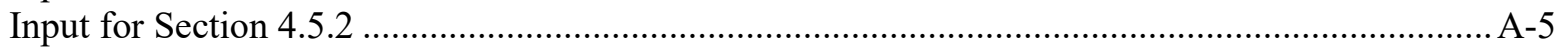

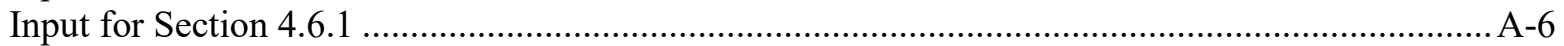

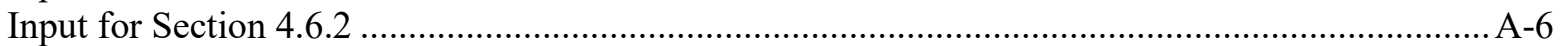

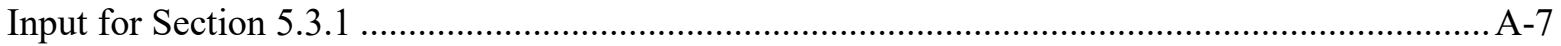

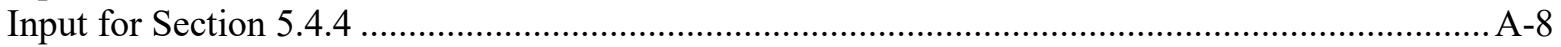

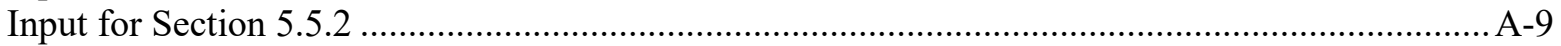

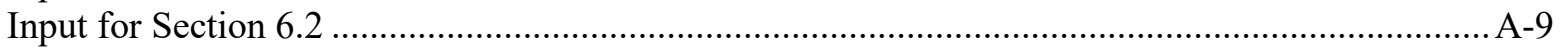

Input for Section 6.3

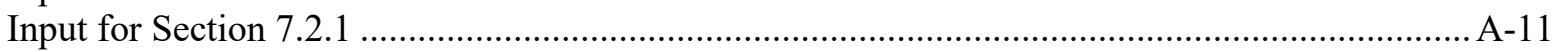

Input for Section 7.2.2

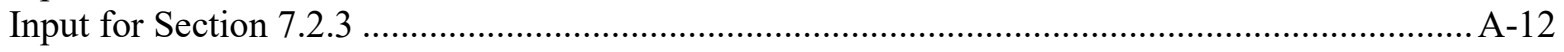

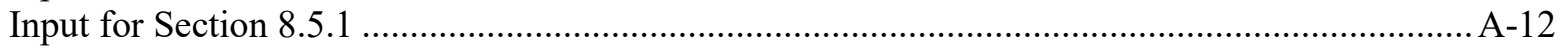

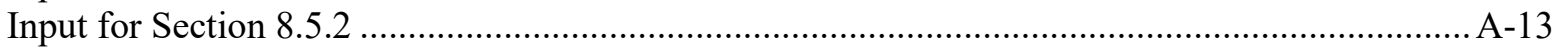

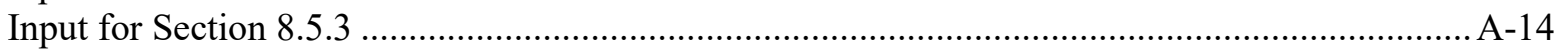





\section{LIST OF FIGURES}

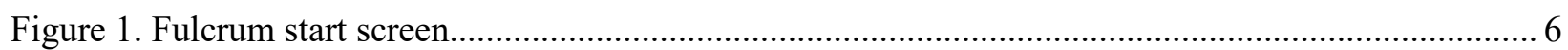

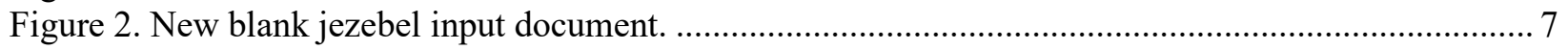

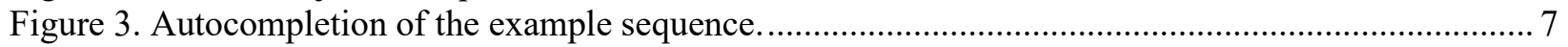

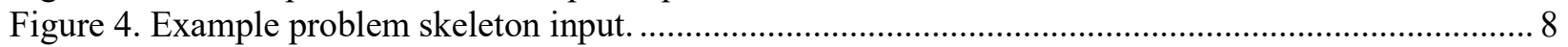

Figure 5. General information for the example problem. ........................................................... 9

Figure 6. Validation panel indicating that required compositions are not present..................................... 9

Figure 7. Example problem Basic Composition autocompletion. ......................................................... 10

Figure 8. Example default basic stdcomp configurable form. ................................................................ 11

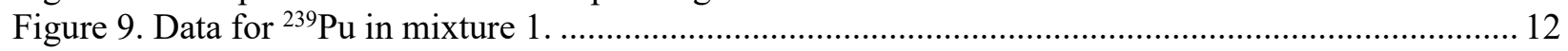

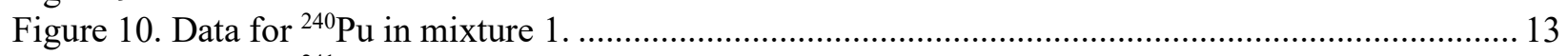

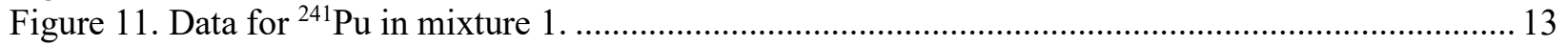

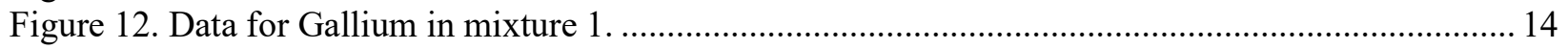

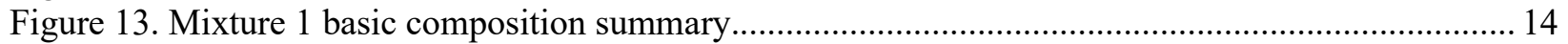

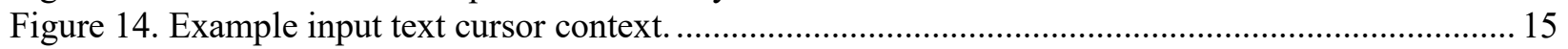

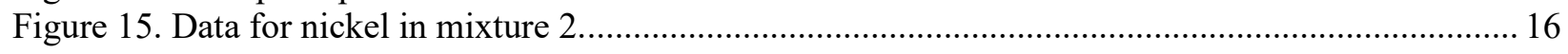

Figure 16. Complete example problem composition block.............................................................. 16

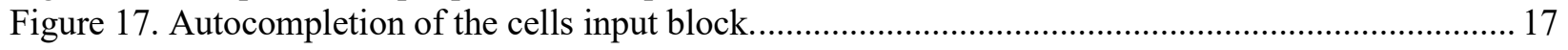

Figure 18. Example autocompleted initial celldata input block................................................................ 17

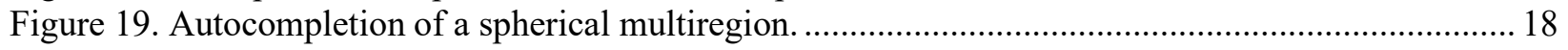

Figure 20. Example autocompleted initial spherical multiregion input.................................................. 18

Figure 21. Example spherical multiregion zone mixture and radius placeholders. ................................ 18

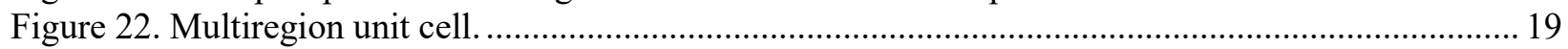

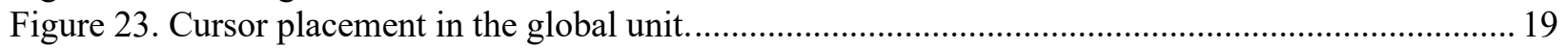

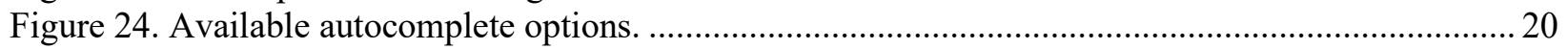

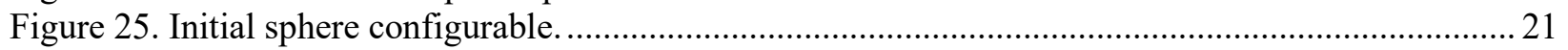

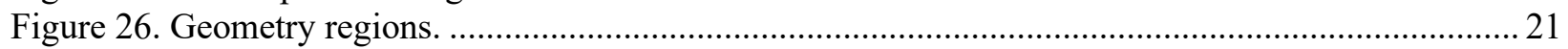

Figure 27. Configurable geometry media autocompletion. ............................................................... 22

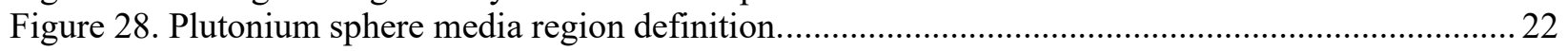

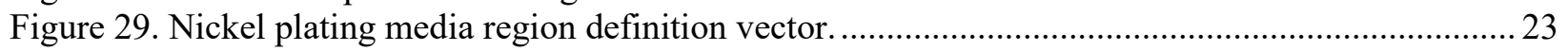

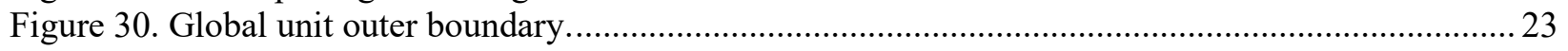

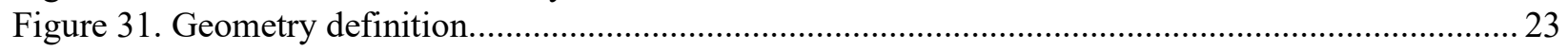

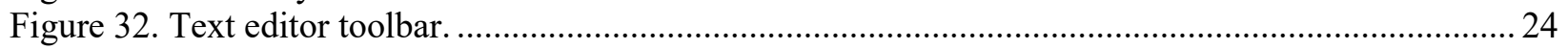

Figure 33. Location of Messages panel and red background color indicating unseen messages................ 24

Figure 34. Messages panel showing completed run......................................................................... 24

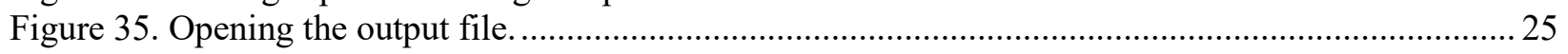

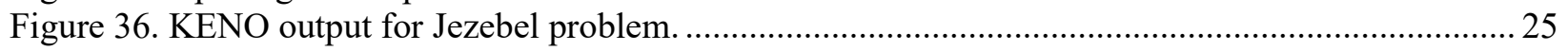

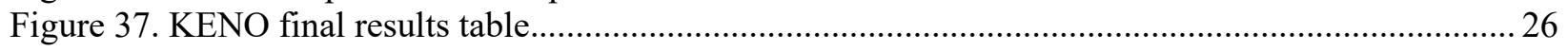

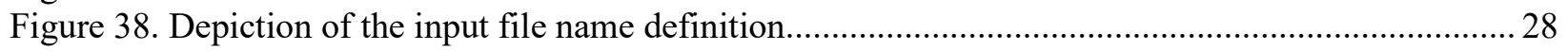

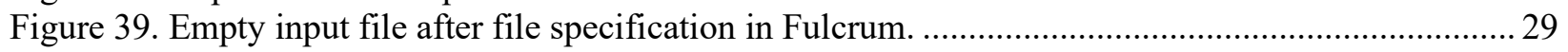

Figure 40. Dropdown menu with the SCALE sequence specification.................................................. 29

Figure 41. Empty input file resulting from the CSAS6 sequence specification. ................................... 30

Figure 42. Specification of problem title and cross section library. ................................................ 31

Figure 43. Accessing the basic standard composition configurable ..................................................... 32

Figure 44. Basic standard composition configurable input initial display.............................................. 33

Figure 45. Basic standard composition configurable input, initial display filled out. ............................... 33

Figure 46. Input result of the standard basic composition configurable input.......................................... 34

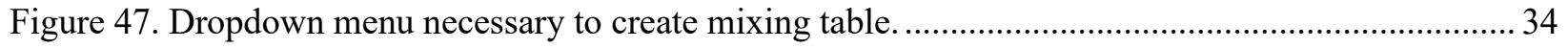

Figure 48. Mixing table results for the uranium metal example.......................................................... 35 
Figure 49. Isotopic composition input prior to user modification.

Figure 50. Isotopic composition input following user modification for the enriched uranium metal case...

Figure 51. Final composition input for the enriched uranium metal case ............................................. 37

Figure 52. Mixing table results for the uranium metal example......................................................... 38

Figure 53. Isotopic composition input following user modification for the enriched uranium oxide

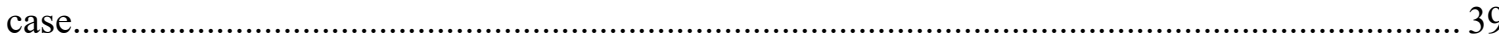

Figure 54. Final composition input for the enriched uranium oxide case............................................ 39

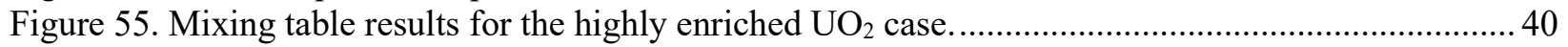

Figure 56. Accessing the basic standard composition configurable. .................................................. 41

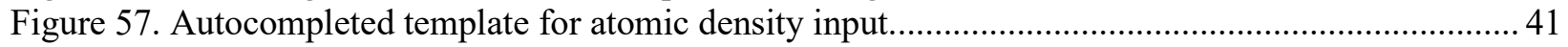

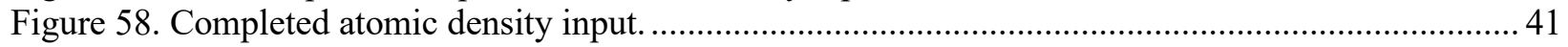

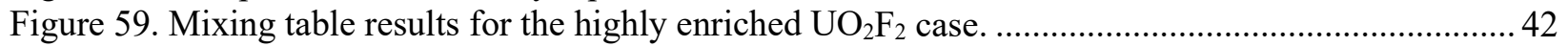

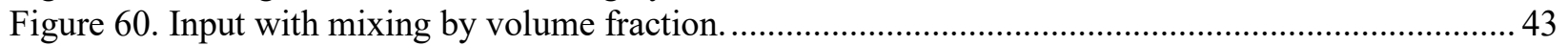

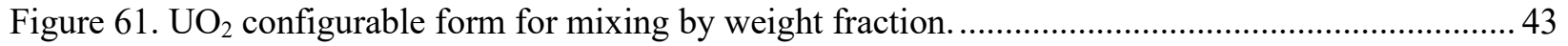

Figure $62 . \mathrm{H}_{2} \mathrm{O}$ configurable form for mixing by weight fraction....................................................... 44

Figure 63. Final material specification input for mixing by volume fraction and mixing by weight

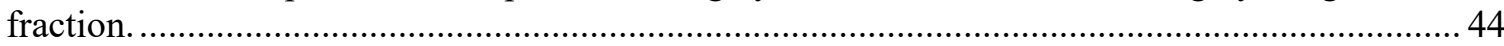

Figure 64. Mixing table output for the mixing by volume fraction and the mixing by weight

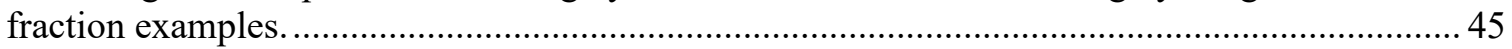

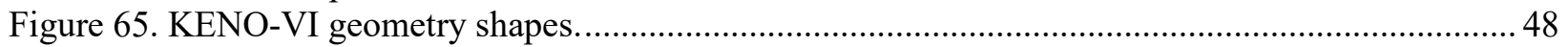

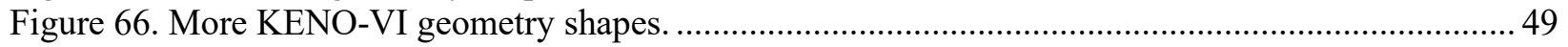

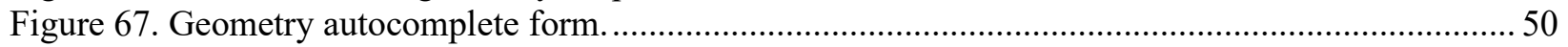

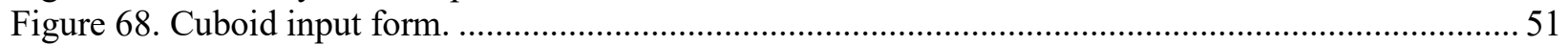

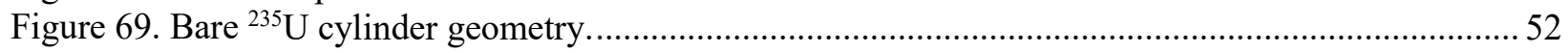

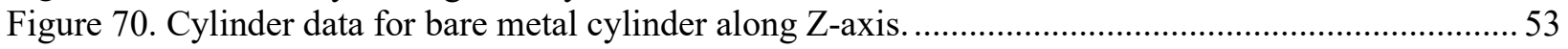

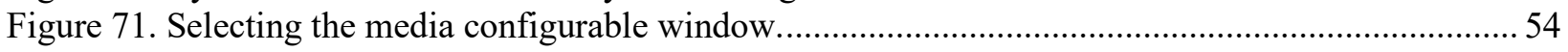

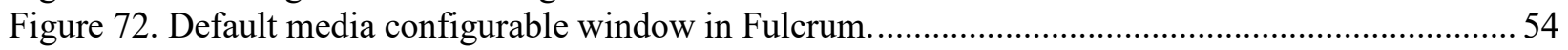

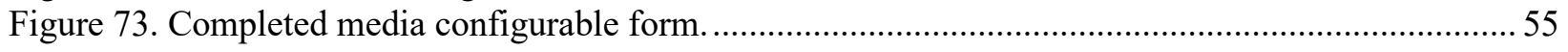

Figure 74. Input file for the bare metal cylinder example ................................................................... 55

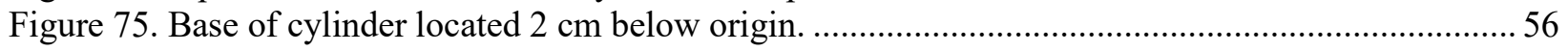

Figure 76. Cylinder data input with base located $2 \mathrm{~cm}$ below origin....................................................5 57

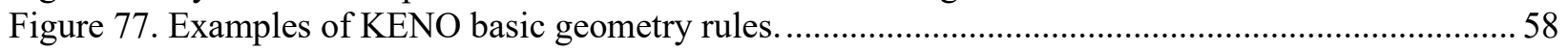

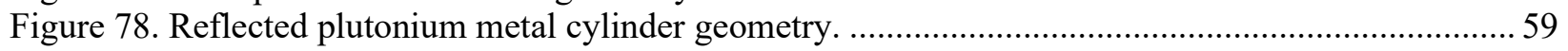

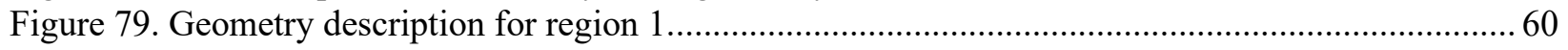

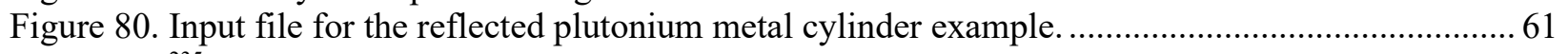

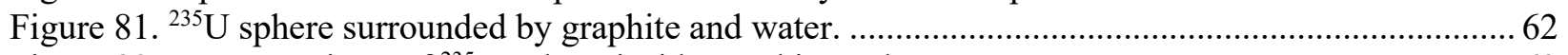

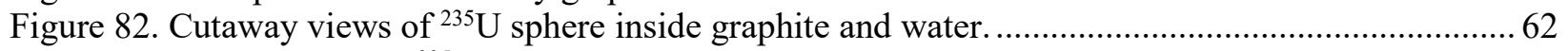

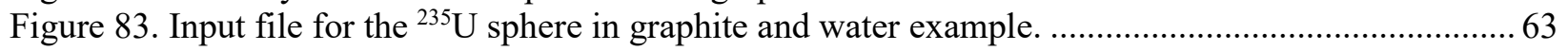

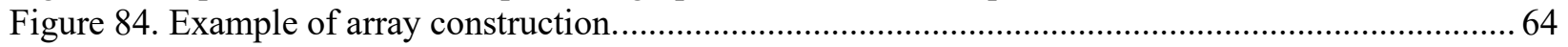

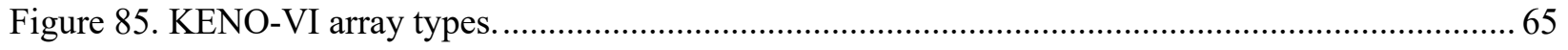

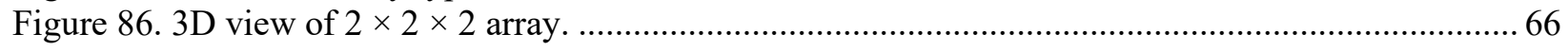

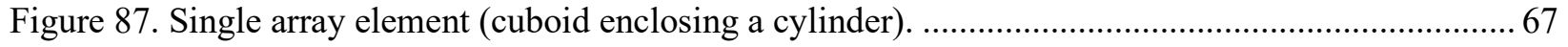

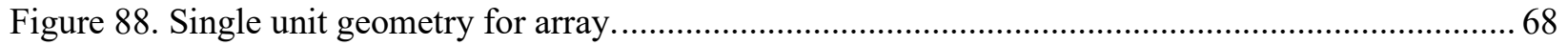

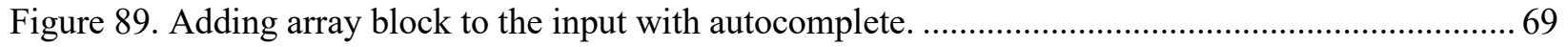

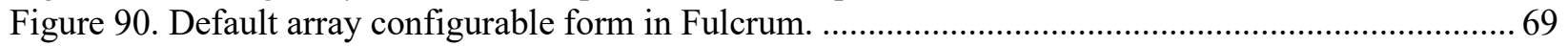

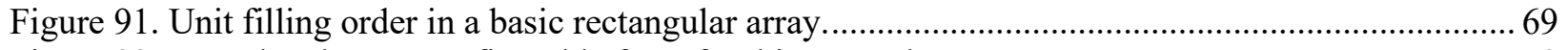

Figure 92. Completed array configurable form for this example.......................................................... 70

Figure 93. Completed array block for this example........................................................................ 70

Figure 94. Autocomplete menu for array contents record. ................................................................ 71

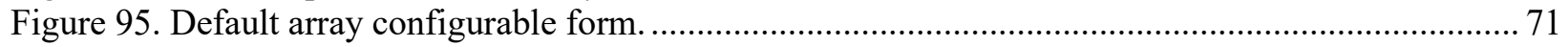


Figure 96. Completed array contents record configurable form....................................................... 72

Figure 97. Geometry form with array record to place array in geometry. ............................................... 72

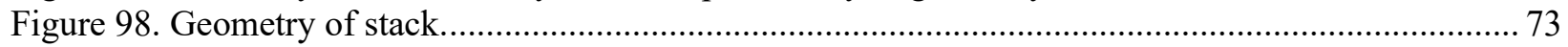

Figure 99. Composition and geometry blocks prior to array definition................................................. 74

Figure 100. Fulcrum configurable form for the array in this example.................................................. 75

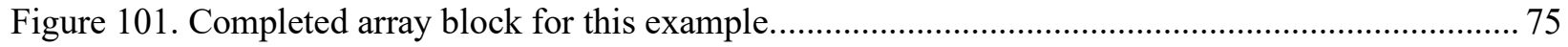

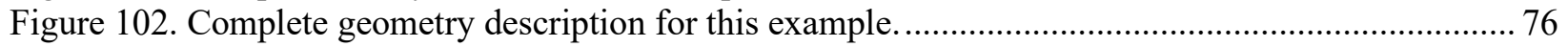

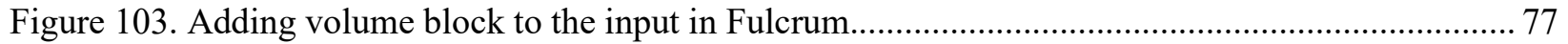

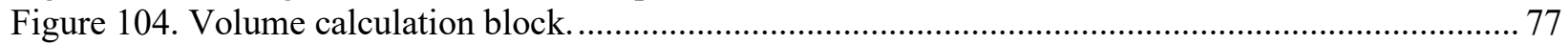

Figure 105. Examples of body truncation using the CHORD keyword................................................... 79

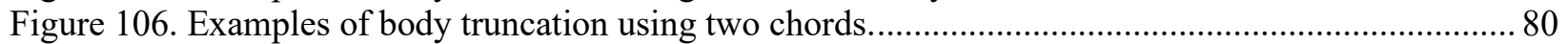

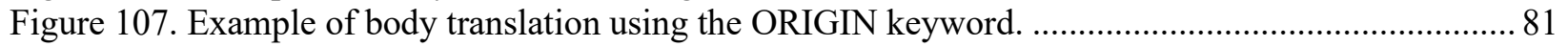

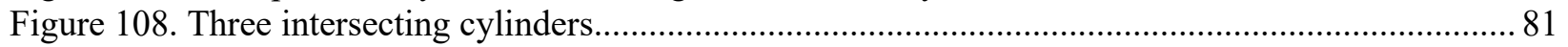

Figure 109. Cylinder configurable form with ORIGIN specification data............................................ 82

Figure 110. Input for three cylinders with ORIGIN data specified. ............................................... 83

Figure 111. Complete media record configurable form for the region inside all three cylinders..............83

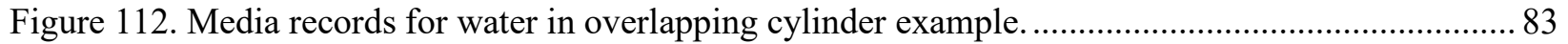

Figure 113. Media records for stainless steel in overlapping cylinder example.................................... 84

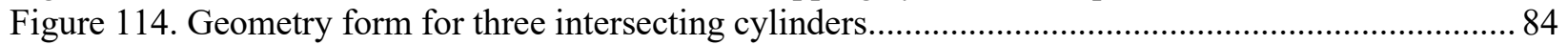

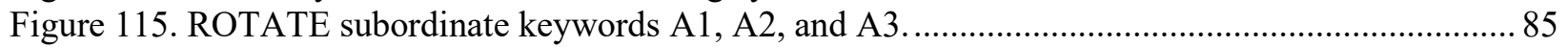

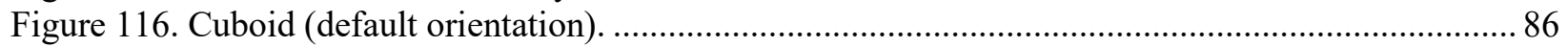

Figure 117. Cuboid rotated 45 degrees counterclockwise around $\mathrm{Z}$-axis (rotate $\mathrm{A} 1=45$ ) ......................86

Figure 118. Cuboid rotated 45 degrees counterclockwise around $X$-axis (rotate $A 2=45$ )...................... 87

Figure 119. Cuboid rotated 45 degrees counterclockwise around $Y$-axis (rotate $A 1=90$ A2 $=45$ )............ 87

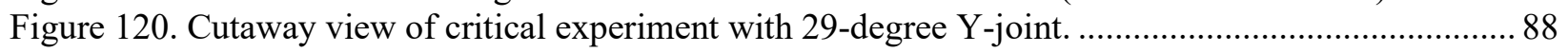

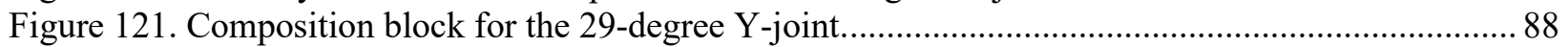

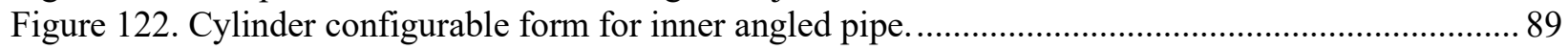

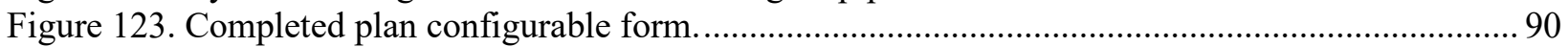

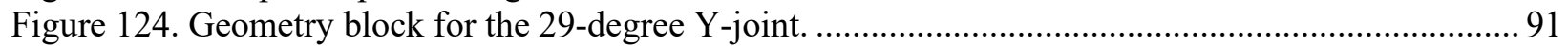

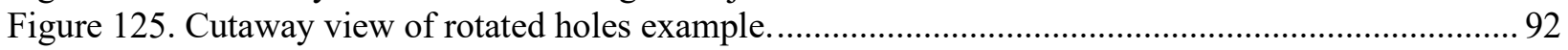

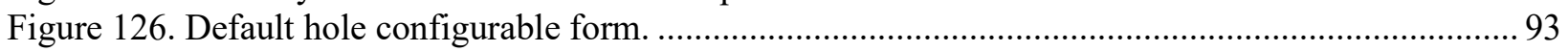

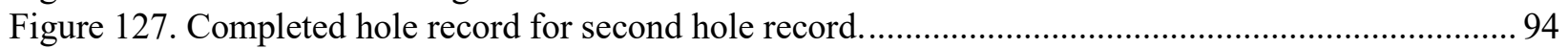

Figure 128. Geometry input for rotated holes example. ........................................................................ 95

Figure 129. SS316 cylinder with U(93.2)C cruciform element. ................................................... 97

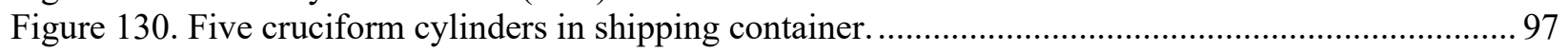

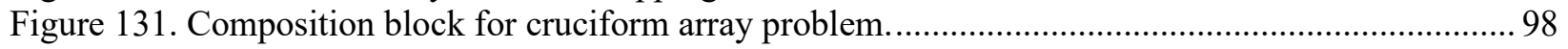

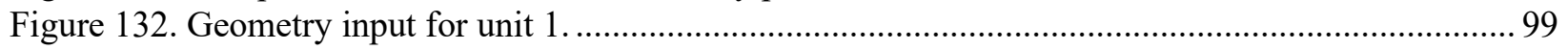

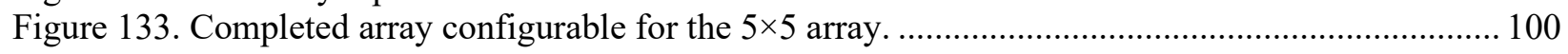

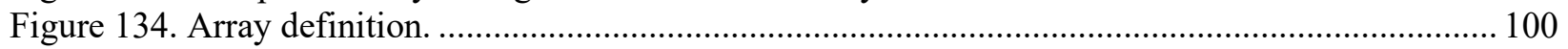

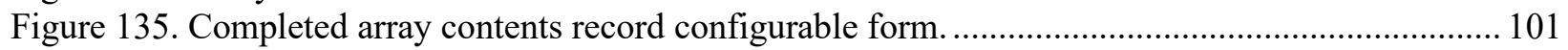

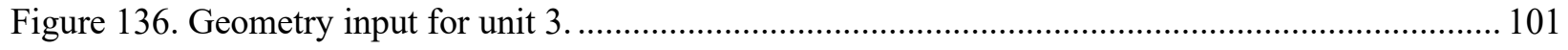

Figure 137. Annular carbon steel cylindrical tank with aluminum-clad uranium cylinders.................... 102

Figure 138. Cutaway view of tank with highlighted edges................................................................. 102

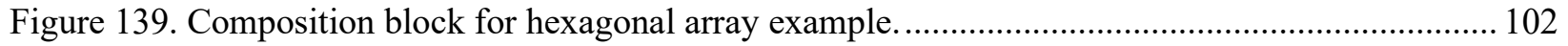

Figure 140. Input for units 1 and 2 for hexagonal array example....................................................... 103

Figure 141. Completed array configurable form for the hexagonal array example............................... 104

Figure 142. Fill statement with additional spacing to help visualize final array geometry. .................... 104

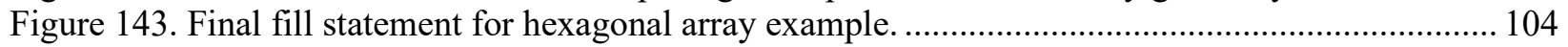

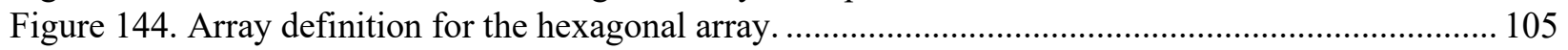

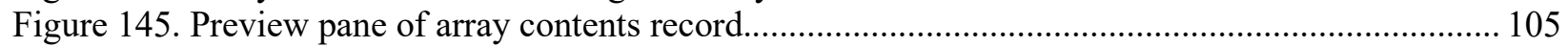

Figure 146. Complete array contents configurable form for the hexagonal array example..................... 106 


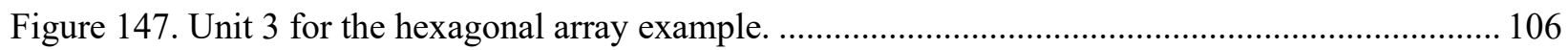

Figure 148. Dropdown menu selection of atom compound configurable............................................. 107

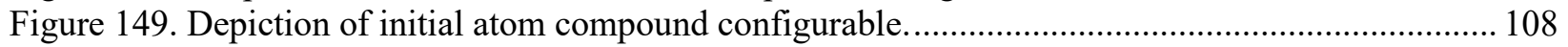

Figure 150. Depiction of element addition portion of the compound configurable ................................. 108

Figure 151. Second depiction of element addition portion of the compound configurable...................... 109

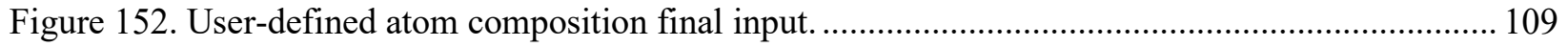

Figure 153. Dropdown menu selection of wtpt compound configurable.............................................. 110

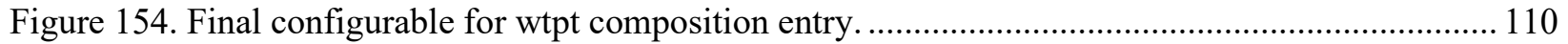

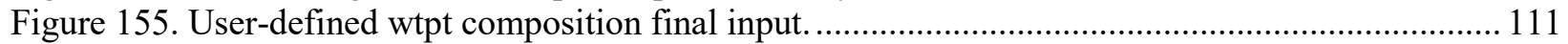

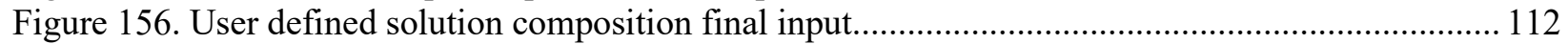

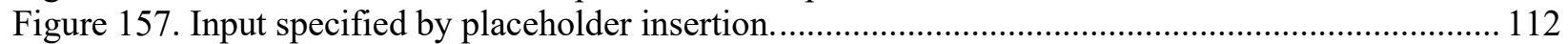

(normally placed on one line by Fulcrum but shown on two lines for readability) ................................ 112

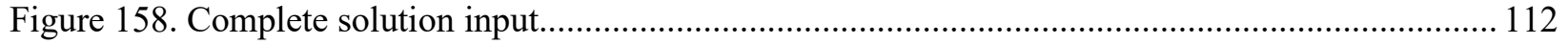

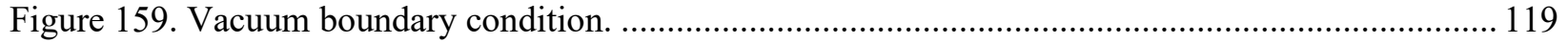

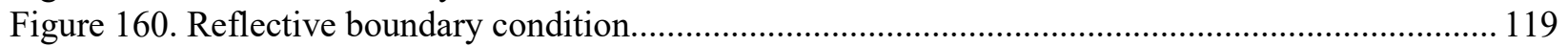

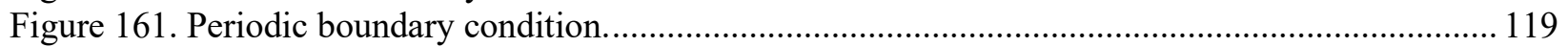

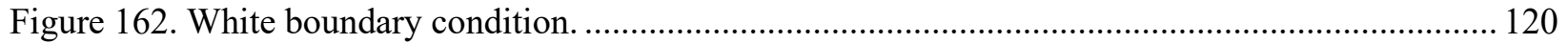

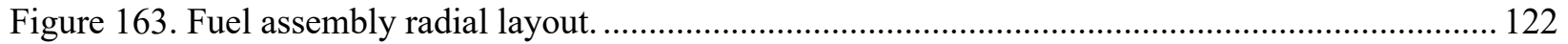

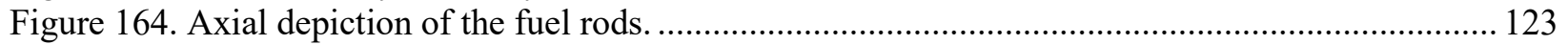

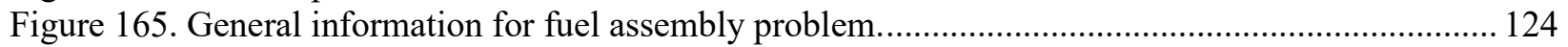

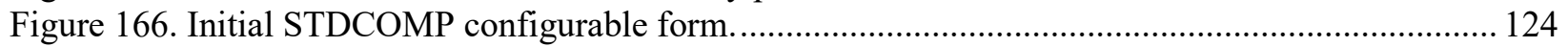

Figure 167. Complete $\mathrm{UO}_{2}$ configurable form, showing an enlarged section of the user-specified

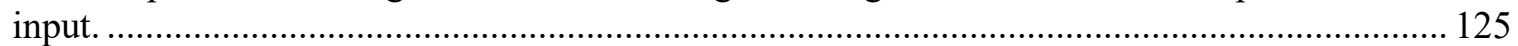

Figure 168. User-specified input portion of the complete zirc2 configurable form............................. 125

Figure 169. Complete COMPOSITION block for the fuel assembly problem..................................... 126

Figure 170. Autocomplete list of additional blocks available for inclusion in the model. ..................... 126

Figure 171. Autocomplete list of cells available within the CELLDATA block.................................... 127

Figure 172. Templated input for LATTICECELL SQUAREPITCH provided by Fulcrum.................... 127

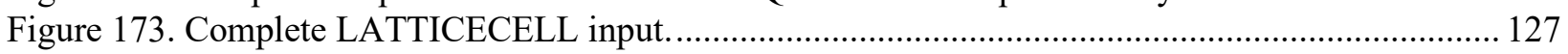

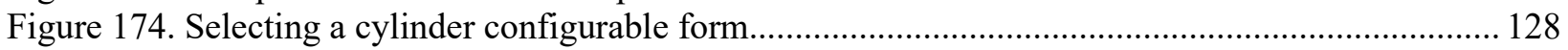

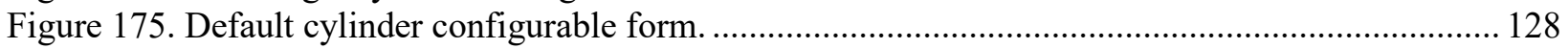

Figure 176. User-specified portion of the completed cylinder configurable form................................. 129

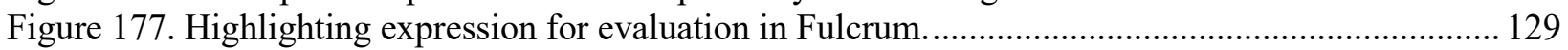

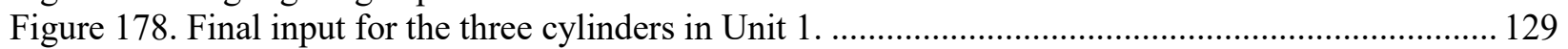

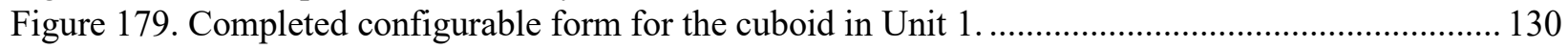

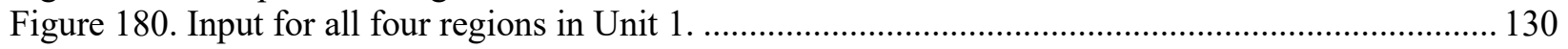

Figure 181. Selecting the MEDIA configurable form in Fulcrum..................................................... 131

Figure 182. Completed MEDIA configurable form placing $\mathrm{UO}_{2}$ in cylinder 10 in Unit $1 \ldots \ldots \ldots \ldots \ldots \ldots \ldots . . . . . . .131$

Figure 183. Completed MEDIA configurable form placing void in cylinder 20 and outside

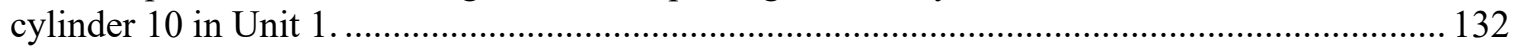

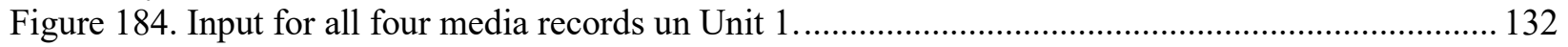

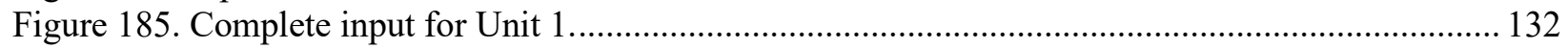

Figure 186. Completed configurable form in Fulcrum for first cylinder in Unit 2 .............................. 133

Figure 187. User-specified input in configurable form for second cylinder un Unit 2 .......................... 133

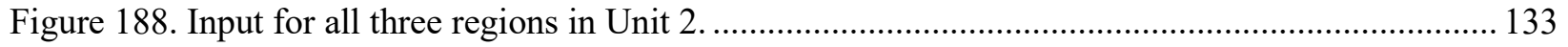

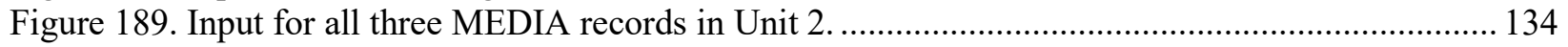

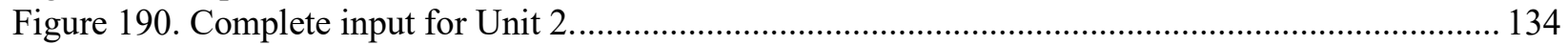

Figure 191. Autocomplete list of available blocks to add to the input file. ......................................... 134

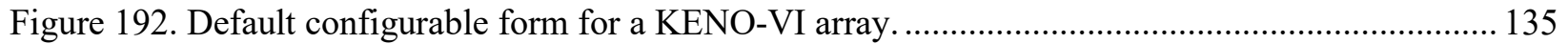

Figure 193. Complete configurable form for the array in the model. ................................................ 136

Figure 194. Complete array specification with guide tube units in FILL block.................................... 136 


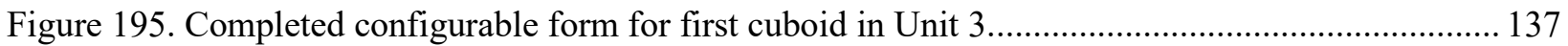

Figure 196. Completed configurable form for second cuboid in Unit 3 ............................................. 138

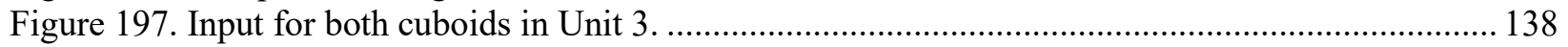

Figure 198. Selecting an ARRAY configurable form in the autocomplete menu in Fulcrum................. 139

Figure 199. Completed configurable form for the array 1 CONTENTS record..................................... 139

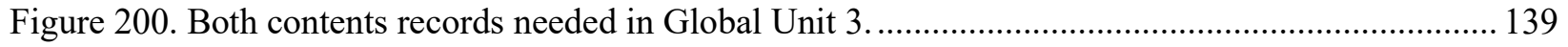

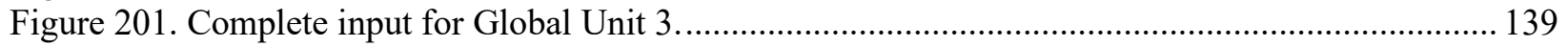

Figure 202. Radial slice of the completed fuel assembly at the model midplane.................................. 140

Figure 203. 3D rendering of the two tanks, with the top half of the model removed to show the

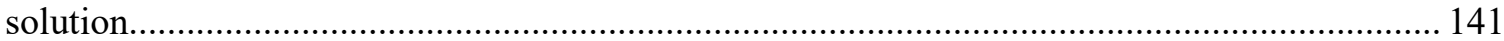

Figure 204. First three lines of the slab tanks model. ...................................................................... 141

Figure 205. Initial template text provided by Fulcrum for a fissile solution. .......................................... 141

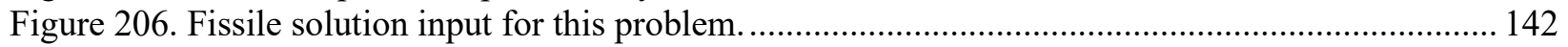

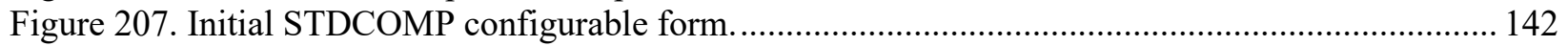

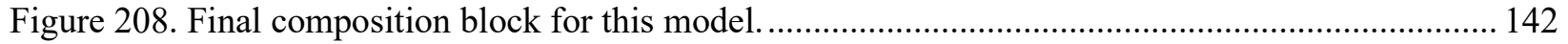

Figure 209. Autocomplete list of available blocks for inclusion in the model. .................................... 143

Figure 210. Autocomplete list of cells within the CELLDATA block................................................ 143

Figure 211. Templated input provided for MULTIREGION SLAB cell by Fulcrum............................ 143

Figure 212. Final CELLDATA block showing the correct input for this model................................... 144

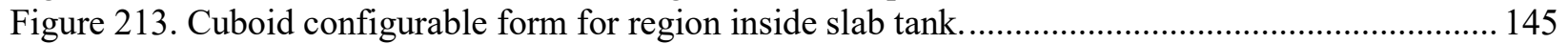

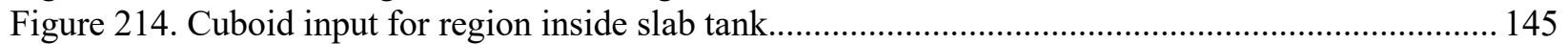

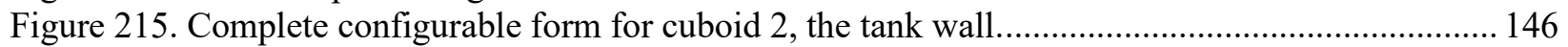

Figure 216. Complete configurable form for cuboid 3, the water reflector............................................ 146

Figure 217. All three cuboids, the only three geometry records needed for this model. ...................... 147

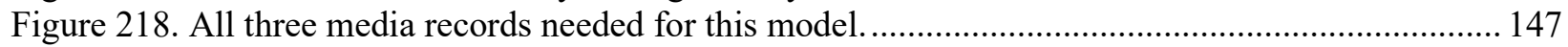

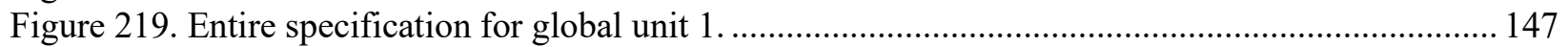

Figure 220. Adding the BOUNDS block from the Fulcrum autocomplete list..................................... 148

Figure 221. Boundary condition specification to apply a reflective boundary condition to the $-\mathrm{X}$

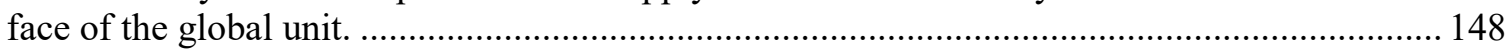

Figure 222. Fulcrum 2D visualization of the axial midplane of the model. ........................................ 148

Figure 223. Detailed view of the $+Y$ end of the axial midplane of the model, showing the end of

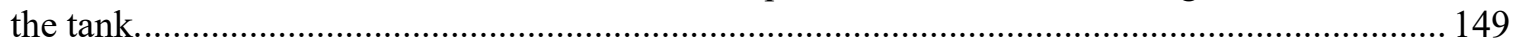

Figure 224. Schematic of individual grain (right) and pebble (left) .................................................... 150

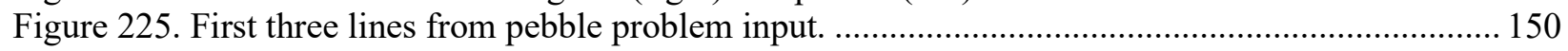

Figure 226. Configurable form for $\mathrm{UO}_{2}$ composition.................................................................. 151

Figure 227. Configurable form for the porous carbon layer composition. ........................................... 152

Figure 228. Input with $\mathrm{UO}_{2}$, porous carbon layer, and inner pyrolytic carbon layer compositions.......... 152

Figure 229. Selecting ATOM mixture configurable form in Fulcrum................................................ 152

Figure 230. ATOM mixture configurable form showing all necessary buttons and fields..................... 153

Figure 231. Complete ATOM composition configurable form for the SiC layer composition................ 153

Figure 232. Mixture definitions for all composition in the fuel grains................................................ 153

Figure 233. Composition definitions for all mixtures in the pebble................................................... 154

Figure 234. Complete configurable form for helium coolant mixture ................................................. 154

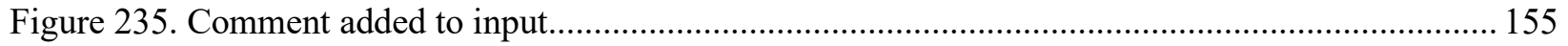

Figure 236. Entire COMPOSITION block for the pebble model........................................................ 155

Figure 237. Autocomplete list of available blocks for inclusion in the model ...................................... 156

Figure 238.Autocomplete list of cells within the CELLDATA block................................................ 156

Figure 239. Templated input provided for the DOUBLET PEBBLE + SPHSQUAREP cell in

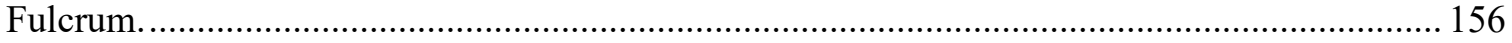

Figure 240. Grain and coating details for the DOUBLEHET unit cell for this model. .......................... 157

Figure 241. Complete DOUBLEHET unit cell specification for this model.......................................... 157 
Figure 242. Selecting the sphere configurable form in Fulcrum. ..................................................... 158

Figure 243. Complete configurable form for the fuel/matrix region of the pebble.............................. 158

Figure 244. All three geometry records for the pebble model. ..................................................... 158

Figure 245. Complete geometry block for the pebble model................................................................ 159

Figure 246. Adding the BOUNDS block to the model in Fulcrum with autocomplete........................... 159

Figure 247. Complete BOUNDS block for the pebble model. ........................................................... 159

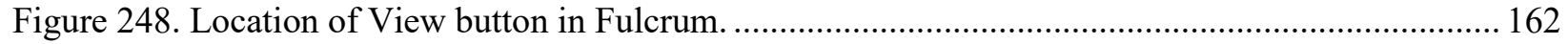

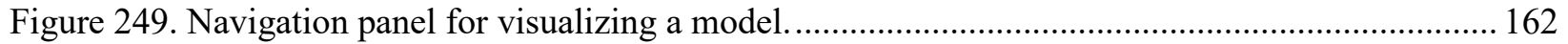

Figure 250. Default plot view of the fuel assembly model in the Fulcrum plotter................................ 162

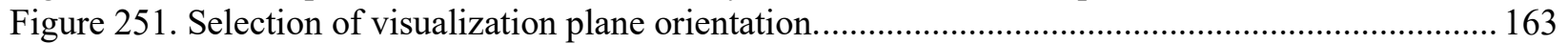

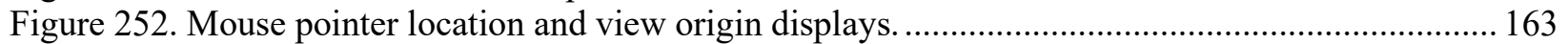

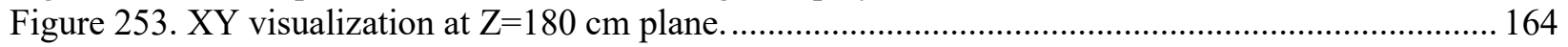

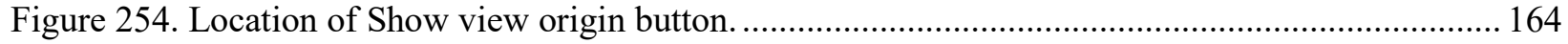

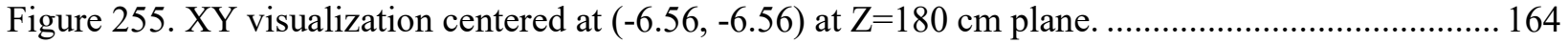

Figure 256. Location of slider to control position of the visualization plane. ........................................ 165

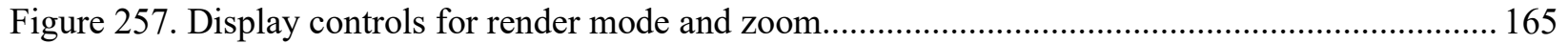

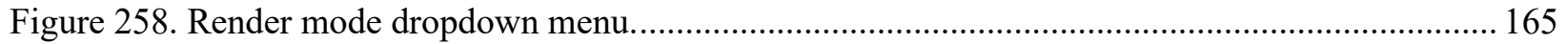

Figure 259. Midplane view of fuel assembly in Material + outline render mode.................................. 166

Figure 260. Midplane view of fuel assembly in Outline render mode................................................ 166

Figure 261. Fulcrum visualization while creating zoom control box. ............................................. 167

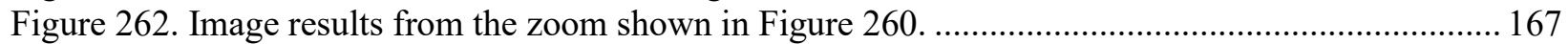

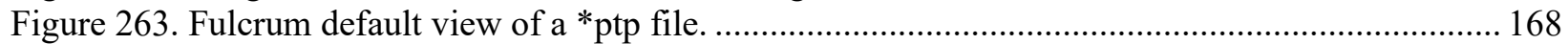

Figure 264. Default view of Shannon entropy by generation plot........................................................ 168

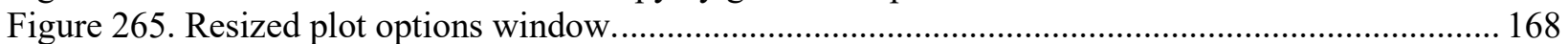

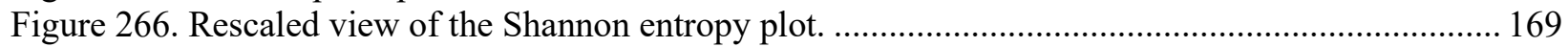

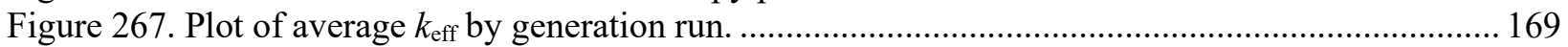

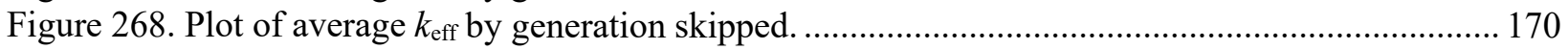

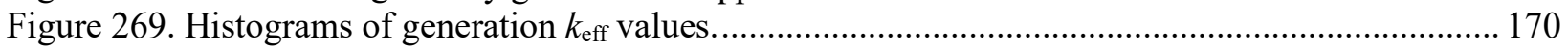

Figure 270. Histogram of generation $k_{\text {eff }}$ values for the last quarter of active generations...................... 171

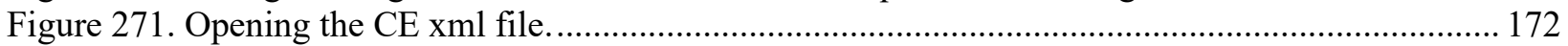

Figure 272. Default Navigation panel after opening a CE library..................................................... 172

Figure 273. Expanded ${ }^{238} \mathrm{U}$ entry showing temperatures at which data are available............................ 172

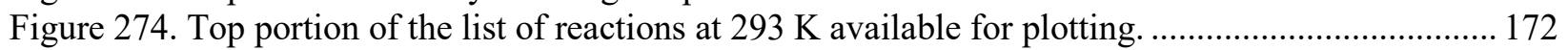

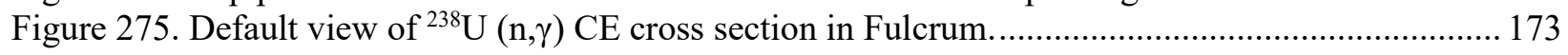

Figure 276. MG library loaded into the Navigation panel below the CE library................................... 173

Figure 277. MG library in the Navigation panel with ${ }^{238} \mathrm{U}$ expanded.................................................. 173

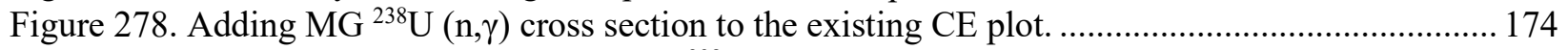

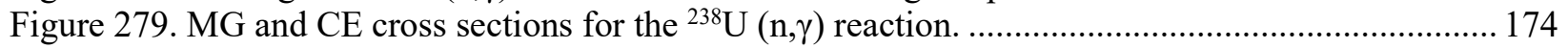

Figure 280. Covariance library loaded into the Navigation panel below the MG library....................... 175

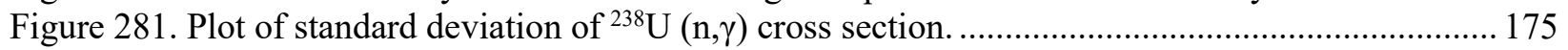

Figure 282. Table of values for standard deviation of ${ }^{238} \mathrm{U}(\mathrm{n}, \gamma)$ cross section..................................... 175

Figure 283. Uncertainty plot with repositioned legend..................................................................... 176

Figure 284. Positioning the uncertainty plot in the upper half of the view window............................... 176

Figure 285. ${ }^{238} \mathrm{U}(\mathrm{n}, \gamma)$ uncertainty and cross sections in a single view in Fulcrum................................ 176

Figure 286. Updated parameter block with $\mathrm{cds}=\mathrm{yes}$ included to enable the neutron production

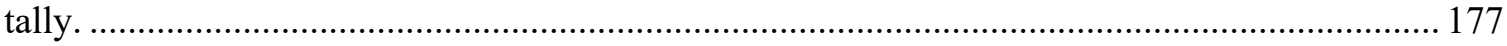

Figure 287. Adding GRID block to specify mesh for the neutron production tally. ............................. 177

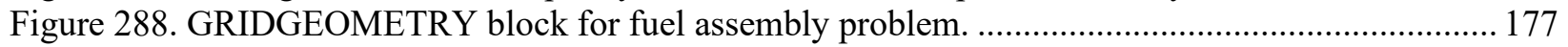

Figure 289. Location of the Meshes button in the Fulcrum visualization window................................. 178

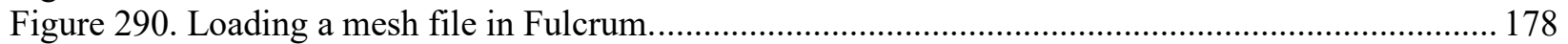

Figure 291. Loaded and expanded 3dmap file for the fuel assembly model. ......................................... 178 
Figure 292. Default view of neutron production rate at the fuel assembly midplane............................ 179

Figure 293. Fuel assembly midplane with colors rescaled to linear bins.............................................. 179

Figure 294. Midplane neutron production rate with mesh boundaries displayed. .................................. 180

Figure 295. Selecting relative uncertainties as the overlay quantity to view........................................ 180

Figure 296. Relative uncertainties for the neutron production rate at the fuel assembly midplane.......... 180

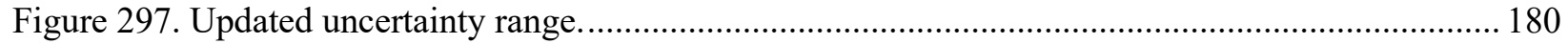

Figure 298. Overlay plot of uncertainties with custom value range................................................. 181

Figure 299. Axial view of the fuel assembly neutron production rate distribution rotated such that

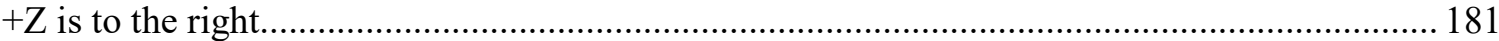

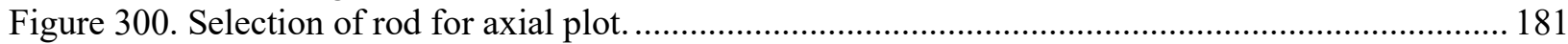

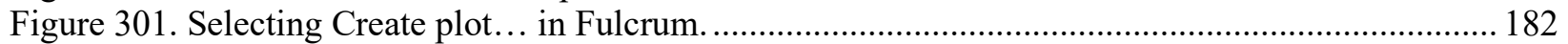

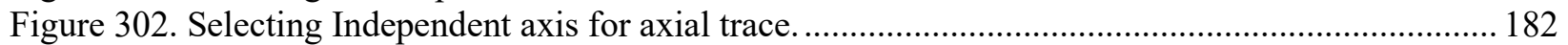

Figure 303. Plot figure generated by Fulcrum and displayed in the Navigation panel.......................... 182

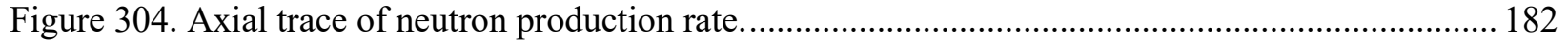

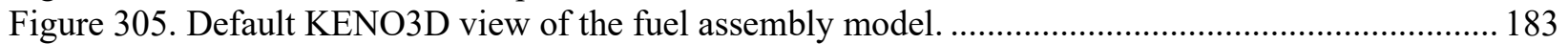

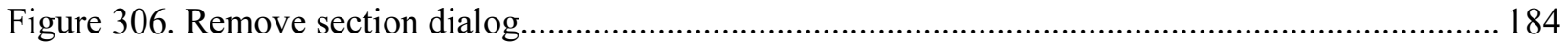

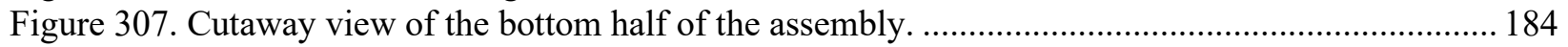

Figure 308. Cutaway view of the bottom half of the assembly with water removed.............................. 185

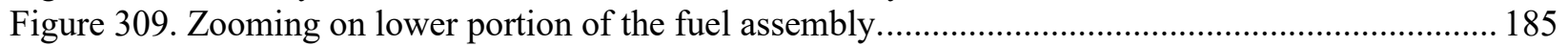

Figure 310 . Zoomed view of cutaway view of the bottom of the fuel assembly with water

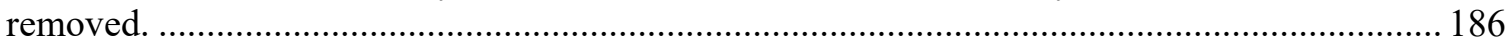

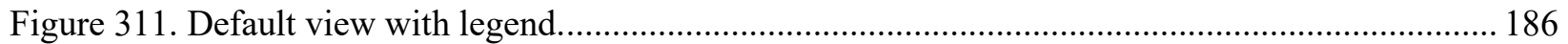

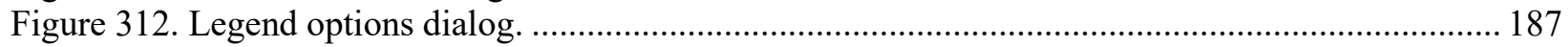

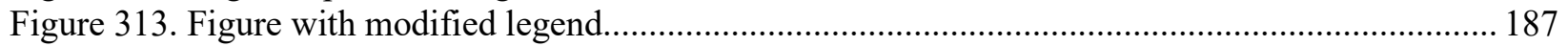

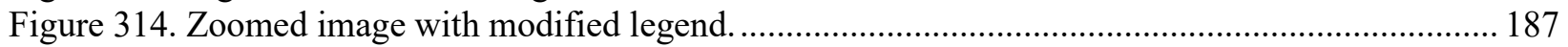

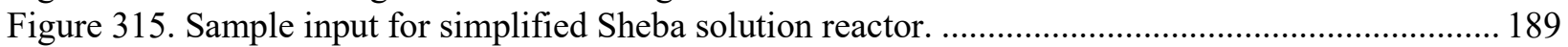

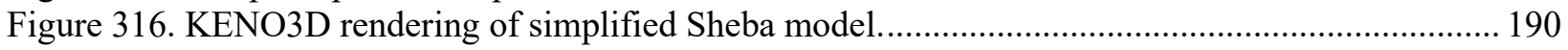

Figure 317. Updated PARAMETER block for KMART calculations................................................ 190

Figure 318. Adding the KMART sequence input after the CSAS sequence input................................ 191

Figure 319. Skeleton KMART input provided by Fulcrum autocomplete. ........................................... 191

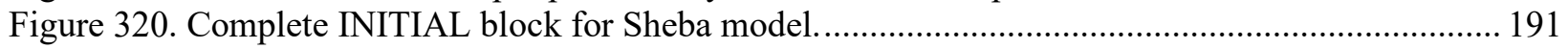

Figure 321. Adding ACTIVITY block to the KMART input............................................................ 192

Figure 322. Complete ATIVITY block for the Sheba model. ................................................................. 192

Figure 323. Complete COLLAPSE block for the Sheba model. ........................................................ 193

Figure 324. Plot Results dialog in KENO3D for selecting KMART results to display.......................... 193

Figure 325. KENO3D rendering of the Sheba model with fission reaction rates................................... 193

Figure 326. KENO3D rendering of the Sheba model with fission reaction rates and color scale............ 194

Figure 327. Detailed fission neutron generation rate $(\mathrm{MT}=1452)$ in Sheba model. ............................... 194 



\section{LIST OF TABLES}

Table 1. SCALE cross section libraries for criticality safety calculations .............................................2 28

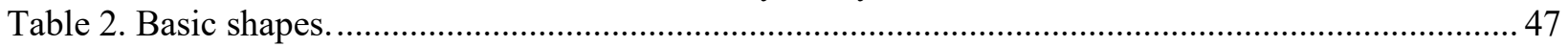

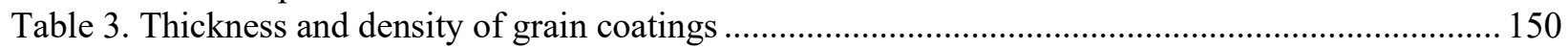

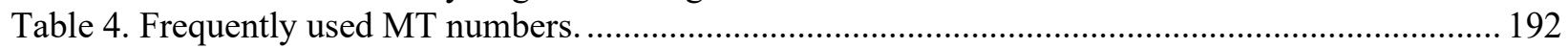





\section{ACRONYMS AND ABBREVIATIONS}

1D

2D

3D

AEC

CE

CSAS

FCM

GUI

HEU

ICSBEP

IEU

INFHOMMEDIUM

$k_{\text {eff }}$

KMART6

LEU

MG

$\mathrm{MoC}$

NEA

NRC

NPG

NSK

OECD

ORNL

OS

PBMR

PMC

SGGP

TRISO

TSL

V7-238GROUP one-dimensional

two-dimensional

three-dimensional

US Atomic Energy Commission

continuous energy

Criticality Safety Analysis Sequence

fully ceramic microencapsulated

graphical user interface

highly enriched uranium

International Criticality Safety Benchmark Experiments Project

intermediate-enriched uranium

infinite homogeneous medium

effective multiplication factor

KENO6 Module for Activity-Reaction Rate Tabulation

low-enriched uranium

multigroup

method of characteristics

Nuclear Energy Agency

US Nuclear Regulatory Commission

neutrons per generation

number of generations skipped

Organization for Economic Co-operation and Development

Oak Ridge National Laboratory

operating system

pebble bed modular reactor

produce multigroup cross sections

SCALE Generalized Geometry Package

tristructural isotropic

thermal scattering law

ENDF/B-VII 238-group library 



\begin{abstract}
The SCALE code system developed at Oak Ridge National Laboratory is widely used and accepted around the world for criticality safety analysis. The well-known KENO-VI three-dimensional Monte Carlo criticality computer code is one of the primary criticality safety analysis tools in SCALE. The KENO-VI primer is designed to help a new user understand and use the SCALE/KENO-VI Monte Carlo code for nuclear criticality safety analysis. It assumes that the user has a college education in a technical field. There is no assumption of familiarity with Monte Carlo codes in general or with SCALE/KENO-VI in particular. The primer is designed to teach by example, with each example illustrating two or three features of SCALE/KENO-VI that are useful in criticality analysis.

The primer is based on SCALE 6.2 and 6.3, which includes the Fulcrum graphical user interface. Each example uses Fulcrum to provide the framework for preparing input data and viewing output results. Starting with a Quickstart section, the primer gives an overview of the basic requirements for SCALE/KENO-VI input and allows the user to quickly run a simple criticality problem with SCALE/KENO-VI. Each following section begins with a list of basic objectives identifying the goal of the section and the individual SCALE/KENO-VI features covered in detail in the section's sample problems. Upon completion of the primer, a new user should be comfortable using Fulcrum to set up criticality problems in SCALE/KENO-VI.

The primer provides a starting point for the criticality safety analyst who uses SCALE/KENO-VI. Complete descriptions are provided in the SCALE/KENO-VI manual. Although the primer is selfcontained, it is intended as a companion volume to the SCALE/KENO-VI training and documentation. The SCALE manual and training schedule are available at https://scale.ornl.gov. The primer provides specific examples of using SCALE/KENO-VI for criticality analysis; the SCALE/KENO-VI manual provides information on the use of SCALE/KENO-VI and all its modules. The primer also contains an appendix with sample input files. In addition, this primer, its errata, and sample inputs are also available at https://code.ornl.gov/scale/primers/kenovi/.
\end{abstract}





\section{INTRODUCTION AND PURPOSE}

\subsection{INTENDED AUDIENCE AND AVAILABLE RESOURCES}

This primer is designed to help the user understand and use the KENO-VI three-dimensional (3D) Monte Carlo code for nuclear criticality safety analysis. KENO-VI is part of the SCALE code system [1]. It assumes that the user has a college education in a technical field. There is no assumption of familiarity with Monte Carlo codes in general or with SCALE and KENO-VI in particular. The primer is designed to teach by example, with each example illustrating two or three features of KENO-VI that are useful in criticality safety analysis. The theory and implementation details of KENO-VI are discussed in Goluoglu et al. [2].

The primer is based on SCALE 6.2 and 6.3, which includes the Fulcrum graphical user interface (GUI), a cross-platform program (available on Windows, Macintosh, and Linux) to assist criticality safety analysts in creating and executing SCALE input files for KENO-VI. Each example uses Fulcrum to provide the framework for data input. Starting with a Quickstart section, the primer gives an overview of the basic requirements for SCALE/KENO-VI input and allows the user to quickly run a simple criticality problem with SCALE/KENO-VI. This section is not designed to explain Fulcrum, the input, or the SCALE/KENO-VI options in detail; rather, it introduces the Fulcrum user interface and some basic concepts that are further explained in following sections. Each following section begins with a list of basic objectives identifying the goal of the section and the individual SCALE/KENO-VI features covered in detail in the section's example problems. It is expected that on completion of the primer, the user will be comfortable using Fulcrum to set up criticality problems in SCALE/KENO-VI. The user will be able to use SCALE/KENO-VI in criticality calculations and will be capable of handling most situations that normally arise in a facility. The primer provides a set of basic input files that can be selectively modified by the user to fit the particular problem at hand.

Although much of the information to perform an analysis is provided in the primer, there is no substitute for understanding the problem and the theory of neutron interactions. The SCALE/KENO-VI code is only capable of analyzing the problem as it is specified: it does not necessarily identify inaccurate modeling of the geometry; nor does it know when the wrong material has been specified. However, the 2D and 3D visualization capabilities of Fulcrum are quite useful for identifying geometry errors. Remember that a single calculation of $k_{\text {eff }}$ and its associated confidence interval with SCALE/KENO-VI or any other code is meaningless without an understanding of the context of the problem, the quality of the solution, and a reasonable idea of what the result should be.

The primer provides a starting point for the criticality safety analyst using SCALE/KENO-VI. Complete descriptions are provided in the SCALE manual [1]. Although the primer is self-contained, it is intended to serve as a companion to the SCALE training courses and manual. The SCALE manual and training schedule is available at https://www.ornl.gov/scale. The primer provides specific examples using SCALE/KENO-VI for criticality safety analyses, whereas the documentation provides information on the use of SCALE and all its modules. The primer also contains appendices that give the user additional information about available cross section libraries in SCALE and example input files. This information is provided in appendices so as not to obscure the basic information illustrated in each example. It is also available at https://code.ornl.gov/scale/primers/kenovi/.

A standard set of notation has been provided to make the primer easy to use. The text is set in Times New Roman font. Information to be directly input is set in Courier font. Characters in the Courier font represent commands, keywords, or data that would be used as computer input. References to items displayed by Fulcrum are highlighted in bold font. Because the primer often references the SCALE 
manual, these references are set in square brackets; e.g., [see SCALE Manual Section Title]. To maximize the applicability of this primer across multiple SCALE releases, the manual section references refer to section titles and not section numbers.

It is hoped that users find the primer useful and easy to read. The user will benefit the most from this tutorial by starting with Section 2: SCALE/KENO-VI Quickstart and proceeding through the rest of the sections in order. Each section assumes that the user knows and is comfortable with the concepts discussed in the previous sections. Although it may be tempting to pick up the primer and immediately go to an example problem that is similar to an analysis requirement, this approach will not provide the user with the background or confidence in analysis that is necessary for accurate, effective implementation of procedures and limits. There is no substitute for a thorough understanding of the techniques used in a SCALE/KENO-VI analysis. A little extra time spent going through the primer and working through the examples will save many hours of possible confusion and frustration later.

\subsection{SCALE OVERVIEW}

The SCALE code system provides modern, robust calculations while reducing requirements for user input and knowledge of the intricacies of the underlying methods. SCALE provides standardized sequences to integrate many modern, advanced capabilities into a seamless calculation that the user controls from a single input file. SCALE's heritage dates back to 1969, when Oak Ridge National Laboratory (ORNL) began providing computational support in the use of the new KENO code to staff at the US Atomic Energy Commission, later the US Nuclear Regulatory Commission (NRC). The NRC has continued to instigate code and data improvements for criticality, shielding, reactor physics, and spent fuel characterization for various NRC applications. The NRC staff provided ORNL with the following general development criteria for SCALE:

1. focus on applications related to nuclear fuel facilities and package designs,

2. use well- established computer codes and data libraries,

3. design an input format for the occasional or novice user,

4. prepare "standard" analysis sequences (control modules) that will automate the use of multiple codes (functional modules) and data to perform a system analysis, and

5. provide complete documentation and public availability.

The concept of SCALE is to provide standardized sequences. Input for the control modules has been designed to be free-form, with extensive use of keywords and engineering-type input requirements. The most important feature of the SCALE system is the capability to simplify the user knowledge and effort required to prepare material mixtures and to perform adequate problem-dependent cross section processing.

Today, SCALE is a comprehensive modeling and simulation suite for nuclear safety analysis and design. SCALE is developed and maintained by ORNL under contract with the NRC, the US Department of Energy (DOE), and the National Nuclear Security Administration (NNSA) to perform reactor physics, criticality safety, radiation shielding, and spent fuel characterization for nuclear facilities and transportation/storage package designs. 


\subsection{CSAS OVERVIEW}

The Criticality Safety Analysis Sequence (CSAS) provides a consolidated, streamlined user input for material information and model geometry specification. The material information input allows users to specify problem materials using easily remembered and easily recognizable keywords that are associated with mixtures, elements, and nuclides provided in the Standard Composition Library. The material information also uses other keywords and simple geometry input specifications to prepare input for the cross section processing module, XSProc. The XSProc module performs problem-dependent cross section processing using the BONAMI, CENTRM, PMC, and XSDRNPM functional modules. A keyword supplied by the user selects the cross section library from a standard set provided in SCALE or designates the reference to a user-supplied library.

CSAS has been developed to automate and standardize various analytic sequences. The control module input format has been designed to help minimize input errors. Upon processing the user-specified input, the SCALE code system immediately prints an input checklist in which the user (or reviewer) can easily establish that the input describes the system to be analyzed. The CSAS control module is the primary criticality safety control module for the calculation of the neutron multiplication factor of a system. Multiple sequences within the CSAS module provide capabilities for a number of analyses, such as modeling a one-dimensional (1D) or 3D system and processing cross sections.

The XSProc module provides cross section temperature correction and resonance self-shielding, as well as energy group collapse and spatial homogenization using the BONAMI, CENTRM, PMC, and XSDRNPM functional modules.

BONAMI performs resonance shielding through the application of the Bondarenko shielding factor method. BONAMI is typically used to process data in the unresolved resonance energy range. As input, BONAMI requires the presence of shielding factor data on the AMPX master library interface.

CENTRM computes continuous-energy (CE) neutron spectra using 1D discrete ordinates or infinite media geometry. CENTRM determines the problem-specific fluxes for processing resonance-shielded multigroup (MG) data by using a 1D or 2D unit cell and then using the spectrum as a problem-dependent weight function for MG averaging. The MG data processing is done by the PMC code, which reads the CENTRM CE flux spectra and cross section data and calculates problem-dependent, group-averaged cross sections over some specified energy range.

XSDRNPM is a 1D discrete ordinates transport code for performing neutron or coupled neutron-gamma calculations. The code has a variety of uses within SCALE. In the CSAS-XSProc sequence, it is used for preparation of cell-averaged cross sections for subsequent system analysis in KENO-VI and for 1D criticality safety analysis.

KENO-VI is a 3D MG Monte Carlo code employed to determine effective multiplication factors ( $k_{\text {eff }}$ ) for multidimensional systems. The basic geometrical bodies allowed for defining the model are cuboids, spheres, cylinders, hemispheres, and cuboidal arrays. The model may be viewed using the visualization capabilities in Fulcrum.

Although the control modules are typically designated by their principal analytic sequence, it should be noted that more than one sequence may exist within a control module. The sequences available in CSAS are discussed in Section 3.

SCALE includes another version of KENO, known as KENO V.a. Since the geometry package for KENO-VI is different from that for KENO V.a, a different control module, CSAS5, provides an 
automated criticality safety sequence using KENO V.a for performing 3D Monte Carlo analyses of simpler geometries, with no support for hexagonal lattices or nonorthogonal geometry. Its simplified geometry system makes it very easy to use and much more computationally efficient than other Monte Carlo criticality safety codes. The geometry models consist of units that are constructed by nesting partial or whole spheres, cylinders, and rectangular cuboids oriented along X-, Y-, and Z-axes. These units can be combined in rectangular arrays or inserted in other units. These restrictions allow for very fast particle tracking, but they still permit users to construct complex geometry models.

KENO-VI was introduced to provide SCALE users with a more general geometry system, known as the $S C A L E$ Generalized Geometry Package (SGGP), for constructing geometry models that are more complex. KENO-VI has a much larger assortment of bodies, including the following:

- cone
- cuboid
- dodecahedron
- elliptical cylinder
- ellipsoid
- hexprism
- papper
- pentlelepiped
- plane
- quadratic
- rotated hexprism
- sphemboid
- $\quad$ wedge, and
- paired planes (parallel to X-, Y-, or Z-axis).

In addition to rectangular arrays, KENO-VI provides the dodecahedral array type, which is a 3D stack of dodecahedra, as well as three hexagonal array types.

The added complexity of the KENO-VI generalized geometry results in longer running times than those required for KENO V.a. In general, a KENO V.a model that has been converted to KENO-VI will require approximately two times the amount of CPU time in KENO-VI as it did in KENO V.a.

The SCALE code system includes several problem-independent MG cross section libraries for criticality analyses. The recommended general-purpose MG library is the 252-group ENDF/B-VII.1 neutron library [SCALE Manual SCALE Nuclear Data Libraries]. A 56-group library is also available for use with thermal systems, but the reduced number of groups does not provide an appreciable speed-up and can lead to large biases, especially for fast-spectrum systems. The CE libraries, based on ENDF/B-VII.0 and ENDF/B-VII.1, are also general-purpose libraries, though KENO execution time is increased in CE mode.

The SCALE XSProc modules used for preparing problem-dependent cross section libraries and performing criticality safety analyses are well established and in routine use by much of the US and international criticality safety communities as the primary computational tool or as a backup/review tool. 


\section{SCALE/KENO-VI QUICKSTART}

\subsection{WHAT YOU WILL BE ABLE TO DO}

- Describe the structure of SCALE/KENO-VI input files.

- Use the Fulcrum user interface to create a SCALE/KENO-VI input file.

- Set up and run a simple criticality problem using SCALE/KENO-VI.

- Find and interpret $k_{\text {eff }}$ information from SCALE/KENO-VI output.

\subsection{SCALE/KENO-VI INPUT FILE}

The SCALE/KENO-VI input file describes the problem geometry, specifies the materials and the neutron source, and defines the control parameters for analyzing the problem. The geometry is constructed by defining objects and their relationship with other objects in a system. Each object can be filled with a material or a void.

A SCALE/KENO-VI input file consists of some or all of the above data, depending on the type of problem being analyzed and the amount and type of output desired. The most user-friendly method for entering the data is to use the Fulcrum user interface.

SCALE interprets an entire line as a comment if it starts with an apostrophe (') in column 1 . The apostrophe must be in column 1, no matter where the text begins on the line. Entirely blank lines are ignored as white space. In-line comments, that is comments starting after other input on a line, are not supported. Comments and blank lines do not impact input processing, though comment lines are printed in the text output file.

\subsection{EXAMPLE PROBLEM}

This section provides enough information to run a simple example problem so that the user can gain confidence in using Fulcrum to enter SCALE/KENO-VI input data right away. This section walks the user through the example problem step by step, explaining each input step. At this stage, it is important that the user enter the problem exactly as it is described. With more experience using Fulcrum and SCALE/KENO-VI, the user may find other more logical ways to set up input files. For example, the user may find alternate methods for the geometry setup.

\subsubsection{Problem Description}

This problem is a bare sphere of delta-phase plutonium metal (density of $15.61 \mathrm{~g} / \mathrm{cc}$ ) with a coating of nickel (also known as Jezebel). Experimental parameters are as follows:

- Delta-phase Pu metal sphere: radius

- Spherical nickel coating: thickness

- $\mathrm{N} 239=$ Atom density of ${ }^{239} \mathrm{Pu}$

- $\mathrm{N} 240=$ Atom density of ${ }^{240} \mathrm{Pu}$

- $\mathrm{N} 241=$ Atom density of ${ }^{241} \mathrm{Pu}$

- $\mathrm{NGa}=$ Atom density of Ga

- $\mathrm{NNi}=$ Atom density of $\mathrm{Ni}$

$$
\begin{aligned}
& =6.38493 \mathrm{~cm} \\
& =0.0127 \mathrm{~cm} \\
& =3.7047 \mathrm{E}-2 \text { atoms } / \mathrm{b}-\mathrm{cm} \\
& =1.751 \mathrm{E}-3 \text { atoms } / \mathrm{b}-\mathrm{cm} \\
& =1.17 \mathrm{E}-4 \text { atoms } / \mathrm{b}-\mathrm{cm} \\
& =1.375 \mathrm{E}-3 \text { atoms } / \mathrm{b}-\mathrm{cm} \\
& =9.1322 \mathrm{E}-2 \text { atoms } / \mathrm{b}-\mathrm{cm}
\end{aligned}
$$




\subsubsection{Fulcrum Interface - General Information}

Now you are ready to begin entering the example problem. First start the Fulcrum user interface. Background information and an overview of the interface design and function are provided in Rearden et al. [3]. You should see a screen that looks like Figure 1. Note that Fulcrum screen captures throughout this document are made from both Mac and Windows computers; associated minor differences in the operating system (OS) specific appearance of windows is neither unexpected nor made consistent. These differences are inherited from the OS and will vary with user OS as well.

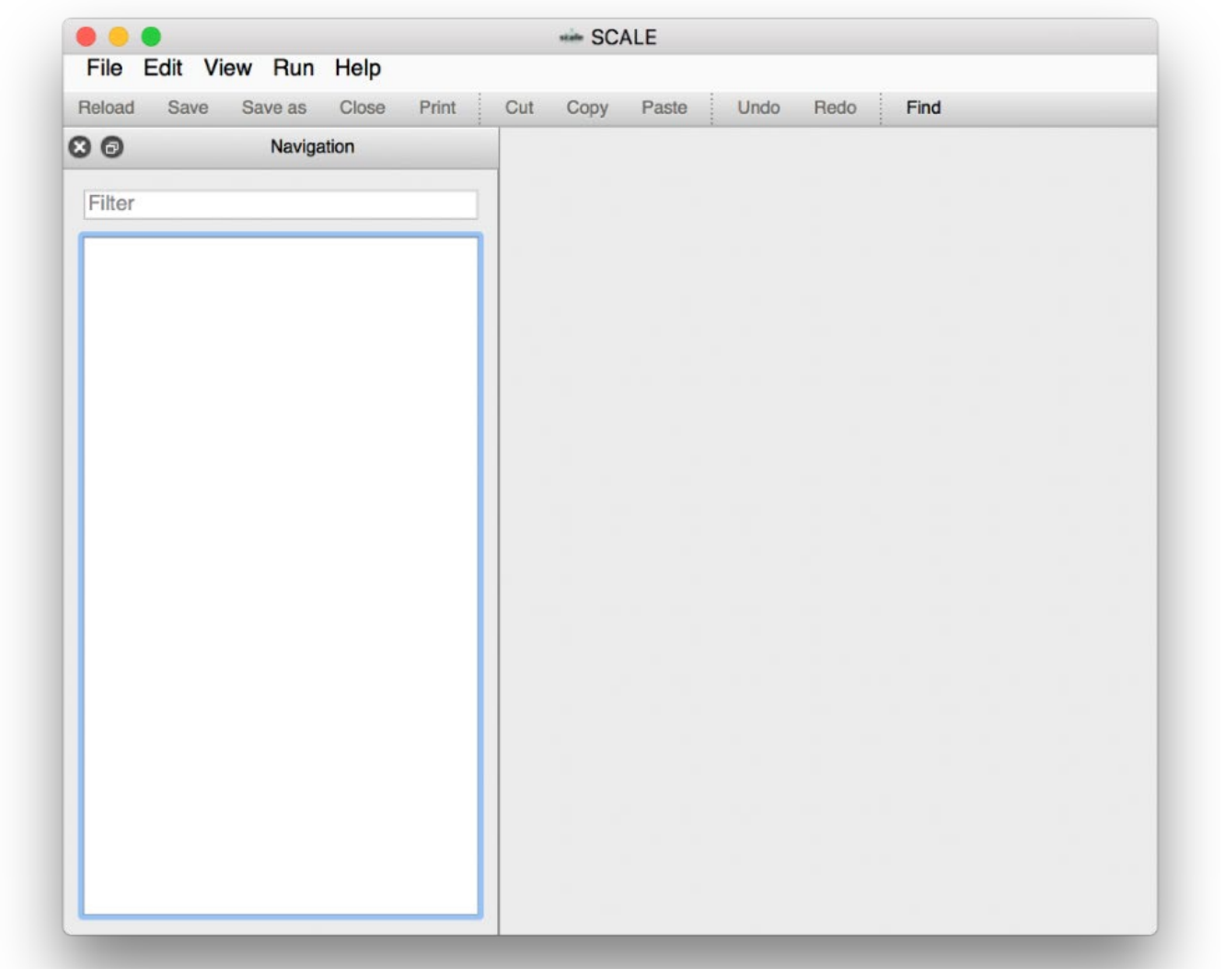

Figure 1. Fulcrum start screen.

Click the File $>$ New File... button on the upper left to display the New file dialog window. In this window, enter the name of your input file with a .inp input file extension. Enter the input file name as jezebel inp and click the Save button. A blank input document will be displayed as shown in Figure 2. 


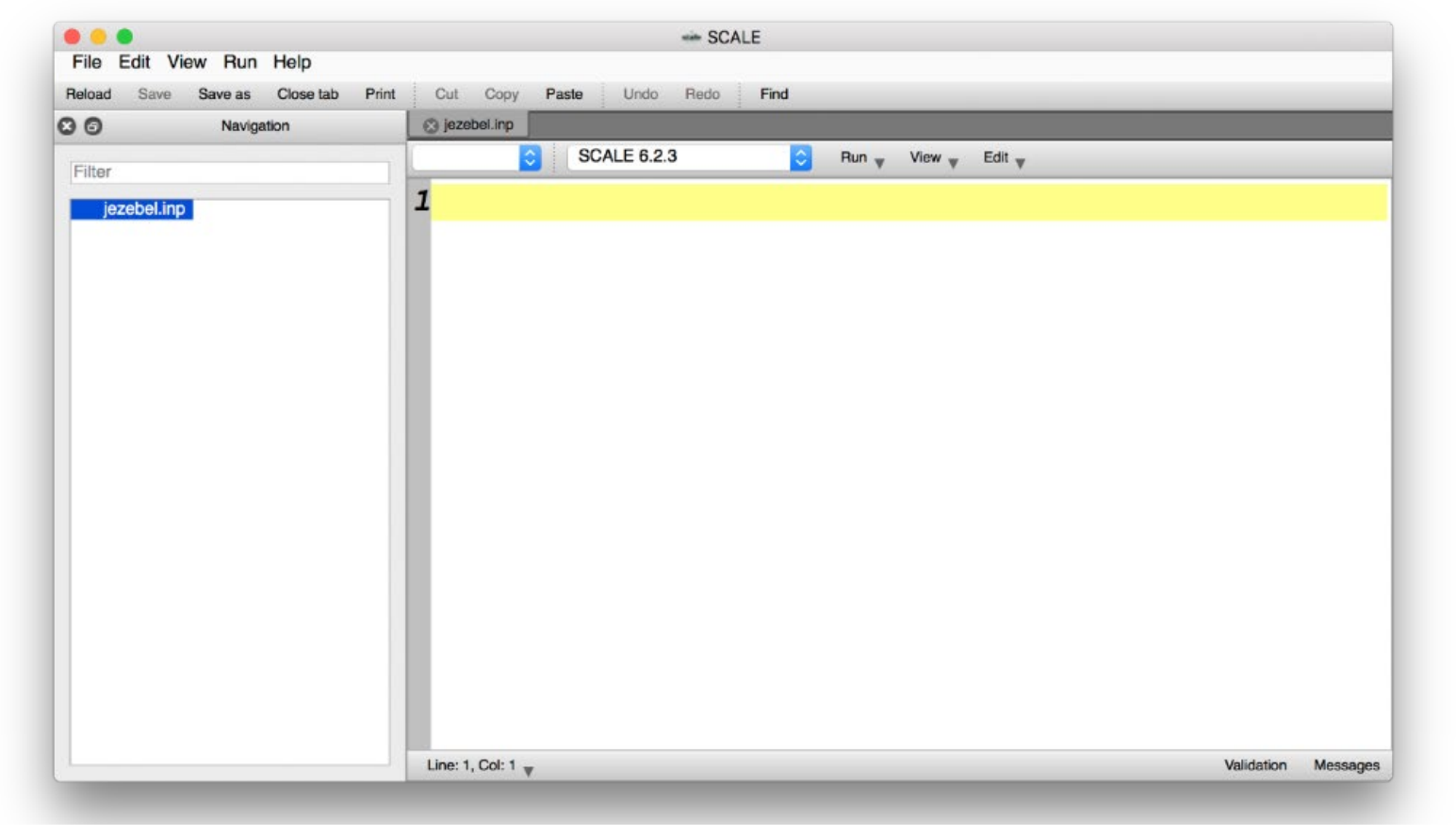

Figure 2. New blank jezebel input document.

The Fulcrum user interface is an advanced text editor with the primary capability of autocompleting input records. This capability is a design feature that allows Fulcrum to scale with the user's experience level, avoiding the inevitable impediments that GUI widgets can present to experienced users. The autocompletion feature is available whenever the cursor is inside the text editor panel, and it is accessible via the Edit > Autocomplete button or the Control-Space (CTRL-SPACE) keyboard combination.

Keep in mind that information presented here that is to be entered into Fulcrum will appear in the Courier font. With the cursor on line 1, press the CTRL-SPACE autocomplete key combination and select the csas6 - Criticality safety analysis using KENO-VI option, as shown in Figure 3.

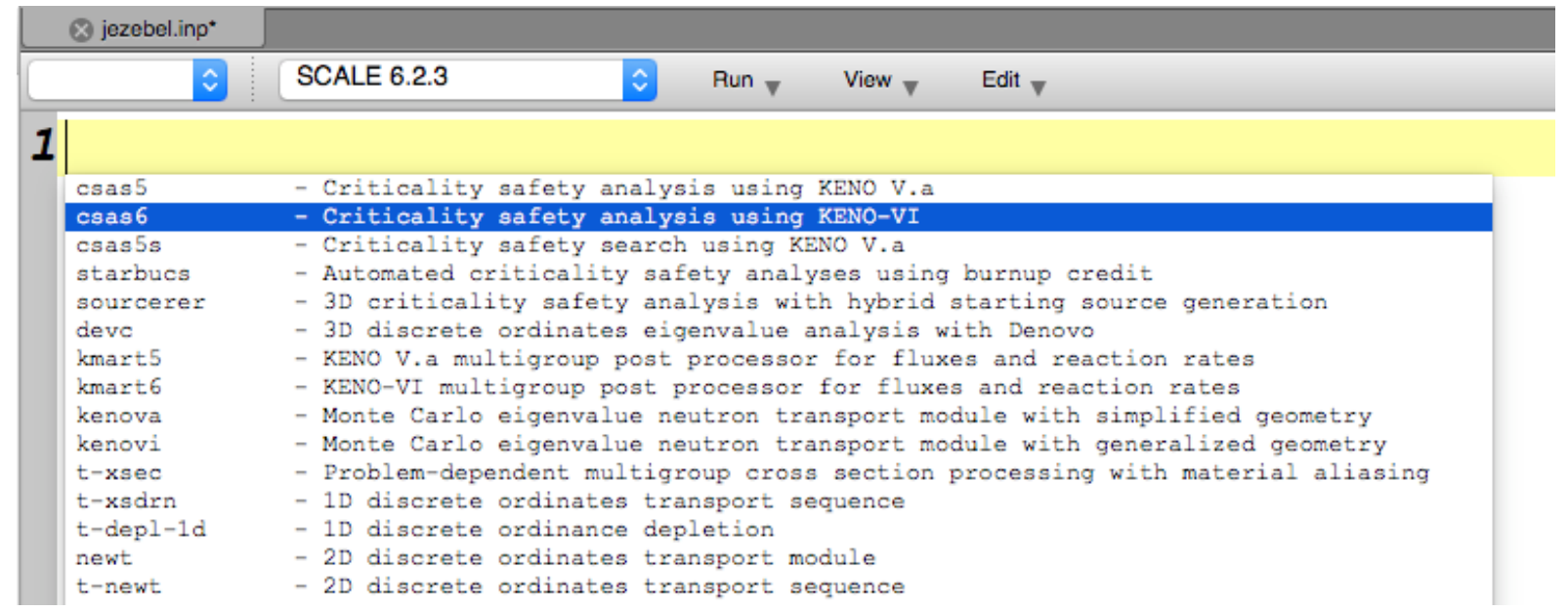

Figure 3. Autocompletion of the example sequence. 
A skeleton CSAS6 input is created with placeholders and empty read blocks, as shown in Figure 4. These blocks streamline the additional updates needed to complete the input specification.

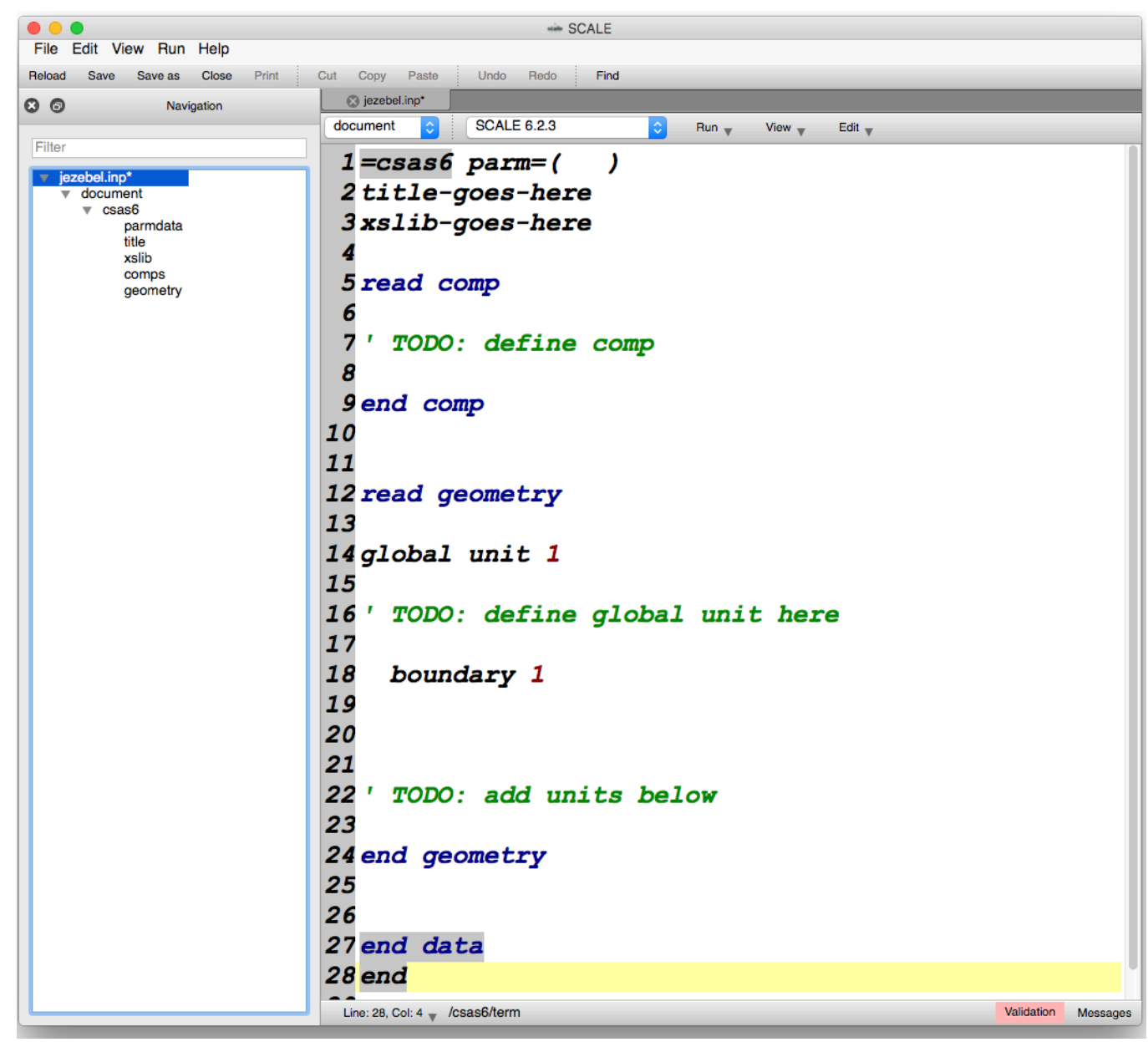

Figure 4. Example problem skeleton input.

With this CSAS6 skeleton available, start entering in the necessary parts of the problem. On line 2, replace the title placeholder, title-goes-here with:

jezebel problem, bare plutonium sphere with nickel shell

On line 3, replace the cross section library placeholder, xslib-goes-here, with the AMPXgenerated 252 neutron group ENDF/B-VII.1 MG cross section library, v7 . 1-252.

Figure 5 shows the CSAS6 input with the title and cross section library entered. 


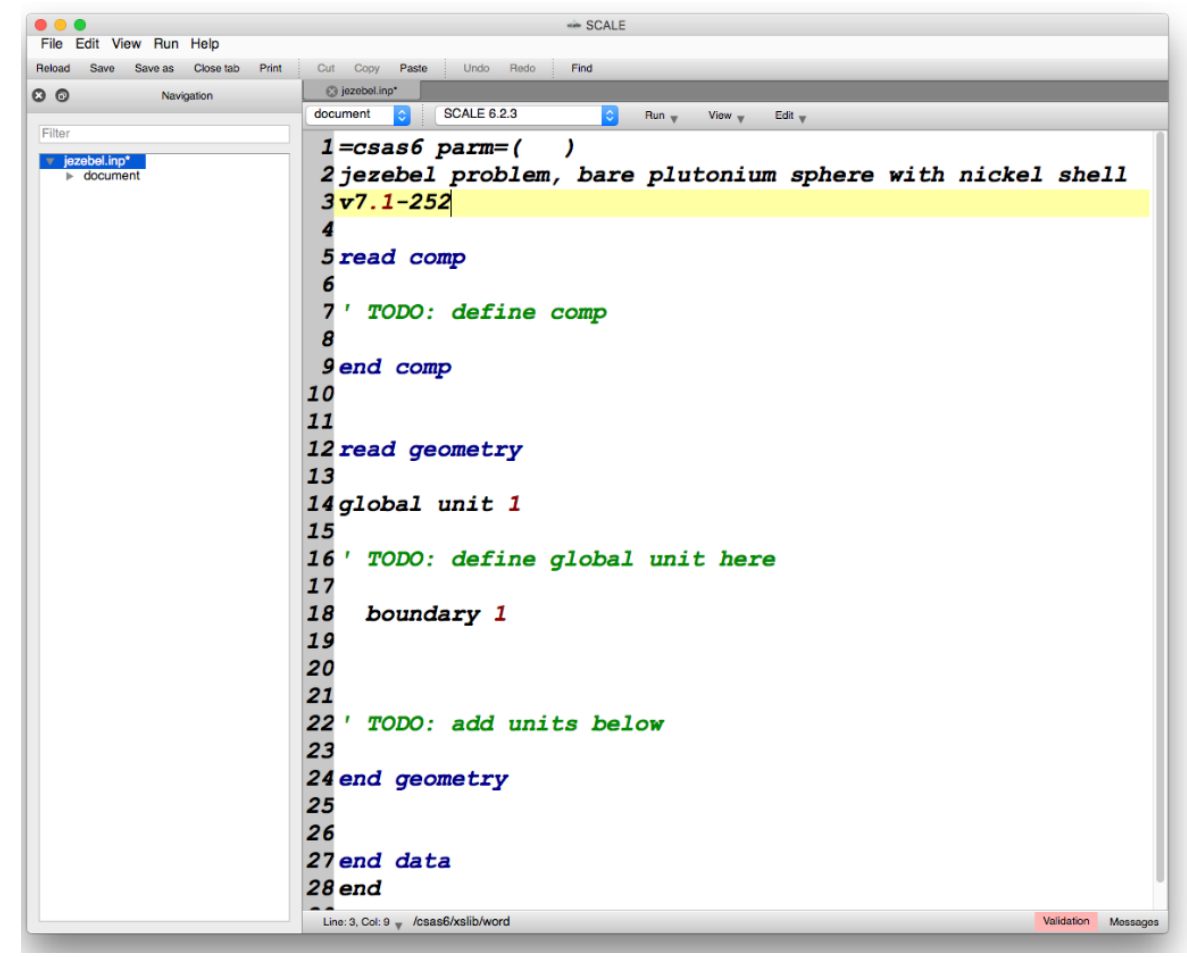

Figure 5. General information for the example problem.

The Validation panel button is located in the lower right corner of the text editor panel. Notice that it has a red background, indicating that messages related to the correctness of the input are available for viewing. Click the Validation button to view the current input messages as depicted in Figure 6.

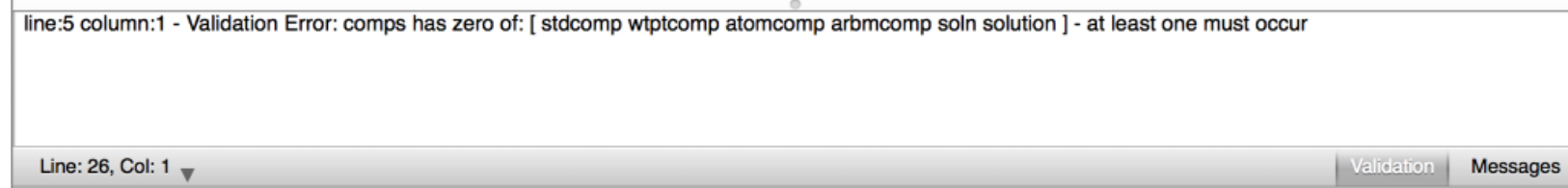

Figure 6. Validation panel indicating that required compositions are not present.

The validation message-

line:5 column:1 - Validation Error: comps has zero of: [ stdcomp wtptcomp atomcomp arbmcomp soln solution ] - at least one must occur

-indicates that on line 5, column 1, the read comp, referred to as the comps input, has no entries specified in the available composition formats of stdcomp wtptcomp atomcomp arbmcomp soln solution. This transitions you to the Materials specification for the example problem shown in Figure 7.

The autocomplete functionality requires Fulcrum to be able to parse the input and identify key components, such as the read and end statements for each block. Malformed input can, in some situations, cause parse errors that preclude autocomplete functionality. These errors are provided in the Validation pane, and repairing these errors will restore autocomplete functionality. 


\subsubsection{Materials}

The next section of input, the read comp block, provides information on the materials in the problem. For the example problem, there are four materials in the core region, and then the nickel shell in the outer region. To enter these, perform autocompletion within the comp block. Placing the text cursor within the read comp block and pressing the CTRL-SPACE autocomplete key combination will display the list of available composition entry formats: the stdcomp - basic + atomic density (configurable) shown in Figure 7 will be used for all problem material entries.

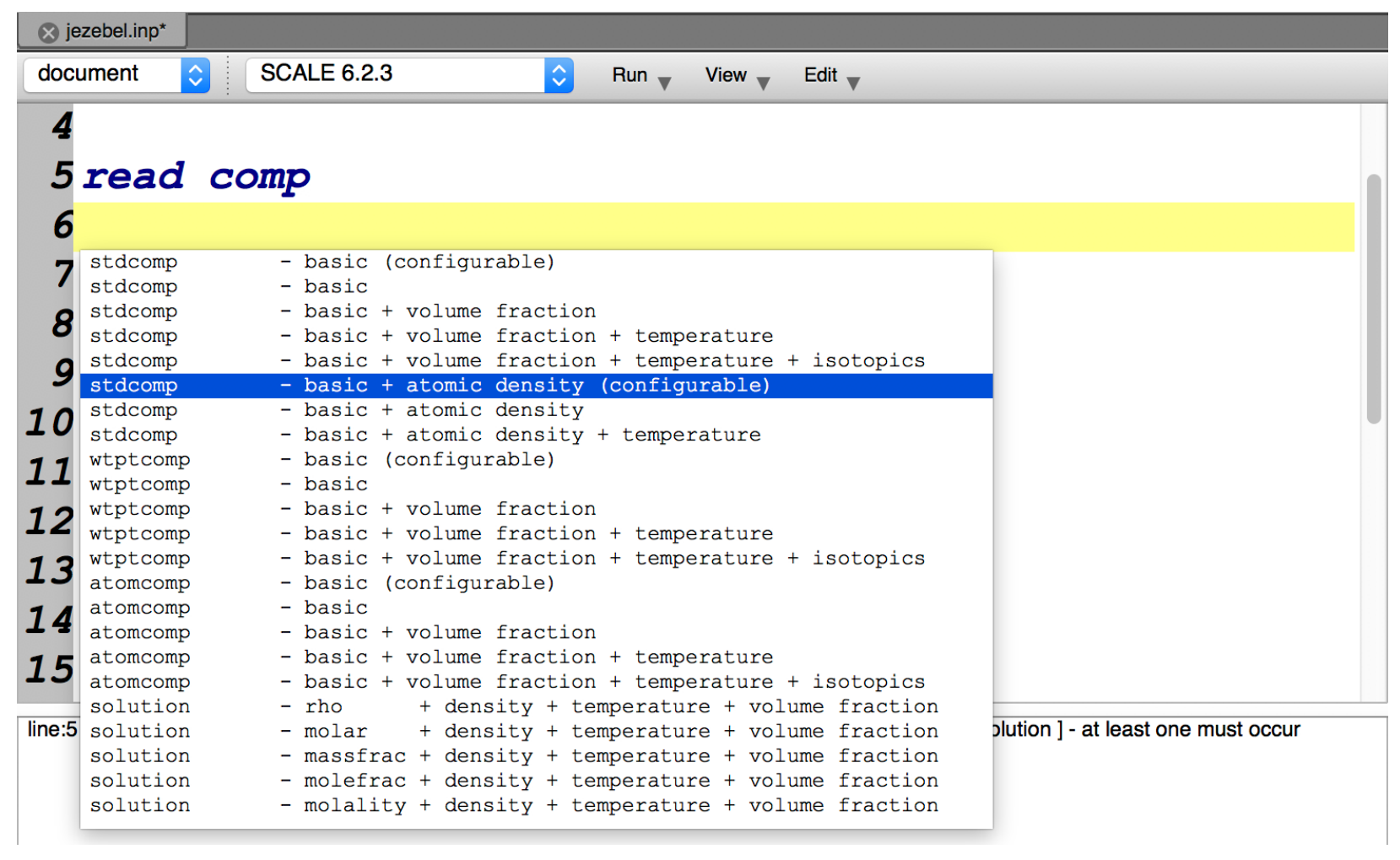

Figure 7. Example problem Basic Composition autocompletion.

For each available composition format, there is a configurable option. The configurable options display a widget with component labels and input preview. The options that are not listed as configurable will insert text variants of composition formats with default placeholders specified. Selecting the stdcomp basic + atomic density (configurable) will display a widget with the default for the Basic Composition, as shown in Figure 8. 


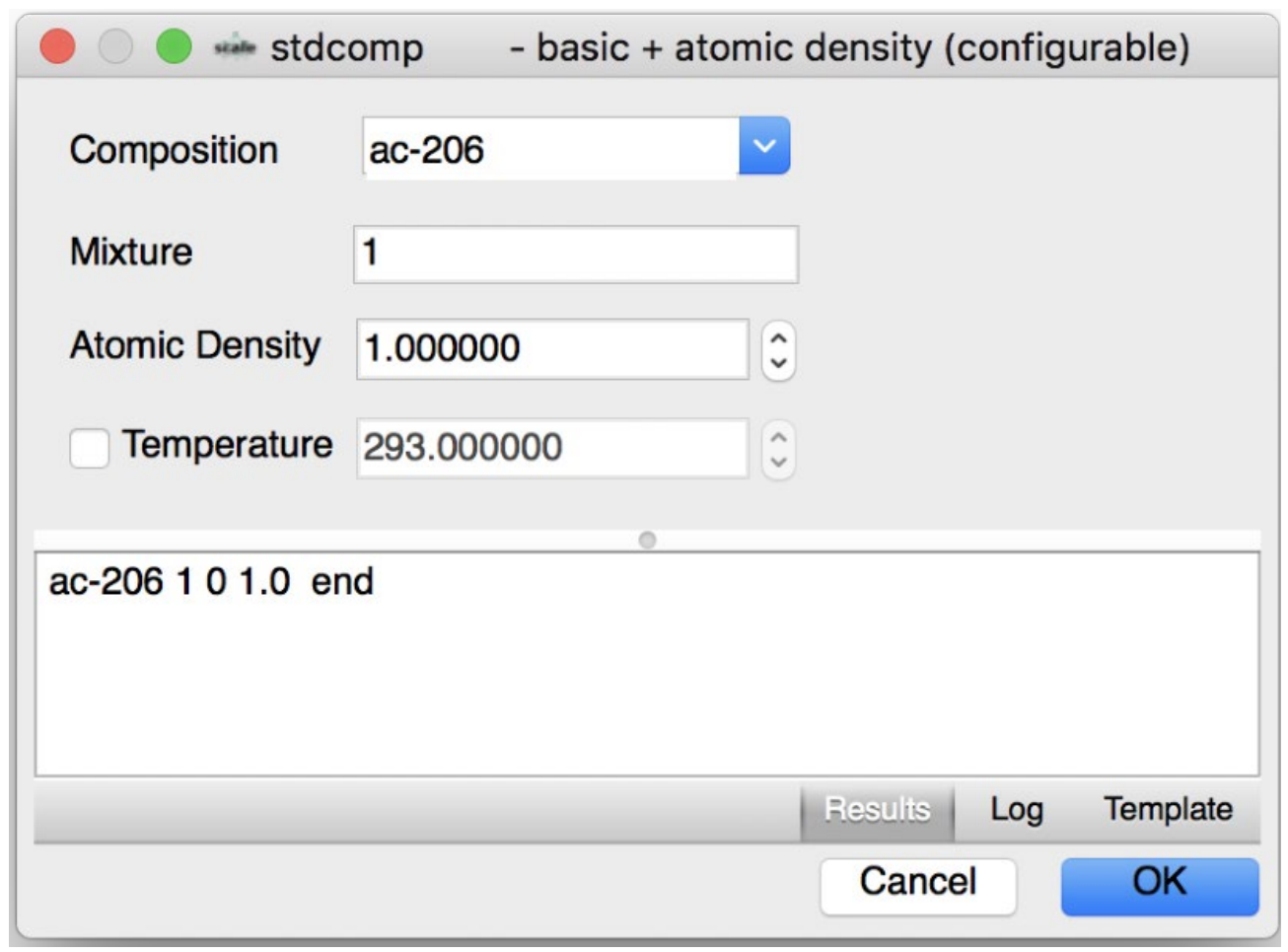

Figure 8. Example default basic stdcomp configurable form.

You will be entering data for ${ }^{239} \mathrm{Pu},{ }^{240} \mathrm{Pu},{ }^{241} \mathrm{Pu}$, gallium, and nickel, all of which are in the Standard Compositions Library and can be entered as Basic Compositions.

The first four materials are constituents of a single alloy and will therefore be part of mixture number 1 . The first four materials are constituents of a single alloy and will therefore be part of mixture number 1 . Four compositions will be entered for mixture 1. The entry for the first material, pu-239, should look like that shown in Figure 9. The Mixture should already be set to 1. Select pu-239 as the Composition from the list of materials in the library. The default Temperature is 293, but to incorporate it, click the Temperature Checkbox to the left of the Temperature label. To enter the atom densities given in the example problem description, you must select Atomic Density value field, and then enter the appropriate value. In this case, enter 0.037047 . 


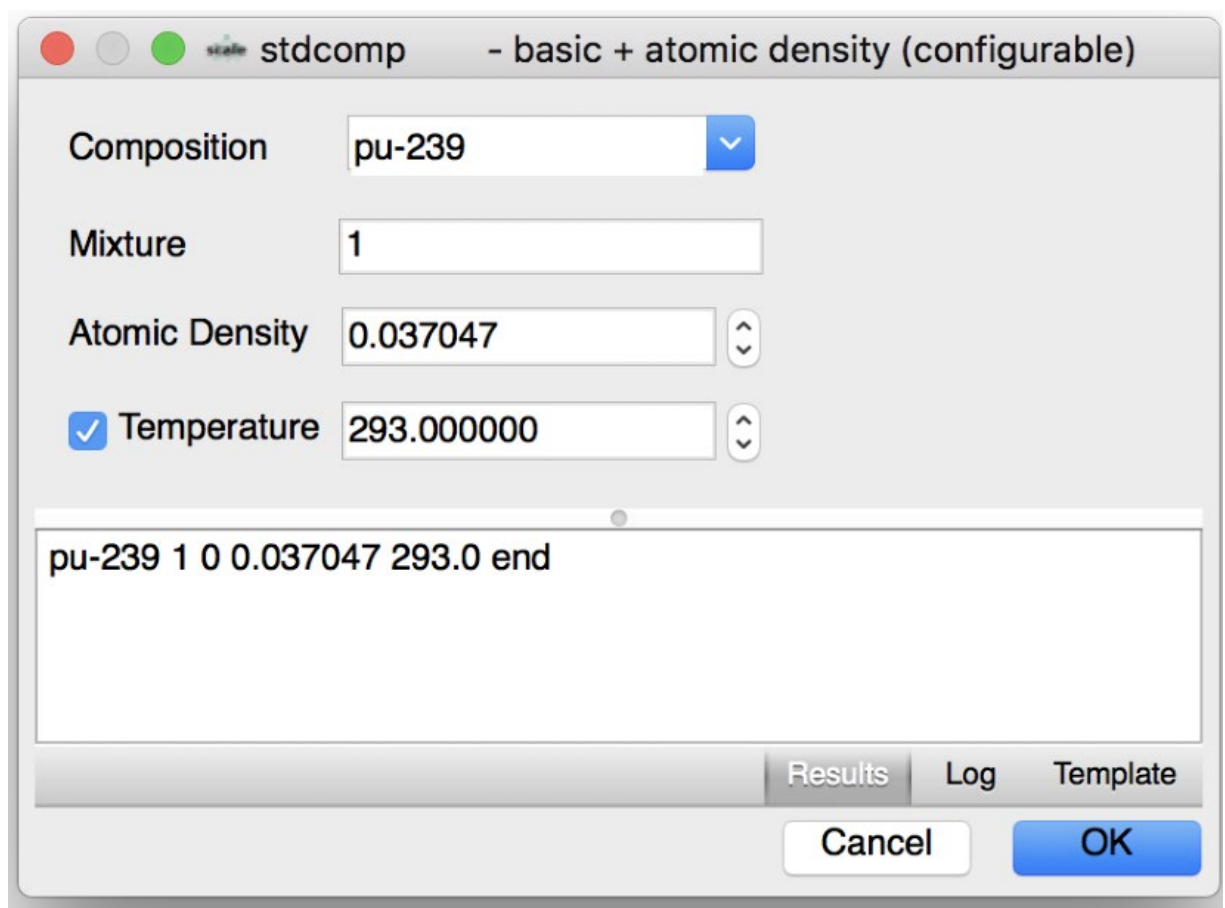

Figure 9. Data for ${ }^{239} \mathrm{Pu}$ in mixture 1.

Select $\mathbf{O K}$ to insert the Result into the document. To enter the data for the remaining materials, autocomplete the stdcomp - basic + atom density (configurable) option. Then modify the Composition Name and Atomic Density. The mixture number should remain the same for each of the four materials. The entries for the next three materials (pu240, pu-241, and ga) look like those shown in Figure 10 through Figure 12. 


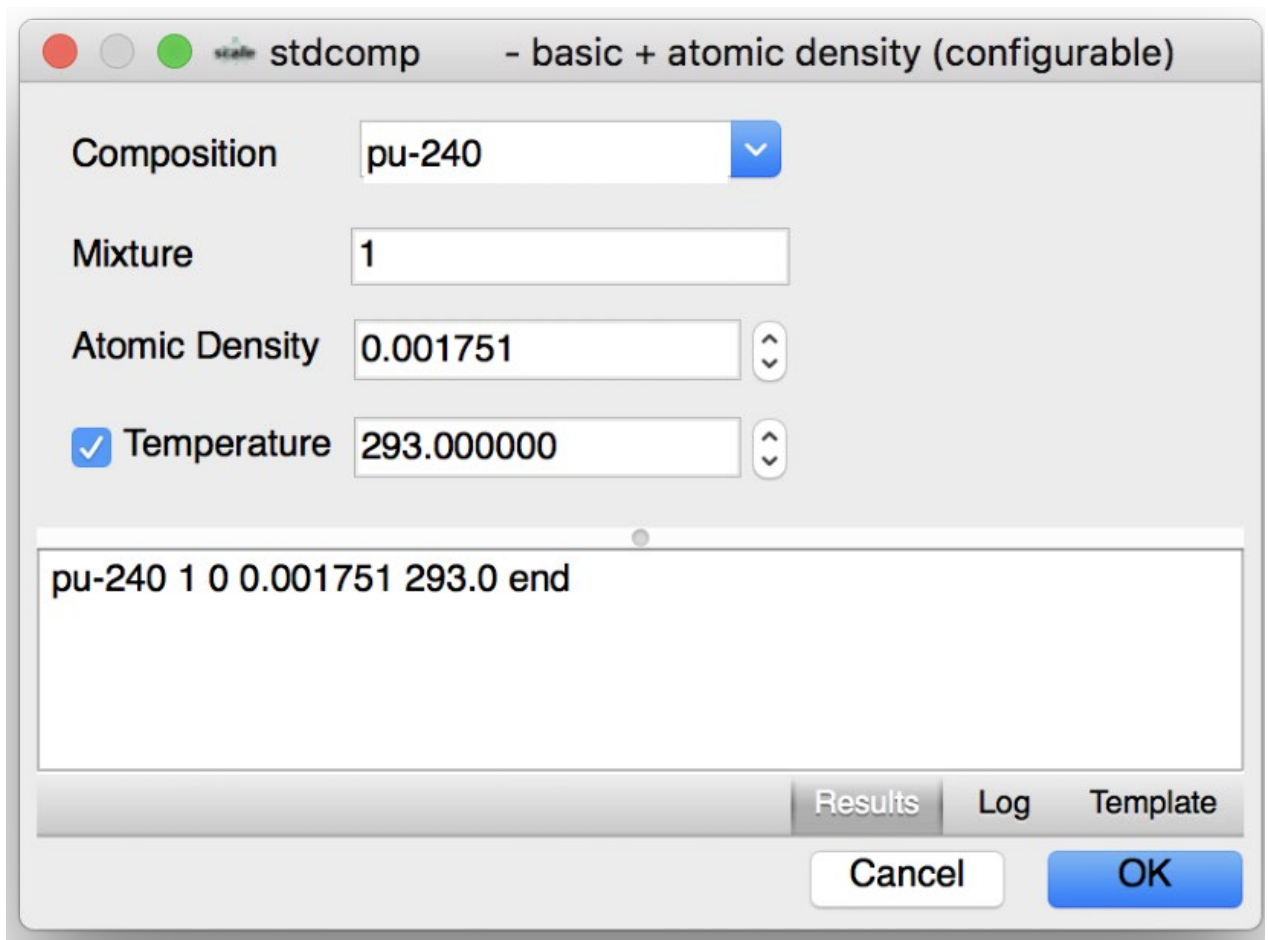

Figure 10. Data for ${ }^{240} \mathrm{Pu}$ in mixture 1.

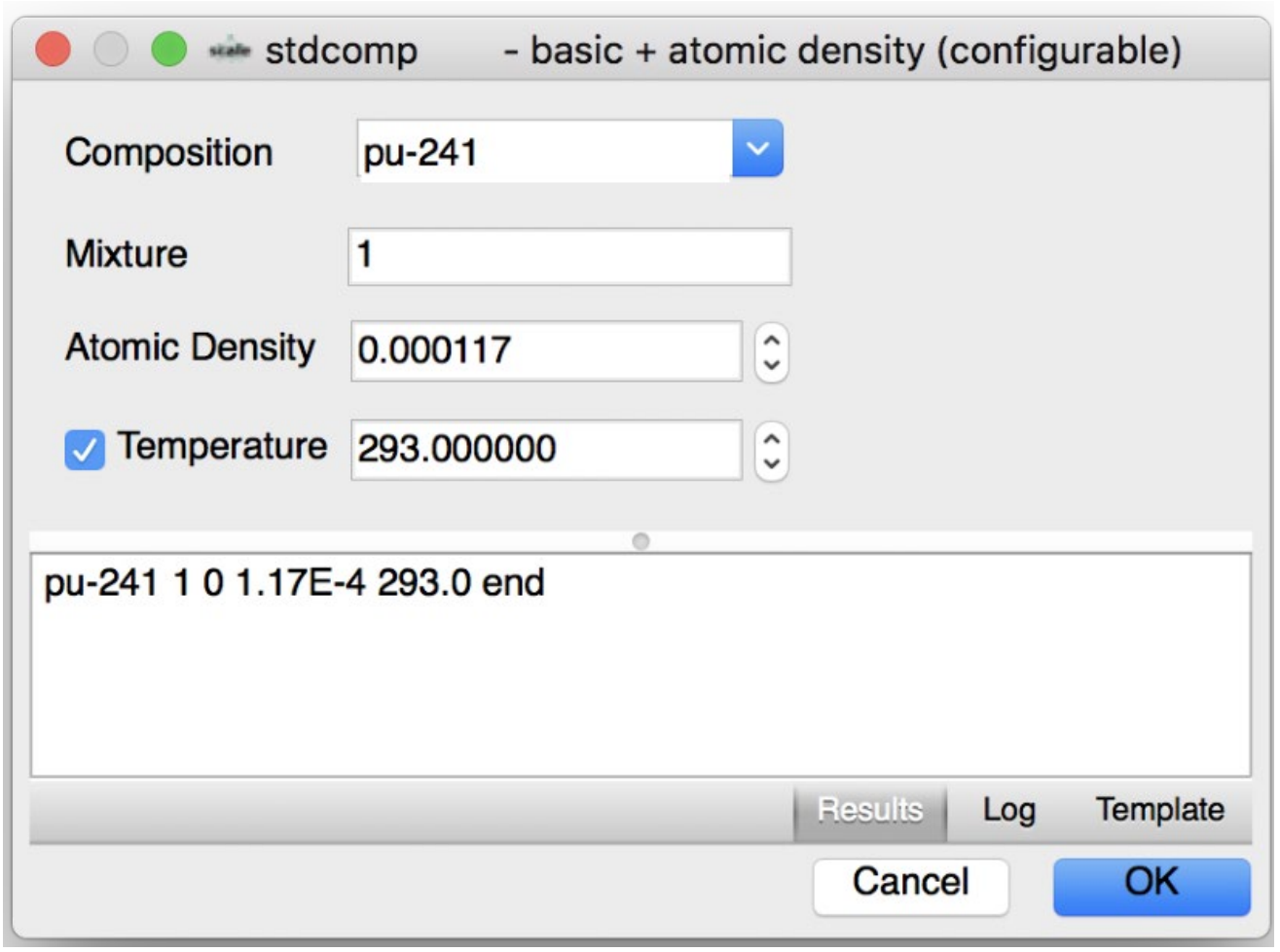

Figure 11. Data for ${ }^{241} \mathrm{Pu}$ in mixture 1. 


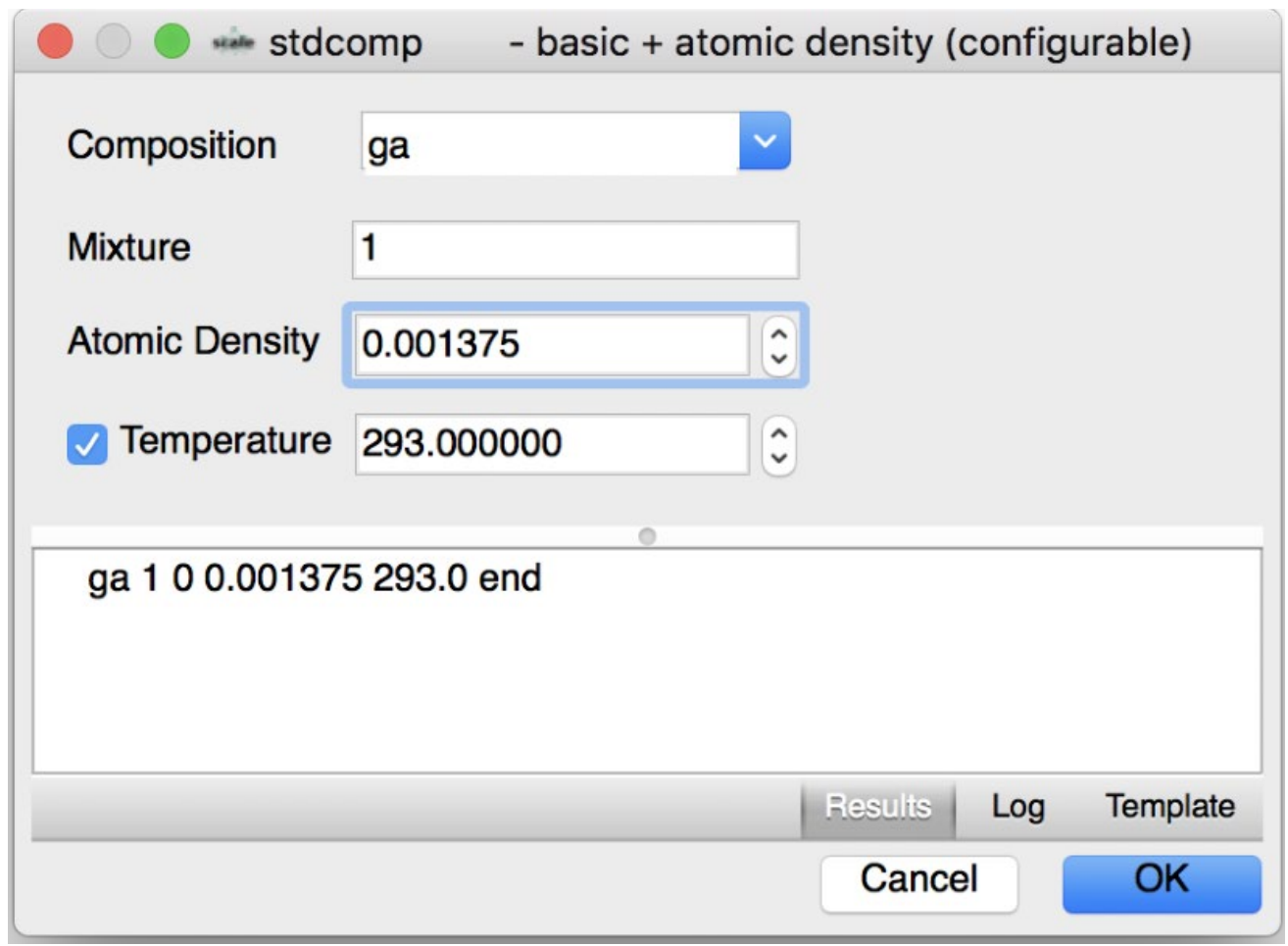

Figure 12. Data for Gallium in mixture 1.

Figure 13 illustrates the composition records for mixture 1.

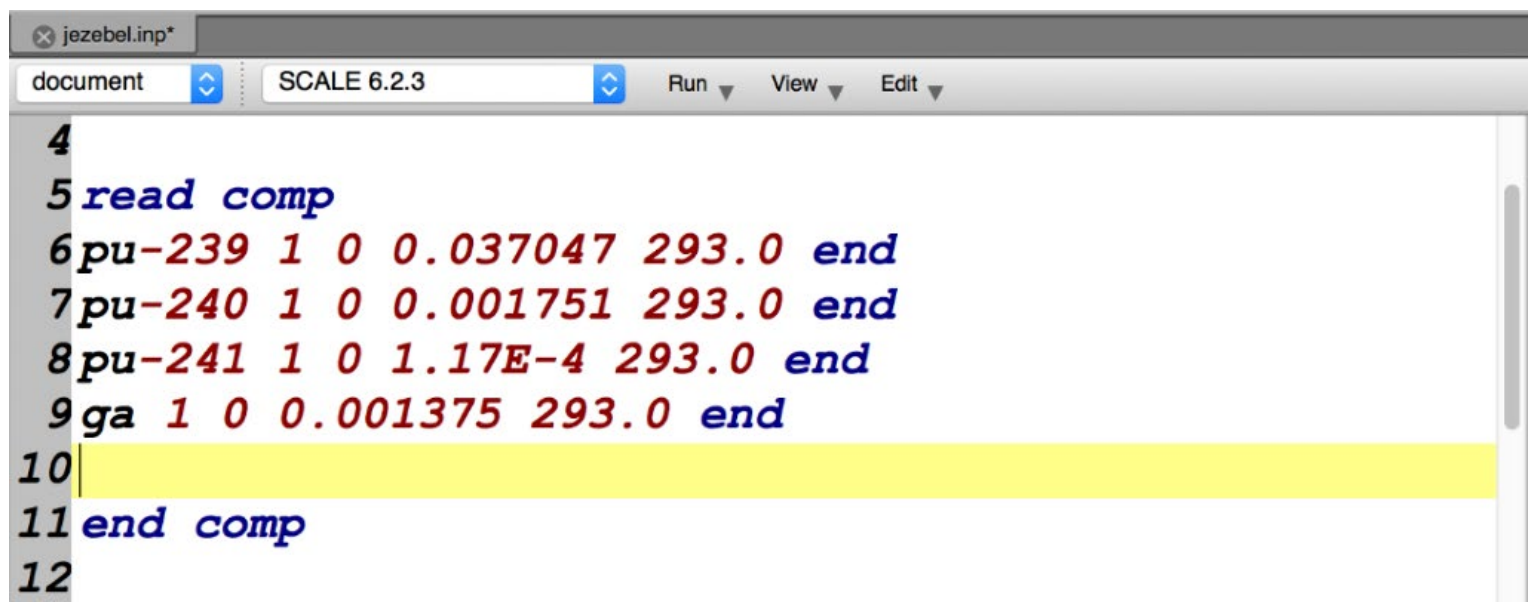

Figure 13. Mixture 1 basic composition summary. 
Because material input can be position dependent, it is important to remember that Fulcrum can assist the user by presenting field labels at any given text cursor position. To determine what the fourth field is in

ga $10 \underline{0.001375} 293.0$ end

place the text cursor on the field and observe the cursor context display the parameter's path /csas6/comps/stdcomp/aden, as shown in Figure 14, at the lower left of the text editor, adjacent to the cursor's line and column numbers.

\section{9 ga 100.001375293 .0 end}

\section{Line: 9 , Col: $8 \nabla$ /csas6/comps/stdcomp/aden}

Figure 14. Example input text cursor context.

This cursor context provides the structure and naming of all SCALE input fields. Aden is the shorthand name for Atomic Density. The context indicates that aden is a field in the stdcomp record within the comps block of the CSAS6 sequence. These are a pseudo-directory listing of SCALE input that can assist you with identifying unlabeled input fields. Parse errors, like those mentioned in Section 2.3.2 that may disable autocomplete capability, also cause the loss of this cursor context. Moving the cursor through the input until cursor context is restored can help identify the location of problematic input causing the parse errors.

The last material to specify is the nickel shell in the outer region. Again, perform an autocompletion of the stdcomp - basic + atomic density (configurable) option to bring up the input entry form. Select ni, and then select Mixture of 2. For the Atomic Density, enter 0.091322 . Use the default Temperature of 293 and click the checkbox to enable its explicit use in the composition input record. The completed screen for nickel should look like that shown in Figure 15. After entering the data for nickel, select $\mathbf{O K}$ to insert the Result into the document. 


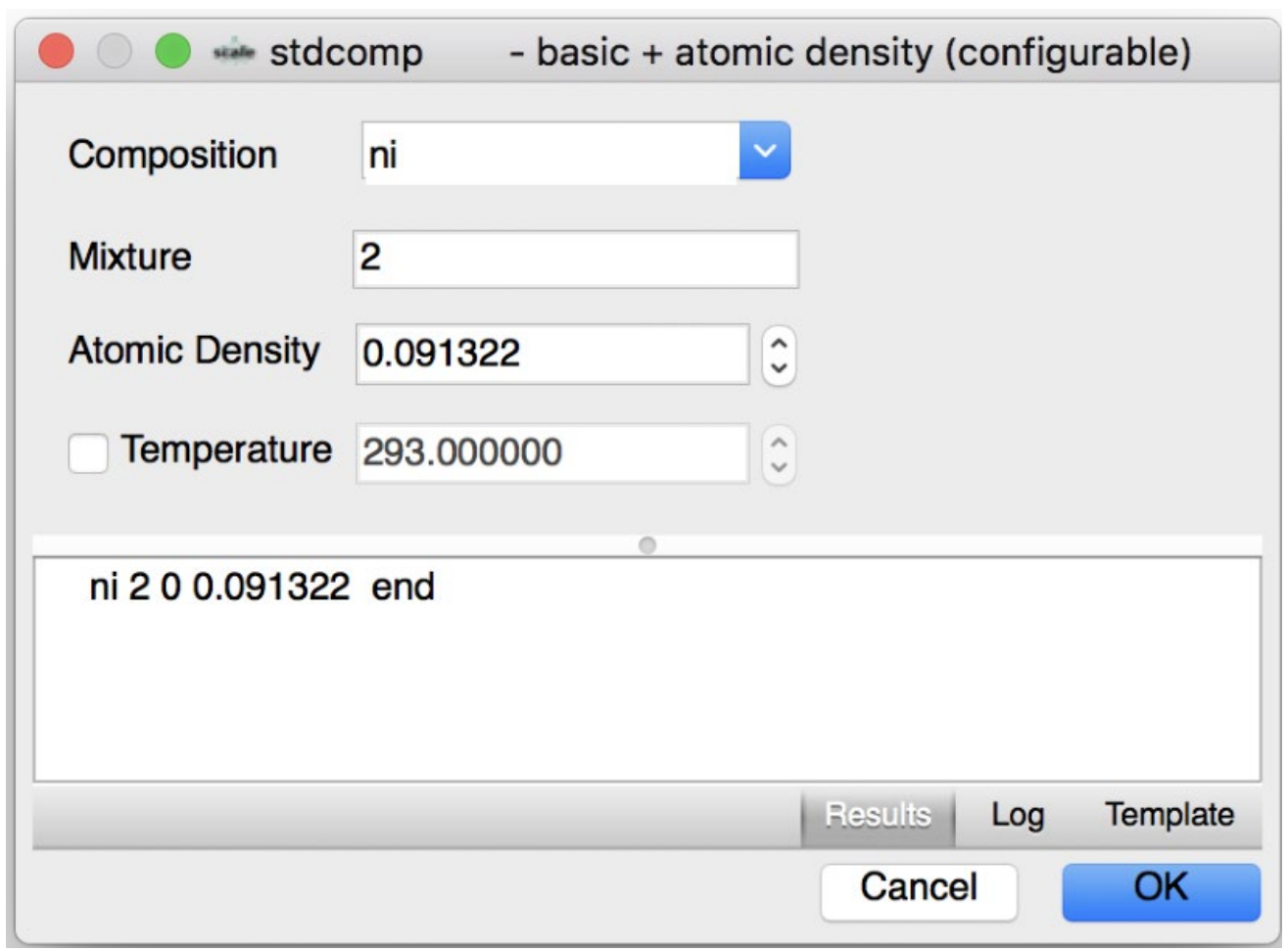

Figure 15. Data for nickel in mixture 2.

Figure 16 illustrates the complete example composition block.

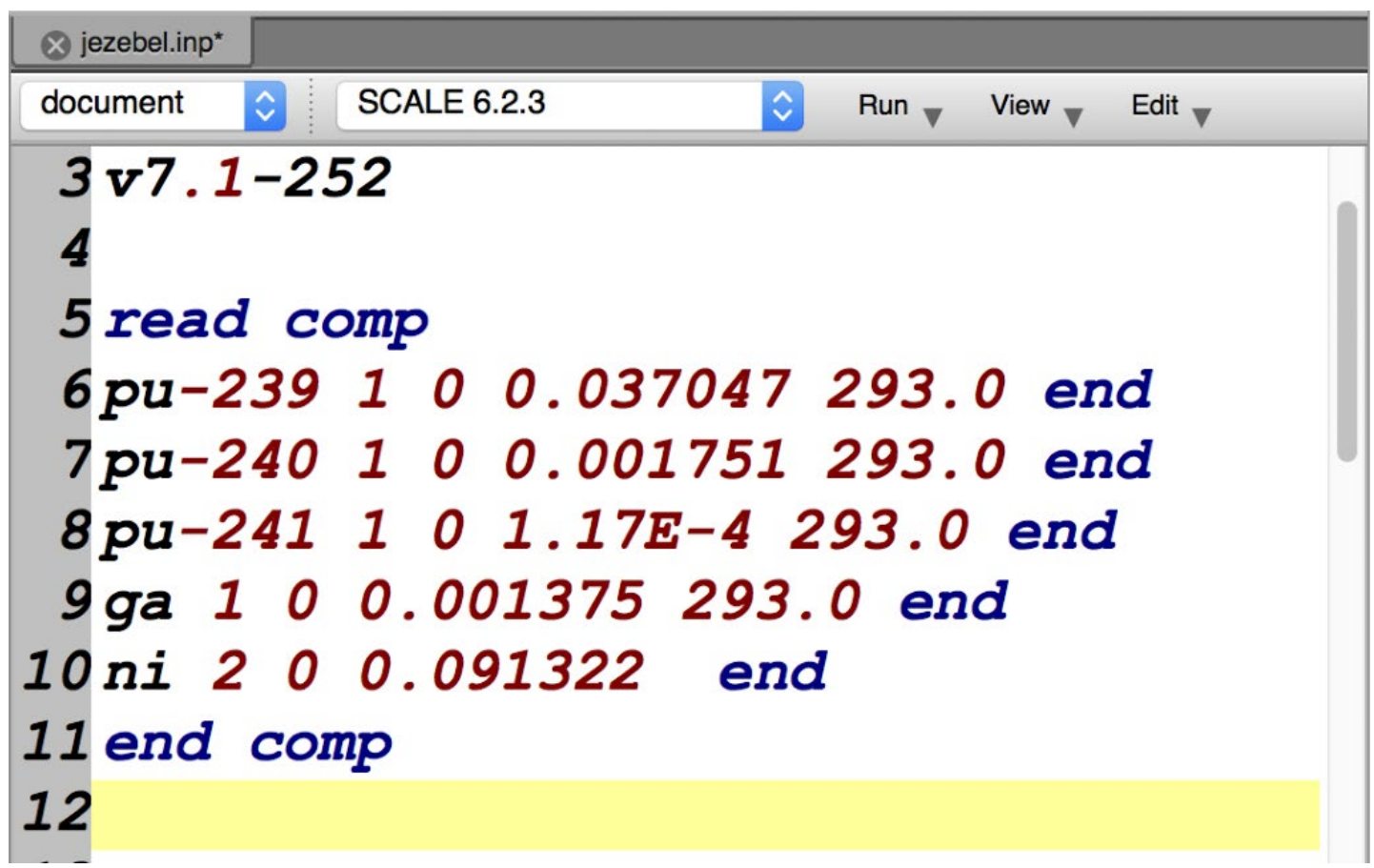

Figure 16. Complete example problem composition block.

Because this example problem uses MG cross sections, a processing method to incorporate spatial affects in the cross section preparation is also specified. The unit cell input parameters are specified in the read 
celldata block. With your text cursor between the composition and geometry blocks, press the CTRLSPACE key combination to display the available input blocks, and select the cells option as shown in Figure 17. Figure 18 illustrates the empty read celldata ready for further autocompletion of the example problem's cell information.

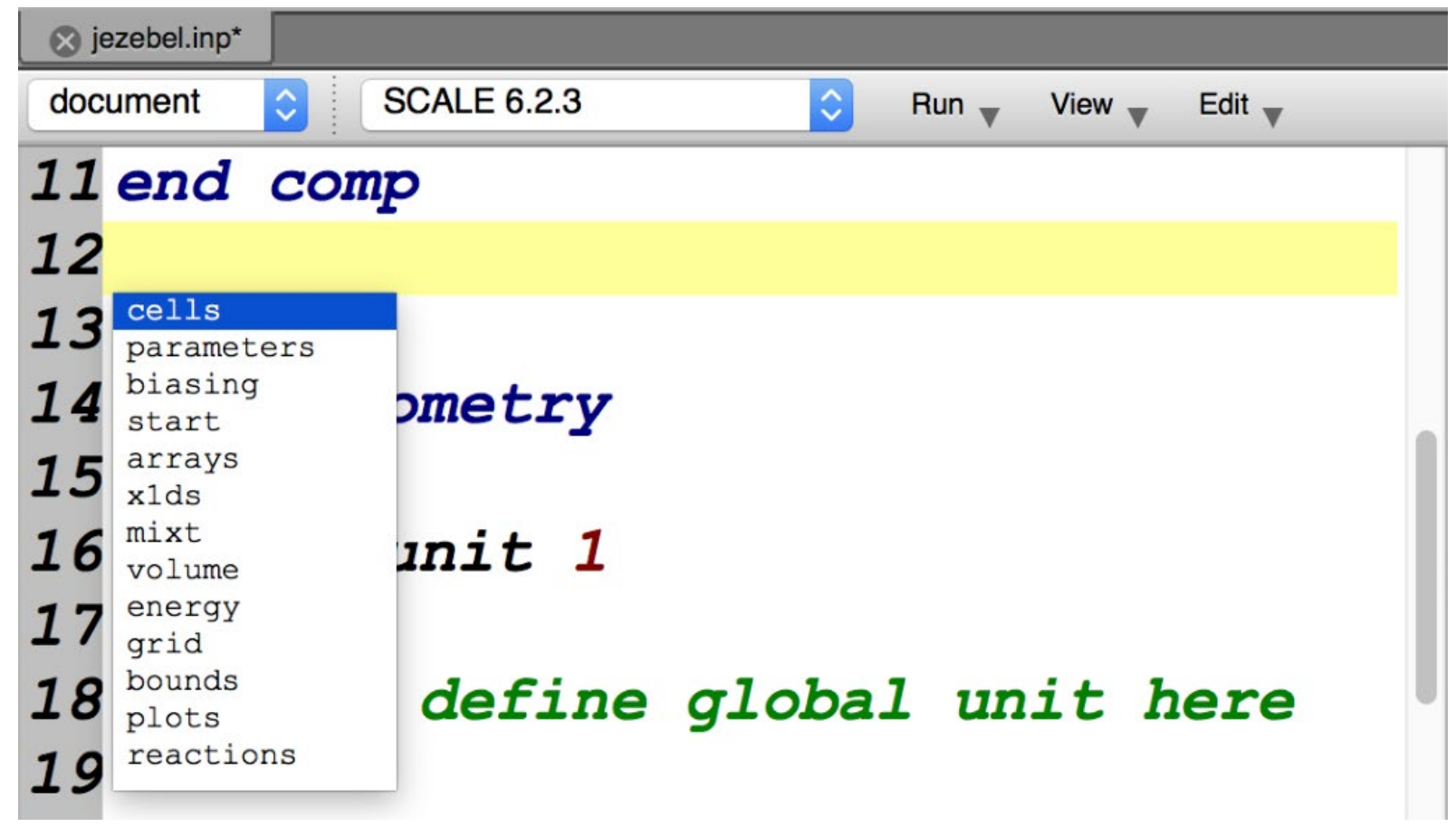

Figure 17. Autocompletion of the cells input block.

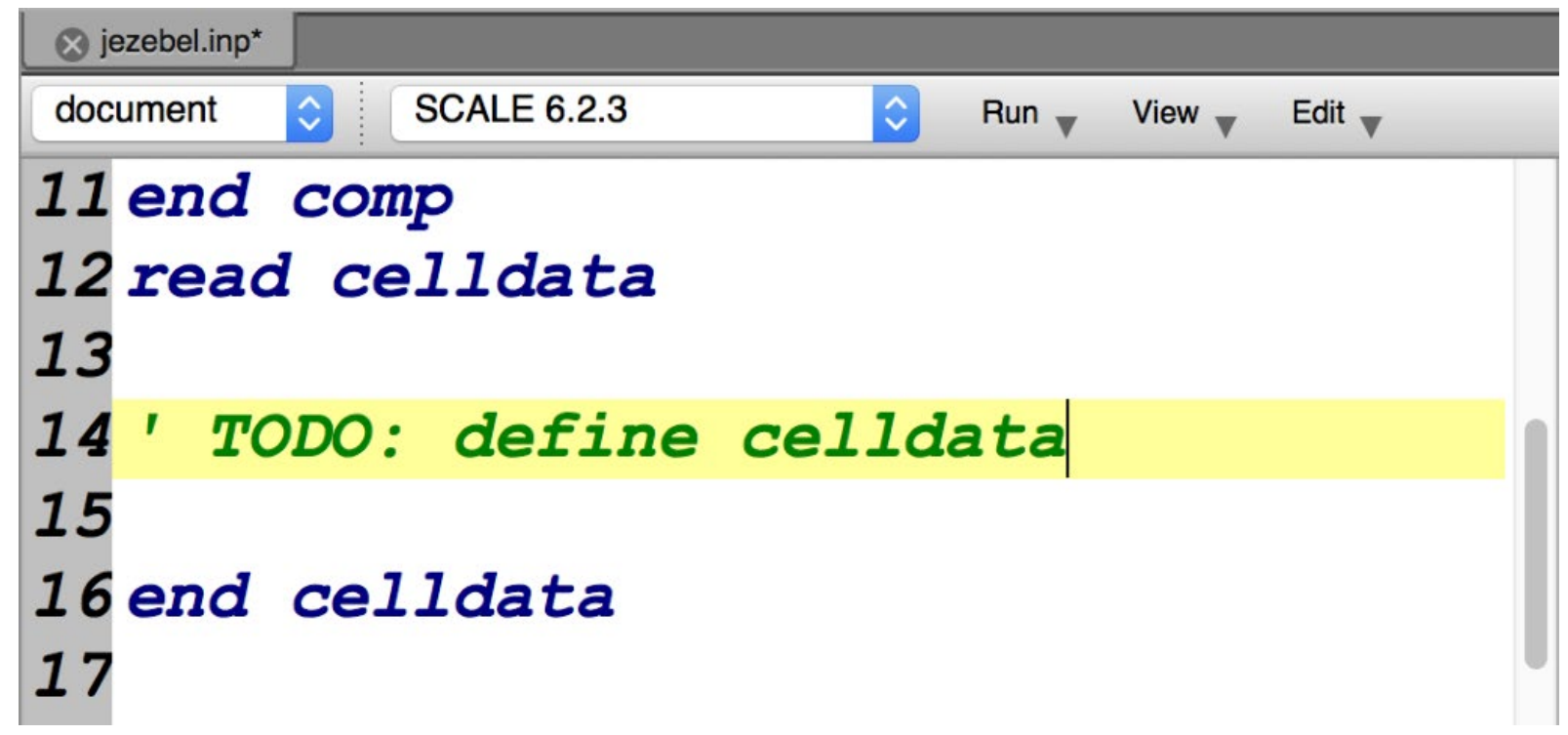

Figure 18. Example autocompleted initial celldata input block. 
With the text cursor inside the celldata block, press the CTRL-SPACE key combination to display the available celldata types, and select the MultiRegion - spherical option as shown in Figure 19. Figure 20 illustrates the default spherical multiregion with placeholders for right or outer boundary, right_bdy, and cell zone mixture-radii pairs. Use the default outer boundary of vacuum; the inner (left) boundary condition of a spherical unit cell must be reflective and cannot be changed.

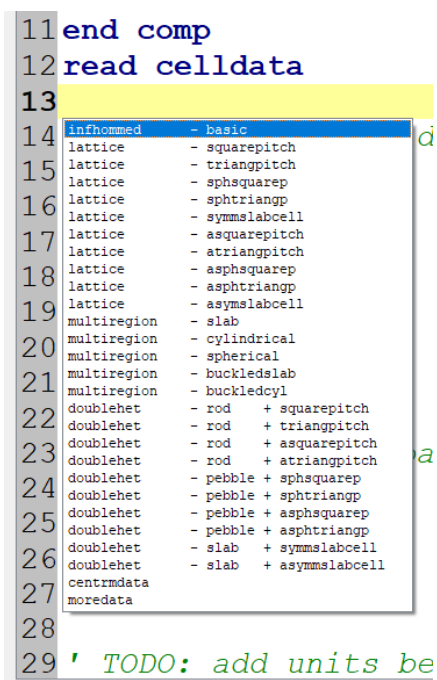

Figure 19. Autocompletion of a spherical multiregion.

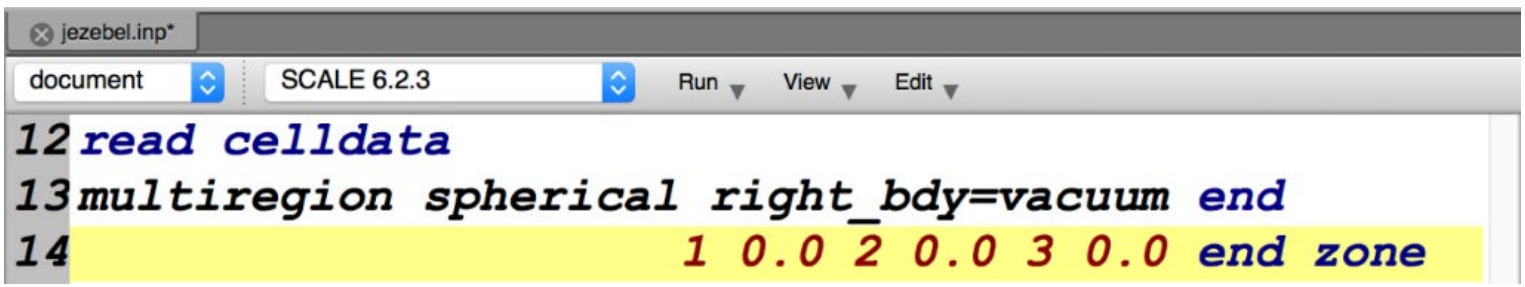

Figure 20. Example autocompleted initial spherical multiregion input.

As a reminder, the text cursor can be used to identify which field is which, as illustrated in Figure 21, which depicts the multiregion zone mixture and radius.

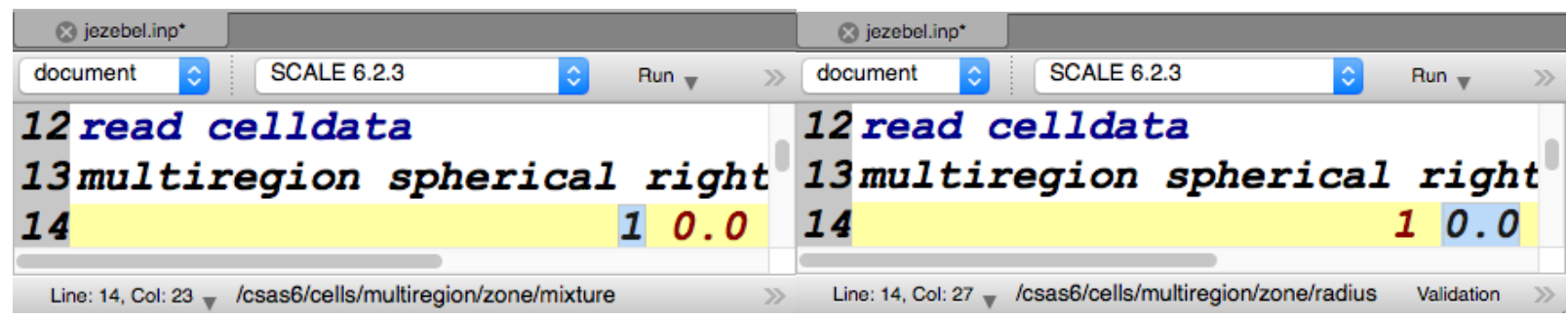

Figure 21. Example spherical multiregion zone mixture and radius placeholders.

The zone mixture-radii pair placeholders need to be updated to be mixture $1\left({ }^{239} \mathrm{Pu},{ }^{240} \mathrm{Pu},{ }^{241} \mathrm{Pu}\right.$, and gallium) with a radius of 6.38493 , and mixture 2 (nickel) with a radius of 6.39763 . The placeholder for mixture 3 can be removed. Figure 22 illustrates the completed example multiregion input. 


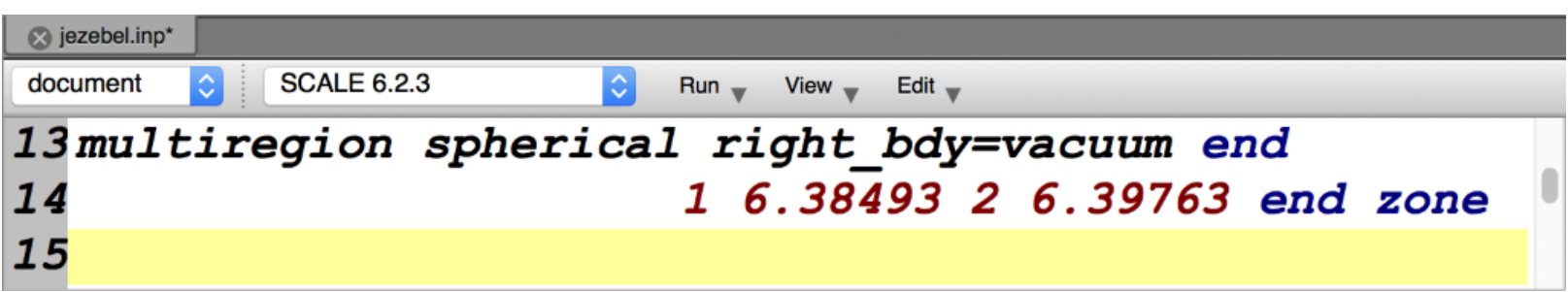

Figure 22. Multiregion unit cell.

\subsubsection{KENO-VI}

Now enter the information required to run a KENO-VI analysis of the problem. Place the cursor inside the global unit in the read geometry block as shown in Figure 23 to prepare for autocompletion of the appropriate geometry for this problem.

\section{3 global unit 1}

24

25' TODO: define global unit here

Figure 23. Cursor placement in the global unit.

Press the CTRL-SPACE key combination to display the available geometry. For this problem, the appropriate geometry is a sphere, so select the sphere - kenovi (configurable) option shown in Figure 24. 


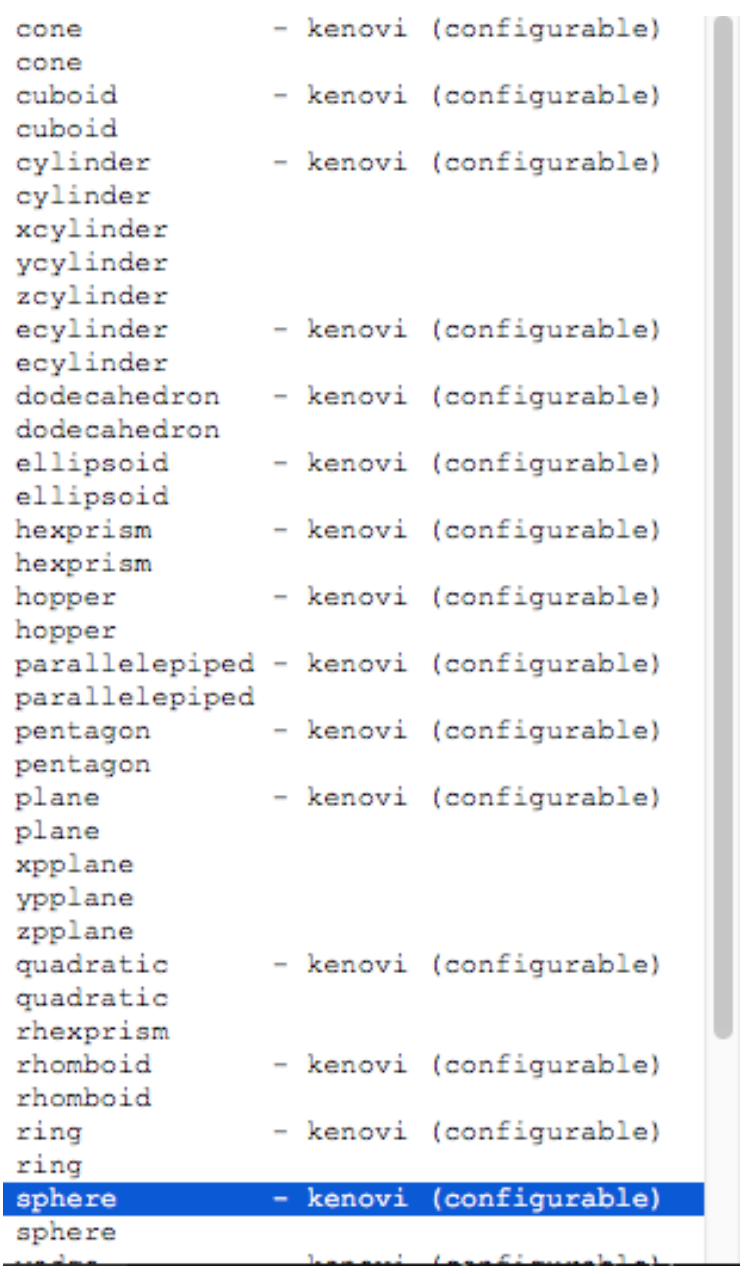

Figure 24. Available autocomplete options.

As shown in Figure 25, select the appropriate Id, 10, enter the radius of 6.38493 , and then select $\mathbf{O K}$. Now add an additional sphere for the nickel outer shell by copying the sphere and pasting it. Update the Id to 20 and the radius to 6.39763 , as shown in the highlighted portion of Figure 26. 


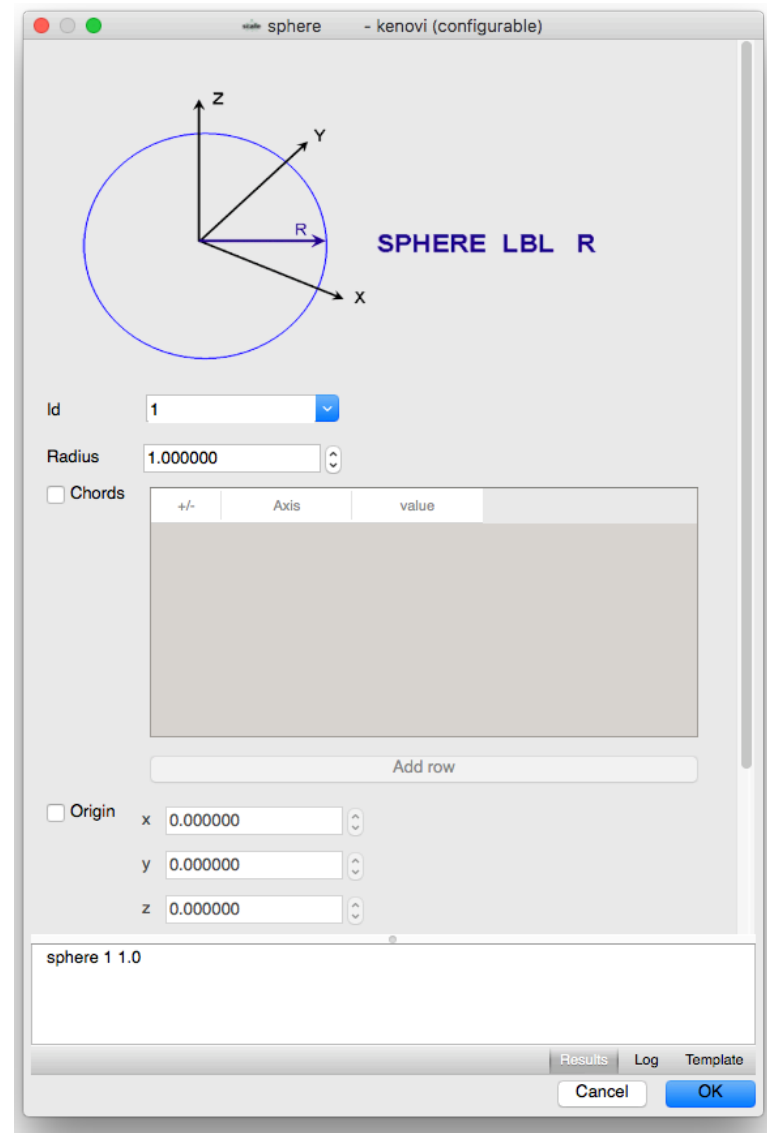

Figure 25. Initial sphere configurable.

\section{2 global unit 1 23 sphere 106.38493 24 sphere 206.39763}

Figure 26. Geometry regions.

Next, describe the contents of the geometry regions. On line 25, press the CTRL-SPACE key combination to display available autocomplete options, and select media - kenovi (configurable), as depicted in Figure 27. 


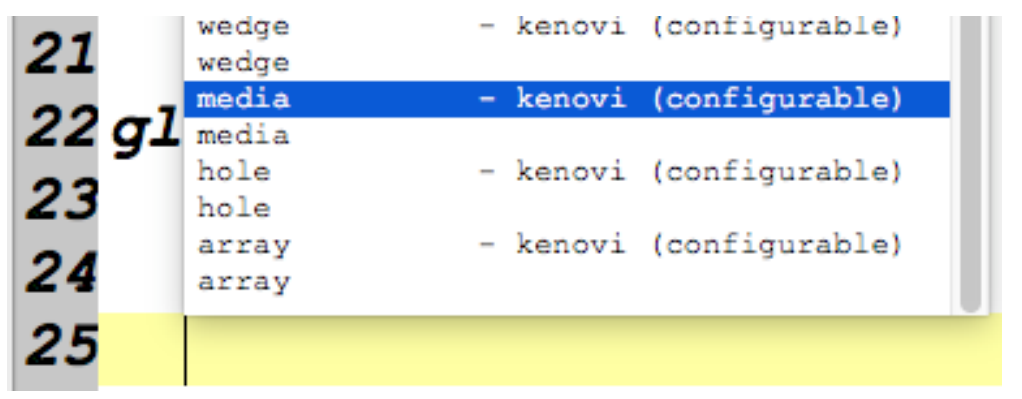

Figure 27. Configurable geometry media autocompletion.

Select the appropriate Material, 1, for ${ }^{239} \mathrm{Pu},{ }^{240} \mathrm{Pu},{ }^{241} \mathrm{Pu}, \mathrm{Ga}$. Click the Add row button to add an entry to the Regions table. Specify the inner sphere id of 10 in the Id cell, and ensure the State field lists inside, as depicted in Figure 28.

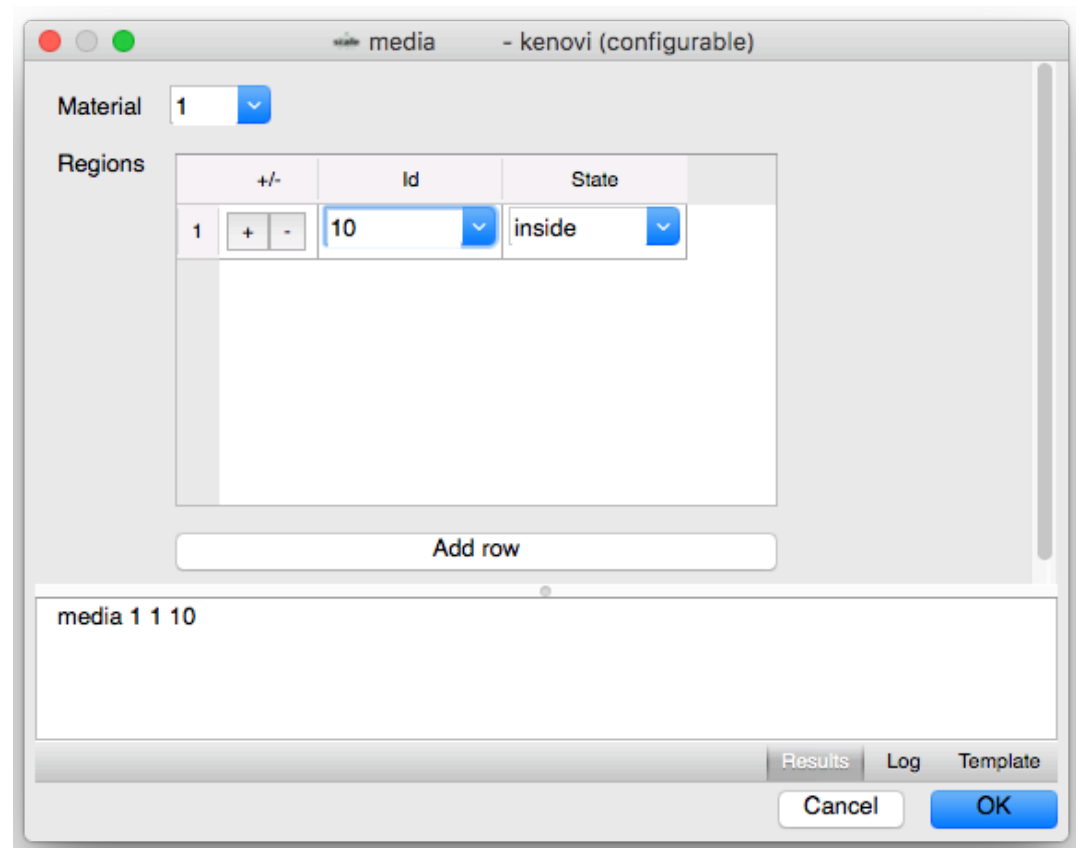

Figure 28. Plutonium sphere media region definition.

To describe the nickel plating outside the plutonium core, perform an additional autocompletion of media - kenovi (configurable) on line 26. Select the appropriate Material, 2, for nickel. Click the Add row button to add an entry to the Regions table. Specify the inner sphere id of 10 in the Id cell and ensure that the State field lists outside. Click the Add row button again to add a second entry to the Regions table. In this second row, specify the outer sphere id of 20 in the Id cell and ensure that the State field lists inside, as depicted in Figure 29. 


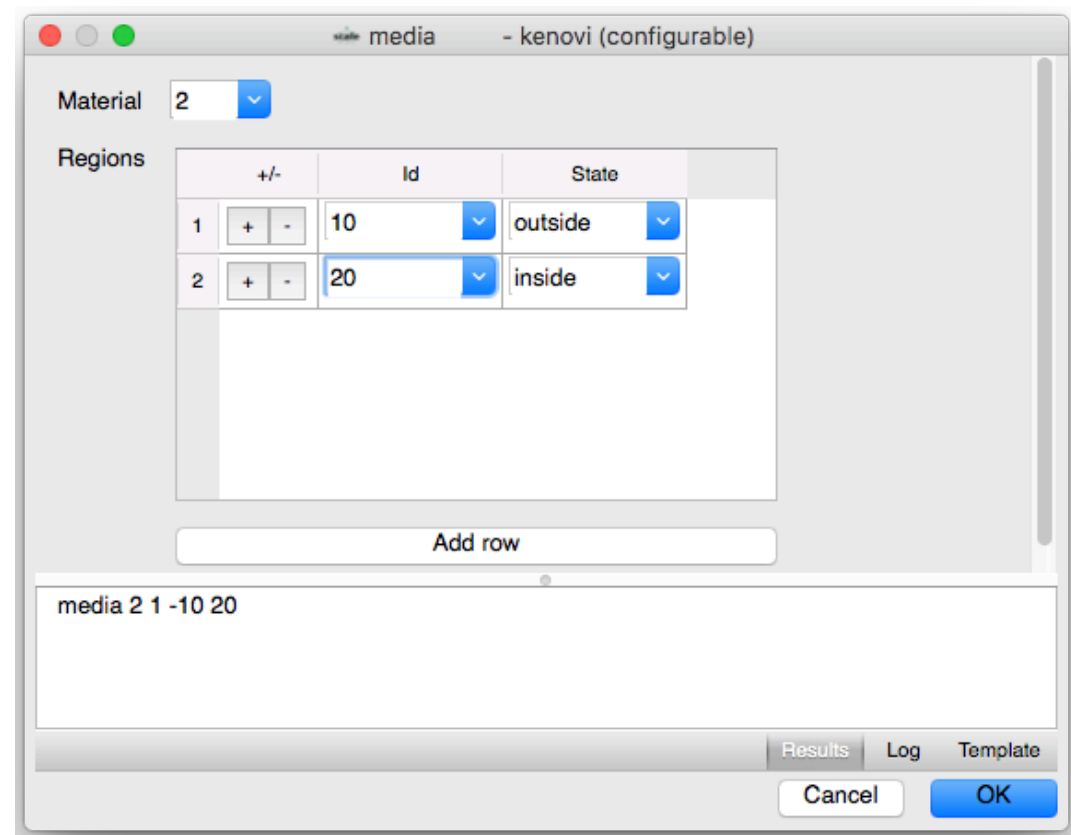

Figure 29. Nickel plating media region definition vector.

Lastly, the outer region boundary of the unit must be defined. Perform an autocompletion on the default boundary region identifier of 1 to display the list of available regions and select the outer sphere, id 20, as depicted in Figure 30.

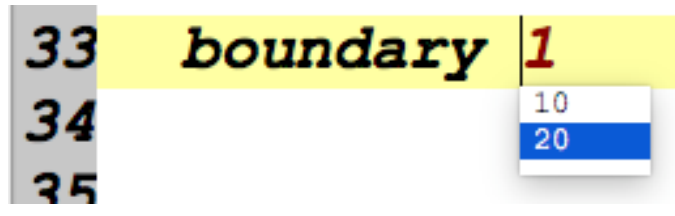

Figure 30. Global unit outer boundary.

The global unit is the unit that defines the entire system to be analyzed and must always be specified. The global unit should look like Figure 31. Note that placeholder comments have been removed in the figure for the purpose of clarity.

22 global unit 1

23 sphere 106.38493

24 sphere 206.39763

25 media 1110

26 media $2 \quad 1-10 \quad 20$

28 boundary 20

29 end geometry

Figure 31. Geometry definition. 
This completes the information necessary to run the sample problem. The next step is to save the input file and run SCALE. Make sure the file is saved by clicking the File $>$ Save.

\subsection{RUNNING SCALE/KENO-VI}

To run SCALE/KENO-VI, click the Run button on the text editor panel's toolbar depicted in Figure 32.

\begin{tabular}{|ll|l|lllll|}
\hline \multicolumn{2}{|c|}{$\otimes$ jezebel.inp } & & & & & \\
\hline \hline document & $\hat{v}$ & SCALE 6.2 & $\hat{v}$ & Run & View & Edit \\
\hline
\end{tabular}

Figure 32. Text editor toolbar.

Upon clicking the Run button, the Messages panel button background color will change to red, indicating that there are unseen messages. The Messages panel is located in the bottom right corner of the text editor, as depicted in Figure 33.

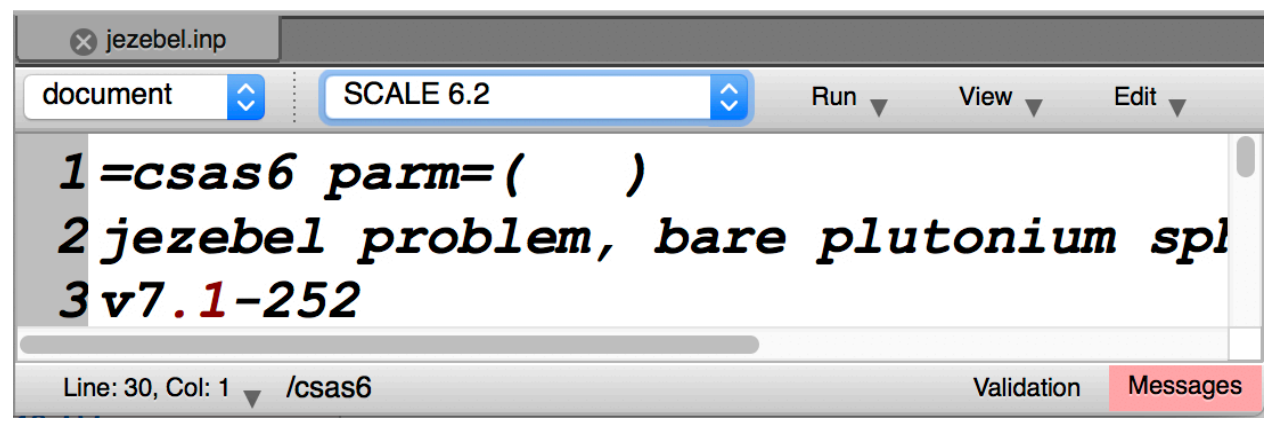

Figure 33. Location of Messages panel and red background color indicating unseen messages.

Click the Messages panel to see the jezebel.inp execution messages, as depicted in Figure 34.

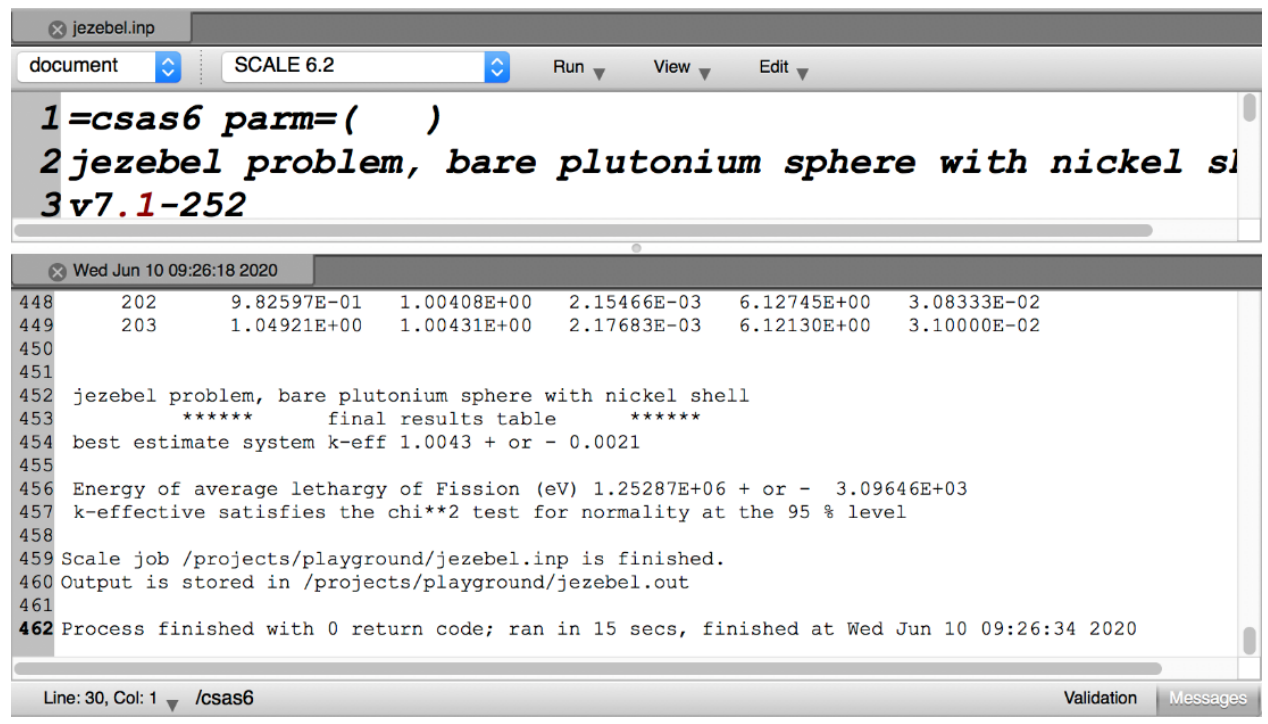

Figure 34. Messages panel showing completed run.

Note that the messages provide a running summary of results and should indicate that the SCALE job is finished. The messages also indicate that the Output is stored in jezebel.out. If you had a different name 
for your input file, then the output will be stored in a file with the .out extension. The elapsed time may not match, as each computer has a different central processing unit (CPU) and disk access speeds.

\subsection{SCALE/KENO-VI OUTPUT}

To view the output, right-click the jezebel.inp file in the Navigation panel, and select Open Associated Files $>$ jezebel.out, as depicted in Figure 35 .

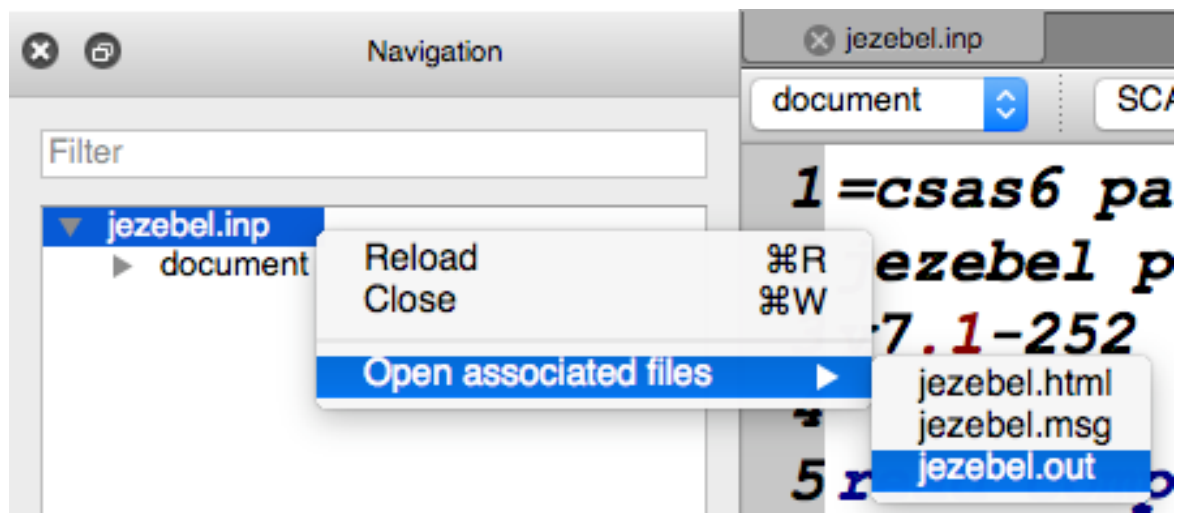

Figure 35. Opening the output file.

The output for this problem consists of the following sections:

- Echo of input

- CSAS information and brief review of input values, cross section processing

- KENO information

This Quickstart section presents a few items in the KENO information section. The first is the information following the line keno messages number k6-123. Click the Find button on the Fulcrum toolbar and enter $k 6-123$. This message states that execution of KENO was terminated due to completion of the specified number of generations. This means that the problem ran to completion based on the generations specified by the user (in this case the default value). The lines below the messages provide information on the neutron lifetime, generation time, nu-bar, average fission group, and energy of the average lethargy causing fission. This information should appear as shown in Figure 36.

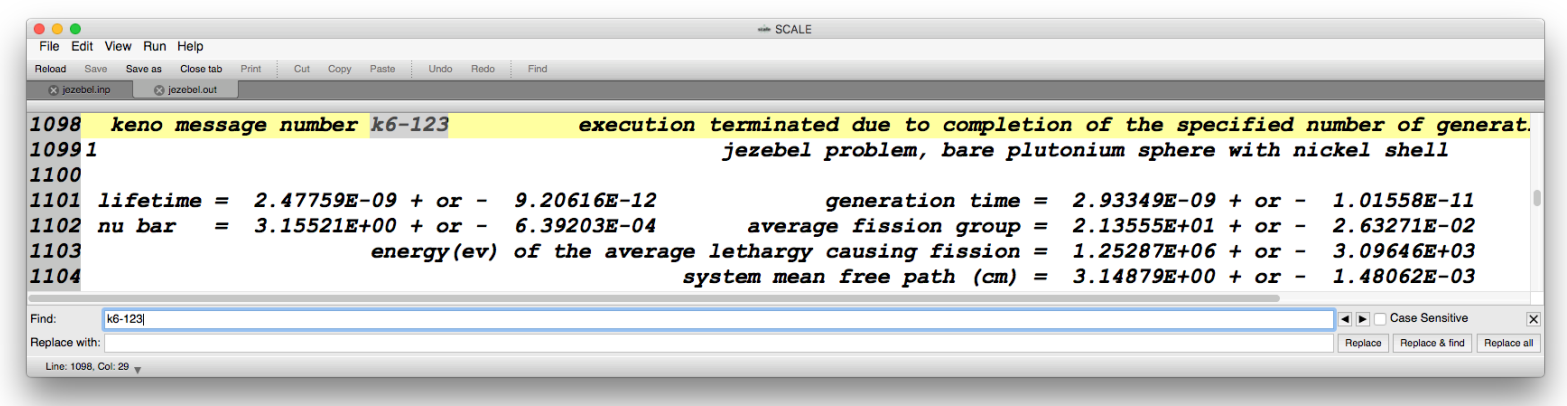

Figure 36. KENO output for Jezebel problem.

Following the neutron parameters table is a table providing the calculated $k_{\text {eff }}$ of the problem vs. the number of initial generations skipped. The final answer for $k_{\text {eff }}$ should be taken from the final results table 
at the end of the output; here the $k_{\text {eff }}$ is labeled as the "best estimate system k-eff." For the example problem, the calculated $k_{\text {eff }}$ is $1.0043 \pm 0.0021$, as shown in Figure 37.

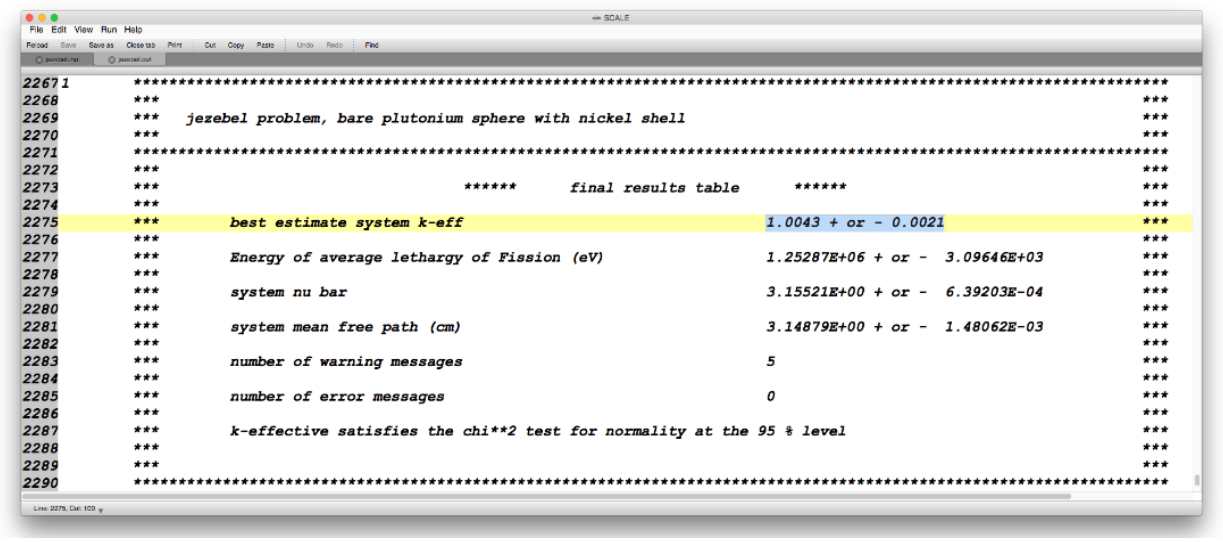

Figure 37. KENO final results table.

\subsection{SUMMARY}

This section has helped you to:

- describe the structure of SCALE/KENO-VI input files and know that there are parts describing the sequence, the materials, and the KENO information;

- use the Fulcrum user interface to create a SCALE/KENO-VI input file;

- set up and run a simple criticality problem using SCALE/KENO-VI; and

- find and interpret $k_{\text {eff }}$ information from your output.

Now that you have successfully run SCALE/KENO-VI, you are ready to learn in detail the options available in each input segment and how to set up more complex problems. The sections that follow present these details in a format similar to that used in this Quickstart section. 


\section{MATERIAL INFORMATION INPUT}

The Quickstart section (Sect. 2), focused on a simple problem with SCALE using the Fulcrum User Interface. From this problem, you gained confidence in using the code and some experience with Fulcrum. This section and subsequent sections provide a more detailed explanation of the commands used in the Quickstart section.

\subsection{WHAT YOU WILL BE ABLE TO DO}

- Define the different criticality sequences used in SCALE.

- Describe the cross section libraries available for criticality safety analyses.

- Use the Fulcrum user interface to provide data on elements, isotopes, compounds, and mixtures of these.

- Interpret basic output information from a SCALE/KENO analysis.

To minimize human error, the SCALE data handling and program flow are automated as much as possible through the use of control modules. These control modules are incorporated into sequences that select the modules required for a particular analysis. For criticality safety work, these CSAS sequences provide automated, problem-dependent, cross section processing, if necessary, for MG calculations, followed by calculation of the neutron multiplication factor for the system being modeled. These control sequences use the cross section processing code CENTRM/PMC to provide resonancecorrected cross sections.

KENO uses the either CE or processed MG cross sections and calculates the $k_{\text {eff }}$ of $3 \mathrm{D}$ system models. The geometric modeling capabilities available in KENO, coupled with the automated cross section processing within the control sequences, allow complex 3D systems to be easily analyzed. The sequences used most often for criticality safety calculations are CSAS5 for KENO V.a calculations and CSAS6 for KENO-VI calculations. This document guides users through the CSAS6 sequence, and [4] is available for CSAS5 users.

\subsection{CROSS SECTION LIBRARIES}

The CSAS sequences (1) use the Standard Composition Library (described later in this section) for specifying the materials and mixtures used in a calculation and (2) provide automatic, problem-dependent cross section preparation prior to the criticality calculation for MG calculations (not necessary for CE calculations). This section describes the cross section libraries most useful for criticality calculations in the SCALE system.

As of the SCALE 6.2.4 release, there are four cross section libraries that are primarily intended for use in criticality safety calculations with SCALE: the (1) CE library based on ENDF/B-VII, (2) the CE library based on ENDF/B-VII.1 (3) the 238-group ENDF/B-VII MG library, and (4) the 252-group ENDF/BVII.1 MG library. There are other cross section libraries available for use in SCALE, but they are not recommended for criticality safety calculations.

When performing calculations with SCALE, the cross section libraries must be referenced using mnemonic identifiers. Each library has multiple acceptable mnemonic names; the names for the criticality safety cross section libraries are listed in Table 1. 
Table 1. SCALE cross section libraries for criticality safety calculations

\begin{tabular}{ll}
\hline \multicolumn{1}{c}{ Mnemonic names } & \multicolumn{1}{c}{ Library } \\
\hline v7-238, v7-238n, v7.0-238n & ENDF/B-VII.0 238-group neutron library \\
v7-252, v7-252n, v7.1-252n & ENDF/B-VII.1 252-group neutron library \\
ce_v7, ce_v7_endf, ce_v7.0_endf & ENDF/B-VII.0 CE library \\
ce_v7.1, ce_v7.1_endf & ENDF/B-VII.1 CE library \\
\hline
\end{tabular}

To perform a KENO calculation with SCALE using the Fulcrum user interface, the user must first generate an input file with an extension that is either .i, .in, or .inp. This is done by clicking the File $>$ New File... button on the upper-left of the screen. Fulcrum will display the New file dialog window. To begin the first material exercise, enter the input file name as uranium_metal.inp, and click the Save button. The entry of the file name is shown in Figure 38 and will result in the blank input file shown in Figure 39.

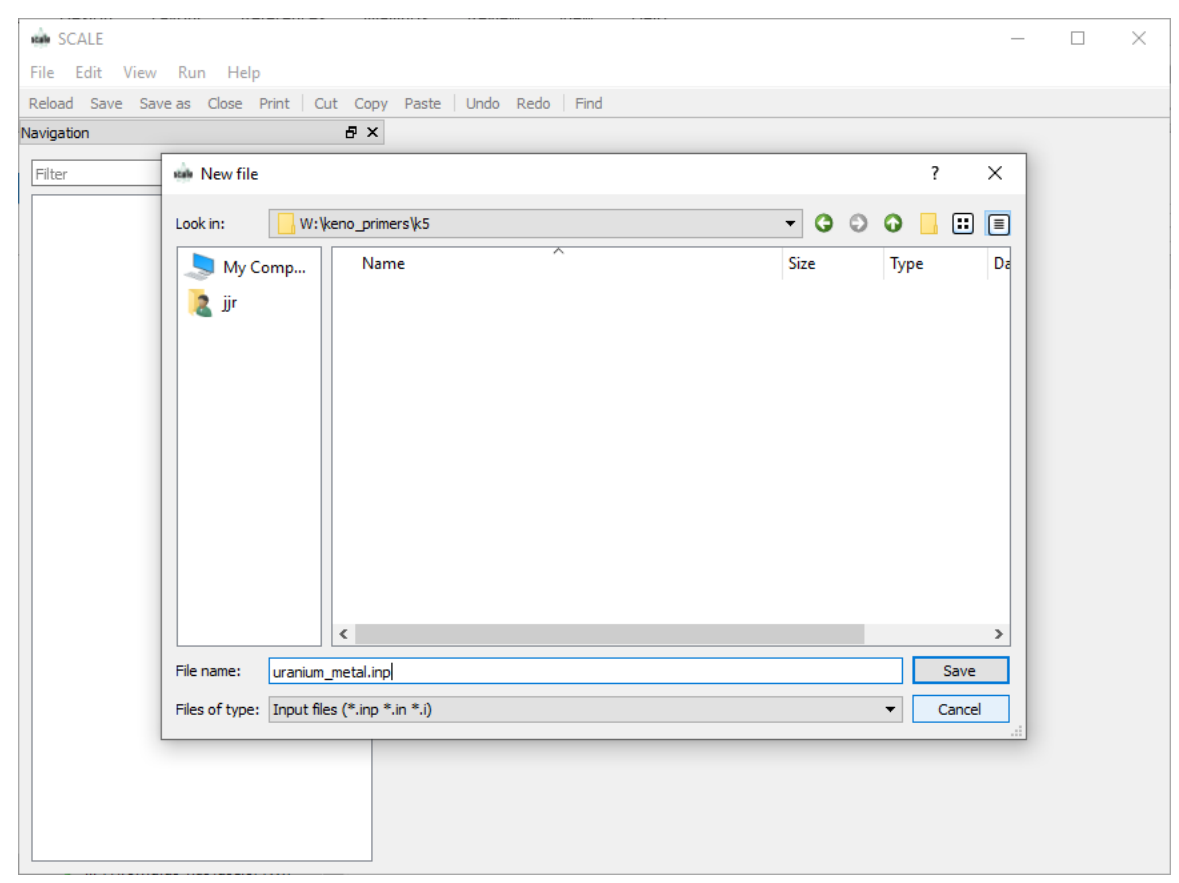

Figure 38. Depiction of the input file name definition. 


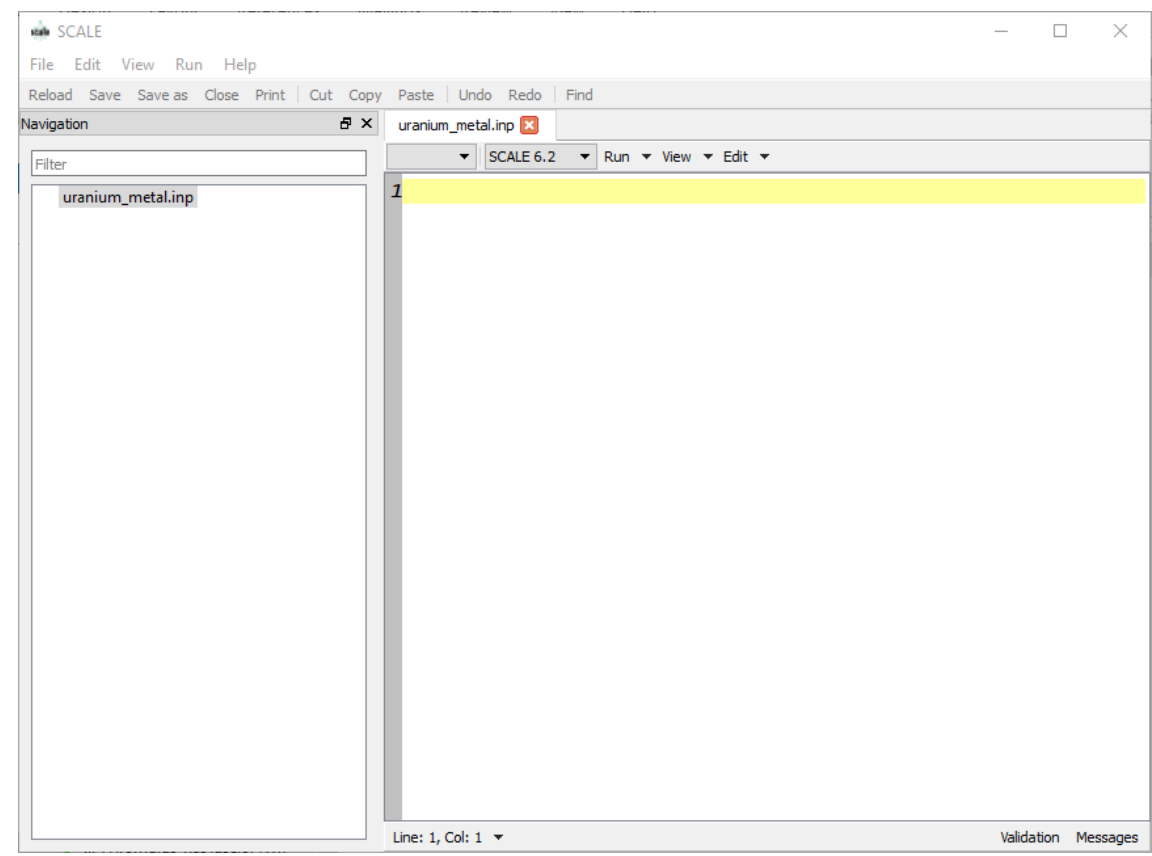

Figure 39. Empty input file after file specification in Fulcrum.

Next, the appropriate sequence must be selected. This is done by pressing CTRL-SPACE inside the input file, which will result in a dropdown menu with all SCALE sequences displayed. Then select the CSAS6 sequence from the dropdown menu and press enter, as shown in Figure 40. Selecting a sequence will result in a CSAS6 input skeleton, as is shown in Figure 41.

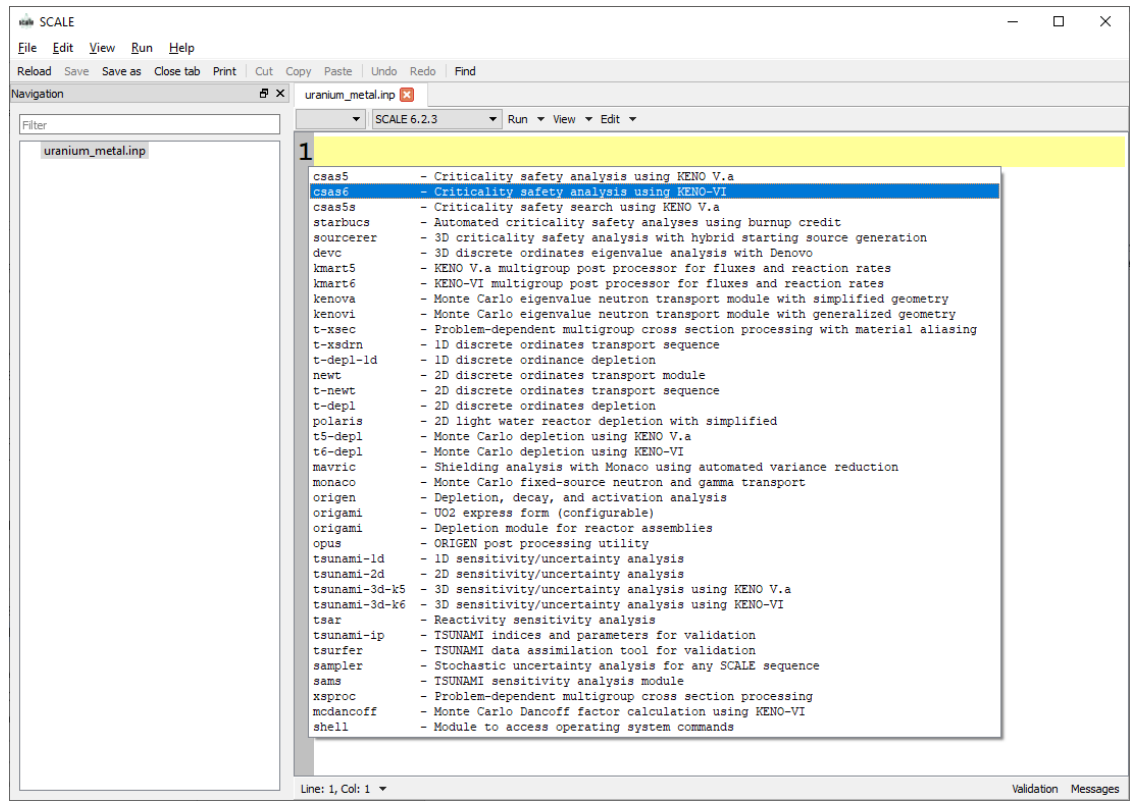

Figure 40. Dropdown menu with the SCALE sequence specification. 


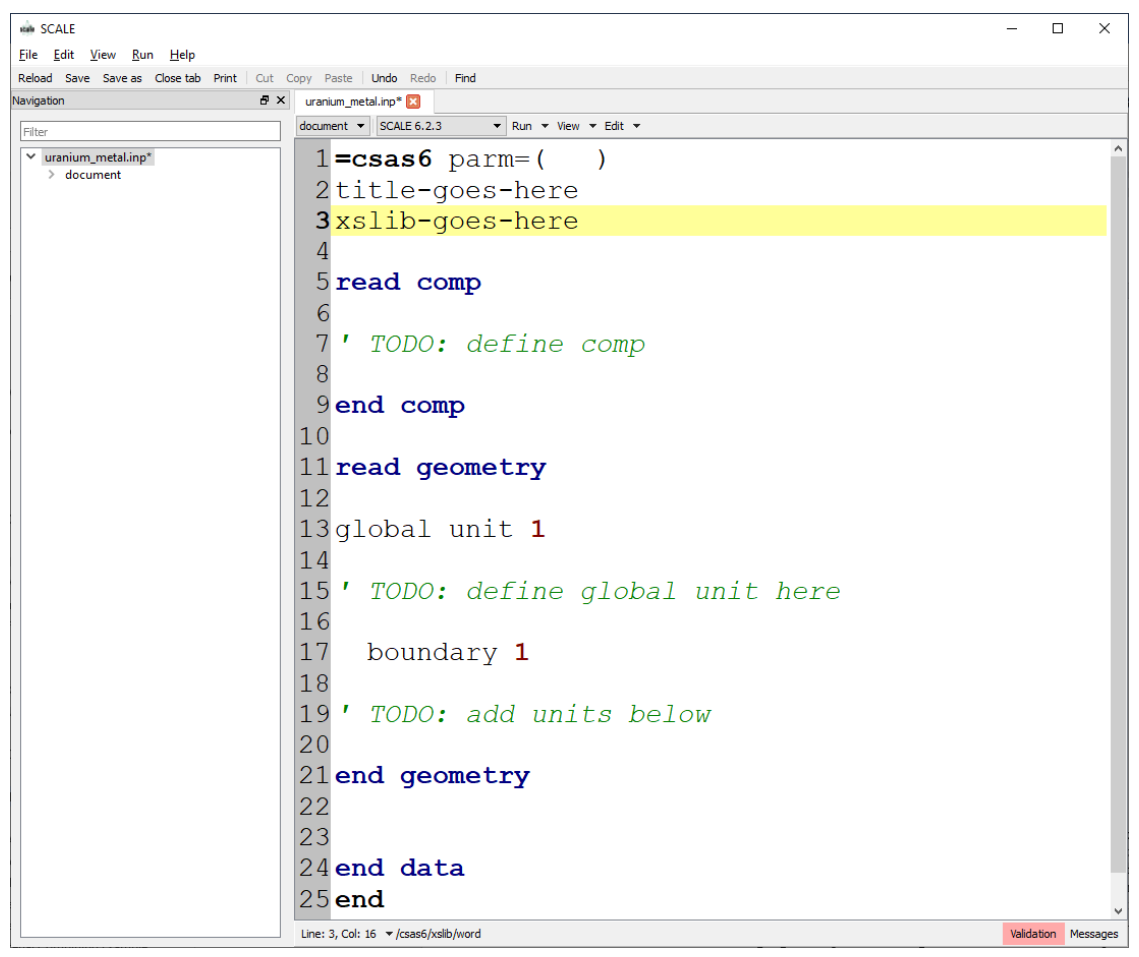

Figure 41. Empty input file resulting from the CSAS6 sequence specification.

Once the empty skeleton is produced, two input placeholders are displayed at the top of the input file on lines 2 and 3. The title of the input is always listed on line 2, which is used to label tables throughout the output. For this example, replace title-goes-here with uranium metal mixture example. The third line is always the cross section library to be used in the calculation. Since cross section processing has not been discussed, select the CE version of the ENDF/B-VII.1 library by replacing xslib-goes-here with ce $v 7.1$ on the third line of input. These modifications to the input file are shown in Figure 42. It can also be seen that the validation tab in the lower right hand portion of the window is highlighted in pink, indicating that there is an error in the input. Clicking on the validation tab opens a pane at the bottom of Fulcrum with the following error message:

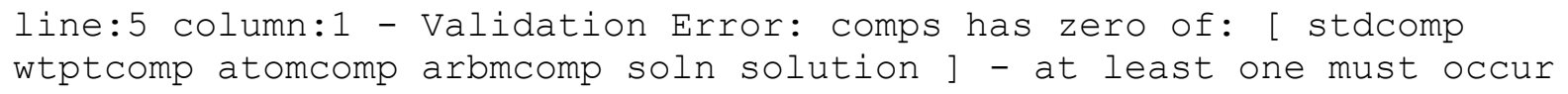

This error message indicates that there are no entries in the composition block. The error message is also shown in Figure 42. 


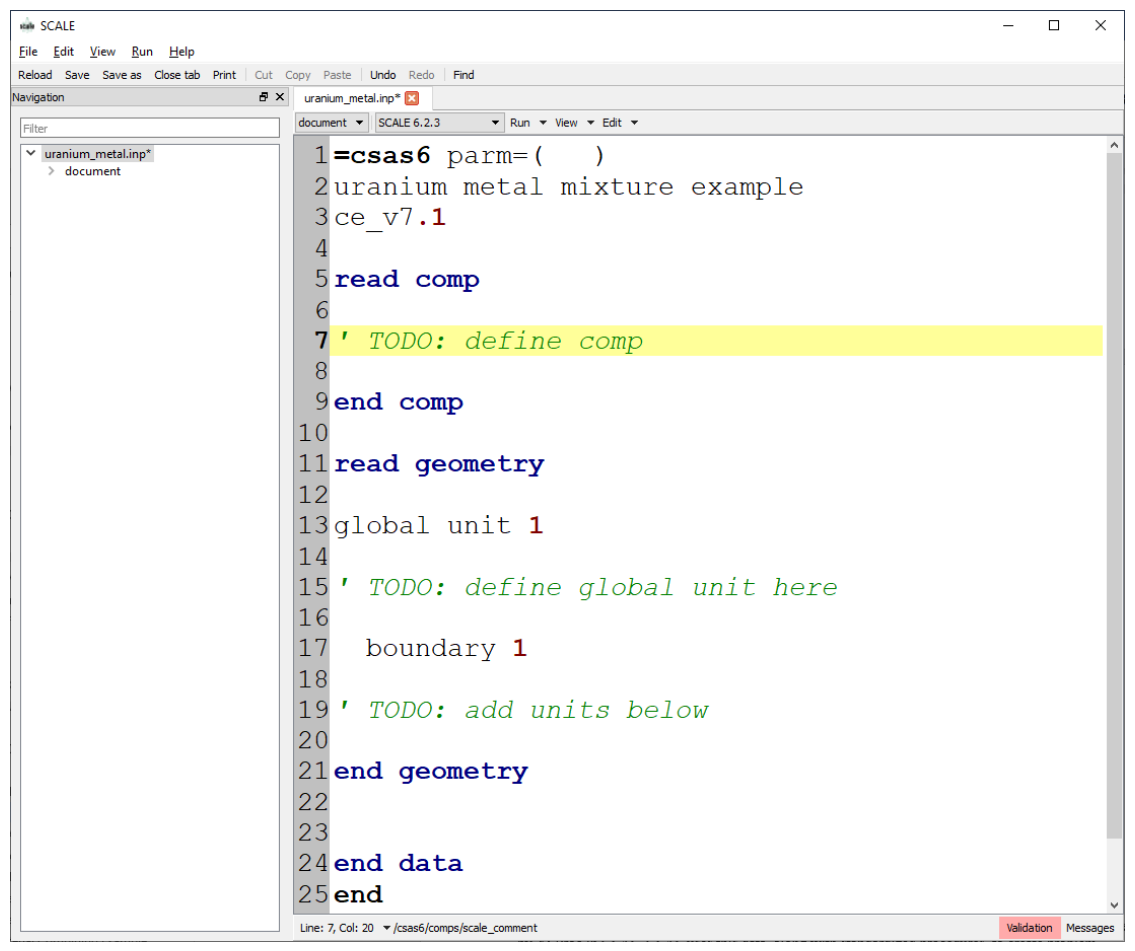

Figure 42. Specification of problem title and cross section library.

\subsection{MATERIAL INPUT}

Information on materials in a system is entered into Fulcrum, which formats the information as required by XSProc in CSAS. CSAS uses this data, along with standardized procedures, to create problemdependent cross section libraries. Input data to Fulcrum identify the materials from the Standard Composition Library and associated physical densities to calculate the number densities (atoms/b-cm) of each material specified in the problem. The number densities are used to develop data used by the downstream codes. These input data include (1) the Standard Composition data used in the standardized number density calculations (a standardized alphanumeric name, mixture number, and other data to define materials, including volume fraction or percent theoretical density, temperature, and isotopic distribution), and (2) the unit cell description defining the materials, dimensions, and boundary conditions of the geometry to be used in the Dancoff factor calculations, the resonance self-shielding calculations, and the flux-weighting cell calculations necessary for cross section processing.

The Standard Composition Library describes the various predefined isotopes, elements (both symbols and full names), compounds, alloys, and other materials that can be used to define the material mixtures for a given problem. A complete description of the materials in the library is found in the SCALE manual [see Standard Composition Library]. The library contains over 600 compounds, alloys, elements, and isotopes that can be used to define the material mixtures for a given problem. Additionally, six fissile solutions are available for which the user can specify the heavy metal, acid, and water components: $\mathrm{UO}_{2}\left(\mathrm{NO}_{3}\right)_{2}, \mathrm{UO}_{2} \mathrm{~F}_{2}$, $\mathrm{Pu}\left(\mathrm{NO}_{3}\right)_{4}, \mathrm{PuF}_{4}, \mathrm{Th}\left(\mathrm{NO}_{3}\right)_{4}$ and $\mathrm{ThF}_{4}$.

When formulating a mixture, it is often necessary to know the density $(\mathrm{g} / \mathrm{cc})$ of the mixture and the weight fractions of the various constituent materials. Note that default densities should not be used for materials containing enriched isotopes, especially light elements with strong absorbers such as boron, $\mathrm{B}_{4} \mathrm{C}$, or lithium. The temperature of a given material can be entered (the default is $293 \mathrm{~K}$, which is room 
temperature). The temperature is used to correctly process resonance data, Bondarenko data, and/or thermal-scattering data. The temperature of a mixture is always specified in Kelvin (K) in SCALE.

\subsection{EXAMPLE MATERIAL SPECIFICATIONS}

Five example problems are used here to illustrate the method for entering material information into Fulcrum. These problems demonstrate composition entries for pure ${ }^{235} \mathrm{U}$ metal, $\mathrm{U}(93.7)$ metal, $\mathrm{U}(93.7) \mathrm{O}_{2}$, $\mathrm{U}(30.3) \mathrm{O}_{2} \mathrm{~F}_{2}$ solution, and a mixture of $\mathrm{UO}_{2}$ and water.

\subsection{1 $\quad{ }^{235} \mathrm{U}$ Metal Composition Input}

The first material will be used to model a bare metal cylinder in Section 4.2.1. This section also uses the input generated in Section 3.2 as a starting point.

Pure ${ }^{235} \mathrm{U}$ is modeled first to demonstrate the use of Fulcrum to enter a single isotope. The density and material composition of the material is shown below.

Core Material $\left(\rho_{\text {mix }}=18.742 \mathrm{~g} / \mathrm{cc}-\right.$ mixture $)$

$\mathrm{U}$ metal (100 percent $\left.{ }^{235} \mathrm{U}\right)$

Temperature of $300 \mathrm{~K}$

\subsubsection{Material input}

To define the uranium metal composition. Move the cursor into the read comp block and press CTRLSPACE to generate the dropdown menu shown in Figure 43.

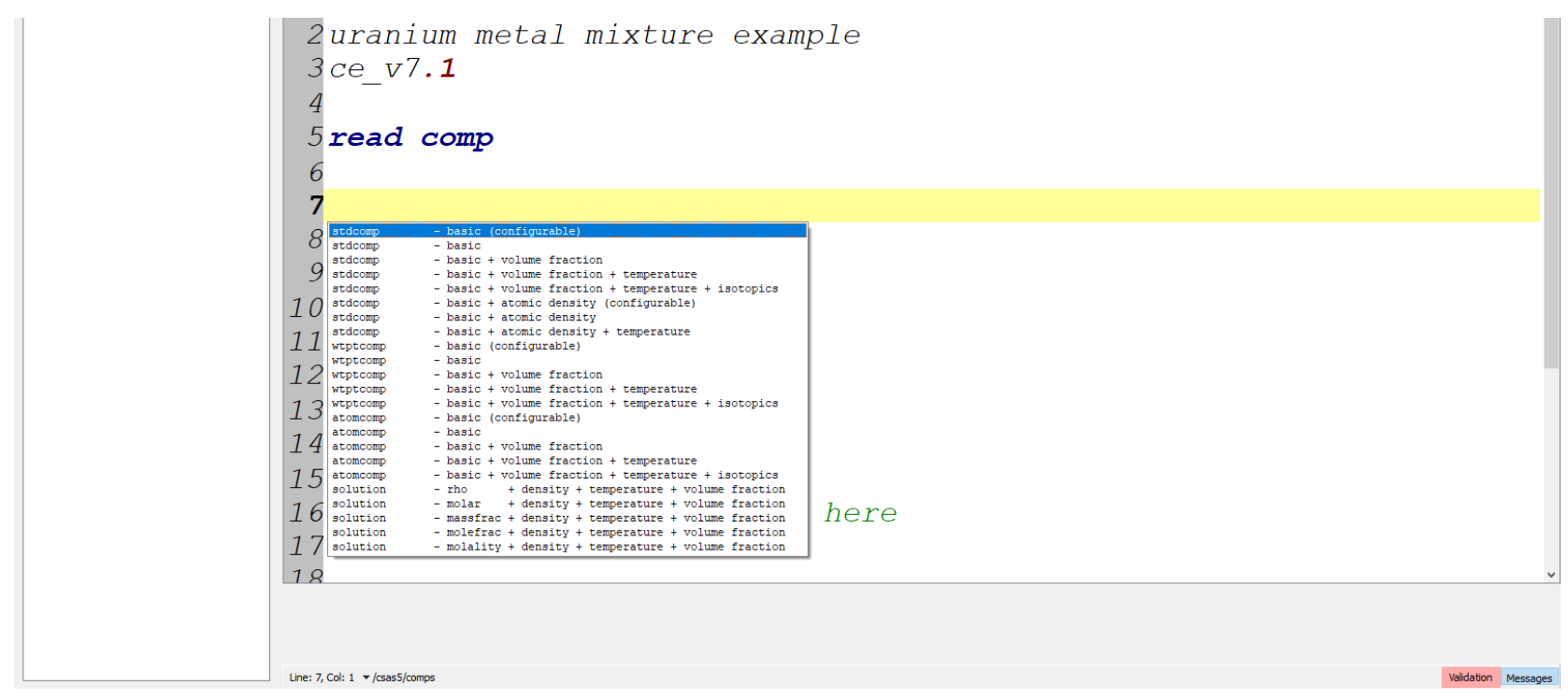

Figure 43. Accessing the basic standard composition configurable.

From the dropdown menu, select stdcomp - basic (configurable). This will generate a fillable form, as shown in Figure 44. 


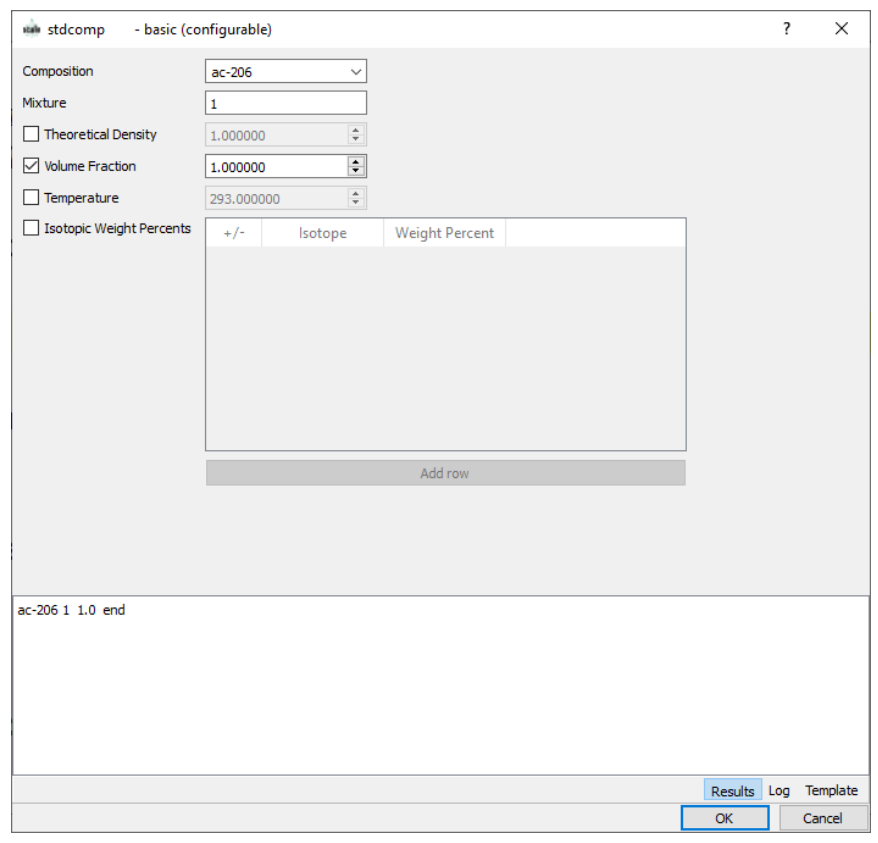

Figure 44. Basic standard composition configurable input initial display.

In the composition dropdown, clicking on the field that contains ac-206 and type $u-235$ over it. Then click the Theoretical Density checkbox and entering the value of 18.742 in place of the default value of 1.0 . Because the material is pure ${ }^{235} \mathrm{U}$, and because we have already specified the density, the default Volume Fraction of 1.0 is appropriate. Modify the temperature by clicking the Temperature box and entering a value of 300 . The final configurable window is shown in Figure 45.

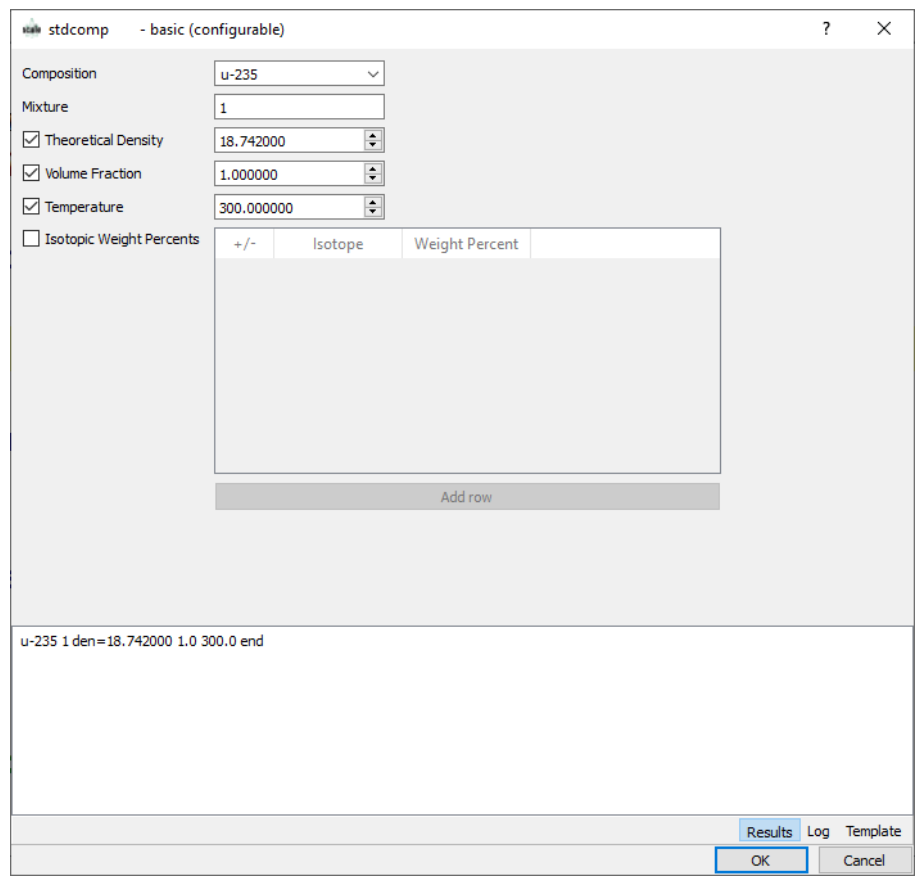

Figure 45. Basic standard composition configurable input, initial display filled out. 
Confirm that the data entry is correct before clicking $\mathbf{O K}$, as the data cannot be modified in the configurable form after it has been entered into the input. Once you have clicked $\mathbf{O K}$ at the bottom of the configurable form, the input file should look like that shown in Figure 46.

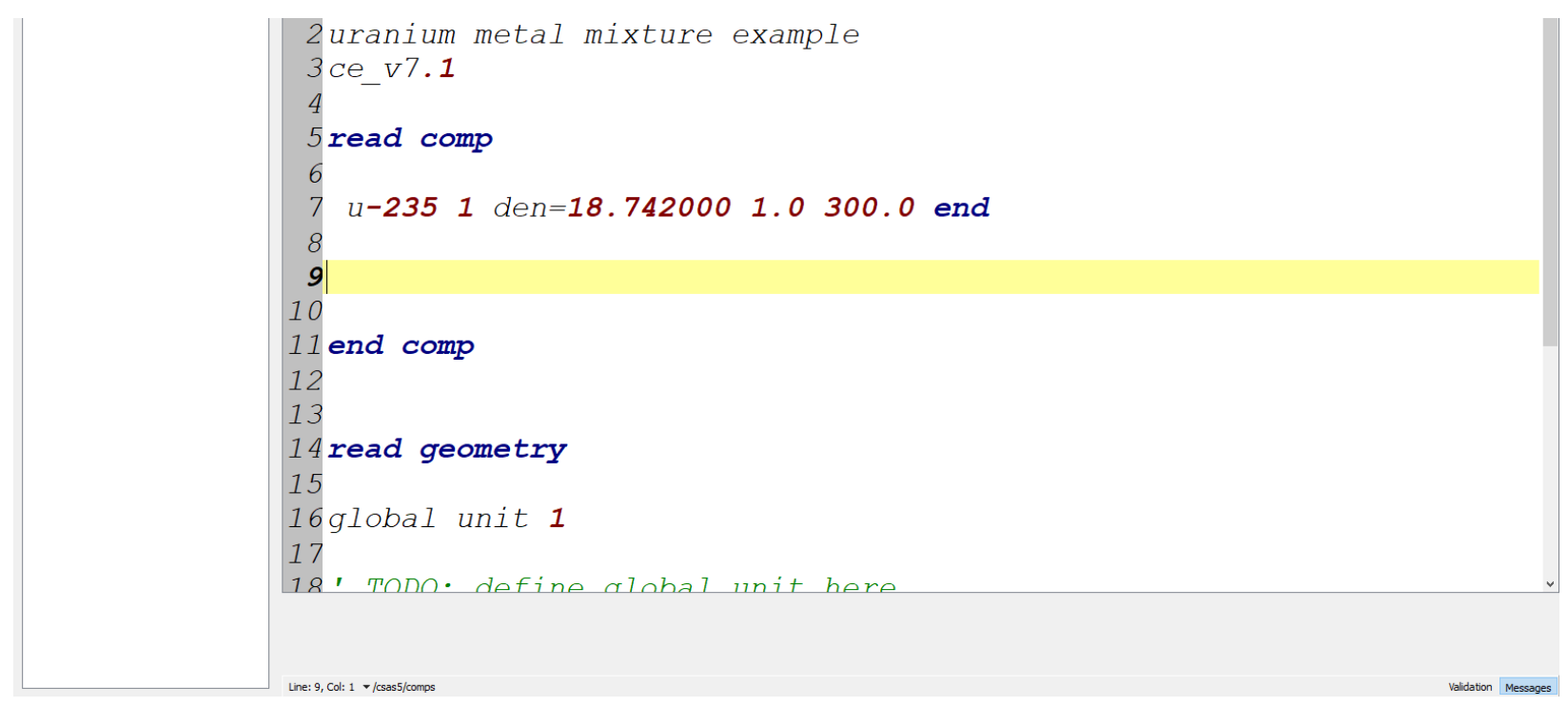

Figure 46. Input result of the standard basic composition configurable input.

\subsubsection{Mixing table output}

At this point there is not enough input specified to perform a KENO-VI calculation, but the atom densities can be generated based on the standard composition input supplied. To do this, generate a MIXING TABLE with CSAS by clicking the downward pointing triangle next to the Run button at the top of the Fulcrum interface and selecting Mixing table from the dropdown menu. A picture of the dropdown menu is provided in Figure 47.

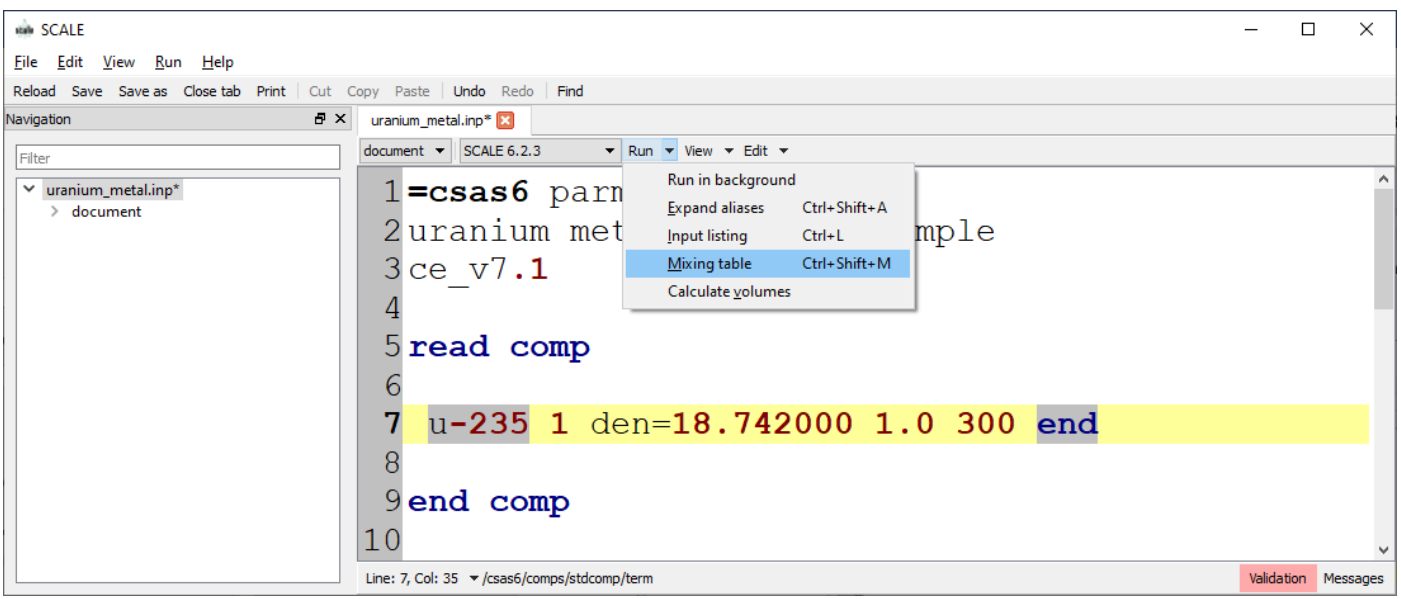

Figure 47. Dropdown menu necessary to create mixing table.

Upon executing the mixing table calculation, the Messages tab at the bottom right-hand corner of the Fulcrum panel should illuminate. Clicking on the Messages tab will reveal the mixing table results, as shown in Figure 48 (enlarged for readability). The resulting mixing table includes the mixture number, mixture temperature, and mixture density, along with the atom density and the nuclide identifier (92235). Geometric specification of problems is covered in Section 4. 


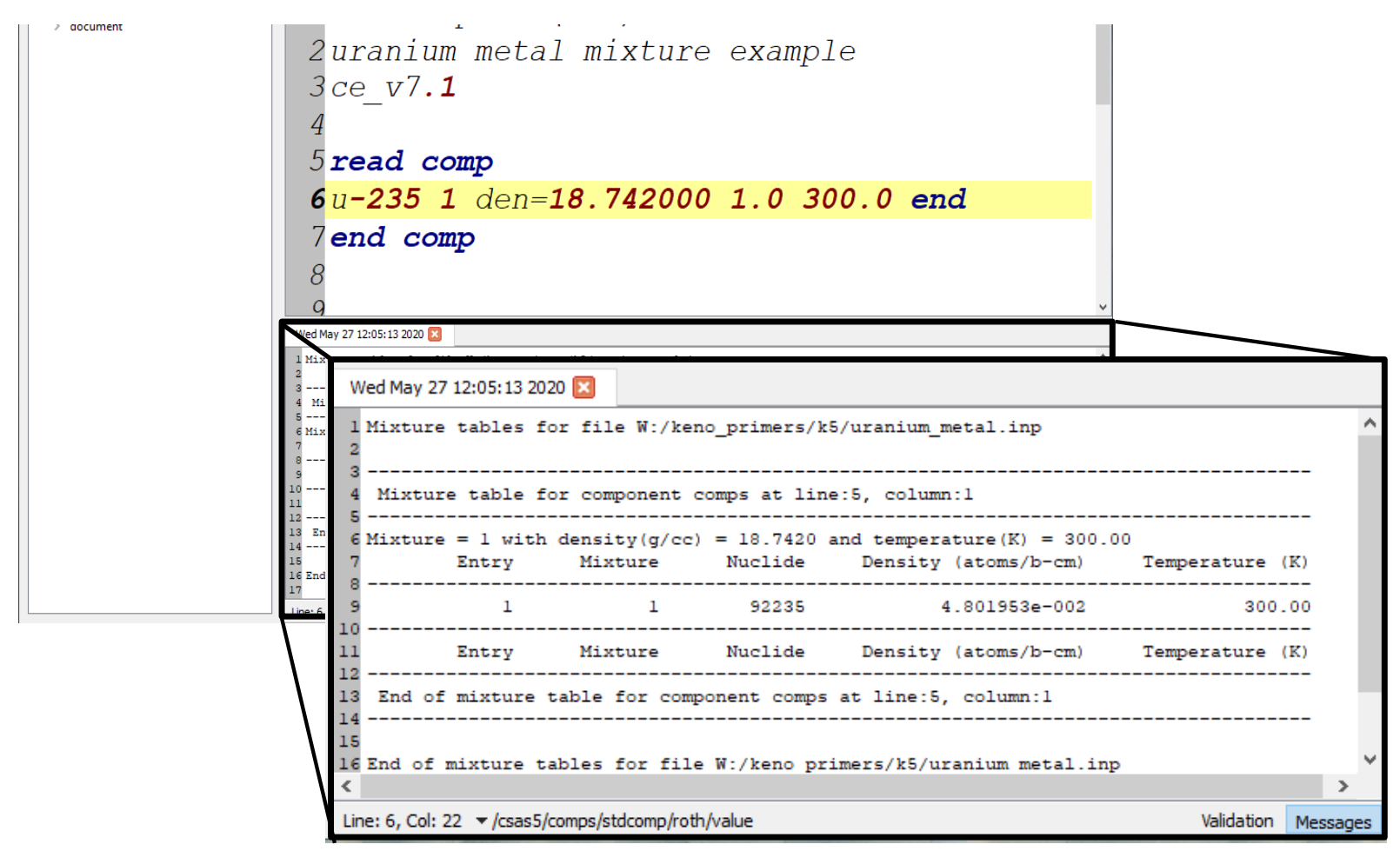

Figure 48. Mixing table results for the uranium metal example.

\subsubsection{U(93.71) Metal Material}

This example changes from material used from pure ${ }^{235} \mathrm{U}$ metal to $U$ metal enriched to $93.71 \mathrm{wt} . \%{ }^{235} \mathrm{U}$. Whereas the first example was composed of a single isotope, this example is a combination of isotopes from the same element. This example can still be specified with a single standard basic composition input, but the isotopic distribution must be modified. For this example, the density of the core material is again $18.742 \mathrm{~g} / \mathrm{cm}^{3}$. Relevant material specification information is as follows:

Core Material $\left(\rho_{\text {mix }}=18.742 \mathrm{~g} / \mathrm{cc}-\right.$ mixture $)$

U metal (93.71 wt.\% ${ }^{235} \mathrm{U}$ and 6.29 wt. $\left.\%{ }^{238} \mathrm{U}\right)$

Temperature of $300 \mathrm{~K}$

\subsubsection{Material input}

As in the ${ }^{235} \mathrm{U}$ metal problem, generate a CSAS6 input skeleton from Fulcrum and specify an appropriate title and cross section library. For this example, replace title-goes-here with enriched uranium metal mixture example.

To define the enriched uranium metal composition, move the cursor into the read comp block, and press CTRL-SPACE to generate the dropdown menu shown in Figure 43. From the dropdown menu, select 
again stdcomp - basic (configurable). This will generate a fillable form, as was shown in Figure 44. This time in the composition dropdown, select the elemental input $u$ by clicking on the blank with ac206 in it and typing over it. Next, adjust the density by clicking Theoretical Density checkbox and entering the value of 18.742 in place of the default value of 1.0 . Because the material is pure $U$, and we have already specified the density, the default Volume Fraction of 1.0 is appropriate. The temperature of the material is also modified by clicking the Temperature box and entering a value of 300. To specify the isotopic composition of the material, click the Isotopic Weight Percents box and then click the Add row button twice. At this point, you should have a configurable form that looks like that shown in Figure 49. Note that you may have to expand the window to see the Add row button.

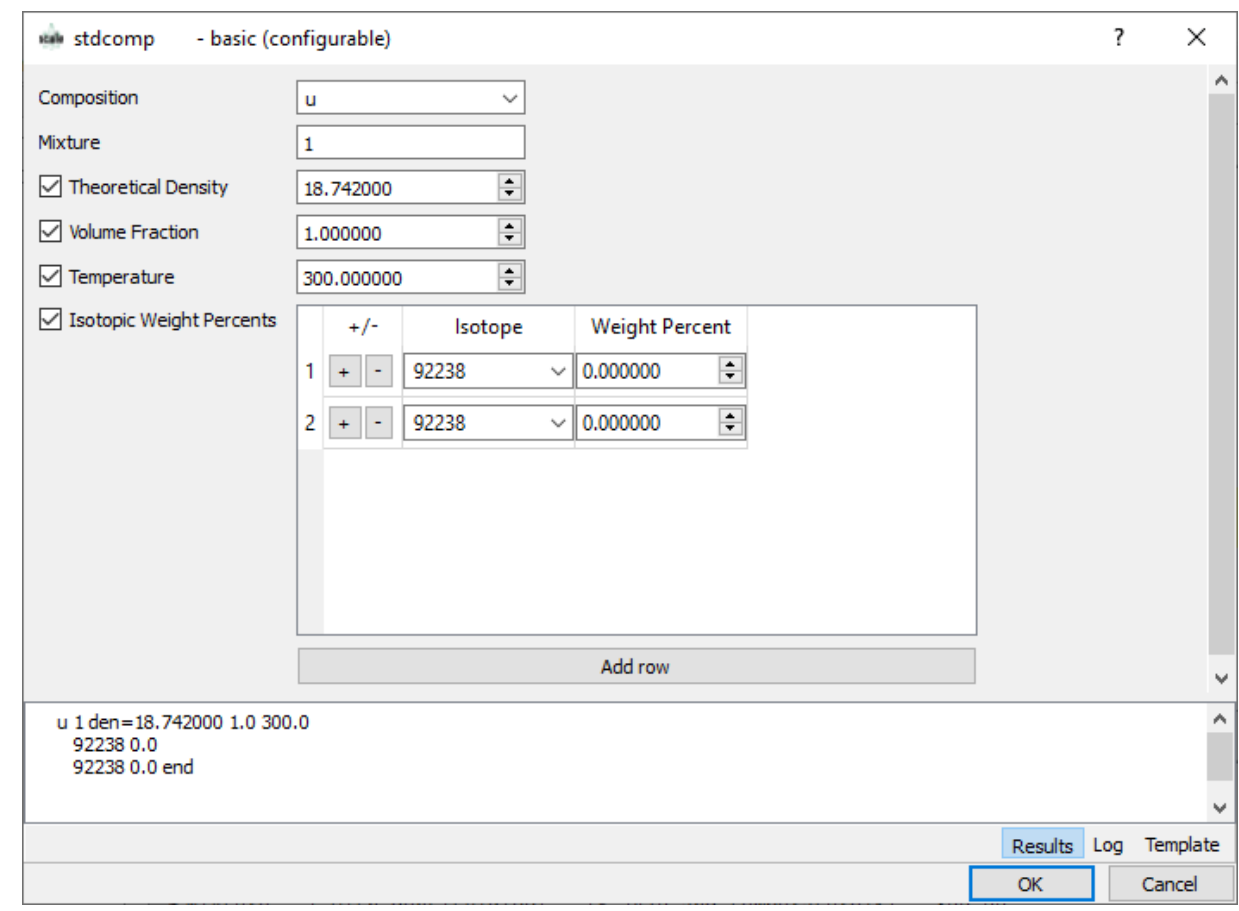

Figure 49. Isotopic composition input prior to user modification.

Now you should select one of the $\mathbf{9 2 2 3 8}$ values listed under the Isotope column, and change the value to the nuclide identifier for ${ }^{235} \mathrm{U}$, which is 92235 . Next, enter the isotopic weight percentages in the Weight Percent column by clicking on the box with the $\mathbf{0 . 0 0 0 0 0 0}$ value inside and over writing it with 93.71 for the ${ }^{235} \mathrm{U}$ entry and 6.29 for ${ }^{238} \mathrm{U}$. The final configurable window is shown in Figure 50. 


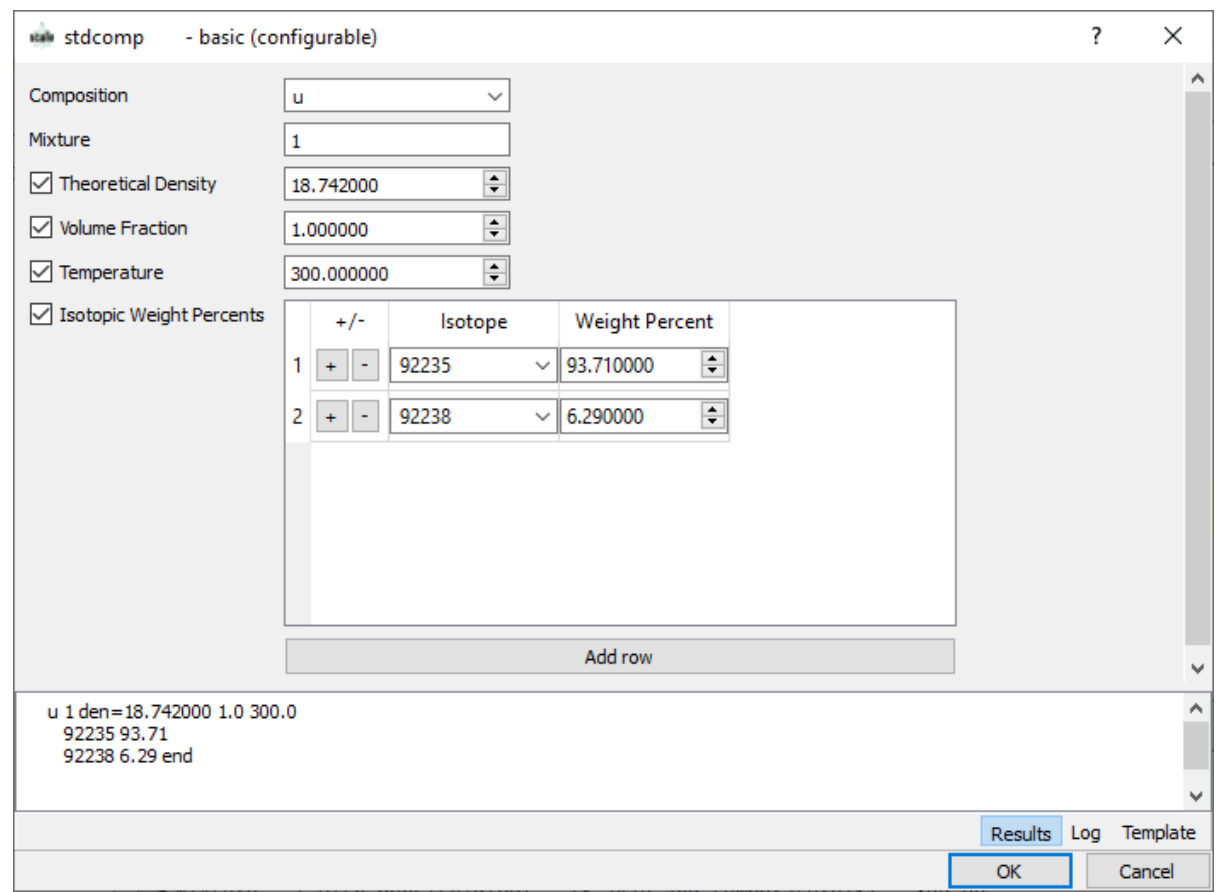

Figure 50. Isotopic composition input following user modification for the enriched uranium metal case.

Confirm that the data entry is correct before clicking $\mathbf{O K}$, as the data cannot be modified in the configurable form after it has been entered into the input. Once you have clicked $\mathbf{O K}$ at the bottom of the configurable form, the input file should look like that shown in Figure 51.

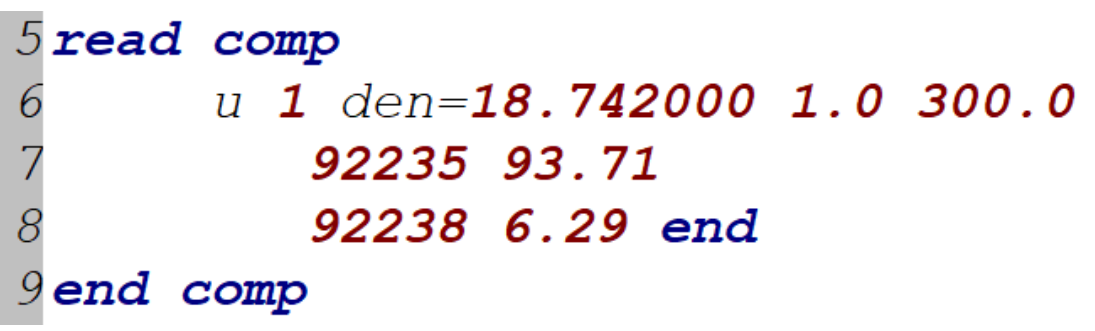

Figure 51. Final composition input for the enriched uranium metal case.

\subsubsection{Mixing table output}

At this point there is not enough input specified to perform a KENO-VI calculation, but the atom densities can be generated based on the standard composition input supplied. To do this, generate a MIXING TABLE with CSAS by clicking the downward pointing triangle next to the Run button at the top of the Fulcrum interface and selecting Mixing table from the dropdown menu. A picture of the dropdown menu for the ${ }^{235} \mathrm{U}$ metal example is provided in Figure 47.

Upon executing the mixing table calculation, the Messages tab at the bottom right-hand corner of the Fulcrum panel should illuminate. Clicking on the Messages tab will reveal the mixing table results, which are shown in Figure 52 (enlarged for readability). The resulting mixing table includes the mixture number, the mixture temperature, and the mixture density, along with atom density and nuclide identifiers (92235 and 92238). Geometric specification of problems is covered in Section 4. 


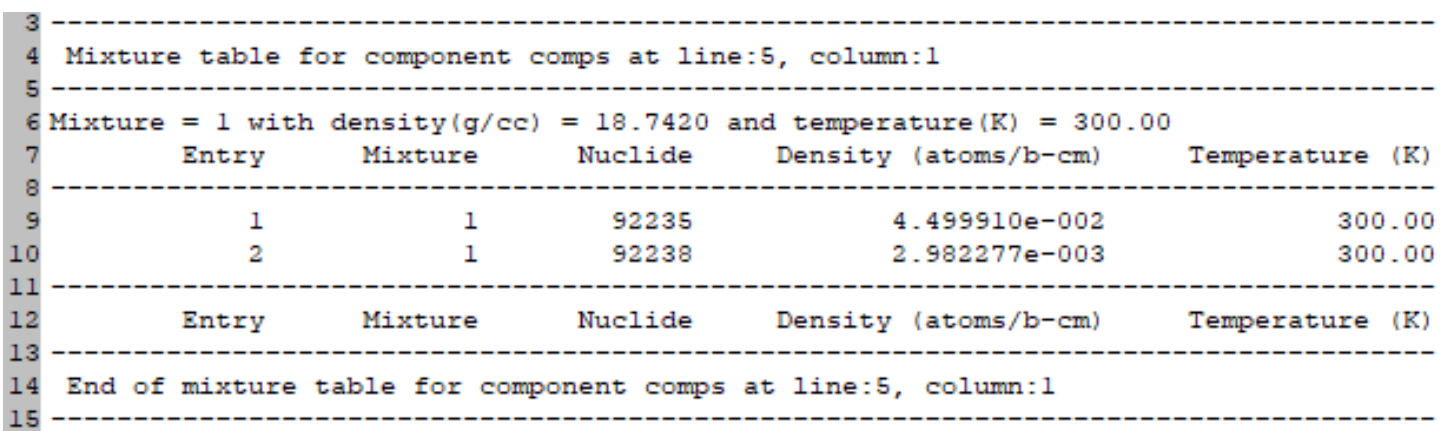

Figure 52. Mixing table results for the uranium metal example.

\subsubsection{Highly Enriched $\mathrm{UO}_{2}$}

This example changes the material from uranium metal to uranium dioxide while keeping the enrichment at $93.71 \%{ }^{235} \mathrm{U}$. The core material has a density of $95 \%$ of the theoretical density of $\mathrm{UO}_{2}$; the theoretical density is $10.96 \mathrm{~g} / \mathrm{cc}$.

Core Material $\left(\rho_{\text {mix }}=95 \%\right.$ of theoretical density $\left.-\mathrm{TD}=10.96 \mathrm{~g} / \mathrm{cc}-\mathrm{mixture}\right)$

$\mathrm{U}\left(93.71\right.$ wt. $\%{ }^{235} \mathrm{U}$ and 6.29 wt. $\left.\%{ }^{238} \mathrm{U}\right)$

It is suggested that you create a new file for this problem and change the title of the problem to highly enriched uranium oxide example.

\subsubsection{Material input}

Once you have the same input skeleton that was used in the previous examples, the first step is to define the uranium oxide composition. Move the cursor into the read comp block and press CTRL-SPACE to generate the dropdown menu similar to what is shown in Figure 43 above. From the dropdown menu, select stdcomp - basic (configurable). This will generate a configurable form, as shown in Figure 44. In the composition dropdown, select uo2 by clicking on the blank with ac-206 and typing over it. Next, adjust the density by either changing the default density or by changing the volume fraction. Since changing the default density has already been demonstrated and the material is specified as a fraction of theoretical density, the volume fraction is modified here. The volume fraction, also known as the density multiplier, is modified by clicking the box next to Volume Fraction and entering a value of 0.96 in place of $\mathbf{1 . 0 0 0 0 0}$ in the adjacent box. Modifying the Volume Fraction to 0.96 results in a density of 0.96 * 10.96, or $10.412 \mathrm{~g} / \mathrm{cm}^{3}$. For this example, leave the temperature of the material at the default value of 293 K. To specify the isotopic composition of the material, click the Isotopic Weight Percents box, and then click the Add row button twice. Note that you may have to expand the configurable window to see the Add row button. Now select one of the 92238 values listed under the Isotope column, and change this value to the nuclide identifier for ${ }^{235} \mathrm{U}$, which is 92235 . Next, enter the isotopic weight percentages into the Weight Percent column by clicking on the box with the $\mathbf{0 . 0 0 0 0 0 0}$ value inside and overwriting it with 93.71 for the ${ }^{235} \mathrm{U}$ entry, and enter 6.29 for ${ }^{238} \mathrm{U}$. The final configurable window is shown in Figure 53. Confirm that the data entry is correct before clicking $\mathbf{O K}$, as the data cannot be modified in the configurable form after it has been entered into the input. After clicking $\mathbf{O K}$ at the bottom of the configurable form, the input file should look like that shown in Figure 54. 


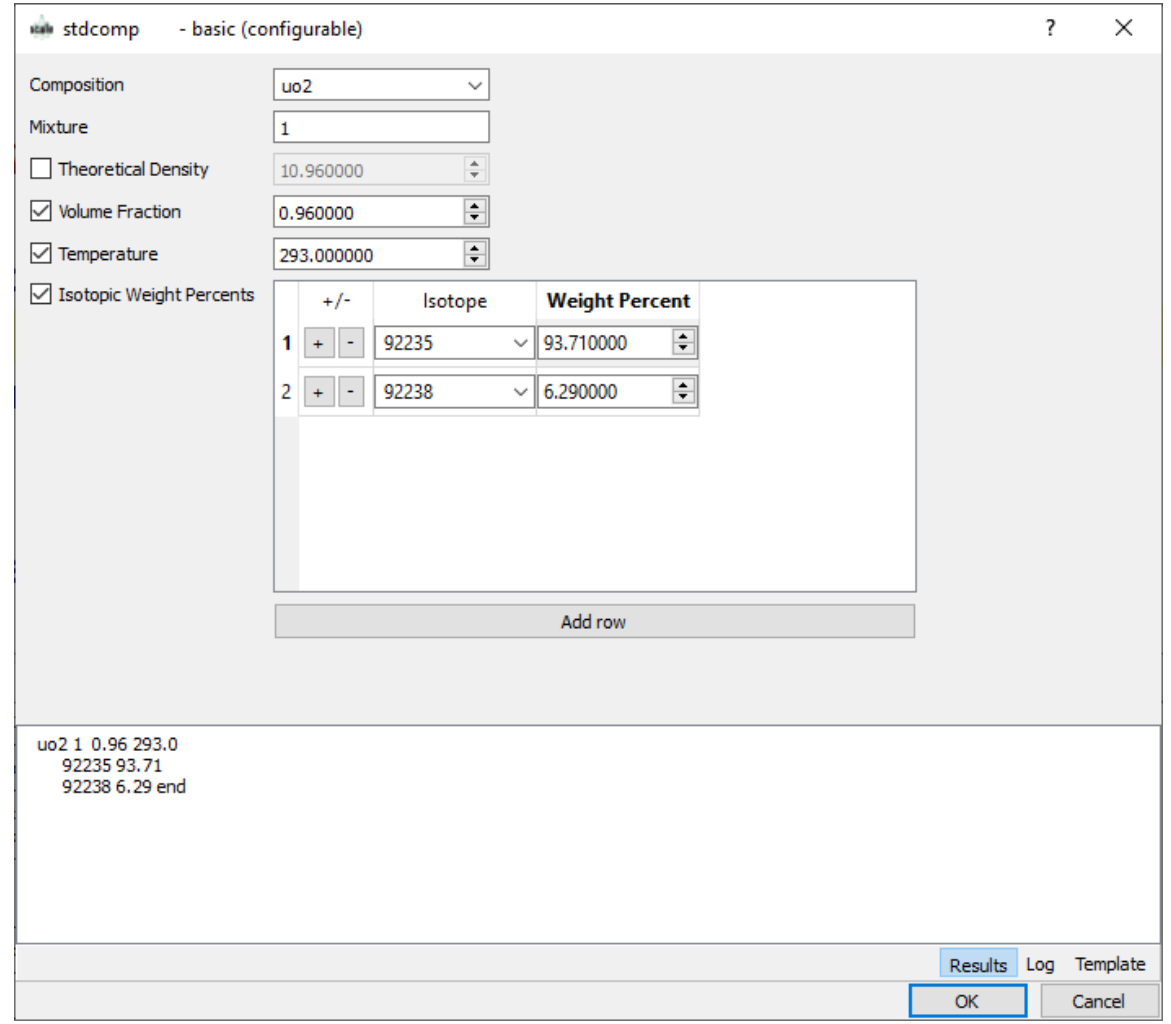

Figure 53. Isotopic composition input following user modification for the enriched uranium oxide case.

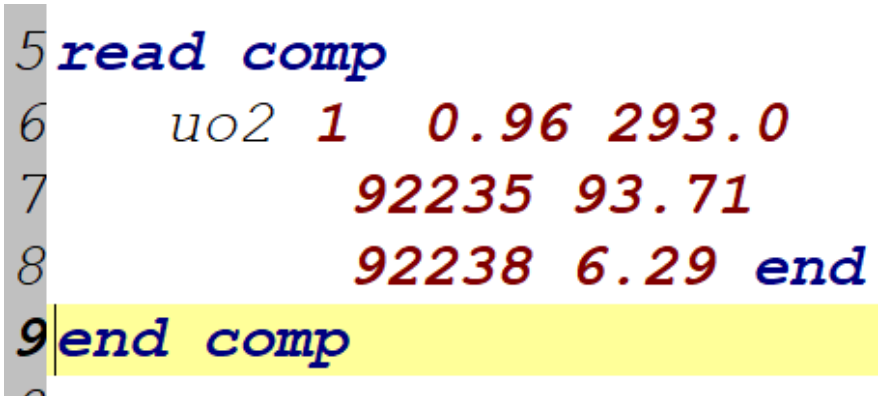

Figure 54. Final composition input for the enriched uranium oxide case.

\subsubsection{Mixing table output}

As encountered in the first two examples, there is not enough input specified to perform a KENO-VI calculation, but the atom densities can be generated based on the standard composition input supplied. Click the downward pointing triangle next to the Run button at the top of the Fulcrum interface and select Mixing table from the dropdown menu. A picture of the dropdown menu for the uranium oxide example is provided in Figure 47.

Upon executing the mixing table calculation, the Messages tab at the bottom right-hand corner of the Fulcrum panel will illuminate. Clicking on the Messages tab will reveal the mixing table results. The mixing table results are shown in Figure 55 (enlarged for readability). The resulting mixing table includes the mixture number, the mixture temperature, and the mixture density, along with the atom density and the nuclide identifiers $(92235,92238,8016,8017$, and 8018). 


\begin{tabular}{|c|c|c|c|c|c|}
\hline Mixture & $\begin{array}{l}=1 \text { with } \\
\text { Entry }\end{array}$ & $\begin{array}{l}\text { density }(g / c c) \\
\text { Mixture }\end{array}$ & $\begin{array}{l}=10.5216: \\
\text { Nuclide }\end{array}$ & $\begin{array}{l}\text { Ind temperature }(\mathrm{K})=293.00 \\
\text { Density (atoms/b-cm) }\end{array}$ & Temperature (K) \\
\hline & 1 & 1 & 8016 & $4.730657 e-002$ & 293.00 \\
\hline & 2 & 1 & 8017 & $1.802009 e-005$ & 293.00 \\
\hline & 3 & 1 & 8018 & $9.721476 \mathrm{e}-005$ & 293.00 \\
\hline & 4 & 1 & 92235 & $2.223716 \mathrm{e}-002$ & 293.00 \\
\hline & 5 & 1 & 92238 & $1.473748 \mathrm{e}-003$ & 293.00 \\
\hline & Entry & Mixture & Nuclide & Density (atoms/b-cm) & Temperature (K) \\
\hline
\end{tabular}

Figure 55. Mixing table results for the highly enriched $\mathrm{UO}_{2}$ case.

\subsubsection{Highly Enriched $\mathrm{U}(30.3) \mathrm{O}_{2} \mathrm{~F}_{2}$ Material}

This example demonstrates the method used to enter material composition information when atomic number density information has been calculated outside of SCALE. This example uses a $\mathrm{U}(30.3) \mathrm{O}_{2} \mathrm{~F}_{2}$ solution from Critical Dimensions of Systems Containing ${ }^{235} U,{ }^{239} U$, and ${ }^{233} U$ (LA-10860) [5] as the example. Later, number densities for a solution will be determined using SCALE's solution input capability. However, the information provided in LA-10860 is formatted in a manner that is not convenient for entry into that method. For now, it is assumed that the number densities of the nuclides in the solution are known to be as follows:

$$
\begin{array}{ll}
\mathrm{N}_{\mathrm{H}} & =0.0597522 \text { atoms-H/b-cm } \\
\mathrm{N}_{\mathrm{O}} & =0.0335605 \text { atoms}-\mathrm{O} / \mathrm{b}-\mathrm{cm} \\
\mathrm{N}_{\mathrm{F}} & =0.0036844 \text { atoms-F/b-cm } \\
\mathrm{N}_{\mathrm{U}-235} & =0.0005637 \text { atoms }-{ }^{235} \mathrm{U} / \mathrm{b}-\mathrm{cm} \\
\mathrm{N}_{\mathrm{U}-238} & =0.0012802 \text { atoms }{ }^{238} \mathrm{U} / \mathrm{b}-\mathrm{cm}
\end{array}
$$

It is suggested that a new file be created for this problem with the title of the problem changed to UO2F2 number density example.

\subsubsection{Material input}

Once you have the same input skeleton that was used in the previous examples, the first step is to define the $\mathrm{UO}_{2} \mathrm{~F}_{2}$ composition. Move the cursor into the read comp block and press CTRL-SPACE to generate the dropdown menu shown in Figure 56. This example will be implemented using the place-holder method of material entry rather than with the configurable forms method that was used in the previous examples. From the dropdown menu, select stdcomp - basic + atomic density. This will place the text shown in Figure 57 into the input file. This text is correctly formatted to produce an atomic density input, but it is generic and must be updated to fit the specifics of the problem. To do this, the text should be altered to change the $\mathbf{0 . 9 8 3 2 5 2}$ atomic density value found in the autocompleted template with 0.0005637 . Next, the line of text that has just been constructed should be copied to four additional lines of input — one for each nuclide in the composition - to accommodate the other constituent materials in the mixture. Those lines should then be modified to have the appropriate elemental or nuclide symbols and atomic densities. It is also necessary for each of the lines to have the same composition numbers, because this links them together in the input processor as a single mixture. The Volume Fraction is set to zero in all cases: this signals SCALE that the information that follows is an atomic density rather another type of input. The final material input should look like Figure 58. 


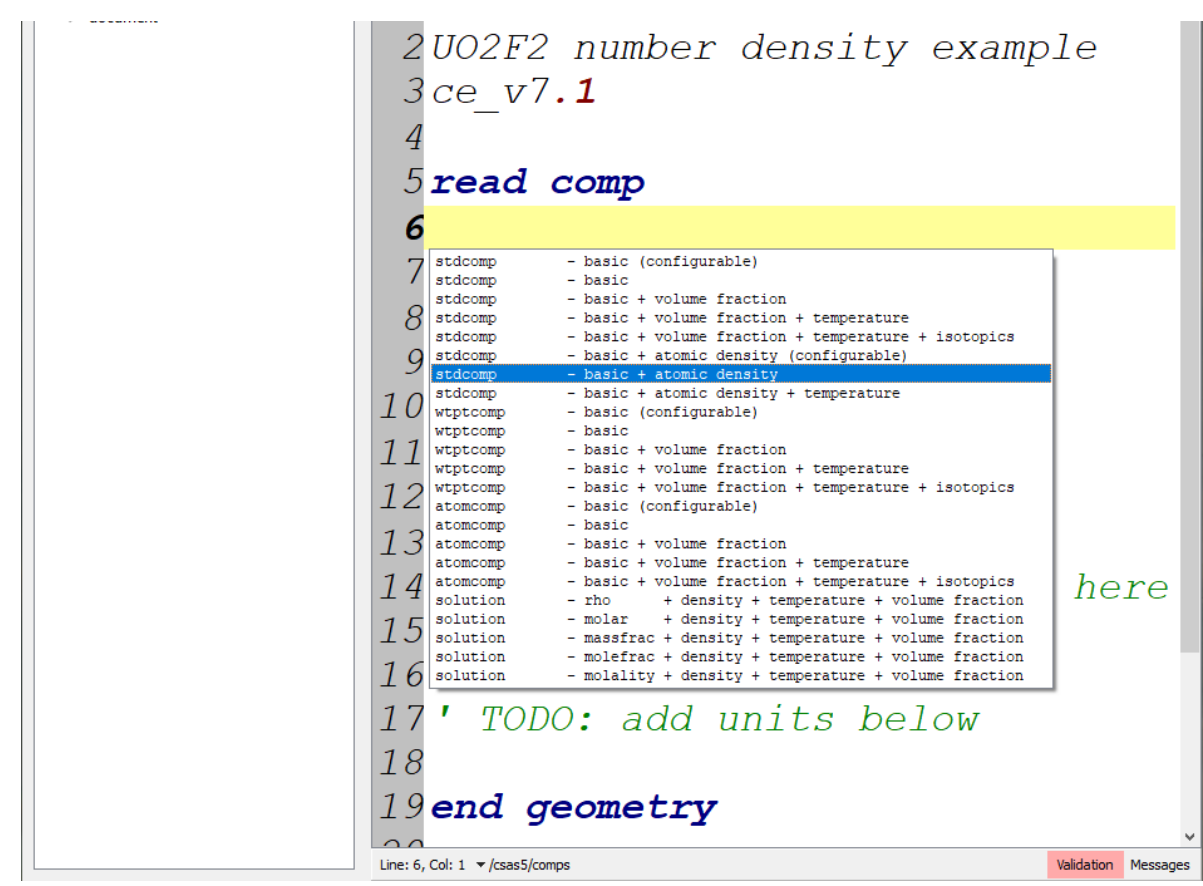

Figure 56. Accessing the basic standard composition configurable.

\section{5 read comp \\ $6 u-23510.0 \quad 0.983252$ end \\ 7 end comp}

Figure 57. Autocompleted template for atomic density input.

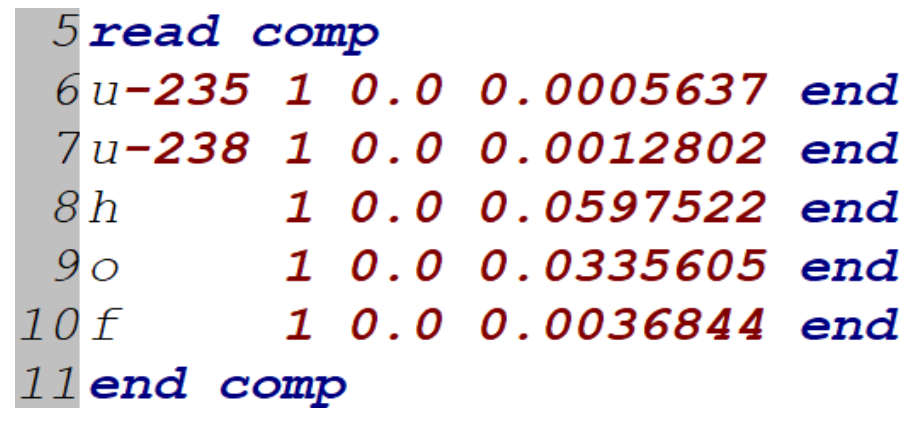

Figure 58. Completed atomic density input.

\subsubsection{Mixing table output}

As in the previous examples, there is not enough input specified to perform a KENO-VI calculation, but the atom densities can be generated based on the standard composition input supplied. Click the downward pointing triangle next to the Run button at the top of the Fulcrum interface and select Mixing table from the dropdown menu. A picture of the dropdown menu for the uranium metal example is provided in Figure 47.

Upon executing the mixing table calculation, the Messages tab at the bottom right-hand corner of the Fulcrum panel will illuminate. Clicking on the Messages tab will reveal the mixing table results, as shown in Figure 59 (enlarged for readability). The resulting mixing table includes the mixture number, 
the mixture temperature, and the mixture density, along with the atom density and nuclide identifiers $(1001,1002,8016,8017,8018,9019,92235$, and 92238).

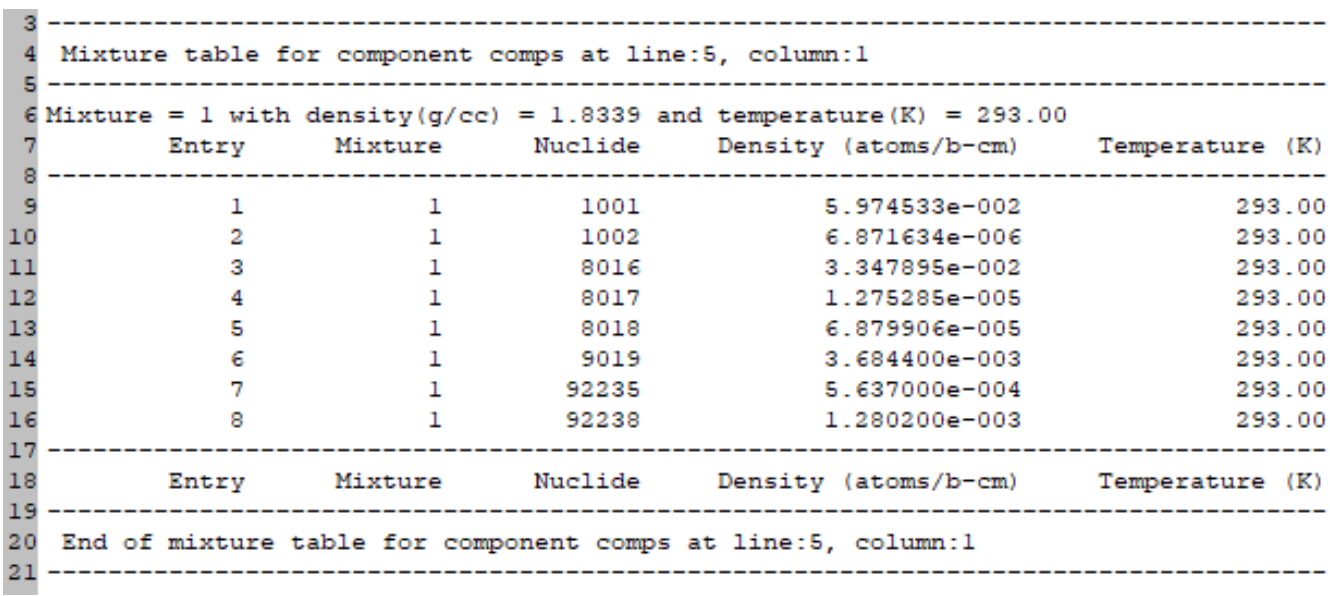

Figure 59. Mixing table results for the highly enriched $\mathrm{UO}_{2} \mathrm{~F}_{2}$ case.

\subsection{5 $\quad \mathrm{H}_{2} \mathrm{O} / \mathrm{UO}_{2}$ Material Combining Example}

This example demonstrates how a user would combine any two basic standard compositions to define a mixture composed of two materials. This method for combining materials is not limited to standard basic compositions; it may also be used with the material types discussed in Section 7. Typically, the description of a material provided in source documents will provide either the volume fractions of individual components of known density or the weight fractions of individual materials with an overall mixture density. For this example, a mixture of water and uranium dioxide is used to illustrate how individual materials can be added to the same mixture. The mixture is $80 \%$ by volume $\mathrm{H}_{2} \mathrm{O}$ and $20 \%$ by volume natural $\mathrm{UO}_{2}$. This is equivalent to a mixture that is 26.703 wt. $\% \mathrm{H}_{2} \mathrm{O}$ and 73.297 wt. $\% \mathrm{UO}_{2}$, with an overall density of $2.9906 \mathrm{~g} / \mathrm{cm}^{3}$. We will model both approaches.

\subsubsection{Material input}

Once you have the same input skeleton that was used in the previous examples, the first step is to define the uranium oxide composition. Move the cursor into the read comp block, and press CTRL-SPACE to generate the dropdown menu similar to what is shown in Figure 43 above. From the dropdown menu, select stdcomp - basic (configurable). This will generate a configurable form, as shown in Figure 44. In the composition dropdown, select uo2 by clicking on the blank with ac-206 in it and typing over it.

Next, adjust the volume fraction to account for the $\mathrm{UO}_{2}$ only making up $20 \%$ of the mixture by clicking the adjustable window next to Volume Fraction and overwriting $\mathbf{1 . 0 0 0 0 0 0}$ with 0.20 . At this point, the $\mathrm{UO}_{2}$ portion of the first entry is complete. Click $\mathbf{O K}$ at the bottom of the configurable form. Next, add the $\mathrm{H}_{2} \mathrm{O}$ portion of the mixture by performing the same process to bring up the composition dropdown menu. Select stdcomp - basic (configurable), and then select $\mathrm{h} 2 \mathrm{O}$ by clicking on the blank with ac-206 in it and typing over it. Next adjust the volume fraction to account for the $\mathrm{H}_{2} \mathrm{O}$ only making up $80 \%$ of the mixture. To do this, click the adjustable window next to Volume Fraction, and overwrite $\mathbf{1 . 0 0 0 0 0 0}$ with 0.80 . It is important that both the $\mathrm{UO}_{2}$ and $\mathrm{H}_{2} \mathrm{O}$ have the same mixture number. This mixture entry will result in a mixture with $0.2 * \rho_{\mathrm{UO} 2}+0.80 * \rho_{\mathrm{H} 2 \mathrm{O}}=0.2 *(10.9600)+0.8 *(0.9982)=2.9906$. Note that this is the value given above for the density. At this point, the input should look like Figure 60. 


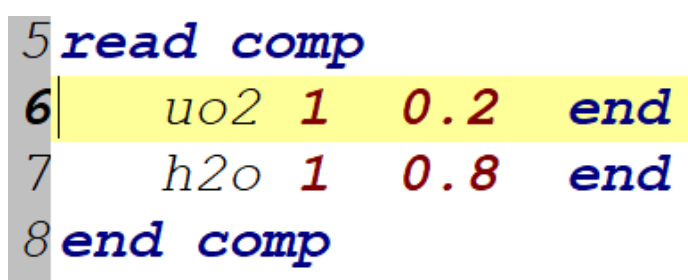

Figure 60. Input with mixing by volume fraction.

Now the same mixture will be specified using the weight fraction method. This is done in a similar manner by using the stdcomp - basic (configurable) once for each of the two compositions, $\mathrm{UO}_{2}$ and $\mathrm{H}_{2} \mathrm{O}$. This first form will be used to enter the $\mathrm{UO}_{2}$ composition. In the $\mathrm{UO}_{2}$ form, the Mixture entry should be changed to 2, the Theoretical Density value changed to 2.9906, and the Volume Fraction changed to 0.732970 . Once this is done, click the OK button at the bottom of the pane. In the $\mathrm{H}_{2} \mathrm{O}$ form, the Mixture entry should also be changed to 2, the Theoretical Density should be changed to 2.9906, and the Volume Fraction should be changed to 0.26703 . This mixture entry will result in a mixture with $0.2 * \rho_{\mathrm{UO} 2}+0.80 * \rho_{\mathrm{H} 2 \mathrm{O}}=0.2 *(10.9600)+0.8 *(0.9982)=2.9906$. Each of the configurable forms is shown in Figure 61and Figure 62, and the final input, including both the mixing by volume fraction and mixing by weight fraction, is shown in Figure 63.

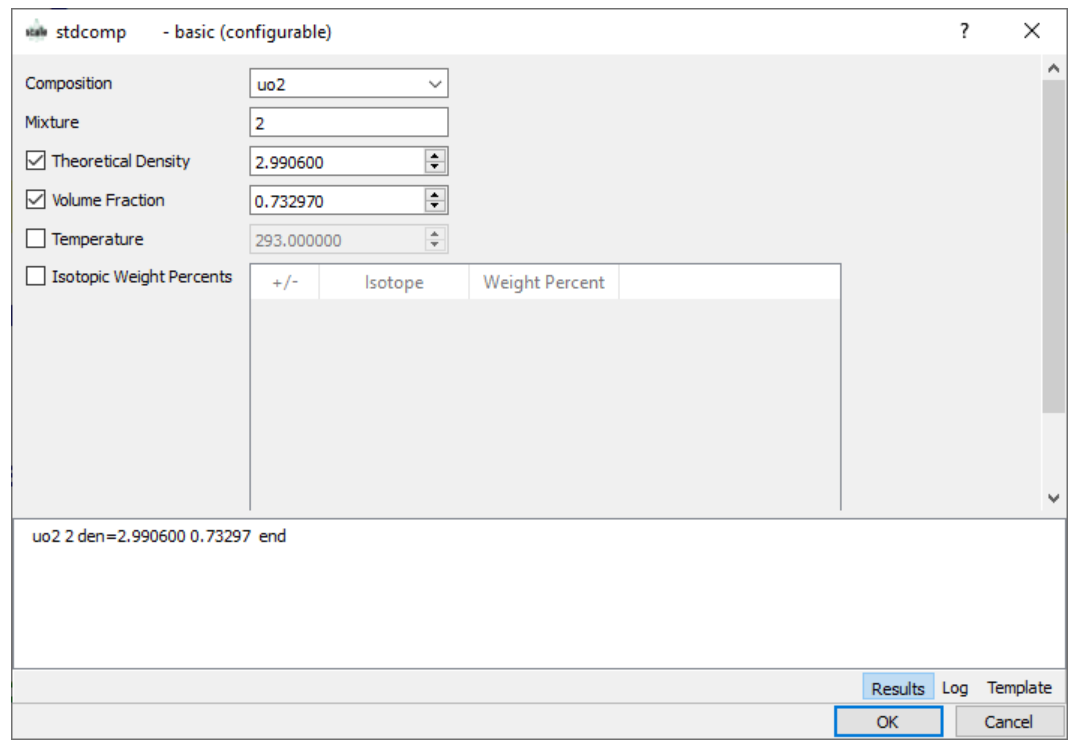

Figure 61. $\mathrm{UO}_{2}$ configurable form for mixing by weight fraction. 


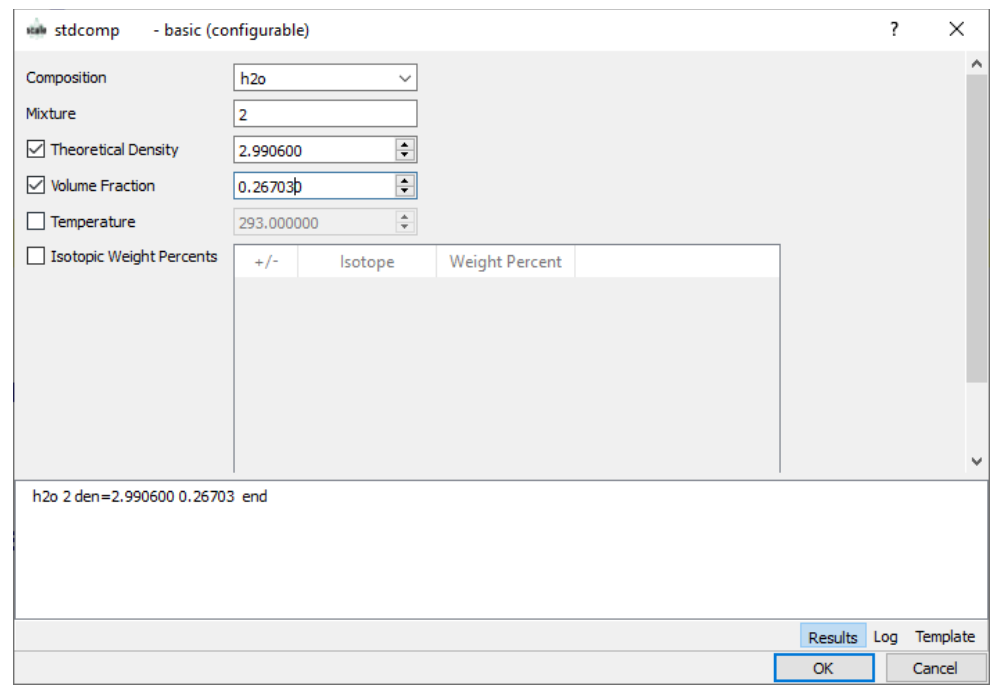

Figure 62. $\mathrm{H}_{2} \mathrm{O}$ configurable form for mixing by weight fraction.

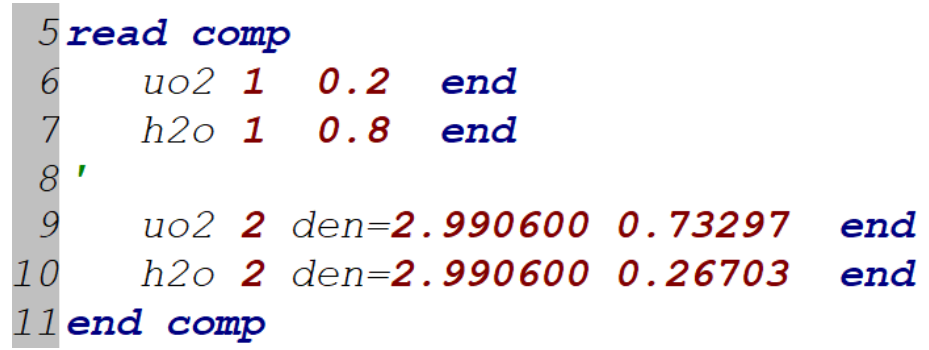

Figure 63. Final material specification input for mixing by volume fraction and mixing by weight fraction.

\subsubsection{Mixing table output}

As in the previous examples, there is not enough input specified to perform a KENO-VI calculation, but the atom densities can be generated based on the standard composition input supplied. Click the downward pointing triangle next to the Run button at the top of the Fulcrum interface and select Mixing table from the dropdown menu. A picture of the dropdown menu for the uranium metal example is provided in Figure 47.

Upon executing the mixing table calculation, the Messages tab at the bottom right-hand corner of the Fulcrum panel should illuminate. Clicking on the Messages tab will reveal the mixing table results. The mixing table results are presented in Figure 64. The resulting mixing table includes the mixture number, the mixture temperature, and the mixture density, along with the atom density and the nuclide identifiers $(1001,1002,8016,8017,8018,92234,92235$, and 92238). 


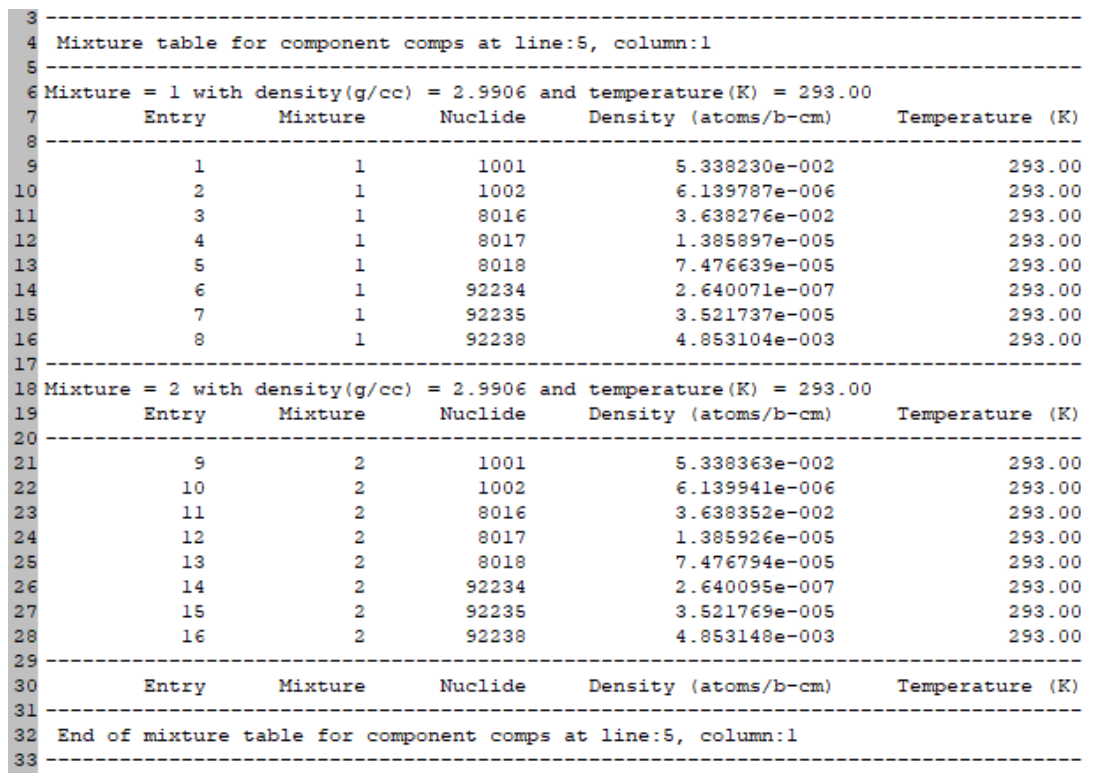

Figure 64. Mixing table output for the mixing by volume fraction and the mixing by weight fraction examples.

\subsection{SUMMARY}

This section has helped you to:

- define the different criticality sequences used in SCALE;

- describe the cross section libraries available for criticality analyses;

- use the Fulcrum user interface to provide data on elements, isotopes, compounds, and mixtures of these types; and

- interpret basic mixing table output information from a SCALE analysis.

Now that you have spent time with the material input, you can proceed to geometry definitions. 



\section{GEOMETRY INPUT}

In Section 2, you modeled a simple geometry with a single material composition using the Fulcrum user interface to provide data on elements, isotopes, compounds, and solutions. This section explains the commands used for basic KENO-VI geometries such as cylinders and spheres and for more complicated geometries such as reflected systems and lattices.

\subsection{WHAT YOU WILL BE ABLE TO DO}

- Use Fulcrum to describe some basic KENO-VI geometry shapes.

- Understand how units are created (including intersecting of shapes).

- Locate and change the location of the origin for shapes and units.

- Create simple arrays consisting of a single unit.

- Create arrays with multiple units of nominally different sizes.

- Use the volume block to calculate volume of regions and/or mixtures.

\subsection{BASIC GEOMETRY SHAPES}

SCALE/KENO-VI uses a set of basic shapes to build the geometry models. One example has already been presented, using Fulcrum to enter data on spheres in Section 2. The sphere and the other basic shapes are described in Table 2 and are illustrated in Figure 65 and Figure 66.

Table 2. Basic shapes.

\begin{tabular}{ll}
\hline \multicolumn{1}{c}{ Keyword } & \\
\hline CONE & Right circular cone, can be truncated \\
CUBOID & Box or rectangular parallelepiped \\
CYLINDER & Right circular cylinder \\
DODECAHEDRON & Surface consisting of 12 rhombi of the same size \\
ECYLINDER & Right elliptic cylinder \\
ELLIPSOID & Surface formed by rotating an ellipse \\
HEXPRISM & Right hexagonal prism (flat up) \\
HOPPER & Truncated square pyramid \\
QUADRATIC SURFACE & Quadric shape defined by a quadratic surface equation \\
PARALLELEPIPED & Six faces composed of parallelograms with opposing parallel faces \\
PENTAGON & Right pentagonal prism \\
PLANE & Arbitrary 2D plane \\
RING & Right annular prism \\
RHEXPRISM & Hexprism rotated 30 degrees about the long axis (point up) \\
RHOMBOID & Six identical faces, each one a rhombus \\
SPHERE & Sphere \\
WEDGE & Right prism with triangular cross section \\
XPPLANE & Two planes perpendicular to the X-axis \\
YPPLANE & Two planes perpendicular to the Y-axis \\
ZPPLANE & Two planes perpendicular to the Z-axis \\
\hline
\end{tabular}




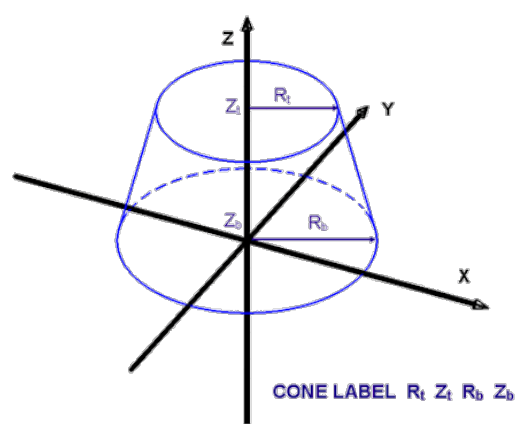

Cone

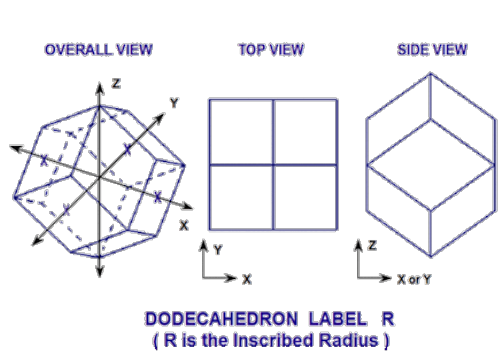

Dodecahedron

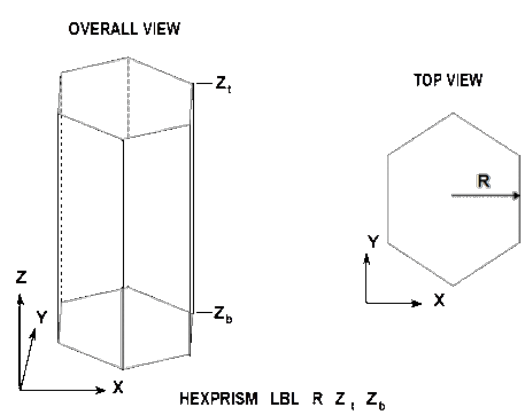

Hexprism

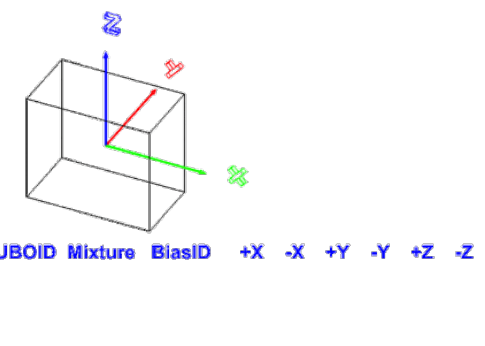

Cuboid

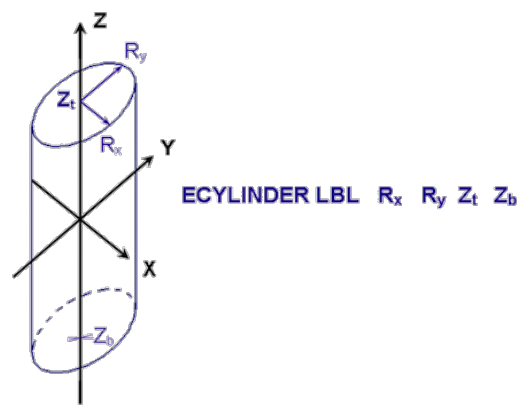

Ecylinder

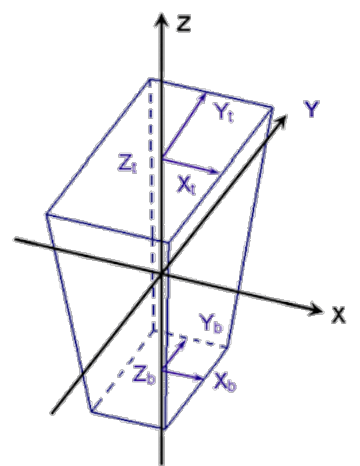

HOPPER LBL $\begin{array}{lllllllll}X_{t} & Y_{t} & Z_{t} & X_{b} & Y_{b} & Z_{b}\end{array}$

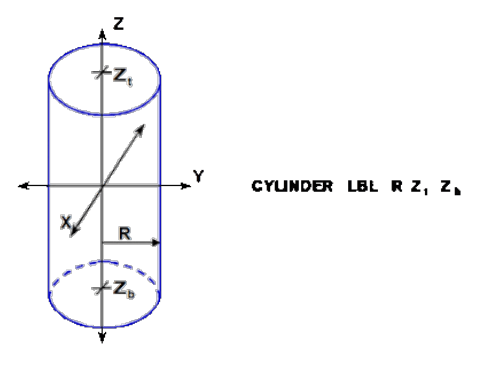

Cylinder

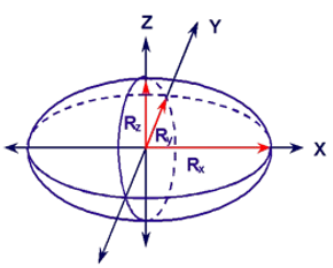

ELLIPSOID LABEL $R_{x} R_{y} R_{x}$

Ellipsoid

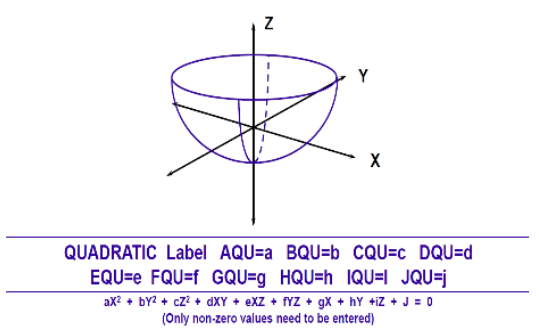

Quadratic Surface

Figure 65. KENO-VI geometry shapes. 


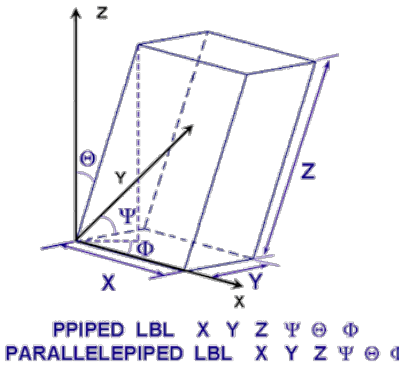

Parallelepiped

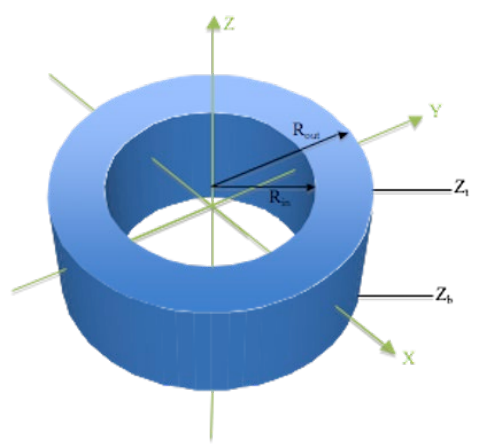

Ring

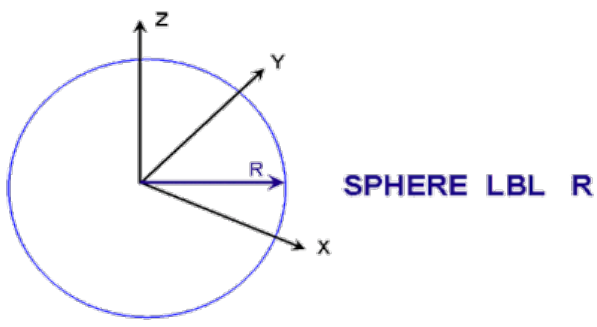

Sphere

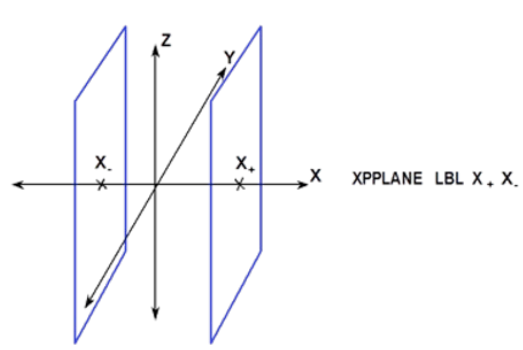

XPPPlane

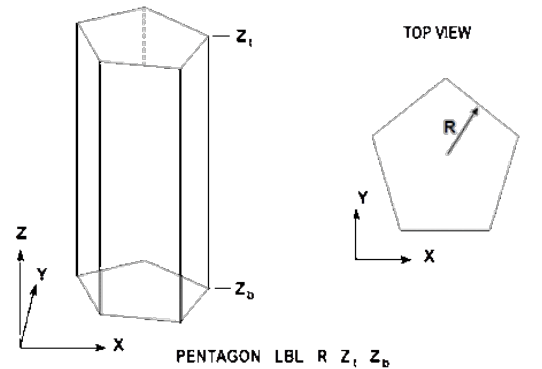

Pentagon

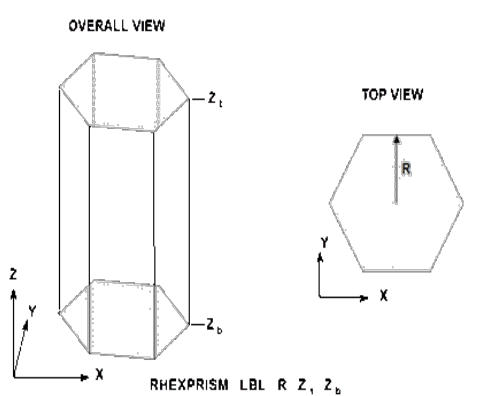

Rhexprism

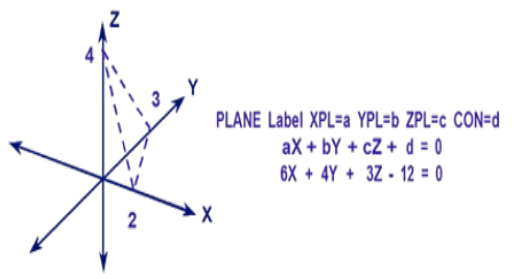

Plane

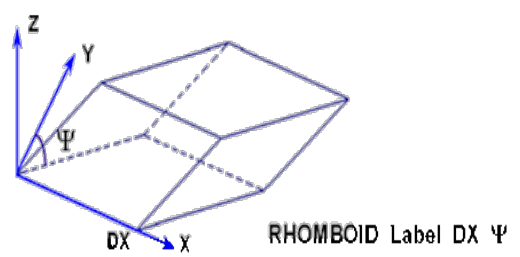

Rhomboid

Wedge LABEL XbASE Xpt Ypt ZLNG

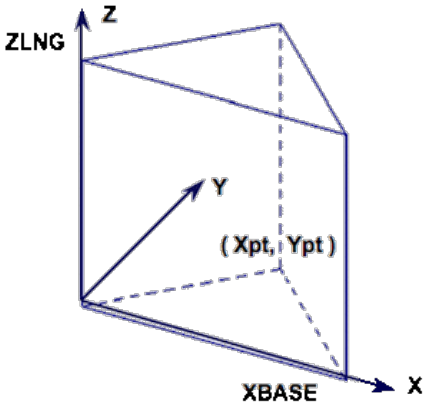

Wedge

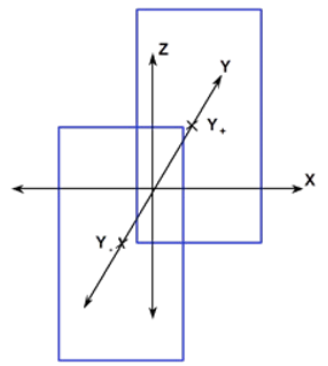

YPPPlane

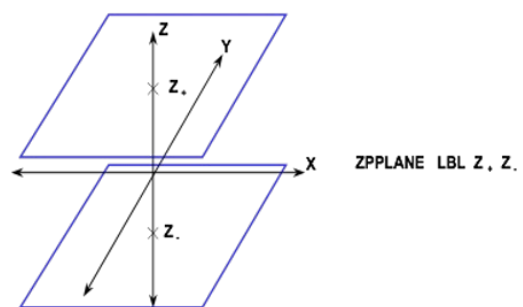

ZPPPlane

Figure 66. More KENO-VI geometry shapes. 
These shapes are the basic geometry options in KENO-VI. Placing the text cursor within the read geometry block and pressing the CTRL-SPACE autocomplete key combination will display the list of shapes available (Figure 67). Each shape has a set of quantitative information that is needed to describe its size and location. Selecting a geometry option brings up the associated shape configurable input form. Figure 68 shows the input form for a cuboid, which is accessed by clicking on cuboid - kenovi (configurable); the other input forms ask for similar information. A description of the options for each basic shape can be found in [see SCALE Manual section KENO-VI Shape Descriptions]. Additional information may be entered to translate (relocate the origin), rotate, or to insert a chord that describes a plane surface that truncates the shape. These options are discussed in Section 5.

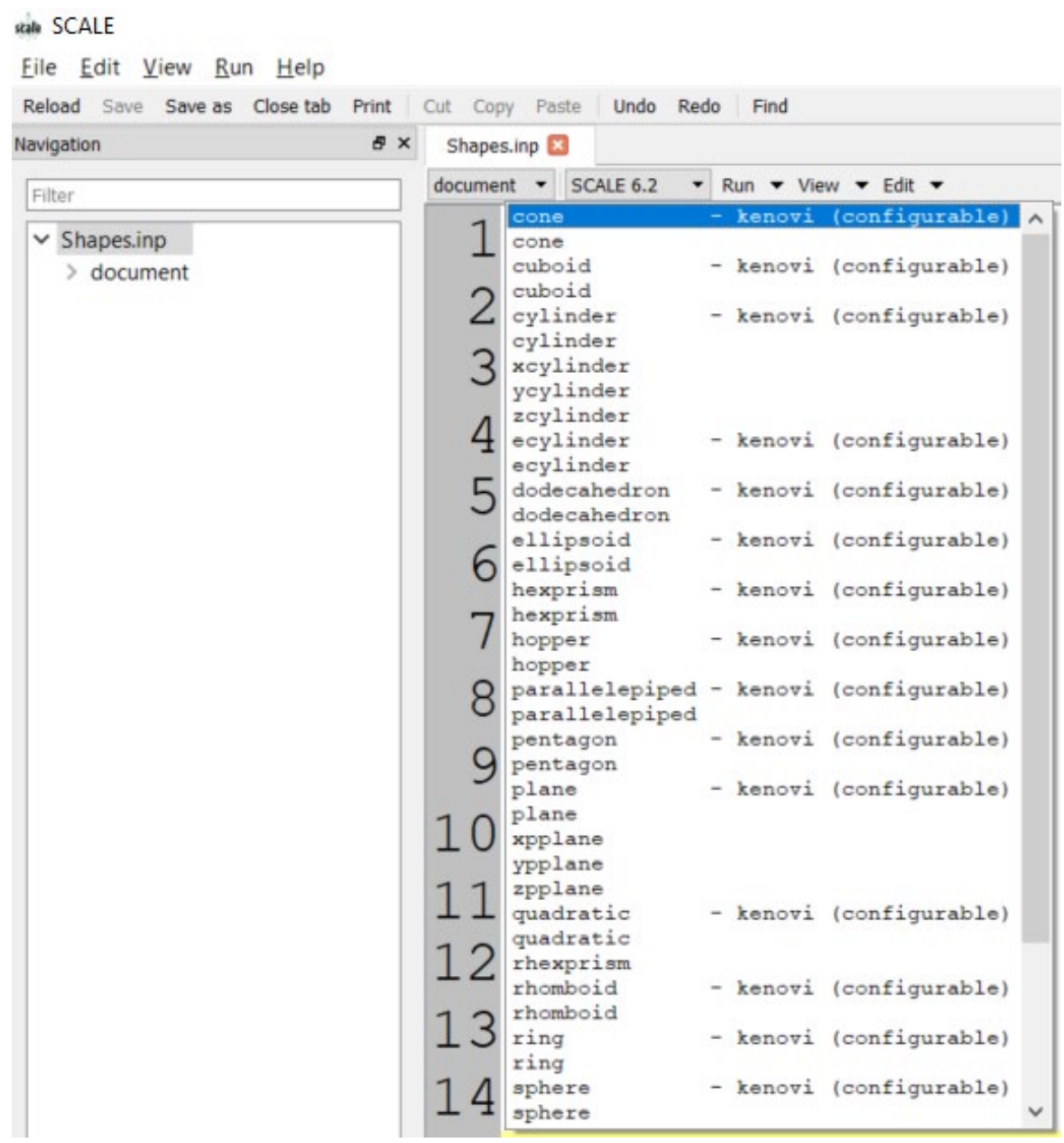

Figure 67. Geometry autocomplete form. 


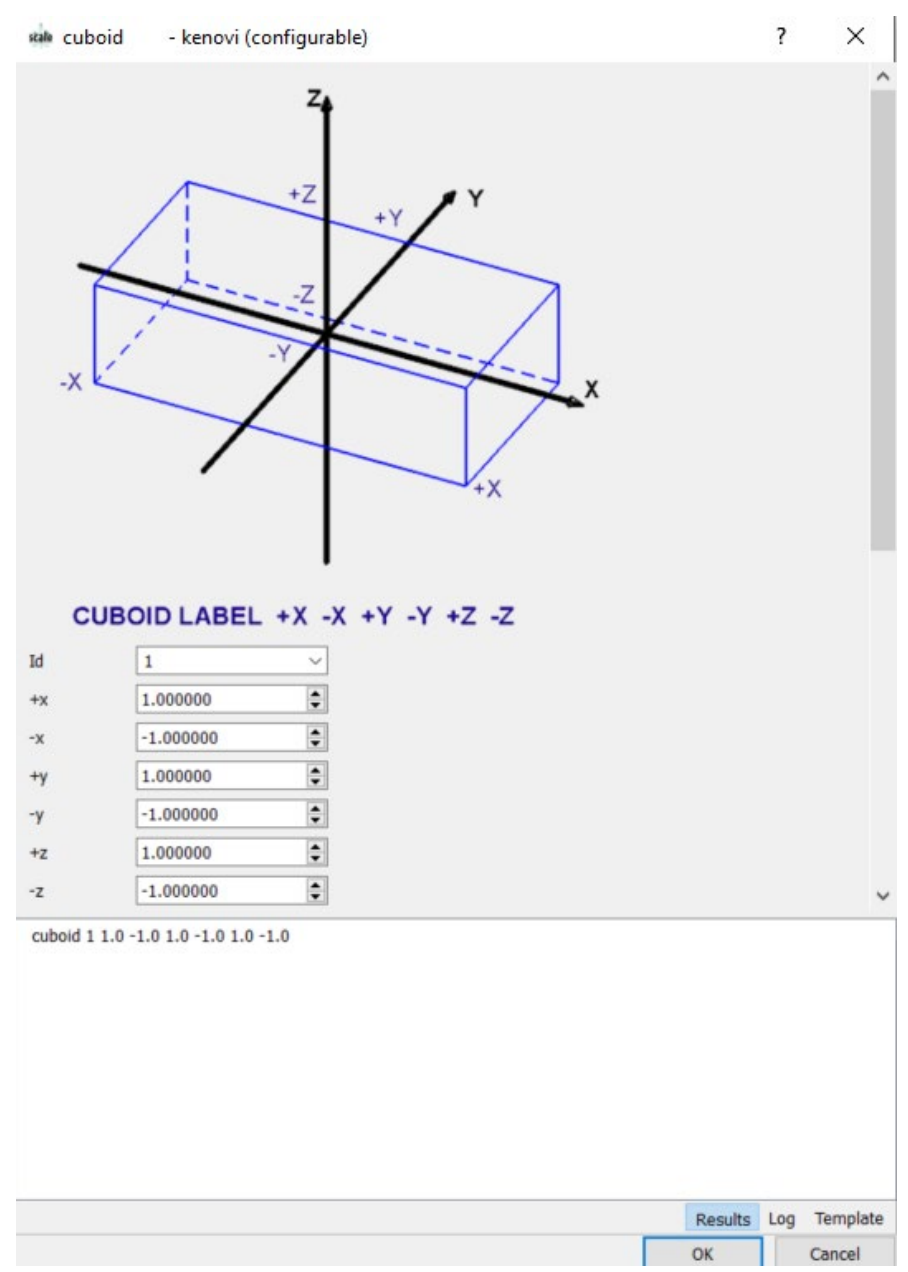

Figure 68. Cuboid input form.

\subsubsection{Simple cylinder examples}

Some sample problems are presented to illustrate how shape parameters are entered using Fulcrum. These problems are based on the pure ${ }^{235} \mathrm{U}$ metal material from Section 3.4.1. Various cylindrical configurations will be examined.

\subsubsection{Bare Metal Cylinder along the Z-axis}

The first example is locating a bare metal cylinder centered on the Z-axis as shown in Figure 69. Based on the knowledge gained in previous sections of this primer, open a new file and enter the title, cross section library, and material information. Note that all problems in this section use the CE ENDF/B-VII.1 library (ce_v7.1). 


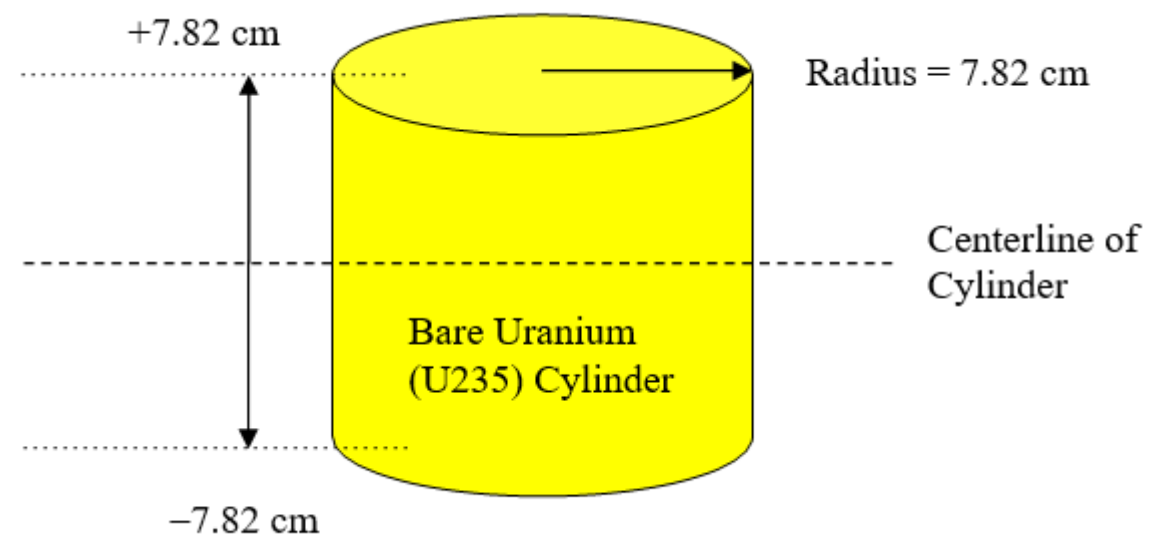

Figure 69. Bare ${ }^{235} \mathrm{U}$ cylinder geometry.

With this simple geometry, only a few entries are needed to complete the KENO geometry input form. Place the text cursor within the read geometry block, between Global Unit 1 and boundary 1 and press the CTRL-SPACE autocomplete key combination to display the list of available shapes shown in Figure 67. To enter geometry information, select the appropriate geometry from the list. For this problem, choose the cylinder - kenovi (configurable) button, which opens the Cylinder input form as shown in Figure 70. Enter 7.82 for Radius, 7.82 for Top, and -7.82 for Bottom. This completes the Cylinder information, as shown in Figure 70. Click on $\mathbf{O K}$ to add the input generated to the input file and return to the main Fulcrum screen. 

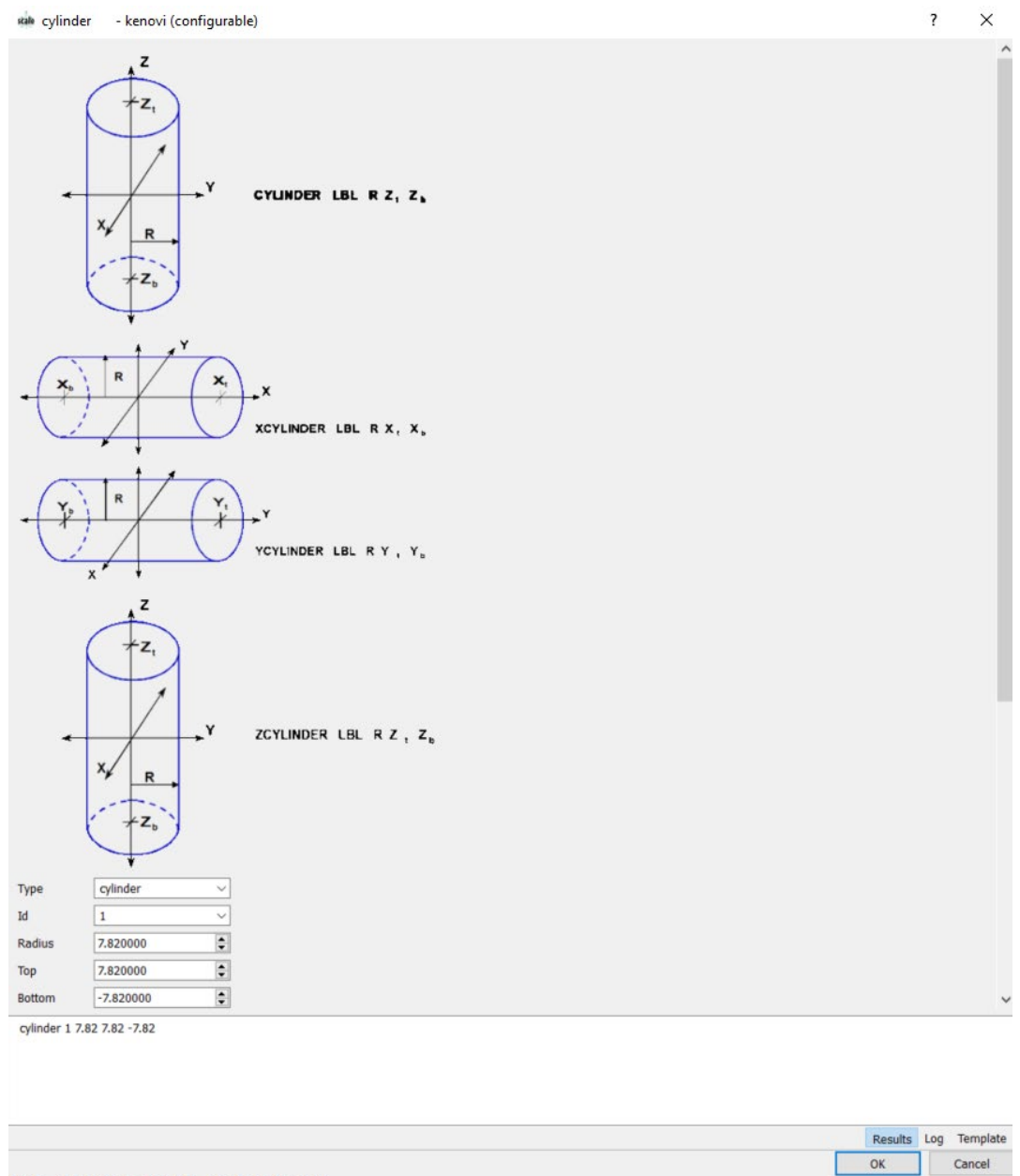

Figure 70. Cylinder data for bare metal cylinder along Z-axis.

The contents of the cylinder must be described next. Now the contents of the regions must be defined using media records. Each media record defines one region in the unit by combining one or more shapes using Boolean logic in a region definition vector. The media record also identifies a mixture to fill the region. There is only one region in the problem, cylinder region 1 , and one mixture, $\mathrm{u}-235$ mixture 1 . Below the cylinder input description, use the CTRL-SPACE autocomplete key combination to display the list of available shapes and scroll to the bottom and select media - configurable, as shown in Figure 71. The media configurable form generated by Fulcrum is shown in Figure 72. Click the Add row button below the Regions pane to add mixture 1 to region 1. The mixture number is selected in the Material dropdown in the upper left corner of the form. The Id column in the Regions pane is the Id number for the shape referenced in the media record, and the State is either inside or outside. For this problem, the default values are correct and, once the input has been confirmed by comparison with Figure 73, press the OK button to add the media record to the input file. The first numerical entry on the media record is the mixture number to be used (mixture number 1). The second is a Bias ID, but only unbiased tracking (Bias ID of 1) is recommended in KENO. The third number 1 is the Region Definition Vector: it states that this region is inside cylinder 1 .

Alternatively, the media record could be added by typing directly in the Fulcrum editor (enter media 1 1 1). Do not enter the media record twice; enter it once by using either the configurable form or typing the text in the input file. 


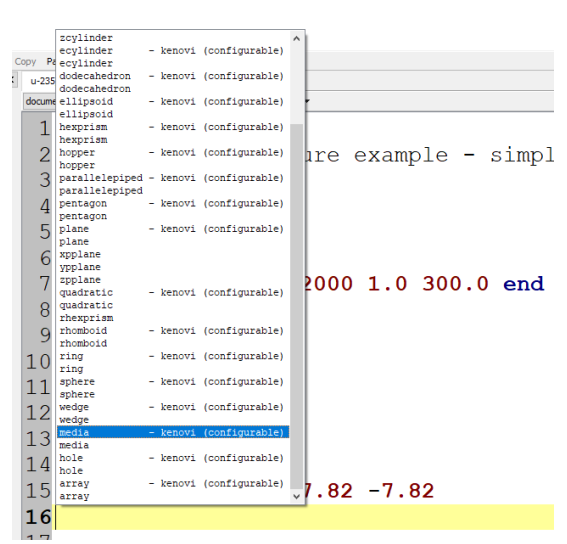

Figure 71. Selecting the media configurable window.

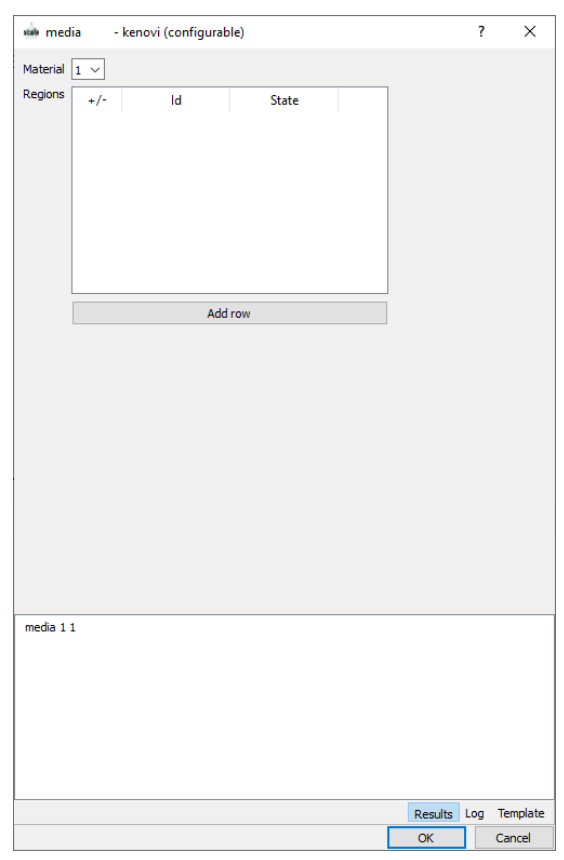

Figure 72. Default media configurable window in Fulcrum. 


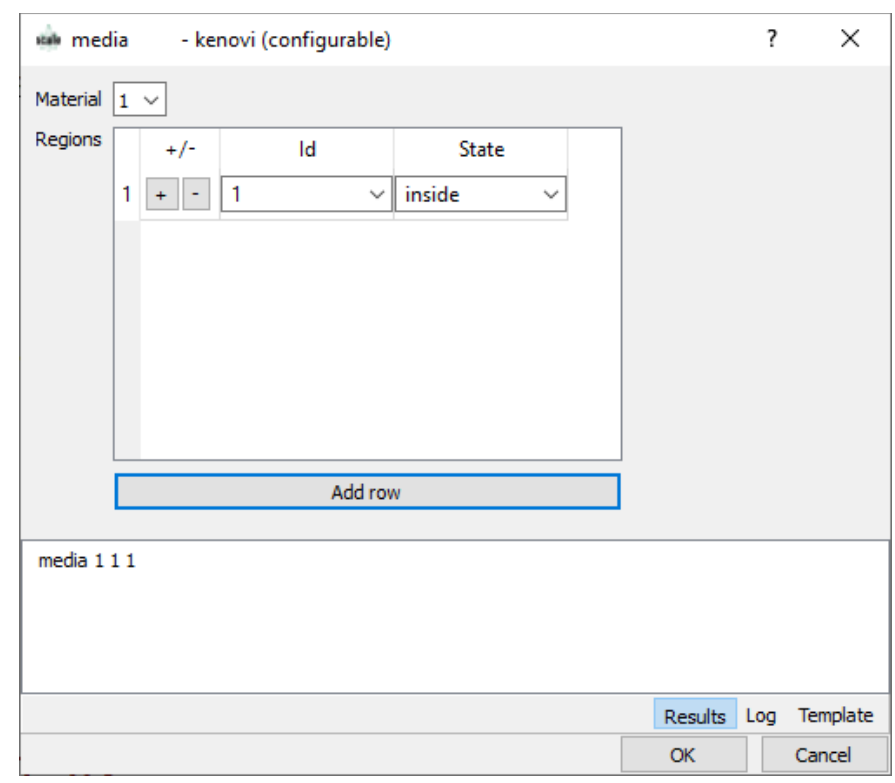

Figure 73. Completed media configurable form.

The boundary record follows the media records, and it defines the outer boundary of the unit using a region definition vector. The outer boundary of the unit was already defined as boundary 1 thanks to the Fulcrum autocompletion. This defines region 1 as the outer boundary of the unit. As stated earlier, the global unit in KENO is the final unit that defines the entire system to be analyzed. This completes the information required for this example. Select Run. Click on messages to allow Fulcrum to show the progress of the calculation. The input file for this example should look like Figure 74.

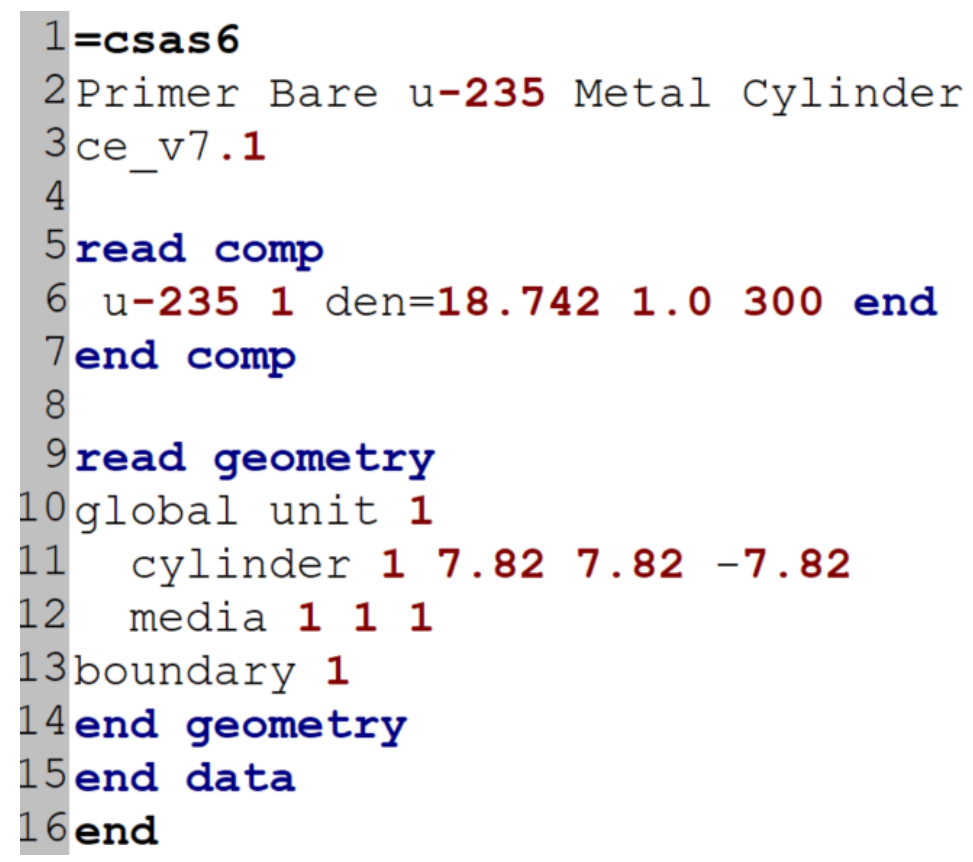

Figure 74. Input file for the bare metal cylinder example.

To make sure the input is correct, check the calculated value of $k_{\text {eff. }}$ The value should be approximately $1.0224 \pm 0.0022$. 


\subsubsection{Bare Metal Cylinder along the Z-axis with Translated Origin}

There are some fissile systems for which the cylinder origin is located at a point other than the center of the cylinder. Moving the origin up and down along the $\mathrm{Z}$-axis is done by changing the values of Top and Bottom. For example, suppose the origin is at a point $2 \mathrm{~cm}$ above the base of the cylinder (see Figure 75).

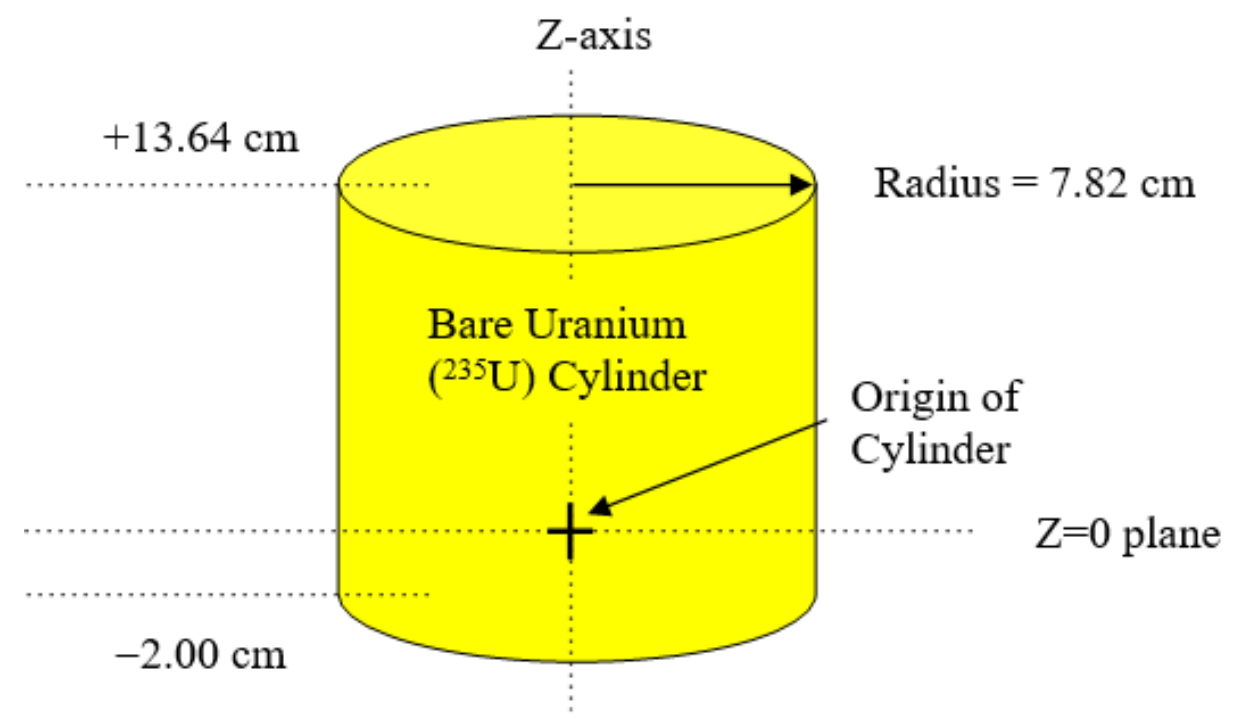

Figure 75. Base of cylinder located $2 \mathrm{~cm}$ below origin.

The cylinder region parameters on the Cylinder input form can be modified from the previous example. Remove the previous cylinder input line and create a new cylinder by placing the text cursor within the read geometry block, between Global Unit 1 and boundary 1, and pressing the CTRLSPACE autocomplete key combination. Select cylinder - kenovi (configurable), which opens the Cylinder input form. Enter 7.82 for Radius, 13.64 for Top, and -2 for Bottom so that the screen looks like Figure 76. 


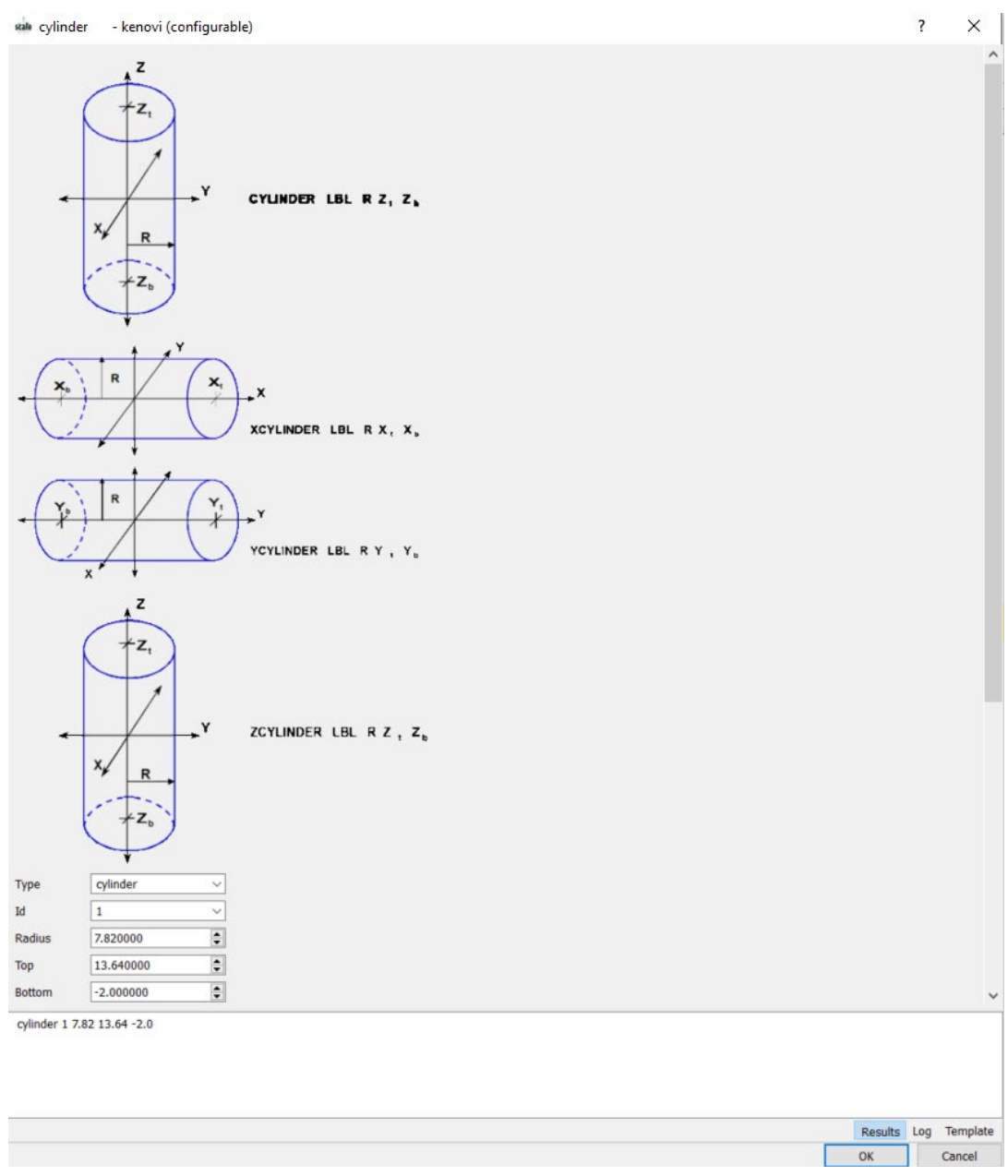

Figure 76. Cylinder data input with base located $2 \mathrm{~cm}$ below origin.

Save the changes in a new input file and execute SCALE. Because no changes were made to the physical system, the $k_{\text {eff }}$ value for this run should be identical to that from the previous run.

\subsection{KENO-VI GEOMETRY RULES}

There are seven basic rules that guide the geometry input to SCALE/KENO-VI.

1. Volumes are built in sections called "units." Each unit is independent of all other units and has its own coordinate system.

2. Units are built using regions. Regions are made using the KENO-VI geometry shapes. The unit boundary must fully enclose all defined regions in the unit. See the example provided in Figure 77.

3. Regions may share boundaries and may intersect. See examples in Figure 77. (Note that intersecting regions are not allowed in KENO V.a.)

4. Regions may be rotated. See example in Figure 77. (Note that rotations are not allowed in KENO V.a.) 
5. A hole is used to place a unit within a region in a different unit. The hole must be completely contained within the region and may not intersect other holes or nested arrays. As many holes as required may be placed in a unit.

6. An array is an ordered stack of units. The touching faces of adjacent units in an array must be the same size. Multiple arrays may be placed directly into a unit by placing them in separate regions. Arrays can also be placed within a unit using holes.

7. A global unit that encloses the entire system must be specified. All geometry data used in a problem are correlated by the global unit coordinate system.

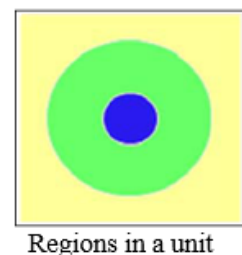

Regions in a unit

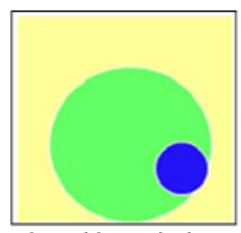

Shared boundaries

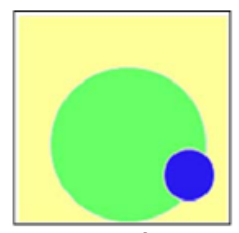

Intersecting regions

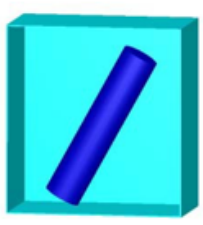

Rotated region

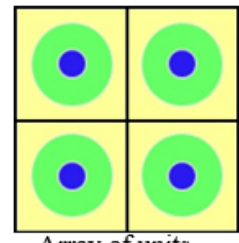

Array of units

Figure 77. Examples of KENO basic geometry rules.

Note that each point in the geometry must be uniquely defined by one content record (media, hole, or array). If more than one media is assigned to any volume, then that volume is "multiply defined." If a region is not assigned in any media record, then that volume is "undefined." Either of these conditions will result in error messages and job termination when a particle is tracked through that volume in KENO-VI.

\subsection{GEOMETRIC ARRANGEMENTS}

Geometric arrangements in KENO-VI are achieved in a manner similar to using a child's building blocks. Each building block is called a unit. Units are constructed of combinations of basic shapes. These shapes can be placed anywhere within a unit but will be truncated by the outer boundary of the unit if they intersect it or are completely outside of it. A unit can be thought of as a container that encloses a number of shapes. A unit encloses one or more basic shapes.

\subsection{NESTED REGIONS IN A UNIT}

To illustrate how to enter KENO geometry information for nested regions in a unit via Fulcrum, two sample problems are included. The first is a reflected cylinder of Pu metal, and the second is a sphere inside a cylinder inside a cube.

\subsubsection{Reflected Pu Metal Cylinder}

The reflected Pu metal cylinder problem comes from page 102 of LA-10860 [5]. The fissile material is $\mathrm{Pu}$ metal (density $=15.44 \mathrm{~g} / \mathrm{cc}$ ), with a maximum of $5.0 \mathrm{wt} \%{ }^{240} \mathrm{Pu}$. The remainder is ${ }^{239} \mathrm{Pu}$. The core has a diameter of $5.72 \mathrm{~cm}$ with an $\mathrm{H} / \mathrm{D}=7.16$, or a critical height of $40.96 \mathrm{~cm}$. Surrounding the core is a graphite reflector (density $=1.60 \mathrm{~g} / \mathrm{cc}$ ) with a thickness of $17.78 \mathrm{~cm}$ in both the radial and axial directions (see Figure 78). 


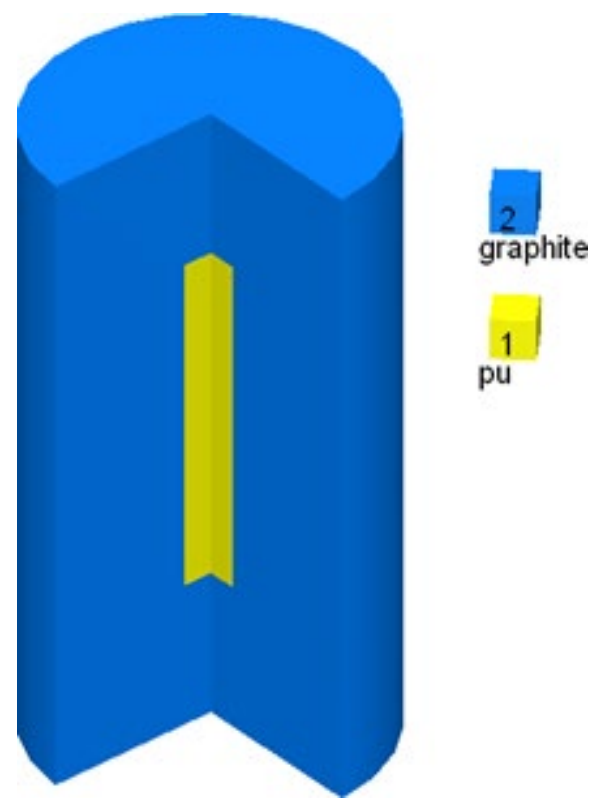

Figure 78. Reflected plutonium metal cylinder geometry.

Based on the knowledge gained in previous sections of this primer, open a new file and enter the title, cross section library, and material information. Specify the isotopic composition for mixture 1 as pu with 95 wt. $\%{ }^{239} \mathrm{Pu}$ and 5 wt. $\%{ }^{240} \mathrm{Pu}$ and a plutonium density of $15.44 \mathrm{~g} / \mathrm{cc}$. Mixture 2 should be c-graphite with a density of $1.6 \mathrm{~g} / \mathrm{cc}$.

For this geometry, there will be two shapes (or regions) in one unit, and that unit will be the global unit for the problem. The inner cylinder containing the plutonium will be described first, and the outer cylinder containing graphite will be described second. The order in which the regions are defined is irrelevant in KENO-VI, so this choice of the inner cylinder first is arbitrary. Place the text cursor within the read geometry block, between Global Unit 1 and boundary 1, and press the CTRLSPACE autocomplete key combination. Select cylinder - kenovi (configurable), which opens the Cylinder input form. Enter 2.86 for Radius, 40.96 for Top, and 0 for Bottom. This puts the origin at the bottom center of the core cylinder. Check that the configurable form should look like that shown in Figure 79 and then click $\mathbf{O K}$. 


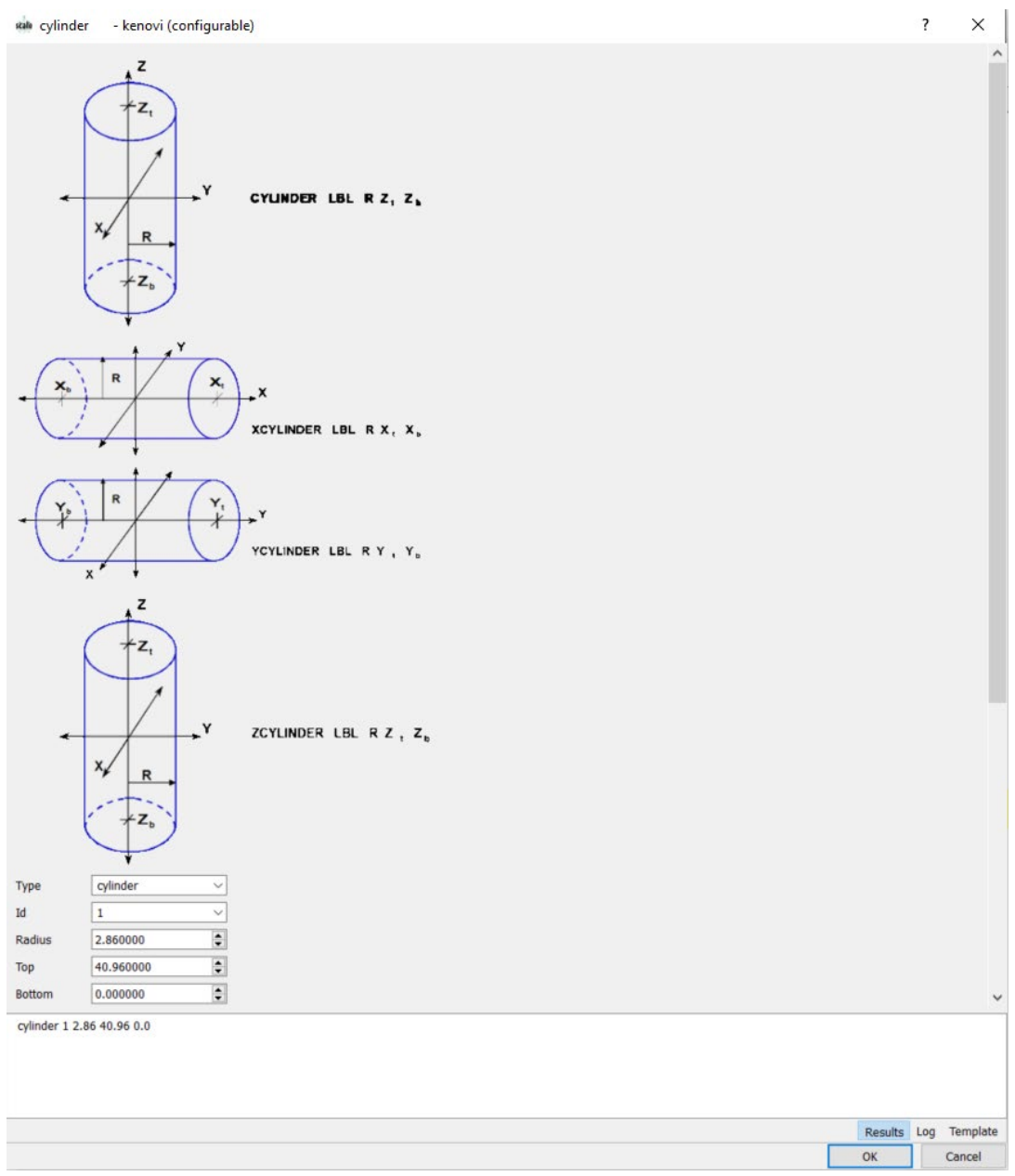

Figure 79. Geometry description for region 1.

To enter the data for the reflector, place the text cursor within the global unit, and press the CTRLSPACE autocomplete key combination. Select cylinder - kenovi (configurable), which opens another Cylinder input form. Change the Id to 2, as the reflector is the second region of the problem. As with the order of entry, the ID numbers for the regions are arbitrary, though in this case must be positive integers. Enter $20.64(=2.86+17.78)$ for Radius, $58.74(=40.96+17.78)$ for Top, and $-17.78 \quad(=0.00-$ 17.78) for Bottom. Then click OK.

The geometry shapes have been defined, so the media records must now be added. Use the CTRL-SPACE autocomplete key combination and select media - kenovi (configurable). Use the configurable form to specify that region 1 is filled with plutonium. Repeat this process to specify that the graphite is inside cylinder 2 but outside cylinder 1 . Cylinder 1 must be excluded to preclude doubly-defined space with the cylinder containing both plutonium and graphite.

The final step is to define the outer boundary of unit 1, which was already defined thanks to the Fulcrum autocompletion as boundary 1 . As the outer region is the reflector, region 2, change to boundary 2 on the line after the media definition. This completes the geometry input (see Figure 80). 


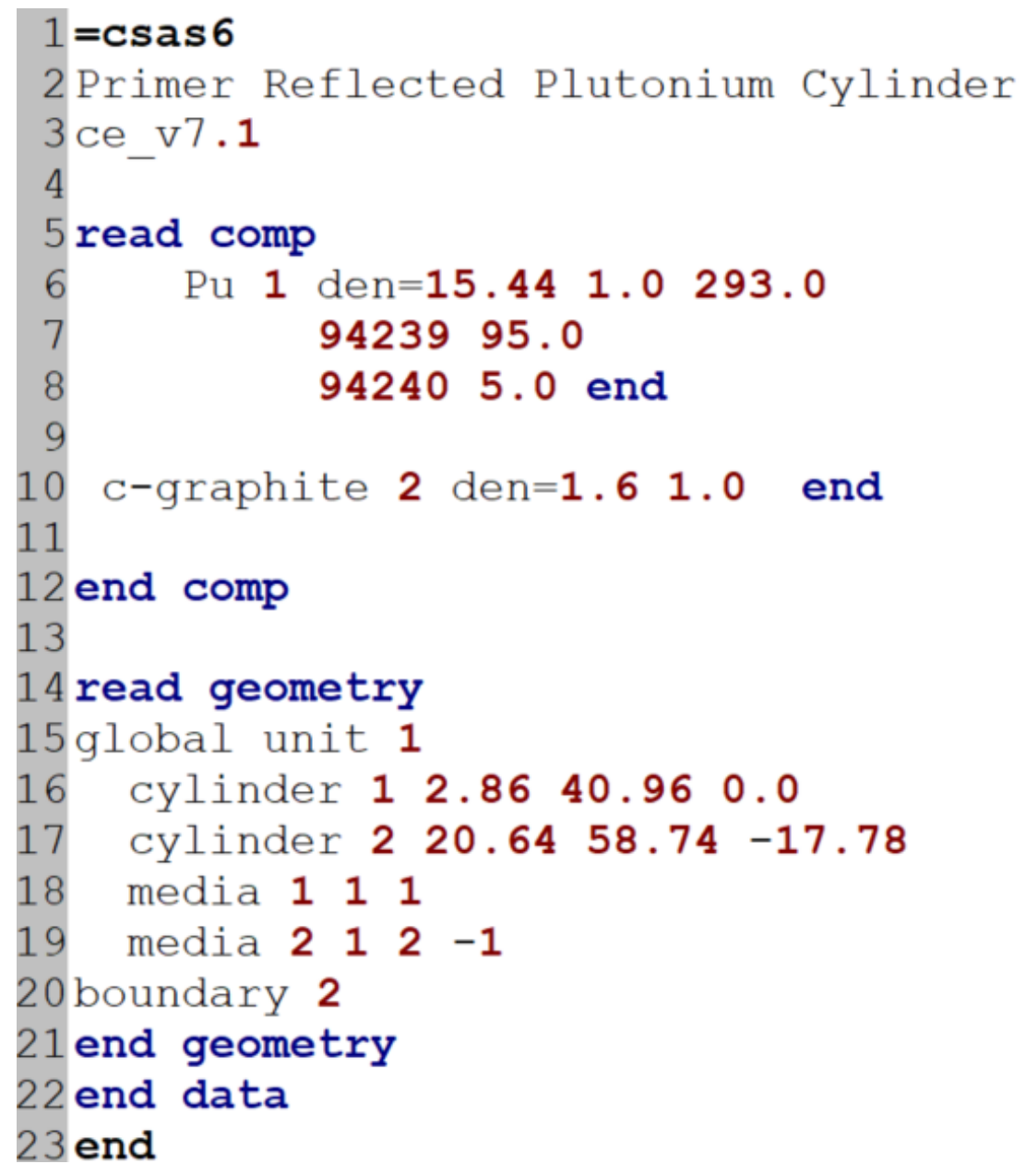

Figure 80. Input file for the reflected plutonium metal cylinder example.

Save the changes in a new input file and execute SCALE. The calculated $k_{\text {eff }}$ value is $0.9790 \pm 0.0021$.

\subsection{2 $\quad{ }^{235} \mathrm{U}$ sphere with Graphite and Water Reflectors}

This problem consists of a metal ${ }^{235} \mathrm{U}$ sphere inside a graphite cylinder inside a cube of water. The center of the cylinder is offset so that the outside edge of the cylinder touches the $-\mathrm{X},-\mathrm{Y}$, and $-\mathrm{Z}$ faces of the cube (see Figure 81). The materials are ${ }^{235} \mathrm{U}$ metal (density $=18.74 \mathrm{~g} / \mathrm{cc}$ ), graphite (density $=1.65 \mathrm{~g} / \mathrm{cc}$ ), and water (default density of $0.9982 \mathrm{~g} / \mathrm{cc}$ ). The ${ }^{235} \mathrm{U}$ sphere radius is $7.0 \mathrm{~cm}$; the graphite cylinder has a radius of $10 \mathrm{~cm}$ and a height of $20 \mathrm{~cm}$. The water cube surrounding the cylinder is $22 \mathrm{~cm}$ on a side. The bottom of the cylinder is coincident with the bottom of the cube. 


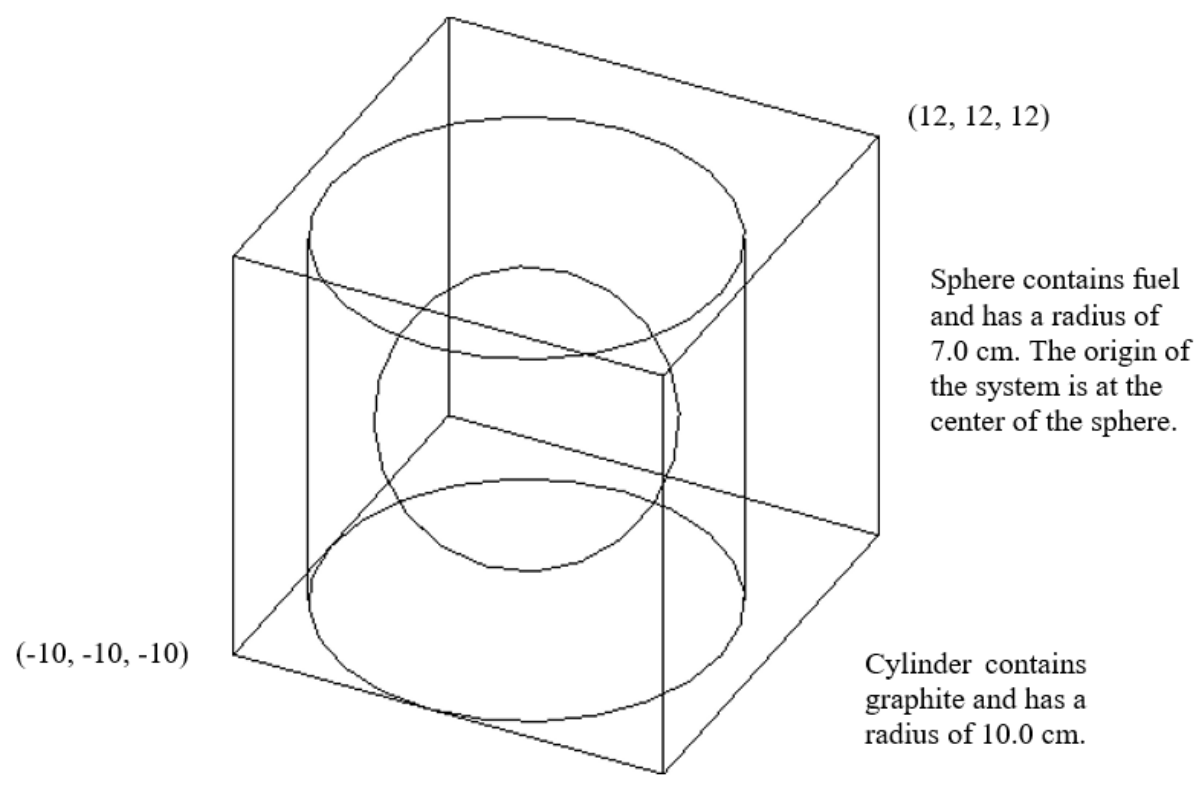

Figure 81. ${ }^{235} \mathrm{U}$ sphere surrounded by graphite and water.

Two cutaway views of the geometry are shown in Figure 82 . The top $3 \mathrm{~cm}$ has been removed from both views to show the graphite cylinder and the sphere inside the water. In the first view, a quarter cut has been removed to reveal the ${ }^{235} \mathrm{U}$ sphere inside the graphite. In the second view, the graphite has been hidden so that the entire ${ }^{235} \mathrm{U}$ sphere can be seen.
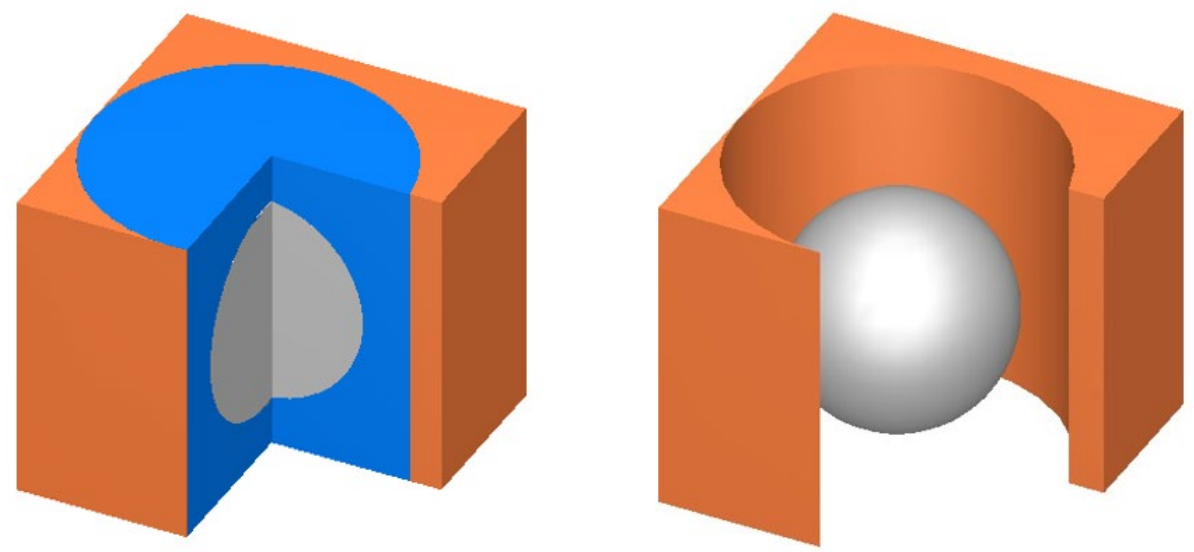

Figure 82. Cutaway views of ${ }^{235} \mathrm{U}$ sphere inside graphite and water.

Using knowledge from previous sections, open a new file and enter the title, cross section library, and material information for the three materials.

For this geometry, there will be three regions in one unit, and that unit will be the global unit for the problem. The uranium sphere will be described first, then the graphite cylinder, and finally, the outer cube containing water. These are nested regions and are entered like those in the previous example. Place the text cursor within the read comp block and press the CTRL-SPACE autocomplete key combination. Select sphere - kenovi (configurable), which opens the Sphere input form. Enter 7 for Radius, and then press $\mathbf{O K}$. To enter the data for the graphite reflector, place your text cursor within the read geometry block, and press the CTRL-SPACE autocomplete key combination. Select cylinder- kenovi 
(configurable), which opens the Cylinder input form. Change the Id to 2, as the reflector is the $2^{\text {nd }}$ region of the problem. Enter 10 for Radius, 10 for Top, and -10 for Bottom. This creates a cylinder centered around the sphere. Click OK. To enter the data for the outside water reflector, place the text cursor within the read comp block and press the CTRL-SPACE autocomplete key combination. Select cuboid- kenovi (configurable), which opens the Cuboid input form. Change the Id to 3, as the reflector is the $3^{\text {rd }}$ region of the problem. The cuboid is slightly off center from the cylinder and sphere, so entering 12 for $+\mathbf{X},+\mathbf{Y}$, and $+\mathbf{Z}$, and -10 for $-\mathbf{X},-\mathbf{Y}$, and $-\mathbf{Z}$ creates a cube with three sides touching the cylinder and the other three sides $2 \mathrm{~cm}$ beyond the cylinder.

The geometry shapes have been defined. Now the contents of the regions must be defined using media records. Use the media configurable form to specify that ${ }^{235} \mathrm{U}$ is inside region 1 . Repeat these steps to create the second region containing the graphite cylinder. For this region, the Region Definition Vector is $2-1$, which indicates that the graphite is inside cylinder 2 but outside sphere 1 . Repeat these steps again to create the third region containing the water. For this region, the Region Definition Vector is $3-2$, which indicates that the water is inside cuboid 3 but outside cylinder 2 .

The final step is to define the outer boundary of unit 1, which was already defined thanks to the Fulcrum autocompletion as boundary 1 . As the outer region is the water cuboid, region 3 , change to boundary 3 on the line after the media definition. This completes the geometry input (see Figure 83). Save the changes in a new input file. Click Run to execute SCALE. The calculated $k_{\text {eff }}$ value is $1.0031 \pm 0.0017$.

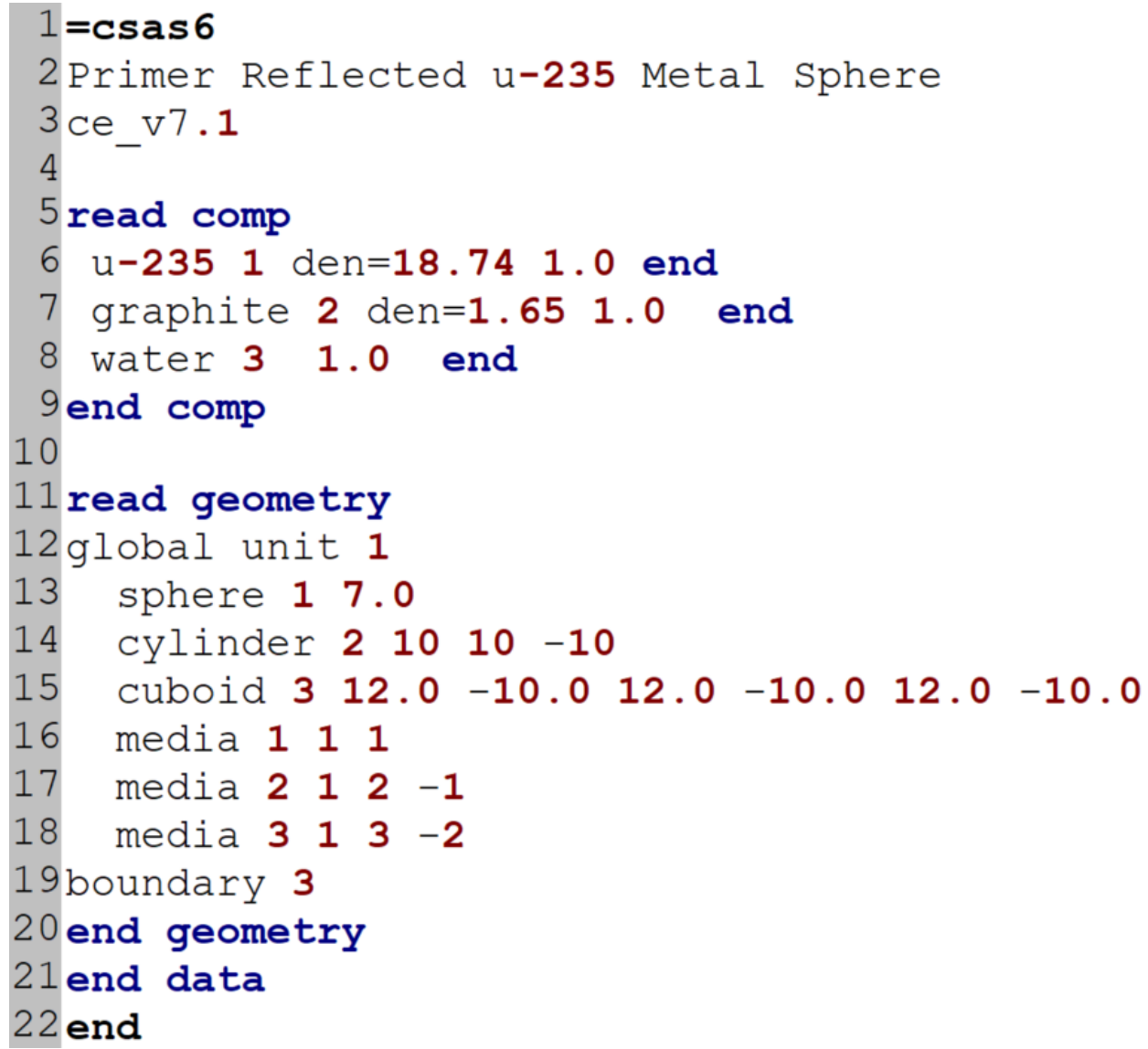

Figure 83. Input file for the ${ }^{235} \mathrm{U}$ sphere in graphite and water example. 


\subsection{ARRAYS}

An array is constructed by stacking units. Each unit in an array or lattice has its own coordinate system; however, all coordinate systems in all units must have the same orientation. All geometry data used in a problem are correlated to the absolute coordinate system by specifying a global unit.

Arrays are created by stacking units that have an outer boundary that matches the array type in which they will be used. For example, the unit must have a cuboid as an outer boundary to be used in a cuboidal array. The size of adjacent faces of adjacent units stacked in this manner must match exactly. See Figure 84 for a typical example.

UNIT 1
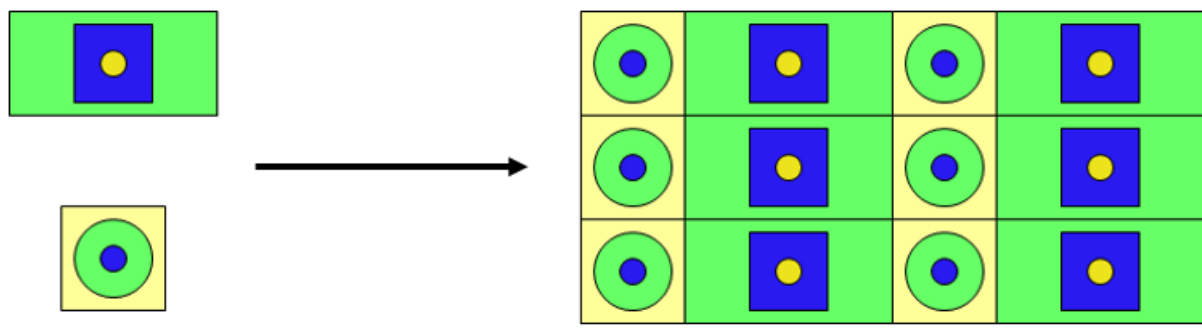

UNIT 2

Figure 84. Example of array construction.

The ARRAY option is provided to allow placing an array within a unit. Arrays of dissimilar units can be created in some cases by stacking units that contain arrays (i.e., "arrays of arrays").

KENO-VI offers five different 3D array types, as shown in Figure 85:

- cuboidal (or square),

- hexagonal (or triangular),

- standard hexagonal,

- rotated hexagonal, and

- dodecahedral. 


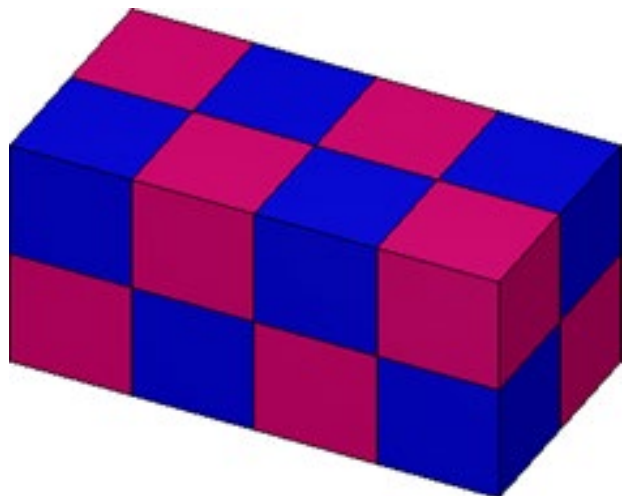

Cuboidal (or Square)

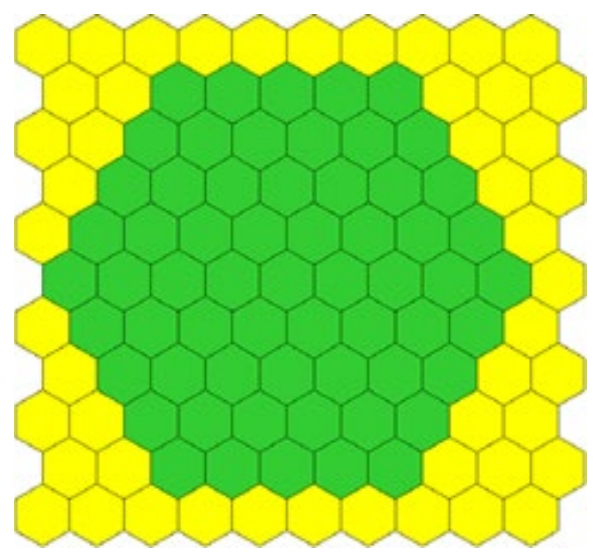

Standard hexagonal

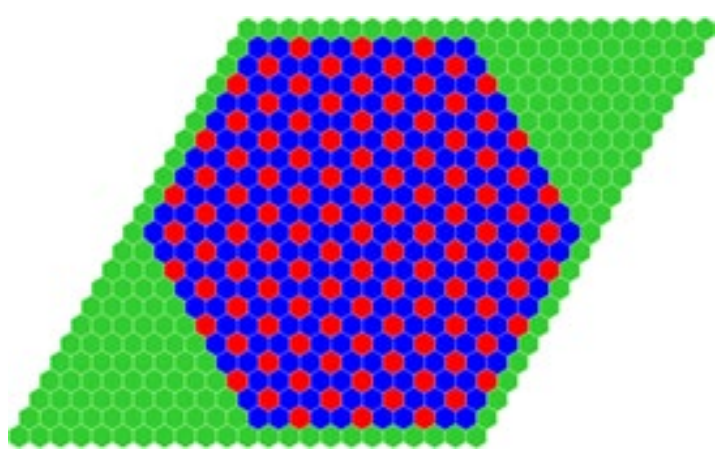

Hexagonal (or triangular)

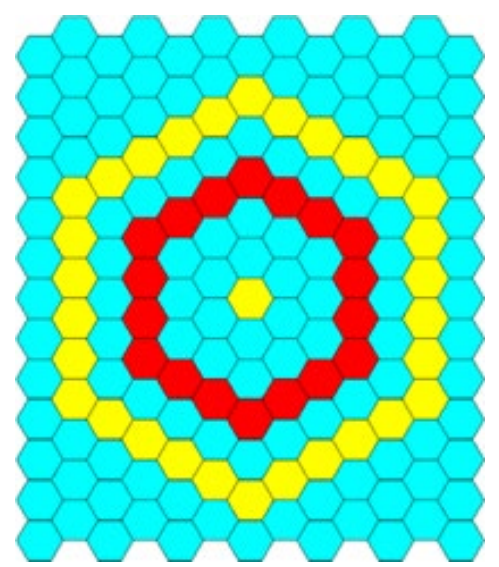

Rotated hexagonal

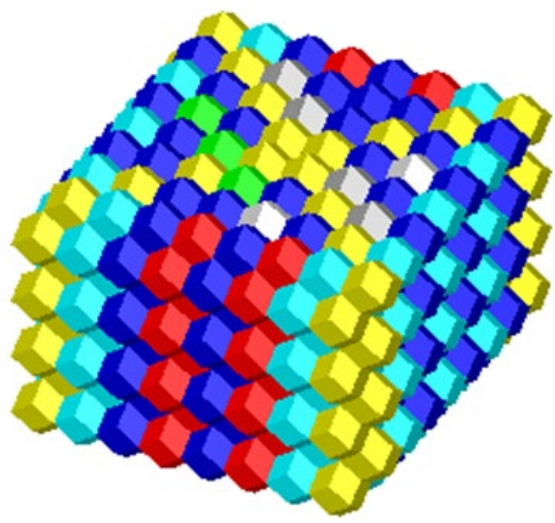

Dodecahedral

Figure 85. KENO-VI array types.

\subsubsection{Arrays with Single Units}

This problem consists of a $2 \times 2 \times 2$ array of bare metal $\mathrm{U}(93.2)$ cylinders. Each cylinder has a radius of $5.748 \mathrm{~cm}$ and an outside height of $10.765 \mathrm{~cm}$. The uranium has a density of $18.76 \mathrm{~g} / \mathrm{cc}$ with $1 \mathrm{wt} . \%{ }^{234} \mathrm{U}$, 93.2 wt. $\%{ }^{235} \mathrm{U}, 0.2 \mathrm{wt} . \%{ }^{236} \mathrm{U}$, and $5.6 \mathrm{wt} . \%{ }^{238} \mathrm{U}$. The pitch in the $\mathrm{X}$ and $\mathrm{Y}$ directions is $13.74 \mathrm{~cm}$, and it 
is 13.01 in the $\mathrm{Z}$ direction. There are no external reflectors or other materials in the array. The geometry is shown in Figure 86.

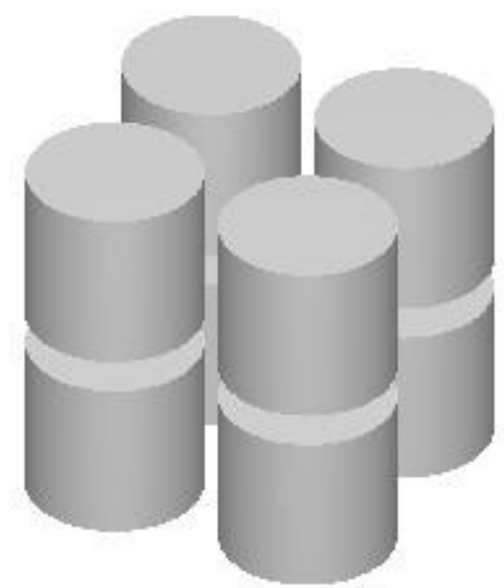

Figure 86. 3D view of $2 \times 2 \times 2$ array.

As mentioned previously, the outside shape of a unit must be a cuboid, a hexprism, or a dodecahedron to be placed in an array. As the constituents of this array are cylinders, they must be enclosed by cuboids to become part of a rectangular array. The dimensions of the cuboid are usually determined by the X-, Y-, and Z-pitch dimensions. For an array of single elements, the cuboid will have an $\mathrm{X}$ dimension equal to the pitch in the $\mathrm{X}$ direction, a $\mathrm{Y}$ dimension equal to the pitch in the $\mathrm{Y}$ direction, and a $\mathrm{Z}$ dimension equal to the pitch in the $\mathrm{Z}$ direction.

Using knowledge from previous sections, open a new file and enter the title, cross section library, and material information for the uranium, including the isotopic composition weight percentages.

This geometry has two regions in one unit, but that unit is not the global unit for the problem. The global unit for this problem will contain the array of eight units. The uranium cylinder will be described first, and then the cuboid enclosing the cylinder to create an element of the array. These are nested shapes and are entered in the same manner as for the previous examples. However, be careful when entering the $Z$ data for both the cylinder and the cuboid. Once the $\mathrm{Z}$ or height information has been entered for the cylinder, the coordinate system and its origin will be fixed. There are three obvious choices for the location of the origin: the bottom center of the cylinder, the middle of the cylinder, or the top center of the cylinder. In some problems, one origin location may make data input for other shapes in a unit easier than other origin locations.

For this problem, choose the origin to be located at the middle of the cylinder, to minimize the arithmetic needed to center the cylinder in the cuboid. This is a frequent choice for a unit which will be used multiple times in an array. The Radius for the cylinder will be 5.748, the Top will be 5.3825, and the Bottom will be -5.3825 (see Figure 79). For the cuboid, the origin is located in the middle in the X, Y, and $\mathrm{Z}$ directions, so the dimensions will be half the pitch: $+\mathbf{X}$ and $+\mathbf{Y}$ will be $6.87,-\mathbf{X}$ and $-\mathbf{Y}$ will be $-6.87,+\mathbf{Z}$ will be 6.505 , and $-\mathbf{Z}$ will be -6.505 . These parameters are sufficient for describing the single array element as shown in Figure 87. 

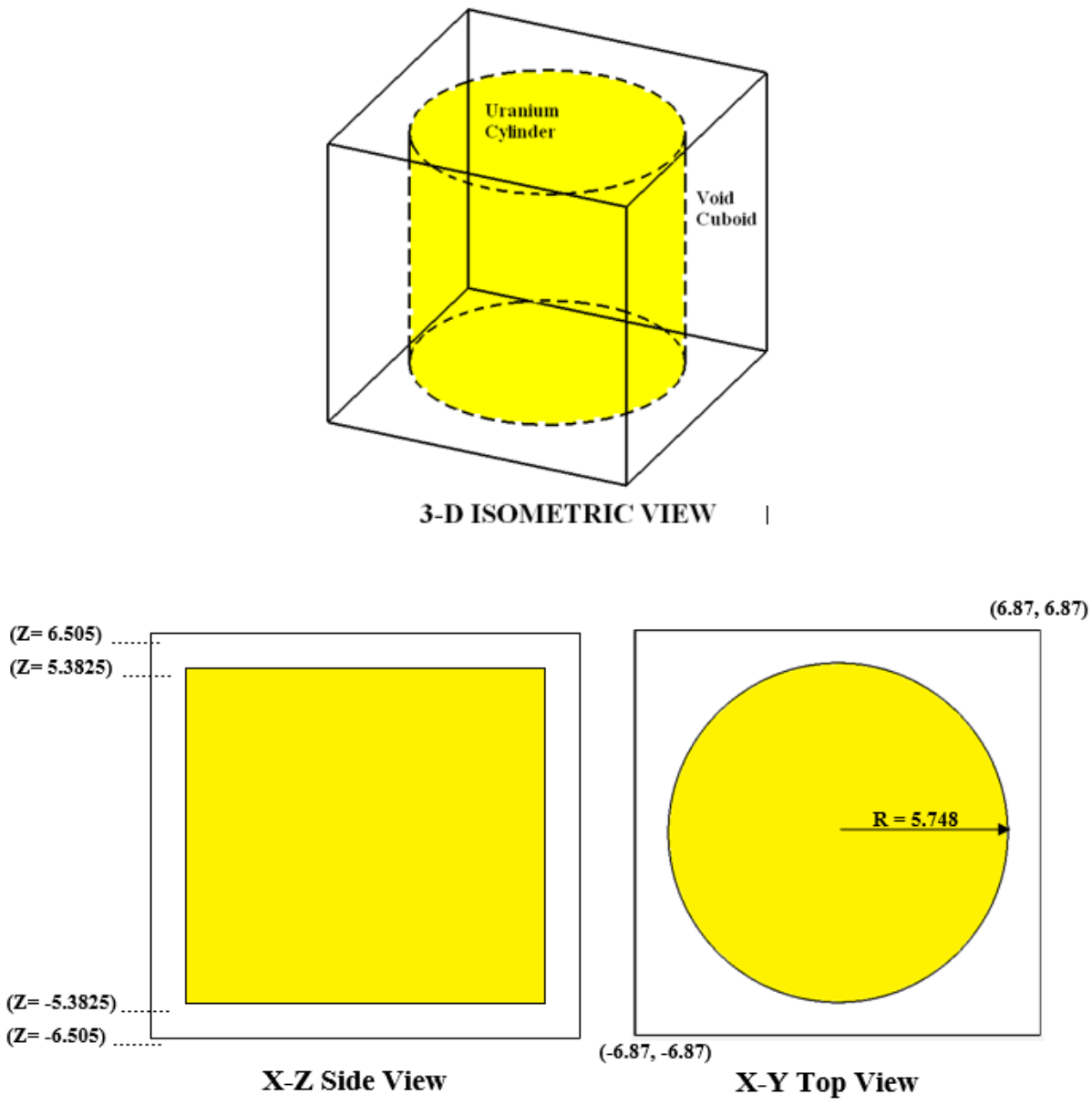

Figure 87. Single array element (cuboid enclosing a cylinder).

Start writing the geometry by placing the text cursor within the read geometry block, between Global Unit 1 and boundary 1, and press the CTRL-SPACE autocomplete key combination. Select cylinder- kenovi (configurable), which opens the Cylinder input form. Enter 5.748 for Radius, 5.3825 for Top, and -5.3825 for Bottom. This puts the origin at the center of the cylinder; this is now the origin for the unit and for all other regions in the unit. To enter the data for the enclosing cuboid, place the text cursor within the read comp block and press the CTRL-SPACE autocomplete key combination. Click on the cuboid- kenovi (configurable) button, which opens the Cuboid input form, to describe the void cuboid. Change the Id to 2. Enter 6.87 for $+\mathbf{X}$ and $+\mathbf{Y},-6.87$ for $-\mathbf{X}$ and $-\mathbf{Y}, 6.505$ for $+\mathbf{Z}$, and -6.505 for $\mathbf{- Z}$. This creates a cuboid around the cylinder with the proper spacing. Before proceeding with the media definition, note that unit 1 will be placed inside an array, so it is not the global unit of the problem. To tell Fulcrum so, remove Global from Global unit 1.

Use two media records to define the two regions (uranium inside the cylinder and void (mixture 0 ) inside the cuboid and outside the cylinder). The final step is to define the outer boundary of unit 1 . The outer boundary of the unit was already defined thanks to the Fulcrum autocompletion as boundary 1 . As the outer region is the void cuboid, region 2, change to boundary 2 on the line after the media definition. When finished, the composition and geometry blocks should look like Figure 88. The Array data will be entered next. 


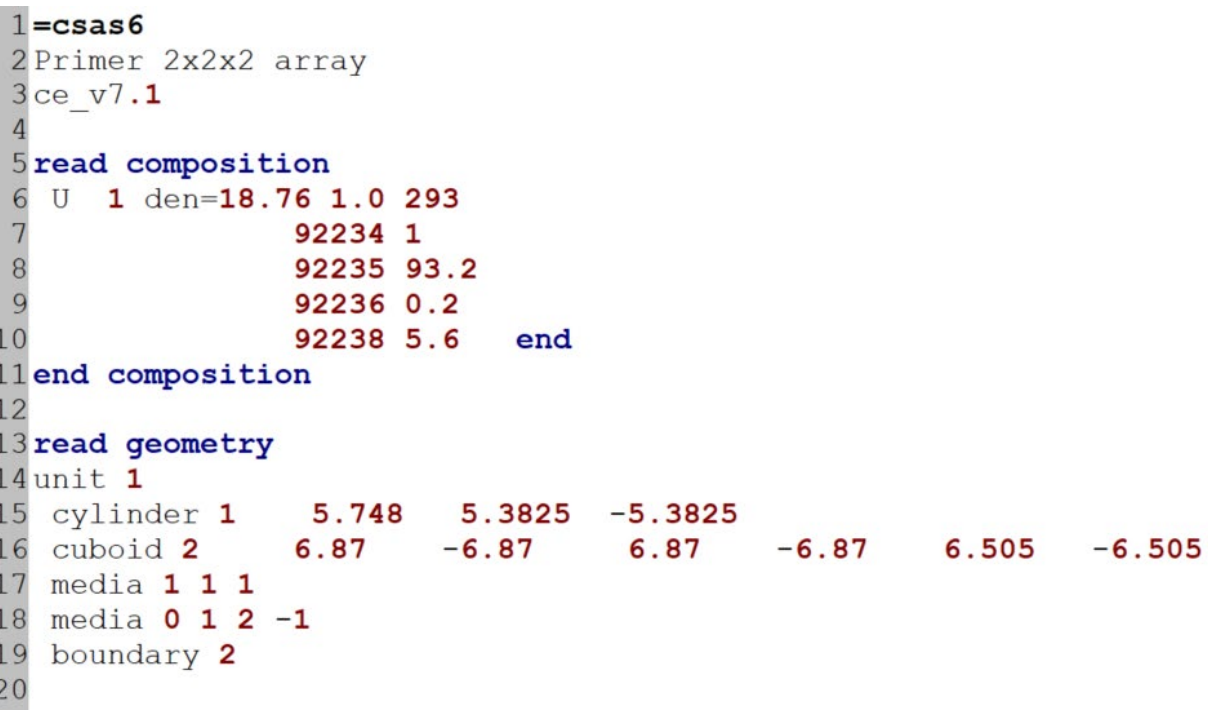

Figure 88. Single unit geometry for array.

Arrays are defined in a separate block in the input file. Each array is specified with an integer ID, the type of array, the number of elements in the $\mathrm{X}, \mathrm{Y}$, and $\mathrm{Z}$ directions, and the units that fill the array. The integer ID is used to reference the array when it is placed into a unit in the geometry block. The type of the array, as discussed above, is one of the five array types supported in KENO-VI: cuboidal, hexagonal, standard hexagonal, rotated hexagonal, or dodecahedral. The outer boundary of the units to be used in this array must match the type of the array. The fill data provides the unit numbers to be placed into the array in the order that they will be used. The unit numbers are read in order of increasing $\mathrm{X}$, then increasing $\mathrm{Y}$, then increasing $\mathrm{Z}$. This order will be demonstrated in this example.

To start describing the array, place the cursor on the line after end geometry and use the CTRLSPACE autocomplete key combination to generate a list of available blocks to add to the input, as shown in Figure 89. Within the empty array block, use the CTRL-SPACE autocomplete key combination to open the configurable array form shown in Figure 90. This array is the first and only array in the problem, so accepting the default entry of 1 in the ara field is recommended. The defaults are also acceptable for gbl (there is no global array in this problem), prt (print a description of this array in the output), and typ (this array is cuboidal). The array is 2 units in the $\mathrm{X}$ direction, 2 units in the $\mathrm{Y}$ direction, and 2 units in the $\mathrm{Z}$ direction, so change nux, nuy, and nuz to 2 . Now that the $2 \times 2 \times 2$ array is defined, it must be filled with units. In this problem, there is only unit 1 . The fill id field specifies the unit with which to initialize the fill data, so the default of $\mathbf{1}$ is appropriate here as well. The order of the units stored in the array using the FILL parameter is $(1,1,1)(2,1,1)(1,2,1)(2,2,1)(1,1,2)(2,1,2)(1,2,2)(2,2,2)$, as shown in Figure 91 . Check that the array configurable form matches the example provided in Figure 92, then press OK to add the array specification to the input file. The completed array block is shown in the input in Figure 93. 


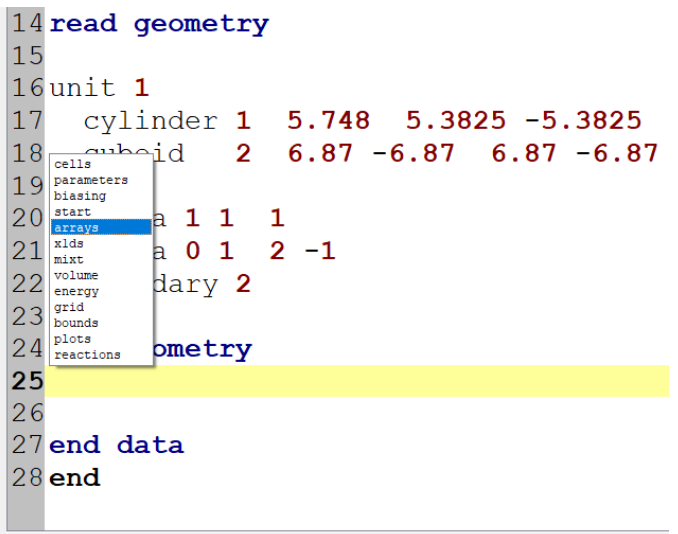

Figure 89. Adding array block to the input with autocomplete.

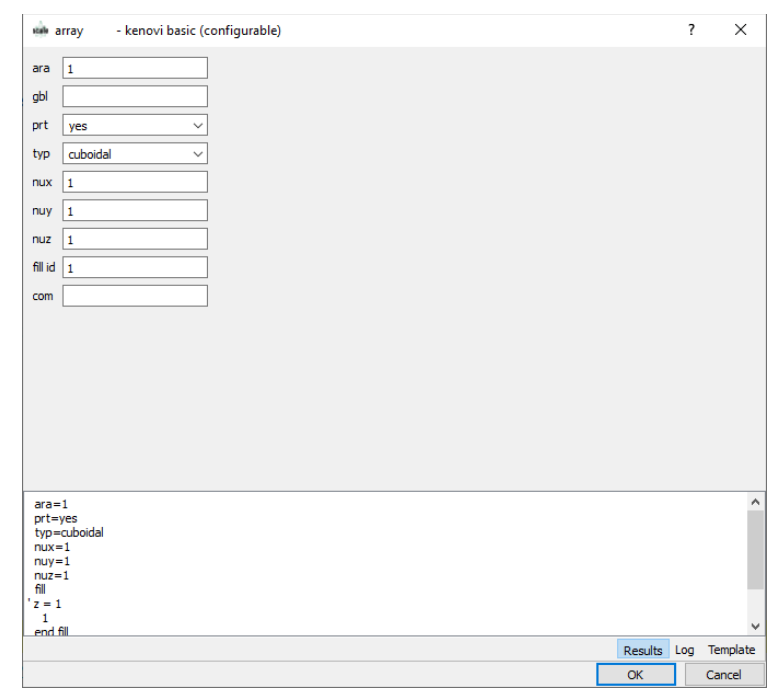

Figure 90. Default array configurable form in Fulcrum.
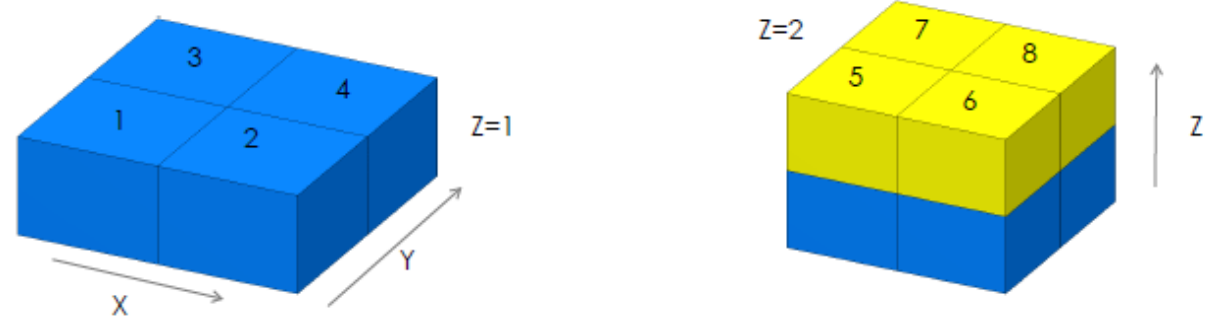

Figure 91. Unit filling order in a basic rectangular array. 


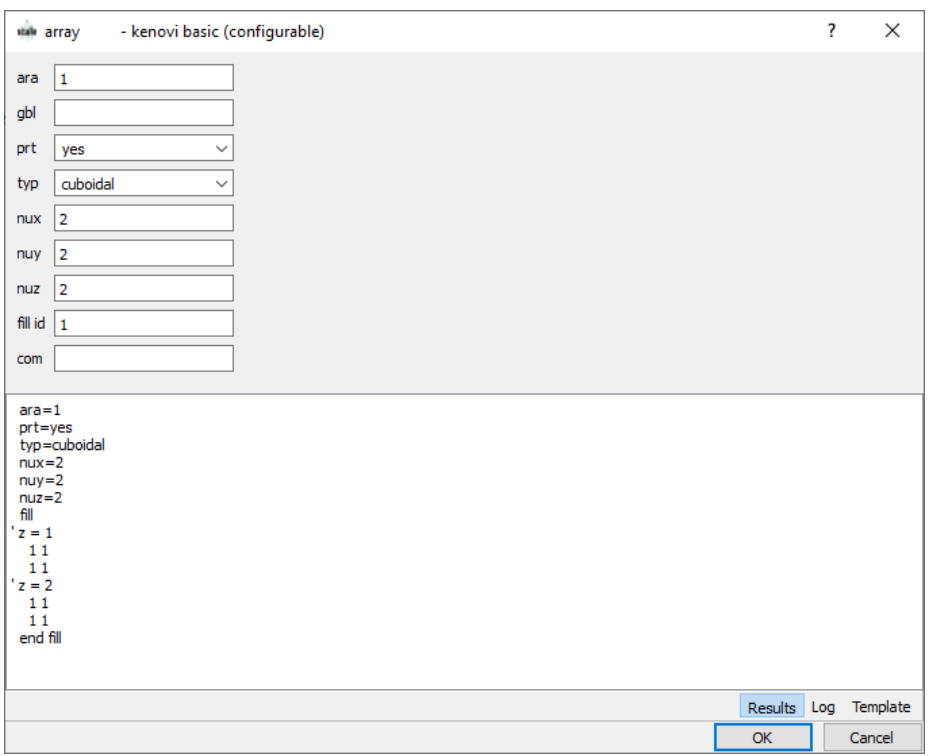

Figure 92. Completed array configurable form for this example.

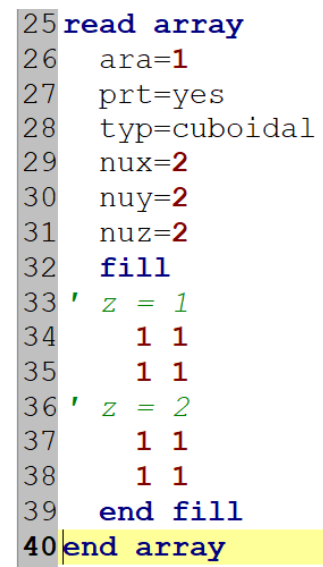

Figure 93. Completed array block for this example.

Return to the geometry definition block and use the CTRL-SPACE autocomplete key combination to add a global unit, then change the unit number to 2 . This unit will be the global unit that contains array 1 . Position the text cursor in the global unit and press the CTRL-SPACE autocomplete key combination. Select the cuboid- kenovi (configurable), which opens the Cuboid input form. Since the cuboid must contain array 1, the dimensions of the cuboid should be the same as those of the array. Because this is a 2 $\times 2 \times 2$ array, simply double the $\mathrm{X}, \mathrm{Y}$, and $\mathrm{Z}$ dimensions of unit 1 . If we center the cuboid on the origin, then the outer dimensions of the cuboid will be \pm 13.74 for $\pm \mathbf{X}$ and $\pm \mathbf{Y}$ and \pm 13.01 for $\pm \mathbf{Z}$. After the dimensions are entered, press the $\mathbf{O K}$ button to close the cuboid form.

The last step in the array definition is to define the placement of array 1 in the cuboid. Similar to the media record, the ARRAY Contents Record must be used to place an array into a region defined in the current unit. To do so, use the CTRL-SPACE autocomplete key combination within the global unit and select array - kenovi (configurable) as shown in Figure 94. The default array configurable form is shown in Figure 95. The Array Id dropdown in the upper left corner of the form selects the array to be added to the geometry; in this case, the default value of $\mathbf{1}$ is used. The Regions pane allows the specification of the Region Definition Vector defining the region of space the array will fill. The data 
entry in this pane is the same as in the media record configurable form discussed above. Press the Add row button to start entering the Region Definition Vector; the array will be placed inside of region 1 so the default information can be accepted. The last section of the input is used to position the array within the specified region. The first part of the Place input selects the element in the array to be used for specifying this position; in this example the xindex, yindex, and zindex of 1 is as easy as any other element in the array. In some cases, there are particular elements of the array, like a central one, that are more useful than others for easily and correctly positioning an array. The $\mathbf{x}, \mathbf{y}$, and $\mathbf{z}$ coordinates specify the position of the origin of the selected unit in the current unit coordinate system. For this example, only unit 1 is used and the origin is at the center of the unit. The origin is thus $6.87 \mathrm{~cm}$ from the $-\mathrm{X}$ and $-\mathrm{Y}$ faces of the unit and $6.505 \mathrm{~cm}$ from the $-Z$ face. The location of these negative surfaces of cuboid 1 have been specified as -13.74 in $X$ and $Y$ and -13.01 in $Z$, so the position of the origin can be determined. For $\mathrm{X}$ and $\mathrm{Y},-13.74+6.87=-6.87$ and for $\mathrm{Z}-13.01+6.505=-6.505$. Therefore, enter -6.87 for $\mathbf{x}$ and $\mathbf{y}$ in the array configurable and -6.505 for $\mathbf{z}$. This completes the array configurable form and, after confirming that it matches that shown in Figure 96, the array contents record can be added to the input file by pressing the $\mathbf{O K}$ button.

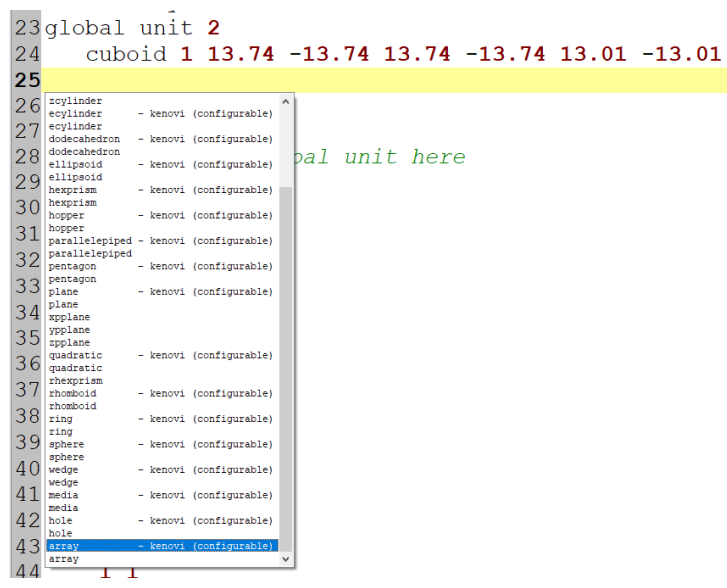

Figure 94. Autocomplete menu for array contents record.

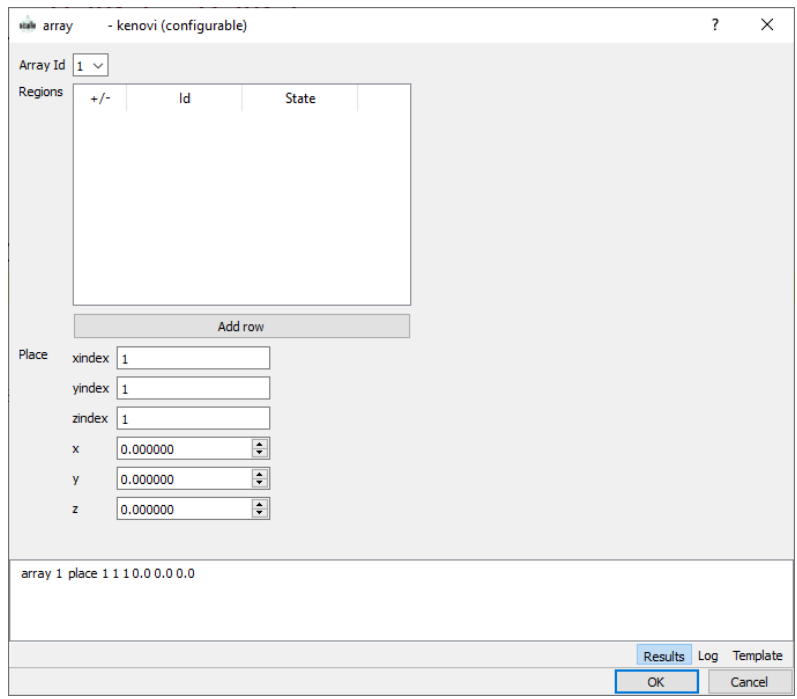

Figure 95. Default array configurable form. 


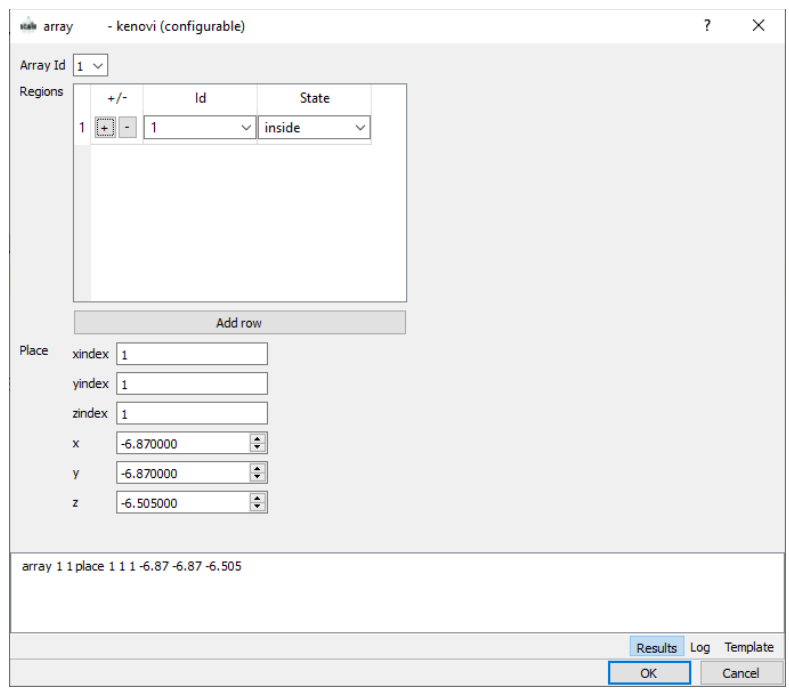

Figure 96. Completed array contents record configurable form.

The final step is to define the outer boundary of unit 2 . The outer region is the cuboid, region 1 . Enter boundary 1 on the line after the array definition. The geometry and array blocks should look like Figure 97. Save the input file and execute SCALE. The calculated $k_{\text {eff }}$ for this critical experiment is $0.9991 \pm 0.0017$.

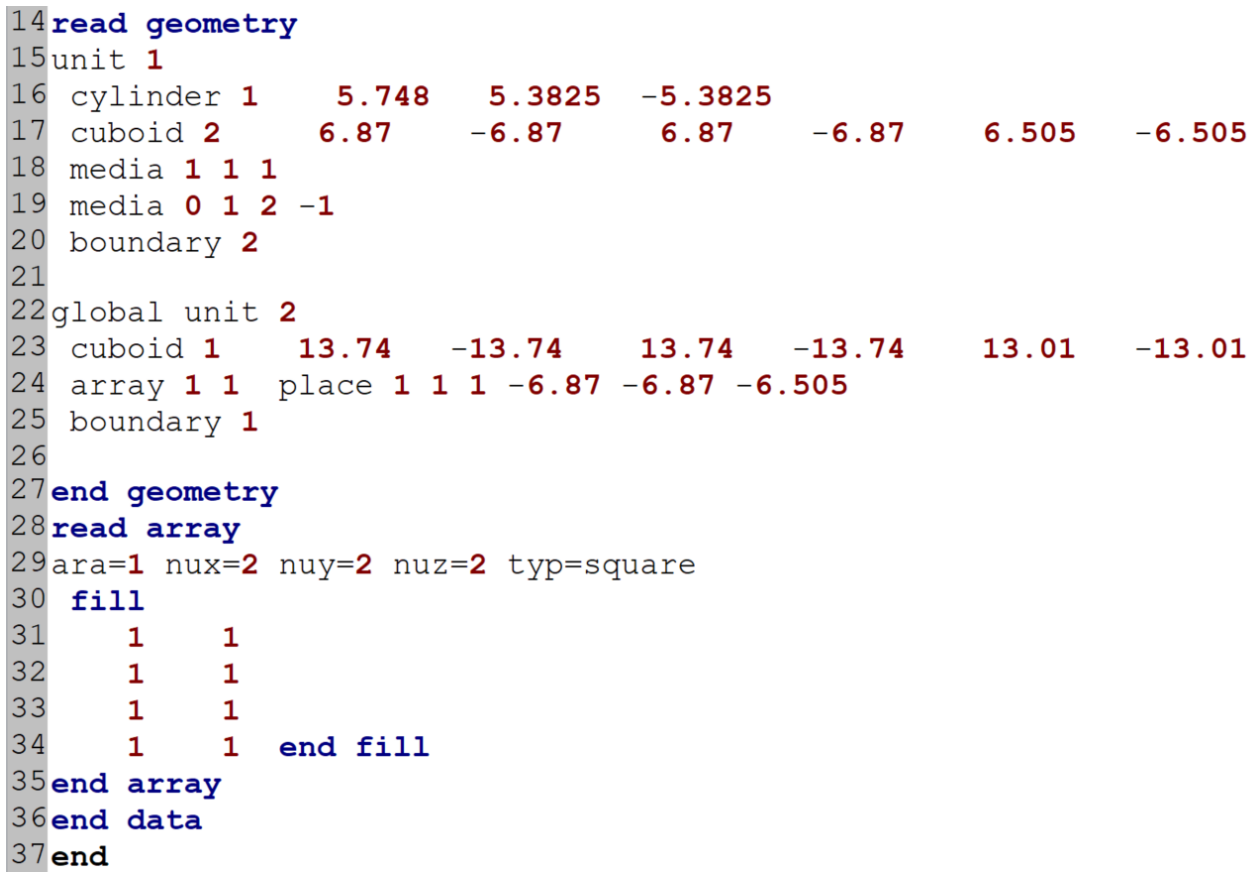

Figure 97. Geometry form with array record to place array in geometry.

\subsubsection{Arrays with Multiple Units of Different Sizes}

This problem consists of a stack of six cylindrical disks covered by a square plate on the top (Figure 98). There are two types of disks; one containing graphite (density $=1.65 \mathrm{~g} / \mathrm{cc}$ ) that is $10 \mathrm{~cm}$ in radius and 2.5 $\mathrm{cm}$ thick, and the other containing $\mathrm{U}(93.2)$ that is $10 \mathrm{~cm}$ in radius and $4 \mathrm{~cm}$ thick. The uranium has a 
density of $18.74 \mathrm{~g} / \mathrm{cm}^{3}$, with 1 wt. $\%{ }^{234} \mathrm{U}, 93.2$ wt. $\%{ }^{235} \mathrm{U}, 0.2 \mathrm{wt} . \%{ }^{236} \mathrm{U}$, and $5.6 \mathrm{wt} . \%{ }^{238} \mathrm{U}$. Progressing upward from the bottom, the disks are graphite, uranium, graphite, uranium, graphite, and uranium. The top plate is aluminum of nominal density, $30 \times 30 \mathrm{~cm}$ square and $3 \mathrm{~cm}$ thick. There are no external reflectors or other materials in the array.
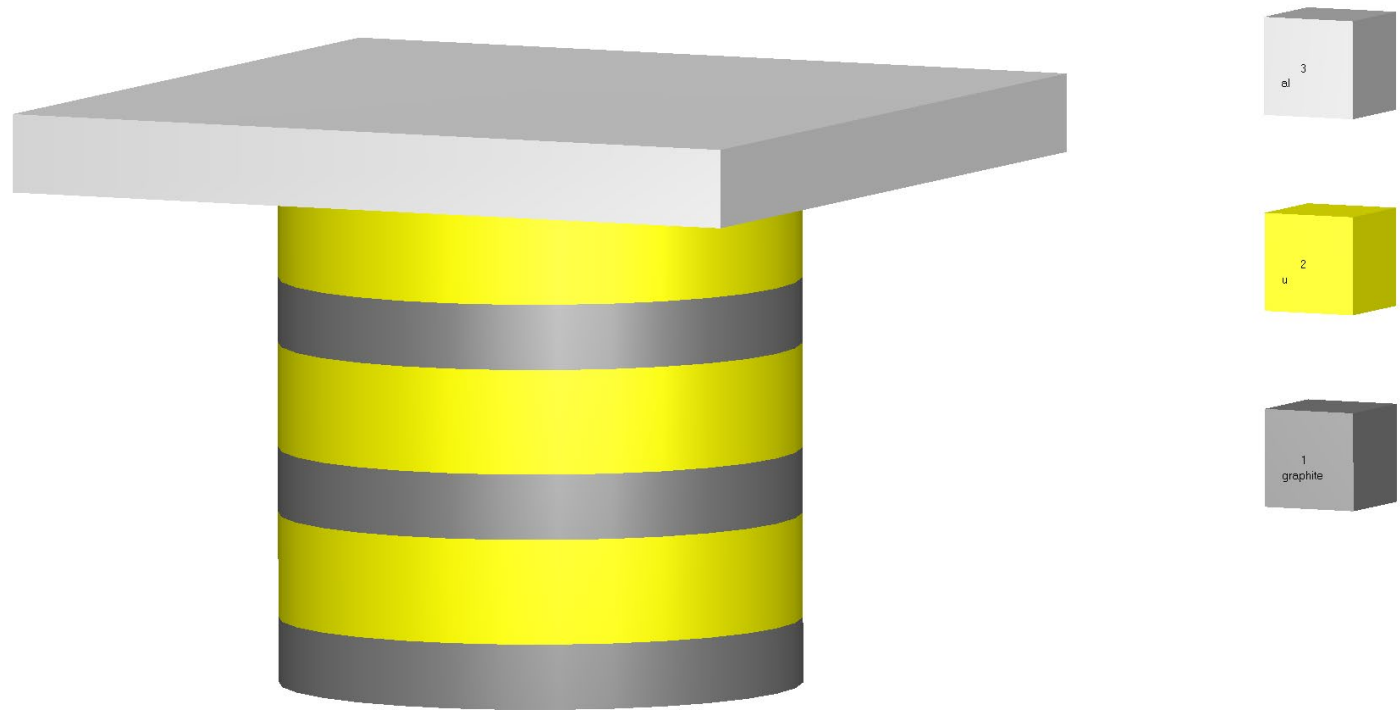

Figure 98. Geometry of stack.

For a unit to be placed in an array, the touching faces of adjacent units must be the same size. This means that the enclosing cuboid for both cylinder types must be the same size in the $\mathrm{X}$ and $\mathrm{Y}$ dimensions as the top plate. Thus, the length of the $X$ and $Y$ sides of the cuboid must be $30 \mathrm{~cm}$. Notice that there is no constraint on the $\mathrm{Z}$ dimension of the cuboid because there are no adjacent faces in that direction. However, to stack the cylinders so that they touch (i.e., no gaps between them), the height of each cuboid surrounding a cylinder must be the same as the height of the cylinder.

Using your knowledge from previous sections, open a new file and enter the title, cross section library, and material information for the graphite, uranium (including the isotopic composition weight percentages), and aluminum.

The first unit will be the graphite cylinder surrounded by a void cuboid so that the unit can be stacked in an array. Start writing the geometry by placing the text cursor within the read geometry block, between Global Unit 1 and boundary 1, and press the CTRL-SPACE autocomplete key combination. Select the cylinder- kenovi (configurable), which opens the Cylinder input form. To describe the graphite cylinder, enter 10 for Radius, 2.5 for Top, and 0.0 for Bottom. This puts the origin for the unit and for all shapes in the unit at the center of the cylinder on its bottom face. To enter the data for the enclosing cuboid, place the text cursor within the read comp block, and press the CTRL-SPACE autocomplete key combination. Select the cuboid- kenovi (configurable), which opens the Cuboid input form, to describe the void cuboid. Change the Id to 2 . Enter 15 and -15 for $+\mathbf{X}$ and $-\mathbf{X}$ and for $+\mathbf{Y}$ and $-\mathbf{Y}$. Enter 2.5 and 0.0 for $+\mathbf{Z}$ and $-\mathbf{Z}$. This creates a cuboid around the cylinder with the proper spacing. Remove Global from Global unit 1 , as unit 1 will be placed in an array.

Use the media records twice to define the two regions. The first region is inside cylinder 1 and is filled with graphite. The second region is inside cuboid 2 and outside cylinder 1 and is filled with void. Change 
boundary 1 to boundary 2 on the line after the media definition to designate the outer boundary as inside cuboid 2.

Now describe the uranium cylinder surrounded by a void cuboid. After the TODO comment boundary 2, create unit 2 by pressing CTRL-SPACE autocomplete key combination and select unit. Change the unit number to 2 . Then press the CTRL-SPACE autocomplete key combination inside the new unit. Again, select the cylinder- kenovi (configurable), which opens the Cylinder input form, to describe the uranium cylinder. Enter 10 for Radius, 4 for Top, and 0.0 for Bottom. To enter the data for the enclosing cuboid, place the text cursor within unit 2 and press the CTRL-SPACE autocomplete key combination. Select cuboid- kenovi (configurable), change the Id to 2 and enter 15 and -15 for $+\mathbf{X}$ and $\mathbf{X}$ and for $+\mathbf{Y}$ and $-\mathbf{Y}$. Enter 4 and 0.0 for $+\mathbf{Z}$ and $-\mathbf{Z}$. This creates a cuboid around the cylinder with the proper spacing. Use the media records twice to define the two regions: uranium in the cylinder and void in the cuboid. Change the boundary record to boundary 2 to designate the outer boundary as inside cuboid 2.

Now describe the aluminum plate by creating unit 3. On the line after boundary 2, create unit 3 by typing unit 3 and on the next line type boundary 1 . Then placing the text cursor between these two lines and press the CTRL-SPACE autocomplete key combination. Select the cuboid- kenovi

(configurable), which opens the Cuboid input form, to describe the aluminum cuboid. Enter 15 and -15 for $+\mathbf{X}$ and $-\mathbf{X}$ and for $+\mathbf{Y}$ and $-\mathbf{Y}$. Enter 3 and 0 for $+\mathbf{Z}$ and $-\mathbf{Z}$. Use a media record to specify that this cuboid contains aluminum. When you are finished, the composition and geometry blocks should look like Figure 99.

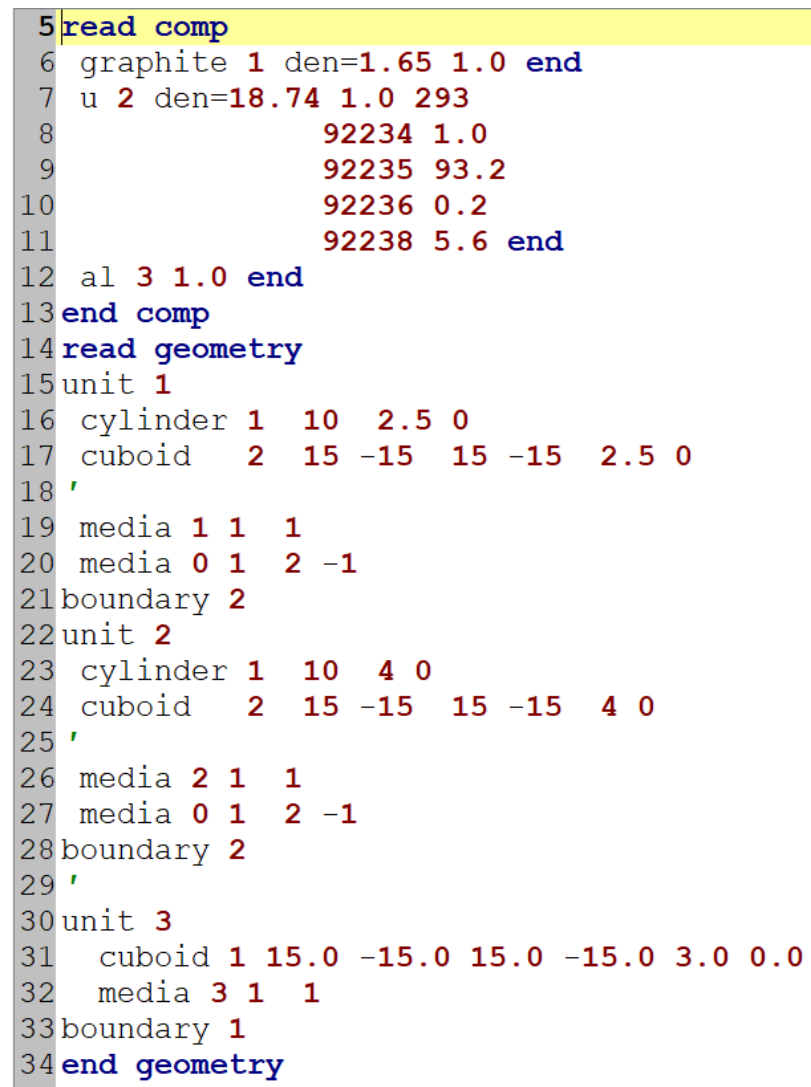

Figure 99. Composition and geometry blocks prior to array definition. 
To start describing the array, place the cursor on the line after end geometry and use the CTRLSPACE autocomplete key combination to generate a list of available blocks to add to the input, as shown in Figure 89. Within the empty array block, use the CTRL-SPACE autocomplete key combination to open the configurable array form shown in Figure 90. This array is the first and only array in the problem, so accepting the default entry of 1 in the ara field is recommended. The defaults are also acceptable for $\mathbf{g b l}$ (there is no global array in this problem), prt (print a description of this array in the output, and typ (this array is cuboidal). This array is a $1 \times 1 \times 7$, so nux and nuy should be left as $\mathbf{1}$ and nuz should be set to 7 . Any initial fill id can be used as several will have to be changed, so accepting the default of 1 is the simplest answer. The completed configurable form is shown in Figure 100; after checking the input press OK to add the array definition to the input file. Axial levels 2, 4, and 6 must be changed to unit 2 to add the uranium unit and axial level 7 must be changed to unit 3 to put the aluminum slab on the top of the array. The final complete array specification is provided in Figure 101.

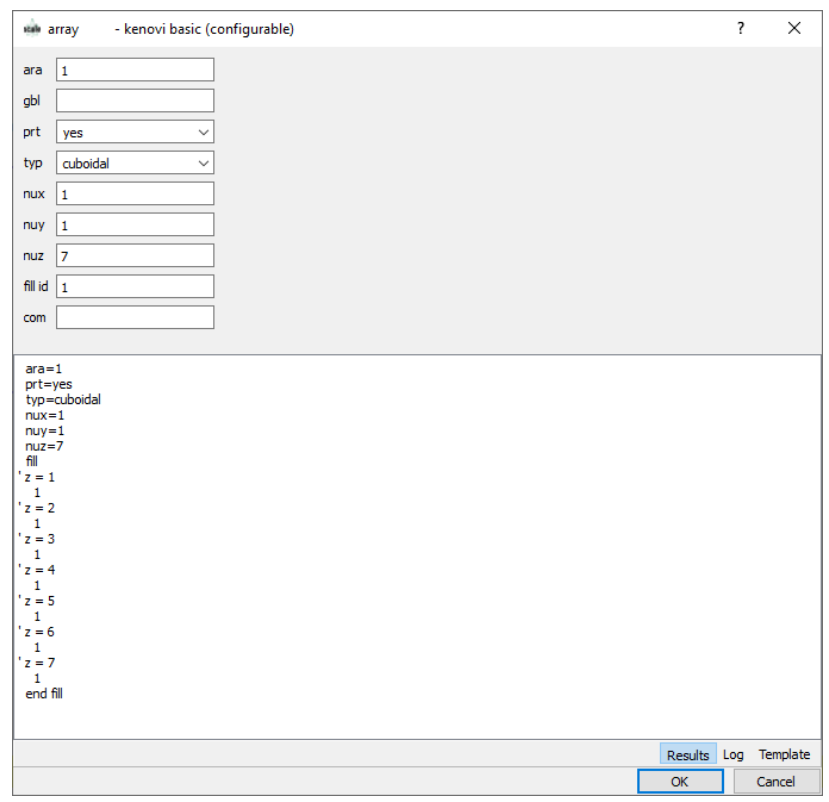

Figure 100. Fulcrum configurable form for the array in this example.

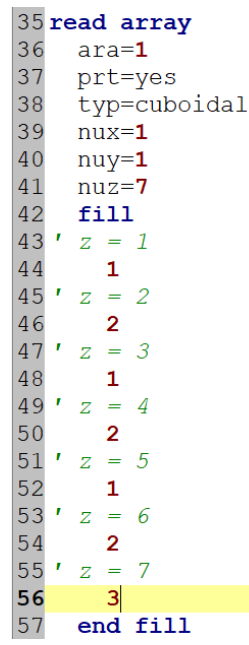

Figure 101. Completed array block for this example. 
Return to the Geometry definition block and enter global unit 4 to create unit 4 and enter boundary 1 on the next line. This unit will be the global unit that contains array 1 . With the text cursor in the unit, press the CTRL-SPACE autocomplete key combination and select the cuboid- kenovi (configurable) button to open the Cuboid input form. Note that the geometry shape that contains an array must have dimensions that are exactly the same as or smaller than the outer dimensions of the array. Otherwise there will be undefined volumes inside the shape but outside the array. Enter the dimensions for the cuboid: \pm 15 for $\pm \mathbf{X}$ and $\pm \mathbf{Y}, 22.5$ for $+\mathbf{Z}$, and 0 for $-\mathbf{Z}$. After the dimensions are entered, press the $\mathbf{O K}$ button to close the cuboid form.

The last step in the array definition is to define the placement of array 1 in the cuboid using the ARRAY Contents Record. To do so, use the CTRL-SPACE autocomplete key combination and select array kenovi (configurable). The Array Id of 1 is correct as the array defined in the array block is declared as array 1. Press the Add row button to start defining the region definition vector for the array contents record. The default values are useful here as well as the array is positioned inside cuboid 1 . The simplest choice for positing this array is the first element in the array which is unit 1 . This is a simple choice because the origin of unit 1 is in the center on the bottom surface of the cylinder; this can be positioned at $(0,0,0)$ to align the entire array correctly in cuboid 1 . To accomplish this, ensure that the xindex, yindex, and zindex fields are all 1 and the $\mathbf{x}, \mathbf{y}$, and $\mathbf{z}$ are 0 . The geometry block should look like Figure 102 . Save the input file and execute SCALE. The calculated $k_{\text {eff }}$ for this critical experiment is $0.9550 \pm 0.0019$.

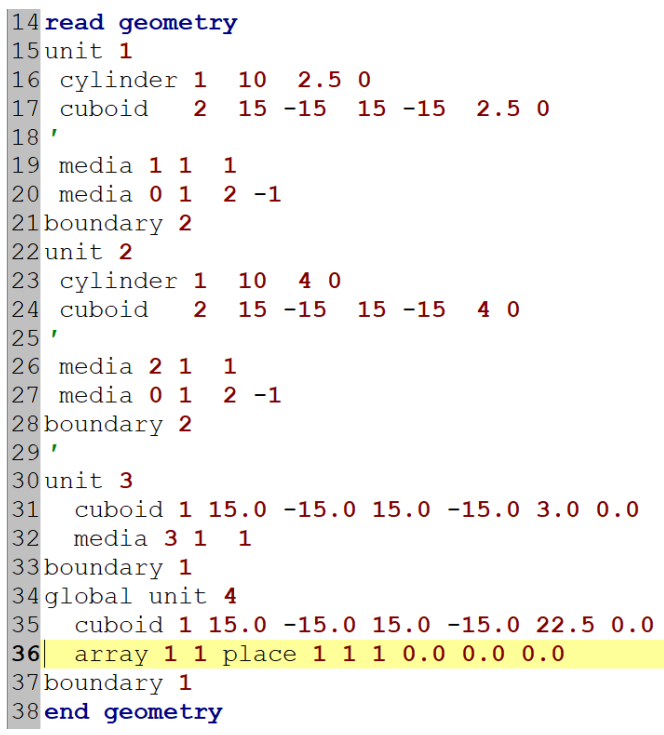

Figure 102. Complete geometry description for this example.

\subsection{VOLUME CALCULATIONS}

In some applications, the knowledge of region and/or mixture volumes is necessary. For example, calculating fission densities or fluxes in a particular cell region requires the code to know the volume of that region. It is possible to calculate the volume by using the volume block. Different ways of calculating volumes are available in the volume block; only one example is introduced in this primer.

In this example, the volumes of the regions from the previous example, the array with multiple units of the same size, will be calculated. Open the input file that was previously saved, start defining the volume block by placing the text cursor after end array, and use the CTRL-SPACE autocomplete key combination to generate a list of available blocks to add to the input. Select volume, as shown in Figure 103. First, use the CTRL-SPACE autocomplete key combination in the volume block to select the 
random volume calculation type. Next, add batches with the CTRL-SPACE autocomplete key combination and set it to 1000 and add points and set it to 1000 . The uncertainty of the calculated volumes is inversely proportional to the total number of samples, which is the product of the points and batches. Finally, a new region must be defined to designate the region of the model in which the volume calculation will be performed. The volume of all regions in the model are desired in this example, so the boundaries of the global unit are used. The syntax in the volume block is different from the geometry block: the positive and negative dimensions are provided in the $\mathrm{x}, \mathrm{y}$, and $\mathrm{z}$ dimensions. Use the CTRLSPACE autocomplete key combination to add $\mathbf{x p}, \mathbf{x m}, \mathbf{y p}, \mathbf{y m}, \mathbf{z p}$, and $\mathbf{z m}$ keywords to the input. The $\mathbf{x p}$ and $\mathbf{y p}$ values should be set to 15, the $\mathbf{x m}$ and $\mathbf{y m}$ values should be set to -15 , $\mathbf{z p}$ should be set to 22.5 and $\mathbf{z m}$ should be set to 0 . Note that these dimensions match exactly with the cuboid defining the boundary of the global unit. The volume block should look like Figure 104. Save the updated input file and launch SCALE. In the output file, the region volumes, mixture volumes, and associated uncertainties are now available, labeled total mixture volume $\left(\mathbf{c m}^{* * 3)}\right.$. The result of the volume calculation of region 1 of unit 3 should be $2.35420 \mathrm{E}+03+/-6.34943 \mathrm{E}+00 \mathrm{~cm}^{3}$, and the volume calculation should only take a fraction of a second. Higher precision volume estimates are not needed for this demonstration.

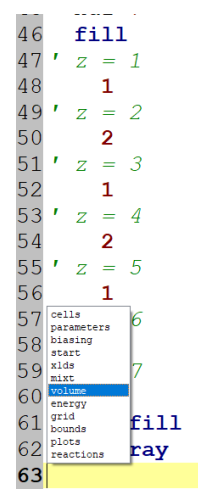

Figure 103. Adding volume block to the input in Fulcrum.

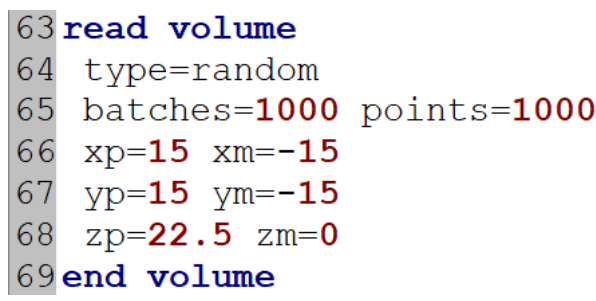

Figure 104. Volume calculation block.

\subsection{SUMMARY}

This section had the following objectives for users:

- to use Fulcrum to describe some basic shapes (sphere, cylinder, cuboid),

- to understand how units are created (including nesting of shapes),

- to locate and change the location of the origin for shapes and units,

- to create simple arrays consisting of a single unit,

- to create arrays with multiple units of nominally different sizes, and

- to use the volume block to calculate volume of regions and/or mixtures.

The next step is to proceed to more advanced geometries. 



\section{ADVANCED GEOMETRY}

The last section focused on some simple geometry problems, including simple array problems. This section provides more advanced geometry options using KENO-VI geometry modification data.

\subsection{WHAT YOU WILL BE ABLE TO DO}

- Use Fulcrum and KENO-VI to describe more advanced geometry models using geometry modification data and holes.

- Use CHORD keyword to truncate a body with a plane perpendicular to a major axis.

- Use ORIGIN keyword to translate the location of a body.

- Use ROTATE keyword to rotate a body to any angle.

- Use HOLE record to place one unit inside another unit.

\subsection{USING THE CHORD KEYWORD TO TRUNCATE A BODY}

The CHORD keyword truncates a geometric volume with a plane perpendicular to a major axis. The location of the cutting plane is specified by the subordinate keywords $+X=,-X=,+Y=,-Y=,+Z=$, and $-\mathrm{Z}=$. Only nonzero data need to be entered. The CHORD keyword applies to the geometry or hole record that immediately precedes it.

Each subordinate keyword specifies a cutting plane perpendicular to the axis in its name (e.g., "+X=" or " $-\mathrm{X}=$ " results in a cutting plane perpendicular to the $\mathrm{X}$-axis). The cutting plane intersects the specified axis at the value that follows the "=" sign. For example, " $+\mathrm{X}=5$ " or " $\mathrm{X}=5$ " specifies a cutting plane perpendicular to the $\mathrm{X}$-axis that crosses the axis at $\mathrm{X}=5$. The positive or negative sign before the axis name indicates on which side of the cutting plane the geometric volume is to be kept. For example, in Figure 105, "- $X=5$ " specifies a cutting plane perpendicular to the $X$-axis at $X=5$ and keeps the volume on the negative side of the plane (i.e., $X \leq 5$ ). Conversely, " $+X=5$ " specifies a cutting plane perpendicular to the $X$-axis at $X=5$ and keeps the volume on the positive side of the plane (i.e., $X \geq 5$ ).

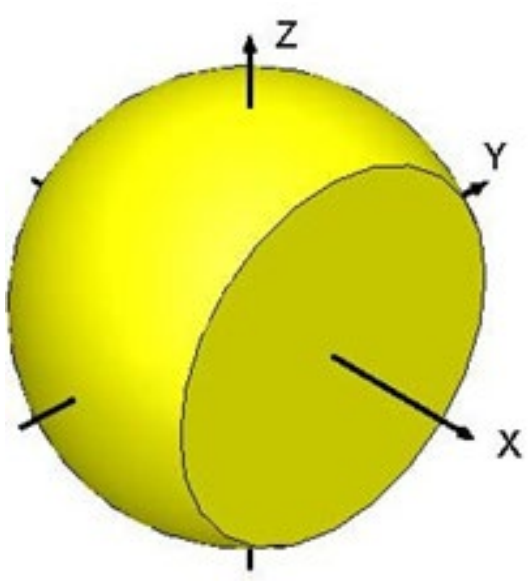

sphere 110.0 chord $-X=5.0$

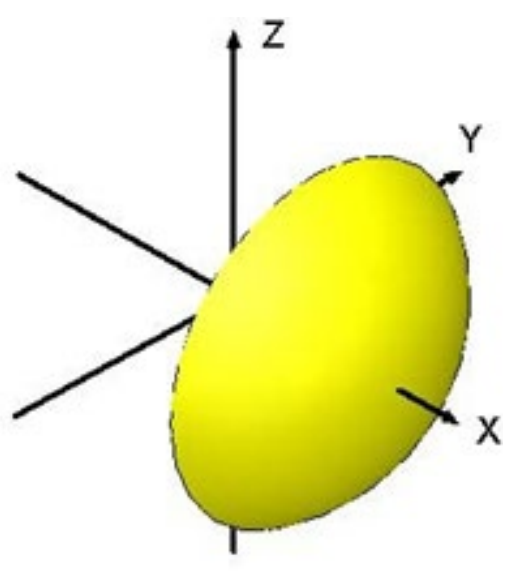

sphere 210.0 chord $+X=5.0$

Figure 105. Examples of body truncation using the CHORD keyword.

It is possible to apply multiple chords to a body by specifying more than one chord and associated subordinate keyword to a body. Figure 106 shows an example in which two chords are applied to a cylinder to obtain a quarter cylinder. The two subordinate keywords " $+X=0$ " and " $+Y=0$ " keep the quarter cylinder where $X \geq 0$ and $Y \geq 0$. Note that the keyword CHORD must be supplied for both cuts. 


\section{$\mathbf{Z}$}

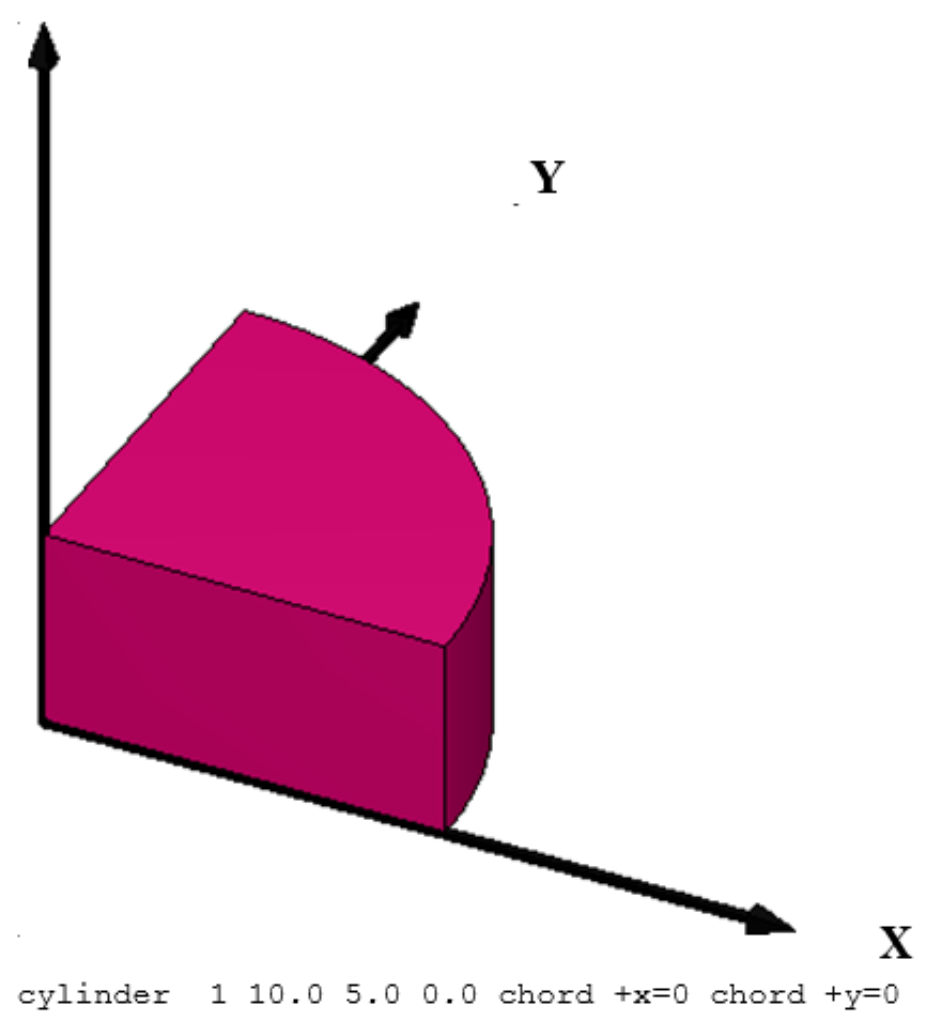

Figure 106. Examples of body truncation using two chords.

\subsection{USING THE ORIGIN KEYWORD TO TRANSLATE A BODY}

The ORIGIN keyword allows translation of the origin of a KENO-VI body to another position within a unit by specifying the $\mathrm{X}, \mathrm{Y}$, and $\mathrm{Z}$ coordinates of the body's origin. The command uses the subordinate keywords $\mathrm{X}=, \mathrm{Y}=$, and $\mathrm{Z}=$. Only nonzero data need to be entered. The ORIGIN keyword applies to the geometry or hole record that immediately precedes it.

A simple example is illustrated in Figure 107, in which the origin of a $10 \mathrm{~cm}$ radius sphere has been translated $10 \mathrm{~cm}$ in $\mathrm{X}, \mathrm{Y}$, and $\mathrm{Z}$. The input syntax for this example is as follows:

$$
\text { sphere } 110.0 \quad \text { origin } x=10.0 \quad y=10.0 \quad z=10.0
$$




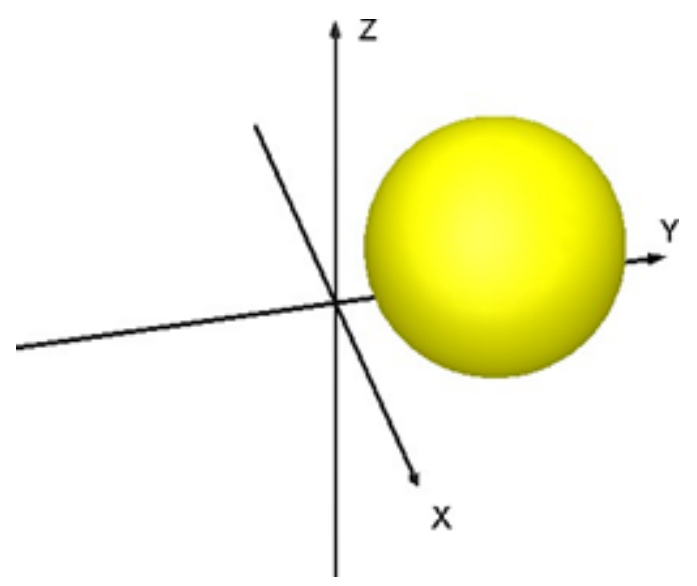

Figure 107. Example of body translation using the ORIGIN keyword.

\subsubsection{Example Using the ORIGIN Keyword to Create Three Intersecting Cylinders}

A more complex example is illustrated in Figure 108, with three intersecting $10 \mathrm{~cm}$ radius cylinders that are $20 \mathrm{~cm}$ tall. Cylinder 1 is translated $4.5 \mathrm{~cm}$ in the positive $\mathrm{Y}$ direction; cylinder 2 is translated $4.5 \mathrm{~cm}$ in the negative $\mathrm{X}$ and $\mathrm{Y}$ directions; cylinder 3 is translated $4.5 \mathrm{~cm}$ in the positive $\mathrm{X}$ and negative $\mathrm{Y}$ directions. The numbers on the top of each colored region are the region definition vectors.

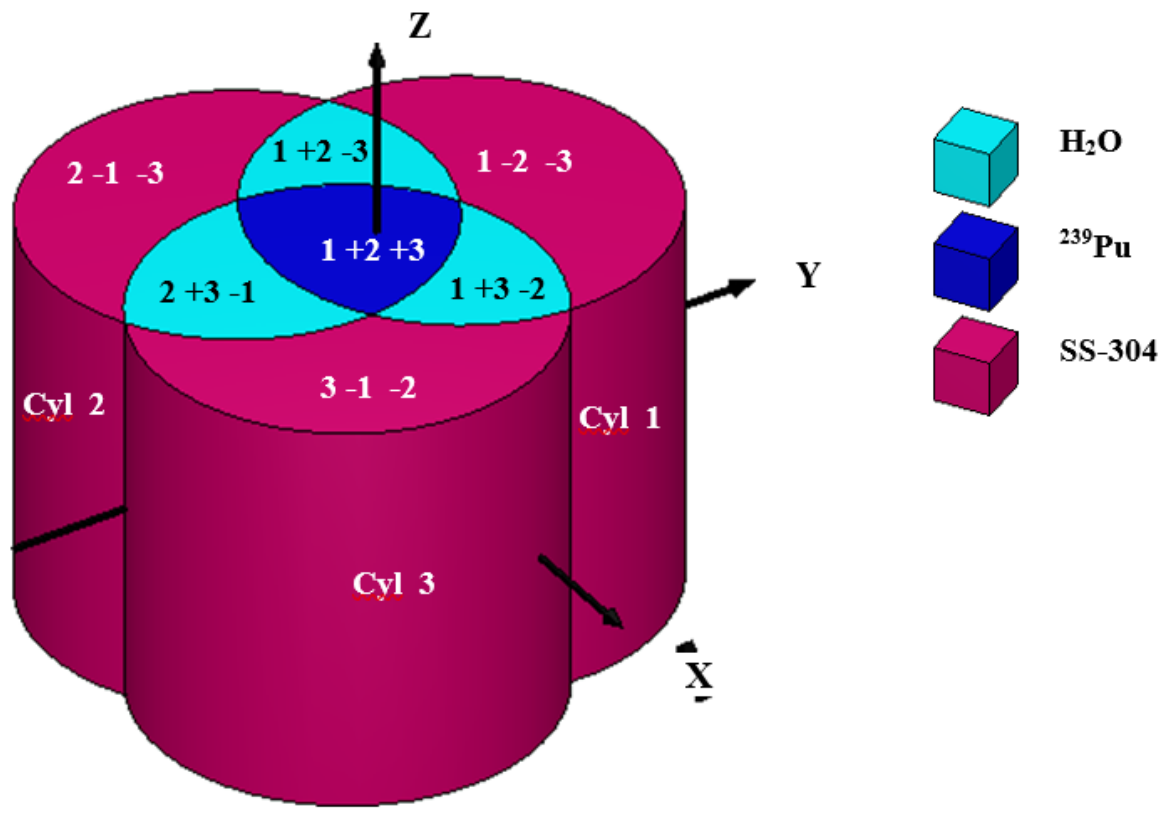

Figure 108. Three intersecting cylinders.

The inner region (dark blue) is ${ }^{239} \mathrm{Pu}$, with a density of $18.5 \mathrm{~g} / \mathrm{cc}$. The surrounding light blue regions contain water. The outer regions (magenta) are stainless steel 304.

Based on the knowledge that you have gained from completing the previous sections of the primer, open a new file in Fulcrum and enter the title, cross section library, and material information. Note that this 
problem uses the CE ENDF/B-VII.1 library (ce_v7.1). Assign ${ }^{239} \mathrm{Pu}(\mathrm{pu}-239)$ as mixture 1, water (h2o) as mixture 2, and stainless steel 304 (ss 304 ) as mixture 3 .

Start the geometry input by placing the text cursor within unit 1, between Global Unit 1 and boundary 1. Press the CTRL-SPACE autocomplete key combination and select cylinder- kenovi (configurable), to open the Cylinder input form. Enter 10 for Radius and Top, and -10 for Bottom. Next, click on the Origin checkbox to specify the origin of the cylinder. Enter 4.5 for the $\mathbf{Y}$ value of Origin to translate the origin of the first cylinder to $(0,4.5,0)$. Click $\mathbf{O K}$ to close the cylinder form, after confirming it matches that shown in Figure 109. Recall that only non-zero data need be entered in the ORIGIN specification; declaring the other coordinates equal to zero is acceptable.

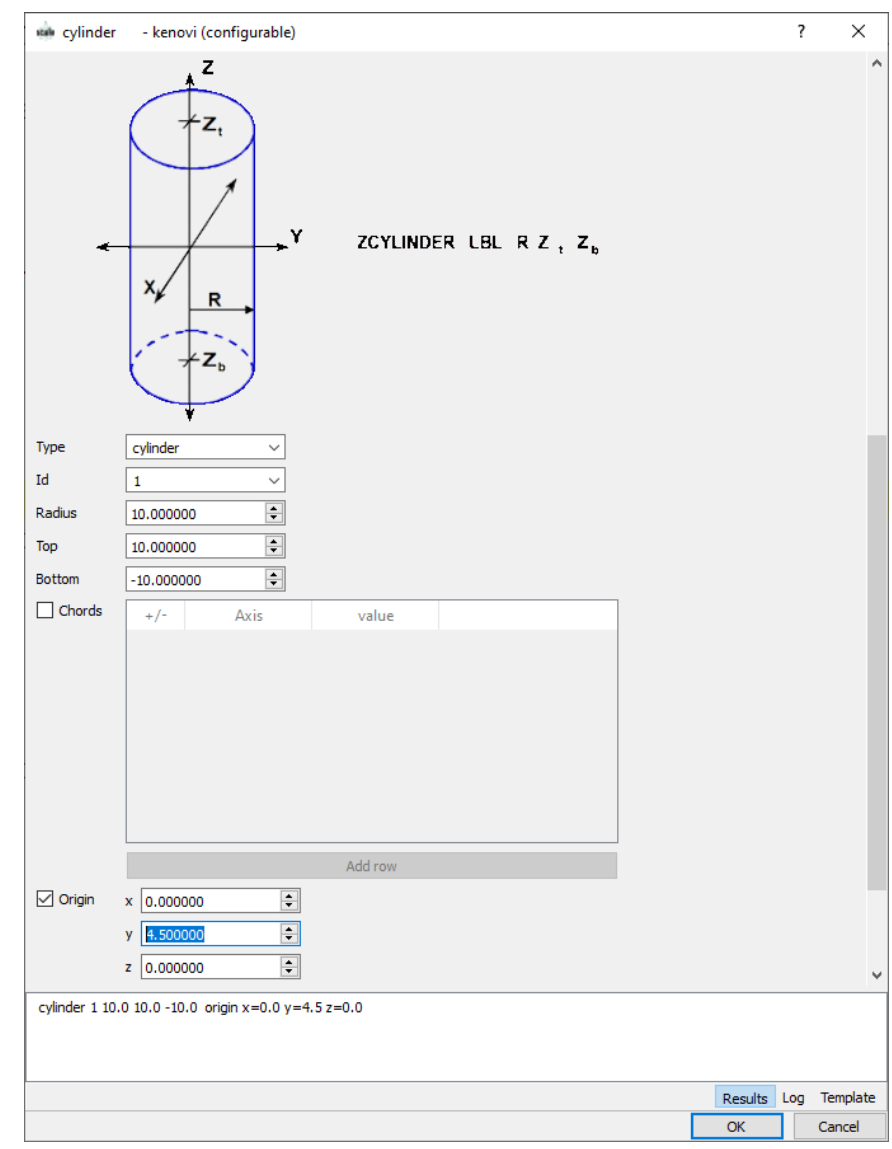

Figure 109. Cylinder configurable form with ORIGIN specification data.

The simplest way to create the other type cylinders is to copy and paste cylinder 1 and update the surface numbers and ORIGIN data. Copy the input for cylinder 1 and paste it on the next line. Change the surface ID to 2 and the ORIGIN data to $x=-4.5$ and $y=-4.5$. Similarly, copy cylinder 2 and paste it. Change the surface ID to 3 and the ORIGIN data to $x=4.5$. On the line after cylinder 3 , press the CTRL-SPACE autocomplete key combination. Select cuboid - kenovi (configurable) to open the Cuboid input form. Change the Id to 4 , enter dimensions of 15 for $+\mathbf{X}$ and $+\mathbf{Y}$ and -15 for $-\mathbf{X}$ and $-\mathbf{Y}$. Set $+\mathbf{Z}$ and $-\mathbf{Z}$ to 10 and -10 , respectively. This region will serve as the outer boundary for the unit, and is sized so that no portion of the three intersecting cylinders will be truncated. The complete set of geometry records is shown in Figure 110. 


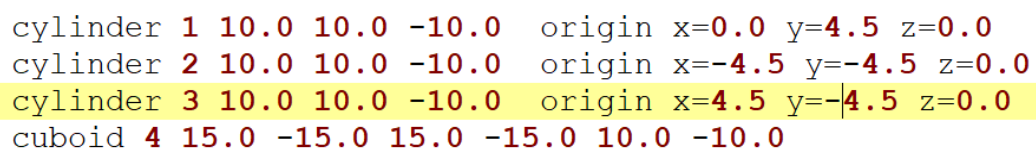

Figure 110. Input for three cylinders with ORIGIN data specified.

The media records are supplied next to fill each region. First, define the ${ }^{239} \mathrm{Pu}$ region in the center. The ${ }^{239} \mathrm{Pu}$ region, mixture 1 , is defined as being inside cylinders 1,2 and 3 at the same time. To create it, press the CTRL-SPACE autocomplete key combination and select media - kenovi (configurable). Press the Add row button below the Regions pane. Add additional rows and declare Material 1 to be inside of Id numbers 2 and 3 as well by typing the numbers into the Id fields. The completed media configurable form is shown in Figure 111; after reviewing the form press the OK button to add the media record to the input. This record fills the region of space that is inside surface 1 and surface 2 and surface 3 with mixture 1 . The point must be simultaneously inside all 3 surfaces to satisfy the media record and be included in this sector.

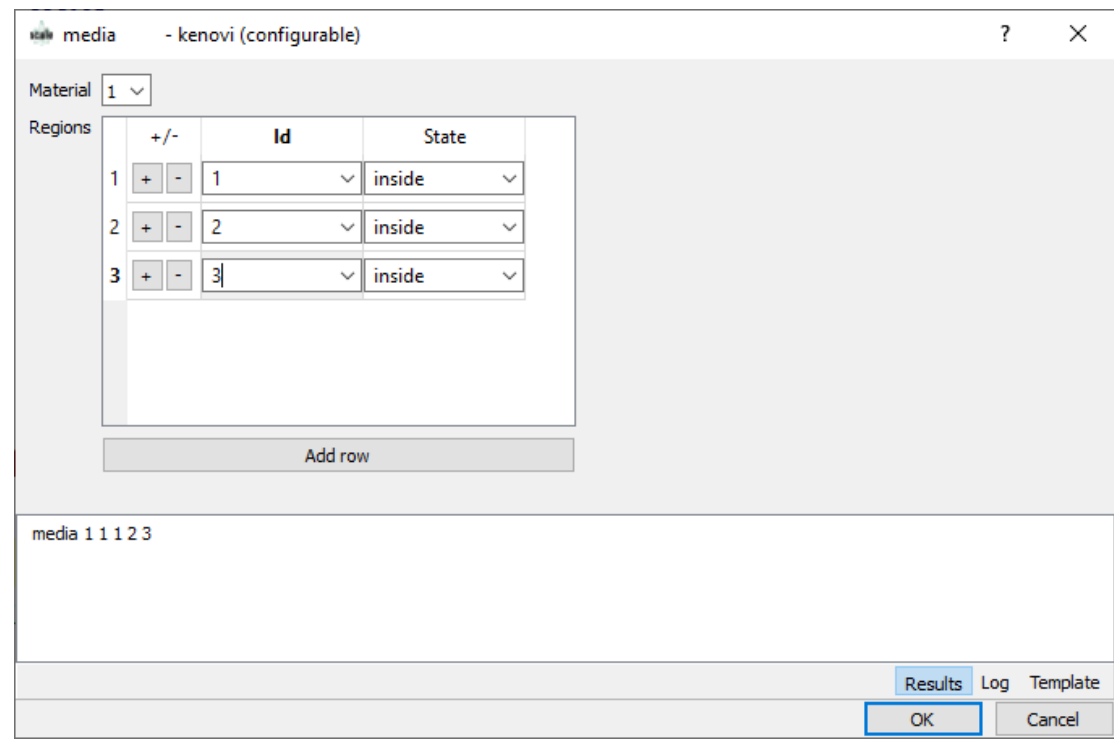

Figure 111. Complete media record configurable form for the region inside all three cylinders.

Next the regions containing water, mixture 2, will be defined. The quickest approach is to copy the existing media record and copy it onto the next line. Change the mixture number from 1 to 2 , and change the sign of surface 1 in the region definition vector from positive to negative. This region will be filled with water and is defined as inside cylinders 2 and 3 but outside cylinder 1 . Copy and paste this record two more times, moving the negative sign in the region definition vector to cylinder 2 and then subsequently to cylinder 3, as shown in Figure 112.

$\begin{array}{lllllll}18 & \text { media } & 2 & 1 & -1 & 2 & 3 \\ 19 & \text { media } & 2 & 1 & 1 & -2 & 3 \\ 20 & \text { media } & 2 & 1 & 1 & 2 & -3\end{array}$

Figure 112. Media records for water in overlapping cylinder example. 
Next the three stainless steel regions will be defined. Again, copy and paste is the fastest way to generate the input. Copy the three water media records defined above and paste them on the following three lines. Change the mixture number for all three records from 2 to 3. This can be accomplished with column highlighting: hold the ALT key and drag the mouse from top to bottom of the three numbers to change. Once all three are highlighted, press 3 and all three mixture numbers are changed at once. Each media record also must have a second negative value added to the region definition vector. For example, change cylinder 2 to negative in the first record, cylinder 3 to negative in the second record, and cylinder 1 to negative in the third record. The three stainless steel media records are shown in Figure 113.

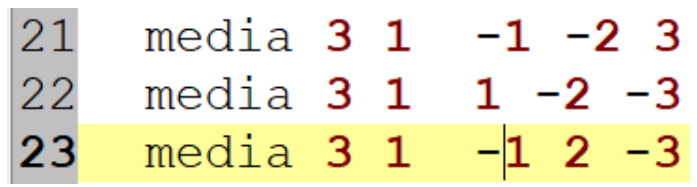

Figure 113. Media records for stainless steel in overlapping cylinder example.

The final media record fills the surrounding void inside cuboid 4 and outside cylinders 1,2 , and 3 . This is done by typing media $\begin{array}{llllllll}0 & 1 & 4 & -1 & -2 & -3\end{array}$. The cuboid is the boundary of the unit, so change boundary 1 to boundary 4 . The geometry form should look like Figure 114. Click the Run button to save the input and run the problem. The calculated $k_{\text {eff }}$ value is $1.3674 \pm 0.0023$.

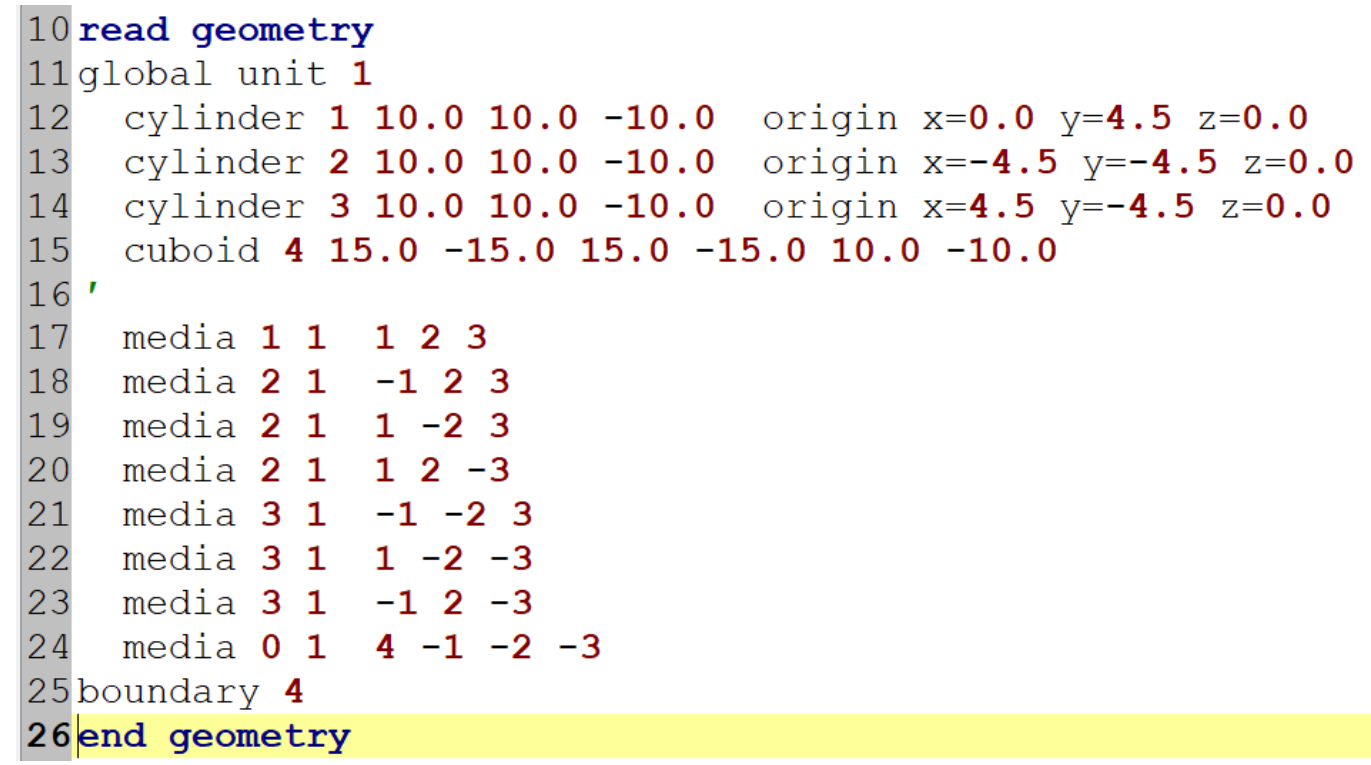

Figure 114. Geometry form for three intersecting cylinders.

\subsection{USING THE ROTATE KEYWORD TO ROTATE A BODY}

The ROTATE keyword allows the rotation of a KENO-VI shape to any angle. It rotates a volume about the origin using the Euler angle-x convention. Like CHORD and ORIGIN, it applies to the geometry or hole record that immediately precedes it. Note that for any geometry record that contains both ORIGIN and ROTATE input, the rotation is performed by KENO-VI first, prior to translating the origin of the body. Any truncation performed by the CHORD keyword will be performed prior to the rotation. The ROTATE keyword has three subordinate keywords that specify the angles of rotation: $A 1=, A 2=$, and $\mathrm{A} 3=$. 
The functions of the three subordinate keywords are illustrated in Figure 115 The operations are performed in order from A1 to A3. First, A1 specifies $\alpha$, the angle (in degrees) to rotate the volume counterclockwise around the Z-axis. The new locations of the X-, Y-, and Z-axes carry the notations $\mathrm{X}^{\prime}$, $Y^{\prime}$, and $Z^{\prime}$. Next, A2 specifies $\beta$, the angle to rotate the volume counterclockwise around the $X^{\prime}$-axis. The new locations of the $X^{\prime}$-, $Y^{\prime}$-, and $Z^{\prime}$-axes are denoted as $X^{\prime \prime}, Y^{\prime \prime}$, and $Z^{\prime \prime}$. Finally, A3 specifies $\gamma$, the angle to rotate the volume counterclockwise around the $Z^{\prime \prime}$-axis. Some examples will help to clarify the use of these keywords.

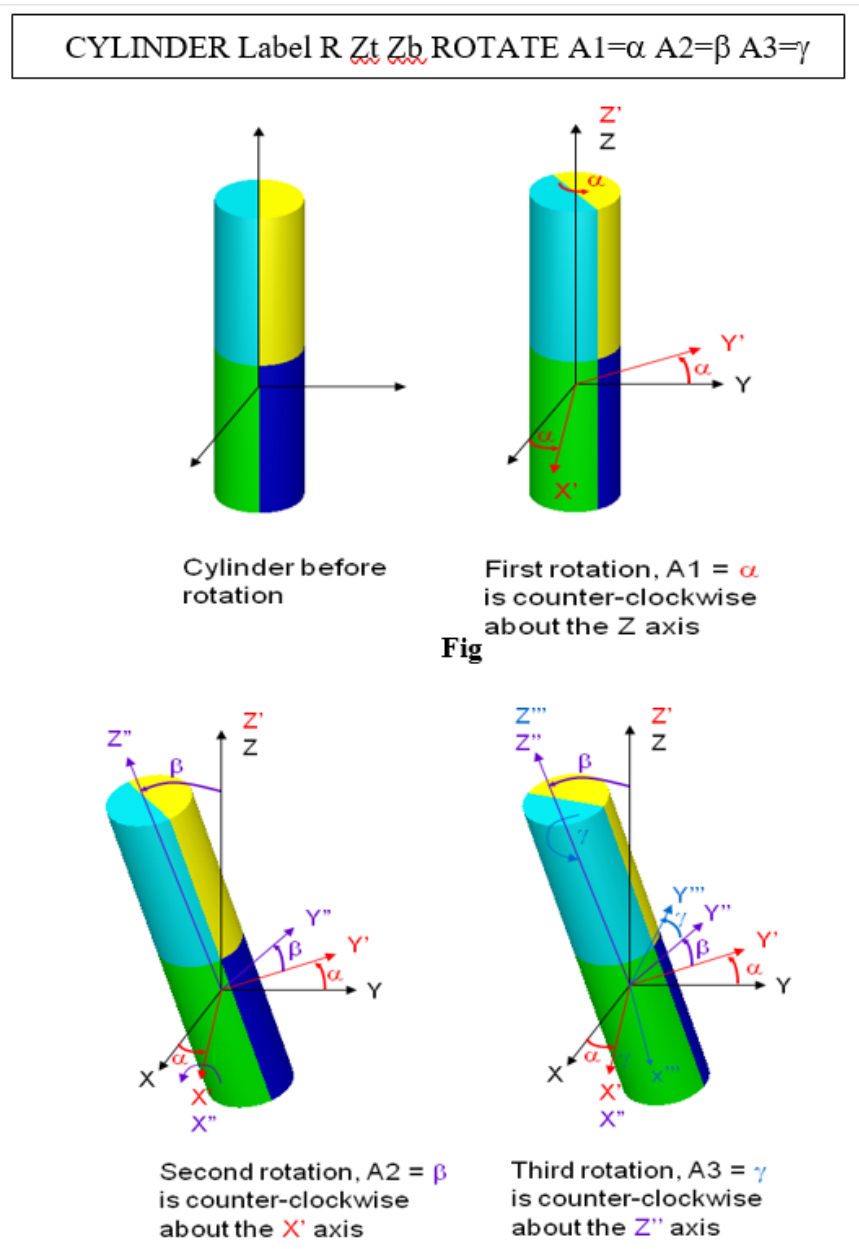

Figure 115. ROTATE subordinate keywords A1, A2, and A3.

\subsubsection{Rotation about the Z-axis}

Rotation around the Z-axis is performed using the keyword A1. The value specified after the keyword "A1=" is the angle of rotation around the Z-axis. Figure 116 shows a cuboid in its default orientation with no rotation. The example in Figure 117 shows the same cuboid with a rotation of 45 degrees counterclockwise around the Z-axis by specifying ROTATE A1 $=45$. Note that a negative value for the angle of rotation would result in a clockwise rotation. 


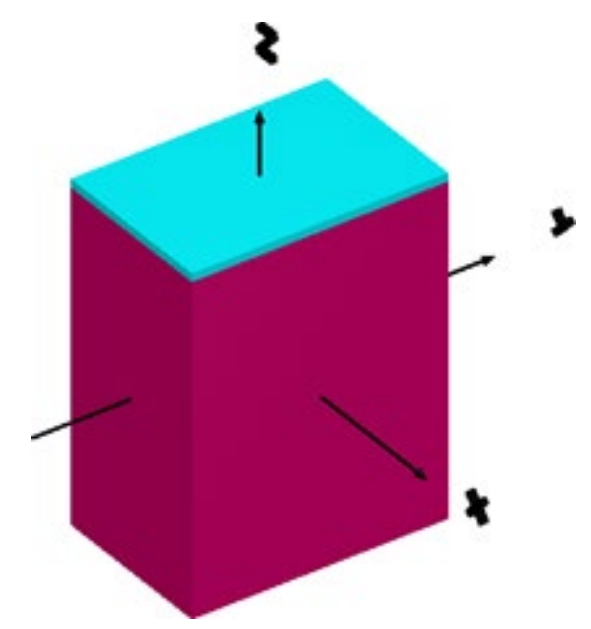

Figure 116. Cuboid (default orientation).

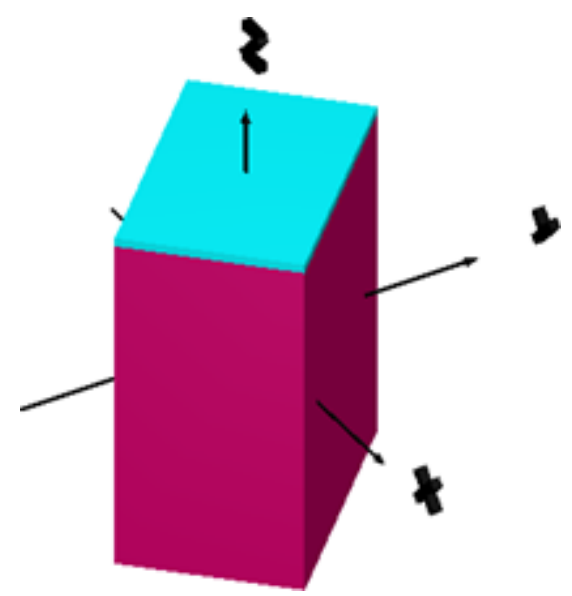

Figure 117. Cuboid rotated 45 degrees counterclockwise around $Z$-axis (rotate $A 1=45)$.

\subsubsection{Rotation about the $\mathrm{X}$-axis}

The keyword A2 is used for rotation around the X-axis. If A1 is not specified there is no rotation about the Z-axis and the $\mathrm{X}$-axis and the $\mathrm{X}^{\prime}$-axis are the same. The example in Figure 118 shows the cuboid from Figure 116 with a rotation of 45 degrees counterclockwise around the $\mathrm{X}$-axis using the keyword ROTATE A2=45. 


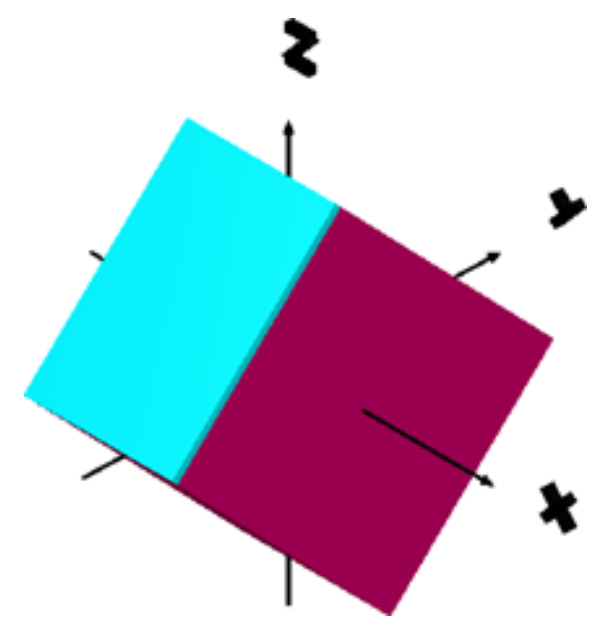

Figure 118. Cuboid rotated 45 degrees counterclockwise around $X$-axis (rotate $A 2=45)$.

\subsubsection{Rotation about the $\mathrm{Y}$-axis}

The subordinate keywords A1 and A2 must be used to perform rotation about the Y-axis. First, a 90 degree counterclockwise rotation around the $Z$-axis is performed $(A 1=90)$ so that the $X^{\prime}$-axis is aligned with the $\mathrm{Y}$-axis. Then the rotation around the $\mathrm{X}^{\prime}$-axis using $\mathrm{A} 2$ will be a rotation around the $\mathrm{Y}$-axis. The example in Figure 119 shows the cuboid from Figure 116 with a 45 degree counterclockwise rotation around the $\mathrm{Y}$-axis using the keywords ROTATE A1=90 A2=45.

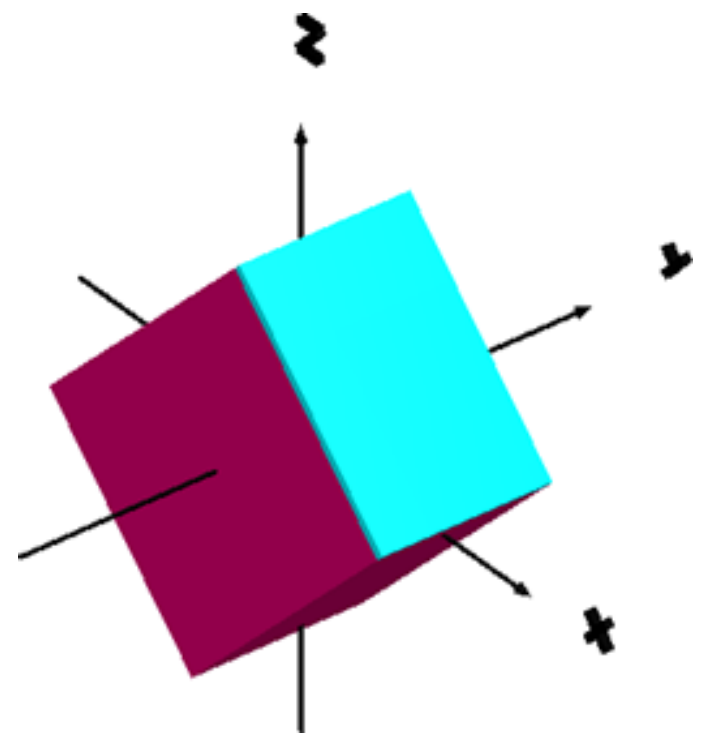

Figure 119. Cuboid rotated 45 degrees counterclockwise around $Y$-axis (rotate $A 1=90$ A2=45).

\subsubsection{Example using ROTATE keyword for Y-angle Joint}

The next example is a critical experiment with intersecting aluminum pipes in the shape of a Y, filled with a $5 \%$ enriched $\mathrm{UO}_{2} \mathrm{~F}_{2}$ solution (Figure 120). The solution contains $907.01 \mathrm{~g} / \mathrm{L}$ of uranium, no excess acid, and has a solution density of $2.0289 \mathrm{~g} / \mathrm{cc}$. The temperature of the materials is of $293 \mathrm{~K}$. The assembly is composed of a $212.1 \mathrm{~cm}$ long vertical pipe and a second pipe that intersects the vertical pipe $76.7 \mathrm{~cm}$ from the outside bottom at an angle of 29.26 degrees with the upper vertical pipe. The center of the vertical pipe at this point of intersection is used as the origin of the geometry, as shown in Figure 120. 
Both pipes have a $13.95 \mathrm{~cm}$ inner radius and a $14.11 \mathrm{~cm}$ outer radius. The vertical pipe is open on the top and $1.3 \mathrm{~cm}$ thick on the bottom. The Y-leg pipe is $126.04 \mathrm{~cm}$ in length, with the sealed end $0.64 \mathrm{~cm}$ thick. Both pipes are fabricated from default density pure aluminum. The solution height is $129.5 \mathrm{~cm}$. From the point where the pipes intersect (see the origin in Figure 120), the assembly is surrounded by water 37.0 $\mathrm{cm}$ in the $\pm X$ directions, $100 \mathrm{~cm}$ in the $+Y$ direction, $-37 \mathrm{~cm}$ in the $-Y$ direction, to the top of the assembly in the $+Z$ direction, and $-99.6 \mathrm{~cm}$ in the $-Z$ direction.

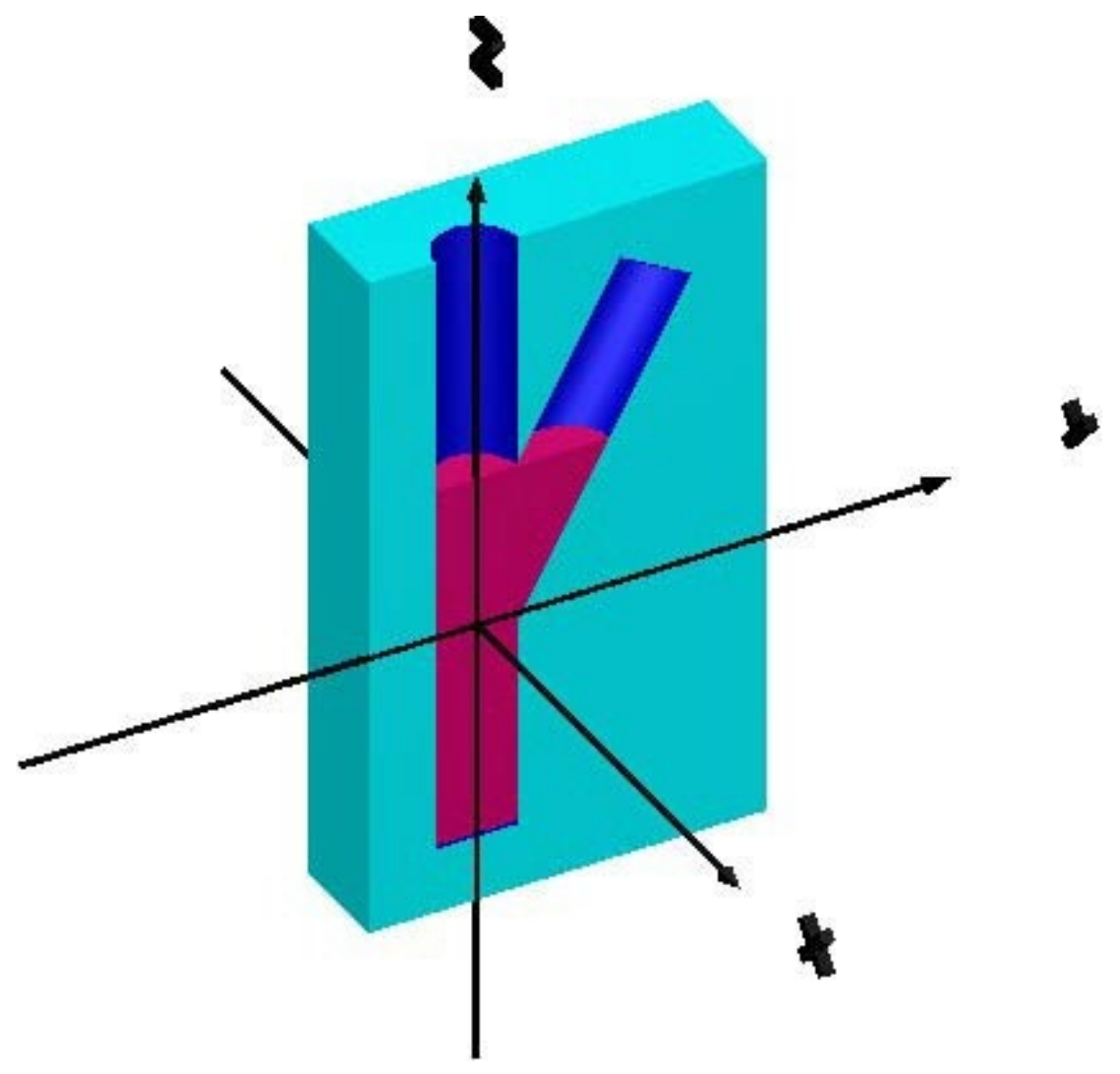

Figure 120. Cutaway view of critical experiment with 29-degree Y-joint.

Based on the knowledge gained from completing the previous sections of the primer, open a new file in Fulcrum and enter the title, cross section library, and material information. The uranium solution is defined with the solution - rho + density + temperature + volume fraction Fulcrum autocomplete option. The completed composition block should look like Figure 121.

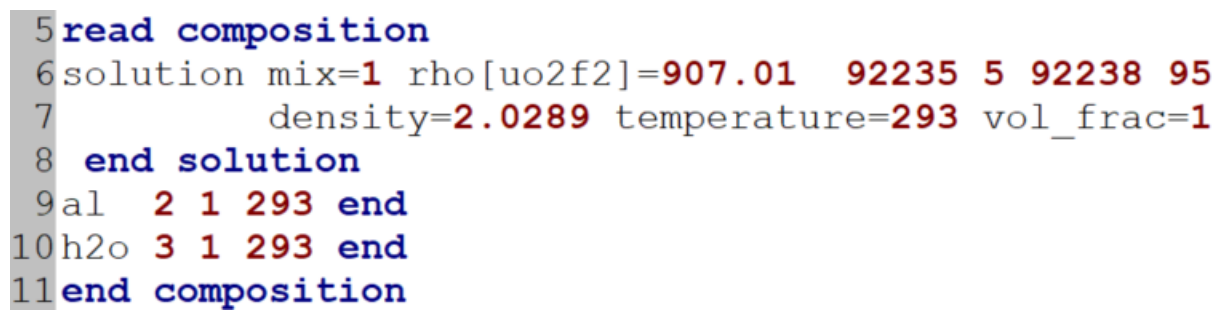

Figure 121. Composition block for the 29-degree Y-joint.

The geometry definition begins with defining two cylinders (inner and outer, respectively) for the vertical pipe. Enter the following data in cylinder configurable forms, typing the input directly in the input, or one configurable form and a copy/paste to define cylinders 1 and 2 : 
- Cylinder 1: 13.95 for Radius, 135.4 for Top and -75.4 for Bottom

- Cylinder 2: 2 for Id, 14.11 for Radius, 135. 4 for Top and -76. 7 for Bottom

Next, define two more cylinders (inner and outer) for the angled pipe. Use the rotate and A2 keywords to rotate the cylinders 29.26 degrees clockwise around the X-axis. On the cylinder form, click on the Rotation checkbox to allow for rotation configuration A2 of the cylinder. The configurable form for the inner cylinder is shown in Figure 122. The following values should be entered for each cylinder.

- Cylinder 3: 3 for Id, 13.95 for Radius, 125.4 for Top and 0.0 for Bottom; rotate $\mathrm{a} 2=-29.26$ for the rotation.

- Cylinder 4: 4 for Id, 14.11 for Radius, 126.04 for Top and 0 . 0 for Bottom; rotate $\mathrm{a} 2=-29.26$ for the rotation.

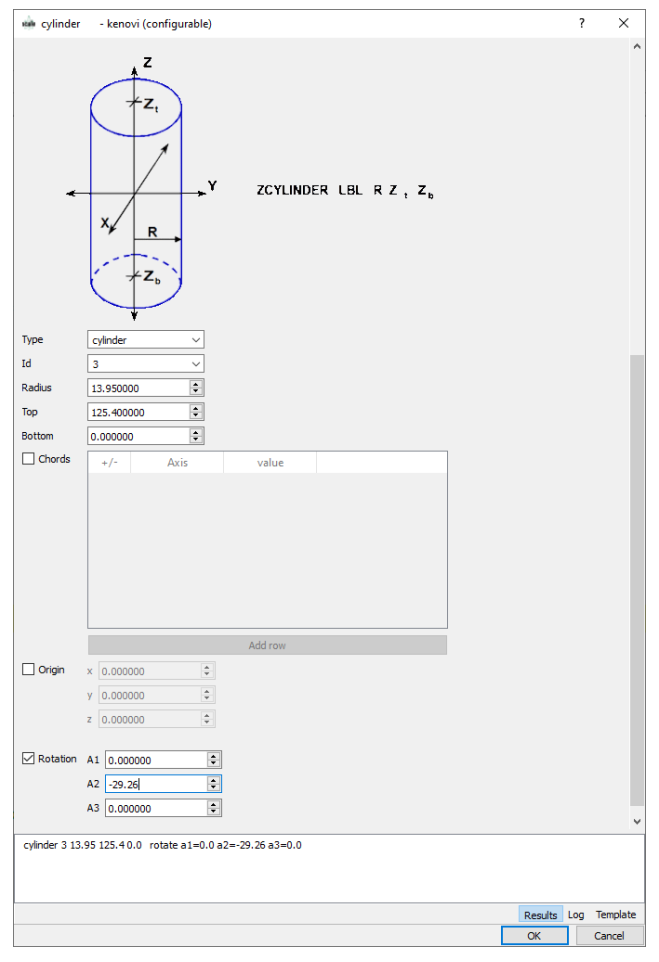

Figure 122. Cylinder configurable form for inner angled pipe.

A horizontal plane is needed to separate the portion of the cylinders filled with solution from the void portions above them. The top of the solution is $52.8 \mathrm{~cm}$ above the origin $(129.5 \mathrm{~cm}$ solution height, 76.7 $\mathrm{cm}$ from the origin to the bottom of cylinder 2), so the plane is perpendicular to the $\mathrm{Z}$-axis and at $\mathrm{Z}=$ 52.8. Press the CTRL-SPACE autocomplete key combination. Click on the plane- kenovi (configurable) button, which opens the Plane input form. Set Id to 5, c to -1 and $\mathbf{d}$ to 52 . 8. This creates a plane corresponding to the equation $-Z+52.8=0$, which is equivalent to the desired plane at $Z=52.8$. The same plane can also be defined with c $(\mathrm{ZPL}=)$ set to 1 and $\mathrm{d}(\mathrm{CON}=)$ set to -52.8 . This alternative definition generates the surface equation $Z-52.8=0$, which is also equivalent to the desired surface of $Z=52.8$. The difference between the equivalent forms is which half space, above the plane or below the plane, is assigned as positive (inside) relative to the surface. The complete plane configurable form is shown in Figure 123. Click $\mathbf{O K}$ to close the plane form and add the geometry record to the input file. 


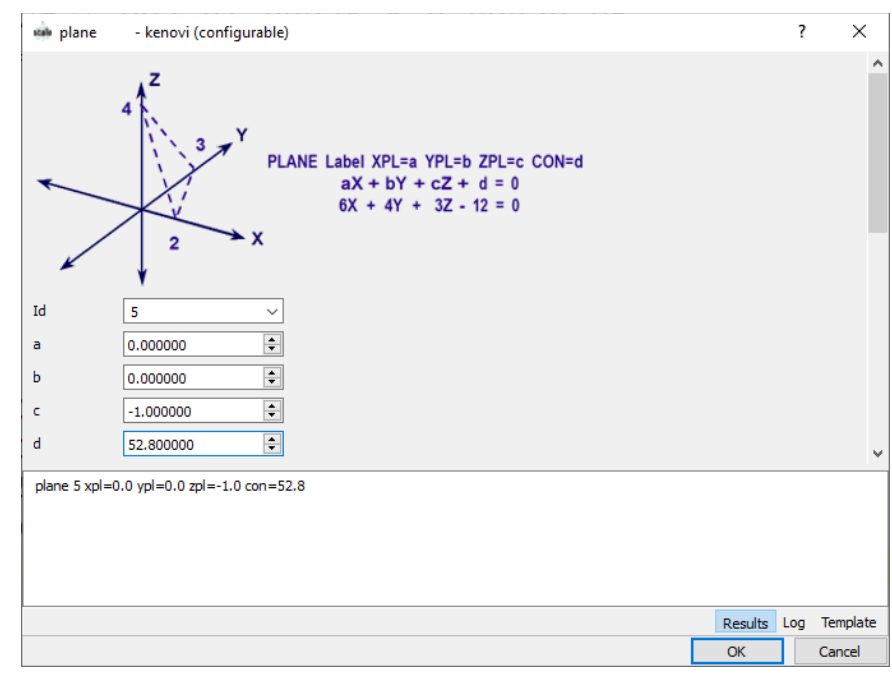

Figure 123. Completed plan configurable form.

The final geometric volume needed is the cuboid for the water reflector. Create a cuboid with the following dimensions: $+\mathbf{X}=37,-\mathbf{X}=-37,+\mathbf{Y}=100,-\mathbf{Y}=-37,+\mathbf{Z}=135.4$, and $-\mathbf{Z}=-99.6$. After you close the cuboid form, define the boundary to be inside cuboid 6 by setting boundary 6 .

Next, define the contents of the geometric volumes using media records. First, define the solution, mixture 1, inside the vertical cylinder and below the plane. Note that the positive side of the plane ("inside" the plane) is the side where the equation $\mathrm{aX}+\mathrm{bY}+\mathrm{cZ}+\mathrm{d}>0$ : in this case $-\mathrm{Z}+52.8>0$. This allows the determination of the positive side of the plane by selecting a known point and evaluating the inequality. For example, in this model the origin can be used $(-1(0)+52.8=52.8)$ to determine that it is on the positive side of the plane. This is the portion of the inner cylinder containing the solution, so the media record needs to define mixture 1 (solution) as inside both cylinder 1 and plane 5 . Enter the media record either with the configurable form or by typing the input directly.

Next, define the contents of the vertical pipe wall. The region definition vector on the media form will be inside cylinder 2 , and it will be outside cylinder 1 and cylinder 3 . Cylinder 3 is excluded for the solution in the side leg. The mixture number for this media record is 2 (al).

The solution in the angled pipe is defined next. The region definition vector on the media form will be inside cylinder 3 and plane 5, and it will be outside cylinder 1 . The mixture number is 1 (uo2f2).

Next, define the contents of the angled pipe wall. The region definition vector on the media form will be inside cylinder 4 , and it will be outside cylinder 3 and cylinder 2 . The mixture number is 2 (al).

The next two media records define the voids in the top of the two pipes. The region definition vector for the void region in the vertical pipe is inside cylinder 1 and outside plane 5 . The region definition vector for the void region in the angled pipe is inside cylinder 3, and outside cylinder 1 and plane 5 . The mixture for both media records is 0 (void).

The final media record is the water reflector. The region definition vector is inside cuboid 6 , and it is outside cylinder 2 and cylinder 4 . The mixture number is $3(\mathrm{~h} 2 \mathrm{o})$. 
Your completed geometry block should look like Figure 124. Click the Run button to save the input and run the case. The calculated $k_{\text {eff }}$ value for this critical experiment is $1.0012 \pm 0.0018$, which is near 1.0 , as expected.

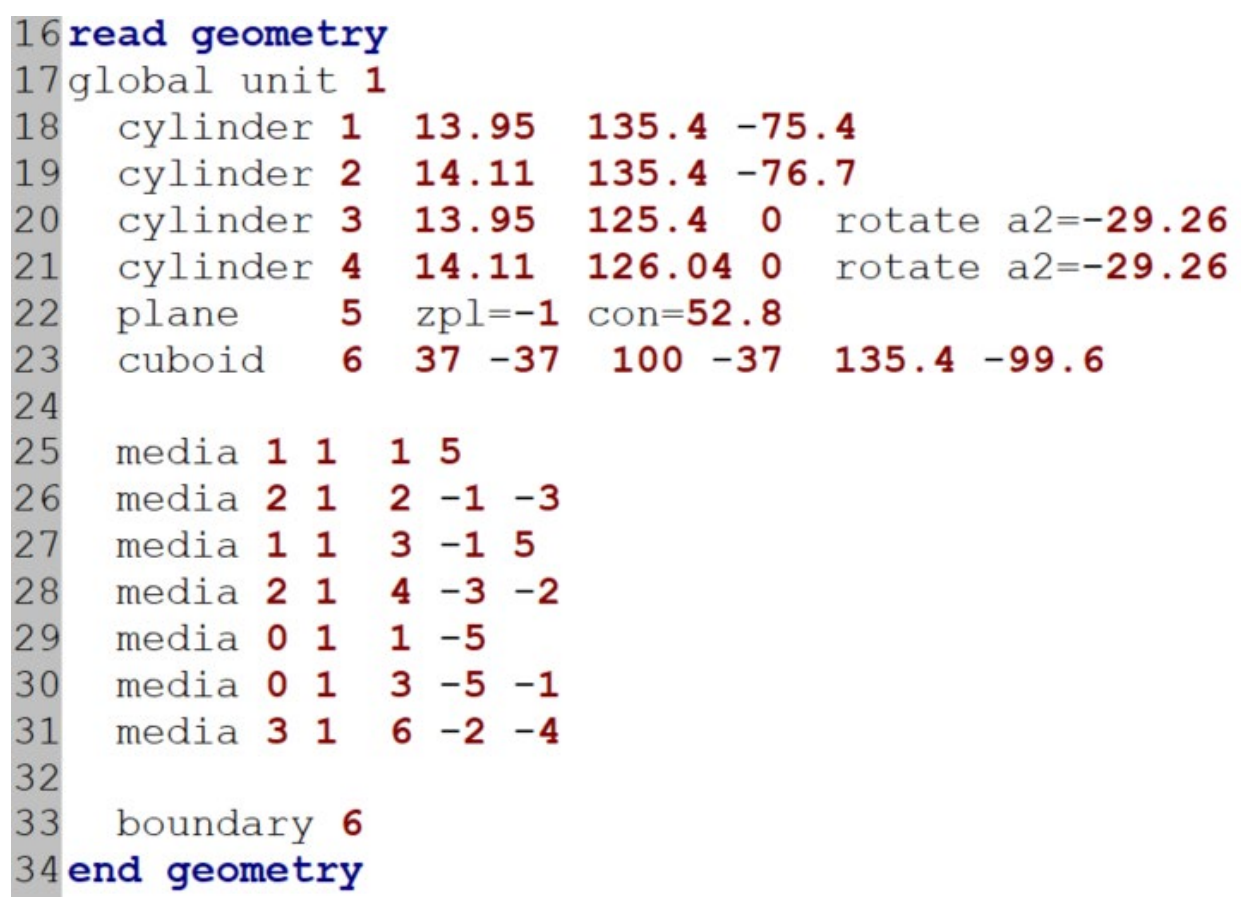

Figure 124. Geometry block for the 29-degree Y-joint.

\subsection{HOLES}

\subsubsection{Introduction}

There are three types of contents records for defining the contents of the geometry regions:

- media,

- arrays, and

- holes.

As shown in Section 4, a media record is used to place a mixture in a specified volume, and an array record is used to place an array in a specified volume. For many problems, the use of media and arrays is adequate to create an accurate model.

However, in some situations, placement of one unit inside another may be necessary or helpful. For those situations, a hole is needed. A hole is used to position a unit within a surrounding unit relative to the origin of the surrounding unit. The keyword, HOLE, is followed by the unit number being placed in the hole. The unit being placed in the hole can be positioned and rotated using the ORIGIN and ROTATE keywords discussed earlier in this section. The origin of the unit being placed as a HOLE is positioned in the current unit coordinate system using the ORIGIN keyword; as with geometry records, only nonzero entries must be provided. The unit being placed as a HOLE is rotated about its origin, not that of the current unit, using the ROTATE keyword. As with the geometry record and ORIGIN keyword, only nonzero entries must be provided. The boundary record of a unit placed in a hole may contain more than 
one geometry label, but all labels must be positive, indicating a location inside the respective geometry bodies.

There are three basic rules for holes:

1. A hole contains a single unit.

2. Holes may share surfaces with but may not intersect other holes, the boundary of the surrounding unit that contains the hole, or an array boundary.

3. Holes may be nested; that is, a unit containing a hole may be placed as a hole inside another unit.

\subsubsection{Simple Hole Example with Rotations}

This example includes five stainless steel 304 boxes that contain plutonium metal $\left(1 \%{ }^{239} \mathrm{Pu}\right.$ and $99 \%$ ${ }^{240} \mathrm{Pu}$ and default density). The boxes are encased in a concrete cylinder. A cutaway view (top half removed) is shown in Figure 125. The dimensions of the plutonium blocks are $10 \times 8 \times 20 \mathrm{~cm}$. The stainless steel box is $1 \mathrm{~cm}$ thick on three sides and on the top and bottom. The fourth side (i.e., the one oriented toward the center of the cylinder for the four outer boxes) is $3 \mathrm{~cm}$ thick. The center of each outer box is spaced $12 \mathrm{~cm}$ in $\mathrm{X}$ and $\mathrm{Y}$ from the center of the cylinder.

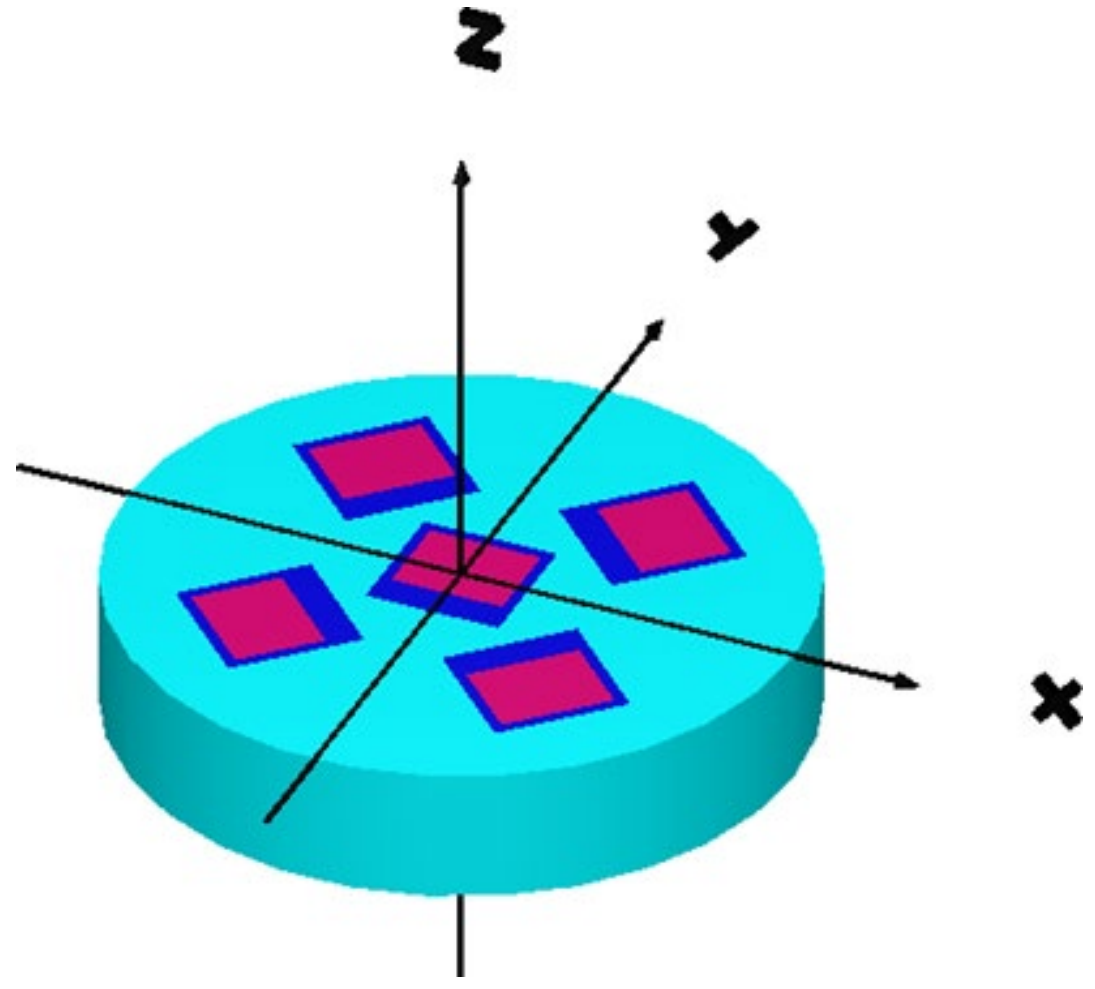

Figure 125. Cutaway view of rotated holes example.

Create a new file with Fulcrum including title, cross section library, and composition information for the plutonium, stainless steel, and concrete. For the concrete, select reg-concrete in the basic compositions list.

Once these data are complete, start the geometry definition. The first unit will be the stainless steel box containing the plutonium. The outer dimensions of the box are $12 \times 12 \times 22$ based on adding the stainless 
steel dimensions to the plutonium block dimensions. Placing the origin of unit 1 at the center of the box will make it easy to position each box as a hole in the concrete cylinder, unit 2.

Start the geometry by deleting the Global label from unit 1 . Then place the text cursor within Unit 1 and press the CTRL-SPACE autocomplete key combination. Select the cuboid - kenovi (configurable), to open the Cuboid configurable form. Enter the following data for the plutonium block: 5 for $\mathbf{X},-5$ for $\mathbf{- X}, 5$ for $\mathbf{Y},-3$ for $-\mathbf{Y}, 10$ for $\mathbf{Z}$ and -10 for $-\mathbf{Z}$. Next, create another cuboid for the stainless steel with the following dimensions: 6 for $+\mathbf{X}$ and $+\mathbf{Y},-6$ for $-\mathbf{X}$ and $-\mathbf{Y}, 11$ for $+\mathbf{Z}$ and -11 for $-\mathbf{Z}$. Set the media record for the plutonium. The plutonium, mixture 1 , is inside cube 1 . Create the second media record inside cuboid 2 and outside cuboid 1 . The mixture number is 2 (ss304). The last step for unit 1 is to change the boundary record region definition vector to 2 .

Create global unit 2. Open the Cylinder input form to define the concrete cylinder, and enter 30 for Radius, 12 for Top and -12 for Bottom. For the media record, set mixture 3 (reg-concrete) inside cylinder 1 . Set the boundary of the problem as the concrete cylinder by writing boundary 1.

Now place the five boxes as holes in unit 2. As mentioned previously, the location of the hole is controlled by placing its origin with respect to the origin of the unit in which it is placed. The first hole will be placed in the center of unit 2 without rotation. To set the first hole, press the CTRL-SPACE autocomplete key combination somewhere in unit 2 and select hole - kenovi (configurable). The default hole configurable form is shown in Figure 126. The Unit Id in the upper left corner of the form should remain 1 since unit 1 is the unit to be inserted as a hole. No further data is needed as the origin of unit 1 should be positioned at the origin of unit 2. Press the OK button to add the hole record to the input file.

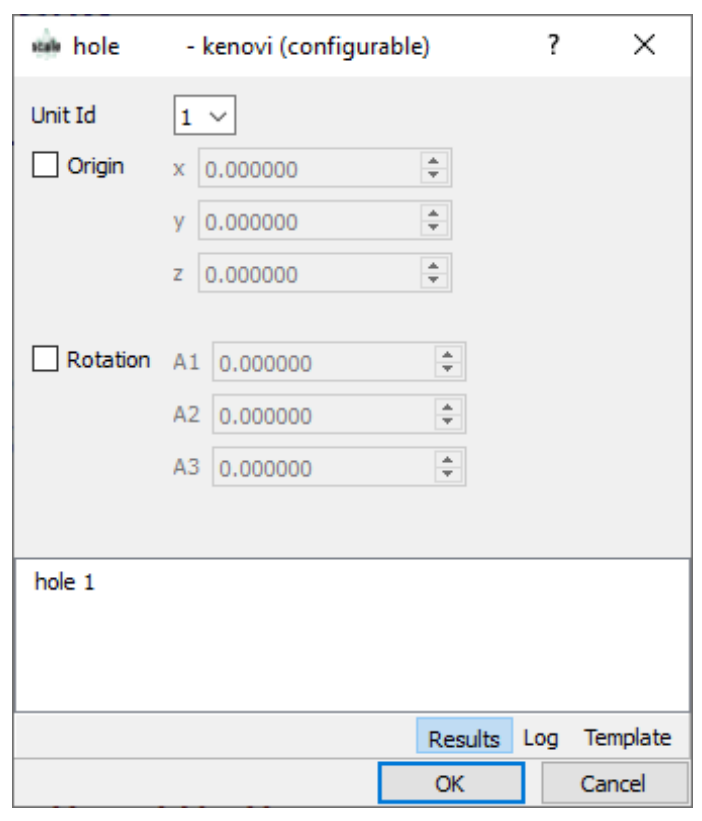

Figure 126. Default hole configurable form.

The next hole will be placed with at $(12,12)$ and it must also be rotated 45 degrees clockwise around the $\mathrm{Z}$ axis for the thick side of the box to face the center of the cylinder as designed. Use the CTRL-SPACE autocomplete key combination somewhere in unit 2 and select hole - kenovi (configurable). Unit 1 is still the applicable Unit Id, but in this case the Origin and Rotation checkboxes must be checked to allow specification of translation and rotation data. $\mathbf{X}$ and $\mathbf{Y}$ should be set to 12, and $\mathbf{A 1}$ should be set to 
-45. After confirming the input with the completed hole configurable form shown in Figure 127 press the OK button to add the hole record to the input file.

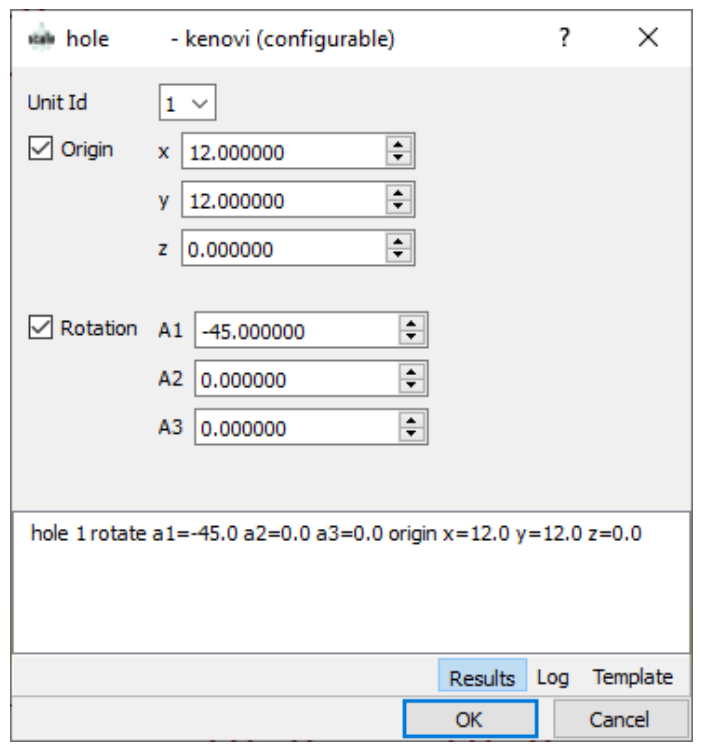

Figure 127. Completed hole record for second hole record.

Continuing clockwise around the cylinder, enter the following data for the remaining holes using the configurable form, directly typing the input, or copy/paste and updating the records:

- Origin is located at $\mathbf{X}=12, \mathbf{Y}=-12$ and Rotate $\mathbf{A 1}=-135$.

- Origin is located at $\mathbf{X}=-12, \mathbf{Y}=-12$ and Rotate $\mathbf{A 1}=135$.

- Origin is located at $\mathbf{X}=-12, \mathbf{Y}=12$ and Rotate $\mathbf{A 1}=45$.

The geometry block may look like Figure 128, depending on the methods used to specify the hole records. After checking that the input data match, click Run to execute SCALE. The calculated $k_{\text {eff }}$ is $1.0406 \pm 0.0018$. 


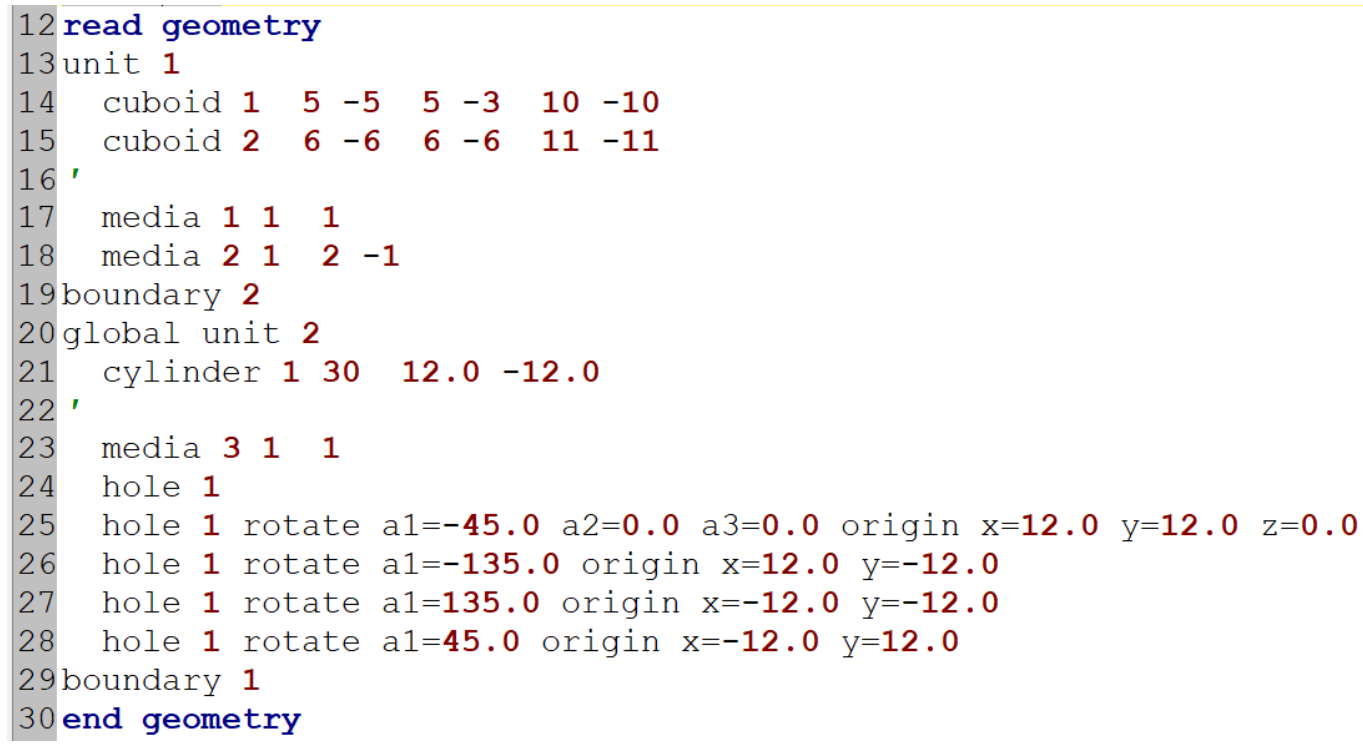

Figure 128. Geometry input for rotated holes example.

\subsection{SUMMARY}

This section has discussed the following:

- to use Fulcrum and KENO-VI to describe more advanced geometry models using geometry modification data and holes,

- to use the CHORD keyword to truncate a body with a plane perpendicular to a major axis,

- to use the ORIGIN keyword to translate the location of a body (i.e., shape),

- to use the ROTATE keyword to rotate a body to any angle, and

- to use the HOLE record to place one unit inside another unit.

The next step is to learn about even more complex geometries. 



\section{ADVANCED ARRAY TOPICS}

\subsection{WHAT YOU WILL BE ABLE TO DO}

- Use Fulcrum and KENO-VI to describe complex geometry models using arrays.

- Remove unwanted portions of arrays using region definition vectors to apply boundaries.

- Build hexagonal arrays.

\subsection{SHIPPING CONTAINER WITH FIVE CYLINDERS OF CRUCIFORM U(93.2)C CERAMIC ELEMENTS}

This problem illustrates how to remove unwanted portions of an array using the region definition vector on the array record. A cruciform-shaped U(93.2)C ceramic element sits in water inside a SS316 cylinder (see Figure 129). Five of these cylinders are then placed inside a larger SS316 cylinder (Figure 130). The cruciform element is $10 \mathrm{~cm}$ on a side, with a width of $1 \mathrm{~cm}$ and a height of $20 \mathrm{~cm}$. Each element is contained in a SS316 cylinder with an inner diameter of $12 \mathrm{~cm}$ and an inner height of $20 \mathrm{~cm}$. The SS316 is $0.1 \mathrm{~cm}$ thick. These cylinders are packed in a cruciform-shaped array with a pitch of $12.4 \mathrm{~cm}$. The five containers are then placed inside a SS316 cylinder with an inside diameter of $40 \mathrm{~cm}$, an inner height of $20.2 \mathrm{~cm}$, and thickness of $0.2 \mathrm{~cm}$.

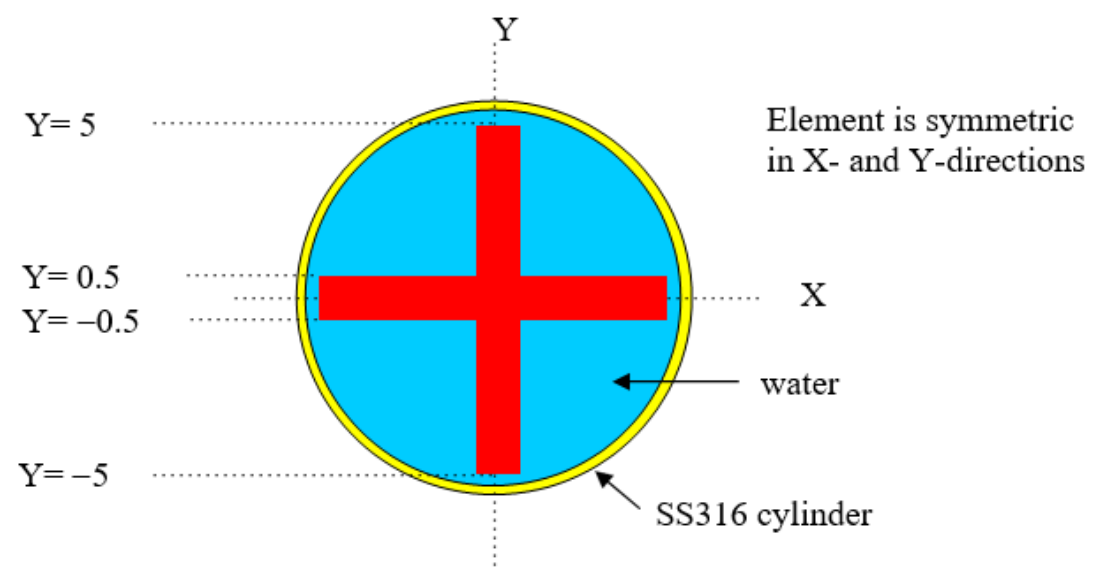

Figure 129. SS316 cylinder with U(93.2)C cruciform element.

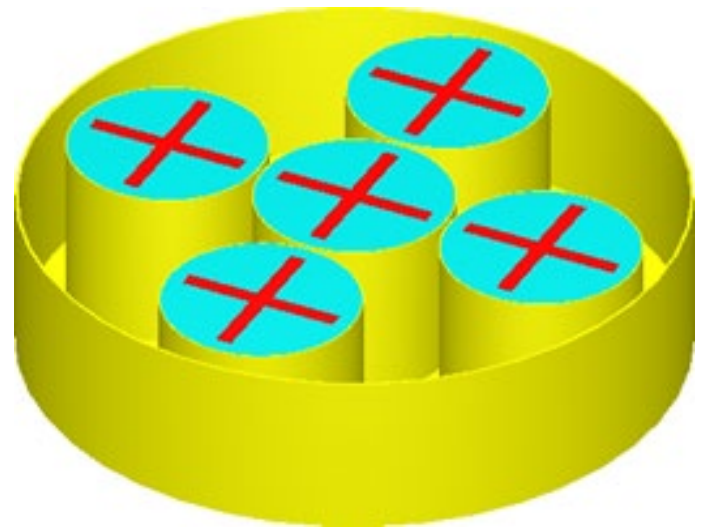

Figure 130. Five cruciform cylinders in shipping container. 
Specify the sequence, title, cross section library and composition descriptions as done in previous sections of this primer. Note that there are basic standard compositions for water, UC, and SS316, which make the composition specifications straightforward. The finished composition block is shown in Figure 131.

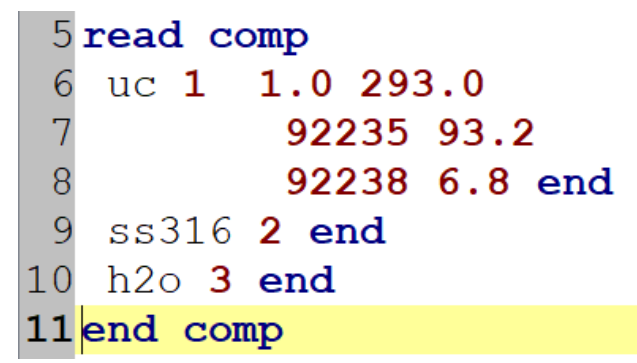

Figure 131. Composition block for cruciform array problem.

The cruciform elements can be formed by two intersecting cuboids. First create unit 1, which contains the cruciform element, water, and the SS316 cylinder. The outside region of unit 1 must ultimately be a cuboid because it will be used in a cuboidal array. First, remove Global from Global unit 1. Then create a cuboid to define the horizontal part of the UC cruciform with $\pm \mathrm{X}$ dimensions of $\pm 5, \pm \mathrm{Y}$ dimensions of \pm 0.5 , and $\pm Z$ dimensions of \pm 10 . This puts the origin in the center of the cruciform so it will be centered in the cylindrical can. Create another cuboid to define the vertical part of the cruciform. Use \pm 0.5 for the $\pm \mathrm{X}$ dimensions, \pm 5 for the $\pm \mathrm{Y}$ dimensions, and \pm 10 for the $\pm \mathrm{Z}$ dimensions. The next volume is the cylinder containing the water, in which the cruciform will be placed. Create a Cylinder, with Id 3, and enter 6 for R, 10 for Top, and -10 for Bottom. Create another Cylinder for the SS316 shell. Use the Id of 4 , and a radius of $6.1 \mathrm{~cm}, 10.1$ for Top, and -10.1 for Bottom. The last volume in unit 1 is the cuboid outside the steel cylinder, which allows the use of this unit in an array. Use the Id 5 for this cuboid. Because the array pitch in the $\mathrm{X}$ and $\mathrm{Y}$ directions is $12.4 \mathrm{~cm}$, the $\pm \mathbf{X}$ and $\pm \mathbf{Y}$ entries are \pm 6.2 (i.e., half of 12.4). The $\pm \mathbf{Z}$ entries are \pm 10.1 (same as the height of the steel cylinder).

Now enter the media records for unit 1 ; this can be done using either the configurable form or by entering the records directly. First is the uranium, mixture 1, in the horizontal cruciform element. Next is the vertical cruciform element, mixture 1, inside cuboid 2 but outside cuboid 1 . Recall that the region of space that is within both cuboids must be included in one but excluded from the other to prevent the space being doubly defined. For the third media record, enter the water by specifying it inside cylinder 3 and outside cuboids 1 and 2. The next media entry is the SS316 cylindrical container: inside cylinder 4 and outside cylinder 3 . The final media record for unit 1 is the void cuboid outside the steel cylinder. Specify it as inside cuboid 5 and outside cylinder 4 . Finally, the boundary record region definition vector must be updated to inside 5. The geometry and contents for unit 1 should look like Figure 132. 


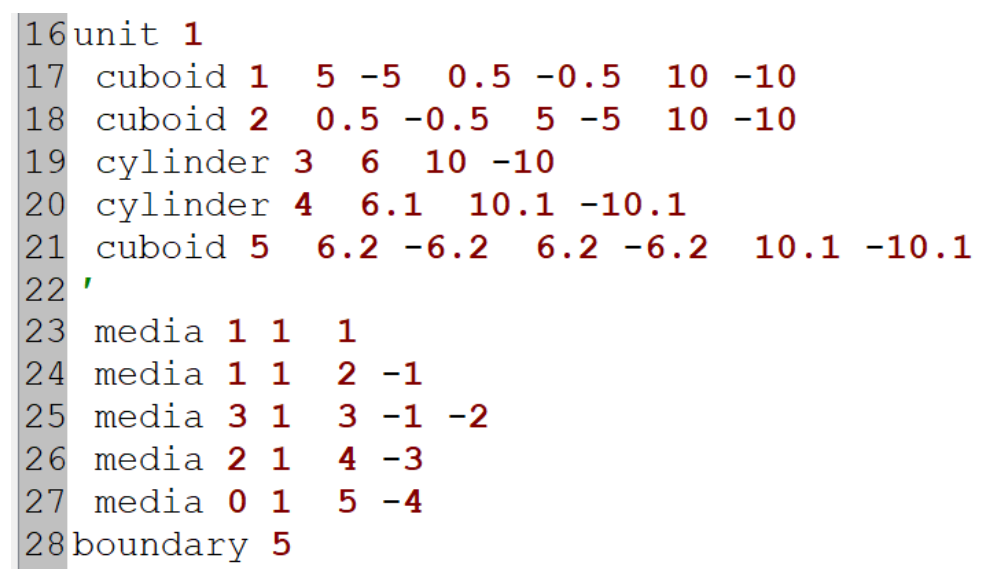

Figure 132. Geometry input for unit 1.

Create a void cuboid unit as unit 2. This unit is needed to complete the array that will be used to position the five cans inside the larger cylinder. Create a Cuboid with the same outer dimensions that were used for the outer cuboid in unit 1. Then define the Media to fill the cuboid with void and define the boundary as inside cuboid 1 to complete unit 2 .

Before defining the global unit, the array to be inserted in that unit must be defined. Note that the five individual containers are positioned to fill the five interior positions in a $3 \times 3$ array, with one unit in the top center position, three units along the center row, and one unit in the bottom center position. Because arrays cannot have empty elements, use unit 2 in the four corner positions of the $3 \times 3$ array. The $3 \times 3$ array does not entirely fill the inner volume of the shipping container. One option is to add a geometry record in the global unit with void filling it to define the space between the array and the shipping container wall. It is also possible to use the shipping container inner surface to truncate elements in the array, so a larger array with more void units can be used as the single contents record in the global unit. Therefore, a larger, $5 \times 5$ array will be constructed.

To start describing the array, place the cursor on the line after end geometry and enter add an array block, either by using the Fulcrum autocomplete capability or by typing the input directly into the input file. A single array will be defined, and is likely most simply started with the CTRL-SPACE autocomplete key combination. The default ara number of 1 can be used, and the defaults for gbl, prt, and typ can also be retained. The values for nux and nuy should be updated to 5, but nuz will still be 1 . There are 25 elements in a $5 \times 5$ array, and only 5 cans so 20 of the 25 locations will be unit 2 . The simplest choice for fill id is therefore 2 , so that only 5 fill entries need to be changed. The completed configurable form for this array is shown in Figure 133. After reviewing the input, press the OK button to add the array definition to the input file. The fill data must still be modified to add unit 1 in the appropriate locations. The central element $(3,3)$ should be changed from 2 to 1 , as should the 4 face adjacent elements so that a total of 5 cans are included in the array. The final array input is shown in Figure 134. 


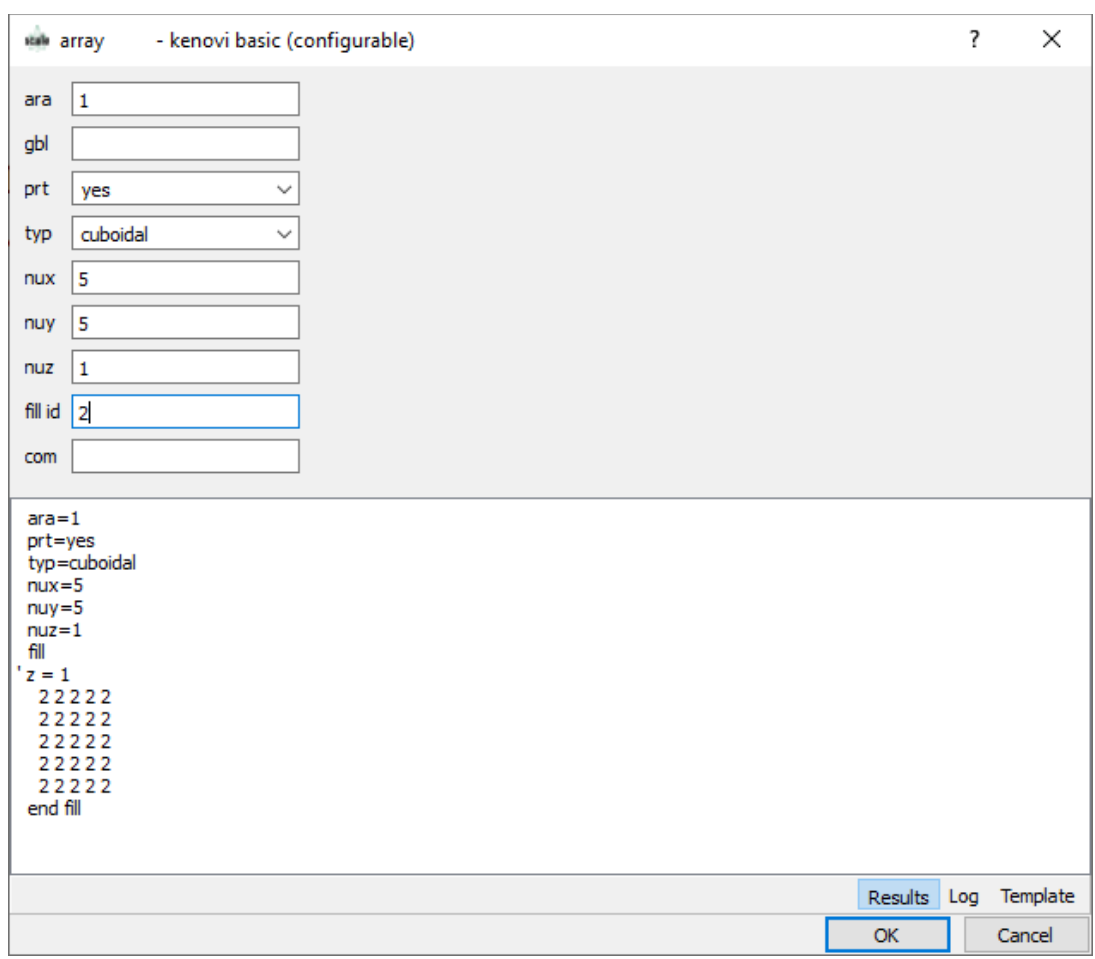

Figure 133. Completed array configurable for the $5 \times 5$ array.

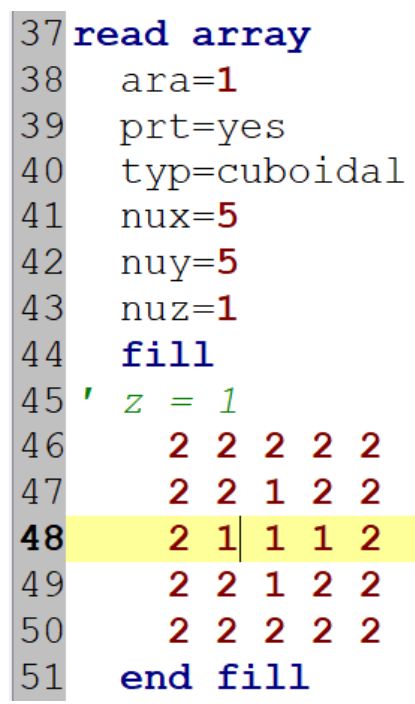

Figure 134. Array definition.

Now return to the geometry block and create a new unit 3 as the global unit. The first geometry record is the inner cylinder of the shipping container. Create a Cylinder and enter 20 for Radius, 10.1 for Top, and -10.1 for Bottom. The next record is the outer cylinder of the container. The shipping container is $0.2 \mathrm{~cm}$ thick, so the dimensions are 20.2 for Radius, 10.3 for Top, and -10.3 for Bottom. With the curser between the unit declaration and the boundary record, the CTRL-SPACE autocomplete key combination to open an array contents record configurable form. The default Array Id in the upper left corner of the form is correct as only array 1 has been defined. Press the Add row button to add a region in the Regions pane; the default values of Inside and Id $\mathbf{1}$ are correct. The simplest way to position the array 
is to use the central element and position it at the origin. So xindex and yindex should be set to 3 and zindex should be left as $\mathbf{1}$. The coordinates for $\mathbf{x}, \mathbf{y}$, and $\mathbf{z}$ can all be left as $\mathbf{0}$. The completed array contents record configurable form is shown in Figure 135; after confirming the input press the OK button to add the record to the input file. Not that the array is larger than the inner volume of the shipping container, but that it is truncated by cylinder 1 . This feature can dramatically simplify the input description for some models.

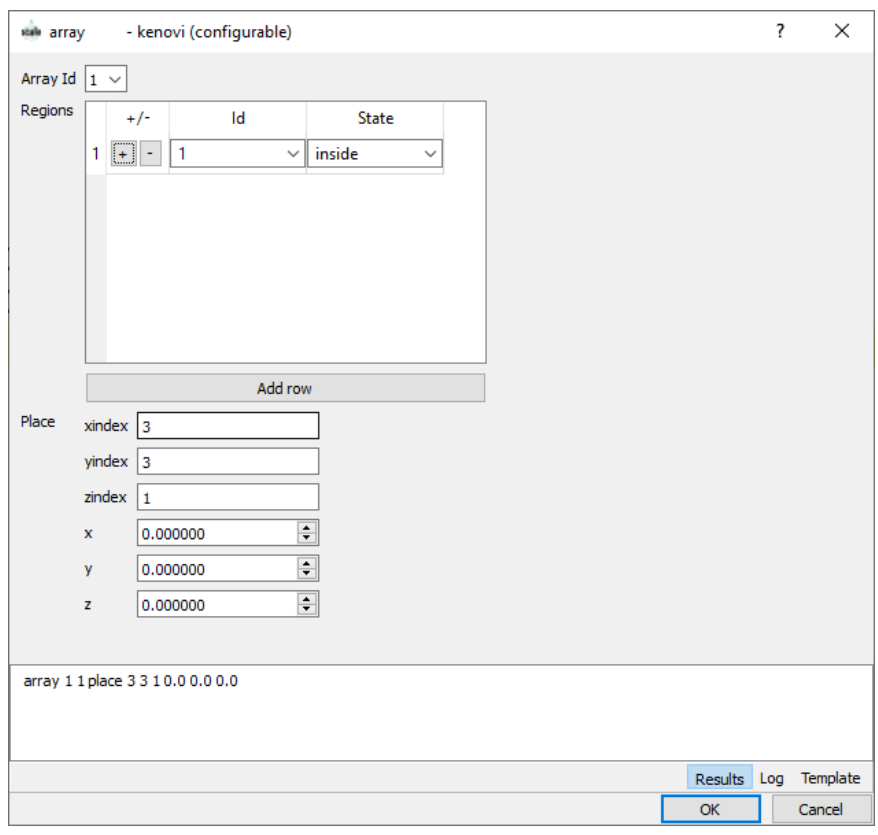

Figure 135. Completed array contents record configurable form.

Now, define the media record for the SS316 outer cylinder, mixture 2 as inside cylinder 2 and outside cylinder 1 . Define the boundary of unit 3 as inside cylinder 2 . The completed input for unit 3 is provided in Figure 136. Save the changes and execute SCALE. The calculated $k_{\text {eff }}$ value is $0.6277 \pm 0.0018$.

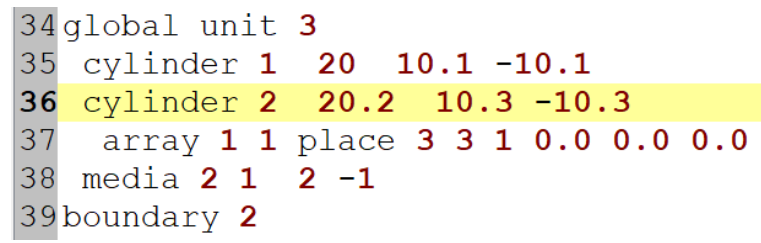

Figure 136. Geometry input for unit 3.

\subsection{HEXAGONAL ARRAY WITH INNER AND OUTER BOUNDARIES}

This next problem illustrates how to create a hexagonal array and remove unwanted portions of the array using both inner and outer boundaries in the region definition vector on the array record. An annular carbon steel cylindrical tank contains six aluminum-clad U(10.0) cylinders submerged in water (Figure 137). The uranium cylinders are $15 \mathrm{~cm}$ in diameter and $18.3 \mathrm{~cm}$ high. The aluminum clad is $0.5 \mathrm{~cm}$ thick on the sides and bottom of the uranium. The cylinders have a center-to-center triangular pitch of $21 \mathrm{~cm}$. The tank has an inner radius of $12 \mathrm{~cm}$ and an outer radius of $31.5 \mathrm{~cm}$. The inside height is $18.8 \mathrm{~cm}$. The tank walls and bottom are $1.5 \mathrm{~cm}$ thick. A cutaway view with highlighted edges in KENO3D (see Section 9.4) is shown in Figure 138. 


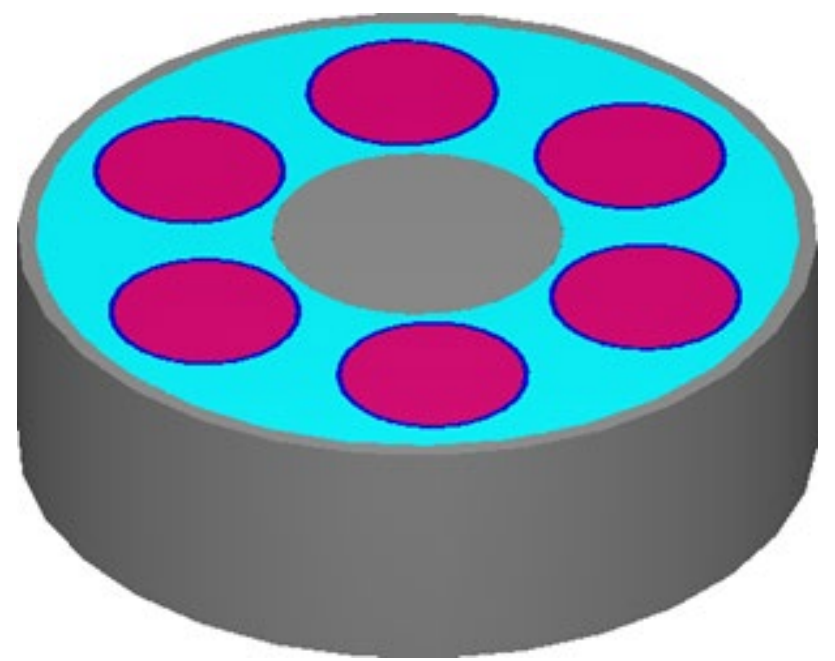

Figure 137. Annular carbon steel cylindrical tank with aluminum-clad uranium cylinders.

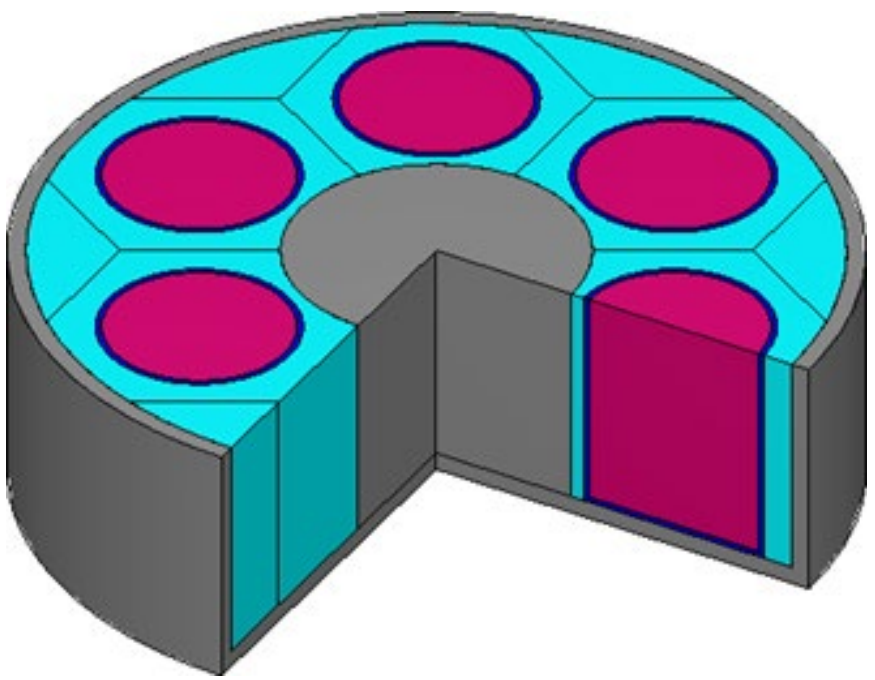

Figure 138. Cutaway view of tank with highlighted edges.

Specify the sequence, title, cross section library and composition descriptions as done in previous sections of this primer. Note that there are basic standard compositions for uranium, aluminum, carbon steel (carbonsteel), and water, which make the composition specifications straightforward. The finished composition block is shown in Figure 139.

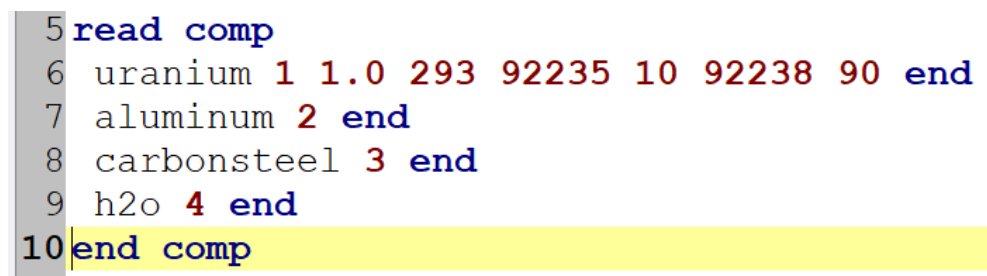

Figure 139. Composition block for hexagonal array example.

The first unit is not the global unit but a uranium cylinder with aluminum clad. Create a first Cylinder by entering the dimensions of the uranium cylinder: 7.5 for Radius, 18.3 for Top, and 0 for Bottom. 
Next enter the data for the aluminum clad cylinder: 8 for Radius, 18.3 for Top, and -0.5 for Bottom. This cylinder must be surrounded with a hexprism of water so that this unit can be placed in a hexagonal array. Create a hexprism and enter 10.5 for Radius, 18.3 for $\mathbf{Z Z}$, and -0.5 for $\mathbf{- Z}$. Note that the radius used to specify a hexprism or rhexprism is the radius of the inscribed circle; this dimension is also referred to as the apothem of the hexagon. The media records for unit 1 place uranium (mixture 1) inside cylinder 1, aluminum (mixture 2) inside cylinder 2 and outside cylinder 1, and water (mixture 4) inside hexprism 3 and outside cylinder 2 . The boundary of unit 1 is inside hexprism 3.

Unit 2 will be a hexprism of water that is so that empty hexagonal array positions can be included around the outside of the unit 1 hexprisms. (Portions of these units are visible in Figure 138.) Create a hexprism and enter 10.5 for Radius, 18.3 for $+\mathbf{Z}$, and -0.5 for $-\mathbf{Z}$. The only media record fills the hexprims with water (mixture 4 ). The boundary is defined by boundary 1 . The geometry and contents records for units 1 and 2 are shown in Figure 140.

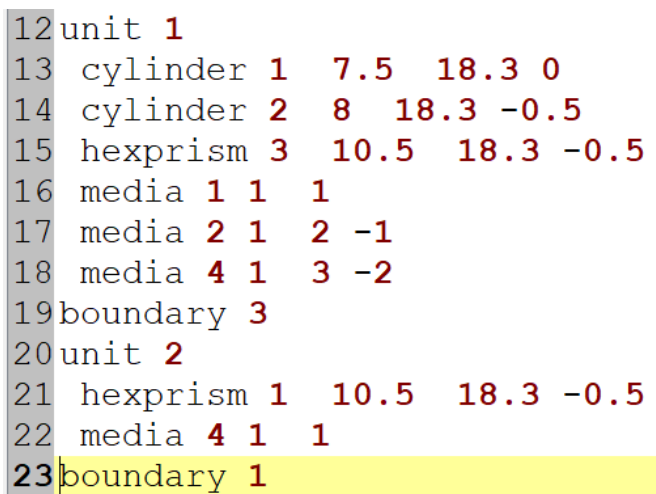

Figure 140. Input for units 1 and 2 for hexagonal array example.

Now the hexagonal array can be defined. Add an array block after the geometry block, and use the CTRL-SPACE autocomplete key combination to begin the specification for array 1. Select triangular from the dropdown for the array type. Sizing the array to be 5 units in the $\mathrm{X}$ and $\mathrm{Y}$ directions, and 1 unit in the $\mathrm{Z}$ direction ensures that it will be large enough to fill the cylinder in the global unit and simplifies selecting the central unit. Specify nux and nuy as 5 and nuz as 1 . The simplest unit to initialize the fill data with is unit 2 since there are 25 elements in the array and only 6 need to be changed to unit 1 . The completed array configurable form is shown in Figure 141. After confirming the input, press the OK button to add the array definition to the input file. Since the triangular (hexagonal) array type shifts to the right with each layer in the $\mathrm{Y}$ direction, adding spaces to the input file can help identify the elements in the array to modify. An example of this spacing, prior to adding unit 1 to the array, is shown in Figure 142. Recall that the order of the Y entries is inverted in the input compared to the model as the entries are read in increasing order (see Figure 91). Either 6 or 7 elements can be changed from unit 2 to unit 1 because the central element will be removed from the array in the model. Changing only 6 entries is slightly simpler; change the third and fourth elements in the $Y=2$ rank of the array, the second and fourth elements in the $\mathrm{Y}=3 \mathrm{rank}$, and the second and third elements in the $\mathrm{Y}=3$ rank. The final fill statement is shown in Figure 143. The complete array definition is shown in Figure 144. 


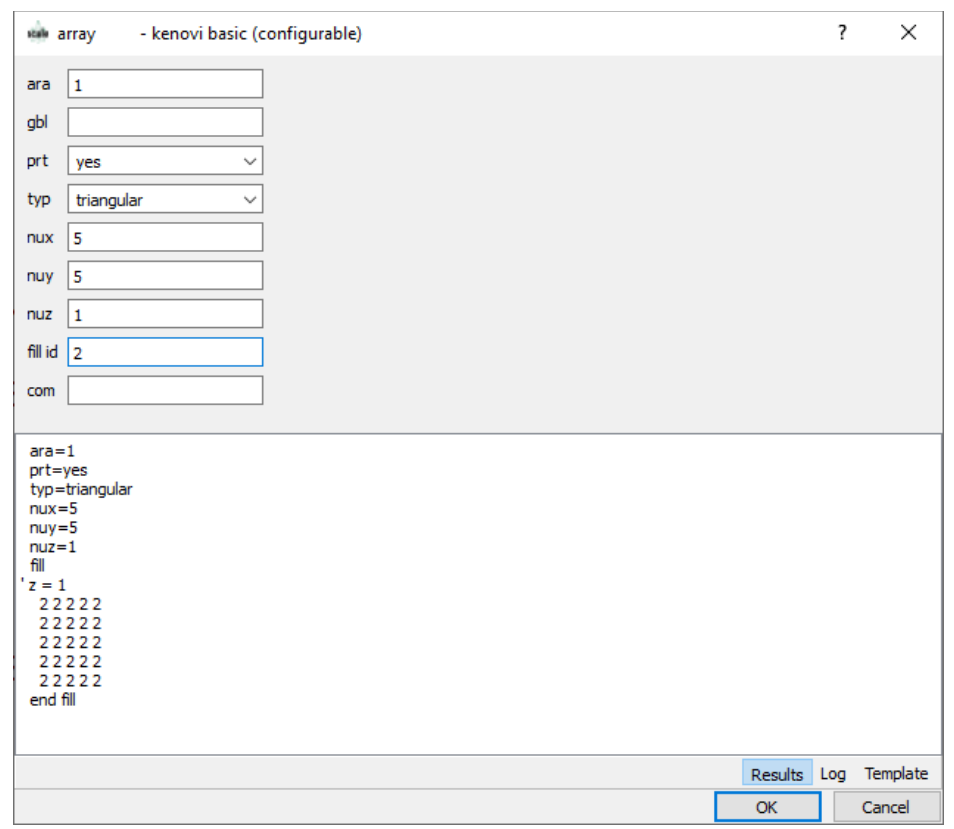

Figure 141. Completed array configurable form for the hexagonal array example.

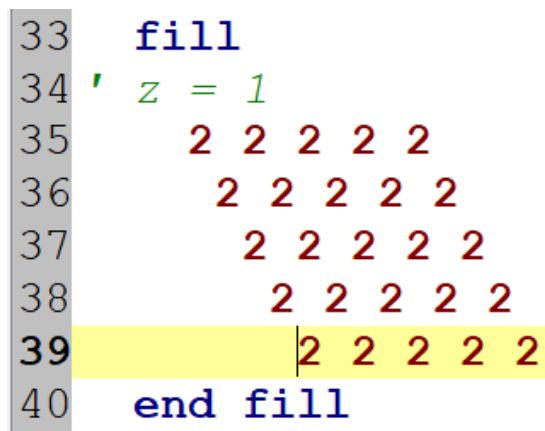

Figure 142. Fill statement with additional spacing to help visualize final array geometry.

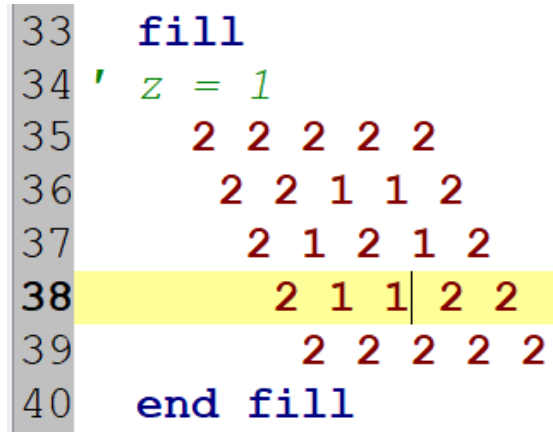

Figure 143. Final fill statement for hexagonal array example. 


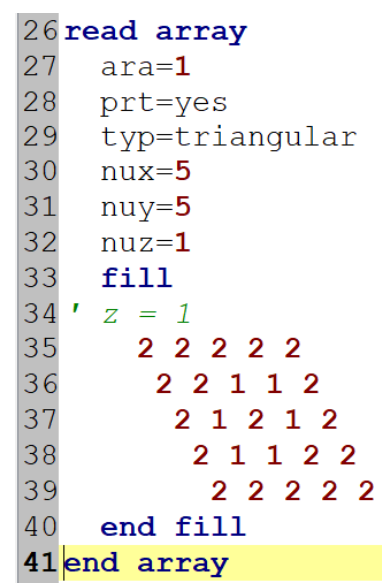

Figure 144. Array definition for the hexagonal array.

Create global unit 3. The first geometry record is the central steel cylinder within the tank. Create a Cylinder, and enter 12 for Radius, 18.3 for Top, and -0.5 for Bottom. The next record is the inner wall of the container, which has a Radius of $31.5 \mathrm{~cm}$ and the Top and Bottom of 18.3 and -0.5 , respectively. The tank is $1.5 \mathrm{~cm}$ thick, so the outer dimensions for the third cylinder are 33 for Radius, 18.3 for Top, and -2 for Bottom.

Next, create the array contents record. The simplest way to do this is by using the array contents configurable form. Click the Add row button to add a region to the Regions pane. Set the first row to be out side Id $\mathbf{1}$ and add a second row to be ins ide Id $\mathbf{2}$. The geometry Id must be typed in the field. Note the region definition vector in the array contents record in the preview pane (as shown Figure 145) now shows that the array will be inside region 2 but outside region 1 . The array must still be positioned and can be positioned with any unit in the array, regardless of whether that unit is used in the model or not. Placing the central element at the origin is the simplest approach for this model, so set xindex and yindex to 3 and leave zindex as $\mathbf{1}$. The $\mathbf{x}, \mathbf{y}$, and $\mathbf{z}$ coordinates of the origin of element $(3,3,1)$ are all $\mathbf{0}$. The complete array contents record is shown in Figure 146; after confirming the input press the OK button to add the contents record to the input file. Now, define the media record to fill the central cylinder with carbon steel cylinder (mixture 3). The outer shell of the tank is also carbon steel (mixture 4) and is inside cylinder 3 and outside cylinder 2 . Define the boundary of unit 3 as inside cylinder 3 . Note that the region inside cylinder 2 but outside cylinder 1 is already filled with array 1 so all of the defined geometry in unit 3 has contents assigned. The completed unit 3 input is provided in Figure 147. Save the changes and execute SCALE. The calculated $k_{\text {eff }}$ value is $0.6182 \pm 0.0016$.

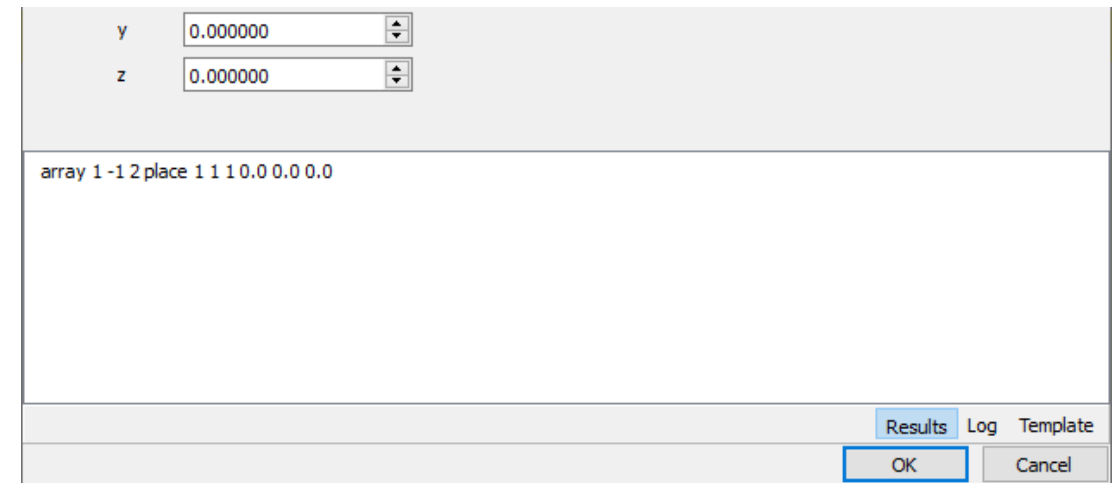

Figure 145. Preview pane of array contents record. 


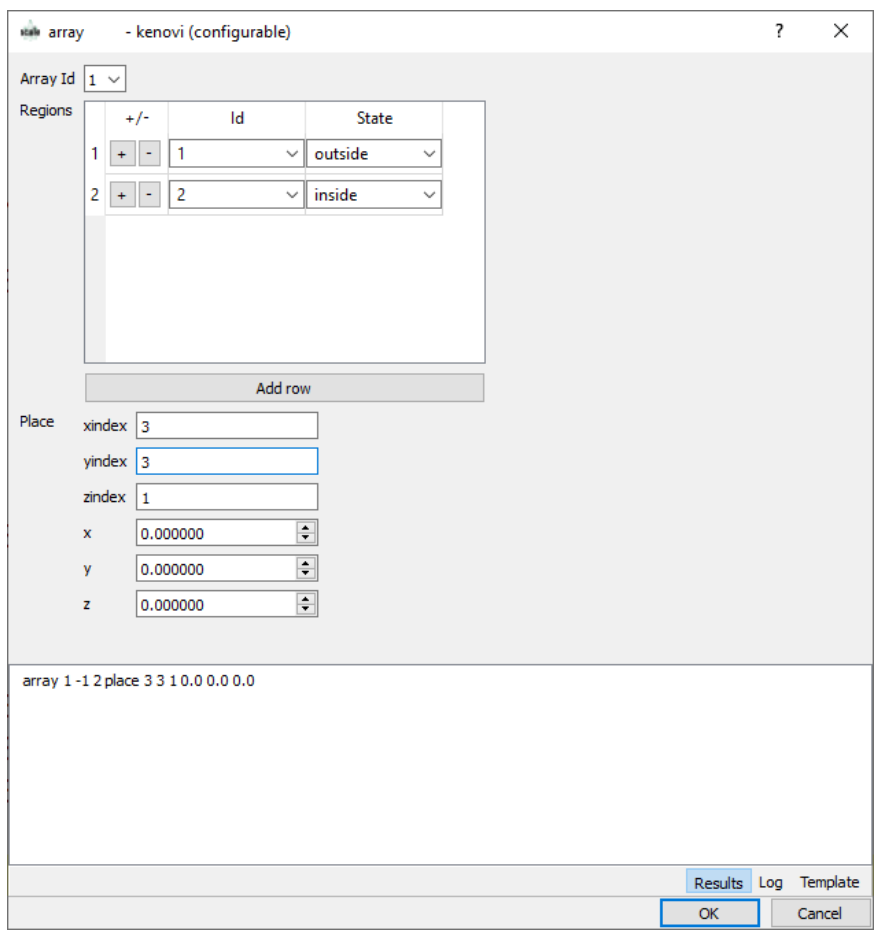

Figure 146. Complete array contents configurable form for the hexagonal array example.

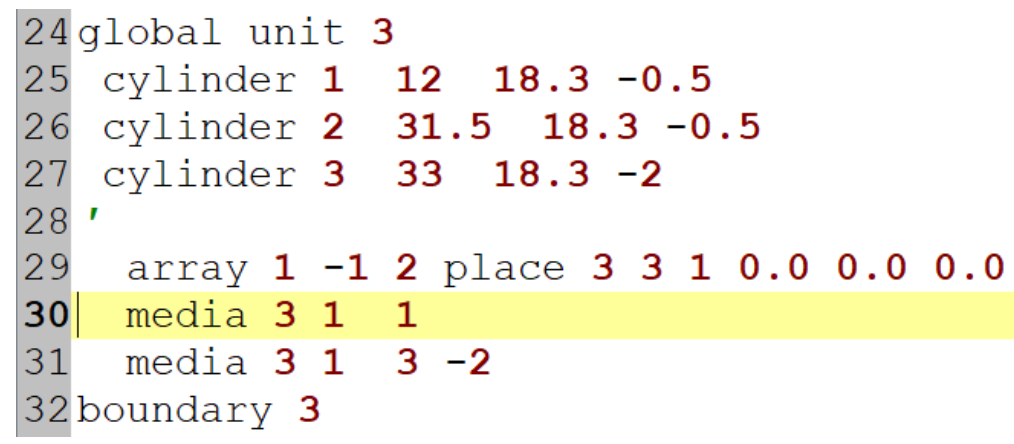

Figure 147. Unit 3 for the hexagonal array example.

\subsection{SUMMARY}

This section has discussed the following:

- to use Fulcrum and KENO-VI to describe complex geometry models using arrays,

- to remove unwanted portions of arrays using region definition vectors to apply boundaries, and

- to build hexagonal arrays. 


\section{NONSTANDARD MATERIALS}

Now that you understand the geometry and basic standard composition input requirements, this section provides an explanation of a few inputs you might find useful for problems involving compounds or other materials not available in the Standard Composition Library.

\subsection{WHAT YOU WILL BE ABLE TO DO}

- Define user-defined (non-standard) materials using Fulcrum.

- Define a mixture by using a combination of user-defined material and a basic standard composition.

\subsection{USER-DEFINED MATERIALS}

Section 3 covered entry of information for elements, isotopes, and compounds found in the Standard Composition Library. However, there are some materials that are not available in the Standard Composition Library in SCALE. These materials can be defined using the Alloy or Mixture (wtpt) or the Compound (atom) configurable forms, or placeholder templates from the material specification menu form used in Section 3 (see, for example, Figure 148). This approach works best for materials in which the chemical composition or weight fraction is provided. If a compound or alloy is in the Standard Composition Library, or if number densities are available for each of the constituents of the desired material, then using the basic standard composition input is likely more efficient and less error prone.

\subsubsection{Description Based on Chemical Formula}

In this example, enter the material information for hydraulic fluid, $\mathrm{C}_{2} \mathrm{H}_{6} \mathrm{SiO}$, with a density of $0.97 \mathrm{~g} / \mathrm{cc}$. Move the cursor into the composition block between Read Comp and End Comp and press CTRLSPACE to trigger the dropdown menu containing the list of available composition configurable and placeholder options. From this list, click select atomcomp - basic (configurable) as shown in Figure 148.

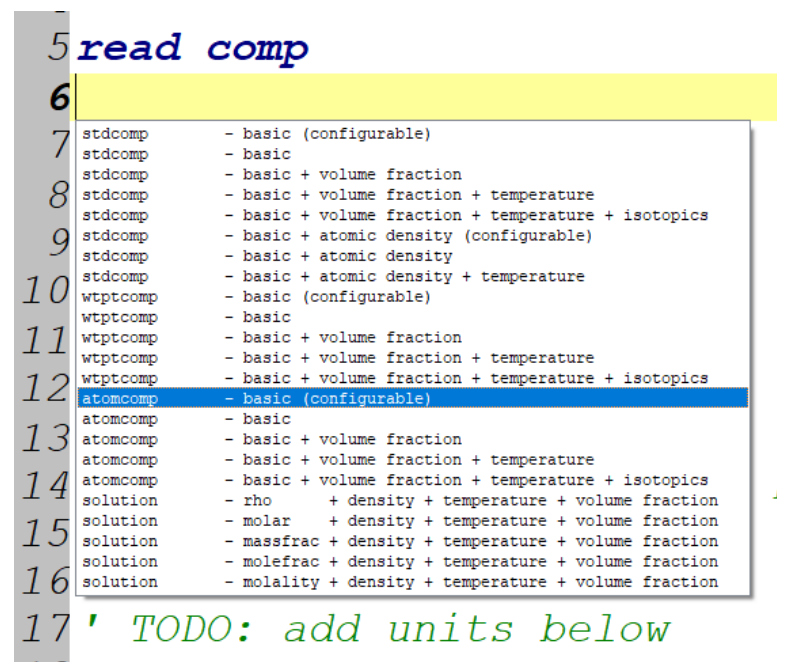

Figure 148. Dropdown menu selection of atom compound configurable.

The configurable from should appear and should look like Figure 149, although you may have to expand the left pane to see the Add button. In the Name portion of the configurable, replace TBD with oil, and enter 0.97 in the Theoretical Density box. Next, click on the Add button in the left pane to begin adding 
the elements to the compound. The configurable form should change to look like Figure 150 below. Using the new right pane, click on the dropdown menu next to Element, select 6000 for carbon, and enter 2 in the Atom Count box below it. This process should be repeated using Element IDs of 1000 , 14000 , and 8000 , and atom counts of 6,1 , and 1 for hydrogen, silicon, and oxygen, respectively, while pressing the Add button between each elemental entry. The final configurable form should look like the one in Figure 151 and create input like that shown in Figure 152.

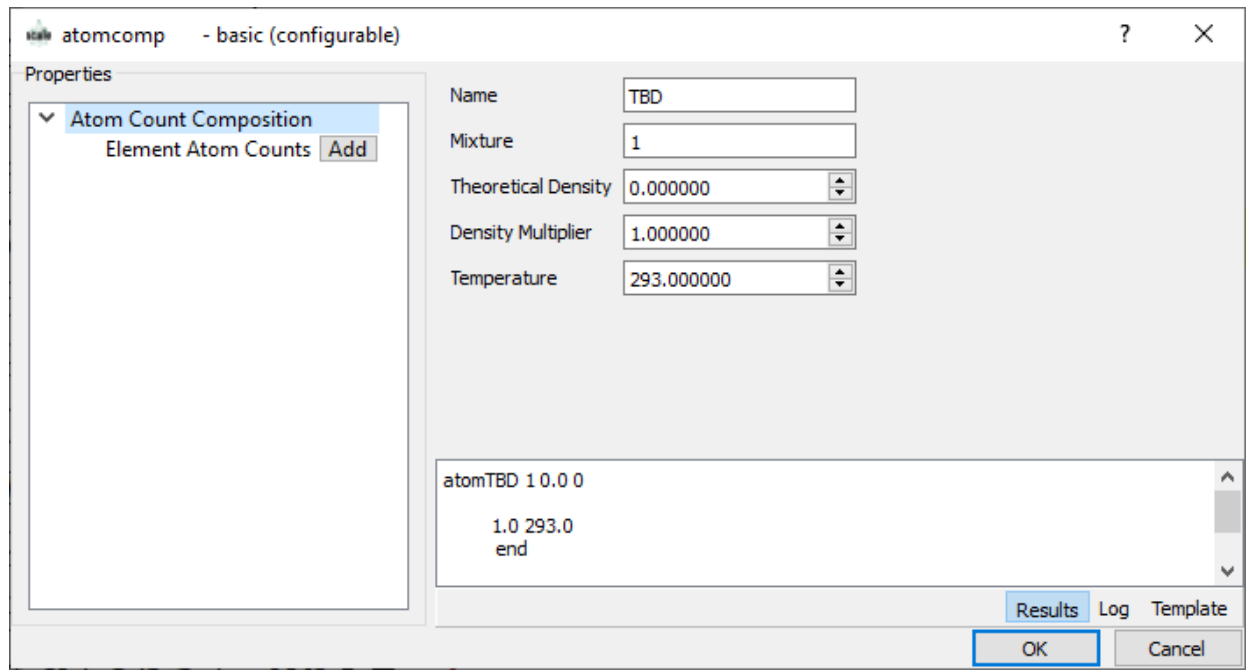

Figure 149. Depiction of initial atom compound configurable.

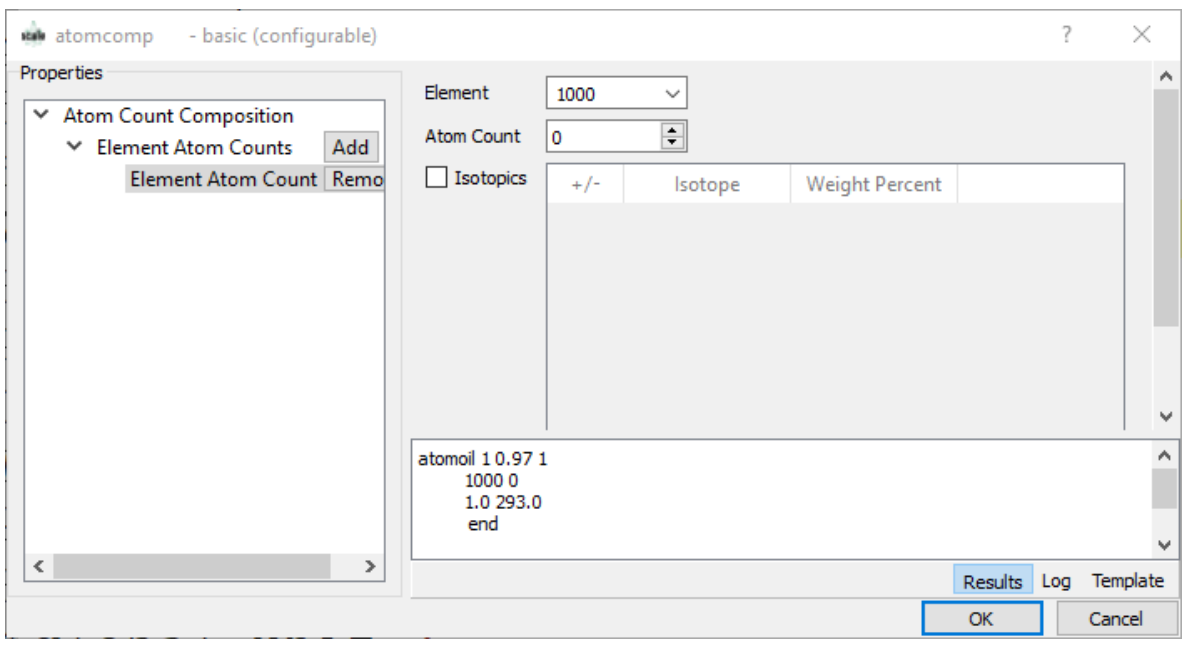

Figure 150. Depiction of element addition portion of the compound configurable. 


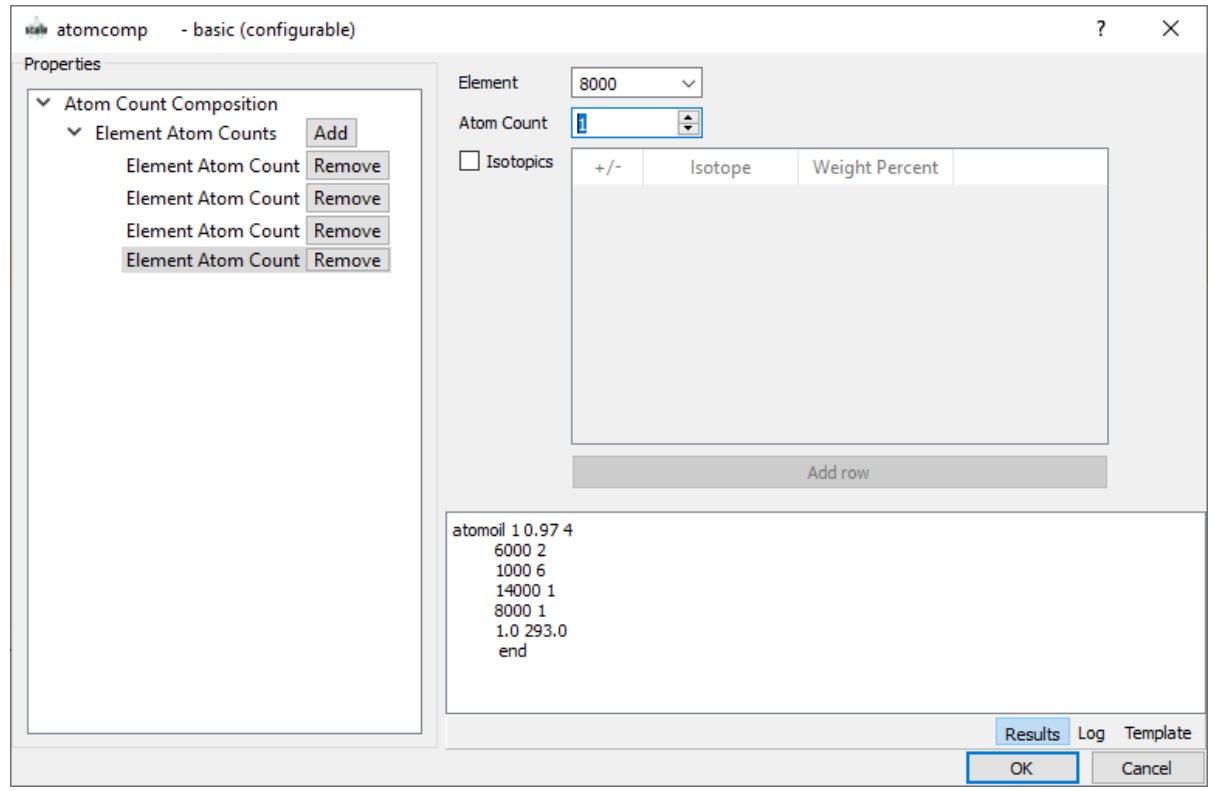

Figure 151. Second depiction of element addition portion of the compound configurable.

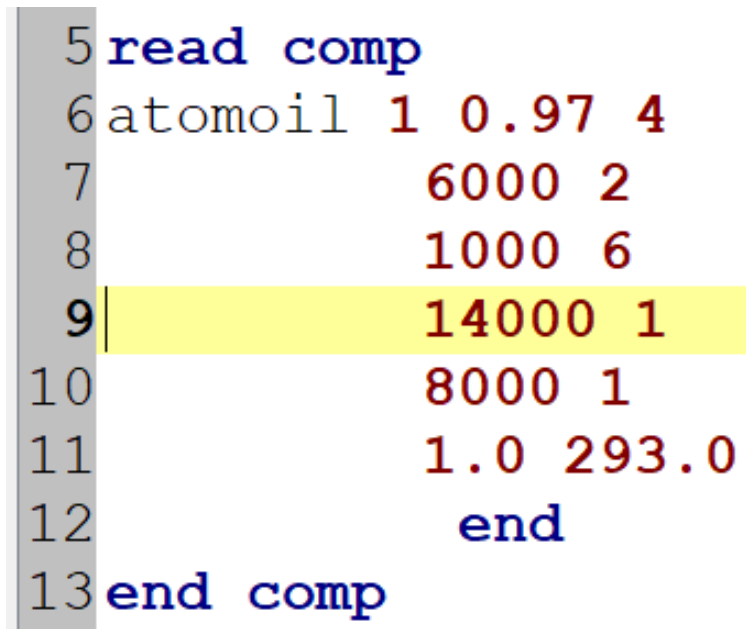

Figure 152. User-defined atom composition final input.

\subsubsection{Description Based on Weight Percent}

In this example, enter the material information for borated aluminum that is $2.5 \mathrm{wt} . \%$ boron and has a density of $2.65 \mathrm{~g} / \mathrm{cm}^{3}$. The boron is $10 \mathrm{wt} . \%{ }^{10} \mathrm{~B}$ and $90 \mathrm{wt} . \%{ }^{11} \mathrm{~B}$. For this material, the weight percents are known, so the information should be entered in terms of weight percent, which is the Alloy or Mix (Wt \%) type. Move the cursor into the composition block between Read Comp and End Comp and press CTRL-SPACE to trigger the dropdown menu containing the list of available composition configurable and placeholder options. From this list, select wtptcomp - basic (configurable), as is shown in Figure 153. 


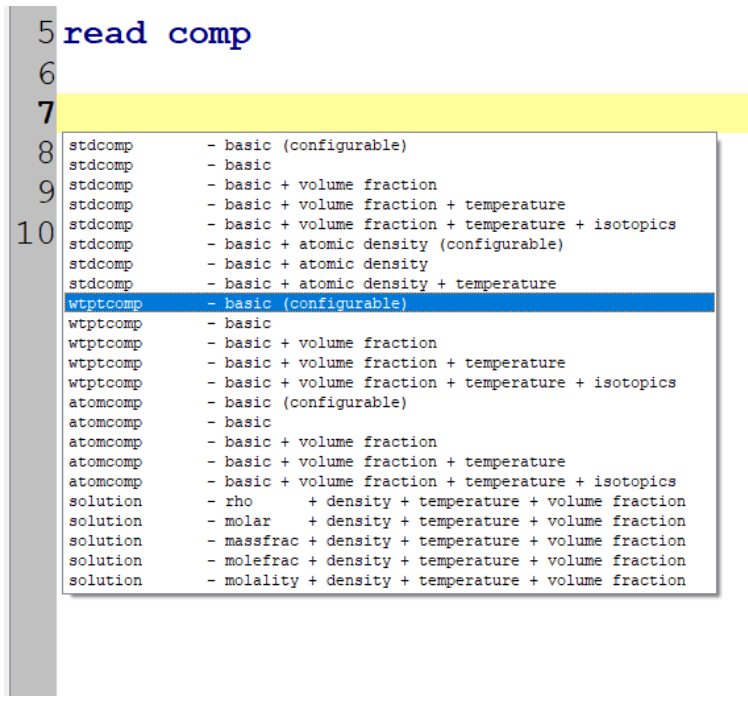

Figure 153. Dropdown menu selection of wtpt compound configurable.

A configurable form similar to that used for the atom compound material (Figure 149) entries will appear. For Composition name, replace TBD with bal to create the material wtptbal. Now enter the Theoretical Density of $2.65 \mathrm{~g} / \mathrm{cc}$ and leave the Density Multiplier and Temperature at the default values. In the left pane of the configurable, click the Add button (the pane may need to be expanded to display the button). This will allow entry of individual nuclides. In the Element dropdown menu, select 13000 for aluminum, and enter 97.5 in the Weight Percent box. Back in the left pane, click the Add button again, select 5000 for boron from the Element dropdown menu, and enter 2.5 for Weight Percent. Because there is an isotopic specification for this example, check the Isotopics box, and click Add row twice. Adjust one of the $\mathbf{5 0 1 0}$ Isotope values to be 5011, enter 90.0 for the Weight Percent value corresponding to 5011, and enter 10.0 in the Weight Percent box corresponding to 5010. When finished, the configurable from should look like Figure 154. If the configurable looks acceptable, click OK, and the final input will look like Figure 155.

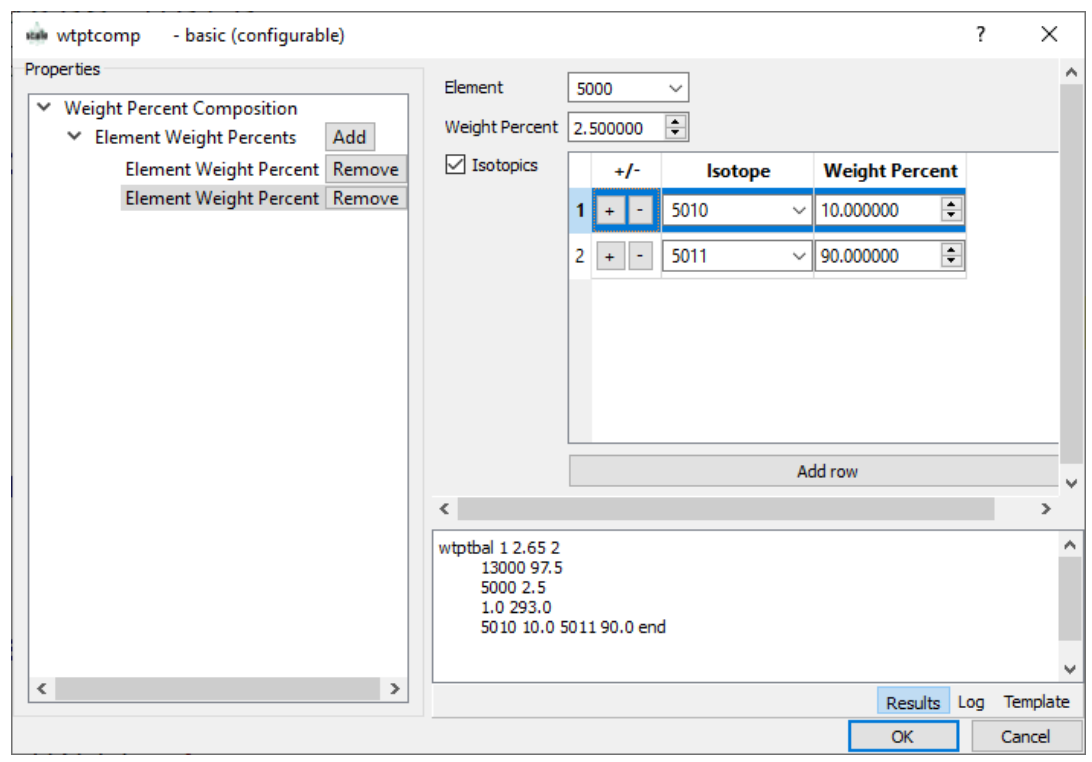

Figure 154. Final configurable for wtpt composition entry. 


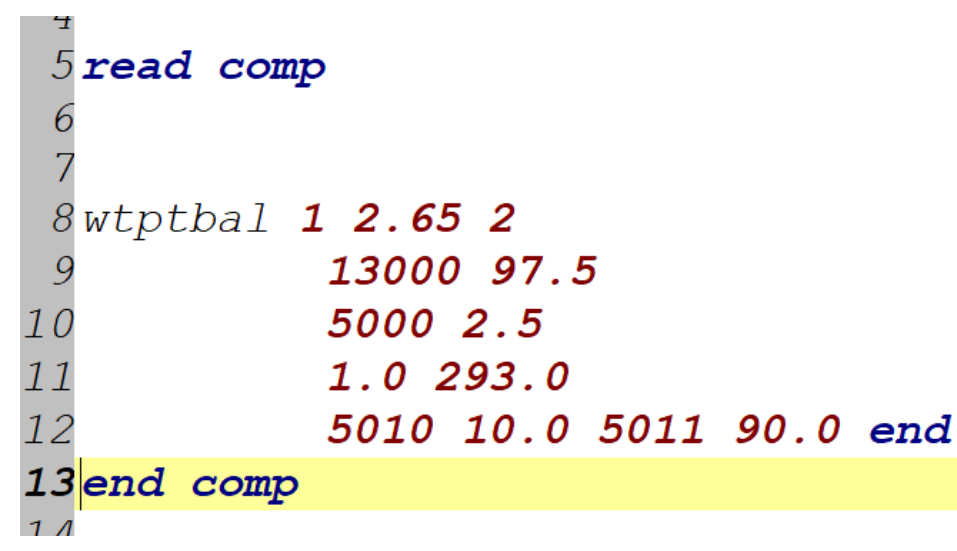

Figure 155. User-defined wtpt composition final input.

\subsubsection{Description Based on Actinide Solution}

The next method of entering material composition information uses SCALE's actinide solution composition specification method. In this example, specify a $\mathrm{UO}_{2} \mathrm{~F}_{2}$ solution containing $907.0 \mathrm{gU} / \mathrm{L}$, with an overall solution density of $2.0289 \mathrm{~g} / \mathrm{cm}^{3}$. The uranium is enriched to $5 \mathrm{wt} .{ }^{2}{ }^{235} \mathrm{U}$; neither ${ }^{234} \mathrm{U}$ nor ${ }^{236} \mathrm{U}$ will be included in this example. At this time, a configurable form for actinide solutions is not present within the Fulcrum interface, so the placeholder method is used to enter this information.

Move the cursor into the composition block between Read Comp and End Comp, and press CTRLSPACE to trigger the dropdown menu containing list of available composition configurable and placeholder options. The dropdown menu is displayed with the five solution specification options shown in Figure 156. The variation placeholder options for solution specification are designed to account for the various methods of expressing the actinide concentration in the solution. In this example, the concentration is expressed in terms of the number of grams of uranium in the solution per liter. The appropriate selection for this set of units is solution - rho + density + temperature + volume fraction. Selecting that option yields the initial input found in Figure 157. 


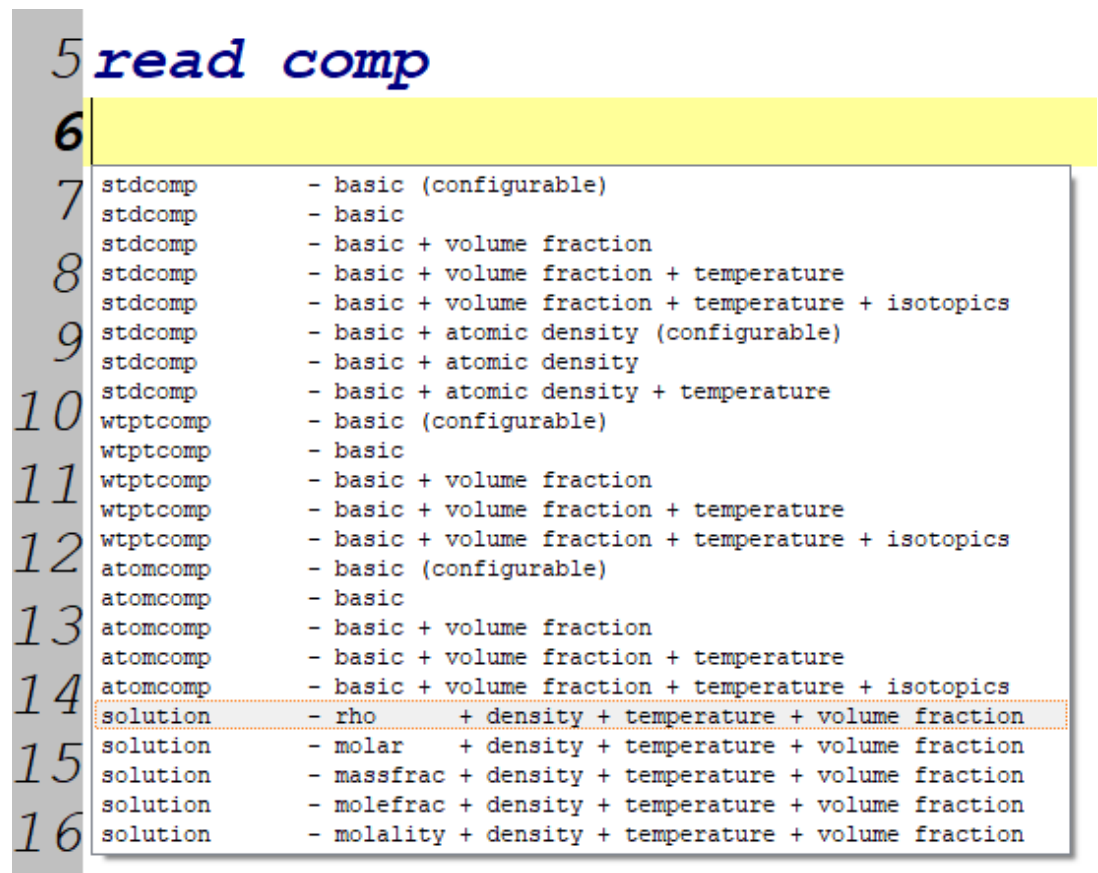

Figure 156. User defined solution composition final input.

\section{5 read comp \\ 6solution $\operatorname{mix}=1$ rho[uo2f2] $=1.0 \quad 9223595.592238 \quad 4.5$ \\ 7 density $=1.0$ temp $=300$ volfrac $=1.0$ end solution \\ 8 end comp}

Figure 157. Input specified by placeholder insertion.

(normally placed on one line by Fulcrum but shown on two lines for readability)

Once the placeholder input has been inserted in the input file, it must be modified to correctly model the composition. In this case, the placeholder has the correct chemical form that accompanies the fissile species, uo2f2, but the number of grams per liter (rho) should be changed from 1.0 to 907.0 . The isotopic specification should be modified from $\mathbf{9 2 2 3 5} 95.5922384 .5$ to 922355.09223895 .0 , and the density of the solution should be modified from $\mathbf{1 . 0}$ to 2.0289 . For this case, there is no excess acid specified; however, if there were, it would be specified as molar [hfacid] =X, where X is the molarity of the acid in the solution. The final solution input is shown Figure 158.

\section{5 read comp \\ 6solution $\operatorname{mix}=1$ rho[uo2f2]=907.0 $92235 \quad 5.09223895 .0$ \\ 7 density $=\mathbf{2} .0289$ temp=300 volfrac=1.0 end solution \\ 8 end comp}

Figure 158. Complete solution input. 


\subsection{SUMMARY}

This section has demonstrated how to use Fulcrum to:

- define user-defined material based on a known chemical formula,

- define user-defined material for a mixture with known weight percents of constituents, and

- define actinide solutions. 



\section{UNIT CELLS AND BOUNDARY CONDITIONS}

The examples thus far have been limited to relatively large homogeneous systems in which the default infinite homogeneous medium unit cell type was appropriate. CE cross sections were also used to avoid any errors associated with improper MG cross section treatment. Cross section processing for resonance self-shielding is only necessary for MG libraries. The resonance self-shielding is a result of using groupaverage cross sections in the transport solution in KENO. In this section, more heterogeneous systems will be presented, along with demonstrations of how to use the other unit cell types appropriately. Transport results determined with CE cross sections can provide a reference solution to ensure that the MG cross section processing has been performed correctly in most cases.

\subsection{WHAT YOU WILL BE ABLE TO DO}

- Understand the difference in cross section processing for infinite homogeneous medium, lattice cell, multiregion, and doubly heterogeneous analyses.

- Identify which unit cell type is appropriate to use for various heterogeneous systems.

- Use Fulcrum to define LATTICECELL, MULTIREGION, and DOUBLEHET unit cells.

- Define different boundary conditions for MULTIREGION and DOUBLEHET unit cells.

- Use boundary conditions to create reflective boundaries for a KENO-VI global unit.

\subsection{CALCULATION TYPES FOR PROBLEM-DEPENDENT CROSS SECTIONS}

XSProc is primarily used to prepare data for creating the problem-dependent cross section libraries required by CSAS. This is particularly important for heterogeneous systems and large regular lattices of slabs, pins, or spheres. XSProc uses a unit cell description to provide information for the resonance selfshielding corrections and the Dancoff corrections (where appropriate) that are applied to the cross sections to create a problem-dependent cross section library. A unit cell is typically a portion of the system containing fuel and, if applicable, moderator that can be used to define the neutron spectrum characteristics for part of the problem.

\subsection{UNIT CELL TYPES}

XSProc offers four types of unit cell calculations: INFHOMMEDIUM, LATTICECELL, MULTIREGION, and DOUBLEHET. Note that multiple unit cells may be defined in a single CSAS/KENO-VI input file. Each mixture number may be used only once in any unit cell, except for void (mixture 0), which can be used as frequently as needed.

\subsubsection{Infinite Homogeneous Medium}

The INFHOMMEDIUM treatment is best suited for large masses of materials in which (1) the size of each material is large compared with the average mean-free path of neutrons in the material, (2) the size of each material is very small compared with the average mean-free path, or (3) the fraction of the material that is within one mean-free path from the surface of the material is very small. An example of the first situation is a large solution tank; the neutron mean-free path may be on the order of a centimeter so a tank would only need to be a few centimeters in diameter to count as "large". This example also satisfies the third condition that a small fraction of the solution is within a mean-free path of the boundary. The second situation is more rare, and a good example is likely to be a fast reactor. In these systems, the mean free path may be several centimeters (or more) and the neutrons do not interact on the same distance scale as the heterogeneity present for heat transfer. Every material specified in the problem 
that is not specified in a unit cell is treated as an infinite homogeneous lump. A system composed of small fuel lumps, such as rods, slabs, or pebbles, should not be treated as an infinite homogeneous medium.

\subsubsection{Latticecell}

The LATTICECELL treatment is appropriate for large arrays of fuel in slabs, pins/rods, or spheres. When the LATTICECELL treatment is used in XSProc, a set of point fluxes is calculated for a set of mesh intervals that make up the unit cell. The Wigner-Seitz equivalent cylindrical cell is used, except for the SQUAREPITCH option, and the cell is assumed to have white boundary conditions. The SQUAREPTICH option for the LATTICECELL treatment uses a 2D method of characteristics (MoC) solver in place of the 1D discrete ordinates solver that is used for other unit cell types. The cross sections for the mixtures specified in the cell are then weighted using these point fluxes and the CE cross sections. Limitations of the LATTICECELL treatment include the following:

1. The cell description is limited to a 1D cell. Most physical systems consist of 3D geometry, but in many instances, a 1D representation is adequate, as in the case of a large array of spheres in a regular lattice, a large array of slabs, or a large array of long uniform pins in a regular lattice. A 1D representation may be inadequate for systems exhibiting a 2D or 3D spatial dependence.

2. The LATTICECELL treatment assumes an infinite array of 1D cells. This assumption is an excellent approximation for large arrays of long fuel pins or large arrays of spheres. The approximation becomes less rigorous for short fuel pins and/or small arrays in which multidimensional spatial dependence or leakage can become important.

\subsubsection{Multiregion}

The MULTIREGION treatment is appropriate for geometric regions in which the geometry effects may be important, but the infinite homogeneous treatment or lattice cell treatment is not general enough. The MULTIREGION unit cell allows more flexibility in the placement of the fuel, but it requires all regions of the cell to have the same geometric shape (i.e., slab, cylinder, sphere, buckled slab, or buckled cylinder). Lattice arrangements can be approximated by specifying a white or reflective boundary condition on the outer boundary. The white boundary should be used for curved outer boundaries (cylindrical of spherical) and the mirror boundary condition should be used for planar boundaries (slab). Limitations of the MULTIREGION cell treatment are listed below.

1. A MULTIREGION cell is limited to a 1D approximation of the system being represented. This constraint is appropriate for a sphere, an infinitely long cylinder, a slab, or an infinite array of slabs.

2. The shape of the outer boundary of the MULTIREGION cell is the same as the shape of the inner regions. Cells with curved outer surfaces cannot be stacked to represent arrays. However, arrays can be represented by making a curved outer region that is of equal volume to the cuboidal outer region and then specifying a white outer boundary condition.

3. The boundary conditions available in a MULTIREGION problem include vacuum (escape at the boundary), reflected (reflected about the normal to the surface at the point of impact), periodic (a particle exiting the surface effectively enters an identical cell having the same orientation and continues traveling in the same direction), and white (isotropic return about the point of impact). Reflected and periodic boundary conditions on a slab can represent real physical situations, but they are not valid on a curved outer surface. 
4. A MULTIREGION cell represents a single cell if the outer boundary has a vacuum boundary condition applied to it. A point flux spectrum is calculated over a set of intervals that make up the unit cell with the incoming flux on the boundary set to zero. This flux spectrum is then used to collapse a set of cross sections.

5. If the outer boundary of a MULTIREGION cell has a boundary condition other than vacuum, then the unit cell approximates an array. A point flux spectrum is calculated over a set of intervals that make up the unit cell with the incoming flux to the outgoing flux on the boundary. This flux spectrum is then used to weight a set of cross sections. For a slab having a periodic or reflected boundary condition, the incoming and outgoing fluxes are exact, but for a curved surface, the incoming flux is approximated by an isotropic return flux.

NOTE: The MULTIREGION cell treatment must be used to apply boundary conditions to your unit cell model. These boundary conditions are completely unrelated to the boundary condition of the outer surface of the KENO-VI model.

\subsubsection{Double-heterogeneous}

DOUBLEHET cells use a specialized XSProc calculational approach to treat resonance self-shielding in "doubly heterogeneous" systems. The fuel for these systems typically consists of small, heterogeneous, spherical fuel particles (grains) embedded in a moderator matrix to form a fuel compact. The fuelgrain/matrix compact constitutes the first level of heterogeneity. Cylindrical (rod), spherical (pebble), or slab fuel elements composed of the compact material are arranged in a moderating medium to form a regular or irregular lattice, producing the second level of heterogeneity. The fuel elements are also referred to as "macro cells." Advanced reactor fuel designs that use tri-structural isotropic (TRISO) or fully ceramic microencapsulated (FCM) fuel require the DOUBLEHET treatment to account for both levels of heterogeneities in the self-shielding calculations. Simply ignoring the double-heterogeneity by volume-weighting the fuel grains and matrix material into a homogenized compact mixture is likely to result in a large reactivity bias.

In the DOUBLEHET cell input, the keywords and the geometry description for grains are similar to those of the MULTIREGION treatment, while the keywords and the geometry for the fuel element (macro-cell) are similar to those of the LATTICECELL treatment. The following rules apply to the DOUBLEHET cell treatment and must be followed. Violation of any rules may cause a fatal error.

1. As many grain types as needed may be specified for each unique fuel element. Note that grain type is different from the number of grains of a certain type. For example, a fuel element that contains both $\mathrm{UO}_{2}$ and $\mathrm{PuO}_{2}$ grains has two grain types. The same fuel element may contain 10,000 $\mathrm{UO}_{2}$ grains and $5,000 \mathrm{PuO}_{2}$ grains. In this case, the number of grains of type $\mathrm{UO}_{2}$ is 10,000 , and the number of grains of type $\mathrm{PuO}_{2}$ is 5,000 .

2. As many fuel elements as needed may be specified, each of which requires its own DOUBLEHET cell. This may be the case for systems with many fuel elements at different fuel enrichments, burnable poisons, etc. Each fuel element may have one or more grain types.

3. Since the grains are homogenized into a new mixture to be used in the fuel element (macro-cell) cell calculation, a unique fuel mixture number must be entered. XSProc creates a new material with the new mixture number designated by the keyword fuelmix $=$, containing all the nuclides that are homogenized. The user must assign the new mixture number in the transport solver geometry (e.g., KENO) input unless a cell-weighted mixture is created. 
4. The type of lattice or array configuration for the fuel-element may be spheres on a triangular pitch (SPHTRIANGP), spheres on a square pitch (SPHSQUAREP), annular spheres on a triangular pitch (ASPHTRIANGP), annular spheres on a square pitch (ASPHSQUAREP), cylindrical rods on a triangular pitch (TRIANGPITCH), cylindrical rods on a square pitch (SQUAREPITCH), annular cylindrical rods on a triangular pitch (ATRIANGPITCH), annular cylindrical rods on a square pitch (ASQUAREPITCH), a symmetric slab (SYMMSLABCELL), or an asymmetric slab (ASYMSLABCELL).

5. If there is only one grain type for a fuel element, the user must enter either the pitch, the aggregate number of particles in the element, or the volume fraction for the grains. The code needs the pitch and will directly use it if entered. If pitch is not given, then the volume fraction (if given) is used to calculate the pitch. If neither the pitch nor the volume fraction is given, then the number of particles is used to calculate the pitch and the volume fraction. The user should only enter one of these items.

If the fuel matrix contains more than one grain type, all types are homogenized into a single mixture for the compact. As for the case of one grain type, the pitch is needed for the spherical cell calculations. However, the pitch by itself is not sufficient to perform the homogenization. Since each grain's volume is known (grain dimensions must always be entered), entering the number of particles for each grain type essentially provides the total volume of each grain type and therefore enables the calculation of the volume fraction and the pitch. Likewise, entering the volume fraction for each grain type essentially provides the total volume of each grain type and therefore enables the calculation of the number of particles and the pitch. Therefore, one of these two quantities must be entered for multiple grain types. In these cases, since pitch is not given, the available matrix material is distributed around the grains of each grain type proportional to the grain volume and is used to calculate the corresponding pitch. Over-specification is allowed as long as the values are not inconsistent to greater than $0.01 \%$.

6. For cylindrical rods and for slabs, fuel height must also be specified. For slabs, the slab width must also be specified.

7. The CENTRM calculation option must be Sn.

\subsubsection{Unit Cell Boundary Conditions}

For the MULTIREGION unit cell and the element level of the DOUBLEHET unit cell, there are four possible boundary conditions. The default for the left or inner boundary is reflected (required for a cylinder or sphere), and the default for the right or outer boundary is vacuum.

\subsubsection{Vacuum Boundary Condition}

A vacuum boundary condition means that no neutrons will re-enter the boundary. Thus, any neutron exiting the system through a vacuum boundary is permanently lost to the system. This condition is shown in Figure 159. (NOTE: In the following figures, a dashed arrow indicates neutrons leaving the system, whereas a solid arrow represents neutrons returning to the system. The length of the arrow is proportional to the number of neutrons, so longer arrows represent more neutrons than shorter arrows.) 


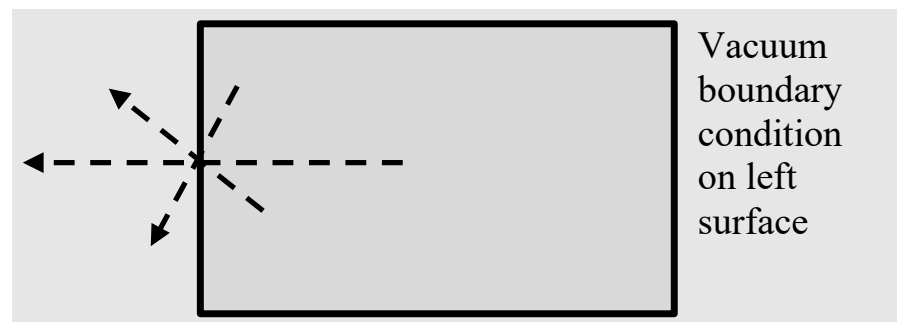

Figure 159. Vacuum boundary condition.

\subsubsection{Reflective Boundary Condition}

For the reflective boundary condition, the incoming angular flux is set equal to the outgoing angular flux in the direction corresponding to mirror or specular reflection. The reflective boundary should not be used on curved surfaces, that is, the outer boundary of a cylinder or sphere. It is the default (and required) for the left (inner) boundary of a cylindrical or spherical system. As shown in Figure 160, a given quantity of neutrons leaving a boundary (dotted line) in a particular direction will be returned (solid line) to the system with the same quantity but at a mirrored angle to the initial leakage direction.

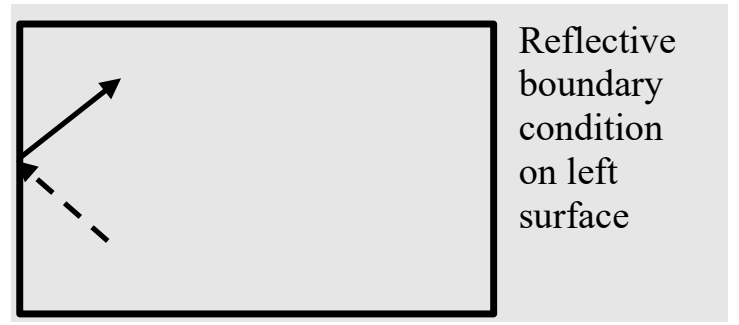

Figure 160. Reflective boundary condition.

\subsubsection{Periodic Boundary Condition}

For the periodic boundary condition, the incoming angular flux on a boundary is set equal to the outgoing angular flux on the opposite boundary. Figure 161 shows the leakage leaving each boundary (dotted lines) being returned at the same quantity and angle on the opposite boundary (solid line of same color). When the periodic boundary condition is used, it must be applied to both opposing boundaries. It should not be used on curved surfaces such as the curved boundary of a cylinder or a sphere. It should also be noted that SCALE only supports periodic boundaries on parallel planes; rotationally periodic boundary conditions are not supported.

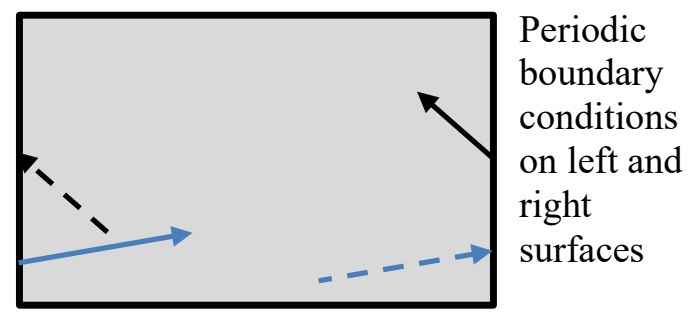

Figure 161. Periodic boundary condition. 


\subsubsection{White Boundary Condition}

For the white boundary condition, the incoming angular fluxes are each set equal to a single value chosen such that the net flow across the boundary is zero. The white boundary provides isotropic return (solid lines) at a boundary (see Figure 162) and is suitable for all geometries. Although the white boundary condition is in a sense nonphysical on a curved surface, it is the best possible approximation of a reflective or repeating boundary condition, such as a lattice cell.

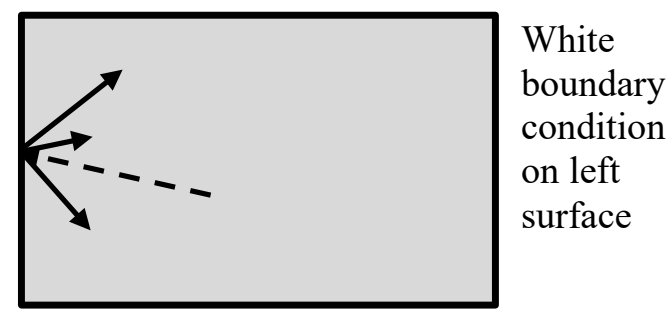

Figure 162. White boundary condition.

\subsection{UNIT CELL SPECIFICATIONS}

Unit cell data are not required for INFHOMMEDIUM. With the LATTICECELL, MULTIREGION, and DOUBLEHET treatments, you must specify the unit cell dimensions and materials to be used in the resonance self-shielding process. The unit cell is always 1D: infinitely long cylinders, infinite slabs, or spheres. Finite cylinders or slabs can be approximated with geometric buckling in MULTIREGION and DOUBLEHET cells. The configuration of the unit cell depends on the treatment and the type of lattice (if the LATTICELL treatment is used).

\subsubsection{Infinite Homogeneous Medium}

No input is required for a mixture to be treated as an infinite homogenous medium. These "cells" can be specified in the input with a single mixture number as the infinite medium to process.

\subsubsection{Latticecell}

For the LATTICECELL treatment, both "regular" and "annular" cells are allowed. These cells are rigorously constrained as to the placement of fuel, gap, clad, and moderators. Materials not used in the cell or other defined cells are treated as infinite homogeneous media. The "regular" cells allow spherical, cylindrical, or symmetric slab fuel regions that are constrained to a central fuel region surrounded by an optional gap, an optional clad, and an external moderator material. The "annular" cells for spherical, cylindrical, or asymmetric slab configurations are constrained to a central (second) moderator material surrounded by a fuel region having an optional gap and optional clad on both sides of the fuel with an external (first) moderator material.

The unit cell data in a LATTICECELL problem are used (1) to provide the dimensions and shape of the lump and the moderator material for resonance cross section processing, (2) to provide lattice corrections for the cross section processing, and (3) to provide information used in creating cell-weighted cross sections when XSDRNPM is executed. CELLMIX= must be specified in the unit cell data if cellweighted cross sections are used in the problem, although the use of cell-weighted cross sections is not recommended. 


\subsubsection{Multiregion}

The unit cell is more flexible for the MULTIREGION treatment than for the LATTICECELL treatment. In fact, the MULTIREGION treatment allows complete freedom in the placement of materials, but it is constrained by shape, as concentric regions of the same shape are required. The MULTIREGION treatment does not account for lattice effects, so it is best used for problems in which lattice effects are not important. To some degree, lattice effects can be approximated with a reflective, periodic, or white boundary condition; these boundary conditions effectively create an infinite array of the defined MULTIREGION cell.

\subsubsection{Double-heterogeneous}

The DOUBLEHET cell type allows for the description of a range of different fuel types that contain grains of fissile material with coatings, subsequently pressed into pebbles or compacts. The grains are assumed to be spherical, but there is no restriction on the number of coating layers surrounding the grain kernel. The input for the compact level is very similar to the MULTIREGION cell, including the spherical, cylindrical, and slab geometries.

\subsubsection{Cell-Weighted Cross Sections}

Cell-weighted cross sections are created by XSDRNPM when CELLMIX= is specified in the unit cell data. This option is not encouraged, but it is maintained for backwards compatibility; the user must ensure that the homogenized unit cell is identical to the real geometry. The unit cell description is then used by XSDRNPM to calculate the eigenvalue of the cell. For LATTICECELL problems, a white boundary condition is assumed. For MULTIREGION problems, the boundary conditions specified in the unit cell are used. The resultant fluxes are used to weight the cross sections of the materials in the cell and create homogenized cell-weighted cross sections that have the characteristics of the heterogeneous cell configuration. Whenever XSDRNPM is executed for cross section processing, only the cell-weighted mixture number can be used in the KENO-VI geometry data. The original mixtures used in the unit cell description cannot be used. For more information on cell-weighting refer to [Cell weighting of MG cross sections] in the Material Specification and Cross Section Processing section of the SCALE manual.

\subsection{PROBLEM DESCRIPTIONS}

Three example problems are used to demonstrate how to enter unit cell data for LATTICECELL, MULTIREGION, and DOUBLEHET unit cell types using Fulcrum. The first problem is a $9 \times 9$ fuel assembly array of $\mathrm{UO}_{2}$ fuel rods with Zircaloy-2 cladding. The second problem involves two parallel, fully reflected SS304 slab tanks containing $\mathrm{U}(93) \mathrm{O}_{2} \mathrm{~F}_{2}$ solution. The third problem is based on an Organization for Economic Co-operation and Development/Nuclear Energy Agency (OECD/NEA) pebble bed reactor benchmark problem.

\subsubsection{Lattice Cell Example: Fuel Assembly}

This problem is a single fuel assembly at $3.5 \mathrm{wt} . \%$ enrichment stored in a pool of water at a temperature of $293 \mathrm{~K}$. There is $15.24 \mathrm{~cm}$ of water above and below the assembly and $7.62 \mathrm{~cm}$ of water on each side of the assembly. The space between fuel rods is full-density water. The guide tubes are made of Zircaloy-2 and have an inner radius of $0.5 \mathrm{~cm}$, a thickness of $0.075 \mathrm{~cm}$, and a length of $387.26 \mathrm{~cm}$. The pitch of the fuel rods and guide tubes is $1.64 \mathrm{~cm}$. The fuel assembly radial layout is provided in Figure 163. 

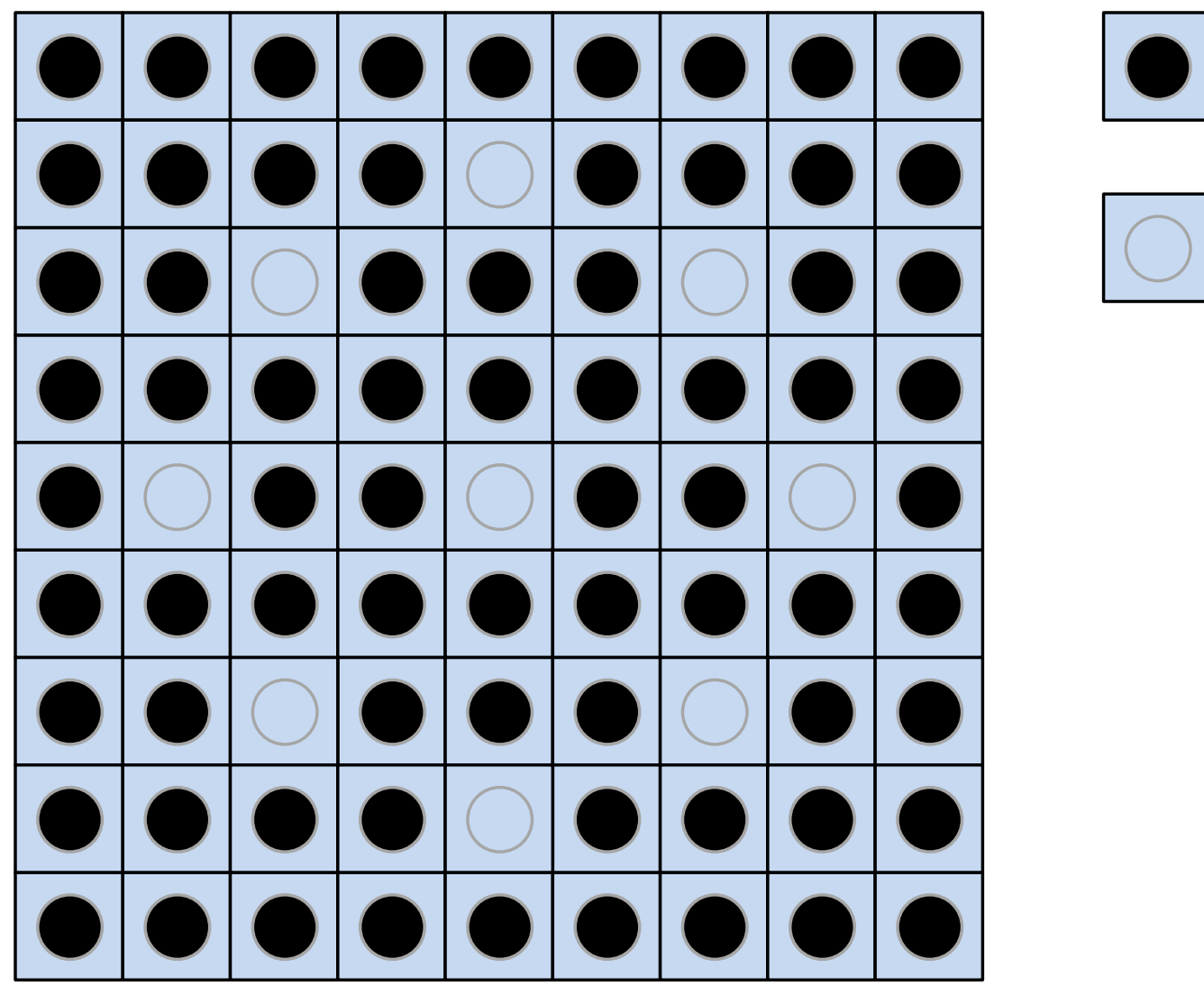

Fuel rod

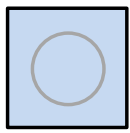

Guide tube

Figure 163. Fuel assembly radial layout.

The fuel pellets have a $0.47 \mathrm{~cm}$ radius and contain $\mathrm{UO}_{2}$ at $95 \%$ of theoretical density. The active fuel length is $365.76 \mathrm{~cm}$. Each fuel rod is made of Zircaloy- 2 with an inner radius of $0.4875 \mathrm{~cm}$ and an outer radius of $0.545 \mathrm{~cm}$. The cladding extends $7.0 \mathrm{~cm}$ above the top of the active fuel, and the ends are capped with solid Zircaloy-2 plugs that have the same outer diameter as the cladding. The top plug is $10.5 \mathrm{~cm}$ long, and the bottom plug is $4.0 \mathrm{~cm}$ long. The inner volume of the cladding tube is modeled containing void. The axial configuration of the fuel rods is shown in Figure 164. 


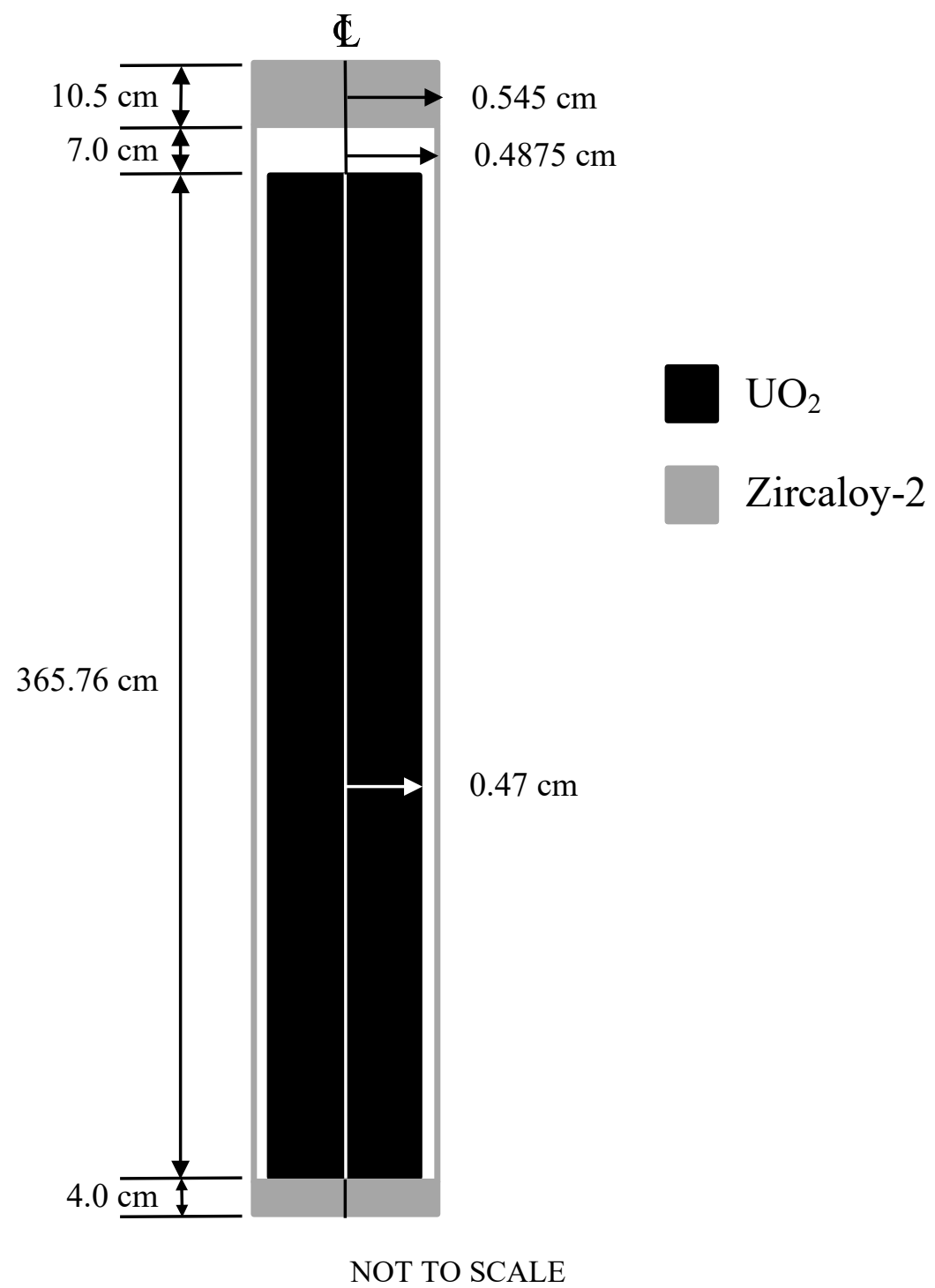

Figure 164. Axial depiction of the fuel rods.

\subsubsection{General and Material Data}

The initial data entry for this example is the same as previous problems. Start Fulcrum and then select the New file... option from the File menu. Select a directory in which to save the model, and name it fuel_assembly.inp. Next, use the autocomplete feature in Fulcrum to create a csas6 - Criticality safety analysis using KENO-VI input. Replace title-goes-here with Unit Cell - Fuel As sembly. Now change xs-lib-goes-here to $\mathrm{v} 7.1-252$ ( $77.1-252 \mathrm{n}$ if using SCALE 6.2, 6.2.1, or 6.2.2). This selects the 252-group neutron cross section library based on ENDF/B-VII.1, and it signals to CSAS6 that this will be a MG KENO calculation. The appropriate cross section processing modules will be called using the input provided in the CELLDATA block, as discussed below in Section 8.5.1.2. The first three lines of the input are shown in Figure 165. 


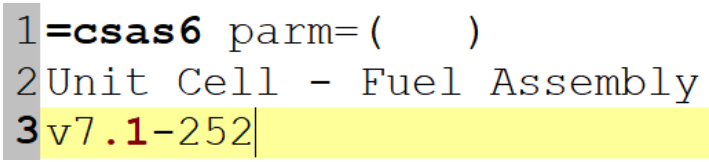

Figure 165. General information for fuel assembly problem.

Next, define the different mixtures needed for the model: $\mathrm{UO}_{2}$, Zircaloy-2, and water. All three materials are available in the SCALE standard composition library, making the definitions much simpler and faster to provide.

First, define the $\mathrm{UO}_{2}$ composition. Move the cursor into the read comp block, and then autocomplete a stdcomp - basic (configurable). This will generate a fillable form as shown in Figure 166. In the composition dropdown menu, select uo2 by opening the dropdown menu and typing uo2. Change the Volume Fraction to 0.95 to set the density multiplier to $95 \%$ of theoretical density. Next, check the box for Isotopic Weight Percents and click the Add row button under the isotopic weight percent pane. Select ${ }^{235} \mathrm{U}$ with its nuclide ID of $\mathbf{9 2 2 3 5}$ and set the Weight Percent to 3.5 . To add ${ }^{238} \mathrm{U}$, click either the Add row button again, or click the + button next to 92235. Set the Weight Percent to 96. 5. The final configurable window is shown in Figure 167. Confirm that the data entry is correct before clicking $\mathbf{O K}$, as the data cannot be modified in the configurable form after it has been entered into the input.

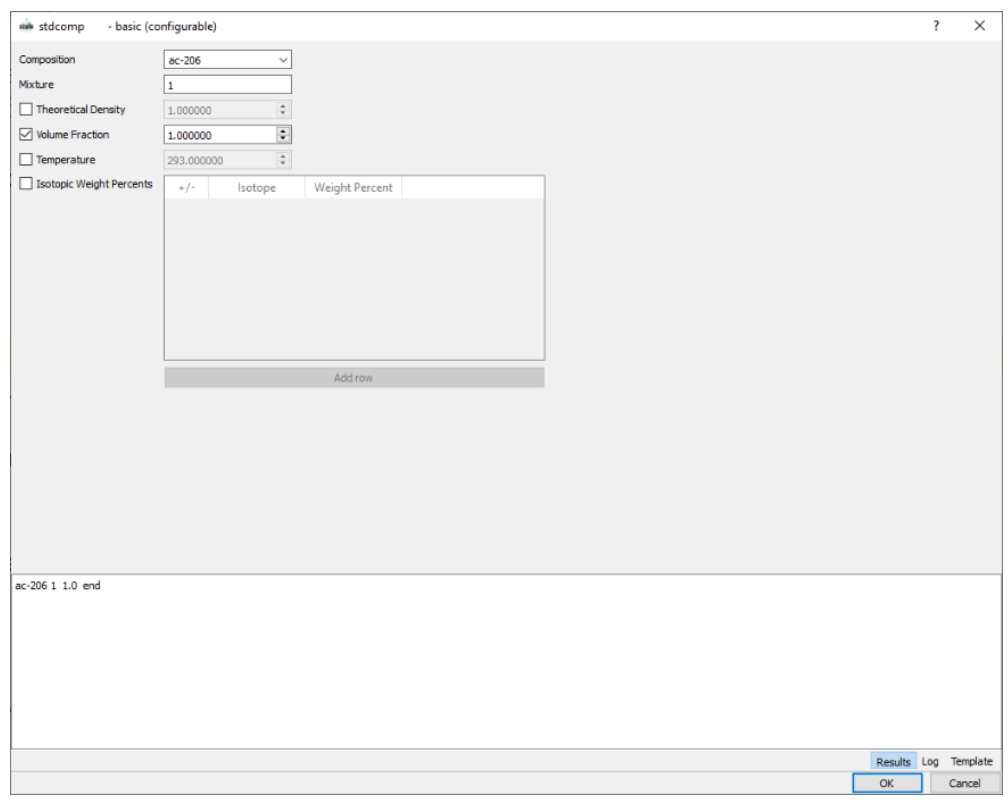

Figure 166. Initial STDCOMP configurable form. 


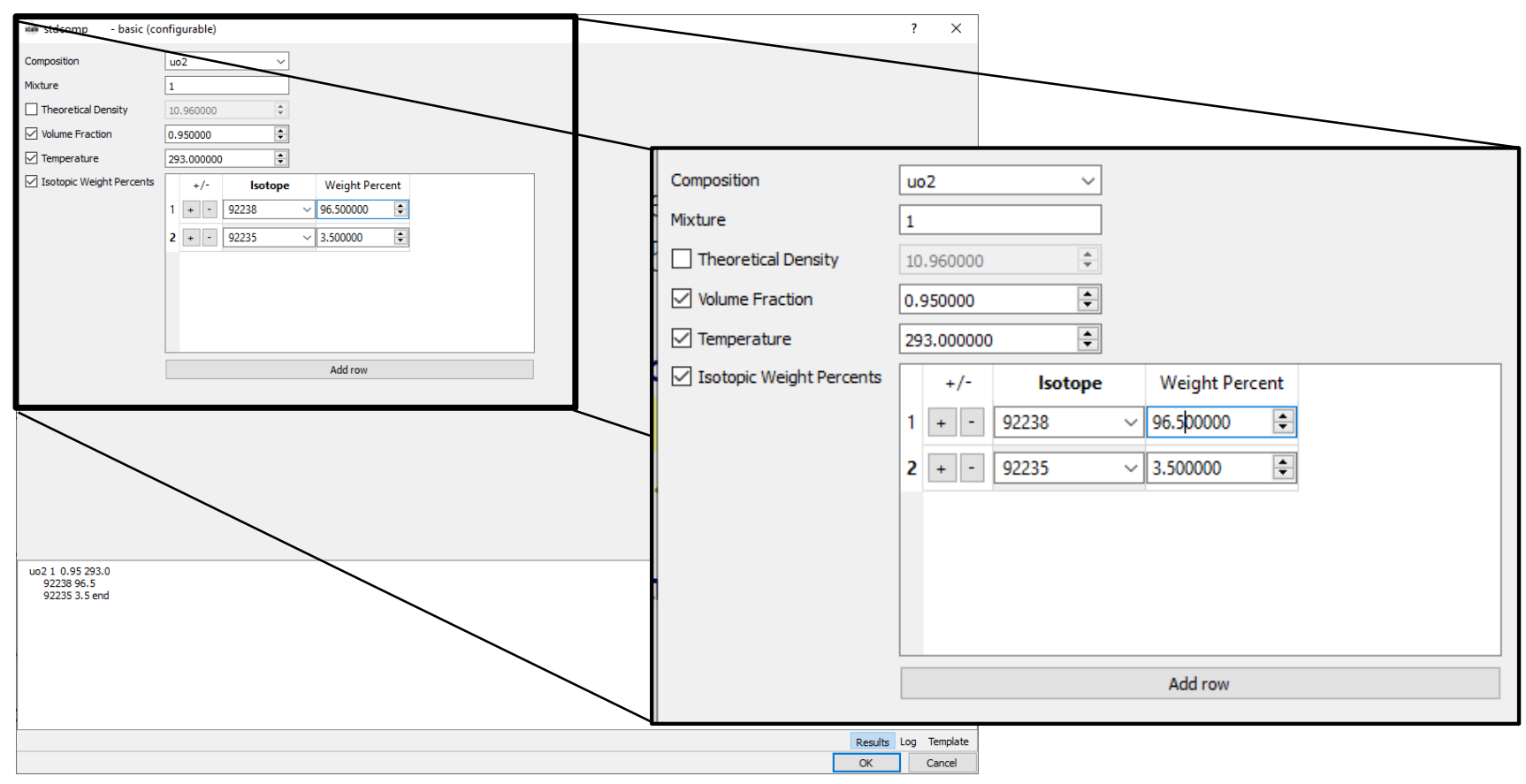

Figure 167. Complete $\mathrm{UO}_{2}$ configurable form, showing an enlarged section of the user-specified input.

Next, repeat this process to generate the Zircaloy-2 composition. Autocomplete a stdcomp - basic (configurable) and select zirc2 from the composition dropdown menu. Change the mixture number to 2 ; remember that if multiple entries are assigned to a single mixture number, then SCALE will create a mixture containing all of these materials. This can be detected by a lack of available mixtures later in the geom block or by checking the mixing table for total mixture density or unexpected isotopes in a specific mixture. No further modifications are needed to the default Zircaloy-2 composition, so the mixture can be added by clicking the $\mathbf{O K}$ button on the configurable form. The user-specified input section of the form is shown in Figure 168.

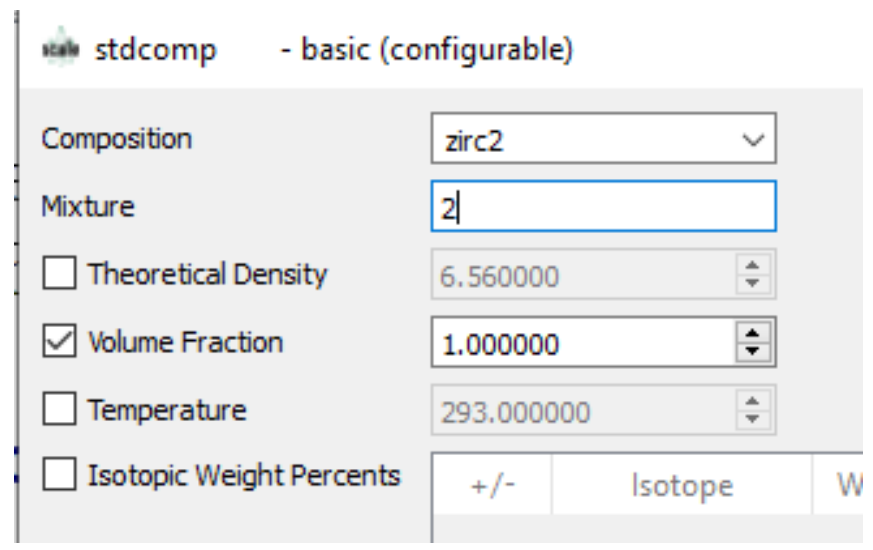

Figure 168. User-specified input portion of the complete zirc2 configurable form.

The water composition is the final mixture to create, and it is also not modified from the defaults provided in the SCALE standard composition library. In many cases such as this, specifying the composition by entering the text directly into Fulcrum is likely faster than using a configurable. In this case, simply type h2o 3 end inside the composition block. This specifies mixture 3 as containing water at the default density $\left(0.9982 \mathrm{~g} / \mathrm{cm}^{3}\right)$ and the default temperature $(293 \mathrm{~K})$. 
The comment TODO: define comp can be removed, as the compositions have been defined. The comment can also be left in the input, as it has no impact on the execution of SCALE. The completed composition block is shown in Figure 169. Note that additional copies of some mixtures should be created in the composition block if needed for multiple unit cell definitions in the CELLDATA block. As discussed in Section 8.5.1.2 below, only a single unit cell is needed for this problem, and correspondingly, no copies of mixtures are needed.

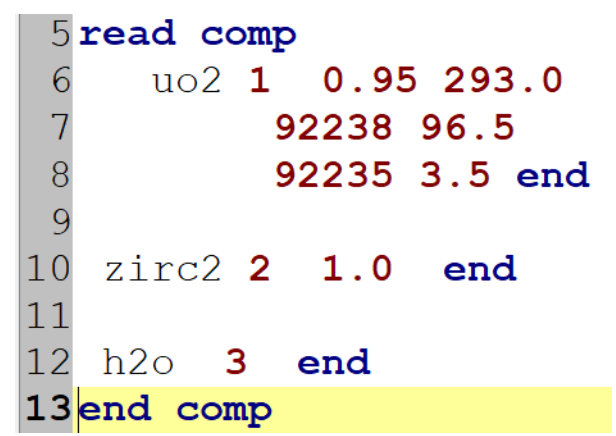

Figure 169. Complete COMPOSITION block for the fuel assembly problem.

\subsubsection{Unit Cell Data}

Now that the material information has been entered, the unit cell information can be specified for cross section treatment. One of the four unit cell types (INFHOMMEDIUM, LATTICECELL, MULTIREGION, DOUBLEHET) will be specified to process the MG cross sections for this problem. An ordered array of moderated fuel pins is well described by a LATTICECELL unit cell, and this will be the option used in this model. The specific array option that best describes the problem is SQUAREPITCH based on the cylindrical rods in a square pitched array. The 3D effects of the plenum space above the fissile material and of the end plugs will be neglected because the unit cell specifications are all 1D cells.

Move the cursor to a line between the composition and geometry blocks and autocomplete to generate a list of other blocks to be entered, as shown in Figure 170. Select cells to instruct Fulcrum to enter a CELLDATA block. There are no configurable forms currently available in the CELLDATA block. Autocompleting in the CELLDATA block provides a list of available unit cell specifications; select the lattice - squarepitch option, as shown in Figure 171. Fulcrum inserts a single line of CELLDATA input, as shown in Figure 172, which must be modified for use in this model.

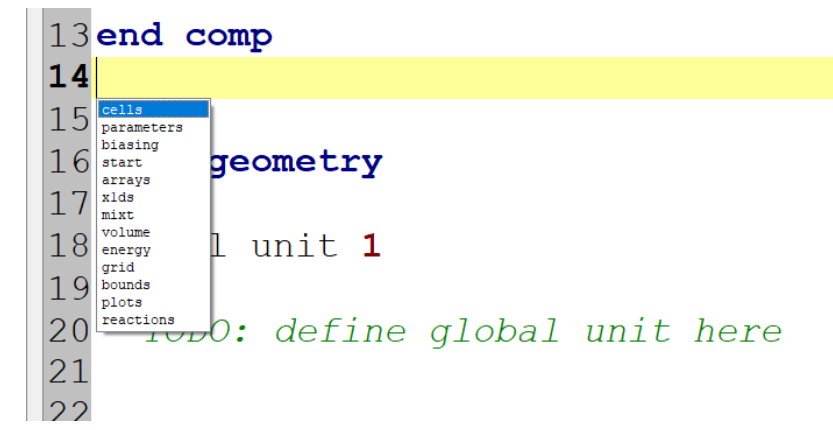

Figure 170. Autocomplete list of additional blocks available for inclusion in the model. 


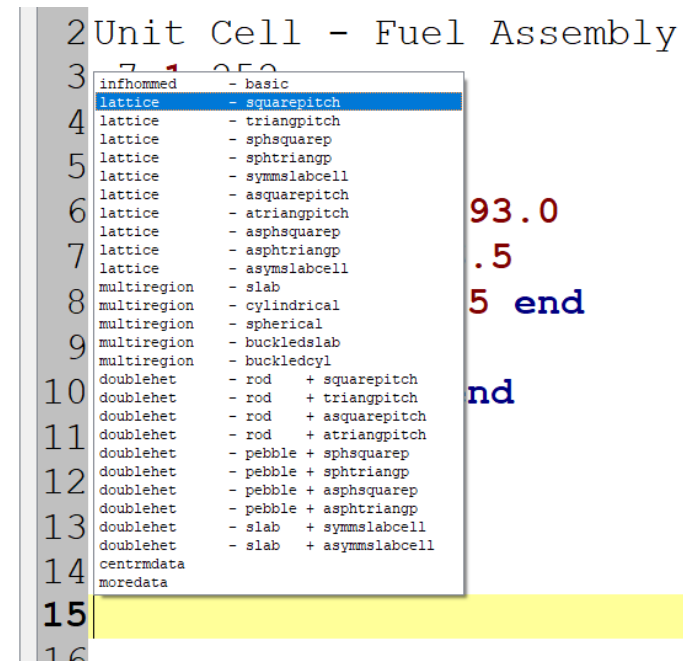

Figure 171. Autocomplete list of cells available within the CELLDATA block.

14 read celldata

15 latticecell squarepitch hpitch=0.0 1 fuelr=0.0 2 gapr=0.0 3 cladr=0.0 4 end

Figure 172. Templated input for LATTICECELL SQUAREPITCH provided by Fulcrum.

The first entry in the LATTICECELL SQUAREPITCH input is hpitch=0.0. This input specifies the half pitch for the unit cell; the full pitch is the spacing between fuel rods, and it can be provided in SCALE by changing the keyword from hpitch to pitch. In this case, the pitch is $1.64 \mathrm{~cm}$, so the half pitch is 0.82 . The single integer provided after the dimension of the half pitch, 1 in the template input, is the mixture number for the material filling the space between the fuel rods. In this model, that is water as specified in the COMPOSITION block as mixture 3. Similarly, fuelr is the radius of the fuel material (fueld is the keyword for the diameter), and the mixture number for the fuel is provided. Replace the fuel radius input with $0.47 \mathrm{~cm}$ and the fuel mixture with 1 . The gap between the fuel and the cladding is modeled as a void, which is always mixture 0 in SCALE. The gapr is equal to the inner radius of the cladding (gapd for the inner diameter), $0.4875 \mathrm{~cm}$. Finally, the cladr is the outer radius of the cladding (cladd for the outer diameter). The relevant dimension is $0.545 \mathrm{~cm}$, and the mixture number for Zircaloy-2 in this model is 2 . This completes the unit cell entry, and since it is the only unit cell specified in this model, it completes the CELLDATA block. The complete entry/block is provided in Figure 173; note that the entry is allowed to span multiple lines and is not complete until end is specified for the cell specification. White space also has no impact in the input, so the input shown is split on to two lines to narrow the figure, and white space is added to align the entries for user convenience.

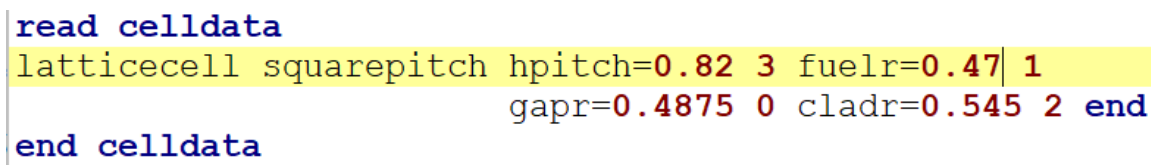

Figure 173. Complete LATTICECELL input.

\subsubsection{KENO-VI Geometry Data}

In this problem, the following three units are needed: Unit 1 will be a fuel rod cell; Unit 2 will be a guide tube cell, and Unit 3 will be the global unit containing the array of rods and the surrounding water. 
The fuel rod unit cell, Unit 1, is the first unit to be defined. The outer boundary of the unit must be a cuboid so that Unit 1 can be included in the array. The regions to be entered are the fuel cylinder, the gap cylinder, the cladding cylinder, and finally the water cuboid. In KENO-VI, there is no requirement for a particular order for the regions in a unit; this is merely a logical progression. This is a significant difference from KENO V.a. The first step in defining Unit 1 is to delete global from the unit declaration. Configurable forms are included for all the shapes available in KENO-VI. To add the cylinder, position the cursor between the unit declaration and the boundary record. Autocomplete in this context provides a list of shapes and their configurable forms and select cylinder - kenovi (configurable), as shown in Figure 174. The default configurable form is shown in Figure 175.

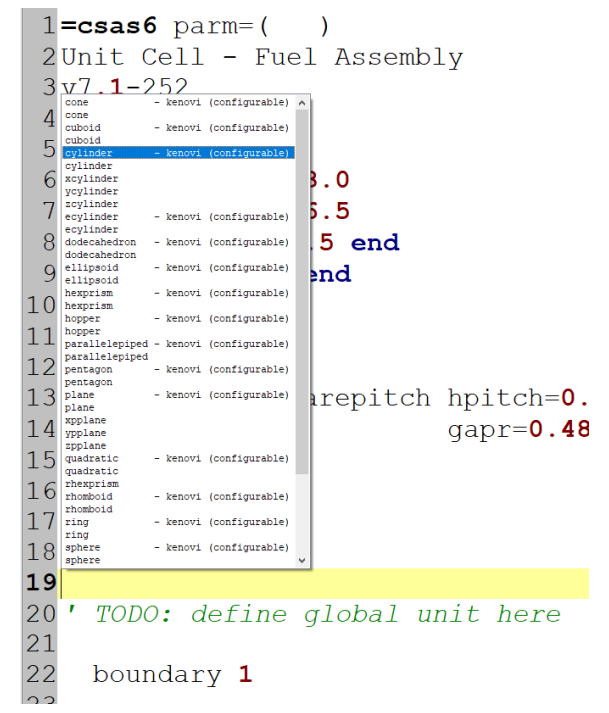

Figure 174. Selecting a cylinder configurable form.

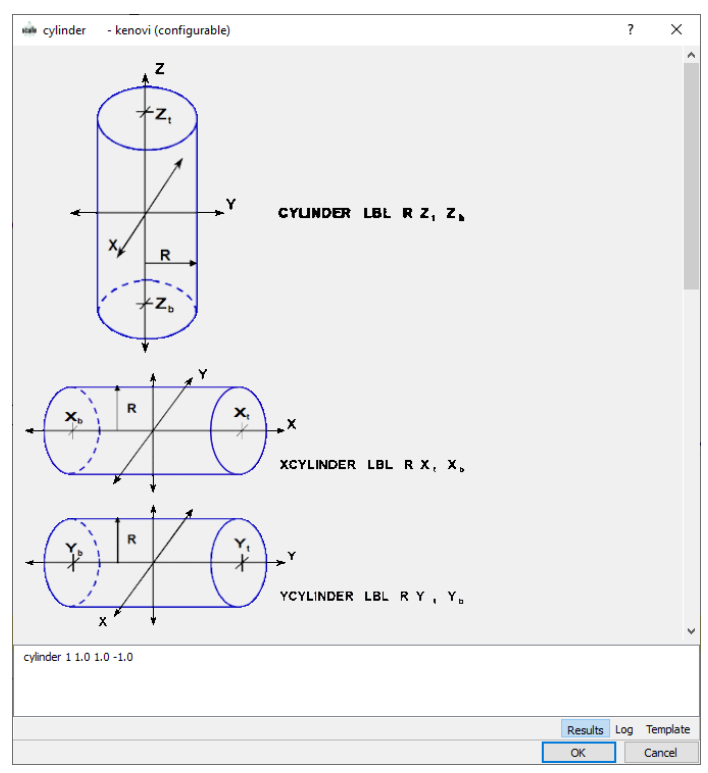

Figure 175. Default cylinder configurable form.

Completing the configurable form will provide the input for a single cylindrical region in Unit 1 . The first dropdown menu (Type) is available because by default, a cylinder in SCALE is aligned parallel to the Zaxis; cylinders parallel to the $\mathrm{X}$ - or $\mathrm{Y}$-axis can also be generated using this form. The input for the second 
dropdown menu is the ID for this region; set this to 10 . The next three fields specify the dimensions of the cylinder; the radius is $0.47 \mathrm{~cm}$. The height of the fissile column is $365.76 \mathrm{~cm}$, but the actual $+\mathrm{Z}$ and $-Z$ entries depend on the selection of the origin in the unit. For this model, the origin will be placed at the bottom of the fissile column, so the $+Z$ entry is $365.76 \mathrm{~cm}$ and the $-Z$ entry is $0 \mathrm{~cm}$. No origin input is needed, as the unit will be built with the centerline of the fuel rod passing through the origin. The complete user-specified input for the cylinder is provided in Figure 176. Press OK to add the cylinder region to the input after checking that the inputs are correct.

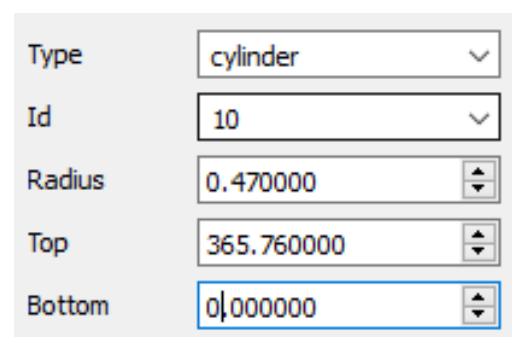

Figure 176. User-specified portion of the completed cylinder configurable form.

The next region in unit 1 is the void inside the cladding and outside the fissile column. The easiest way to complete this portion is to copy and paste the existing entry and change the dimensions. Assign this second cylinder an ID of 20; it has a radius of $0.4875 \mathrm{~cm}, \mathrm{a}+Z$ surface of $372.76 \mathrm{~cm}$, and it has a $-Z$ surface of $0 \mathrm{~cm}$.

The cladding cylinder can also be added by copying the previous cylinder and pasting it into the unit. The ID number for the Zircaloy- 2 cladding should be 30 , and the radius is $0.545 \mathrm{~cm}$. The $+Z$ surface for the void cylinder is 372.76 , and the end plug length is $10.5 \mathrm{~cm}$. Enter $372.76+10.5$, highlight the expression as shown in Figure 177, and evaluate the expression by selecting the Edit dropdown menu and clicking Evaluate. Enter the $-Z$ surface as $-4.0 \mathrm{~cm}$ to represent the lower end plug. The completed input for the three cylinders is shown in Figure 178.

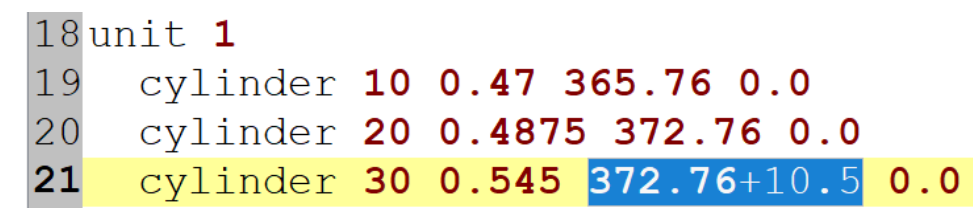

Figure 177. Highlighting expression for evaluation in Fulcrum.

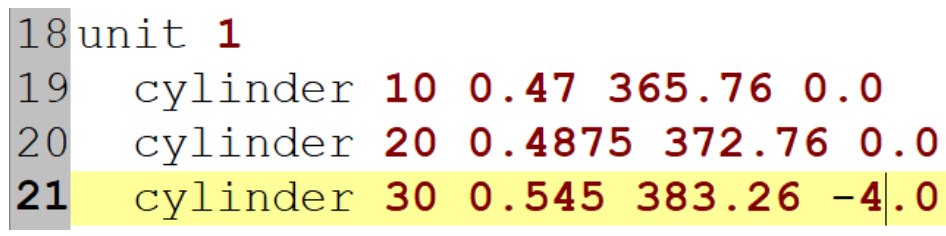

Figure 178. Final input for the three cylinders in Unit 1.

The last region required in Unit 1 is the cuboid to define the unit cell for use in the array. The $\mathrm{X}$ and $\mathrm{Y}$ dimensions will be equal to plus and minus the half pitch of the array. This will ensure that the appropriate spacing is generated when two unit cells are arrayed together. The $\mathrm{Z}$ extent must be at least that of the fuel rod, but it could include the water above and below the assembly. In this case, the water will be added in the global unit. The cuboid can be added with a configurable form; place the cursor anywhere between the unit $\mathbf{1}$ declaration and the boundary record for the unit, and then autocomplete 
and select cuboid - kenovi (configurable). Change the ID to 40 , and then set the $+\mathbf{x}$ and $+\mathbf{y}$ values to 0.82 and the $-\mathbf{x}$ and $\mathbf{- y}$ values to -0.82 . The $+\mathbf{z}$ face is 383.26 and the $\mathbf{- z}$ face is -4.0 . The completed configurable form is shown in Figure 179; after confirming that the input is correct, press $\mathbf{O K}$ to add the cuboid definition to the input. The complete set of 4 region inputs is shown in Figure 180.

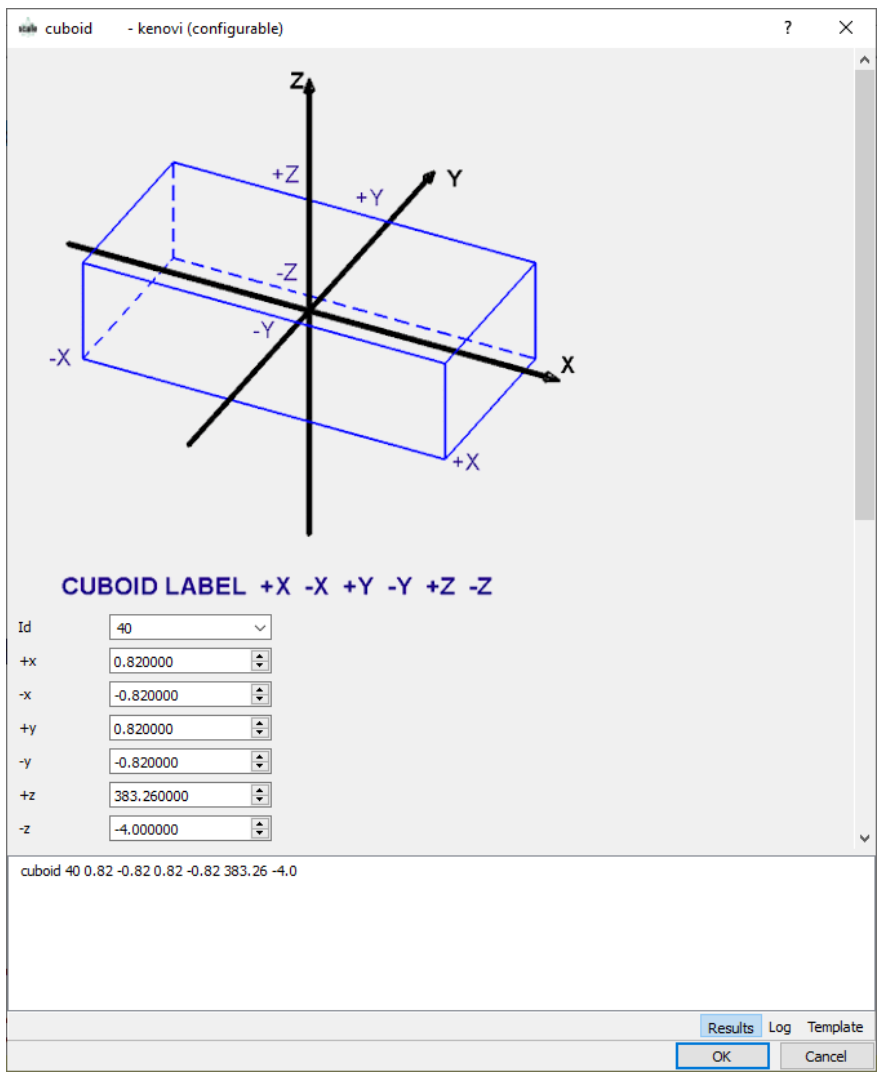

Figure 179. Completed configurable form for the cuboid in Unit 1.

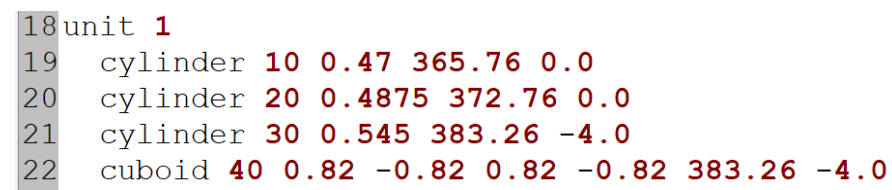

Figure 180. Input for all four regions in Unit 1.

All of the regions needed in Unit 1 have been entered; at this point, media records can be specified to fill the regions with the appropriate mixtures. Autocomplete can be used to select media - kenovi

(configurable), as shown in Figure 181, to generate a configurable form for media entry. The first entry will be the fissile material, which is Mixture 1 and needs to be placed inside cylinder 10. By default, mixture 1 is filled in the Material field; click the Add row button at the bottom of the Regions pane to add a line to the pane for use. Change the Id to 10, and leave the State dropdown menu set to inside. As shown in the Results pane, this will generate a MEDIA record placing Mixture 1 inside region 10. After confirming that the configurable form is correct, as shown in Figure 182, press the OK button to add the MEDIA record to the input. The next MEDIA record will be used to fill the gap space inside cylinder 20 but outside cylinder 10 with void. Generate another MEDIA configurable form and change the Material to 0 . Click the Add row button and set the Id to 20 with a state of inside; add another row and set the Id to 10 and the state to outside. The completed MEDIA configurable form is shown in Figure 183; after 
confirming that the input is correct, press the OK button to add the MEDIA record to the input. The third MEDIA record will place Zircaloy-2 in the cladding region. The fastest way to add this record is to copy the previous MEDIA record and paste it into Unit 1. Change the mixture number from 0 to 2, and then set the region definition vector to $30-20$ (inside region 30 and outside region 20). The last MEDIA record fills cuboid 40 with water. Again, copy and paste a previous MEDIA record, and then change the mixture number to 3 and the region definition vector to $40-30$ (inside region 40 and outside region 30). Recall that there is no requirement to enter the MEDIA records in any order relative to each other or relative to the region definitions. All four MEDIA records are shown in Figure 184.

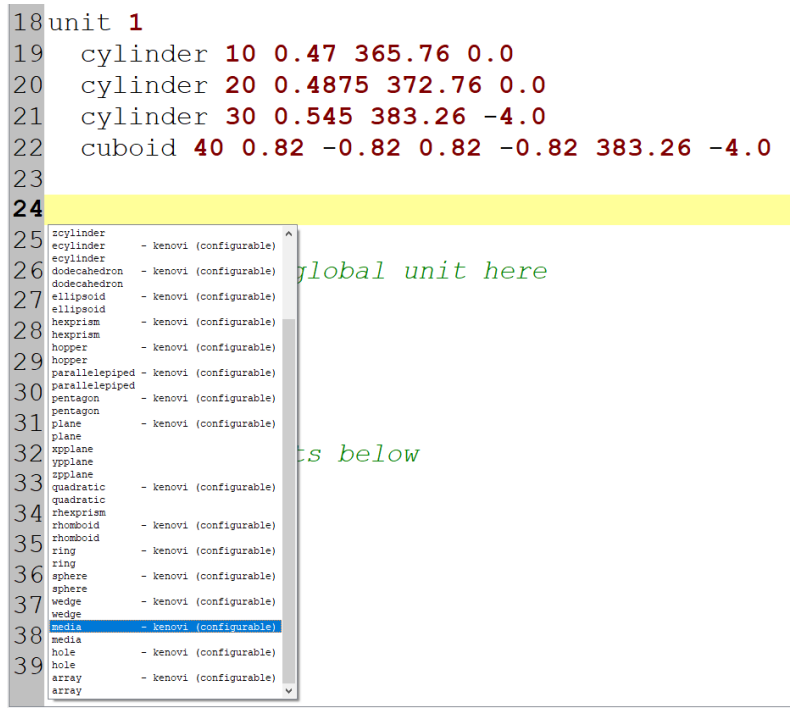

Figure 181. Selecting the MEDIA configurable form in Fulcrum.

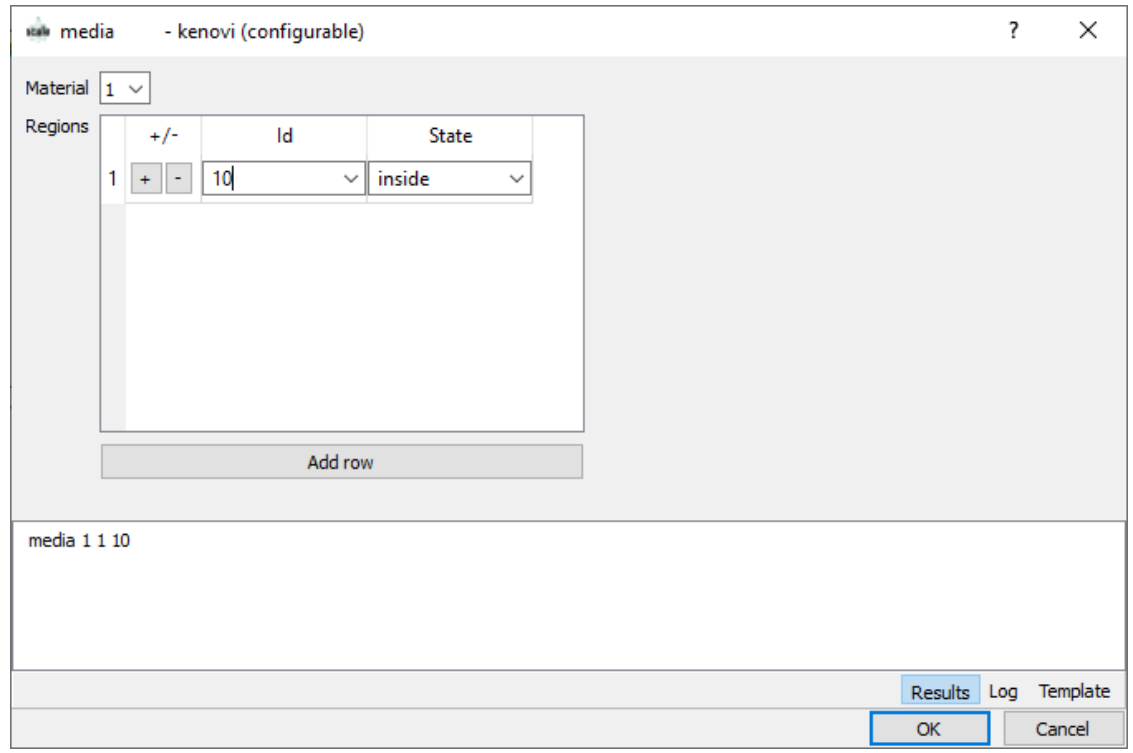

Figure 182. Completed MEDIA configurable form placing $\mathrm{UO}_{2}$ in cylinder 10 in Unit 1. 


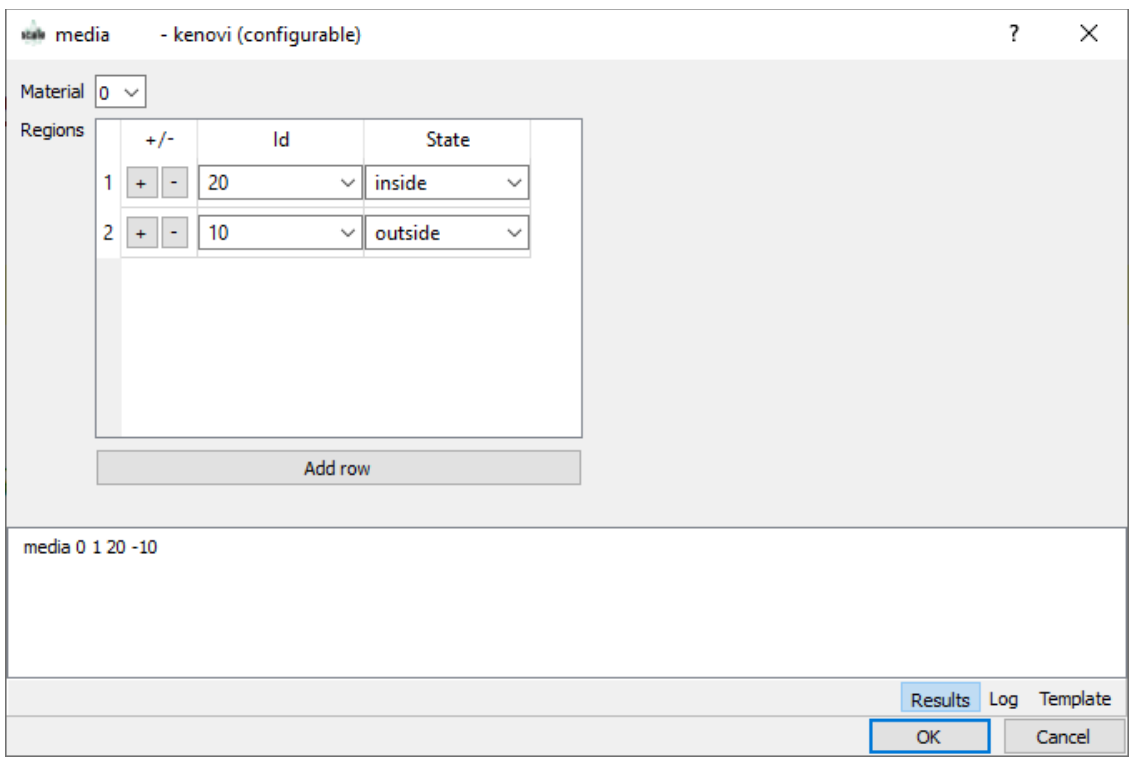

Figure 183. Completed MEDIA configurable form placing void in cylinder 20 and outside cylinder 10 in Unit 1.

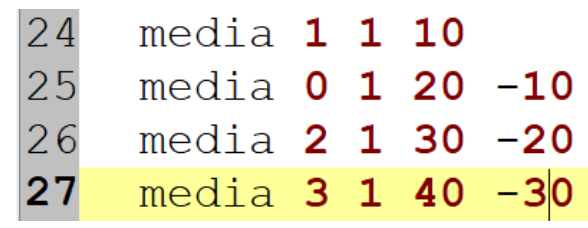

Figure 184. Input for all four media records un Unit 1.

The last step to complete the definition of Unit 1 is to update the boundary record. The default record generated by Fulcrum is for the boundary to contain all of space inside surface 1, but there is no surface 1 in this unit. The outermost region in Unit 1 is cuboid 40, which contains the water outside the fuel rod. Change the region definition vector for the boundary record to 40 (all of space inside region 40 is part of Unit 1). The complete input for Unit 1 is shown in Figure 185; most comments and blank lines have been removed to clarify the figure. Neither comments nor blank lines have any effect on SCALE input processing.

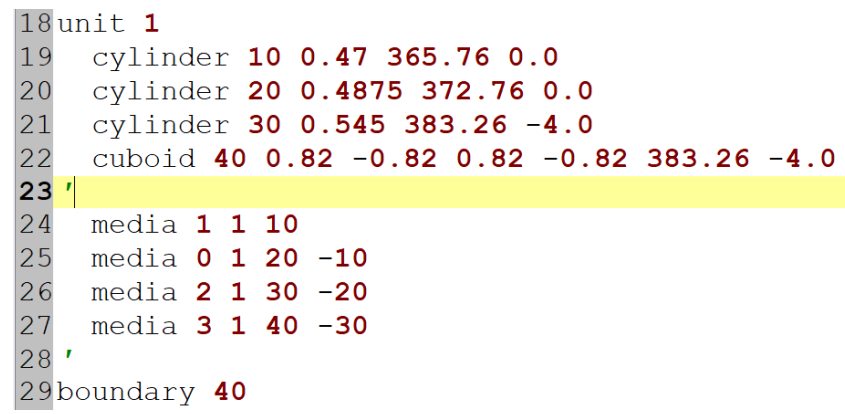

Figure 185. Complete input for Unit 1.

The second unit needed for this problem is the guide tube unit. The outer boundary again must be a cuboid so that this unit can be used in the array, and the cuboid must be the same size as the Unit 1 cuboidal outer surface so that the matching faces are the same size in all cases. This unit only requires two 
cylinders for the water inside the guide tube and for the guide tube itself. The outer cuboid will be the third and final region in Unit 2.

Place the cursor after the boundary record for Unit 1 and enter unit 2 . Use autocomplete to generate a configurable form for the first cylinder; set the Id to 10 and the Radius to 0.5 , the top to 387.26 , and the bottom to 0 . The completed input form is shown in Figure 186; after confirming that the input is correct, press $\mathbf{O K}$ to add the cylinder specification to the input. The second cylinder can also be added with a configurable form; set the Id to 20, the Radius to 0.575 , and the top and bottom are again 387.26 and 0, respectively. The user-specified input for this region is shown in Figure 187; after confirming that the input is correct, press OK to add the cylinder specification to the input. The final surface needed in Unit 2 is the cuboid for water outside of the guide tube. This surface (cuboid 40) can be copied from Unit 1 and pasted in Unit 2; the $\pm \mathrm{z}$ dimensions must be changed to 387.26 and 0 , respectively, after being pasted into Unit 2 . The complete set of three region inputs is shown in Figure 188.

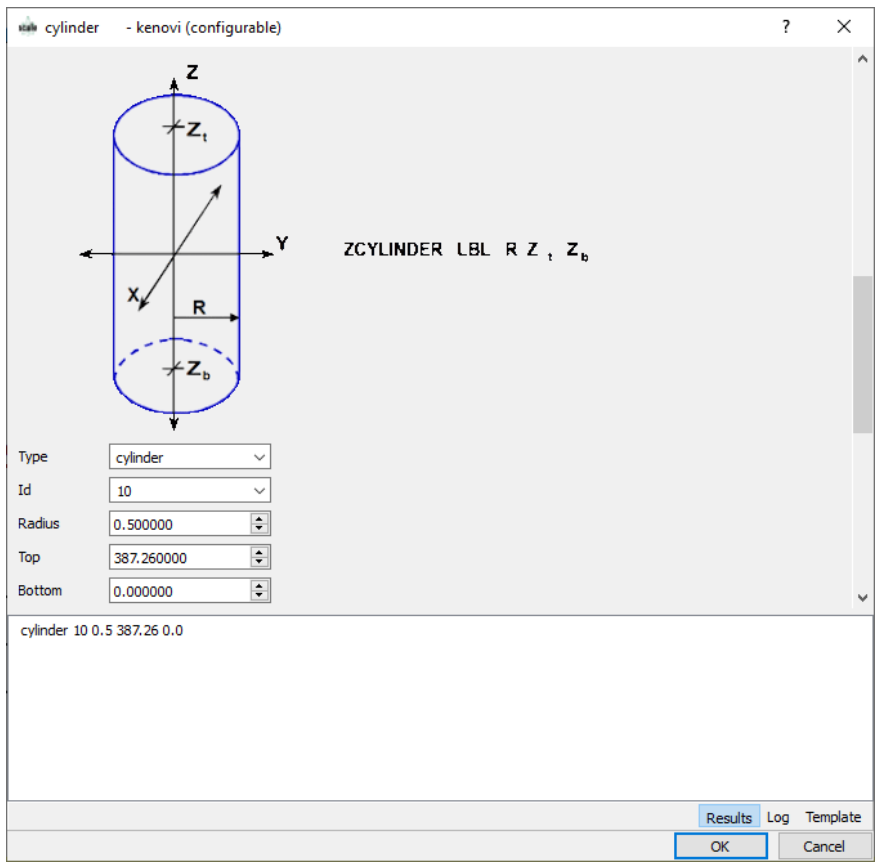

Figure 186. Completed configurable form in Fulcrum for first cylinder in Unit 2.

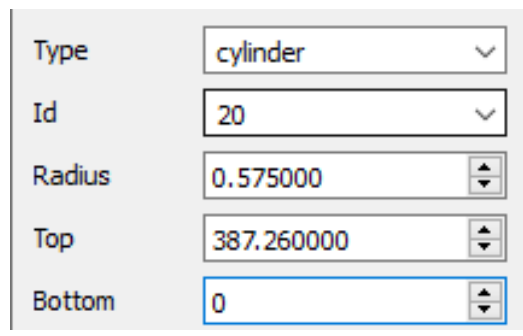

Figure 187. User-specified input in configurable form for second cylinder un Unit 2.

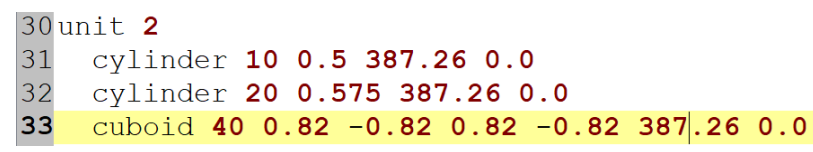

Figure 188. Input for all three regions in Unit 2. 
All of the regions needed in Unit 2 have been entered; at this point, media records can be specified to fill the regions with the appropriate mixtures. The MEDIA entries for this unit are straightforward: the first is the water inside the guide tube, the second is the Zircaloy- 2 forming the guide tube, and the third is the water outside the guide tube. The first MEDIA record can be entered directly in Unit 2 as media 31 10, which fills surface 10 (the first cylinder in the unit) with water. Similarly, the second MEDIA record,

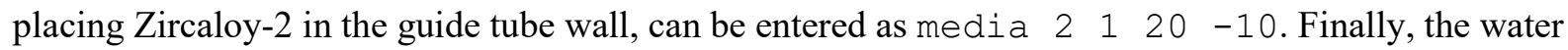
outside the guide tube can be entered as media $3 \quad 1 \quad 40$-20. All three MEDIA records are shown in Figure 189. Finally, the boundary record can be added; as with Unit 1, the outer surface is cuboid 40. Enter boundary 40 after the MEDIA records to complete Unit 2. The complete Unit 2 input is shown in Figure 190.

$\begin{array}{llllll}35 & \text { media } & 3 & 1 & 10 & \\ 36 & \text { media } 2 & 1 & 20 & -10 \\ 37 & \text { media } & 3 & 1 & 40 & -20\end{array}$

Figure 189. Input for all three MEDIA records in Unit 2.

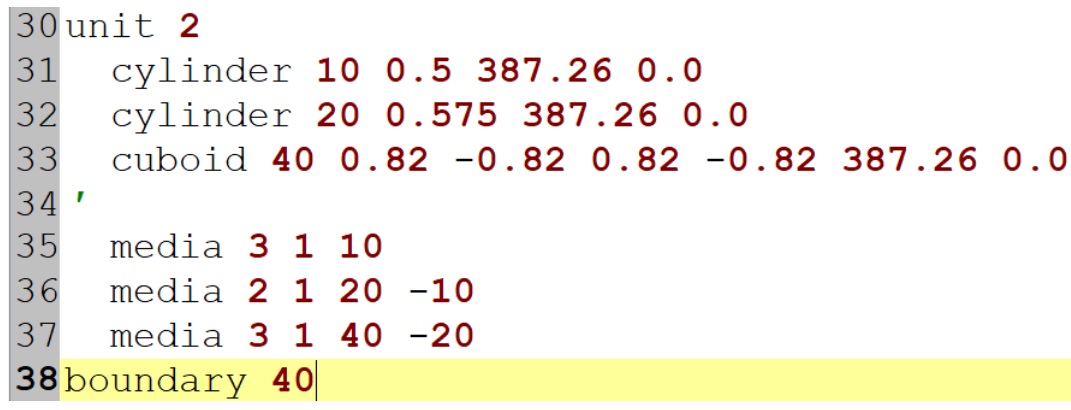

Figure 190. Complete input for Unit 2.

The next step is to create an array of units 1 and 2 for the fuel assembly. As discussed in Section 4.6, the faces that touch between units placed in an array in KENO-VI must be the same size. The X and Y faces of both units 1 and 2 extend from $-0.82 \mathrm{~cm}$ to $0.82 \mathrm{~cm}$, for a total length of $1.64 \mathrm{~cm}$. In the axial dimension, Unit 1 extends from $-4 \mathrm{~cm}$ to $383.26 \mathrm{~cm}$, a total length of $387.26 \mathrm{~cm}$. Unit 2 extends from 0 $\mathrm{cm}$ to $387.26 \mathrm{~cm}$, which matches the Unit 1 axial extent exactly. The dimensions are the same, so the units can be used together in an array. Note that the $\mathrm{X}$ and $\mathrm{Y}$ faces must match because both $\mathrm{X}$ and $\mathrm{Y}$ faces touch in various places within the array. Also note that the $Z$ dimensions are the same length despite having different coordinates. The array need not be specified in the ARRAY block before it is included in the geometry block, but it is often simpler to do this. The ARRAY block can be added with autocomplete once the cursor is positioned outside the geometry and all other blocks. An example is shown in Figure 191. Select arrays from the list of available blocks to add.

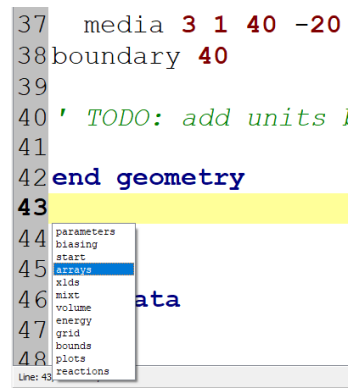

Figure 191. Autocomplete list of available blocks to add to the input file. 
The only option for autocomplete within the array block is for an array - kenovi basic (configurable); select this option to launch an array configurable form like that shown in Figure 192. The array number (ara) can remain as 1, and the gbl field can remain blank, as this will not be a global array. The prt option controls printing the contents of the array in the output file; yes is the default option and is generally retained. PRT is only set to no for particularly large, uniform arrays which generate large edits that are not helpful in understanding the model. The Typ remains cuboidal, as this is an array of units with cuboids as outer boundaries. The nux, nuy, and nuz entries specify the number of units in the array in the $\mathrm{X}, \mathrm{Y}$, and $\mathrm{Z}$ directions, respectively. The array for the fuel assembly being modeled here is a $9 \times 9$ element array with only one axial level. nux and nuy are therefore 9 , and nuz is 1 . The fill id option allows the user to select a unit with which to populate the ARRAY FILL; in this case, 1 is the better choice, as 72 of the elements in the array are Unit 1, while 9 are Unit 2. This completes the user-specified input for this array, as shown in Figure 193. After reviewing the input to confirm that it is correct, press OK to add the array to the input. The final step is to add Unit 2 in the appropriate places within the ARRAY FILL to place guide tubes within the fuel assembly. Recall that the processing of entries is first in $\mathrm{X}$, then in $\mathrm{Y}$, and finally in Z. The Fulcrum configurable form inserts the text in NUY rows that have NUX columns as a convenience for the user in creating the correct FILL entries, but this is not required in KENO input. The first row to be read will be the $Y=1$ row, and the last row will be the $Y=9$ row, so the input is essentially upside down relative to the model. The fuel assembly layout is symmetric, so this is not a concern for this model but is worth noting. The final ARRAY data for this problem, including the FILL entries with the guide tubes (Unit 2), are shown in Figure 194. Note that the TODO comment and extra blank lines have been removed to save space in this document, but they have no impact on KENO if they remain in the input.

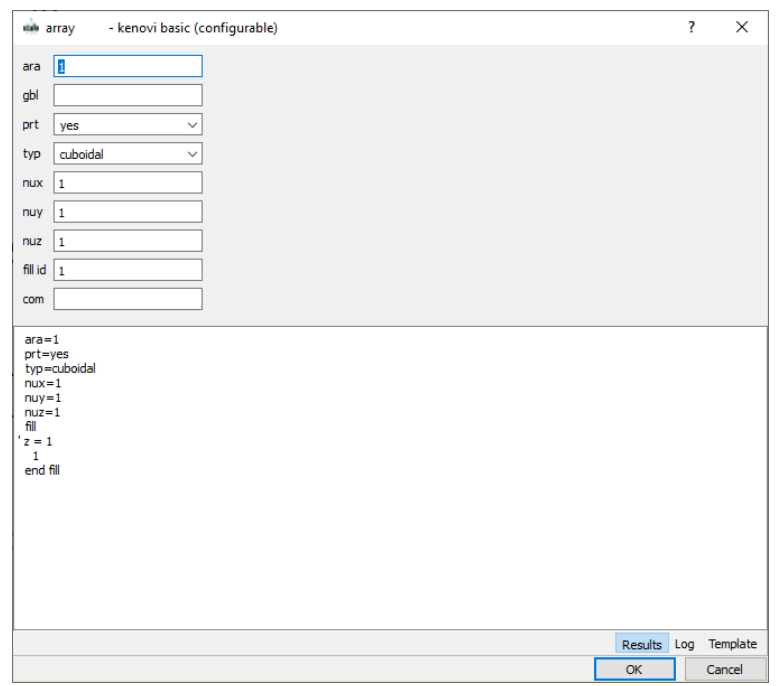

Figure 192. Default configurable form for a KENO-VI array. 


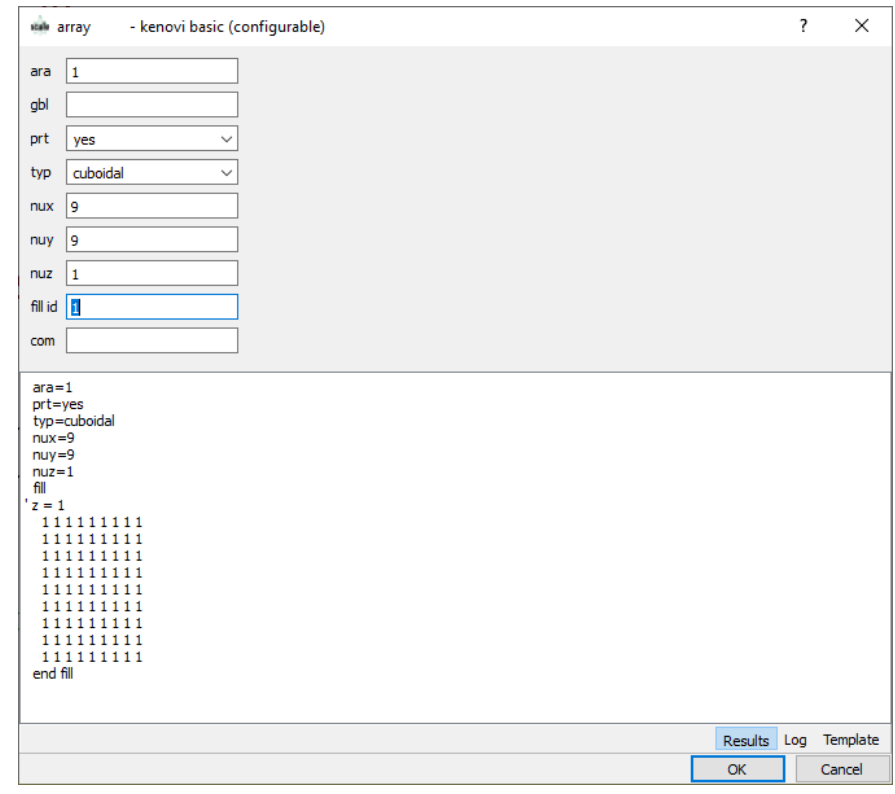

Figure 193. Complete configurable form for the array in the model.

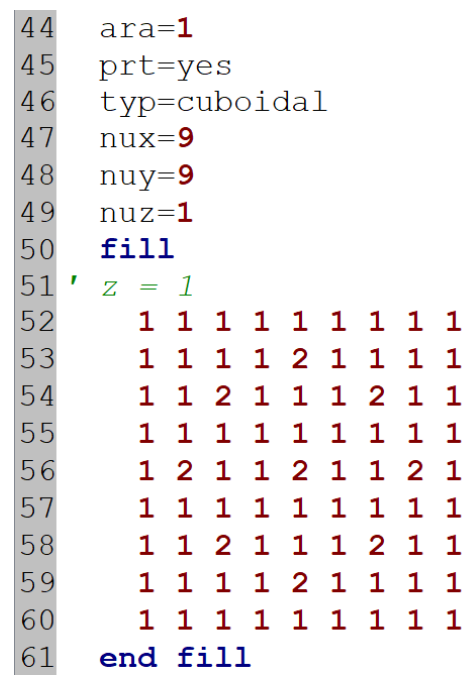

Figure 194. Complete array specification with guide tube units in FILL block.

The array for the fuel assembly can be inserted into a unit in the GEOMETRY block now that it has been created. An array cannot be placed into one of the units that has been used in it, so a new unit is needed. This will be Unit 3 and will be the global unit. The array must be placed inside a region that it fills completely, as discussed in Section 4.6. Then a subsequent region in this unit will be added for the water above, below, and around the fuel assembly.

The first step is to create global unit 3. This can be done by placing the cursor directly above end geometry and using autocomplete to add global_unit. Autocomplete provides global unit 1 , so the unit number must be changed manually to 3 . The array is 9 units wide in $\mathrm{X}$ and $\mathrm{Y}$, with each unit having a width of $1.64 \mathrm{~cm}$, so the total width of the array is $14.76 \mathrm{~cm}$. The height of the array is $387.26 \mathrm{~cm}$. Placing the origin of the unit at the middle of the array will make adding the water region simpler, so a cuboid with $X$ and $Y$ dimensions of $\pm 7.38 \mathrm{~cm}$ and $Z$ dimensions of \pm 193.63 can be created to contain the array. Autocomplete can be used to generate a configurable form of a cuboid by selecting cuboid - 
kenovi (configurable) from the list. Leave the Id as 1 and enter the dimensions provided for the dimensions of the cuboid. The completed configurable form is shown in Figure 195. After confirming that the input is correct, press the $\mathbf{O K}$ button to add the cuboid specification to the input file. A second cuboid is needed to contain the water around the assembly in the problem specification. This region was stated to be $7.62 \mathrm{~cm}$ in $\mathrm{X}$ and $\mathrm{Y}$ and $15.24 \mathrm{~cm}$ in $\mathrm{Z}$, adding reflection on all 6 faces of the array. The dimensions for this new cuboid are therefore $\pm 15 \mathrm{~cm}$ in both $X$ and $Y$ and $\pm 208.87 \mathrm{in} Z$. This cuboid can also be added using a configurable form, this time with Id 2. The completed configurable form is shown in Figure 196. After confirming that the input is correct, press the $\mathbf{O K}$ button to add the cuboid specification to the input file. The complete input for these two regions is shown in Figure 197.

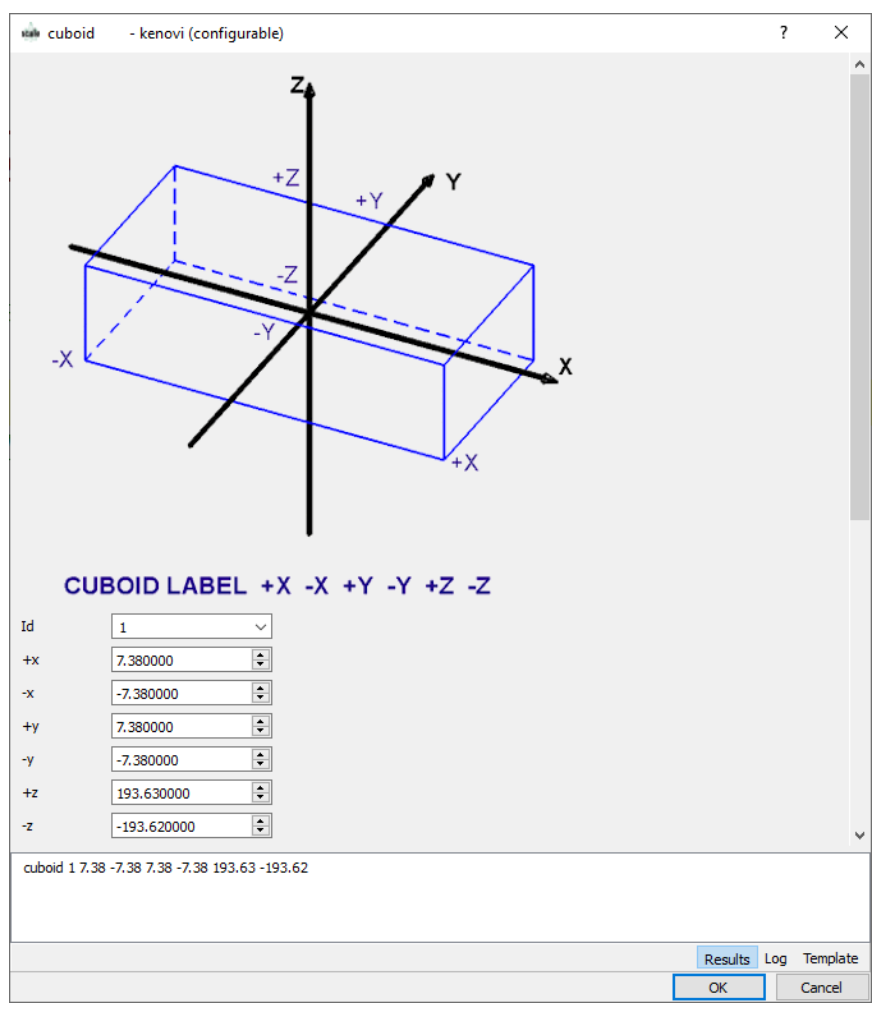

Figure 195. Completed configurable form for first cuboid in Unit 3. 


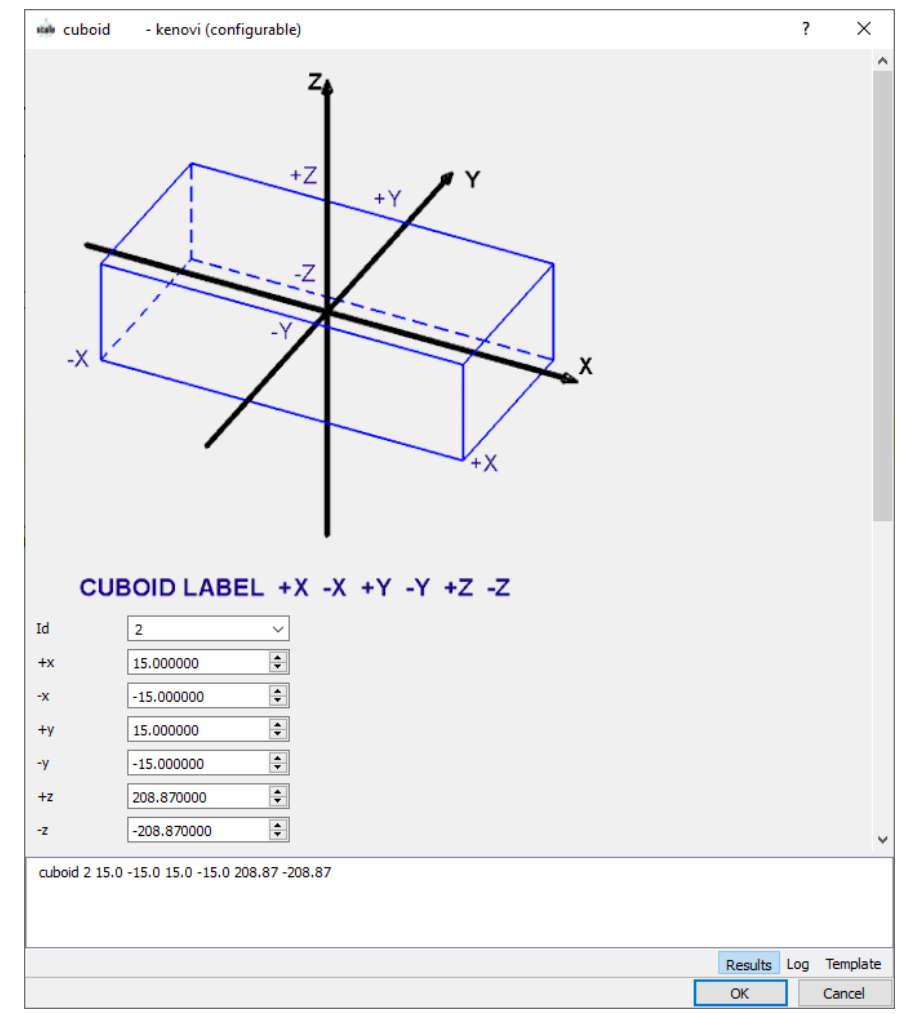

Figure 196. Completed configurable form for second cuboid in Unit 3.

41 global unit 3

42 cuboid $1 \quad 7.38 \quad-7.38 \quad 7.38 \quad-7.38 \quad 193.63-193.62$

43 cuboid $2 \quad 15.0 \quad-15.0 \quad 15.0 \quad-15.0 \quad 208.87 \quad-208.87$

Figure 197. Input for both cuboids in Unit 3.

Now the contents of the regions defined in Unit 3 must be specified. The first cuboid (cuboid 1) will contain the array and the second cuboid (cuboid 2) will contain the water reflector. Cuboid 1 has been sized to be completely filled by array 1; recall from Section 4.6 that an ARRAY must be placed inside a region and must fill it completely. Array 1 will be positioned inside cuboid 1 using the PLACE entries on the ARRAY CONTENTS record; cuboid 1 is specified such that the center of the array will be at the origin of the unit. The central element of array 1 is element $(5,5,1)$, which is a guide tube and thus Unit 2. The origin of Unit 2 is specified to be at the radial center of the guide tube and the bottom of the unit. The $\mathrm{X}$ and $\mathrm{Y}$ coordinates of the origin in the Unit 3 coordinate system are therefore $(0,0)$ so that the origin stays at the center of the UNIT. The origin of Unit 2 must be placed at $-193.62 \mathrm{~cm}$ in the $Z$ direction such that the axial middle of the array is at $Z=0$ in the Unit 3 coordinate system. This information can be provided in an array configurable form (Figure 198); select array - kenovi (configurable) from the autocomplete list. The Array Id is 1, so no change is needed to the first field in the form. Press the Add row button at the bottom of the Regions pane to add a row to the pane; this array is being placed inside of region 1, so no changes are needed to the Id or State fields. As discussed above, the xindex and yindex should be set to 5, and the zindex is 1 . Finally, the $\mathbf{x}$ and $\mathbf{y}$ fields remain 0 , and the $\mathbf{z}$ field is set to -193.62 . The completed configurable form for the array 1 CONTENTS record (geometry block) is shown in Figure 199. Press the OK button to add the specification to the input file after confirming that all inputs are correct. The next step is to add a MEDIA record to place the water

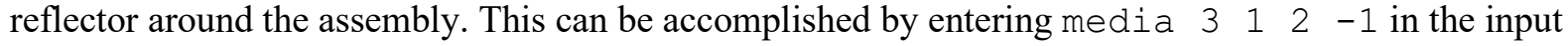
file. Recall that region 1 is filled with the array, so the MEDIA record must only specify that the water (mixture 3) be placed inside of region 2 and outside of region 1. The two CONTENTS records for Unit 3 
are shown in Figure 200. Finally, the boundary record must be corrected; the outer boundary of Unit 3 is surface 2, not surface 1. The complete input for global unit $\mathbf{3}$ is shown in Figure 201; as before, additional comments and blank lines have been removed for the sake of clarity in this document.

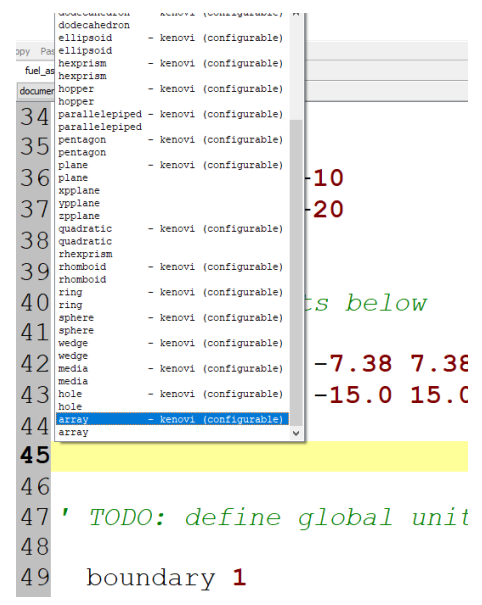

Figure 198. Selecting an ARRAY configurable form in the autocomplete menu in Fulcrum.

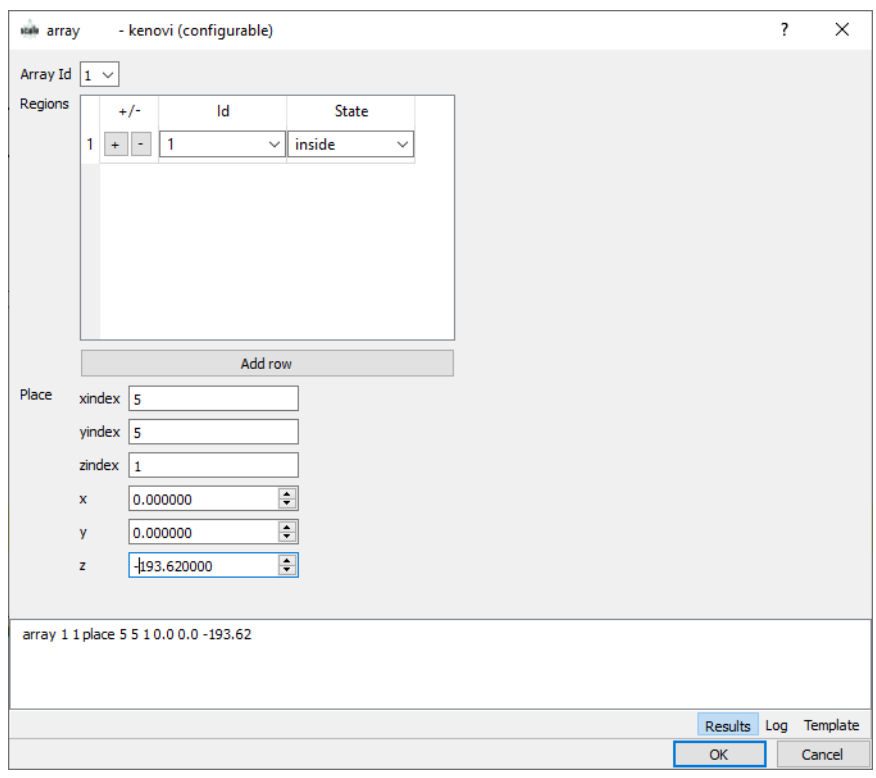

Figure 199. Completed configurable form for the array 1 CONTENTS record.

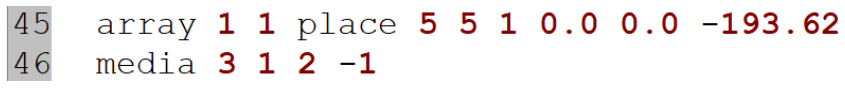

Figure 200. Both contents records needed in Global Unit 3.

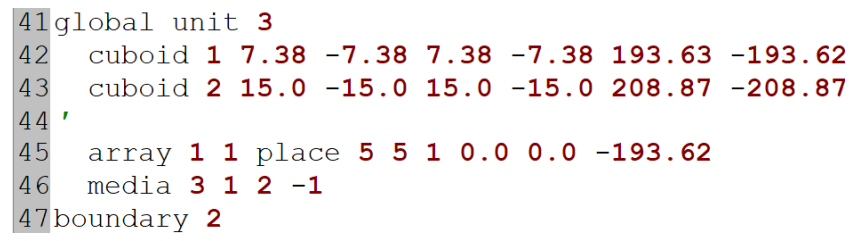

Figure 201. Complete input for Global Unit 3. 
Fulcrum visualization can now be used to check the model; a more complete description of Fulcrum's geometry visualization capabilities is provided in Section 9.2.1. To launch the visualization, click the View button between the Run and Edit buttons just above the text input screen. The default view is an $\mathrm{XY}$ slice through the axial center of the model, as shown in Figure 202.

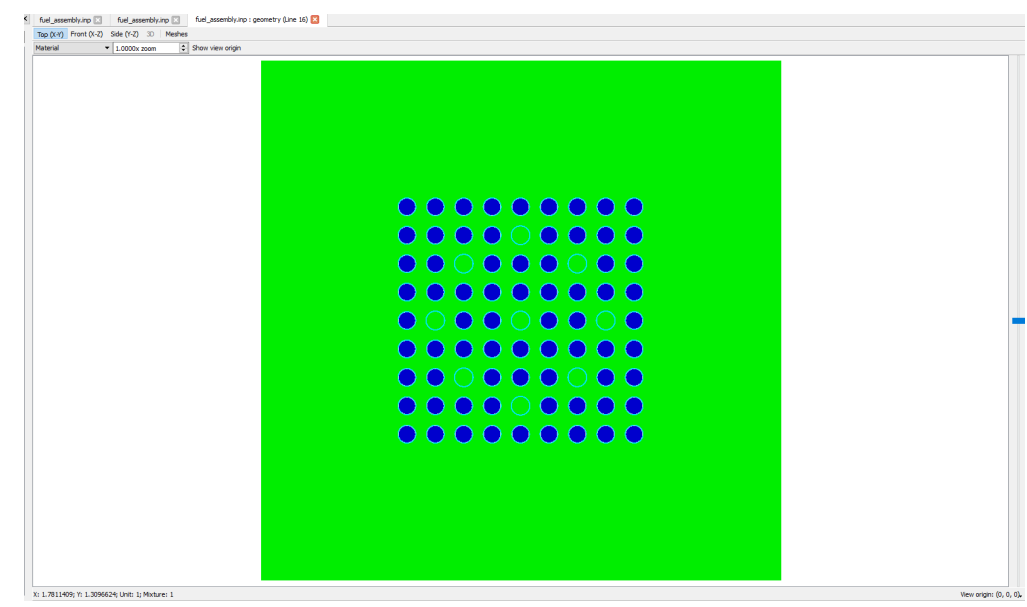

Figure 202. Radial slice of the completed fuel assembly at the model midplane.

Now save all changes and run SCALE by clicking the Run button at the top of the Fulcrum window. The default run parameters of 1,000 particles per generation, 203 total generations, and 3 discarded generations will be used if no specific values are chosen by adding a PARAMETER block. These parameters yield a $k_{\text {eff }}$ estimate of $0.6915 \pm 0.0018$; exact values will vary, as different computers and different operating systems generate different random numbers. A more rigorous calculation that simulates 10,000 particles per generation for 282 total generations and that skips the first 50 results in a $k_{\text {eff }}$ estimate of $0.69087 \pm 0.00049$. This calculation repeated with CE cross sections yields a $k_{\text {eff }}$ of $0.69168 \pm 0.00059$; the difference from the MG calculation is $0.00082 \pm 0.00077$. This difference is only $\sim 1.1$ standard deviations and thus is statistically insignificant. The LATTICECELL processing used in this problem provides sufficiently accurate cross sections to reproduce the CE solution.

\subsubsection{Multiregion example: Parallel slab tanks}

This problem consists of two parallel slab tanks constructed of SS304 that is $0.5 \mathrm{~cm}$ thick. The material in the tanks is a $\mathrm{U}(93) \mathrm{O}_{2} \mathrm{~F}_{2}$ solution with a fuel density of $459 \mathrm{~g} / \mathrm{L}$, a solution density of $1.566 \mathrm{~g} / \mathrm{cm}^{3}$, and no excess acid. Each slab tank has internal dimensions of $5 \mathrm{~cm}$ thick by $300 \mathrm{~cm}$ long by $150 \mathrm{~cm}$ high. Each tank is reflected by $5 \mathrm{~cm}$ of water on each side in the X direction (i.e., $10 \mathrm{~cm}$ of water between the tanks). There is no reflector material above the tanks or on the ends of the tanks. A 3D rendering of the system is shown in Figure 203. 

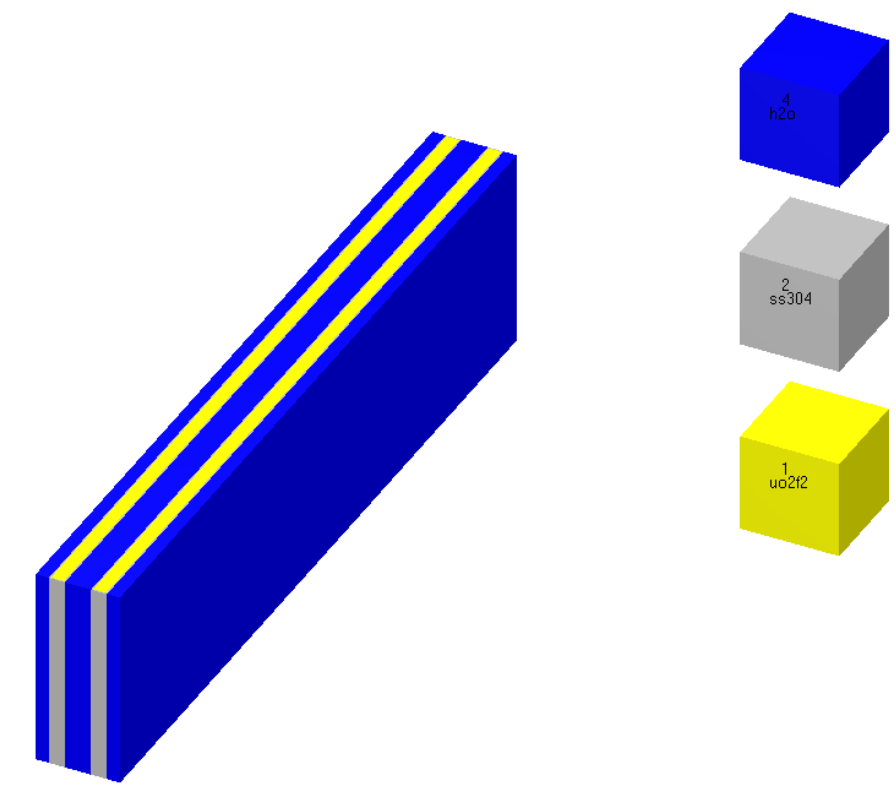

Figure 203. 3D rendering of the two tanks, with the top half of the model removed to show the solution.

\subsubsection{General and Materials Data}

The initial data entry for this example is the same as in previous problems. Start Fulcrum and then select the New file... option from the File menu. Select a directory where the model will be saved, and name it slab_tanks. inp. Next, use the autocomplete feature in Fulcrum to create a csas6 - Criticality safety analysis using KENO-VI input. Replace title-goes-here with Unit Cell - Slab Tanks. Now change xs-lib-goes-here to $\mathrm{v} 7.1-252$ ( $\mathrm{v} 7.1-252 \mathrm{n}$ if using SCALE 6.2, 6.2.1, or 6.2.2). This selects the 252-group neutron cross section library based on ENDF/B-VII.1 and signals to CSAS6 that this will be a MG KENO calculation. The appropriate cross section processing modules will be called using the input provided in the CELLDATA block as discussed in Section 8.5.2.2. The first three lines of the input are shown in Figure 204.

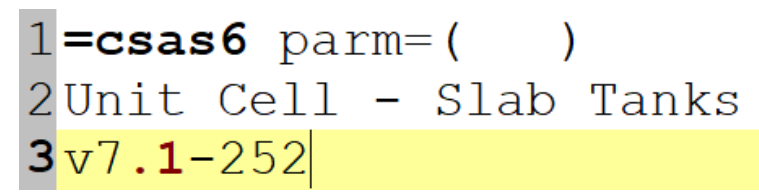

Figure 204. First three lines of the slab tanks model.

The materials information will be entered for the fissile solution and standard basic compositions for stainless steel and water. Position the cursor in the COMPOSITION block and select the solution - rho + density + temperature + volume fraction option. No configurable forms are available for fissile solution input, so template text is provided in the input, as shown in Figure 205. In the figure, the input has been divided onto two lines to allow better presentation in this document, but this has no impact on how CSAS processes the input. Change the concentration of the solution (rho[uo2f2]) from 1.0 to 459 , the ${ }^{235} U$ (92235) wt.\% to 93, and the ${ }^{238} U$ (92238) wt.\% to 7. Set the density of the solution to 1.566 and the temperature (temp) to $293 \mathrm{~K}$. The updated, applicable solution input is shown in Figure 206.

$$
\begin{aligned}
& 5 \text { solution } \operatorname{mix}=1 \text { rho[uo2f2] }=1.0 \quad 9223595.592238 \quad 4.5 \\
& 6 \text { density }=1.0 \text { temp=300 volfrac=1.0 end solution }
\end{aligned}
$$

Figure 205. Initial template text provided by Fulcrum for a fissile solution. 


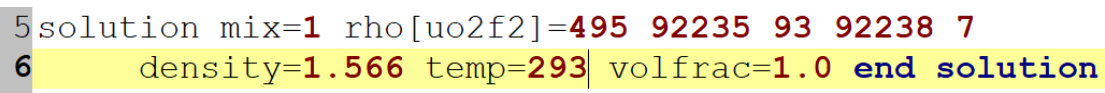

Figure 206. Fissile solution input for this problem.

The stainless steel 304 and water compositions can both be added via configurable forms for basic standard compositions. The initial standard basic composition configurable is shown in Figure 207. Type ss 304 into the Composition box in the configurable form, set the Mixture number to 2, and press OK to add the specification to the SCALE input. Two ss 304 and two h2o mixtures must be created because a unique mixture number is required for each region in the unit cell. The easiest way to do this is to copy and paste the existing ss304 entry and change the mixture number to 3. Another standard basic composition configurable form can be used to add the first water composition. Type h2o into the Composition box in the configurable form, set the Mixture number to 4, and press $\mathbf{O K}$ to add the specification to the SCALE input. As with the stainless steel, copy and paste the mixture and change the mixture number from 4 to 5. The complete composition block is shown in Figure 208.

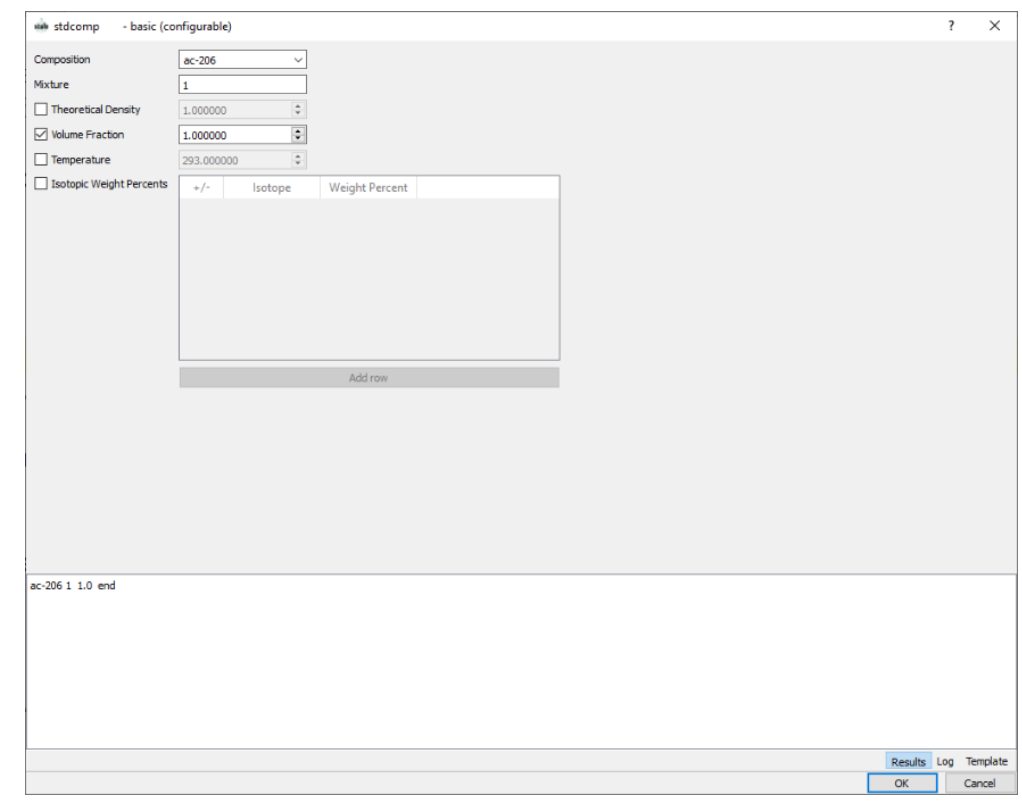

Figure 207. Initial STDCOMP configurable form.

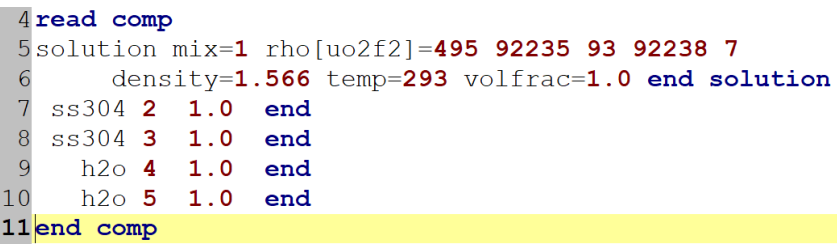

Figure 208. Final composition block for this model.

\subsubsection{Unit Cell Data}

The unit cell information for the cross section treatment can be specified now that the material information has been entered. One of the four unit cell types (INFHOMMEDIUM, LATTICECELL, MULTIREGION, DOUBLEHET) will be specified to process the MG cross sections for this problem. A MULTIREGION SLAB cell will allow cross section processing to be performed on almost the exact geometry for this problem. While INFHOMMEDIUM may result in acceptable answers, the fact that the 
tanks are thin relative to their length and height makes MULTIREGION a better option. On the other hand, the tanks are wide enough and tall enough that the infinite slab approach should yield good results, and the BUCKLEDSLAB option is not needed. In this case, the left boundary can be set as reflective to allow specification of one side of the problem to represent both tanks.

To add the CELLDATA block, place the cursor between the COMPOSITION and GEOMETRY blocks and autocomplete for a list of available blocks to add. Select cells, as show in Figure 209. There are no configurable forms currently available in the CELLDATA block. Autocompleting in the CELLDATA block provides a list of available unit cell specifications; select the multiregion - slab option as shown in Figure 210. Fulcrum inserts generic CELLDATA input, as shown in Figure 211, which must be modified for use in this model.

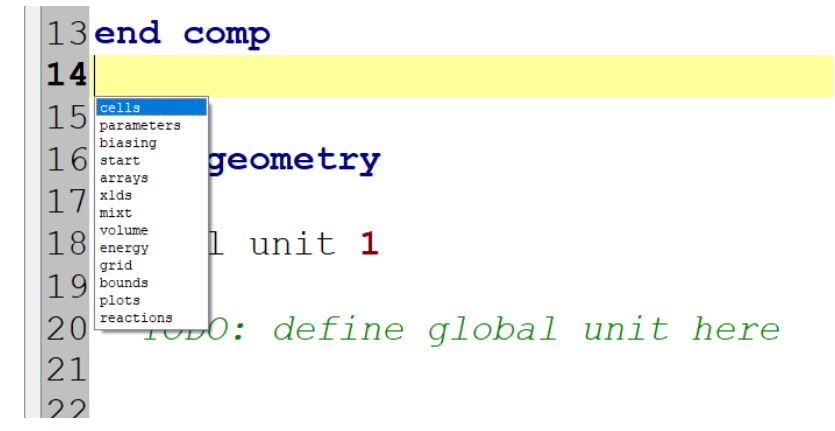

Figure 209. Autocomplete list of available blocks for inclusion in the model.

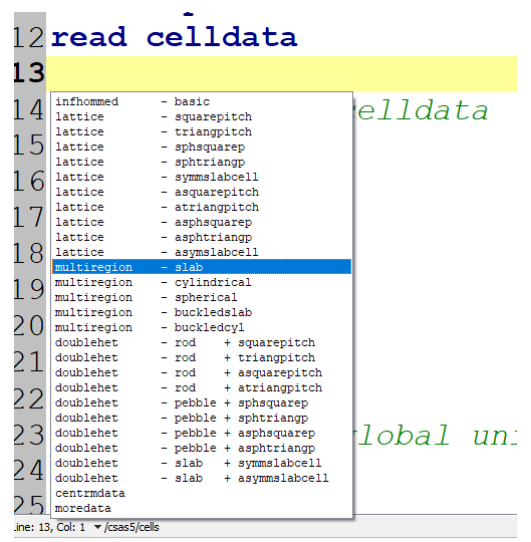

Figure 210. Autocomplete list of cells within the CELLDATA block.

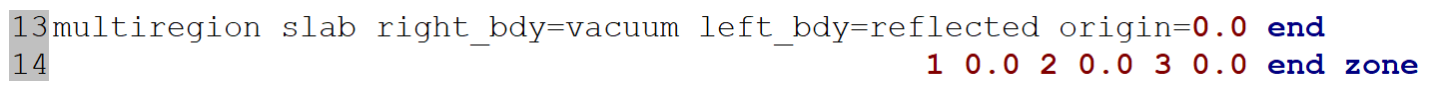

Figure 211. Templated input provided for MULTIREGION SLAB cell by Fulcrum.

The templated input must now be replaced with the appropriate input for this problem. The geometry to be constructed in the CELLDATA block will be one tank, with the associated water around the tank. The right boundary (right_bdy) will therefore remain a vacuum boundary condition, and the left boundary (left_bdy) will remain a reflected boundary condition. The specification of the origin at 0.0 can remain or be removed; it has no effect, as it is explicitly specifying the default value. The remaining inputs are the zones present, from left to right, represented as infinite slabs. These regions are water, tank wall, solution, tank wall, and water; each will be specified with the appropriate mixture number. The distances to be specified are those from the left boundary of the cell to the right boundary of the region; these inputs must be monotonically increasing, as they represent the accumulating distance and not the thickness of the 
relevant regions. The zone inputs to be provided are as follows: $\begin{array}{llllllll}5 & 5.0 & 3 & 5.5 & 1 & 10.5 & 2 & 11.0\end{array}$ 4 16.0. The input is not white-space dependent and can be entered on a single line as provided, or it can be entered in columns as shown in Figure 212. This arrangement is more convenient, makes it easier to track which mixtures have been used, and helps to ensure that the correct increasing dimensions have been provided. Note that different water mixtures and different stainless steel mixtures are used to meet the requirement that each mixture is used in only one region of one cell in the CELLDATA block. These duplicate mixtures will not be used in the KENO-VI geometry, but they are necessary for cross section processing. If the neutron flux, and therefore the MG cross sections, were significantly different between the two sides of the tank, then the different mixtures should be used in the respective regions in the KENO-VI geometry.

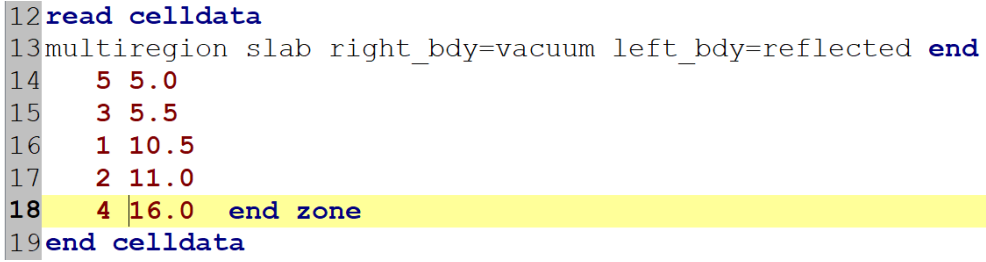

Figure 212. Final CELLDATA block showing the correct input for this model.

\subsubsection{KENO-VI Geometry Data}

This model requires only a single unit and will use a reflecting boundary condition to reduce the amount of the problem that must be specified. The entire geometry could be specified using an array, but this model provides a demonstration of using boundary conditions to simplify the geometry specification for the model. In this case, three regions are required: the solution, the tank, and the water outside the tank.

The first region is the inner volume of the tank, containing the solution. This is a cuboid that is $5 \mathrm{~cm}$ thick in the $\mathrm{X}$ direction, $300 \mathrm{~cm}$ long in the $\mathrm{Y}$ direction, and $150 \mathrm{~cm}$ tall in the $\mathrm{Z}$ direction. This region can be added with a cuboid configurable form in Fulcrum. The appropriate dimensions can be added to the form; the origin of the model is arbitrary and is positioned in the center of the fissile solution in the form shown in Figure 213. After checking the input to confirm that it is correct, press $\mathbf{O K}$ to enter the cuboid entry into the input file. Figure 214 shows the cuboid entry in the input file. 


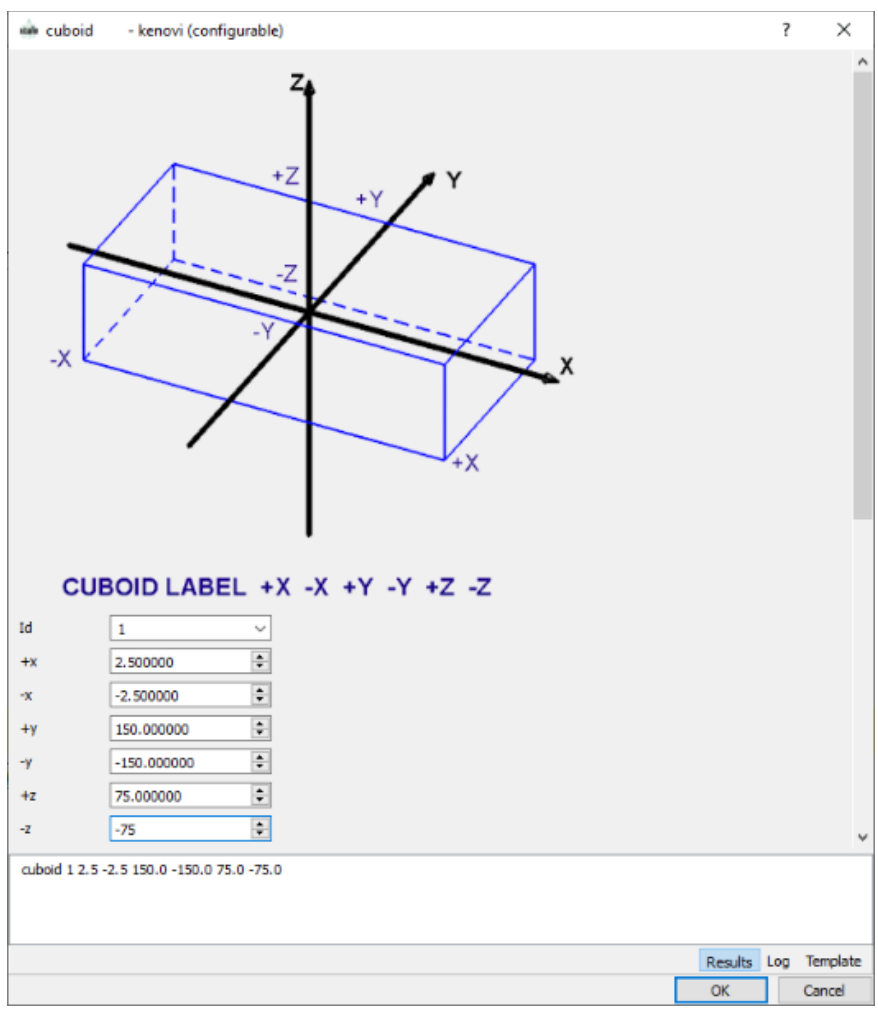

Figure 213. Cuboid configurable form for region inside slab tank.

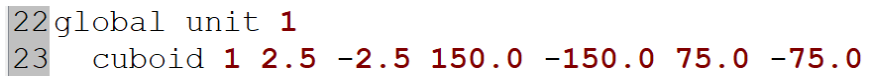

Figure 214. Cuboid input for region inside slab tank.

The next region is the tank wall, which is $0.5 \mathrm{~cm}$ thick in all 6 directions. The cuboid can be added with a configurable form with an Id of 2. The dimensions of cuboid 2 are \pm 3.0 in the X direction, \pm 150.5 in the $\mathrm{Y}$ direction, and \pm 75.5 in the $\mathrm{Z}$ direction. The final configurable form for this region is shown in Figure 215; after confirming that all input is correct, press the OK button to add cuboid 2 to the input. The final region is the water outside the tank on the $+\mathrm{X}$ and $-\mathrm{X}$ faces. These water regions are $5 \mathrm{~cm}$ on each side of the tank, so the dimensions are \pm 8 . This cuboid can be added via a configurable form as cuboid 3, as shown in Figure 216, after the inputs have been confirmed to be correct. All the geometry records are shown in Figure 217. 


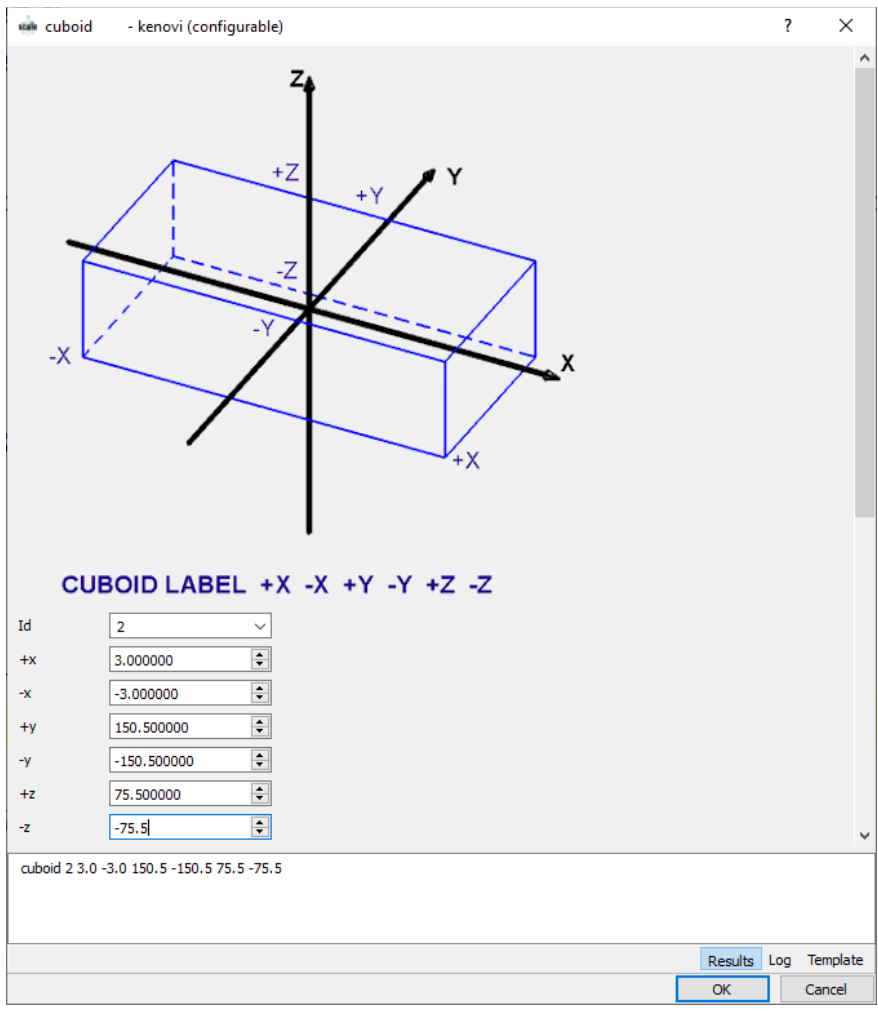

Figure 215. Complete configurable form for cuboid 2, the tank wall.

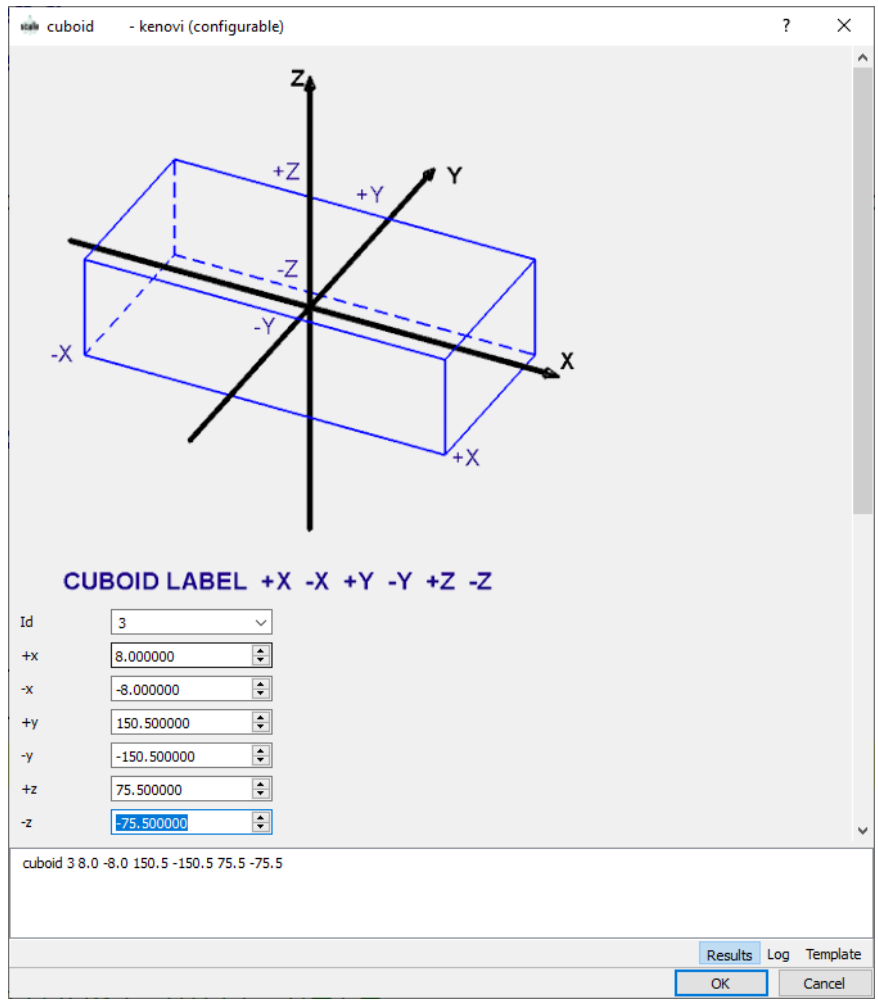

Figure 216. Complete configurable form for cuboid 3, the water reflector. 


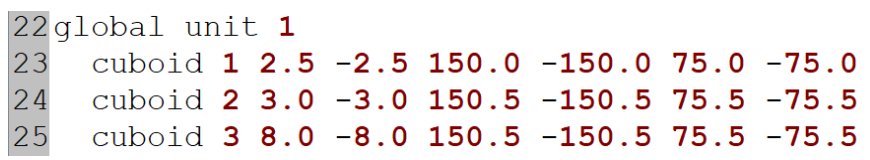

Figure 217. All three cuboids, the only three geometry records needed for this model.

Next, the contents records must be added. In this case, three MEDIA records are needed to add the solution, the stainless steel, and the water into the appropriate cuboids. The first record can be added as media 111 , to add the solution (mixture 1) into cuboid 1. The input can be added directly or by using a media configurable form. The second record, which can also be added either directly or via configurable form, places the stainless steel (mixture 2) inside the tank wall but outside the inside volume

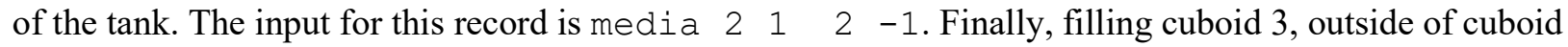
2 , with water (mixture 4) can be accomplished with media $\begin{array}{llllll}4 & 1 & 3 & -2 \text {. All three contents records are }\end{array}$ shown in Figure 218. The last entry to complete global unit 1 is to update the boundary record. Cuboid 3 forms the outer boundary of the unit, so the boundary record should be updated to boundary 3 . The entire specification for global unit 1 is provided in Figure 219.

$\begin{array}{llllll}27 & \text { media } & 1 & 1 & 1 & \\ 28 & \text { media } & 2 & 1 & 2 & -1 \\ 29 & \text { media } & 4 & 1 & 3 & -2\end{array} \mid$

Figure 218. All three media records needed for this model.

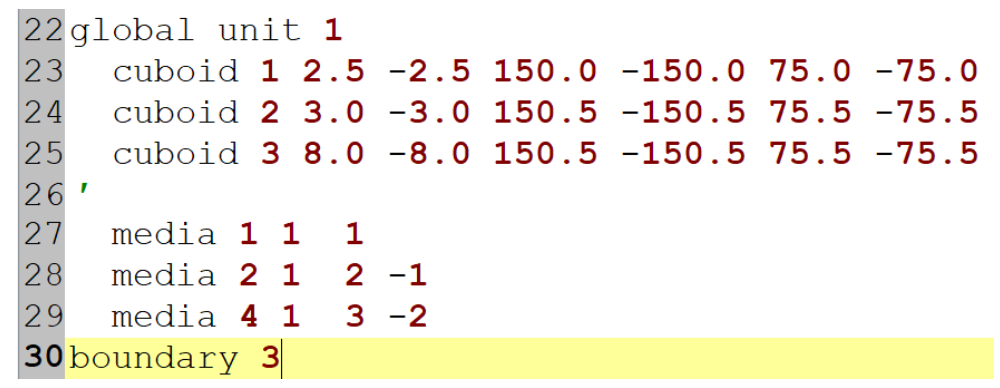

Figure 219. Entire specification for global unit 1.

The model with a single tank explicitly represented in it can be made to represent a system with two tanks by adding a reflective boundary condition to one of the $\mathrm{X}$ surfaces. This returns a neutron through the $5 \mathrm{~cm}$ water region it just traversed, so it must travel through a total of $10 \mathrm{~cm}$ of water to return to the tank. This exactly describes the problem as stated above. The boundary conditions on the outer surface of the global unit are supplied in the BOUNDS block. The BOUNDS block can be added by autocomplete if the cursor is placed outside of any other blocks, as shown in Figure 220. No autocomplete is available in Fulcrum in the BOUNDS block, but the options are described in [Albedo data] in the KENO section of the manual. The default boundary condition in KENO-VI is vacuum, so only one $\mathrm{X}$ face needs to be changed. The boundary condition is changed by specifying a face code and the boundary condition to apply to that face. For cuboid outer boundaries, the KENO V.a face codes may be used. For instance, the face code for the positive $\mathrm{X}$ face is $+\mathrm{XB}=$, and for the negative $\mathrm{X}$ face it is $-\mathrm{XB}=$. For cuboids and all other shapes, each face of the surface can be referenced via a number; the list of these face numbers for each surface is provided in [Face codes and surface numbers for entering boundary (albedo) conditions] in the [Albedo data] section of the KENO manual. A mirror boundary condition can be specified with the keywords MIRROR or REFLECT, or a shortened form of either. For this model, the KENO V.a style input would be $-\mathrm{XB}=\mathrm{REFL}$. In the more general approach, the number of the $-\mathrm{X}$ face of a cuboid is 2 , and the input is then surface (2)=REFL. This approach is shown in Figure 221. 


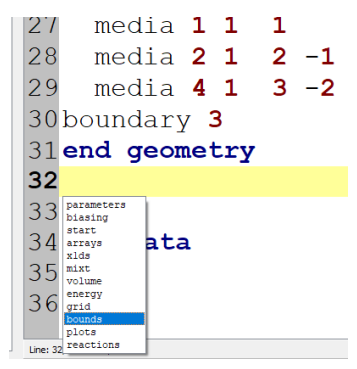

Figure 220. Adding the BOUNDS block from the Fulcrum autocomplete list.

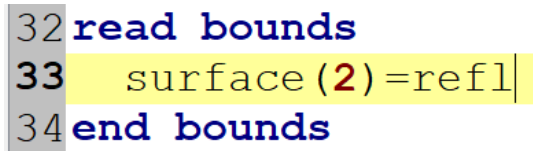

Figure 221. Boundary condition specification to apply a reflective boundary condition to the $-X$ face of the global unit.

The model is complete, and a Fulcrum 2D view of the entire model is shown in Figure 222. A magnified view of one end of the model is shown in Figure 223; the color scheme in these figures is the same as that used in Figure 203: yellow for $\mathrm{UO}_{2} \mathrm{~F}_{2}$ solution, gray for stainless steel, and blue for water. There is no indication in the geometry rendering that a reflective boundary condition has been applied to the -X face of the model.

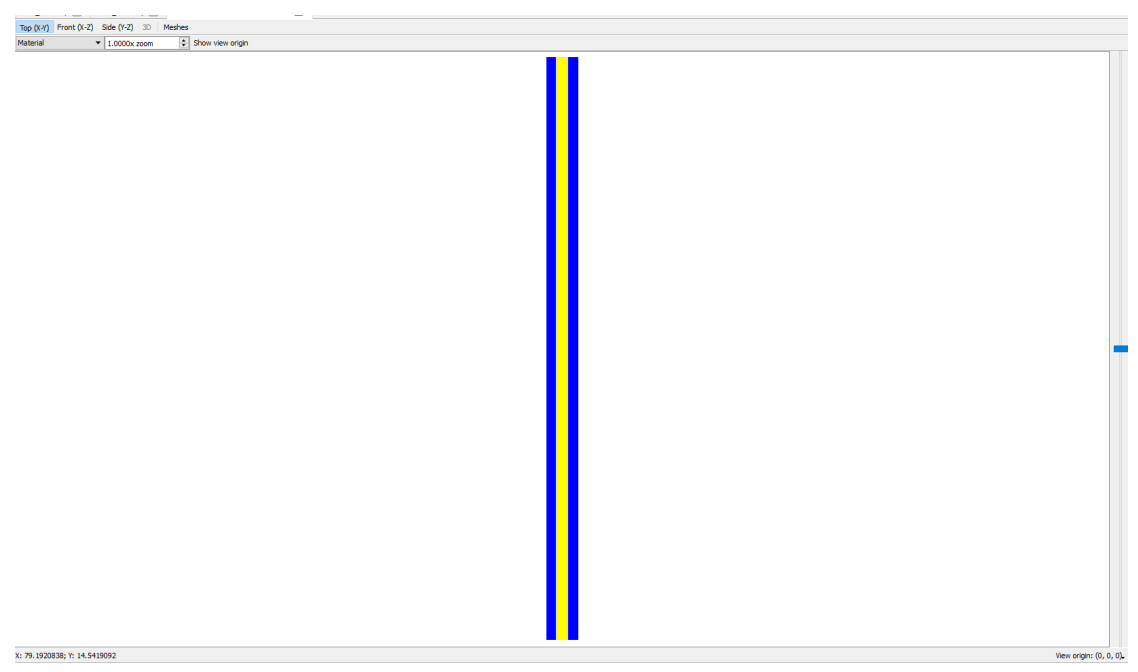

Figure 222. Fulcrum 2D visualization of the axial midplane of the model. 


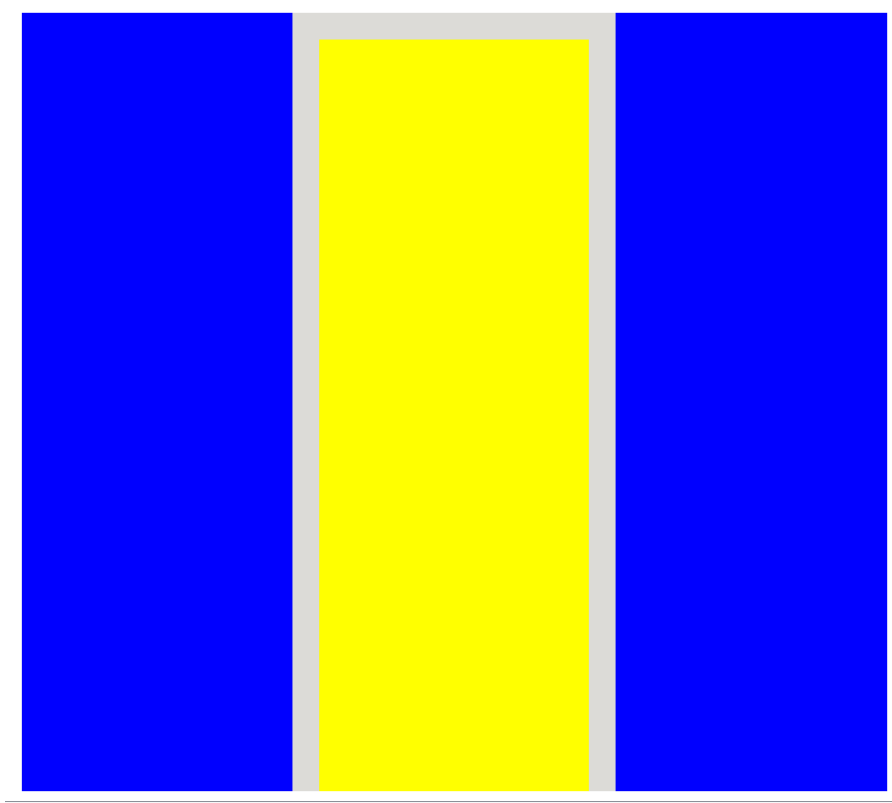

Figure 223. Detailed view of the $+Y$ end of the axial midplane of the model, showing the end of the tank.

Now save all changes and run SCALE by clicking the Run button at the top of the Fulcrum window. The default run parameters of 1,000 particles per generation, 203 total generations, and 3 discarded generations will be used if no specific values are chosen by adding a PARAMETER block. These parameters yield a $k_{\text {eff }}$ estimate of $0.9519 \pm 0.0022$; exact values will vary, as different computers and different operating systems generate different random numbers. A model using an array to explicitly represent both tanks resulted in a $k_{\text {eff }}$ estimate of $0.9497 \pm 0.0021$. The difference between these results is $0.0022 \pm 0.0030$; since this difference is less than one standard deviation, the estimates are statistically equivalent. This demonstrates that the model with the single explicit tank and the reflective boundary condition can be used in place of the more complicated geometry with both tanks explicitly represented.

A more rigorous calculation, simulating 10,000 particles per generation for 377 total generations and skipping the first 50 , resulted in a $k_{\text {eff }}$ estimate of $0.94895 \pm 0.00049$. This calculation repeated with CE cross sections yields a $k_{\text {eff }}$ of $0.94841 \pm 0.00056$; the difference from the MG calculation is $0.00054 \pm$ 0.00074. This difference is less than one standard deviation, so the estimates are therefore statistically equivalent. The MULTIREGION processing used in this problem provides sufficiently accurate cross sections to reproduce the CE solution.

\subsubsection{DOUBLEHET Example: Infinite, Square-pitched Array of Pebbles}

This sample problem is based on a pebble bed modular reactor (PBMR) computational benchmark organized by the OECD/NEA [6]. Each fuel pebble contains 15,000 TRISO grains in a graphite matrix, and each grain is $\mathrm{UO}_{2}$ with an enrichment of $9.6 \mathrm{wt} . \%{ }^{235} \mathrm{U}$, and the balance is ${ }^{238} \mathrm{U}$. The $\mathrm{UO}_{2}$ density is $10.4 \mathrm{~g} / \mathrm{cm}^{3}$, and the radius of the fuel grain is $0.02397 \mathrm{~cm}$. Each kernel has four coatings: a porous carbon layer, a pyrolytic carbon layer, a silicon carbide layer, and another pyrolytic carbon layer. The dimensions and densities of these layers is provided in Table 3 . The radius of the fuel region is $2.5 \mathrm{~cm}$, the total pebble radius is $3.0 \mathrm{~cm}$, and the pitch of the pebbles is $6.1 \mathrm{~cm}$. The outer layer of the pebble is composed of graphite; the matrix and pebble outer layer graphite has a density of $2.1 \mathrm{~g} / \mathrm{cm}^{3}$. Schematics of the grain and pebble are shown in Figure 224. Helium gas is the coolant flowing through the pebble bed at a density of $0.0061 \mathrm{~g} / \mathrm{cm}^{3}$; for simplicity in this problem, all materials are assumed to be at $700 \mathrm{~K}$. 
Table 3. Thickness and density of grain coatings

\begin{tabular}{|l|c|c|}
\hline Coating material & Material density & Coating thickness $(\boldsymbol{\mu m})$ \\
\hline Porous carbon & 1.05 & 95 \\
\hline Pyrolytic carbon & 1.90 & 40 \\
\hline Silicon carbide & 3.18 & 35 \\
\hline Pyrolytic carbon & 1.90 & 40 \\
\hline
\end{tabular}

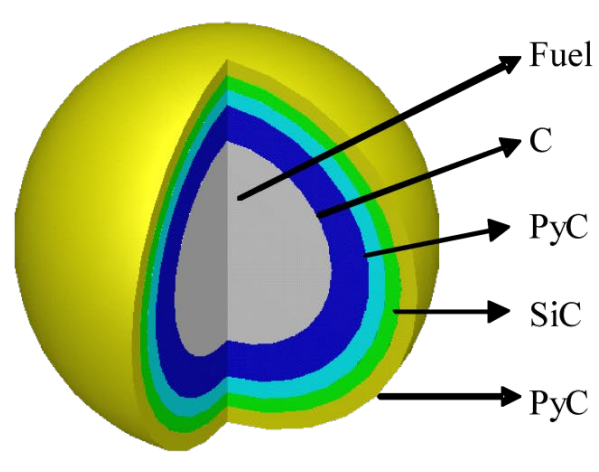

Grain

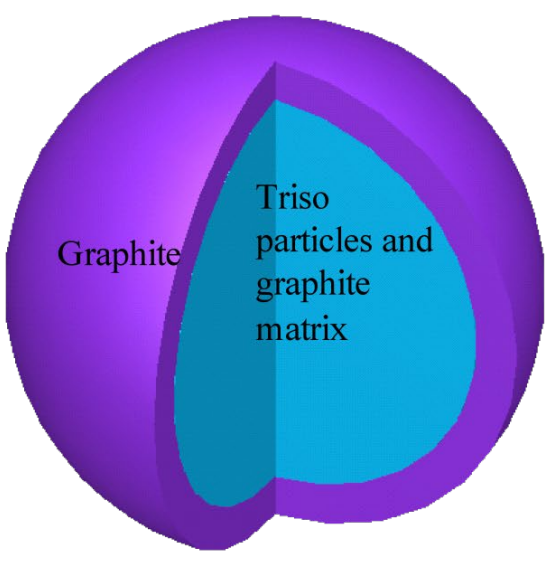

Pebble

Figure 224. Schematic of individual grain (right) and pebble (left).

\subsubsection{General and materials data}

The initial data entry for this example is the same as previous problems. Start Fulcrum and then select the New file... option from the File menu. Select a directory where the model will be saved, and name it pebble. inp. Next, use the autocomplete feature in Fulcrum to create a csas6 - Criticality safety analysis using KENO-VI input. Replace title-goes-here with Unit Cell - Pebble. Now change xs-lib-goes-here to $\mathrm{v} 7.1-252$ ( $\mathrm{v} 7.1-252 \mathrm{n}$ if using SCALE 6.2, 6.2.1, or 6.2.2). This selects the 252group neutron cross section library based on ENDF/B-VII.1 and signals to CSAS6 that this will be a MG KENO calculation. The appropriate cross section processing modules will be called using the input provided in the CELLDATA block as discussed in Section 8.5.3.2 below. The first three lines of the input are shown in Figure 225.

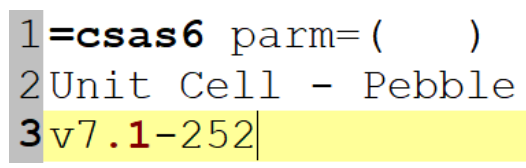

Figure 225. First three lines from pebble problem input.

A number of different mixtures must be specified in the COMPOSITION block because each mixture can only be used in one region in the CELLDATA block. A single $\mathrm{UO}_{2}$ mixture is needed, as well as the porous carbon mixture, two pyrolytic carbon mixtures, a SiC layer, a graphite matrix, a graphite pebble layer, and a helium coolant mixture. 
The first mixture to define is the $\mathrm{UO}_{2}$ fuel mixture. $\mathrm{UO}_{2}$ is a basic standard composition, so the configurable form can be used to create the composition input. With the cursor in the COMPOSITION block, choose stdcomp - basic (configurable) to launch the configurable form. Type uo 2 into the Composition field. Check the box for Theoretical Density and enter $10.4 \mathrm{~g} / \mathrm{cm}^{3}$ in the associated field; leave the Volume Fraction as 1.0. Check the box for Temperature and enter $700 \mathrm{~K}$ in the associated field. Check the box for Isotopic Weight Percents and click the Add row button at the bottom of the pane. Select 92235 for the Isotope and enter $9.6 \mathrm{wt}$ \%. Add another row by clicking the + button on the $\mathbf{9 2 2 3 5}$ row or the Add row button and select $\mathbf{9 2 2 3 8}$ and 90.4 wt.\%. After confirming that the input is correct, press the $\mathbf{O K}$ button to add the $\mathrm{UO}_{2}$ composition definition to the input. A picture of the final configurable form is provided in Figure 226.

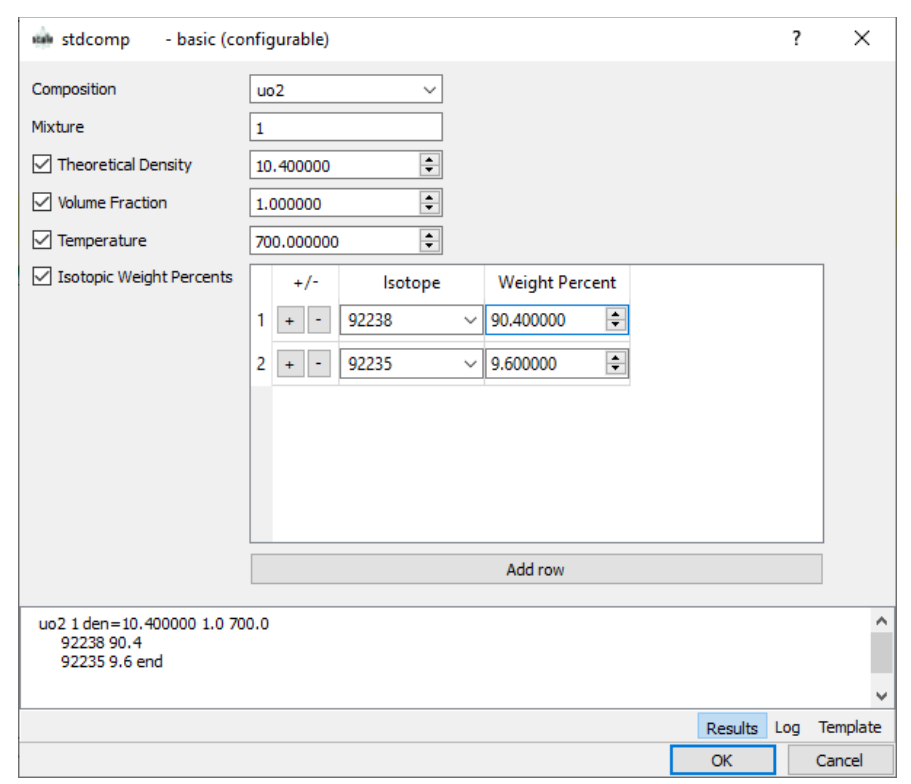

Figure 226. Configurable form for $\mathrm{UO}_{2}$ composition.

The next four mixtures will be for the coatings on the grain. The porous and pyrolytic carbon layers will be specified as graphite to use the graphite thermal scattering law (TSL) data, which is the most appropriate option available in ENDF/B-VII.1. The $\mathrm{SiC}$ layer will be created as an ATOM mixture.

Create a new standard basic composition configurable form and enter graphite in the Composition field. Make this Mixture 2, check the Theoretical Density check box, and enter $1.05 \mathrm{~g} / \mathrm{cm}^{3}$ in the associated field. Check the Temperature box and enter $700 \mathrm{~K}$ in the field. After checking the input, press the $\mathbf{O K}$ button to add the composition input to the CSAS input. A picture of the final configurable form is provided in Figure 227. Once the mixture has been defined in the COMPOSITION block, copy the line of input and paste it on the next line. Change the mixture number to 3 and the theoretical density to $1.90 \mathrm{~g} / \mathrm{cm}^{3}$. The updated composition block is shown in Figure 228. 


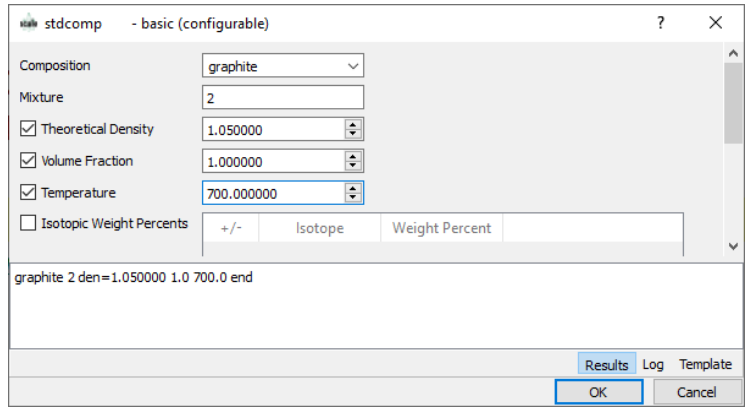

Figure 227. Configurable form for the porous carbon layer composition.

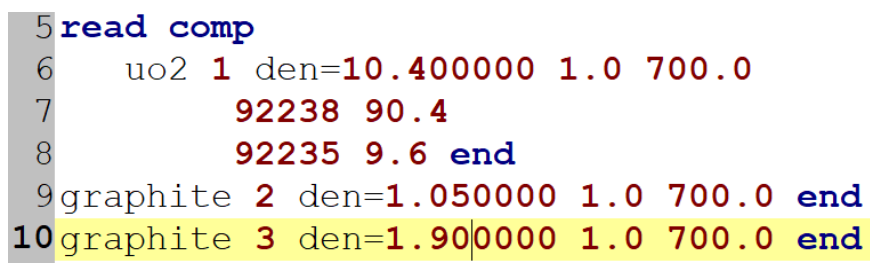

Figure 228. Input with $\mathrm{UO}_{2}$, porous carbon layer, and inner pyrolytic carbon layer compositions.

Mixture 4 will be the $\mathrm{SiC}$ layer; $\mathrm{SiC}$ is not a standard basic composition, but it can be entered as an ATOM composition because it has a chemical formula. With the cursor below the three mixtures already defined but still in the COMPOSITION block, autocomplete and select atomcomp - basic (configurable), as shown in Figure 229. It may be necessary to resize the configurable window, and especially the Properties pane, to show the Add button for Element Atom Counts. An empty but appropriately sized ATOM composition configurable form is shown in Figure 230. Change the text in the Name field to SiC and set this as Mixture 4. Set the Theoretical Density to $3.18 \mathrm{~g} / \mathrm{cm}^{3}$ and the Temperature to $700 \mathrm{~K}$. Click the Add button next to Element Atom Counts, select $\mathbf{1 4 0 0 0}$ from the Element dropdown menu to select $\mathrm{Si}$, and set the Atom Count to 1. Click the Add button next to Element Atom Counts again, select $\mathbf{6 0 0 0}$ from the Element dropdown menu to select $C$, and again set the Atom Count to 1. After checking the input, press the OK button to add the $\mathrm{SiC}$ composition to the input. The complete configurable form is shown in Figure 231; note that the carbon data are showing since it is impossible to display both the $\mathrm{Si}$ and $\mathrm{C}$ pages at the same time.

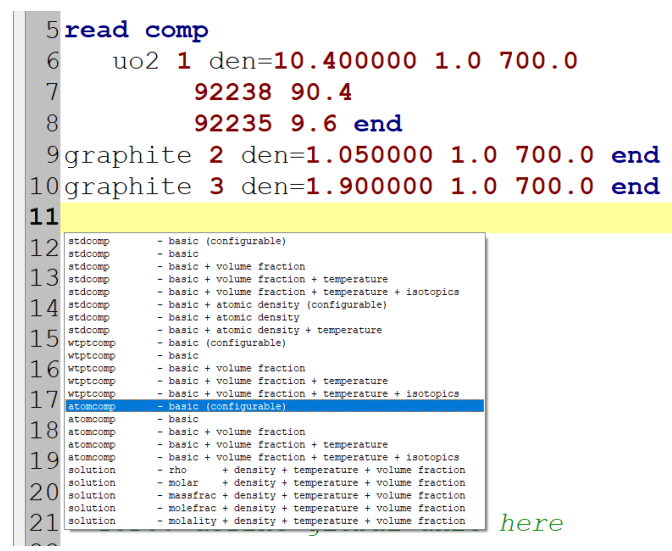

Figure 229. Selecting ATOM mixture configurable form in Fulcrum. 


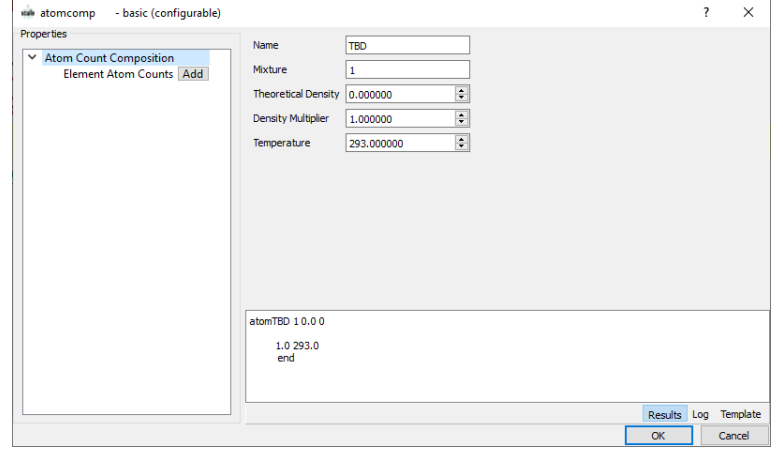

Figure 230. ATOM mixture configurable form showing all necessary buttons and fields.

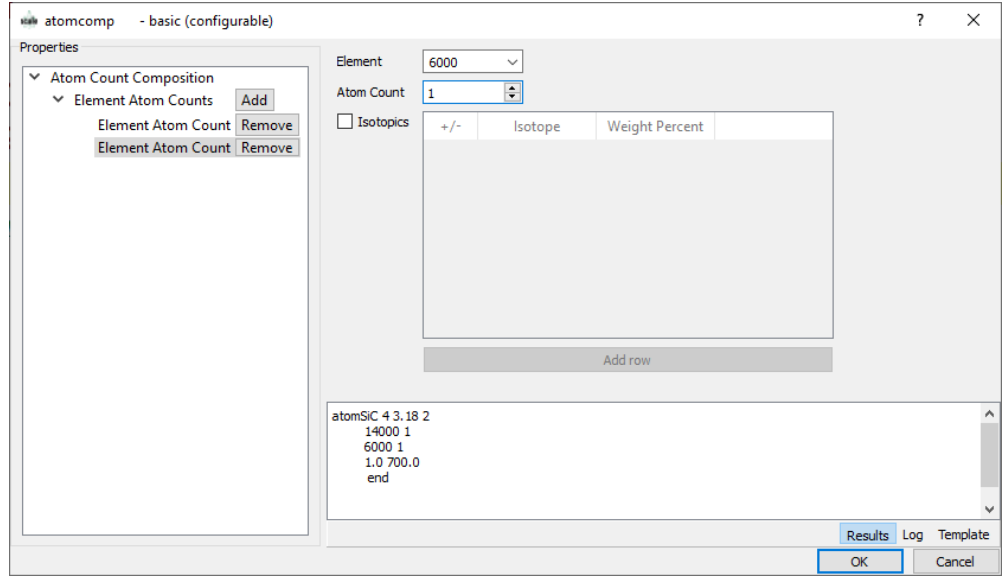

Figure 231. Complete ATOM composition configurable form for the SiC layer composition.

Copy the input for the inner pyrolytic carbon layer (mixture 3) and paste it below the atomSiC input. The only necessary modification to the input is to change the mixture number to 5 . Figure 232 shows the first five mixture inputs in the COMPOSITION block. The next two compositions needed are the graphite for the matrix and the outer coating of the pebble. These can also be created by copying the mixture 5 graphite and pasting it into the input. These mixtures should be 6 for the matrix and 7 for the outer layer of the pebble. Both these mixtures have a density of $2.1 \mathrm{~g} / \mathrm{cm}^{3}$. The increased COMPOSITION block is shown in Figure 233. This completes the mixtures needed for the pebble definition.

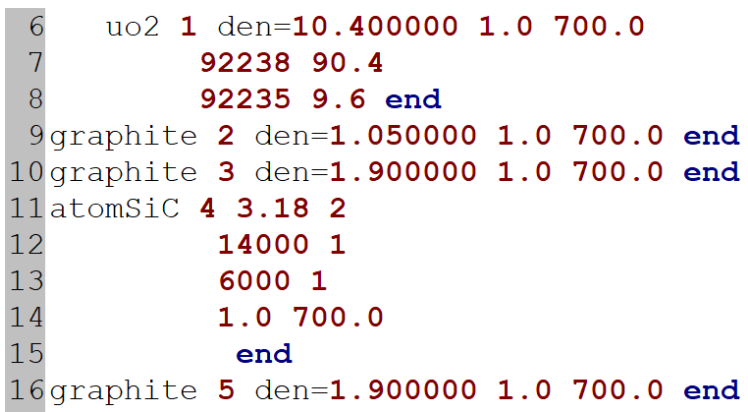

Figure 232. Mixture definitions for all composition in the fuel grains. 


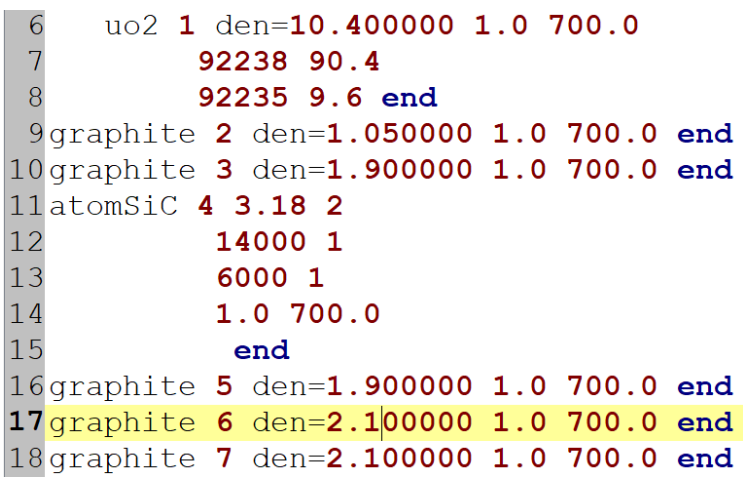

Figure 233. Composition definitions for all mixtures in the pebble.

The last mixture definition for this problem is the helium coolant. This can be added as a standard basic composition using a configurable form. Use the Composition name he (be sure to select the element and not one of the isotopes), set the Mixture number to 8, check the box for Theoretical Density, and set it to $0.0061 \mathrm{~g} / \mathrm{cm}^{3}$. Finally, check the box for Temperature and set it for $700 \mathrm{~K}$. After checking the input, press the $\mathbf{O K}$ button to add the helium definition to the input; the complete configurable form is shown in Figure 234.

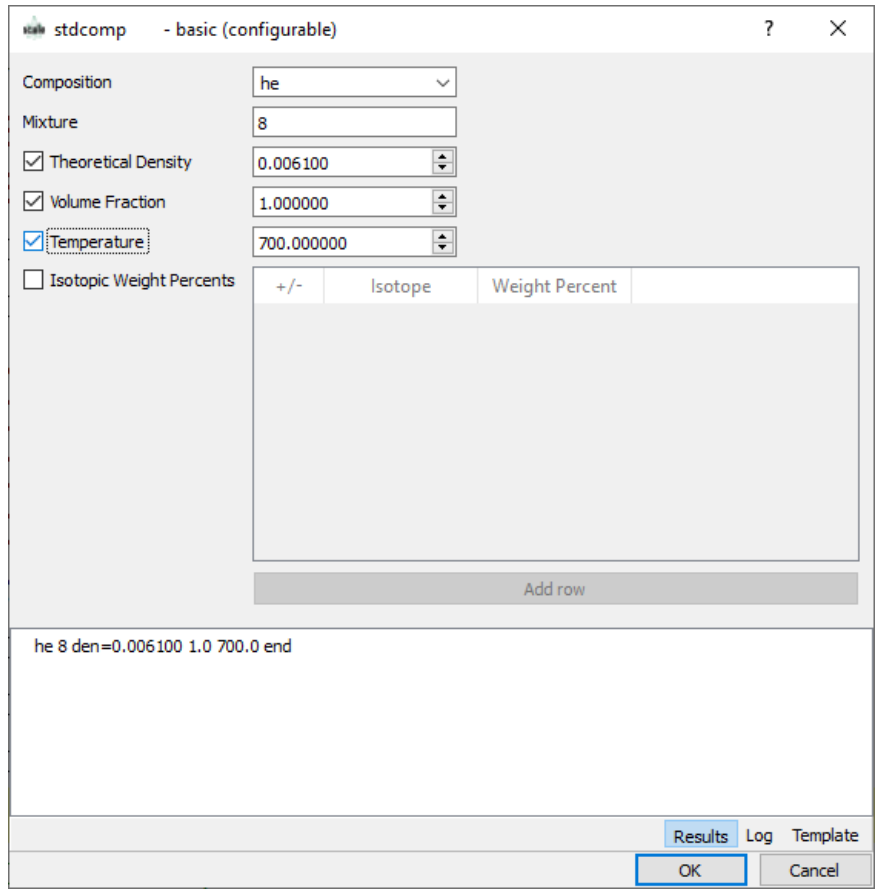

Figure 234. Complete configurable form for helium coolant mixture.

Comments should be added in the input to clarify which mixtures are to be used for which regions. This will make future updates and reviews much easier. Recall that SCALE interprets an entire line as a comment if it starts with an apostrophe (' ) in column 1. Insert a blank line above the graphite mixture that is Mixture 2 by placing the cursor in column one and pressing enter. Move back to this new blank line, place an apostrophe in column 1, and enter the following comment: Mixture 2 is porous graphite layer. The updated input is shown in Figure 235. Repeat this process to add comments for Mixture 3('Mixture 3 is inner pyrolytic carbon layer), Mixture 4 (' Mixture 4 is silicon carbide layer), Mixture ('Mixture 5 is outer pyrolytic carbon 
layer), Mixture 6(' Mixture 6 is graphite matrix), and Mixture 7(' Mixture 7 is graphite pebble cladding).

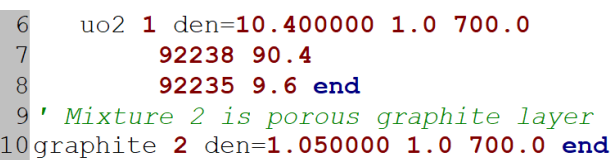

Figure 235. Comment added to input.

This completes the mixture specifications in the COMPOSITION block for this model. The complete input block is shown in Figure 236. Blank lines and extraneous comments have been removed from the input for clarity in the figure.

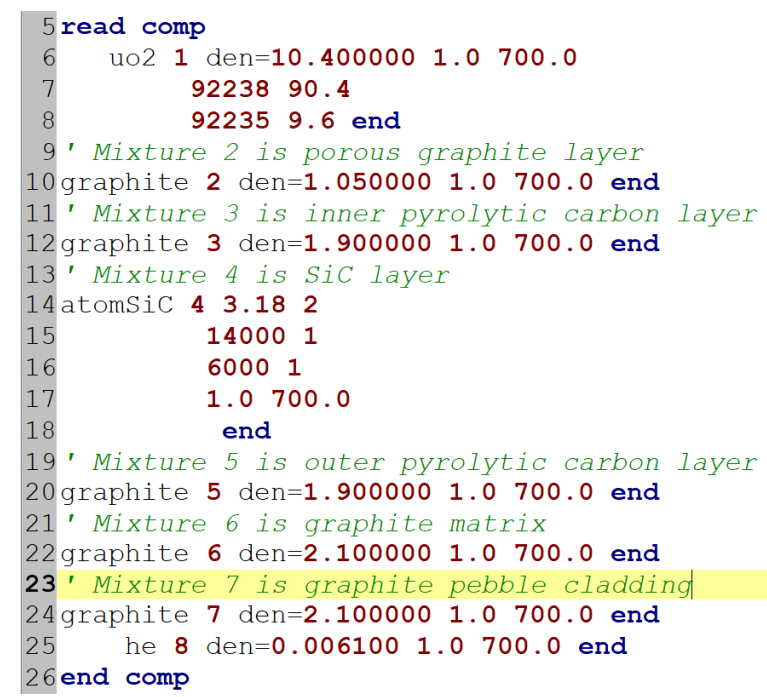

Figure 236. Entire COMPOSITION block for the pebble model.

\subsubsection{Unit cell data}

The unit cell information for the cross section treatment can be specified now that the material information has been entered. One of the four unit cell types (INFHOMMEDIUM, LATTICECELL, MULTIREGION, DOUBLEHET) will be specified to process the MG cross sections for this problem. A DOUBLEHET cell will allow cross section processing to be performed accurately for this problem. The individual grain and coatings will be specified, and the PEBBLE option will allow the description of the pebble and unit cell boundary conditions to approximate the infinite array of pebbles.

To add the CELLDATA block, place the cursor between the COMPOSITION and GEOMETRY blocks and autocomplete for a list of available blocks to add. Select cells as show in Figure 237. There are no configurable forms currently available in the CELLDATA block. Autocompleting in the CELLDATA block provides a list of available unit cell specifications; select the doublehet - pebble + sphsquarep option as shown in Figure 238. Fulcrum inserts generic CELLDATA input, as shown in Figure 239, which must be modified for use in this model. 


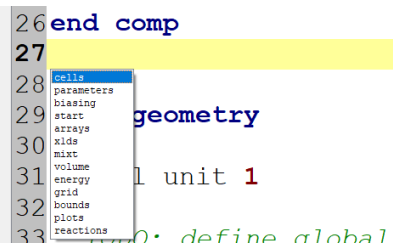

Figure 237. Autocomplete list of available blocks for inclusion in the model

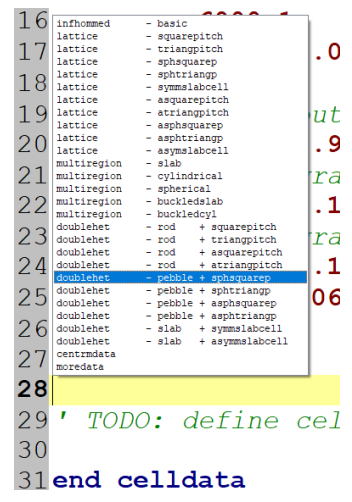

Figure 238.Autocomplete list of cells within the CELLDATA block.

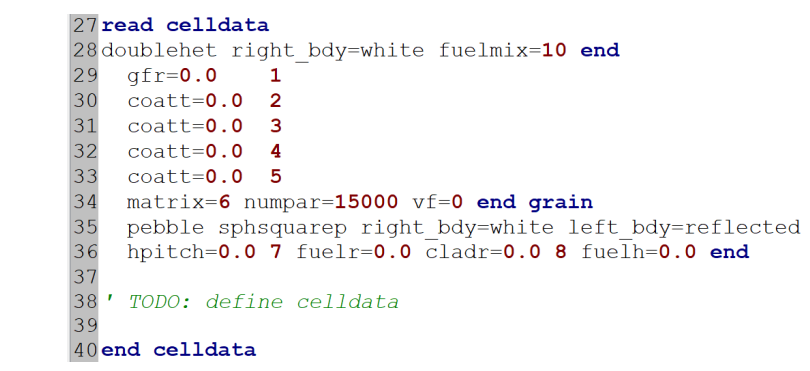

Figure 239. Templated input provided for the DOUBLET PEBBLE + SPHSQUAREP cell in Fulcrum.

The first line declares the cell type as DOUBLEHET, as intended, and it specifies the right boundary condition of the grain cell as white. This white boundary is the desired boundary condition because it simulates a single grain in an infinite array of grains, which is a good approximation for the grains in the matrix. The fuelmix specified is the mixture number to be used for the homogenized cross sections for the fuel/matrix region; it must be a number that is not already specified in the COMPOSITION block, so $\mathbf{1 0}$ is an acceptable number to use for this model. The next section of input provides the fuel grain and coating dimensions and mixture numbers. The template input includes the radius of the fuel grain and the thicknesses of the coatings. Alternatively, the diameter can be entered for the fuel grain with the keyword gfd. The coatings can be specified with coatr or coatd to provide the monotonically increasing radii or diameters of the coatings; there is no practical limit on the number of coatings that can be used in a DOUBLEHET cell specification. The template keywords are convenient for the problem details provided in Section 8.5.3. The fuel grain radius (gfr) should be set to 0.02397 ; the $\mathrm{UO}_{2}$ fuel is mixture 1, so no change is necessary. Four coatings are included in the template input, and this model also includes 4 coatings. The relevant mixture numbers are also 2 through 5 for this model. Only the coating thicknesses provided in Table 3 need to be entered. The input is in centimeters, but the thicknesses are provided in $\mu \mathrm{m}$, so the entries are $0.0095,0.0040,0.0035$, and 0.0040 , respectively. The next line specifies the matrix containing the coated grains, and mixture 6 (graphite) is the desired mixture for this model. The templated number of particles in each pebble (15000) is also applicable to this model. The $\mathbf{v f}=\mathbf{0}$ input 
should be removed; the volume fraction can be specified in lieu of the number of particles. The end of the grain specification is declared with end grain; multiple different types of grains could be used in a single DOUBLEHET cell in CSAS. The completed grain section of the input is provided in Figure 240.

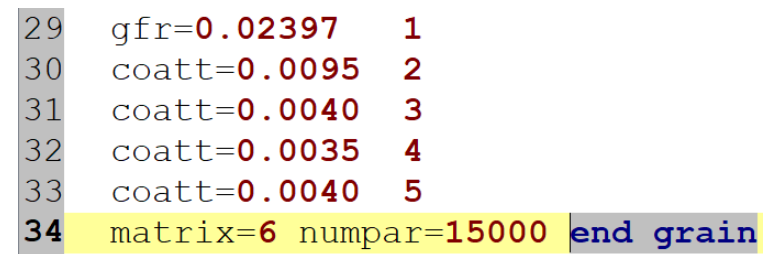

Figure 240. Grain and coating details for the DOUBLEHET unit cell for this model.

The last section of the input deals with the pebble level of the problem. The keyword pebble specifies that this DOUBLEHET cell is based on pebbles and not cylindrical compacts or slabs. The type of pebble cell is sphsquarep for spherical bodies in a square pitched array; the options for these geometries are essentially the same as for the MULTIREGION cell type. The white boundary condition is applied to approximate an infinite array of pebbles, and the left boundary is specified as reflected because it is the inner "surface" of the sphere. The half-pitch (hpitch) for this model is $3.05 \mathrm{~cm}$, and mixture $8(\mathrm{He})$ is the mixture for the region between the pebbles. The fueled region has a radius (fuelr) of $2.5 \mathrm{~cm}$; no mixture number is specified here because the homogenized cross sections for the fuel/matrix region are calculated by the code and assigned to the mixture number provided earlier in the input for the fuelmix parameter. The cladr input provides the outer radius of the overall pebble and the mixture number for the material around the fuel/matrix fuel region; in this model, the radius is $3.0 \mathrm{~cm}$, and the "cladding" is mixture 7 . The fuelh=0 input should be removed since it specifies the height of a cylindrical or slab compact. The final DOUBLEHET unit cell specification is provided in Figure 241; as before, extraneous comments and blank lines have been removed for simplicity.

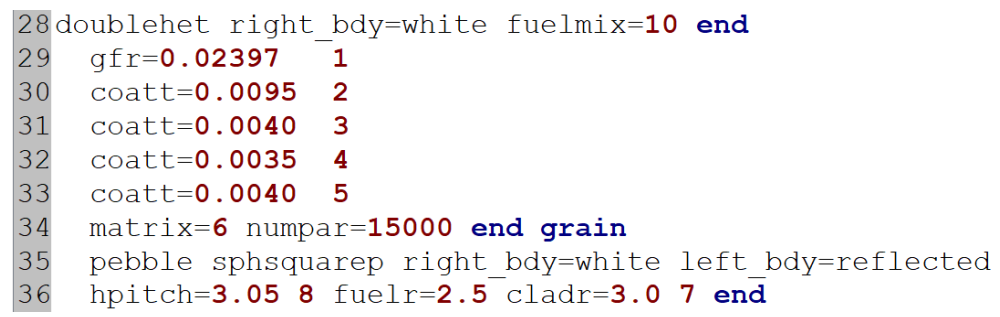

Figure 241. Complete DOUBLEHET unit cell specification for this model.

\subsubsection{KENO-VI Geometry data}

The KENO-VI data for this problem consists of a single unit containing a single pebble and the helium coolant around it. Reflective boundary conditions will be applied to all 6 faces of the cuboid to approximate an infinite array of pebbles. This problem differs from the previous two example problems because it contains a homogenized region for the fuel/matrix region inside the pebble. The homogenization in this case significantly accelerates tracking because it eliminates tracking through the 15,000 explicit grains.

The innermost region of the problem is the homogenized fuel/matrix region. This is a sphere with a radius of $2.5 \mathrm{~cm}$, followed by graphite "cladding" and the cuboid with the helium coolant. The initial sphere can be added via a configurable form by placing the cursor in the global unit and selecting sphere - kenovi (configurable) as shown in Figure 242. Set the Id to 10 and the radius of this region is $2.5 \mathrm{~cm}$; the completed configurable form is shown in Figure 243. After confirming that the input is correct, press the 
OK button to enter the sphere definition in the CSAS input. The next region is the graphite "cladding," or outer layer, on the pebble. Add this second region in the input by entering sphere 203.0 or by using a sphere configurable form. The final region is a CUBOID filled with helium for the region between the pebbles; the dimension in $\pm \mathrm{X}, \pm \mathrm{Y}$, and $\pm \mathrm{Z}$ is $3.05 \mathrm{~cm}$. Add the cuboid by entering cuboid 303.05 $-3.053 .05-3.053 .05-3.05$ or by using a cuboid configurable form. This completes the geometry records as shown in Figure 244.

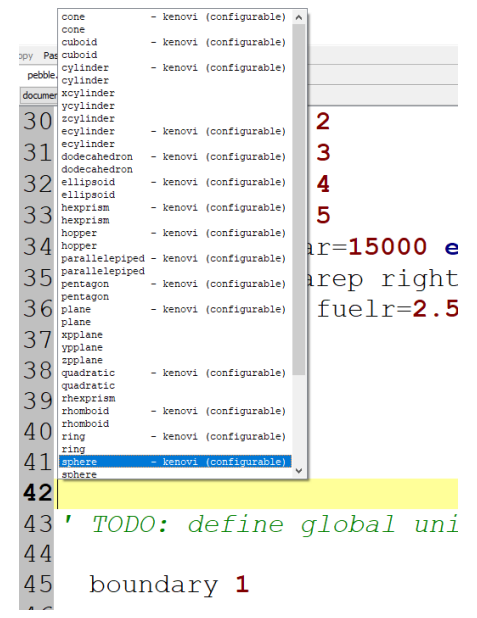

Figure 242. Selecting the sphere configurable form in Fulcrum.

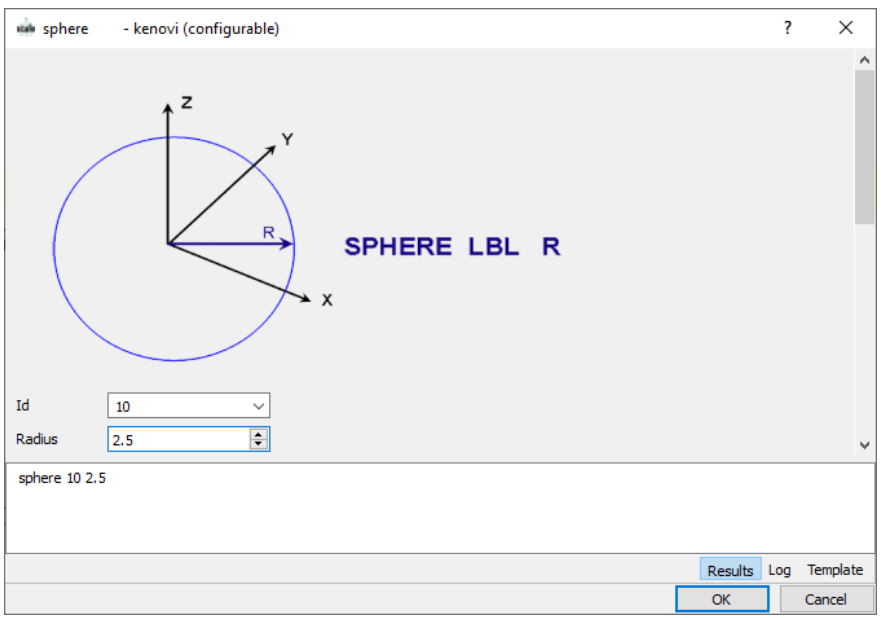

Figure 243. Complete configurable form for the fuel/matrix region of the pebble.

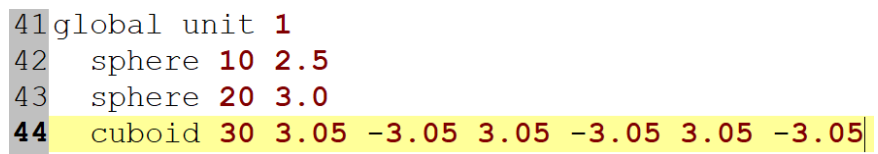

Figure 244. All three geometry records for the pebble model.

The next step is to define the contents records, which in this case are all MEDIA records. The first media record fills the innermost sphere with the homogenized mixture of grains and matrix generated in the CELLDATA block and assigned to Mixture 10. The media entry can be directly entered as media 10 110 or through the use of a configurable form. The next region is the graphite "cladding" on the pebble, which is mixture 7. Again, the media record can be entered directly as text in Fulcrum as media 71 
$20-10$ or with the configurable form. The media record specifies that graphite is to be placed inside the larger sphere but outside the smaller inner sphere. The final region is the helium coolant outside the pebble, which is mixture 8 . The text input is media $8 \quad 1 \quad 30-20$, or this can be entered using the media - kenovi (configurable) form. This input fills the volume inside the cuboid but outside the larger sphere with helium. The last entry in the GEOMETRY block is the boundary record. This must be updated to boundary 30, as everything within cuboid 30 is part of the model. The complete GEOMETRY block is shown in Figure 245.

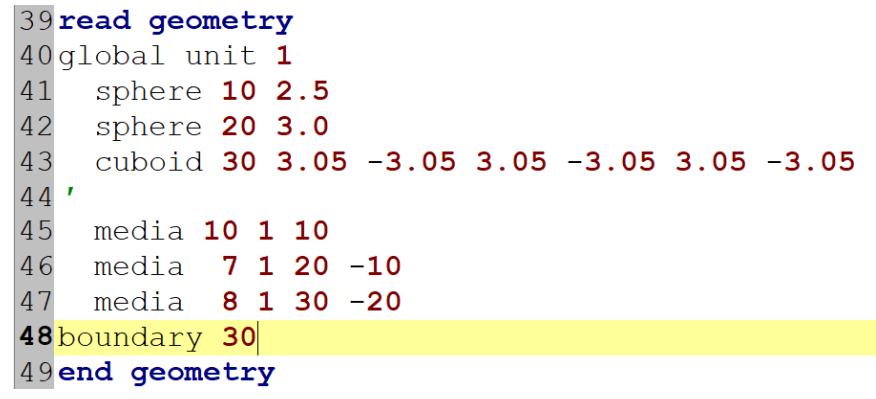

Figure 245. Complete geometry block for the pebble model.

The last step is to add reflective boundary conditions to all 6 faces in the problem; recall that the global unit boundary conditions are specified in the BOUNDS block. To add the BOUNDS block, position the cursory outside the other blocks and use the Fulcrum autocomplete function to add a new block, as shown in Figure 246. Autocomplete is not available in the BOUNDS block. The most efficient way to set all the faces to reflective boundaries is to use the cuboid face code from the first portion of the Face Codes and Surface Numbers table [see SCALE Manual Section Albedo data] and enter all=refl. The more explicit method is to specify the boundary condition on each face of the cuboid. This approach is shown in Figure 247. Both methods will set all 6 faces of the cuboid to reflective boundary conditions and generate equivalent models.

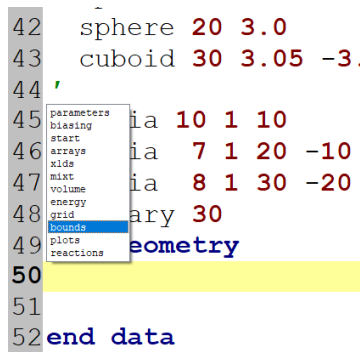

Figure 246. Adding the BOUNDS block to the model in Fulcrum with autocomplete.

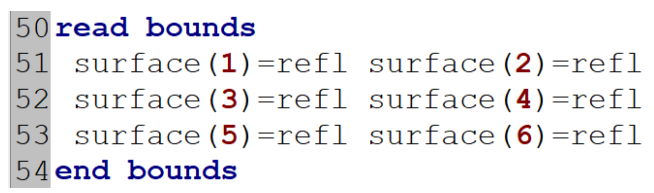

Figure 247. Complete BOUNDS block for the pebble model.

Now save all changes and run SCALE by clicking the Run button at the top of the Fulcrum window. The default run parameters of 1,000 particles per generation, 203 total generations, and 3 discarded generations will be used if no specific values are chosen by adding a PARAMETER block. These parameters yield a $k_{\text {eff }}$ estimate of $1.5358 \pm 0.0020$; exact values will vary, as different computers and different operating systems generate different random numbers. A more rigorous calculation simulating 
10,000 particles per generation for 197 total generations, skipping the first 50, resulted in a $k_{\text {eff }}$ estimate of $1.53833 \pm 0.00049$. Homogenized cross sections cannot be generated for CE calculations, so the more difficult explicit model would need to be built to check this model. The explicit representation of the 15,000 grains brings additional challenges in terms of distribution and truncation; these issues are not discussed here, as they are beyond the scope of this primer.

\subsection{SUMMARY}

This section has demonstrated:

- the difference in cross section processing for Infinite Homogeneous Medium, Lattice Cell, Multiregion, and Double Heterogeneous analyses;

- identification of the appropriate unit cell type to use for various heterogeneous systems;

- using Fulcrum to define LATTICECELL, MULTIREGION, and DOUBLEHET unit cells;

- defining different boundary conditions for a MULTIREGION unit cell; and

- using boundary conditions to create reflective boundaries for the KENO-VI global unit. 


\section{PLOTTING AND VISUALIZATION}

A strongly recommended practice for all KENO users is to visually check the geometry models prior to performing calculations. This section discusses the use of the 2D and 3D plotting and visualization tools available with KENO-VI and Fulcrum.

\subsection{WHAT YOU WILL BE ABLE TO DO}

- Generate 2D color plots of KENO-VI geometry models.

- View 2D source convergence diagnostics generated by KENO-VI.

- Generate 2D plots of nuclear data used in KENO-VI calculations.

- Generate and display mesh-based data in Fulcrum.

- Interactively view 3D wireframe and rendered images of KENO-VI geometry models using KENO3D.

- Plot calculated results overlain on the 3D geometry model using KMART6 and KENO3D.

\subsection{CREATING AND VIEWING 2D PLOTS WITH FULCRUM}

The Fulcrum interface contains a robust interface for visualizing 2D data, including geometry plots, cross section data, scattering and covariance matrices, and generic plot data. This section introduces the plotting capabilities of Fulcrum; a more complete description can be found in Lefebvre's "Advanced User Interface Capabilities" [7].

\subsubsection{Geometry}

CSAS and KENO-VI have historically had a capability to produce plots of 2D KENO-VI geometry slices. These plots can be generated without performing a calculation by using the PARM=CHECK option on the first line of the CSAS/KENO-VI input. This option provides the user with the capability to check their geometry model prior to performing calculations [see SCALE Manual Section Plot data].

An easier approach to generating 2D plots normal to the X-, Y-, and Z-axes is to use the 2D plotting capability in Fulcrum. This capability is used in Sections 8.5.1.3 and 8.5.2.3 to display models as they are being built. The geometry viewer can be launched by clicking the View button near the top of the editor window, by pressing $\mathrm{C} t r l+S h i f t+V$, or by right clicking the geometry block name in the navigation panel and selecting Visualization. These options are shown in Figure 248 and Figure 249 using the fuel assembly model from Section 8.5.1. This model is used to demonstrate the features of the Fulcrum geometry plotting capabilities, but the features are applicable to all models. Note that Fulcrum will not launch visualization without a global unit in the model, and some parse and validation errors may also prevent visualization. Check the Messages pane in Fulcrum if a global unit exists and the geometry plotter does not launch. 


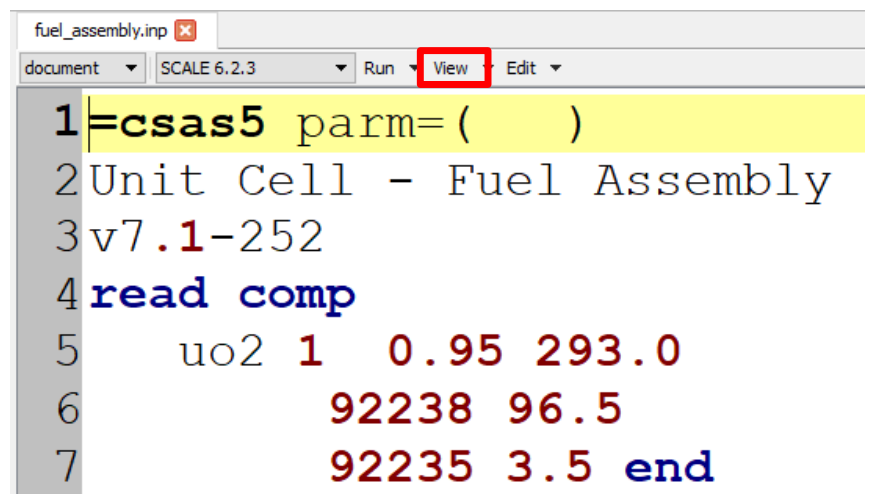

Figure 248. Location of View button in Fulcrum.

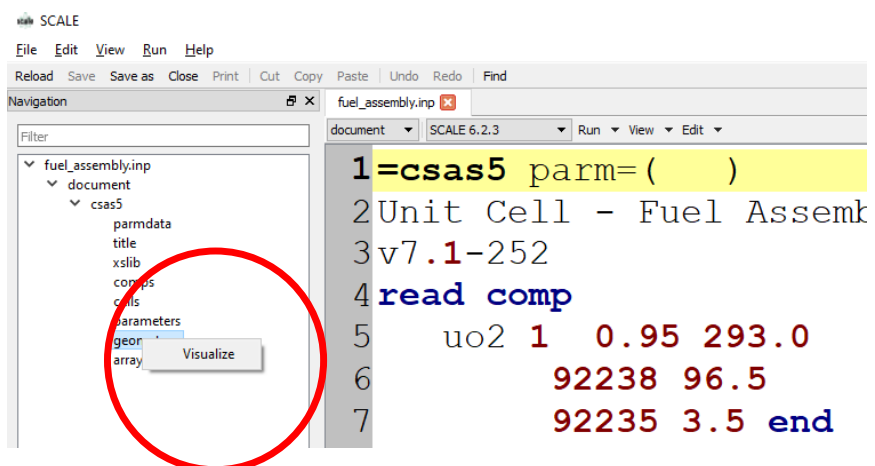

Figure 249. Navigation panel for visualizing a model.

The default initial slice presented in the plotter is an XY plot through the midplane of the model. The default view is shown in Figure 250; note that the plotter is launched in a separate tab within Fulcrum. The orientation of the plot view can be selected from the list of buttons just above the view window to the left edge of the tab. The three choices are Top (X-Y), Front (X-Z), and Side (Y-Z), as shown in Figure 251.

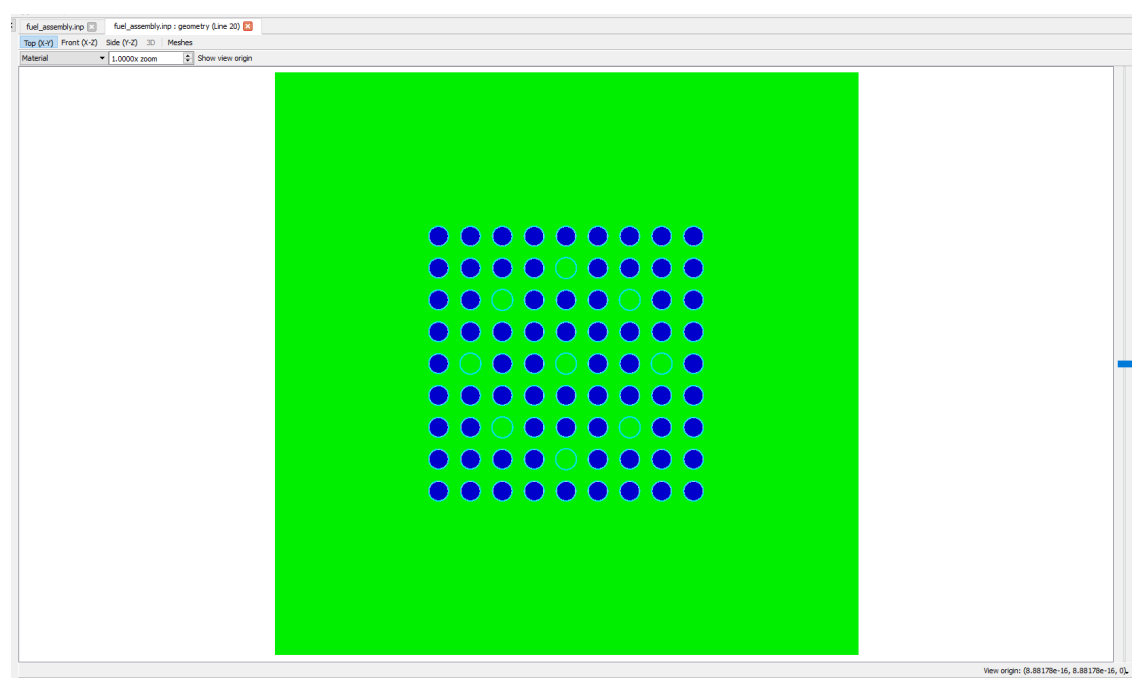

Figure 250. Default plot view of the fuel assembly model in the Fulcrum plotter. 


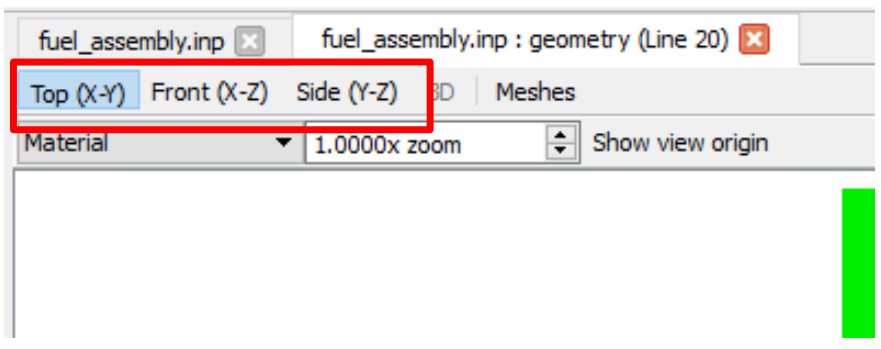

Figure 251. Selection of visualization plane orientation.

The bottom frame of the visualization window displays the current mouse pointer location on the bottom left, and the origin of the current view is shown on the bottom right, as presented in Figure 252. The view origin can be set by clicking on the View origin field in the lower right corner of the visualization frame. Entering one number will set the location of the visualization plane along the axis perpendicular to it. For example, click on the View origin field and enter 180. For the fuel assembly model, this creates an XY plane through the upper plenum of the fuel rods, as shown in Figure 253; note that the View origin has a $\mathrm{Z}$ coordinate of $\mathbf{1 8 0}$. Entering two numbers in the View origin field re-centers the view in the current plane at the specified coordinates. For example, click on the View origin field and enter $-6.56-6.56$. For the fuel assembly model, this centers the view on the center of the fuel rod in the lower left corner of the assembly; click the Show view origin button on the top of the visualization frame (Figure 254), and a crosshair will appear displaying the exact center of the view. Note that the View origin display shows -6.56 -6.56 180, as shown in Figure 255. Entering three numbers in the View origin field centers the visualization plane at the coordinates entered in $\mathrm{X}, \mathrm{Y}$, and $\mathrm{Z}$ as expected. Finally, the slider on the right side of the frame (blue rectangle on Windows and white circle on Mac), shown in Figure 256, allows control of the visualization plane along the axis to which it is perpendicular. For example, sliding up and down the frame controls the $\mathrm{Z}$ elevation of an $\mathrm{XY}$ visualization plane.

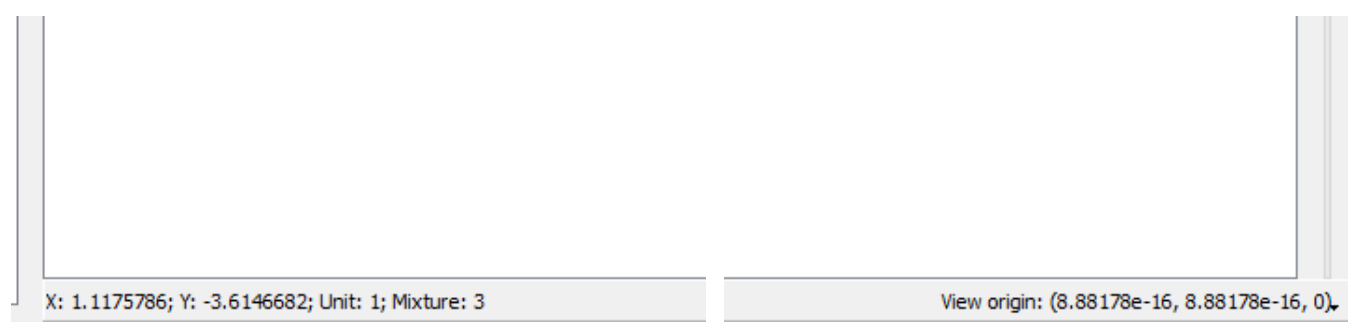

Figure 252. Mouse pointer location and view origin displays. 


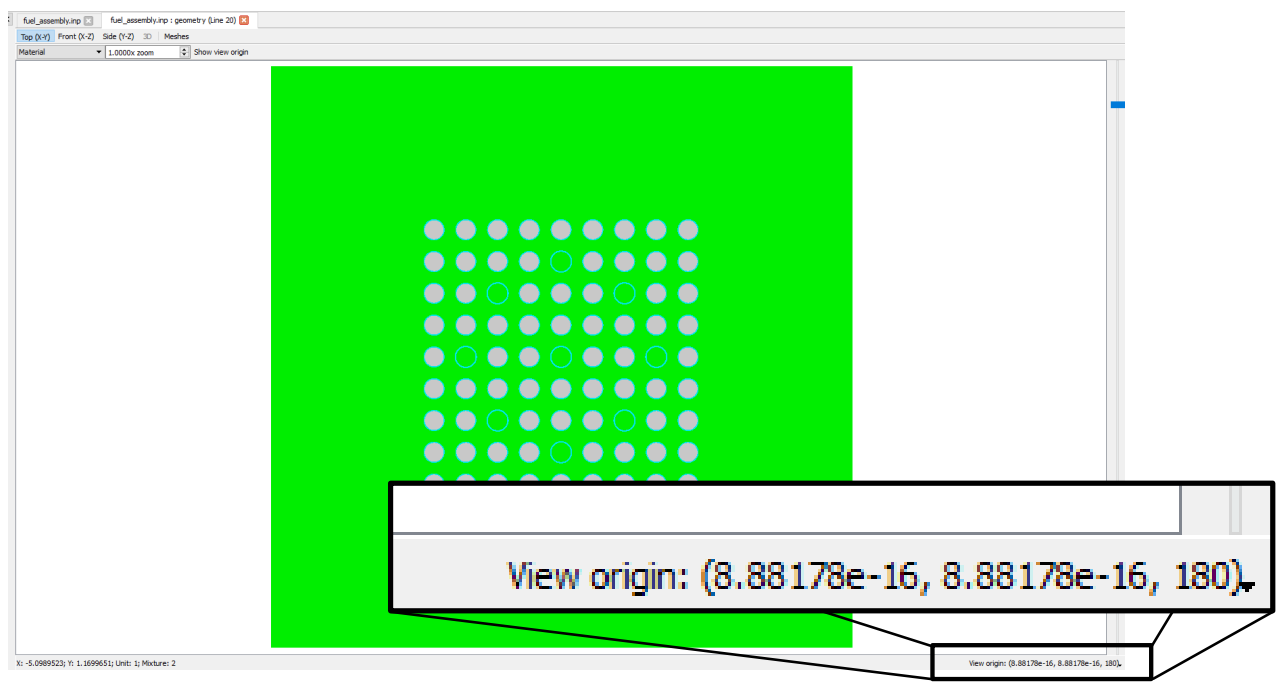

Figure 253. $X Y$ visualization at $Z=180 \mathrm{~cm}$ plane.

\begin{tabular}{|c|c|c|c|c|}
\hline \multicolumn{2}{|c|}{ fuel_assembly.inp $\mathrm{X}$} & \multicolumn{3}{|c|}{ fuel_assembly.inp : geometry (Line 20) $x$} \\
\hline $\operatorname{Top}(X-Y)$ & Front $(X-Z)$ & Side $(Y-Z) \quad 3 D$ & Meshes & \\
\hline Material & & $1.0000 \times$ zoom & 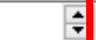 & Show view origin \\
\hline
\end{tabular}

Figure 254. Location of Show view origin button.

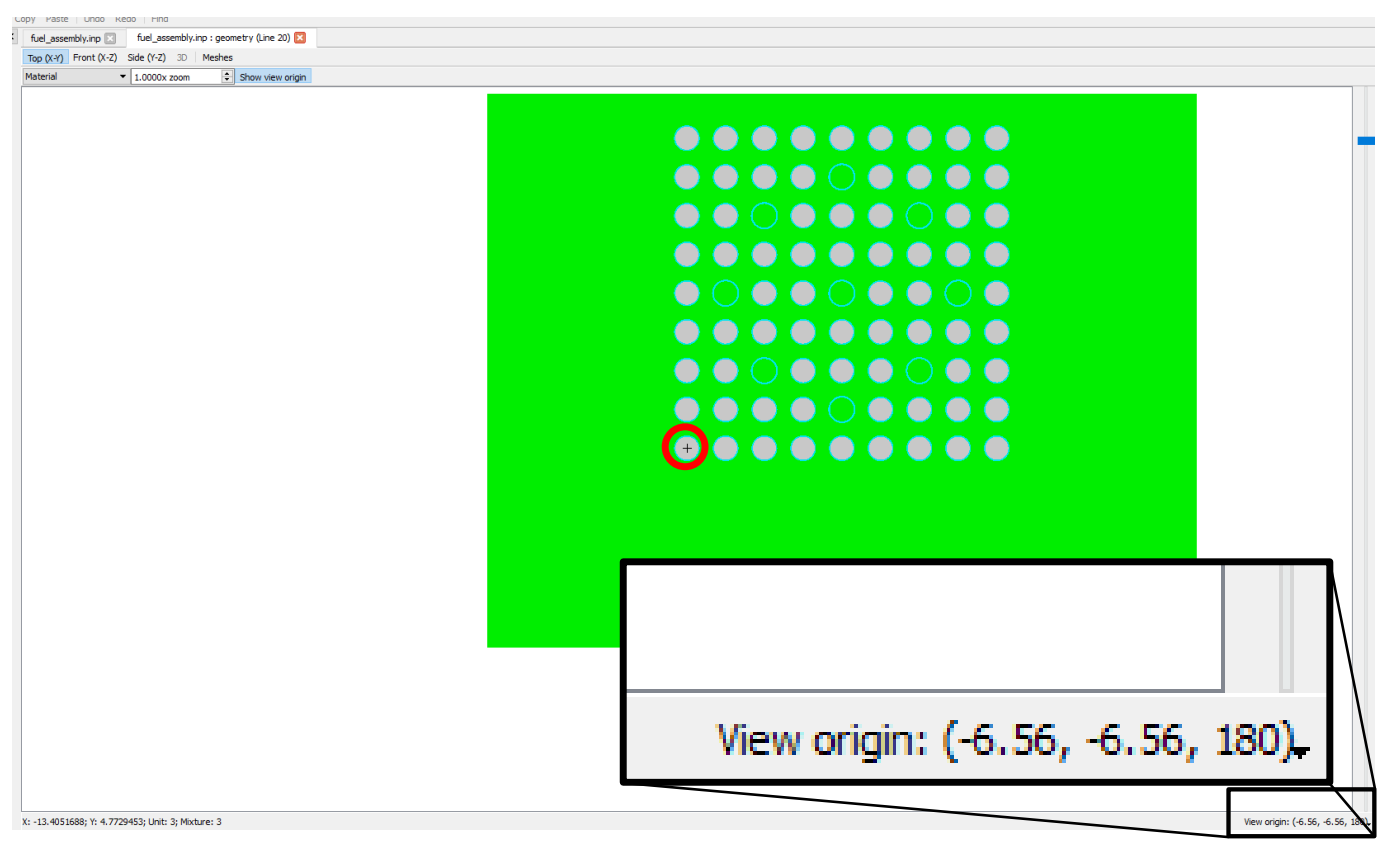

Figure 255. $X Y$ visualization centered at $(-6.56,-6.56)$ at $Z=180 \mathrm{~cm}$ plane. 


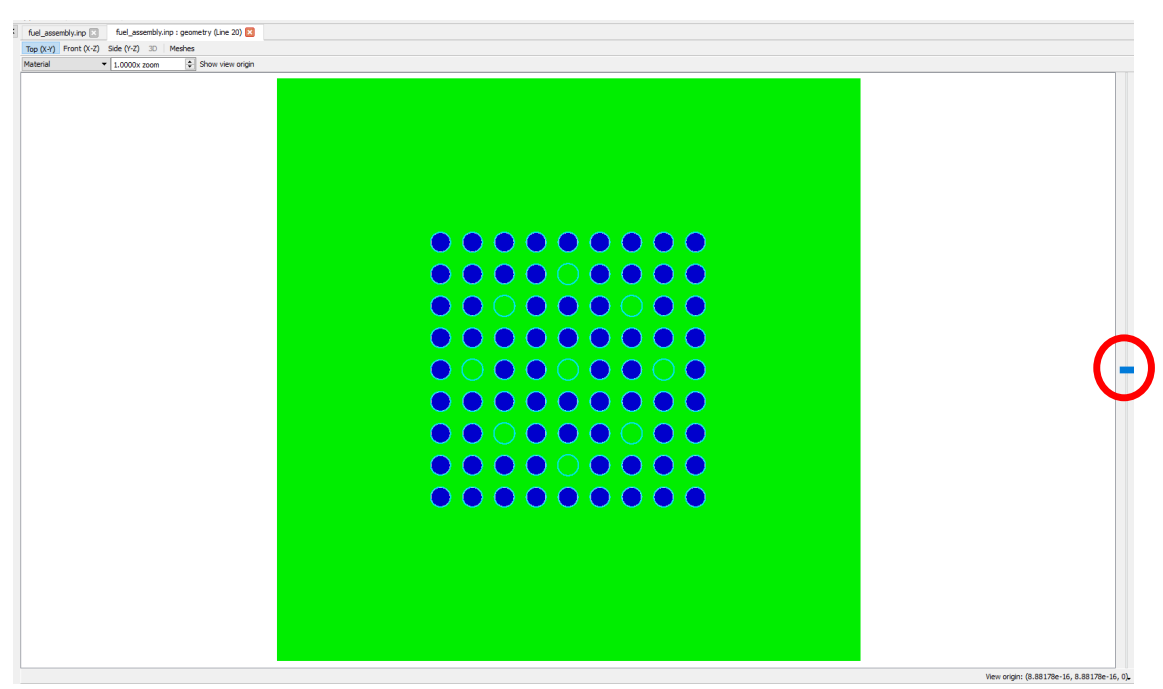

Figure 256. Location of slider to control position of the visualization plane.

Two key visualization controls are on the top of the visualization frame and control the render mode and zoom level of the current view. These controls are shown in Figure 257. The render mode dropdown menu is shown in Figure 258; the first three options are discussed here, and Overlay and Overlay + boundaries are discussed in Section 9.3. The default render mode is Material, and it colors the view according to the mixture number (material) in each region. No region boundaries are shown between regions of the same material unless the Material + outline render mode is selected. An example of this is shown in Figure 259; note that the unit boundaries are now shown in the array, as well as the outer boundary of the array. Region boundaries for the fuel pellet, gap, and cladding are also shown. The third option is for Outline only and displays the region boundaries in the color of the mixture in that region. An example is provided in Figure 260.

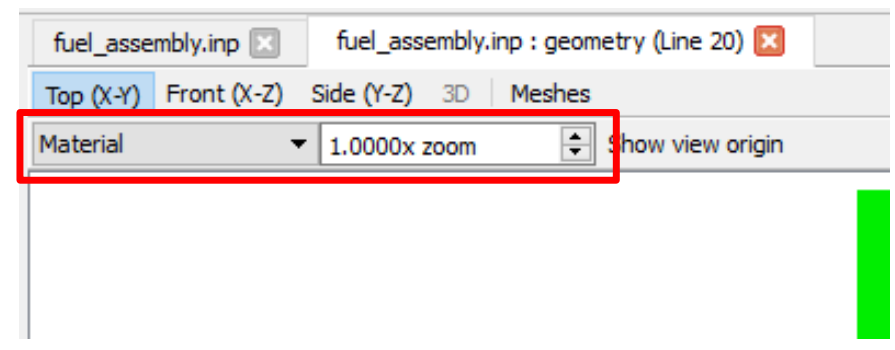

Figure 257. Display controls for render mode and zoom.

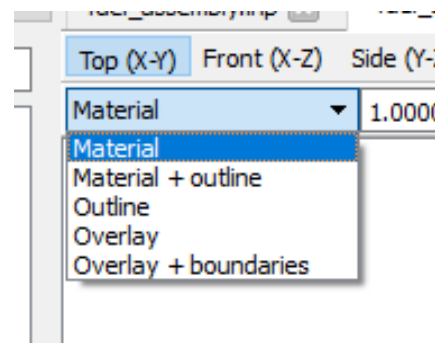

Figure 258. Render mode dropdown menu. 


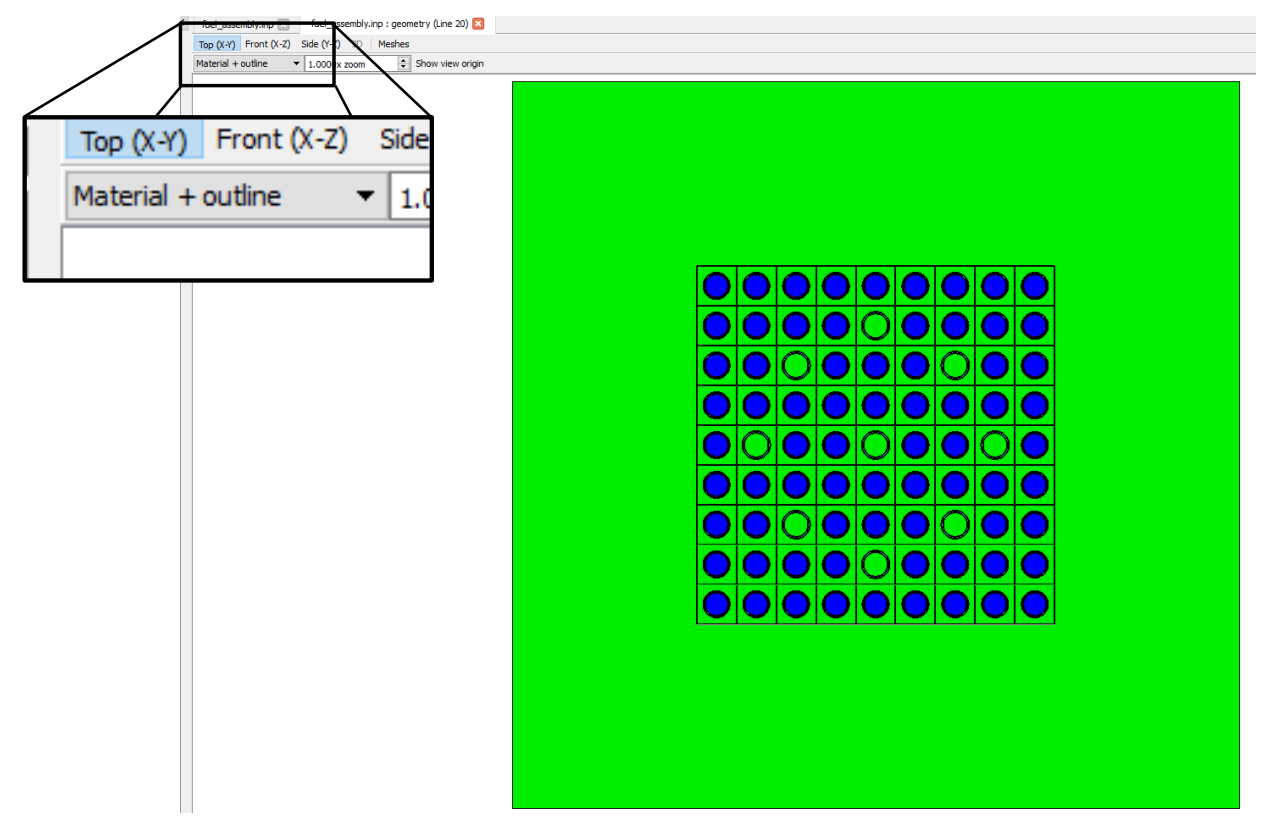

Figure 259. Midplane view of fuel assembly in Material + outline render mode.

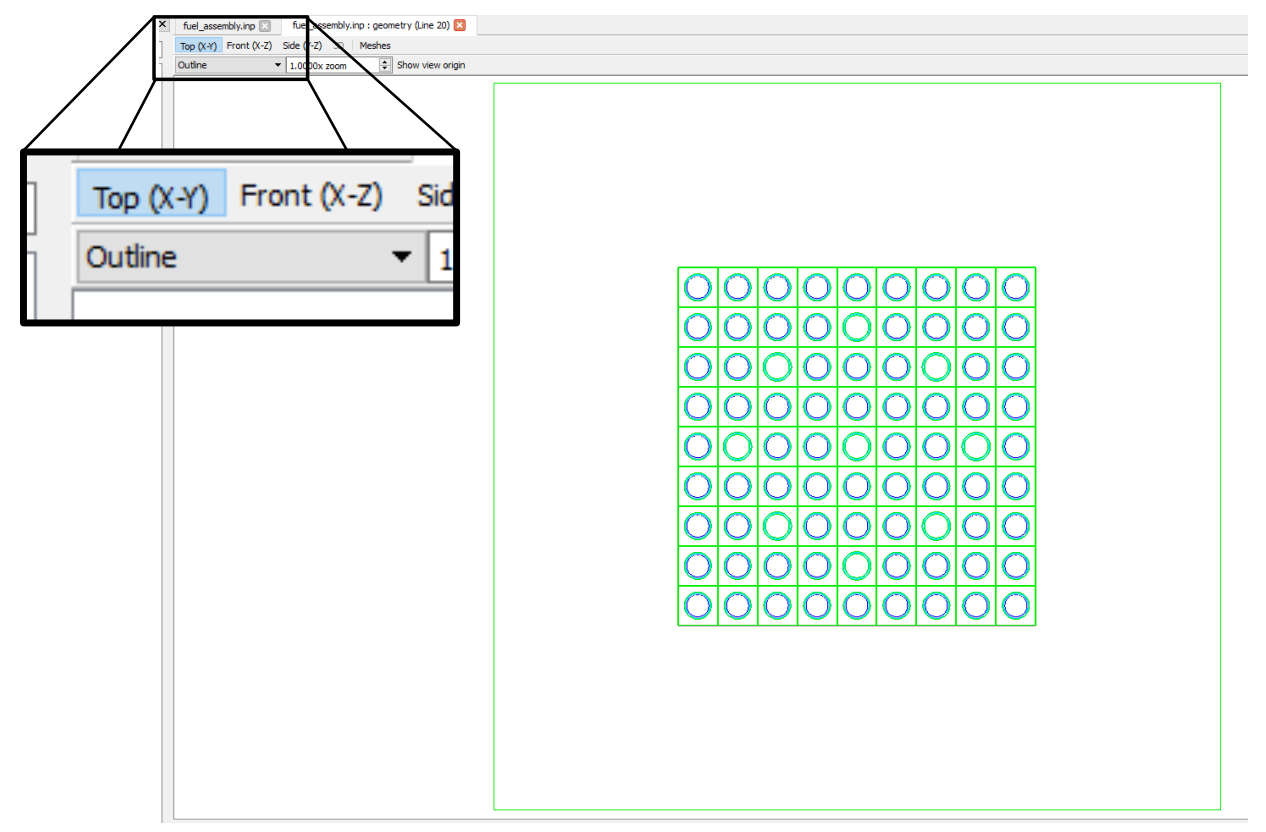

Figure 260. Midplane view of fuel assembly in Outline render mode.

There are two primary ways to control the zoom level of the image. The first is the zoom field at the top of the frame, as shown in Figure 257 above. A zoom factor can be typed directly into the field, or the arrows can be used to increase or decrease the zoom factor. The second primary zoom control is to left click and drag from the upper left corner to the lower right corner of a box. Fulcrum displays the box as it is created, as shown in Figure 261, and then attempts to fit this box into the view window as shown in Figure 262. Typically, the vertical distance is fit to the view window, so the zoomed image will likely be wider than the zoom box. To reset to the default zoom level, click and drag from the lower right to the upper left corner of a box. Fulcrum does not display the box because the image is being returned to the default view of the current visualization plane. 


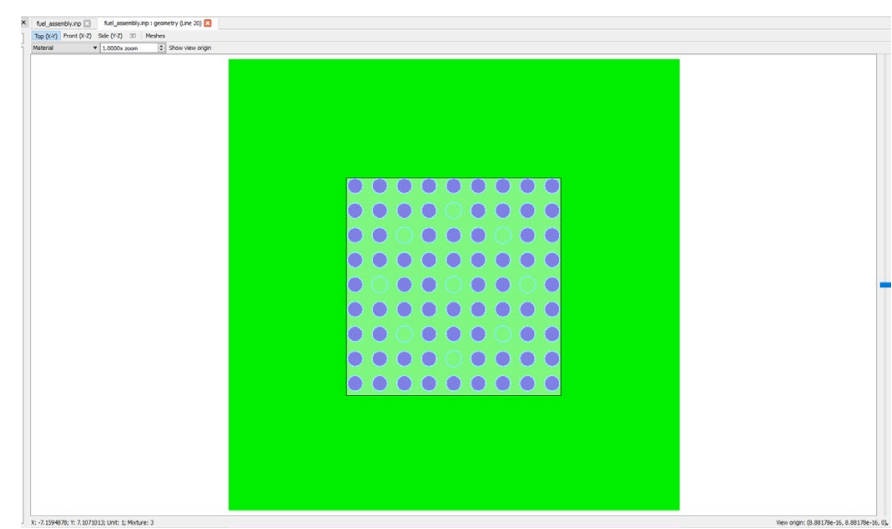

Figure 261. Fulcrum visualization while creating zoom control box.

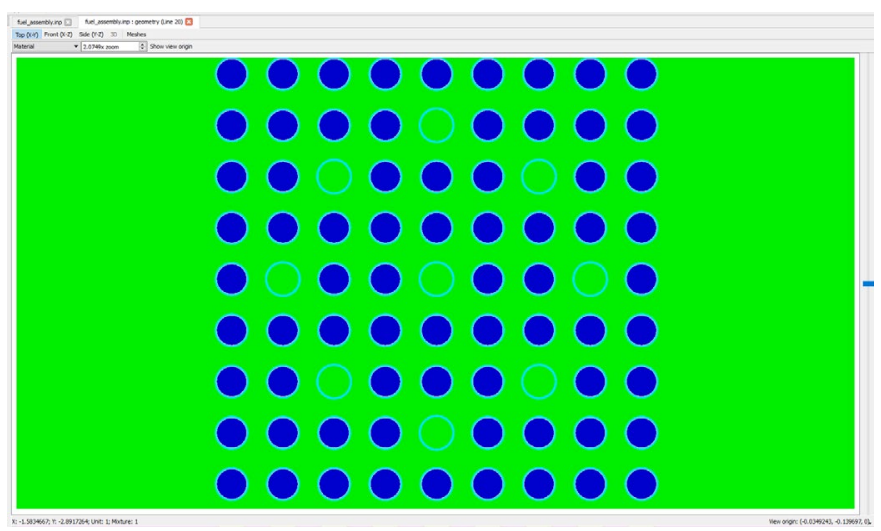

Figure 262. Image results from the zoom shown in Figure 260.

The last visualization control to discuss here is panning; there are two methods that can be used to pan the image. One is to hold the Shift key, left click on the image, and drag it to a new location. The image is translated as far as the mouse moved and is then redrawn; the image does not move as it is being translated. The second option is to double left click at a point on the image that will become the new view origin. The image is then redrawn with the selected point as the view origin.

\subsubsection{KENO Convergence Summary Plots}

KENO convergence summary plots are generated with the HTML output and can be opened directly in Fulcrum. A series of *ptp files is created in the htmd directory when execution is complete. Three different categories of *ptp files exist: "errorbars," "freqgen," and "ShannonEntropy." Each of these types of plots is discussed briefly below.

The most obvious plot type is ShannonEntropy. This file contains the Shannon entropy calculated for each generation in a format that can be plotted in Fulcrum. After the calculation has been executed, click on File > Open file and navigate into the htmd directory associated with the input, in this case "fuel_assembly.htmd." Double click on ShannonEntropy00001.ptp, and Fulcrum opens the file in the text editor, as shown in Figure 263. As highlighted in the figure, a small plot icon appears next to the word document in the Navigation panel. This icon indicates an entry in the Navigation panel that can be double clicked to generate a plot; double click on the word document to plot the Shannon entropy data for this problem. The resulting figure is shown in Figure 264. It may be difficult to assess convergence on this scale. To reset the $\mathrm{Y}$-axis scale, right click on the figure and select Plot options. Adjust the window so that all the options are visible, as shown in Figure 265, and select Axes from the pane on the left. 
Scroll down to Y-axis and Range Min and Range Max; set the Range Min to 4 and the Range Max to 6 . The updated plot is shown in Figure 266, indicating that the source may converge by approximately generation $50-75$.

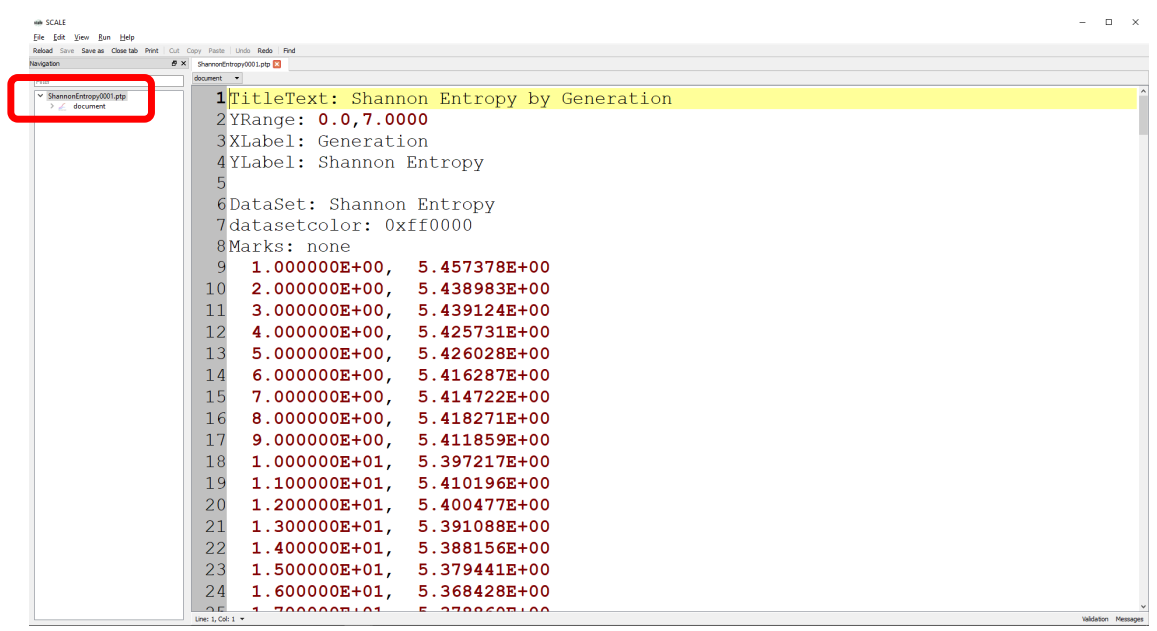

Figure 263. Fulcrum default view of a *ptp file.

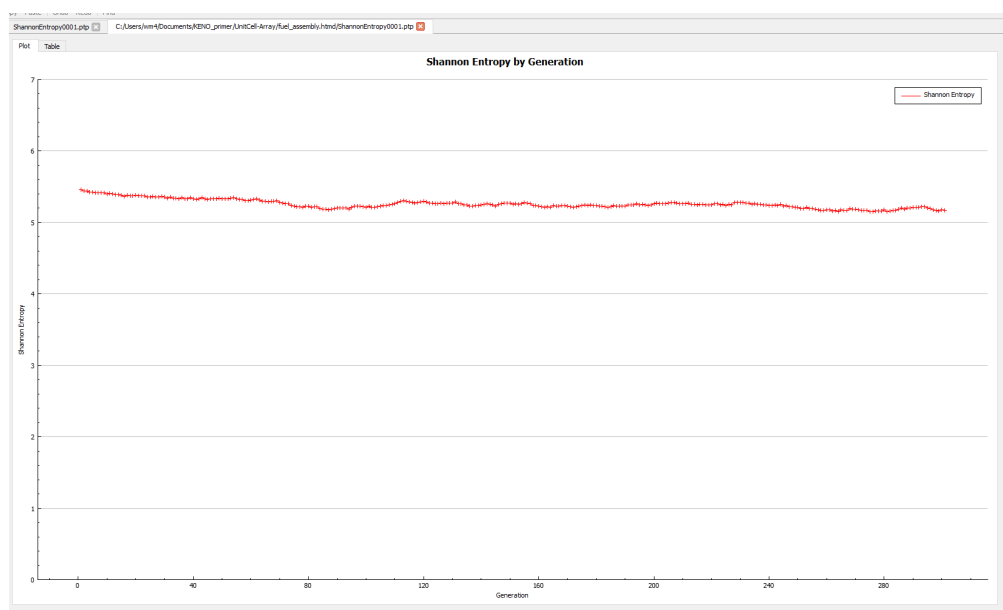

Figure 264. Default view of Shannon entropy by generation plot.

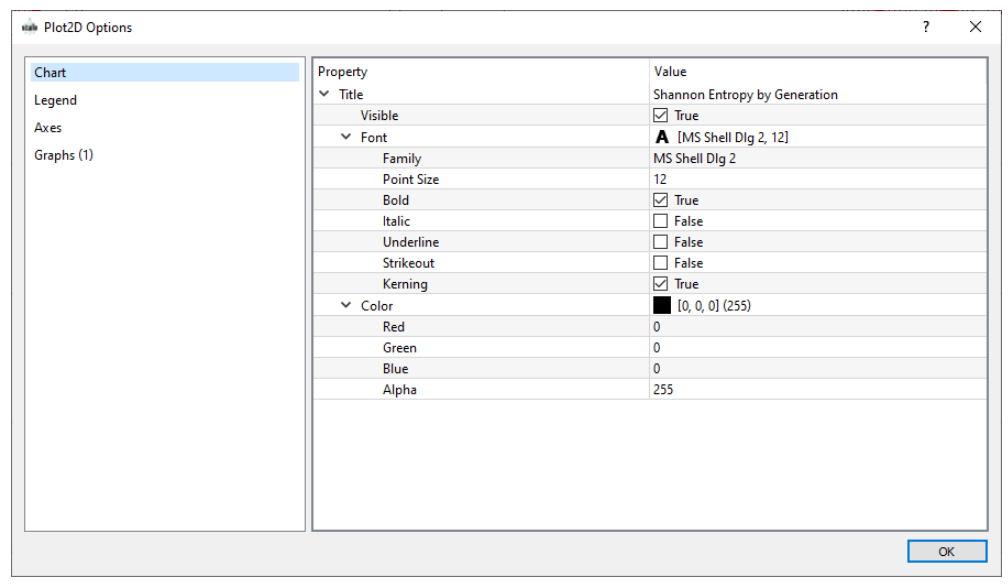

Figure 265. Resized plot options window. 


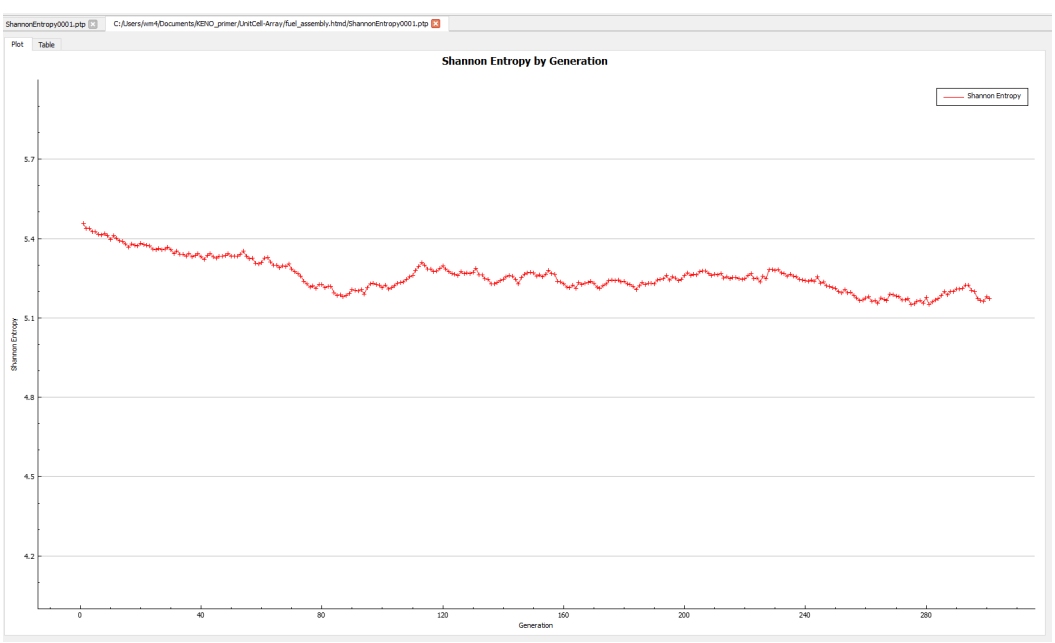

Figure 266. Rescaled view of the Shannon entropy plot.

The next plots to discuss are those labeled "errorbars0001.ptp" and "errorbars20001.ptp." These plots provide the $k_{\text {eff }}$ convergence summary based on the number of generations run (0001) and the number of generations skipped (20001). To view a plot, click on File $>$ Open file (or press Ctrl+o) and select errorbars0001.ptp. Fulcrum will by default attempt to open a file from the same directory used previously. As before, the document is opened in the editor. To plot the data, double-click on document. As shown in Figure 267, the average $k_{\text {eff }}$ is plotted as a circle, and the uncertainty bars correspond to a number of different confidence intervals. Note that the plot is showing the average $k_{\text {eff }}$ value for each number of generations run, not the individual generation $k_{\text {eff }}$ values. The $k_{\text {eff }}$ convergence summary for this problem indicates acceptable convergence for this simulation. Similarly, the second plot (errorbars20001.ptp) is shown in Figure 268. This provides the average $k_{\text {eff }}$ as a function of generation skipped, and it also shows excellent $k_{\text {eff }}$ convergence behavior.

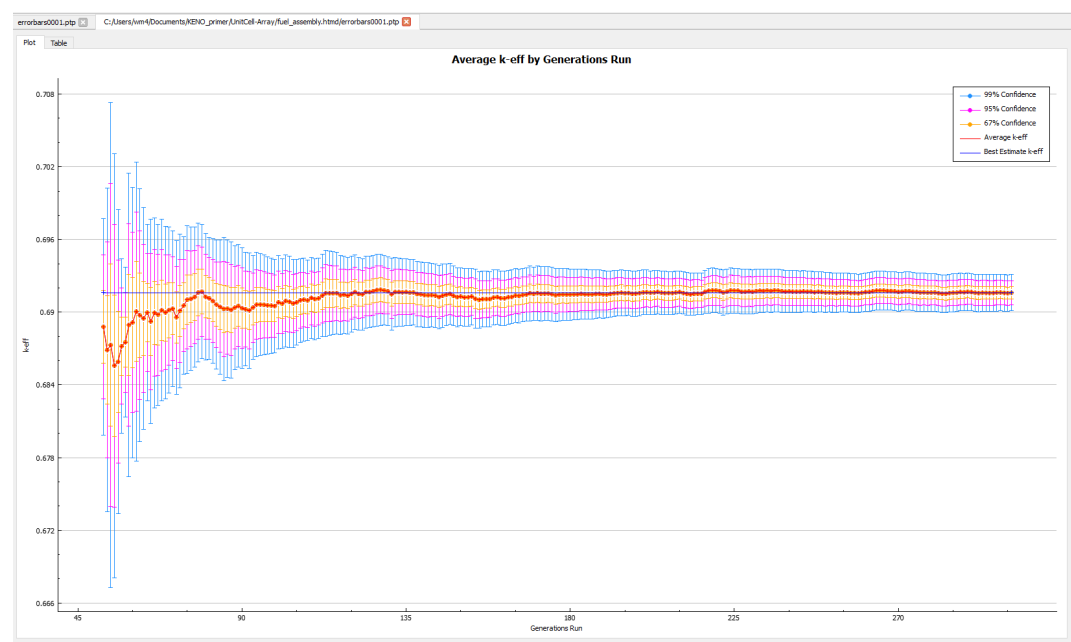

Figure 267. Plot of average $\boldsymbol{k}_{\text {eff }}$ by generation run. 


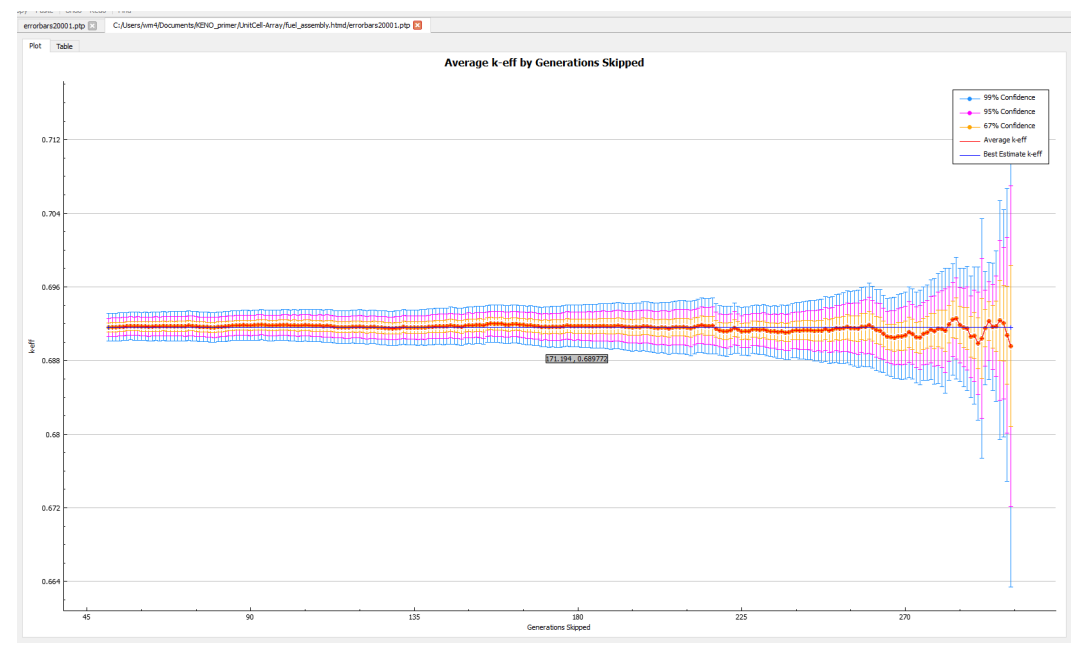

Figure 268. Plot of average $\boldsymbol{k}_{\text {eff }}$ by generation skipped.

The final set of plots is the "freqgen" series; these plots are histograms binning generation $k_{\text {eff }}$ values. If source convergence is achieved prior to the start of active generations, then the distribution of generation $k_{\text {eff }}$ values should follow a normal distribution, as the only variations are random. Separate histograms are generated over all of the active generations, over the last three quarters of active generations, the last half of active generations, and the last quarter of active generations. The 5 plots available are labeled 0001 , $10001,20001,30001$, and 40001. The summary plot with all 4 histograms combined is on "freqgen0001.ptp" and is shown in Figure 269. The histogram for the last quarter of active generations (40001) is shown in Figure 270. It appears that convergence was achieved during the discarded generations because no large shifts are evident among the histograms in Figure 269. Each histogram can be reviewed separately if desired.

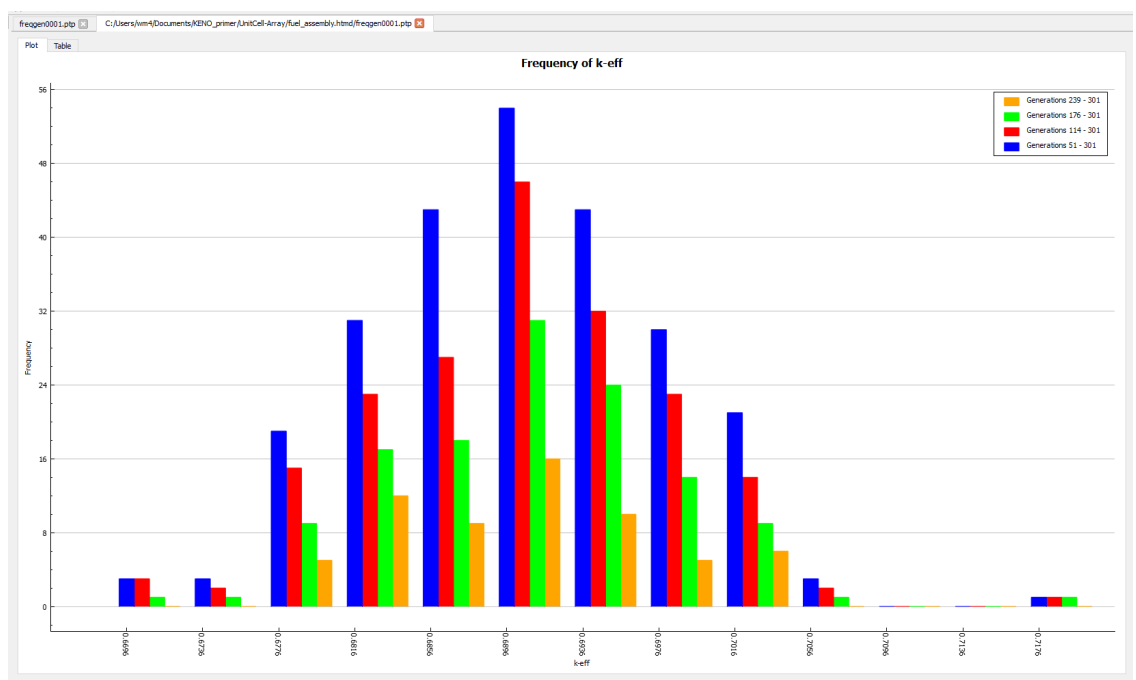

Figure 269. Histograms of generation $\boldsymbol{k}_{\text {eff }}$ values. 


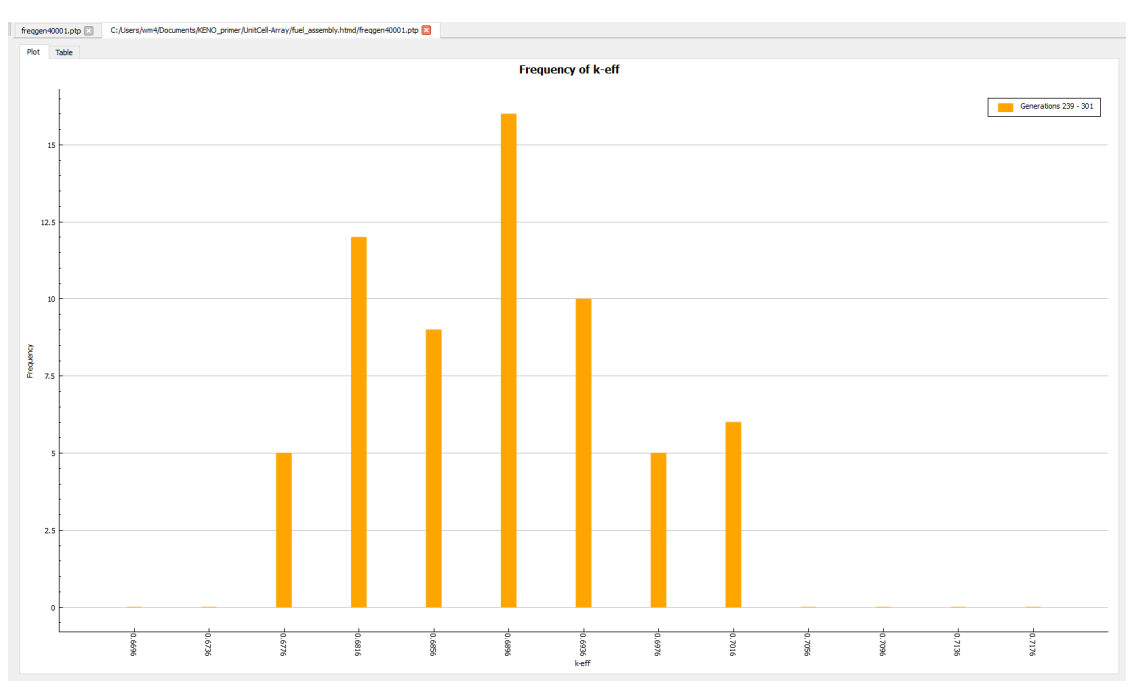

Figure 270. Histogram of generation $\boldsymbol{k}_{\text {eff }}$ values for the last quarter of active generations.

\subsubsection{Cross Sections and Other Nuclear Data in Fulcrum}

Three types of data plotting will be discussed in this section for various 1D data plots within Fulcrum: CE cross section data, MG cross section data, and MG uncertainty data. All of these data are 1D in that they are plotted against energy. Advanced data visualizations for 2D data, scattering, covariance, and correlation matrices are available within Fulcrum but are not covered in this primer. See Lefebvre's "Advanced User Interface Capabilities" [7] for more information on these advanced options.

There are no enforced naming conventions for nuclear data libraries in SCALE, so there are no defined extensions for the different types of libraries. Therefore, each type of library must be opened using the appropriate option from the Fulcrum File menu. Fulcrum issues an error that it is unable to open a library with the requested format if a mismatch occurs, such as when attempting to open a MG library after selecting File $>$ Open continuous-energy library.

Select File $>$ Open continuous-energy library to open a CE library. Fulcrum will open a files navigation window in the default data installation location; if the data were installed to another path, then navigate to that directory. Open ce_v7.1_endf.xml, as shown Figure 271; this file contains the path information to the actual CE cross section files contained in the cekenolib directory. Fulcrum will follow these data paths to plot the data as directed, but first the available isotopes and temperatures will be displayed in the Navigation panel, as shown in Figure 272. Next, expand the Neutron data and scroll down to expand u238. Note that the isotopes available are listed alphabetically and that available temperatures in Kelvin are displayed after expansion of the $\mathbf{u - 2 3 8}$ entry, as shown in Figure 273. Next, double-click on the $\mathbf{2 9 3}$ entry to generate the list of reaction cross sections available at that temperature, as shown in Figure 274. Scroll down and expand the $\mathbf{u - 2 3 8} \mathbf{~ m t = 1 0 2} \mathbf{n}$,gamma entry, and then double-click on $\mathbf{u}-\mathbf{2 3 8} \mathbf{~ m t = 1 0 2} \mathbf{~ n}$, gamma $293 \mathrm{~K}$ xs to plot the $(\mathrm{n}, \gamma)$ cross section for ${ }^{238} \mathrm{U}$. An example of the plot is shown in Figure 275; note that, as is typical for cross section plots, the data are displayed on a log-log scale. 


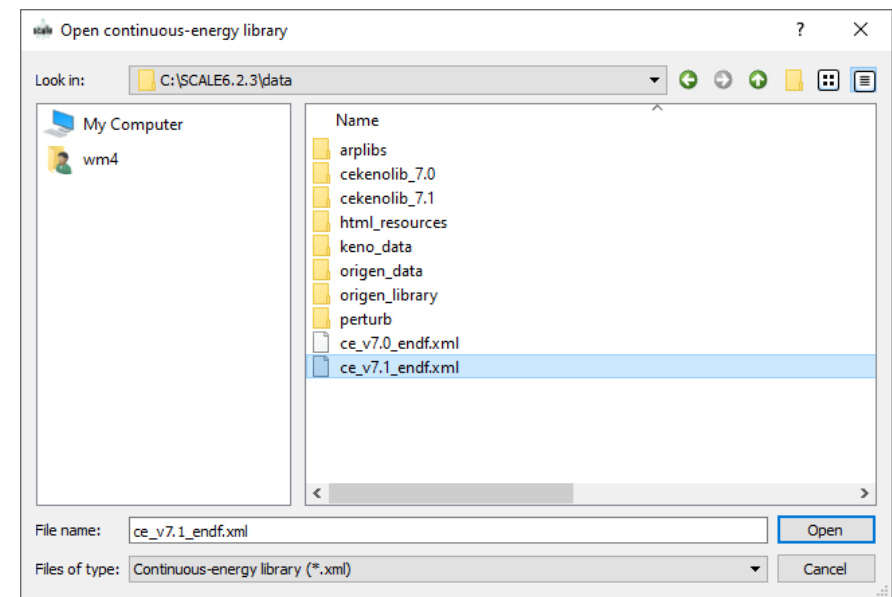

Figure 271. Opening the CE xml file.

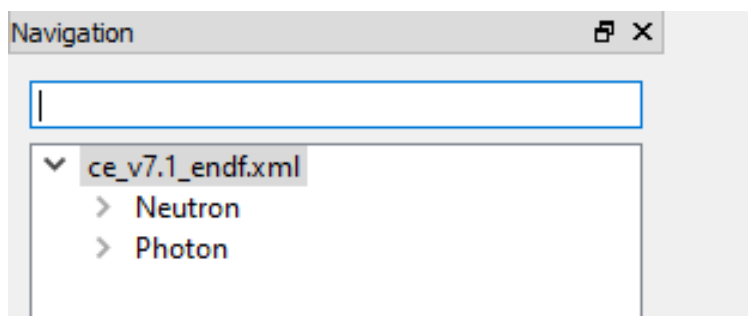

Figure 272. Default Navigation panel after opening a CE library.

$$
\begin{array}{r}
\vee u-238 \\
293 \\
565 \\
600 \\
900 \\
1200 \\
2400
\end{array}
$$

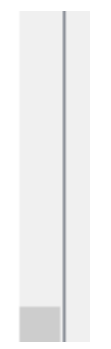

Figure 273. Expanded ${ }^{238} \mathrm{U}$ entry showing temperatures at which data are available.

$$
\mid \begin{aligned}
& \vee u-238 \\
& \vee 293 \\
&>\mathrm{u}-238 \mathrm{mt}=1 \text { total } \\
&>\mathrm{u}-238 \mathrm{mt}=2 \text { elastic } \\
&>\mathrm{u}-238 \mathrm{mt}=3 \text { nonelastic } \\
&>\mathrm{u}-238 \mathrm{mt}=4 \mathrm{n}, \mathrm{n}^{\prime} \\
&>\mathrm{u}-238 \mathrm{mt}=16 \mathrm{n}, 2 \mathrm{n} \\
&>\mathrm{u}-238 \mathrm{mt}=17 \mathrm{n}, 3 \mathrm{n} \\
&>\mathrm{u}-238 \mathrm{mt}=18 \text { fission } \\
&>\mathrm{u}-238 \mathrm{mt}=27 \text { absorption } \\
&>\mathrm{u}-238 \mathrm{mt}=37 \mathrm{n}, 4 \mathrm{n} \\
&>\mathrm{u}-238 \mathrm{mt}=51 \mathrm{n}, \mathrm{n}^{\prime}(1)
\end{aligned}
$$

Figure 274. Top portion of the list of reactions at $293 \mathrm{~K}$ available for plotting. 


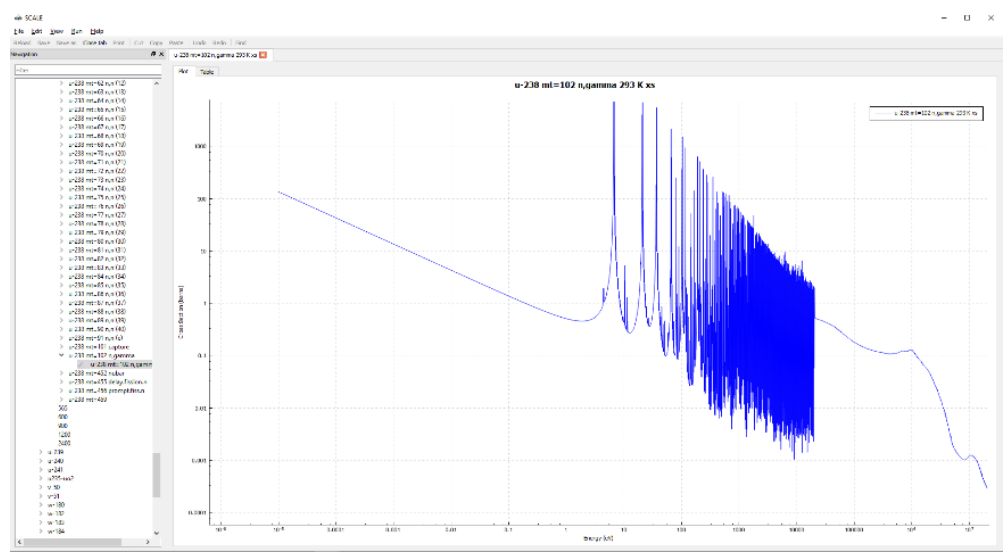

Figure 275. Default view of ${ }^{238} \mathbf{U}(n, \gamma) \mathrm{CE}$ cross section in Fulcrum.

Fulcrum can display MG and CE cross sections on the same plot because the unit (barns) is the same. Select File $>$ Open multigroup library and select scale.rev04.xn252v7.1 for the 252-group library based on ENDF/B-VII.1. This library opens expanded to the isotopes with neutron cross sections in the library, as shown in Figure 276. The libraries are listed alphabetically in the Navigation panel, so in this case, the MG library is listed below the CE library. Scroll down to expand u-238, Neutron, and Reaction XSecs, as shown in Figure 277. Scroll down to the $\mathbf{u - 2 3 8} \mathbf{~ m t = 1 0 2} \mathbf{n}$,gamma entry, right click on it, and select Add graph to "u-238 mt=102 n,gamma 293 K xs", as shown in Figure 278. The resulting plot comparing the MG and CE cross sections is shown in Figure 279.

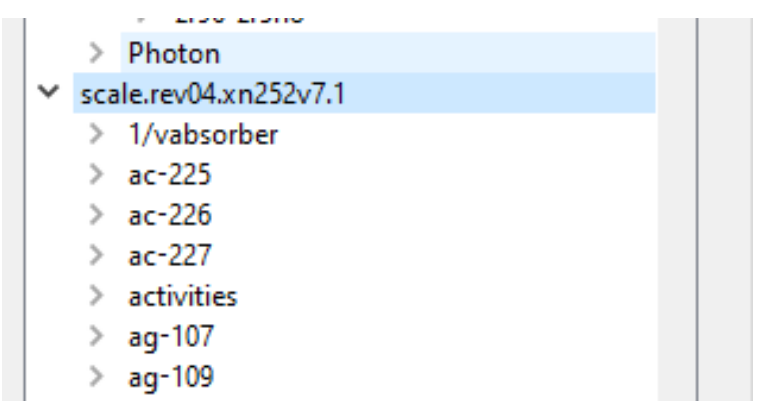

Figure 276. MG library loaded into the Navigation panel below the CE library.

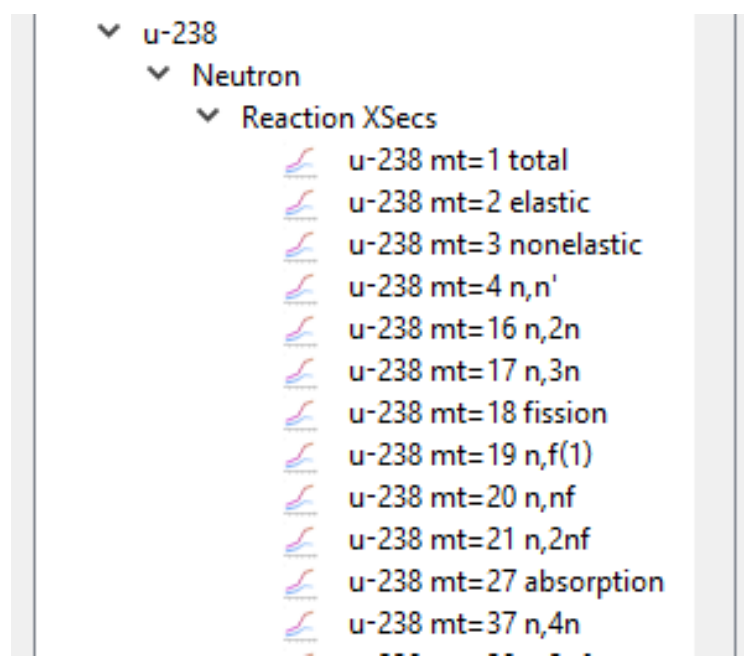

Figure 277. MG library in the Navigation panel with ${ }^{238} \mathrm{U}$ expanded. 


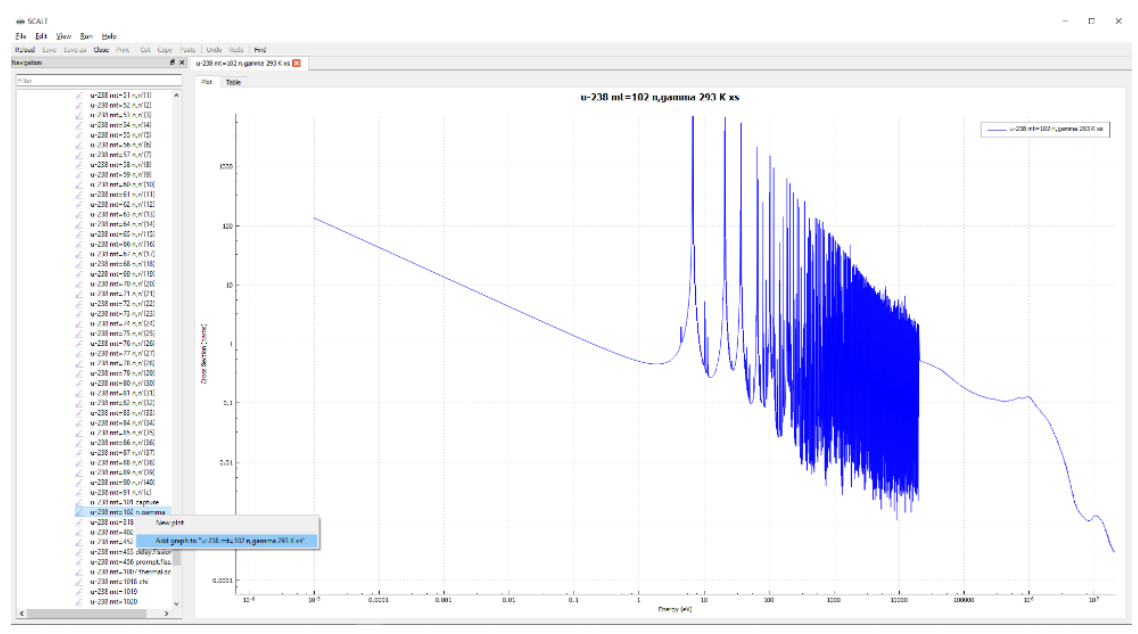

Figure 278. Adding MG ${ }^{238} \mathrm{U}(\mathrm{n}, \gamma)$ cross section to the existing CE plot.

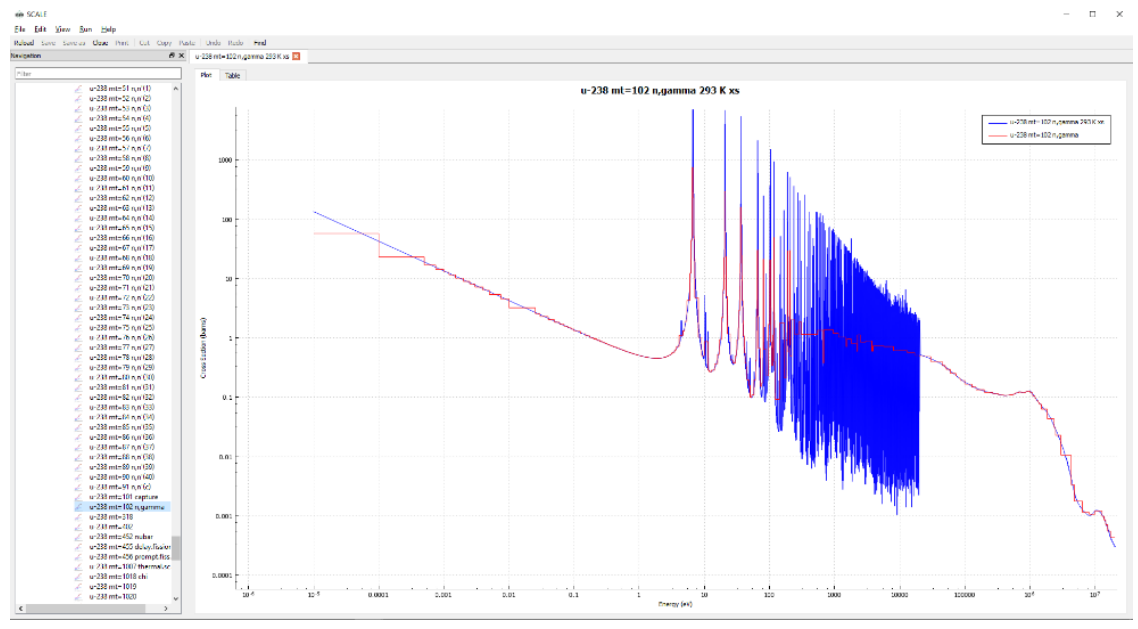

Figure 279. MG and CE cross sections for the ${ }^{238} \mathrm{U}(\mathrm{n}, \gamma)$ reaction.

The last type of data visualization covered here is covariance data. As mentioned previously, only plotting of the cross section uncertainty (the square root of the diagonal elements of the covariance matrix) will be discussed here. The uncertainty data cannot be plotted on the same figure as cross section data because the unit used for the uncertainty data (relative uncertainty [\%]) is not the same as that for the cross section data (barns). To load the covariance data, select File $>$ Open covariance library and select scale.rev08.56groupcov7.1 for the 56-group covariance library based on ENDF/B-VII.1 and other sources. This is the default covariance library for use in TSUNAMI in the SCALE 6.2 releases. The covariance library loads in the Navigation panel below the MG library because of its higher revision number; see Figure 280. Scroll down to $\mathbf{u - 2 3 8}$, expand the entry, and expand $\mathbf{m t}=\mathbf{1 0 2} \mathbf{n}$,gamma. Double click u-238 $\mathbf{~ m t = 1 0 2} \mathbf{n}, \mathbf{g a m m a}-$ Std dev by energy to create a new plot with the relative standard deviation for this reaction as a function of energy, as shown in Figure 281. Just above the plot there are tabs labeled Plot and Table; on windows the tabs are on the left side above the plot and on Mac they are in the center of the top ribbon. Clicking the Table tab provides a table of the points and data used in generating the plot, as shown in Figure 282. This plot of the standard deviation of the cross section is the square root of the diagonal of the covariance matrix, but it is still quite useful for showing the evaluated uncertainty in the cross section. Left click on the Plot tab to return to the plot. Left click on the legend in the upper right-hand portion of the plot and drag it to the left so that it does not overlap with the plot, as shown in Figure 283. Note that the legend in data plots in Fulcrum can be positioned in one of 9 
locations: along the left, center, or right and at the top, middle, or bottom of the view window. Finally, left click on the tab for the covariance plot labeled $\mathbf{u}-\mathbf{2 3 8} \mathbf{~ m t}=\mathbf{1 0 2} \mathbf{n}$,gamma - Std dev by energy, and drag it down to create a top/bottom split view showing both the cross section and covariance plots. Figure 284 shows the screen while the plot is being repositioned, and Figure 285 shows the resulting view of both plots. In this configuration, the cross section and its uncertainty can be compared directly.

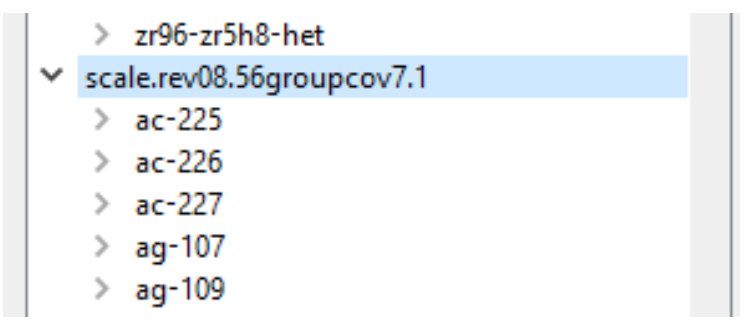

Figure 280. Covariance library loaded into the Navigation panel below the MG library.

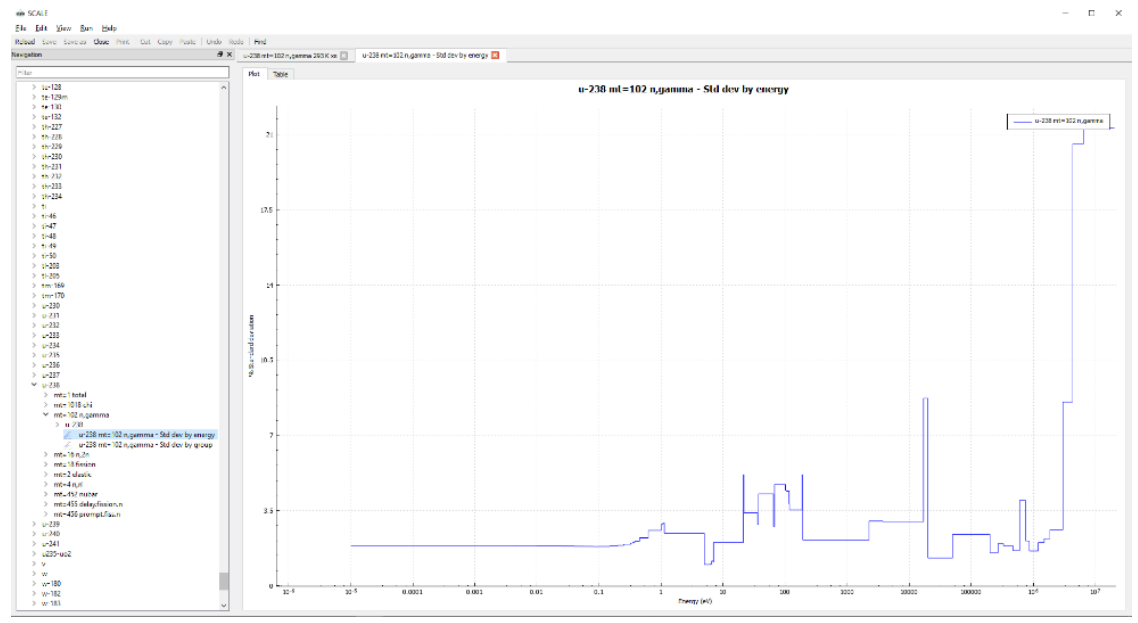

Figure 281. Plot of standard deviation of ${ }^{238} \mathbf{U}(\mathbf{n}, \gamma)$ cross section.

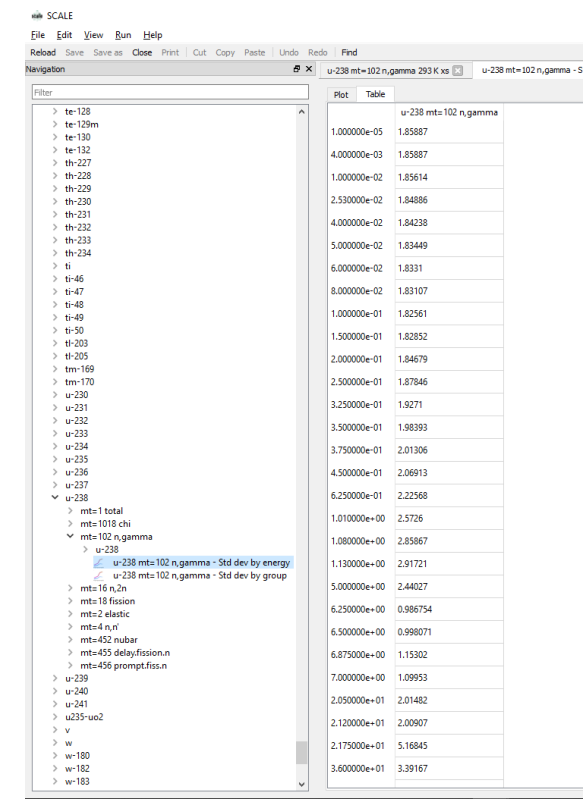

Figure 282. Table of values for standard deviation of ${ }^{238} U(n, \gamma)$ cross section. 


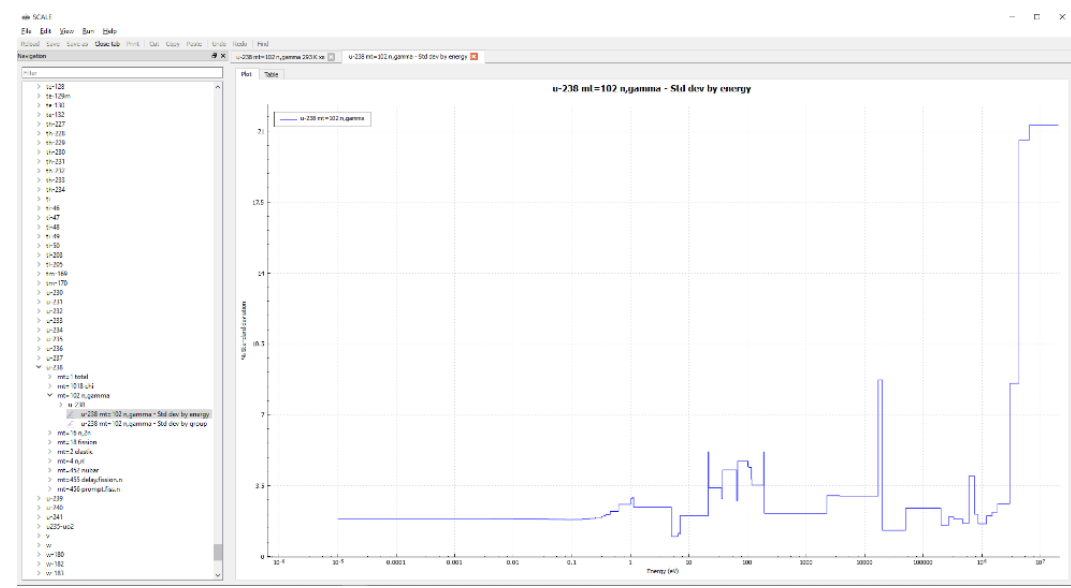

Figure 283. Uncertainty plot with repositioned legend.

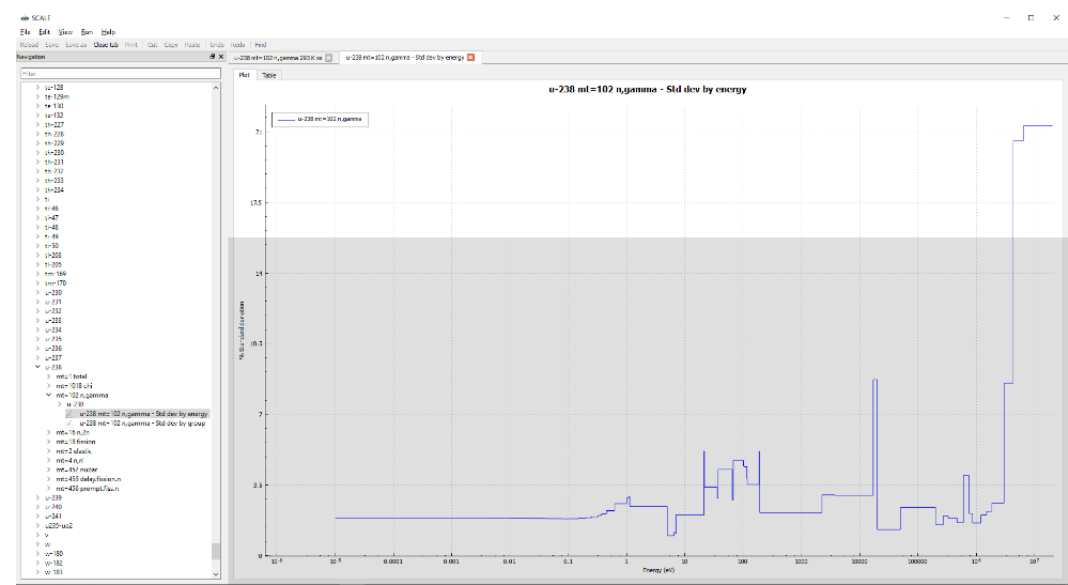

Figure 284. Positioning the uncertainty plot in the upper half of the view window.

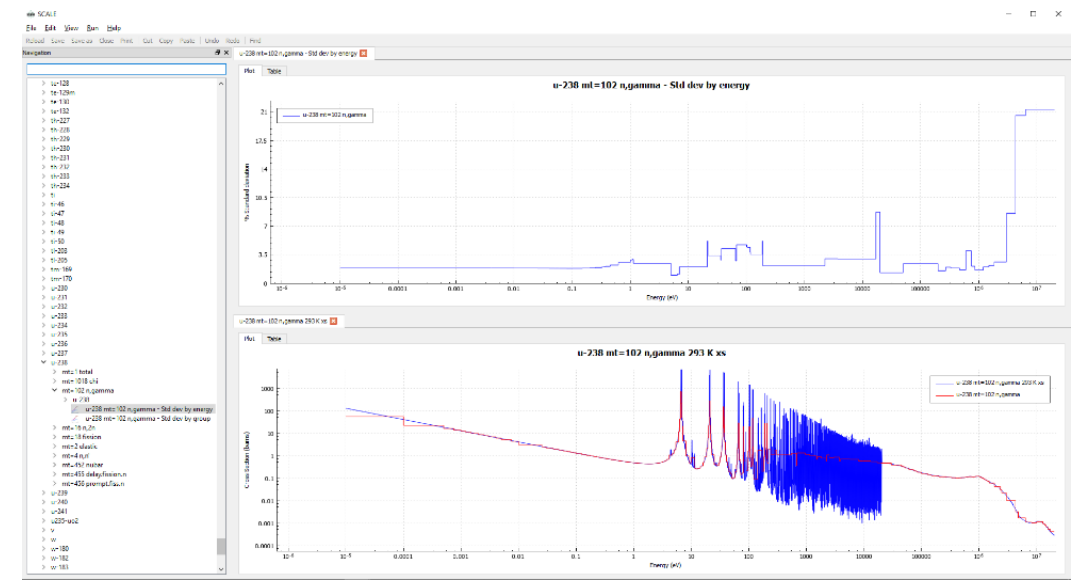

Figure 285. ${ }^{238} \mathrm{U}(\mathrm{n}, \gamma)$ uncertainty and cross sections in a single view in Fulcrum.

The zoom and pan controls for nuclear data plots are different from those used in geometry visualization. Zoom is controlled by the scroll wheel: scrolling up zooms in and scrolling down zooms out. The image can be panned by simply left clicking on the plot and moving the mouse. The default image can be restored by right clicking on the plot and selecting Fit graphs. 


\subsection{PLOTTING MESH DATA IN FULCRUM}

The primary mesh-based data generated and used in KENO is the neutron production edit enabled by the $c d s=y e s$ entry in the PARAMETER block. The mesh is specified in the GRIDGEOMETRY block, and the neutron production in each voxel is tallied by KENO and written to a *3dmap file. This file can be opened in Fulcrum and the mesh data can be plotted on top of the Fulcrum 2D geometry visualization. This capability will be demonstrated here by specifying a mesh on the fuel assembly problem from Section 8.5.1; the mesh will be equal to the fuel rod pitch in $\mathrm{X}$ and $\mathrm{Y}$ and 4 inches $(10.16 \mathrm{~cm})$ in $\mathrm{Z}$.

To create the input, add the parameter $c d s=y e s$ to the PARAMETER block. The PARAMETER block can be added to the KENO input outside of the other blocks by typing it directly or by entering it using autocomplete. The updated parameter block is shown in Figure 286, including cds=yes and an increased number of particles per generation (NPG), skipped generations (NSK), and total generations (GEN). Next, the GRIDGEOMETRY block must be added. Insert a blank line after end array, and use autocomplete to add the grid block to the input as shown in Figure 287. Mesh planes can be added explicitly or by specifying a range for linear interpolation; only Cartesian mesh planes are supported in

KENO at this time. Unfortunately, autocomplete is not supported in Fulcrum in the GRIDGEOMETRY block. The mesh over the fuel assembly will be specified with a series of linear interpolation specifications. In each case, the input is the keyword, the number of intervals, and the minimum and maximum values within which the interpolation will be performed. Individual mesh planes can also be added explicitly in the GRIDGEOMETRY block. The radial mesh to locate each rod in a separate interval is therefore xlinear $9-7.387 .38$ and ylinear $9-7.38$ 7.38. Similarly, the axial mesh can be specified as zlinear $36-182.88$ 182.88; the complete input specification for the GRIDGEOMETRY block is shown in Figure 288. Finally, run the KENO simulation. Larger numbers of particles and the associated longer runtimes will reduce the uncertainties in the local neutron production tallies and result in smoother distributions. The results shown below are based on a calculation which simulated 50,000 neutrons per generation for 2,450 generations, skipping the first 50 .

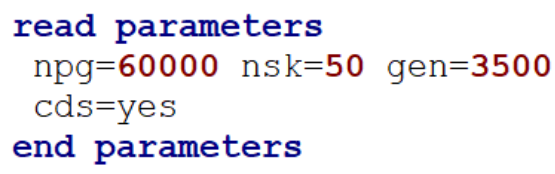

Figure 286. Updated parameter block with $\mathrm{cds}=\mathrm{yes}$ included to enable the neutron production tally.

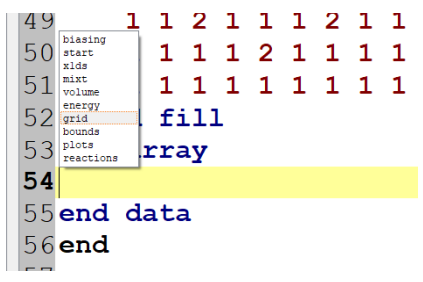

Figure 287. Adding GRID block to specify mesh for the neutron production tally.

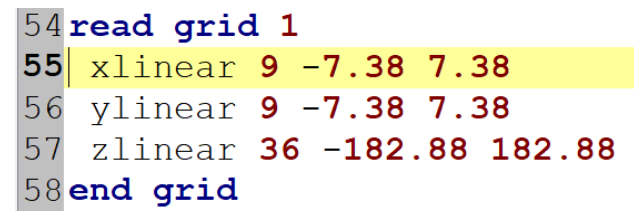

Figure 288. GRIDGEOMETRY block for fuel assembly problem.

After execution, the results can be visualized in Fulcrum. Open the input and visualize it, as discussed in Section 9.2.1. Click the Meshes button, which is located next to the view orientation buttons at the top 
middle of the visualization window, as shown in Figure 289. This opens the meshes pane and allows management of different mesh-based SCALE data formats. In this case, the neutron production rate is written to a *3dmap file; to load it into Fulcrum, right click in the meshes pane and select Load mesh file, as shown in Figure 290. The *3dmap file will be located in the same directory as the input, and in this case it will be named "fuel_assembly.fissionSource.3dmap." Once the mesh file is loaded, the available data sets are shown. Expand fission rate as shown in Figure 291; note that the neutron generation rate has been tallied by group, along with absolute and relative uncertainties. Scroll to the bottom of the list for total reaction rates and uncertainties and click the check box for fission rate - total. Next, change the render mode from Material to Overlay to display the total neutron production rate on the default midplane view, as shown in Figure 292. Scroll down in the mesh view control panel on the left hand side of the viewing window and change the Scale from Logarithmic to Linear. This shifts the color scale onto a linear scale, and the rates displayed on the geometry appear more realistic, as shown in Figure 293.

\begin{tabular}{|c|c|c|c|c|}
\hline \multicolumn{2}{|c|}{ fuel_assembly.inp $\mathrm{X}$} & \multicolumn{3}{|c|}{ fuel_assembly.inp : geometry (Line 21) $X$} \\
\hline $\operatorname{Top}(X-Y)$ & Front $(X-Z)$ & Side $(Y-Z) \quad 3 D$ & Meshes & \\
\hline Material & & $1.0000 \times$ zoom & $\div$ & Show view origin \\
\hline
\end{tabular}

Figure 289. Location of the Meshes button in the Fulcrum visualization window.

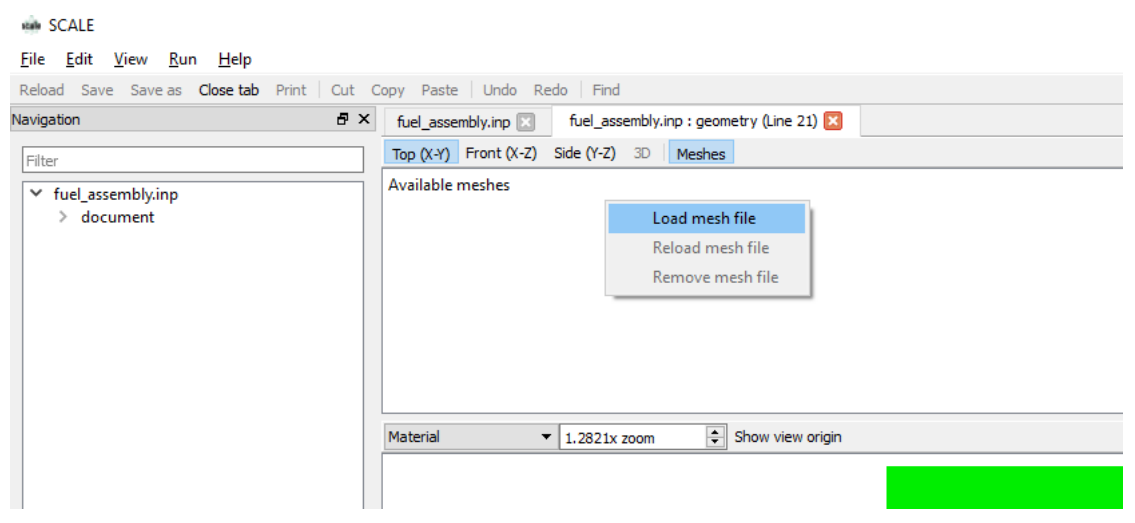

Figure 290. Loading a mesh file in Fulcrum.

\begin{tabular}{|c|c|c|c|c|}
\hline \multicolumn{2}{|c|}{ fuel_assembly.inp $\mathrm{x}$} & \multicolumn{3}{|c|}{ fuel_assembly.inp : geometry (Line 21) $\mathbf{x}$} \\
\hline $\operatorname{Top}(X-Y)$ & Front $(x-Z)$ & Side $(Y-Z)$ & Meshes & \\
\hline \multicolumn{5}{|c|}{ Available meshes } \\
\hline \multirow{2}{*}{\multicolumn{5}{|c|}{$\begin{array}{l}\vee \text { fuel_assembly.fissionSource.3dmap } \\
\vee \text { fission rate }\end{array}$}} \\
\hline & & & & \\
\hline \multicolumn{5}{|c|}{$\square$ group 1} \\
\hline \multicolumn{5}{|c|}{$\square$ group 1 - abs. uncert. } \\
\hline \multirow{2}{*}{\multicolumn{5}{|c|}{$\square$ group 1 - rel. uncert. }} \\
\hline & & & & \\
\hline \multicolumn{5}{|c|}{$\square$ group 2 - abs. uncert. } \\
\hline \multirow{2}{*}{\multicolumn{5}{|c|}{$\begin{array}{l}\square \text { group } 2 \text { - rel. uncert. } \\
\square \text { group } 3\end{array}$}} \\
\hline & & & & \\
\hline Material & & $1.2821 \times \mathrm{zoom}$ & 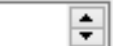 & Show view origin \\
\hline
\end{tabular}

Figure 291. Loaded and expanded 3dmap file for the fuel assembly model. 


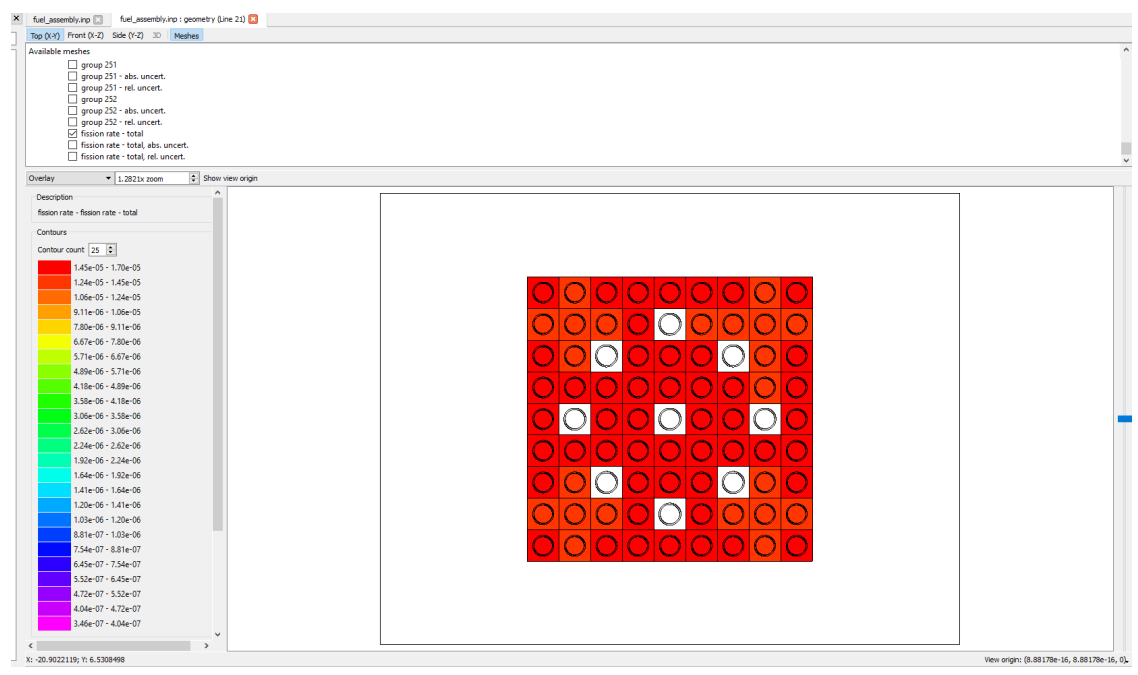

Figure 292. Default view of neutron production rate at the fuel assembly midplane.

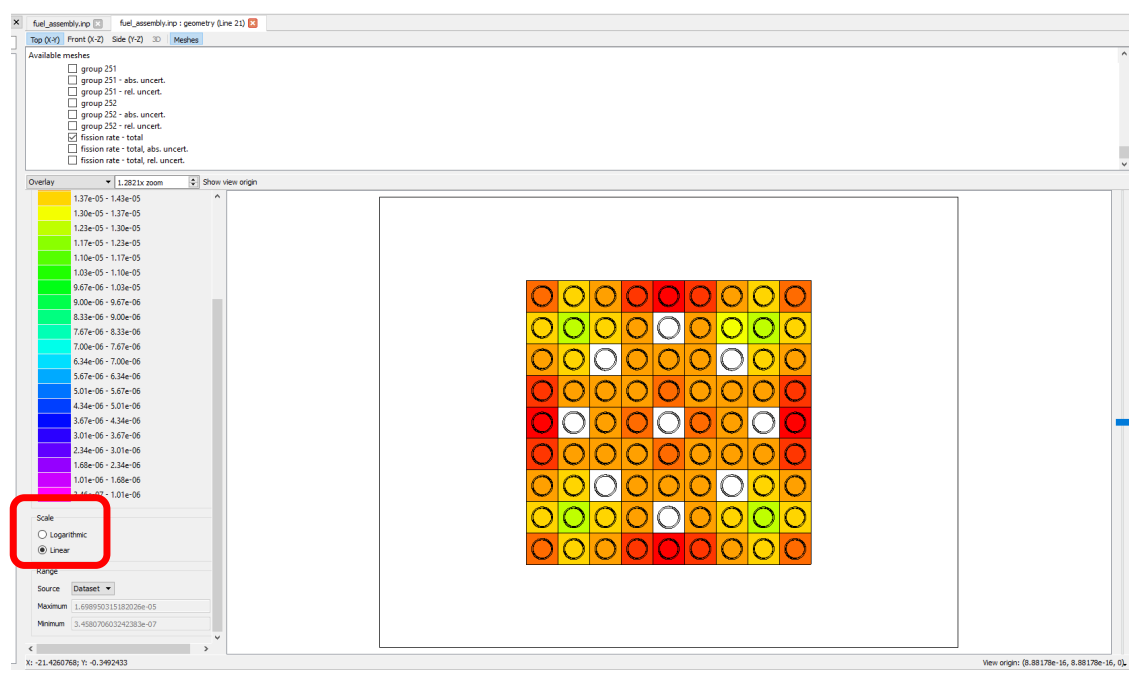

Figure 293. Fuel assembly midplane with colors rescaled to linear bins.

The mesh itself can also be displayed in Fulcrum. Change the Render mode dropdown menu from Overlay to Overlay + boundaries to show the mesh planes. It may be necessary to change the zoom factor slightly to display all mesh planes, as shown in Figure 294. The uncertainties in the mesh tallies can also be displayed; check the box for fission rate - total, rel. uncert. in the meshes pane as shown in Figure 295. The resulting updated figure is shown in Figure 296. The color scale can also be specified to allow better contrast for the data being displayed. In the lower portion of the mesh view control panel, change the Source dropdown in the Range box from Dataset to Custom, as shown in Figure 297. It is clear that all the uncertainties are in the lowest bin, so set the Minimum to $7.5 e-4$ and the Maximum to $1 e-3$, as shown in Figure 297. The updated plot shown in Figure 298 provides a better view of how the uncertainty varies in this specific plane. 


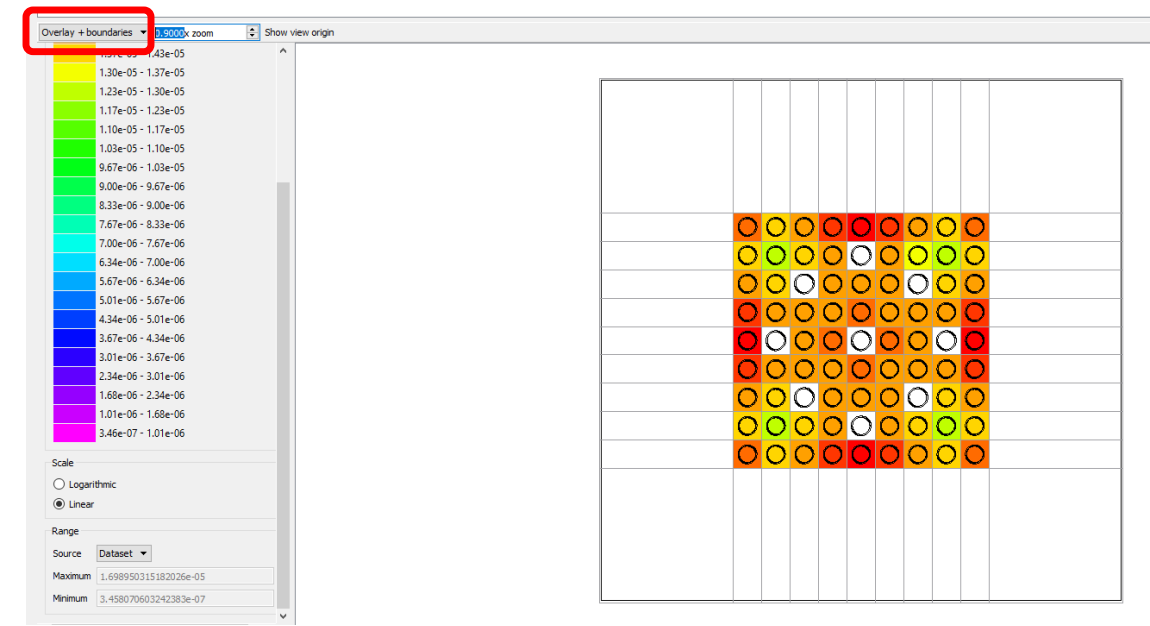

Figure 294. Midplane neutron production rate with mesh boundaries displayed.

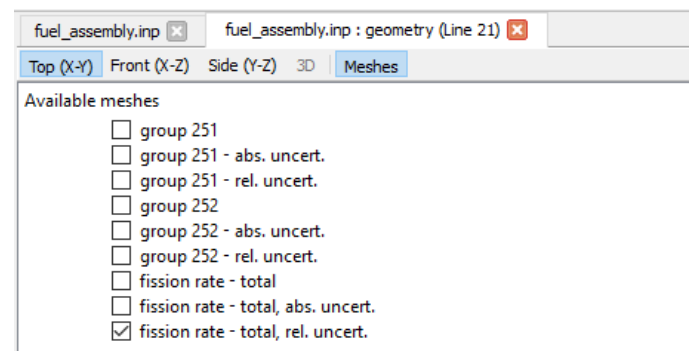

Figure 295. Selecting relative uncertainties as the overlay quantity to view.

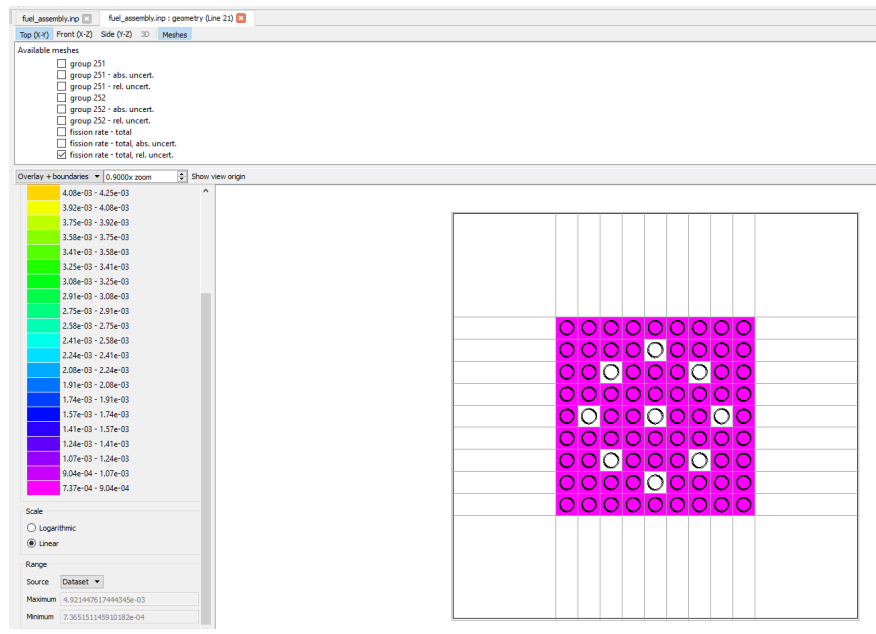

Figure 296. Relative uncertainties for the neutron production rate at the fuel assembly midplane.

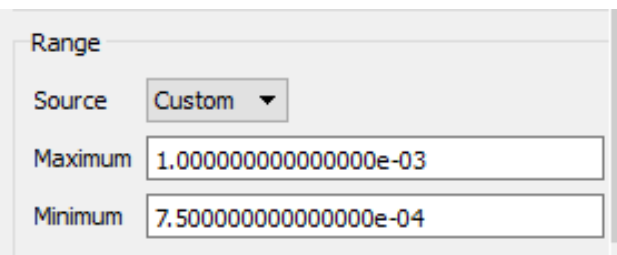

Figure 297. Updated uncertainty range. 


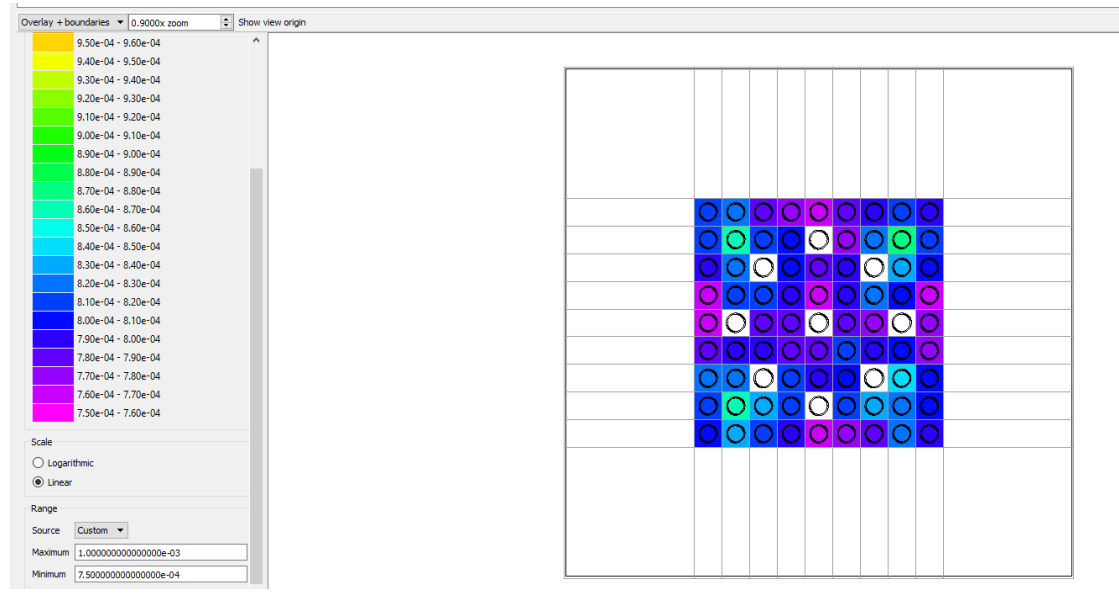

Figure 298. Overlay plot of uncertainties with custom value range.

The visualization controls discussed in Section 9.2.1 allow for detailed review of the neutron production rates throughout the model. The XY visualization plane can be moved axially with the slider or by setting the plane in the View origin field. As shown in Figure 299, axial slices can be viewed by selecting the XZ or $\mathrm{YZ}$ orientation buttons. Check the box to overlay fission rate - total again in the meshes pane and reset the Source dropdown in the Range control box to Dataset.

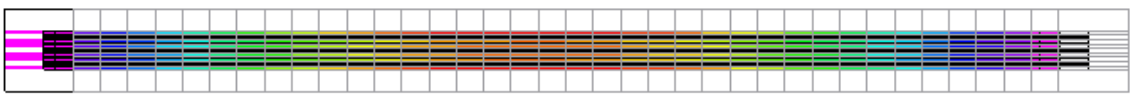

Figure 299. Axial view of the fuel assembly neutron production rate distribution rotated such that $+Z$ is to the right.

The last capability that will be highlighted here is the ability to generate 1D plots from the mesh data displayed in Fulcrum. The example here will be for an axial trace along a single rod, but plots can also be generated along a row ( $\mathrm{X}$ axis), column (Y axis), or as a function of energy. Right click on the middle rod of the left-most column of the assembly, as indicated in Figure 300, and select Create plot (Figure 301). Select the $\mathbf{z}$ axis as the Independent axis in the Options box of the subsequent window as shown in Figure 302. Fulcrum creates a new file and opens the text version of that file in a separate tab in much the same way that the convergence summary plot files open, as discussed in Section 9.2.2 and shown in Figure 263. Double click the word document in the Navigation panel as shown in Figure 303, and Fulcrum will generate a new plot as shown in Figure 304.

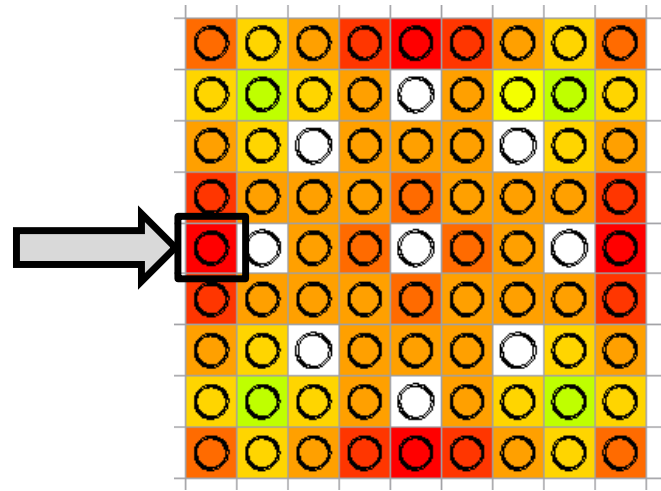

Figure 300. Selection of rod for axial plot. 


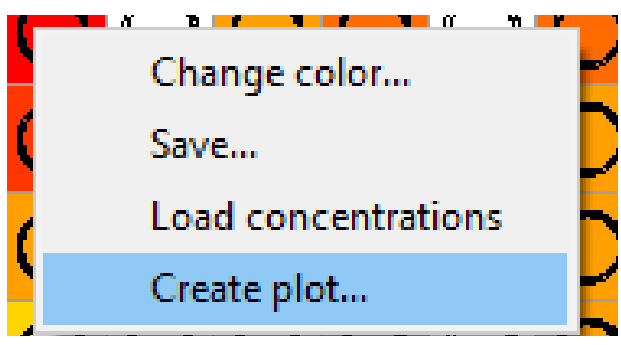

Figure 301. Selecting Create plot... in Fulcrum.

\begin{tabular}{|c|c|c|}
\hline velu Fulcrum & $?$ & $>$ \\
\hline \multicolumn{3}{|l|}{ Instructions } \\
\hline \multicolumn{3}{|c|}{$\begin{array}{l}\text { You have selected the mesh cell } 2,6,20 \text {. For } \\
\text { the } 2 \mathrm{D} \text { plot, please select the independent } \\
\text { axis and how to space the data. }\end{array}$} \\
\hline \multicolumn{3}{|l|}{ Options } \\
\hline Independent axis & $z$ axis & $\nabla$ \\
\hline Plot using & values & $\boldsymbol{\nabla}$ \\
\hline Divide by axis interval widths & none & $>$ \\
\hline OK & \multicolumn{2}{|c|}{ Cancel } \\
\hline
\end{tabular}

Figure 302. Selecting Independent axis for axial trace.

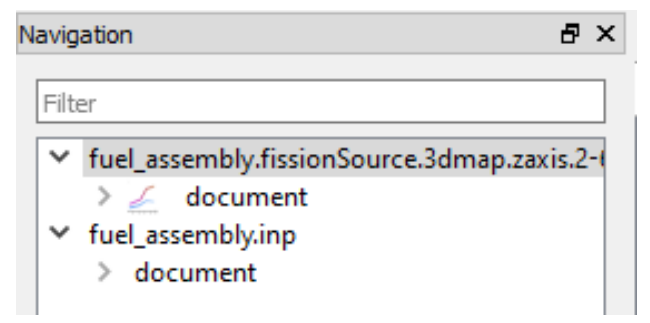

Figure 303. Plot figure generated by Fulcrum and displayed in the Navigation panel.

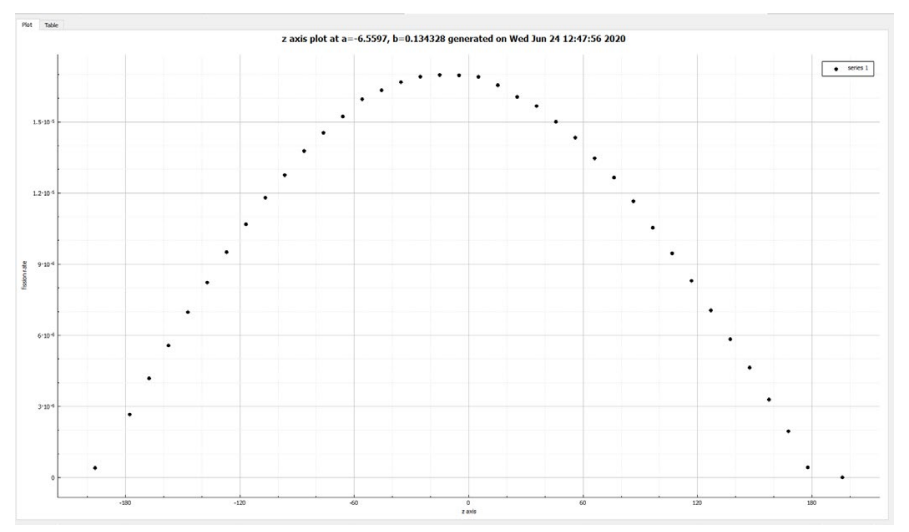

Figure 304. Axial trace of neutron production rate. 


\subsection{INTERACTIVE 3D VISUALIZATION WITH KENO3D}

KENO3D enables KENO-VI users to interactively display their 3D geometry models. KENO3D only runs on the Windows operating system. The interactive options include the following:

- Shaded or wireframe images

- Standard views, such as top view, side view, front view, and isometric (3D) view

- Rotating the model

- Zooming in on selected locations

- Selecting parts of the model to display

- Editing colors and displaying legends

- Displaying properties of any unit in the model

- Creating cut-away views

- Removing units from the model

- Copying images to clipboard or saving images to common graphics formats

KENO3D reads CSAS/KENO-VI input files. It attempts to verify that the KENO geometry input is "legal," that is, KENO3D checks that the input conforms to the code guidelines. KENO3D prints a warning message for illegal geometry input, and if possible, it displays the illegal KENO geometry to facilitate debugging of the input. KENO3D has a fully integrated help system to aid users in learning how to use it. Only a brief demonstration showing how to use KENO3D will be presented here. Users are strongly encouraged to follow the tutorials in the KENO3D help files to quickly learn how to use the many powerful options. KENO3D has several options that provide users with increased flexibility in visualizing portions of a model in greater detail or for visualizing parts of larger models that may be too big to provide a useful image when viewed in their entirety.

To open KENO3D, double-click the keno3d.exe file located in the KENO3D directory within the SCALE install directory. Next, to display the $9 \times 9$ fuel assembly descried in Section 8.5.1, select File > Open... (or $\mathrm{Ctrl+o}$ ), navigate to the directory containing the input, and select fuel_assembly.inp. The default isometric projection is shown in Figure 305.

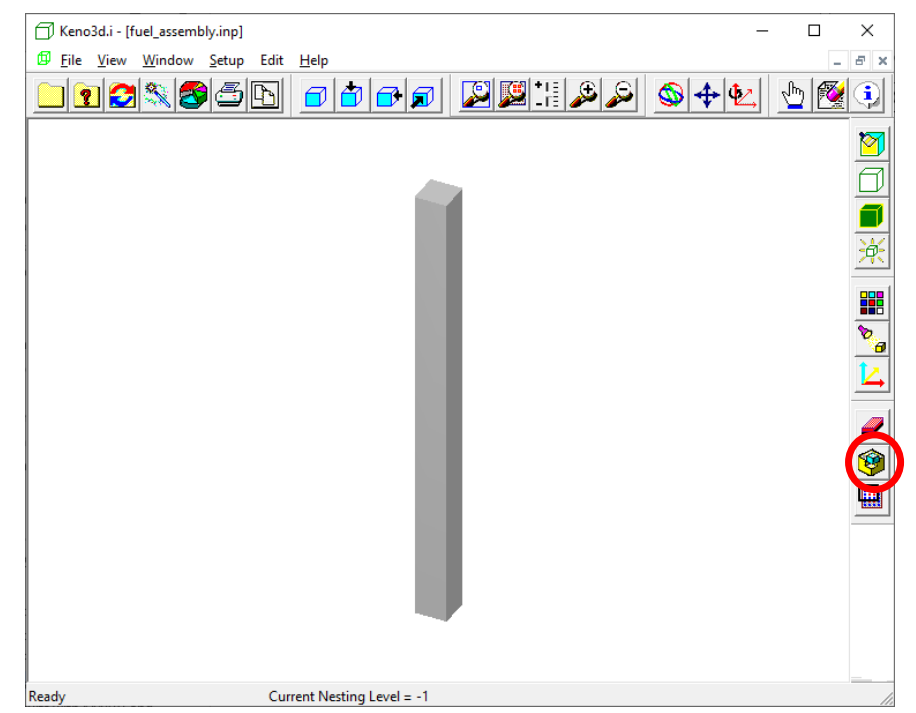

Figure 305. Default KENO3D view of the fuel assembly model. 
This isometric view shows the water reflector that surrounds the assembly. Next, click on the Remove a Section button (see circle in Figure 305 above). This opens the Section to remove from model dialog form shown in Figure 306. Click on the Top half radio button under Section to Remove and click OK. This action removes the top half of the model. Click the Remove a Section button again and click OK to remove the Front-right quarter. Click on the blue magnifying glass button (Zoom all) on the top toolbar. The image should now look similar to that shown in Figure 307.

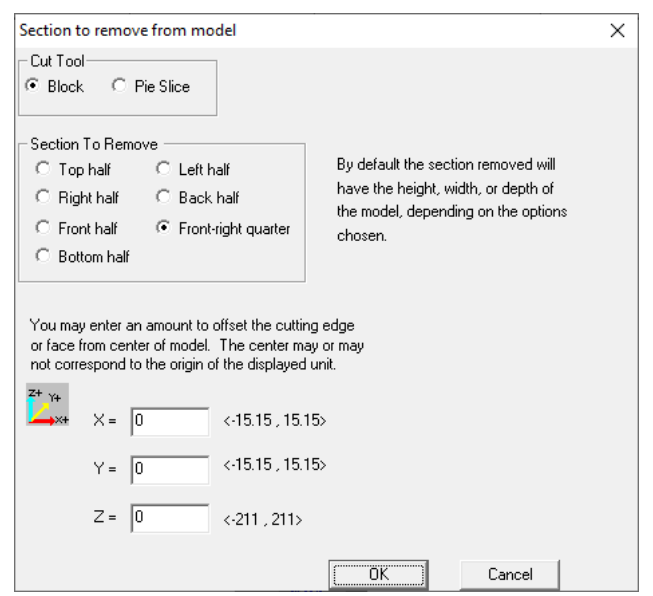

Figure 306. Remove section dialog.

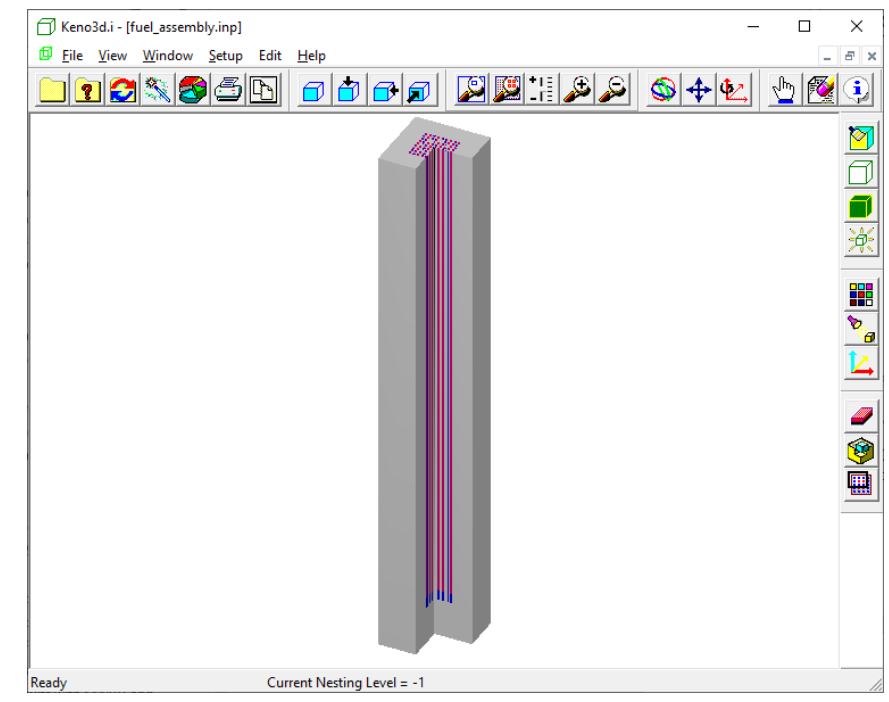

Figure 307. Cutaway view of the bottom half of the assembly.

Next, hide the water so that the fuel rods are more easily visible. Click on the Hide mixture (eraser) button on the top toolbar and select 0 void and 3 h2o under Select mixture(s) to hide and click OK. The image now looks like that presented in Figure 308. 


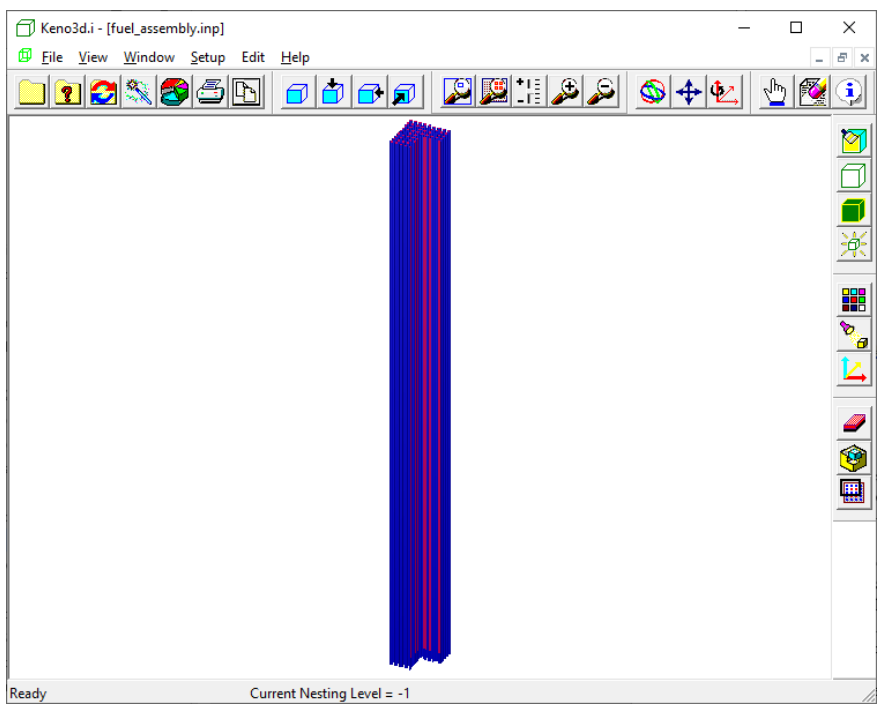

Figure 308. Cutaway view of the bottom half of the assembly with water removed.

To zoom in on the bottom part of the fuel assembly to see the details more clearly, click on the Zoom Window button (red magnifying glass on the top toolbar). Then left-click and drag to make a rectangle around the bottom portion of the assembly (Figure 309). Once the window is satisfactory, left click again to zoom in. The resulting image should look like that shown in Figure 310.

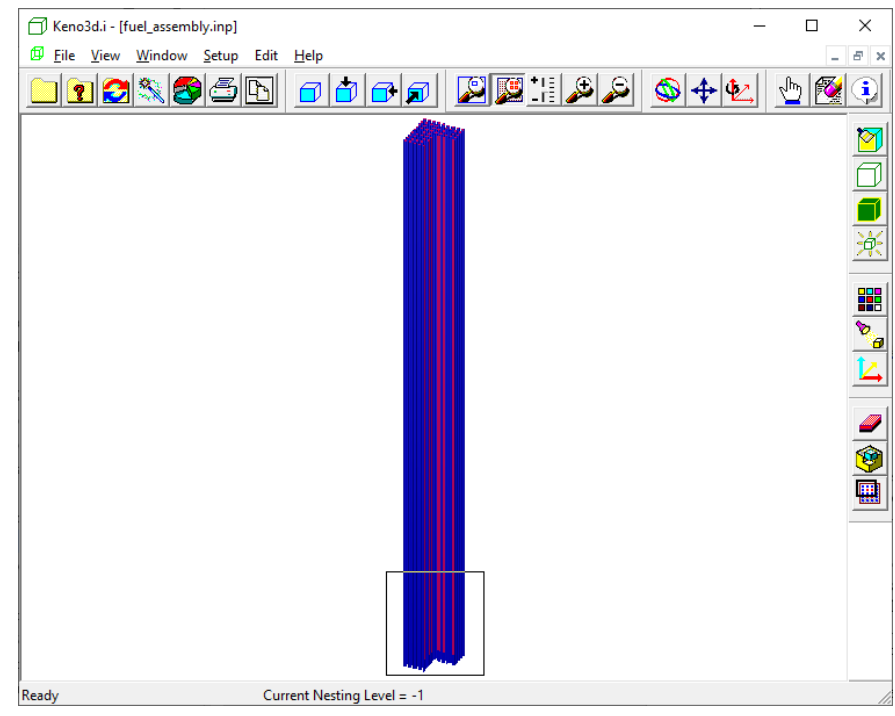

Figure 309. Zooming on lower portion of the fuel assembly. 


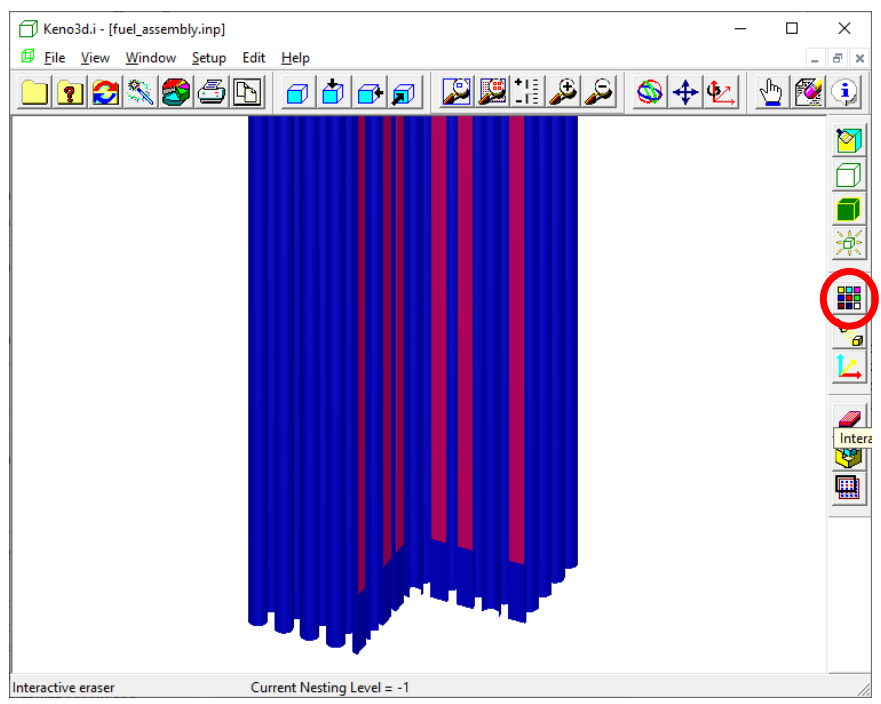

Figure 310. Zoomed view of cutaway view of the bottom of the fuel assembly with water removed.

Next, display the legend by clicking the Display legend button, circled in Figure 310 above. Click OK in the Legend options dialog for an image like that shown in Figure 311.

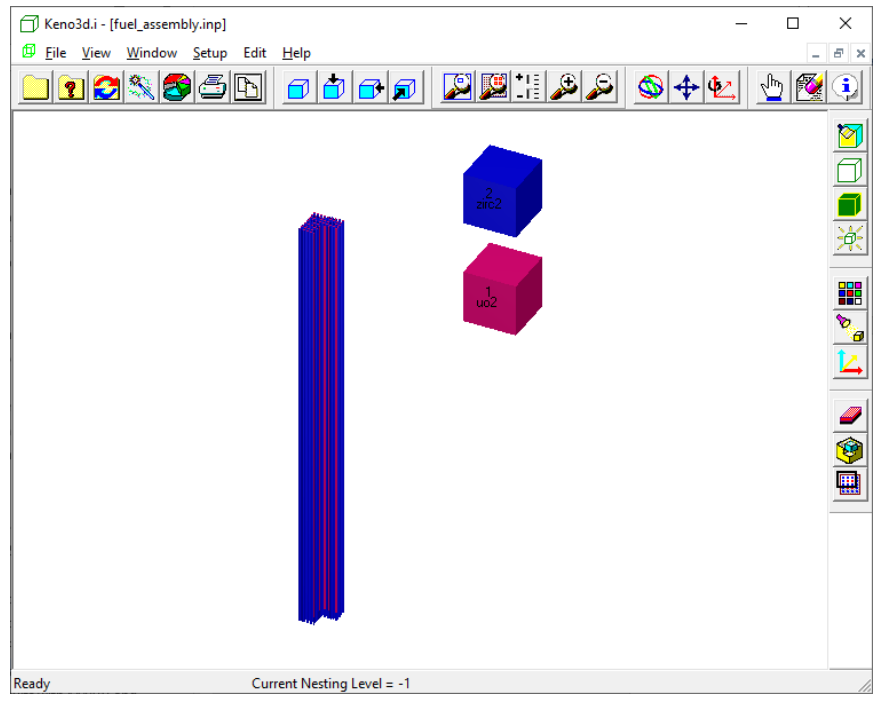

Figure 311. Default view with legend.

Adding the legend has removed the focus on the zoom window and has returned the view of the bottom half of the fuel assembly. In addition, the legend is too large and is not positioned near the bottom of the fuel assembly. This can be fixed by changing the display legend options. Click on the Display legend button again. Change the Body Width and Gap between bodies to 4. Set the Upper left corner of legend to $\mathbf{X}=20, \mathbf{Y}=20$, and $\mathbf{Z}=-180$. Set the Size of the Legend Text to 8 (Figure 312). Click OK, and the screen will look like Figure 313. Finally, zoom in on the lower portion of the fuel assembly again to generate an image like that shown in Figure 314. 


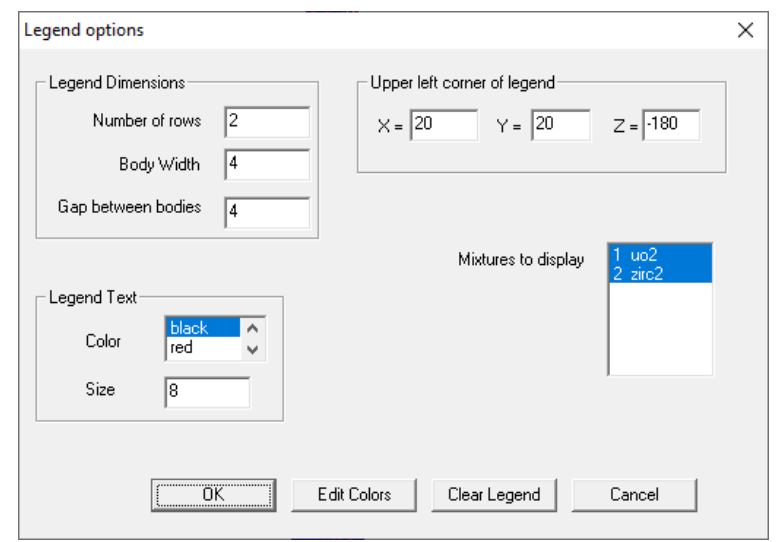

Figure 312. Legend options dialog.

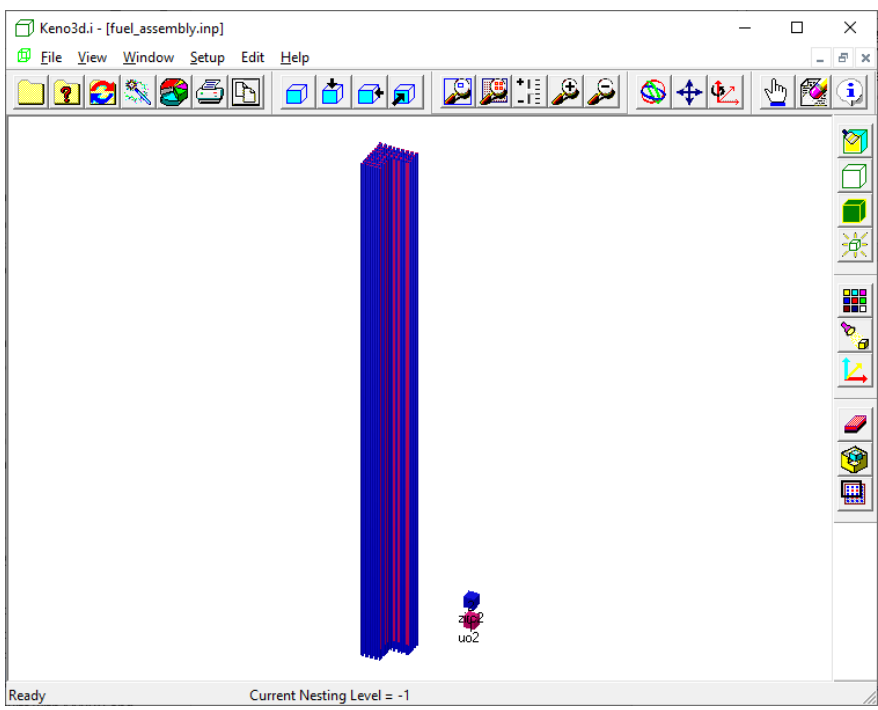

Figure 313. Figure with modified legend.

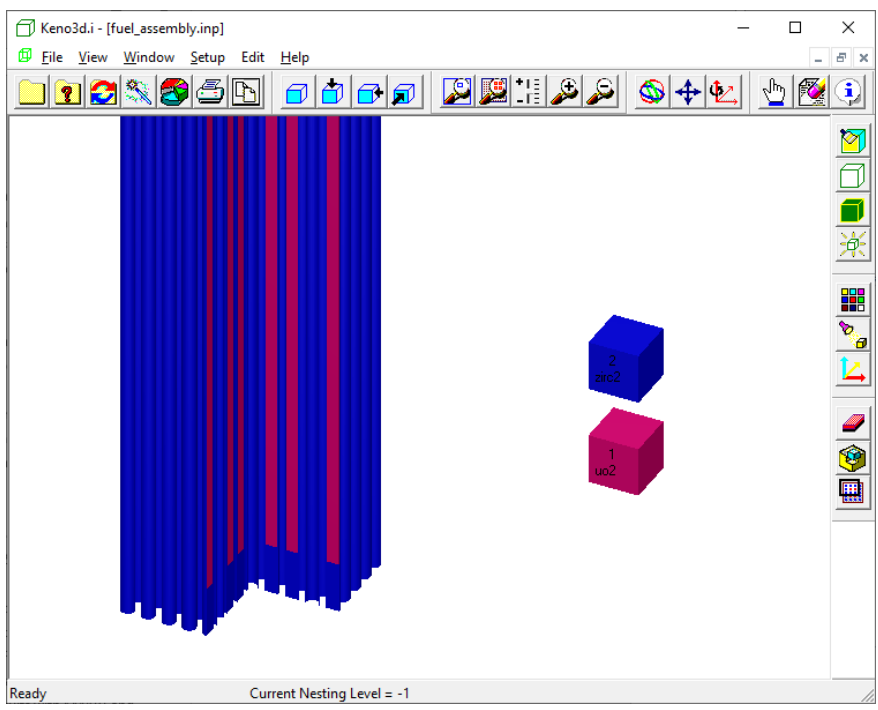

Figure 314. Zoomed image with modified legend. 


\subsection{PLOTTING CALCULATED RESULTS WITH KMART AND KENO3D}

KMART6 (KENO Module for Activity-Reaction Rate Tabulation Version 6) is a tool that post-processes information from a KENO-VI run to provide reaction rates by nuclide and the collapsing and printing of fluxes. See the [SCALE manual section titled KMART5 and KMART6] for details on KMART. KENO3D has the capability to plot the calculated results produced by KENO-VI/KMART6 overlaid on the 3D geometry model. The resulting * kmt file also contains the data in text tables, which are often easier to read than the output KMART adds to the standard output file.

The KENO-VI user must be aware of a few items when using KMART.

- KMART uses a restart file for its evaluations, so the user must make sure that a restart unit number $(\mathbf{W R S}=)$ is identified, and the number of generations between restart $(\mathbf{R E S}=)$ must be greater than zero. Both of these values are set in the PARAMETER block.

- If detailed reaction rates or fluxes are desired, then the user must modify the geometry input to create finer regions to provide more detail for the plot, because KENO-VI collects calculated data on a unit and region basis. The level of detail in the results is directly dependent upon the level of detail in the individual geometry regions.

- Region volumes are highly desired to normalize activities so that low reaction rates in large volumes do not appear to be more important than high reaction rates in small volumes. Volume calculations in CSAS6 are discussed in Section 4.7.

The Sheba solution reactor will be used as an example in this section. A simple KENO-VI input is provided in Figure 315. A view of the model in KENO3D is shown in Figure 316. In this example, the input file name is sheba2.inp.

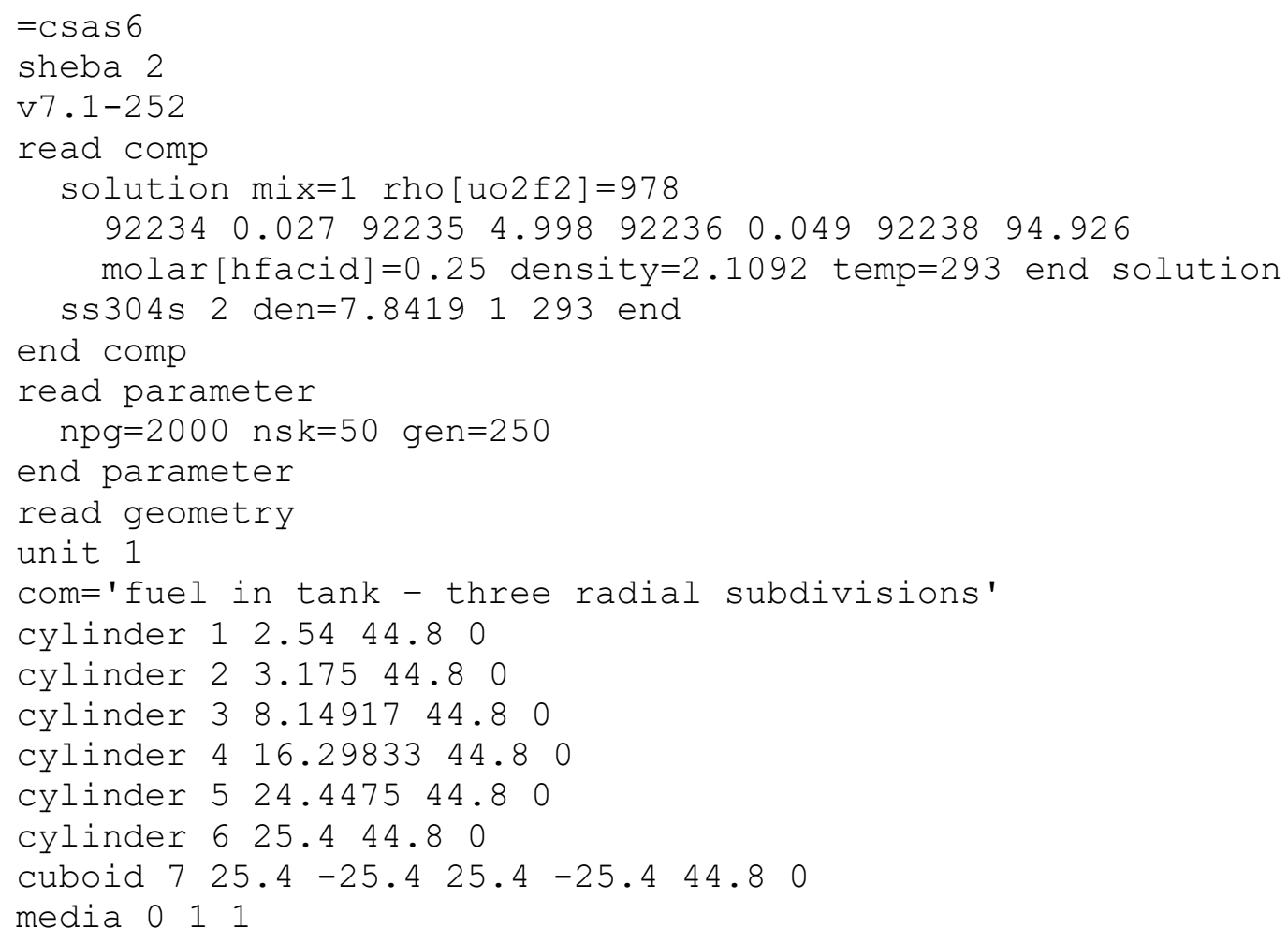




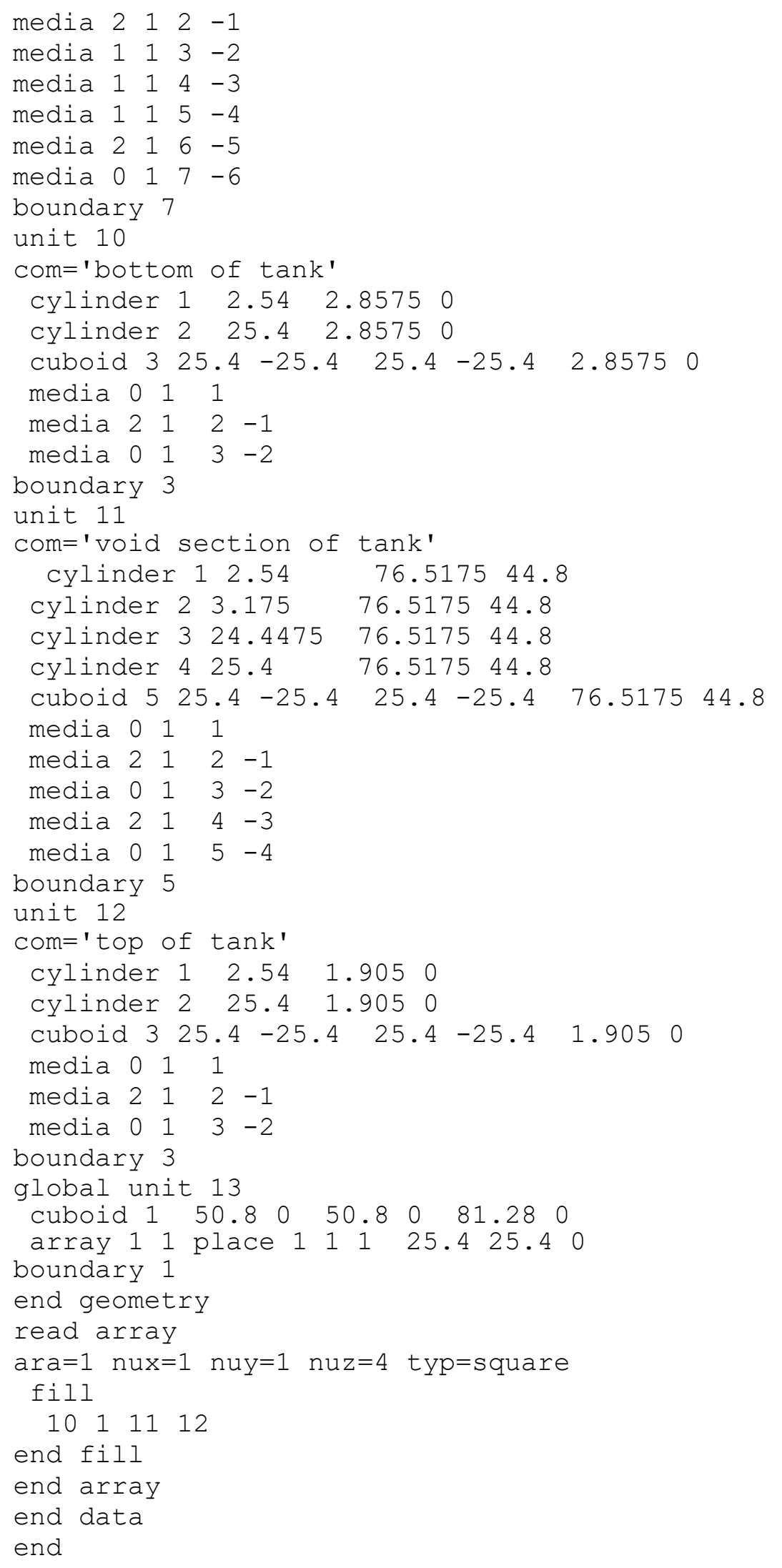

Figure 315. Sample input for simplified Sheba solution reactor. 


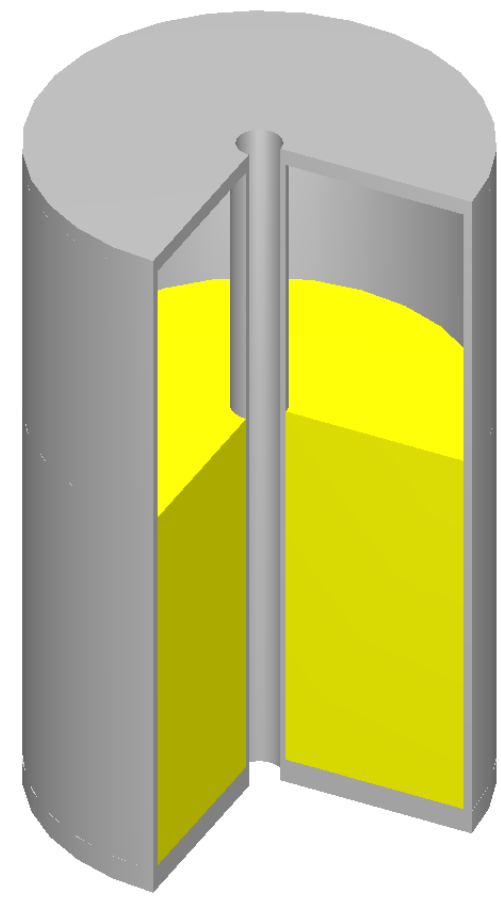

Figure 316. KENO3D rendering of simplified Sheba model.

The next step is to add parameters to write the restart file and add the flux edits to be used by KMART. The flux edits are enabled by specifying $f l x=y e s$ in the PARAMETER block, either by typing it in directly or using Fulcrum autocomplete. The restart file must be written to a specific unit after a specific number of generations. The restart file can be written at any interval of generations, or it can be written at the end of the calculation. In this case, it only needs to be written at the end of the calculation, so set $R E S=250$ in the PARAMETER block. Finally, set $W R S=35$ in the PARAMETER block as well; unit 35 is not used for any other purpose within CSAS by default, so it is available for the restart file. The final PARAMETER block is shown in Figure 317.

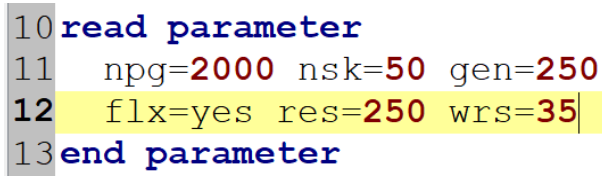

Figure 317. Updated PARAMETER block for KMART calculations.

The next step is to provide the input for KMART itself; KMART is a separate sequence from CSAS, so the input will be provided after the final end terminating the CSAS input. The stacked cases, CSAS and KMART, will be run in a single SCALE execution, so the temporary directory containing the restart file and cross section library will remain intact. Add a blank line to the end of the input and use autocomplete (CTRL-SPACE) to add a KMART6 sequence, as shown in Figure 318. The skeleton KMART input is shown in Figure 319. 


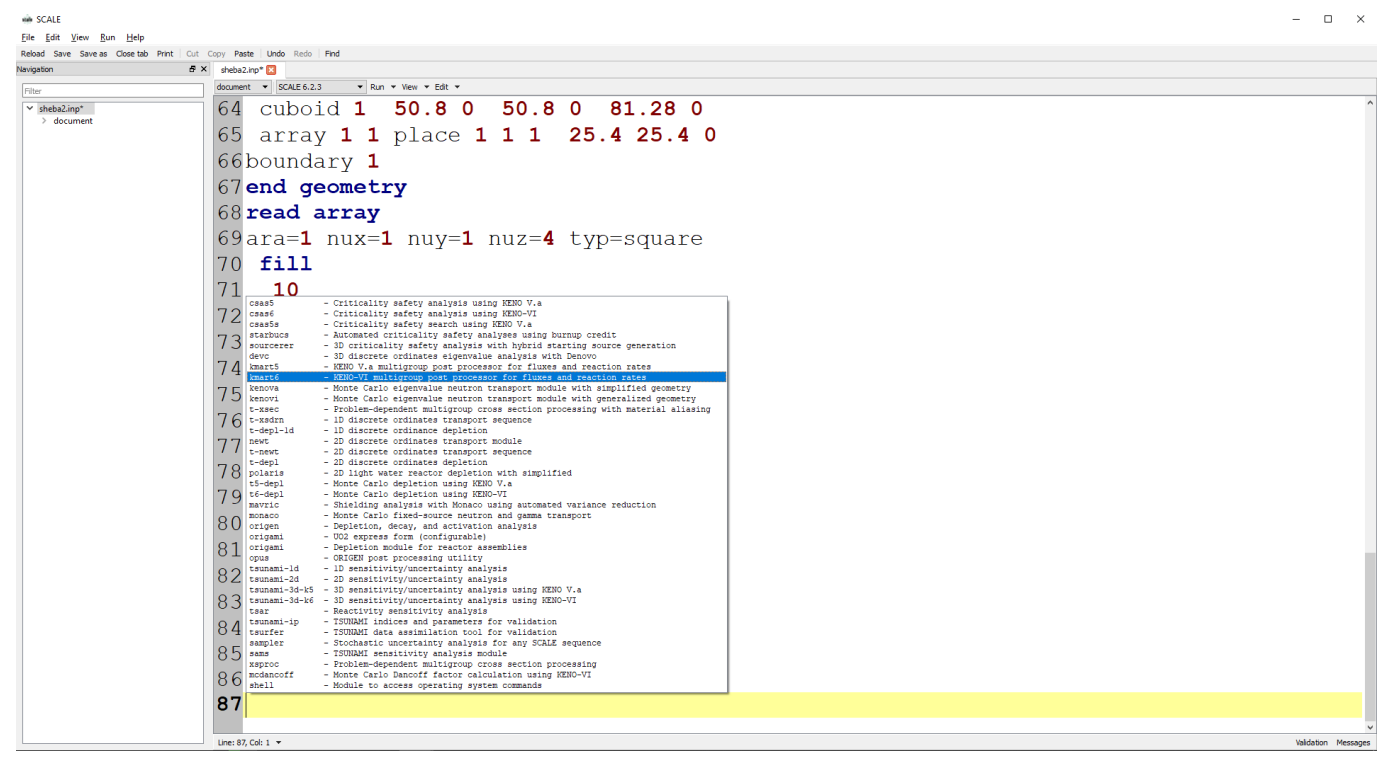

Figure 318. Adding the KMART sequence input after the CSAS sequence input.

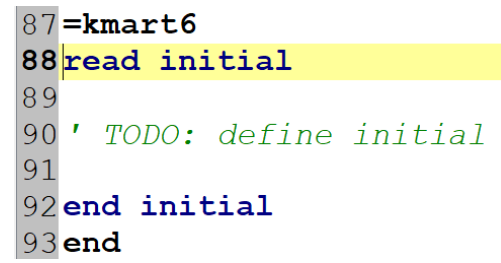

Figure 319. Skeleton KMART input provided by Fulcrum autocomplete.

The first block, and the only one provided by Fulcrum, is the INITIAL block. This block is analogous to the PARAMETER block in KENO. Two inputs are needed: the unit numbers for the restart file and the cross section library used in the KENO calculation. The cross section library is written on unit 4 in all CSAS calculations, so the specification to provide is xunit $=4$. In this example, the restart file has been written to unit 35, so the input specification for that is kunit=35. KMART generates activities by group by declaring actbygrp in the INITIAL block. Normalizing the activities by region volumes is also frequently helpful to prevent small reaction rates in large regions from appearing more important than large reaction rates in small volumes of high importance; this option is triggered with the input rrpvol. The last input in the INITIAL block in this example is the unit number and filename to which KENO3D data will be written. Both of these inputs are preceded by the keyword keno3d. Any unused unit number is acceptable; in this example, use 40 . The KMART filename must be the same as the input filename, but a kmt extension is necessary for KENO3D to recognize it. In this example, use the filename sheba2.kmt. The complete KENO3D input specification is therefore keno3d 40 sheba2. kmt. The complete INITIAL block input is provided in Figure 320, with extra blank lines and extraneous comments removed for clarity and brevity.

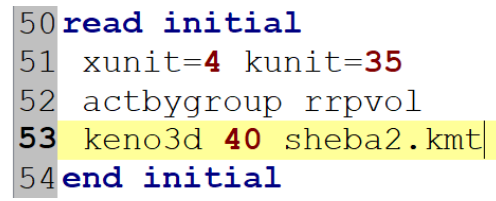

Figure 320. Complete INITIAL block for Sheba model. 
The next input block to supply for the KMART sequence is the ACTIVITY block. This block provides the specific nuclides and reactions for which activities will be calculated. The nuclides are specified by nuclide ID number; these numbers can be found in the Standard Composition Library section of the SCALE manual. The MT numbers for specific reactions of interest can be found in [MT Reaction Types on SCALE Cross section Libraries], an appendix to the SCALE Cross Section Libraries section of the manual. Some common MT numbers are provided in Table 4. In this example, activities will be generated only for absorption in ${ }^{1} \mathrm{H}$ and fission in ${ }^{235} \mathrm{U}$. The ACTIVITY block can be added to the KMART input via autocomplete, as shown in Figure 321. The nuclide ID for ${ }^{1} \mathrm{H}$ is 1001, and the MT number for absorption is 27, so the first input in the ACTIVITY block is 1001 27. Similarly, the nuclide ID for ${ }^{235} \mathrm{U}$ is 92235 , and the MT number for fission is 18 ; so the input is 92235 18. The complete ACTIVITY block is shown in Figure 322.

Table 4. Frequently used MT numbers.

\begin{tabular}{|c|c|}
\hline MT Number & Reaction Description \\
\hline 1 & Total \\
\hline 2 & Elastic scattering \\
\hline 18 & Total fission \\
\hline 27 & Total absorption \\
\hline 1452 & $\begin{array}{c}\text { Neutron generation from fission } \\
(v \times \text { fission })\end{array}$ \\
\hline
\end{tabular}

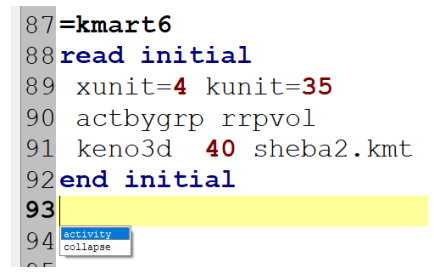

Figure 321. Adding ACTIVITY block to the KMART input.

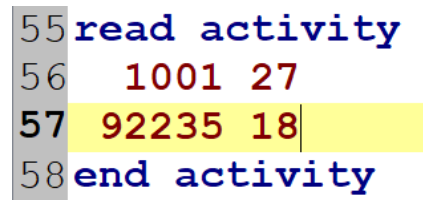

Figure 322. Complete ATIVITY block for the Sheba model.

The last input block is an optional block to collapse the results into fewer groups, primarily to make display and analysis of the data more manageable. This is accomplished in the COLLAPSE block by providing the last fine group number to be included in each broad group. The fine group structures are provided in the SCALE Cross Section Libraries section of the SCALE Manual. In this example, a threegroup structure will be used for the collapsed, broad-group edits: a fast group cutoff at $100 \mathrm{keV}$, an intermediate group cut off at $1 \mathrm{eV}$, and a thermal group extending to the bottom of the library at $10^{-5} \mathrm{eV}$. First, add the COLLAPSE block using autocomplete in Fulcrum. Then add the three bottom energy groups for the broad groups: lastg $=45$, lastg $=190$, and lastg $=252$. The final COLLAPSE block is shown in Figure 323. 


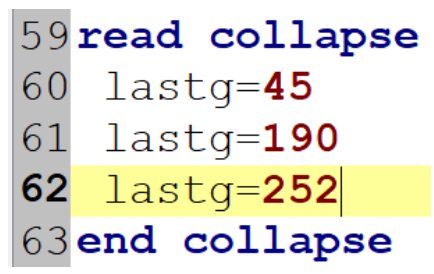

Figure 323. Complete COLLAPSE block for the Sheba model.

The input is now complete and can be executed in SCALE. A *kmt file is also generated and returned alongside the output file. With KENO3D open and displaying the model as shown in Figure 316 above, for example, the KMART data can be visualized by selecting View > Plot KMart Data. This generates a Plot Results dialog, as shown in Figure 324, providing available data for plotting. Select U-235 (MT=18) and click Generate Plot to generate an image such as shown in Figure 325. Finally, click the Show legend button and click OK, as described in Section 9.4, to display the color scale for the fission rates as shown in Figure 326.

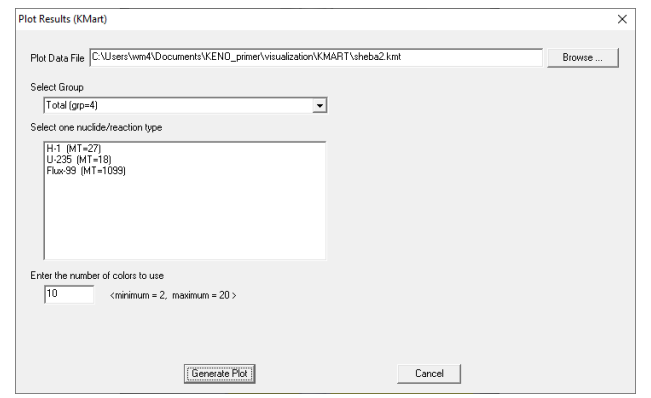

Figure 324. Plot Results dialog in KENO3D for selecting KMART results to display.

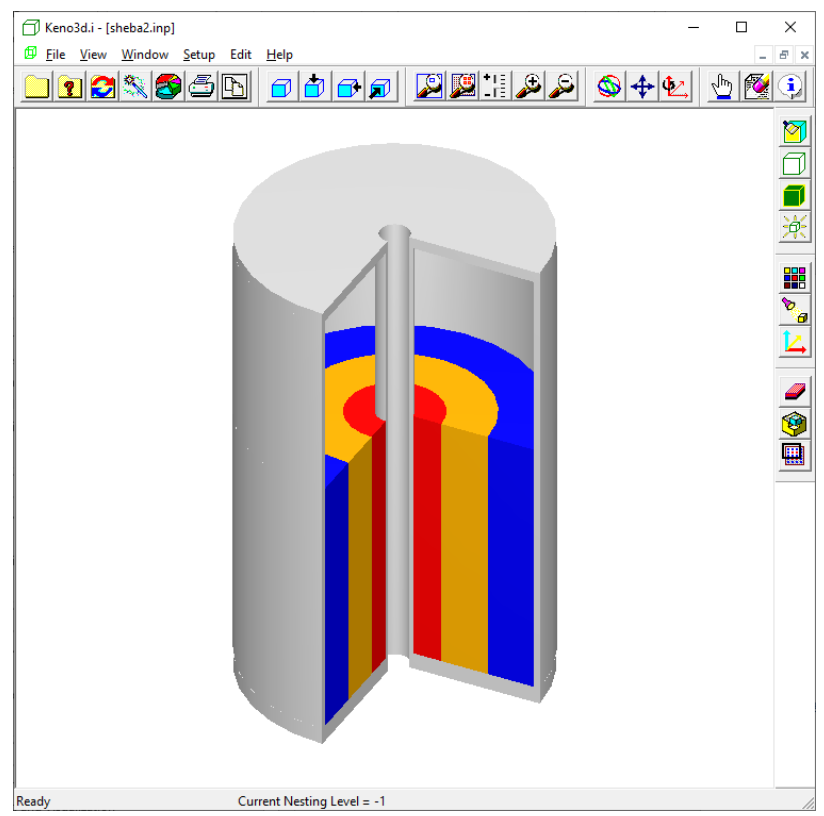

Figure 325. KENO3D rendering of the Sheba model with fission reaction rates. 


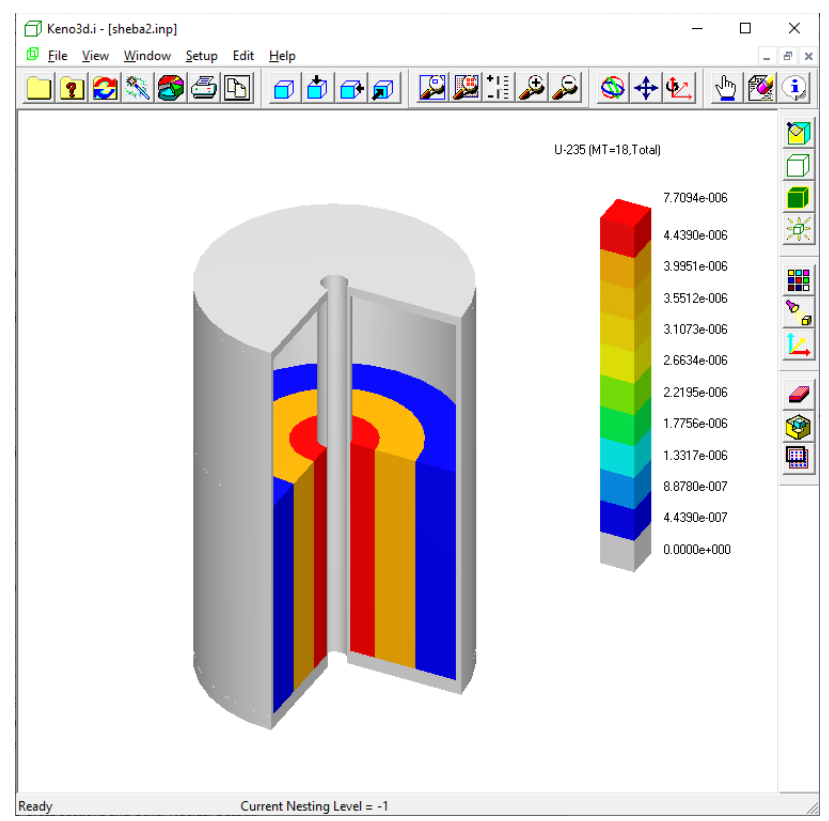

Figure 326. KENO3D rendering of the Sheba model with fission reaction rates and color scale.

The addition of more refinements axially and radially can improve the resolution of the reported fluxes and reaction rates. An example with 45 axial slices, each containing 21 radial divisions, is shown in Figure 327. This is significantly more detailed than the single axial slice with 3 radial divisions used here: the detailed model can be developed using the geometry and array constructs discussed in Sections 4.5 and 4.6.

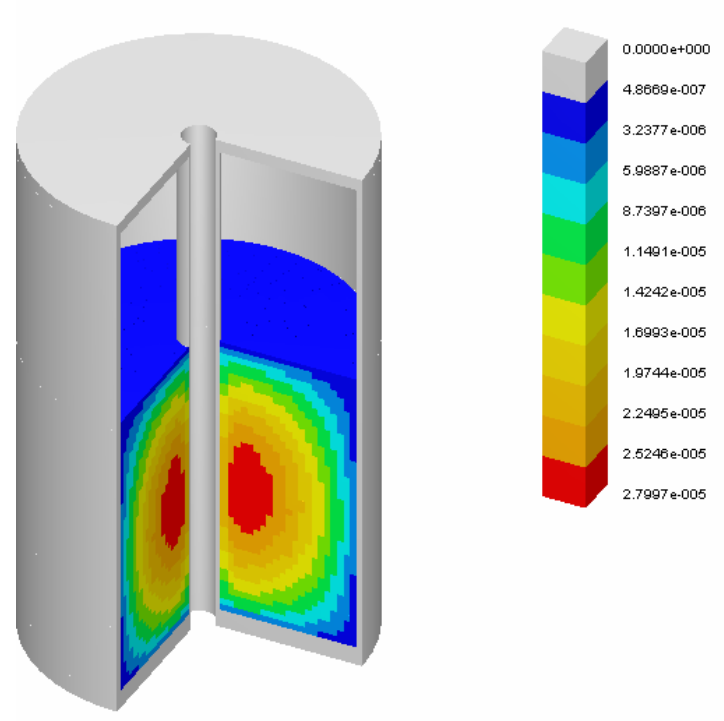

Figure 327. Detailed fission neutron generation rate $(\mathrm{MT}=1452)$ in Sheba model. 


\subsection{SUMMARY}

This section has provided an overview of the following capabilities within SCALE and Fulcrum:

- generating 2D color plots of KENO-VI geometry models,

- viewing 2D source convergence diagnostics generated by KENO-VI,

- generating plots of 1D nuclear data used in KENO-VI calculations,

- generating and display mesh-based data in Fulcrum,

- interactively viewing 3D wireframe and rendered images of KENO-VI geometry models using KENO3D, and

- plotting calculated results overlain on the 3D geometry model using KMART and KENO3D. 



\section{CONCLUSION}

This primer provides an introduction to the KENO-VI Monte Carlo transport code within the CSAS6 sequence for use in calculating the neutron multiplication factor, also known as the $k_{\text {eff }}$ eigenvalue, primarily for nuclear criticality safety analyses. Aside from serving as a quick start guide, the primer specifies compositions in SCALE, describes model geometry, explains problem-specific cross section processing for MG transport problems, and presents instructions for plotting and visualizing models and data. A number of other resources exist to expand on the information presented here, including:

- $\quad$ The SCALE manual

- SCALE training - check for information and updates at: https://www.ornl.gov/scale/scaletraining

- The SCALE web page: https://www.ornl.gov/scale

- A Google discussion group (email scalehelp@ornl.gov for information on joining the group)

- Direct user assistance via scalehelp@ornl.gov 



\section{REFERENCES}

1. W. A. Wieselquist, R. A. Lefebvre, and M. A. Jessee, Eds., SCALE Code System, ORNL/TM2005/39, Version 6.2.4, Oak Ridge National Laboratory, Oak Ridge, TN (2020).

2. S. Goluoglu, L. M. Petrie, M. E. Dunn, D. F. Hollenbach, and B. T. Rearden, "Monte Carlo Criticality Methods and Analysis Capabilities in SCALE," Nucl. Technol. 174(2), pp. 214-235 (2011).

3. B. T. Rearden, B. R. Langley, A. B. Thompson, and R. A. Lefebvre, "Fulcrum User Interface for SCALE 6.2," Proceedings of NCSD 2017: Criticality Safety - pushing boundaries by modernizing and integrating data, methods, and regulations, Carlsbad, NM (2017).

4. K. Bekar, J. B. Clarity, M. Dupont, R. A. Lefebvre, W. J. Marshall, and E. M. Saylor, KENO V.a Primer: Performing Calculations Using SCALE's Criticality Safety Analysis Sequence (CSAS5) with Fulcrum, ORNL/TM-2020/1664, Oak Ridge, TN (2020).

5. H. C. Paxton and N. L. Pruvost, Critical Dimensions of Systems Containing ${ }^{235} U,{ }^{239} \mathrm{Pu}$, and ${ }^{233} \mathrm{U}$, LA10860-MS, Los Alamos, NM (1987).

6. "PBMR Coupled Neutronics/Thermal-hydraulics Transient Benchmark: The PBMR-400 Core Design, Volume I - The Benchmark Definition,” NEA/NSC/DOC(2013)10, Paris, France (2013).

7. R. A. Lefebvre, “Advanced User Interface Capabilities," presented at the 2020 SCALE Users' Group Workshop, Oak Ridge, TN (2020). Available at https://www.ornl.gov/file/advanced-user-interfacecapabilities-tutorial/display, as of September 4, 2020. 

APPENDIX A. INPUT FILES FOR EXAMPLE PROBLEMS 



\section{APPENDIX A. INPUT FILES FOR EXAMPLE PROBLEMS}

\section{INPUT FOR SECTION 2.3}

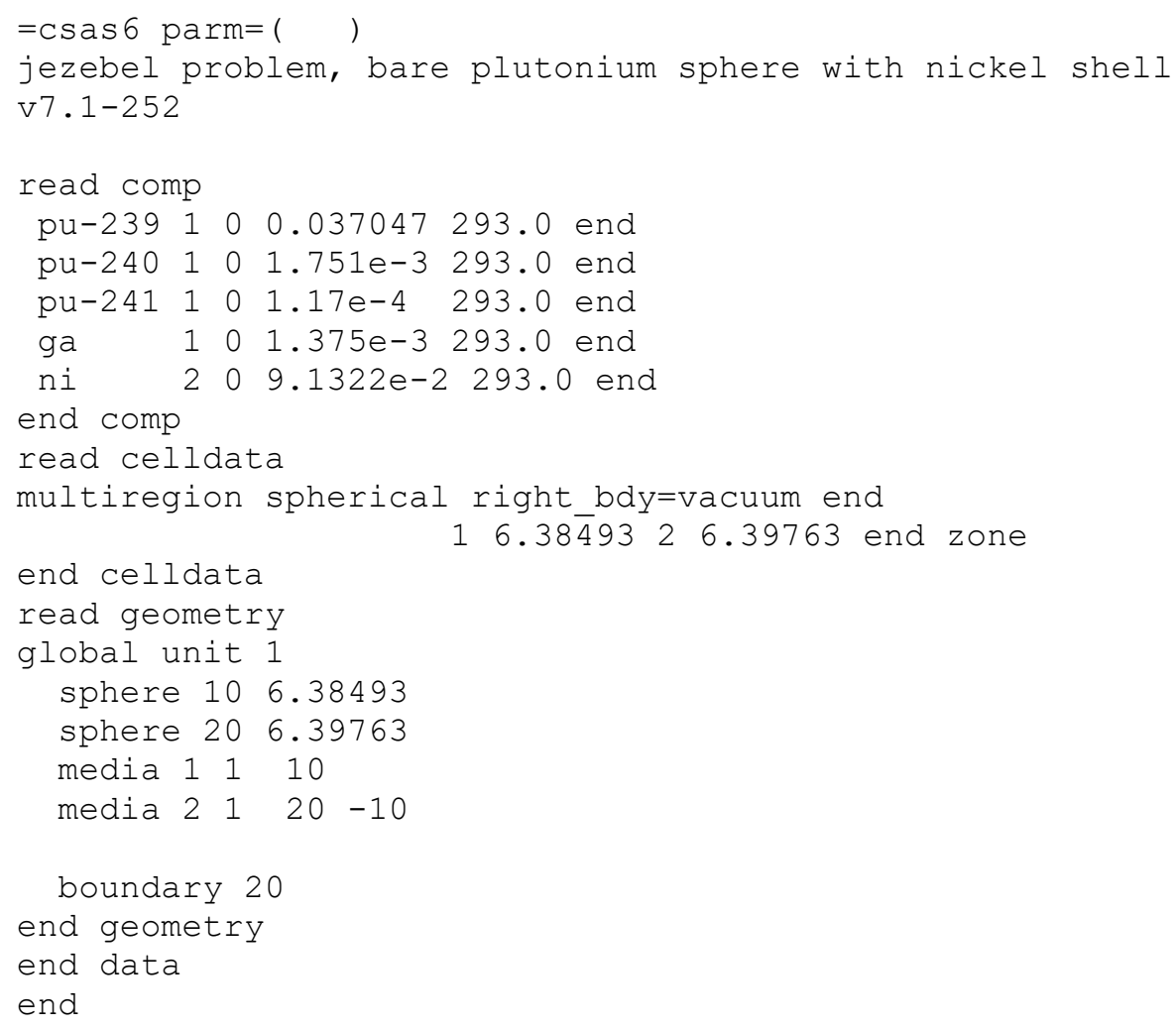

\section{INPUT FOR SECTION 3.4.1}

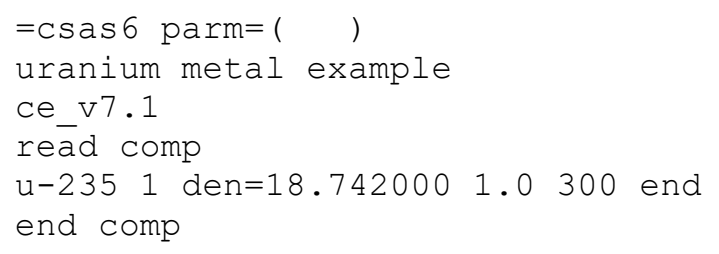

INPUT FOR SECTION 3.4.2

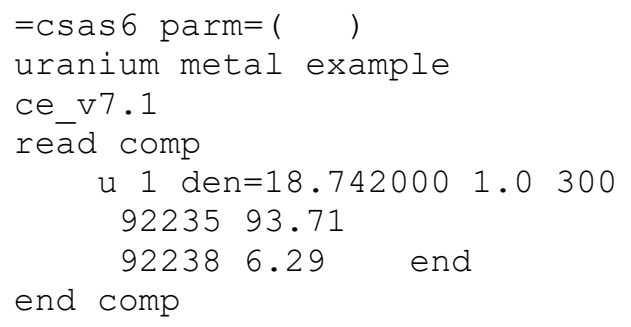




\section{INPUT FOR SECTION 3.4.3}

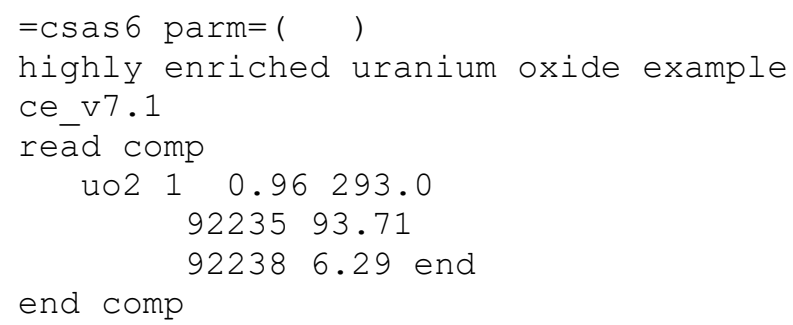

\section{INPUT FOR SECTION 3.4.4}

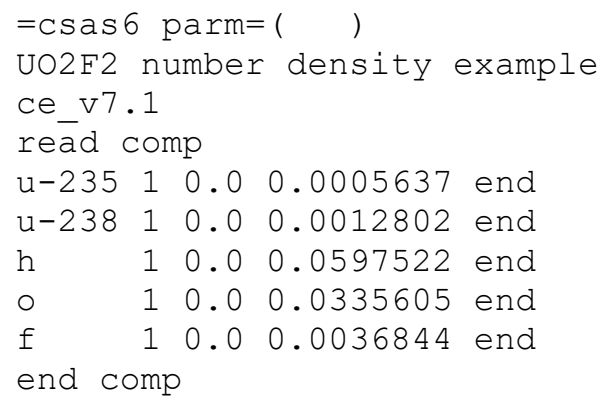

INPUT FOR SECTION 3.4.5

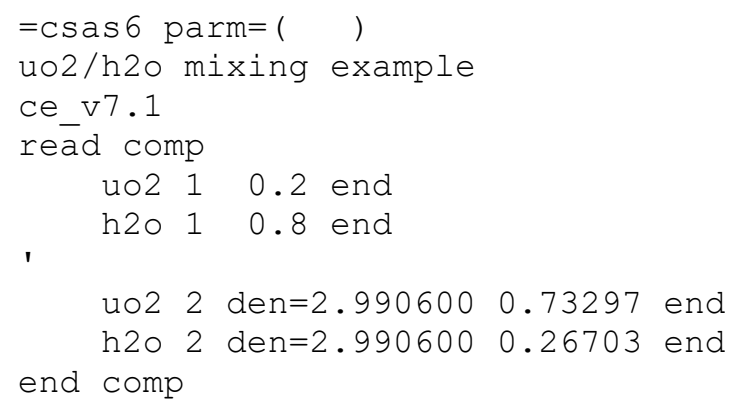

\section{INPUT FOR SECTION 4.2.1.1}

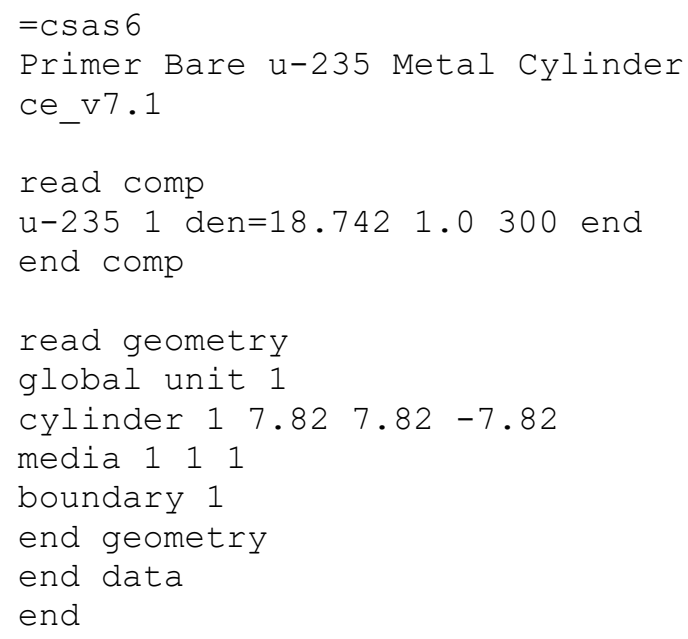




\section{INPUT FOR SECTION 4.2.1.2}

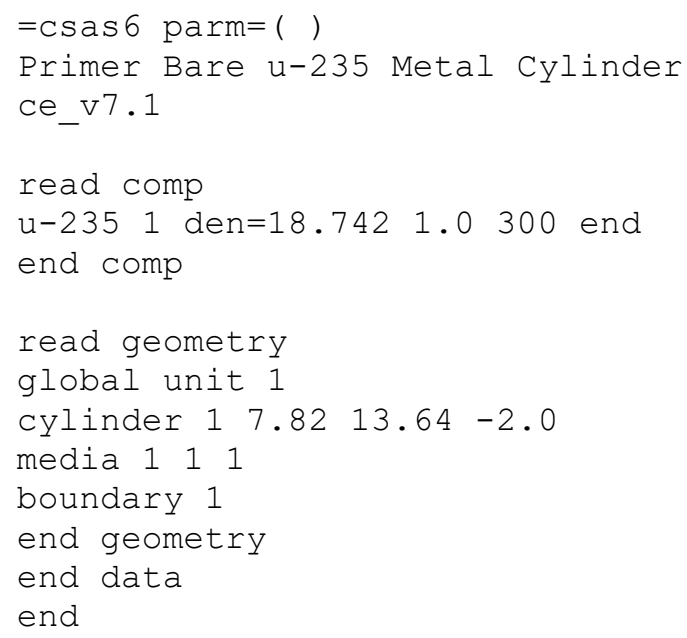

\section{INPUT FOR SECTION 4.5.1}

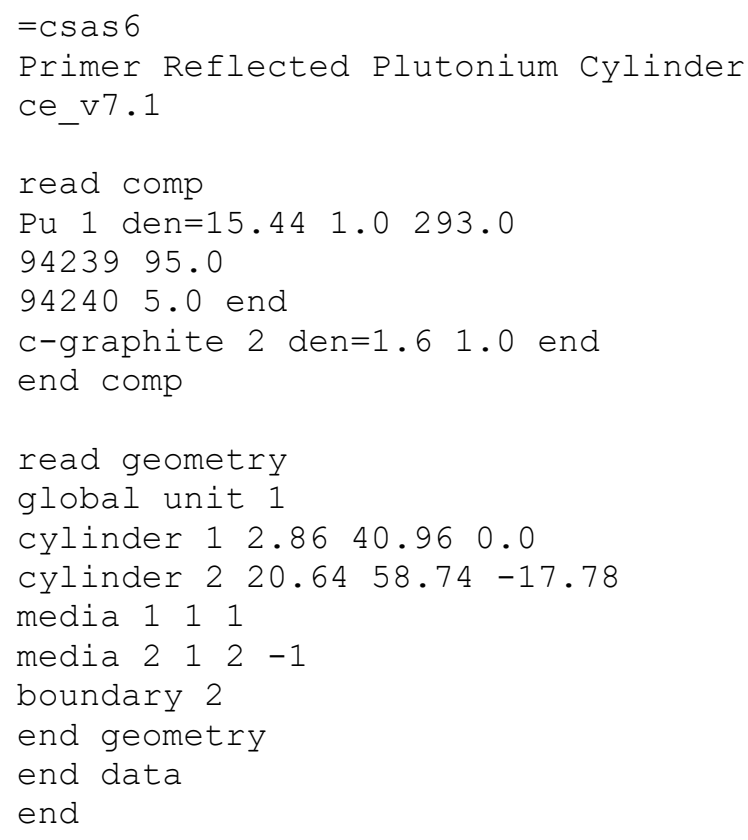

INPUT FOR SECTION 4.5.2

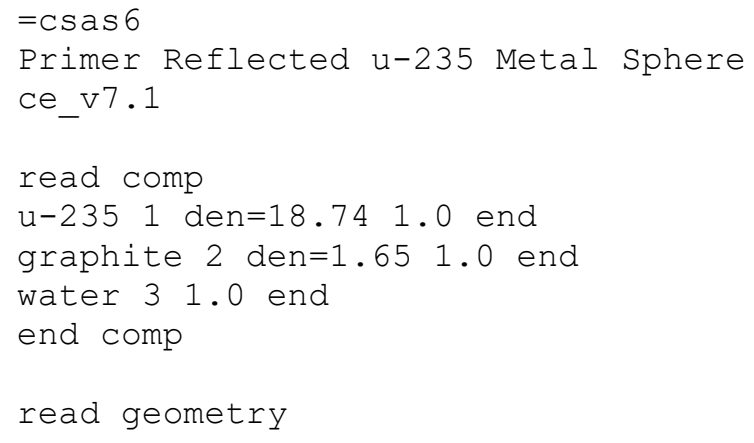




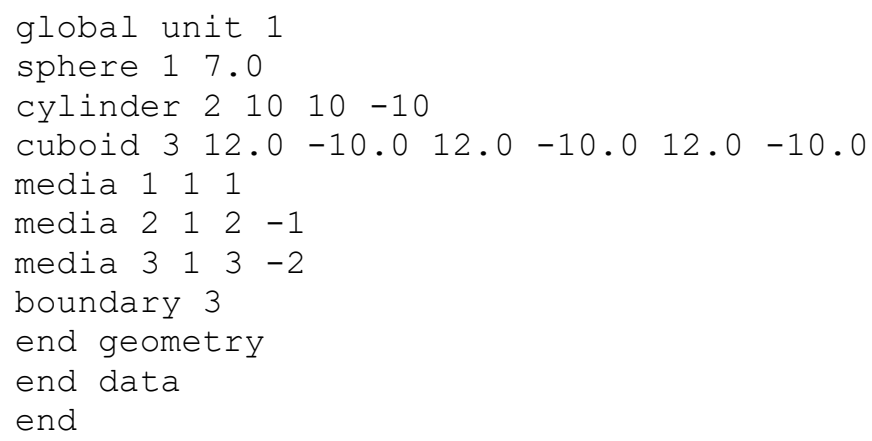

\section{INPUT FOR SECTION 4.6.1}

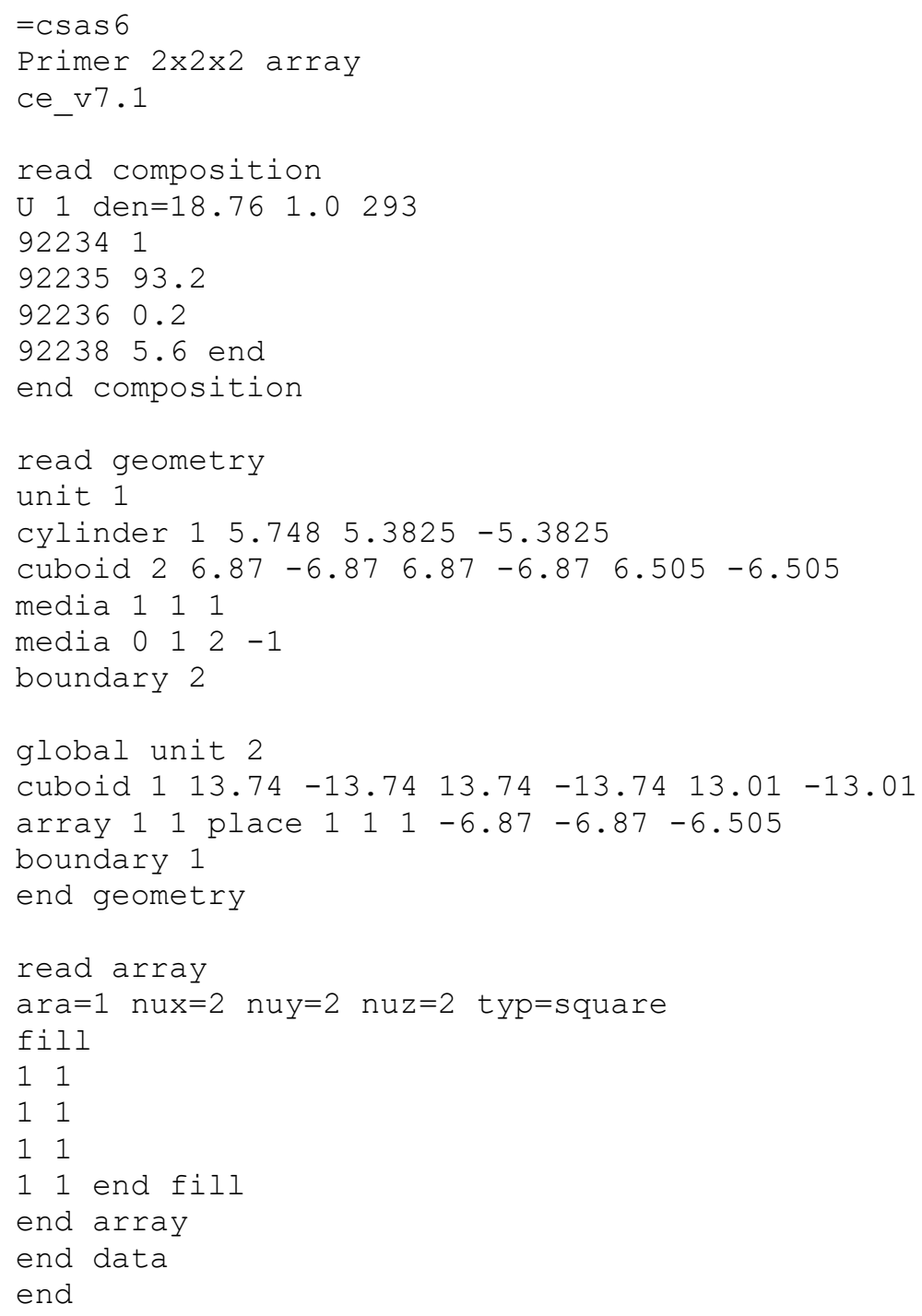

\section{INPUT FOR SECTION 4.6.2}

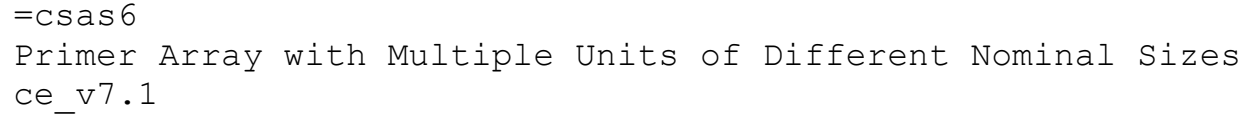




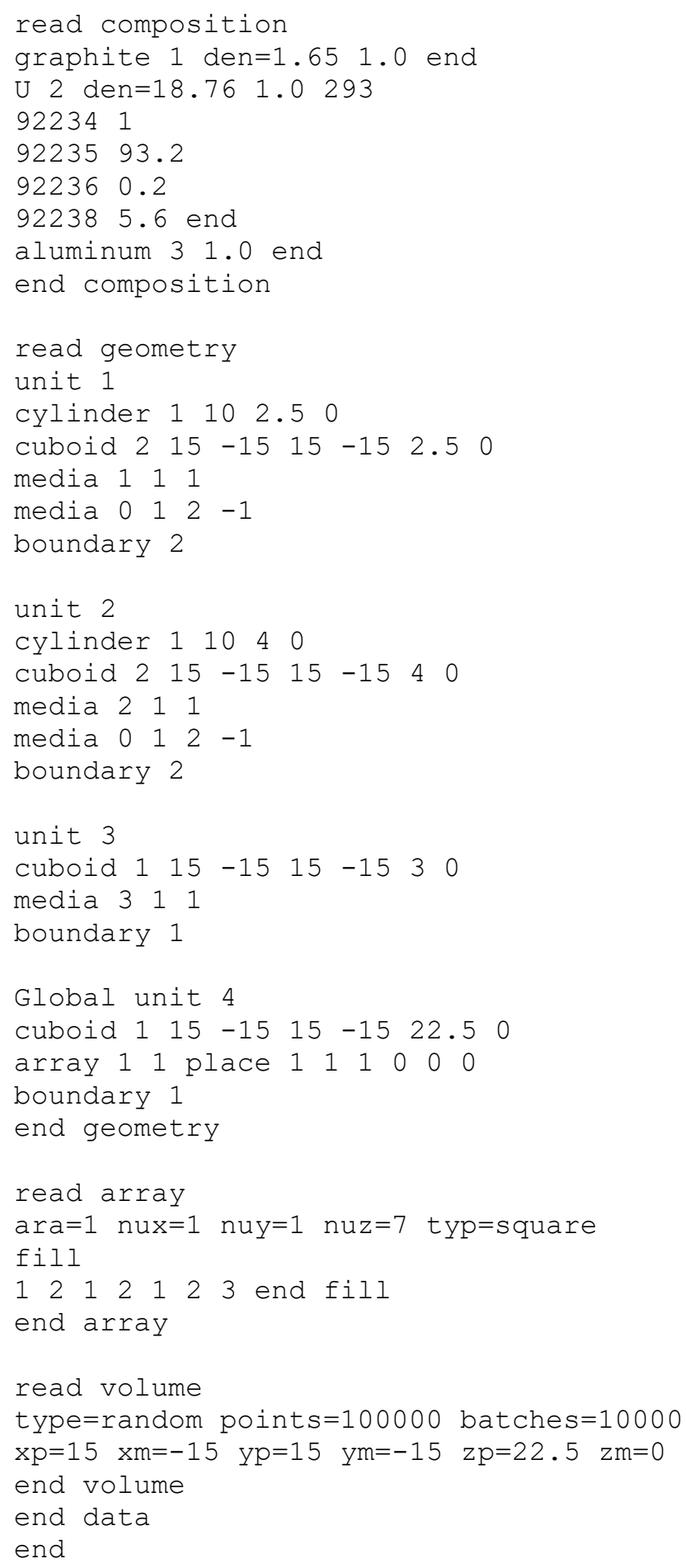

\section{INPUT FOR SECTION 5.3.1}

$=\operatorname{csas} 6$

Primer Three intersecting cylinders ce_v 7.1 


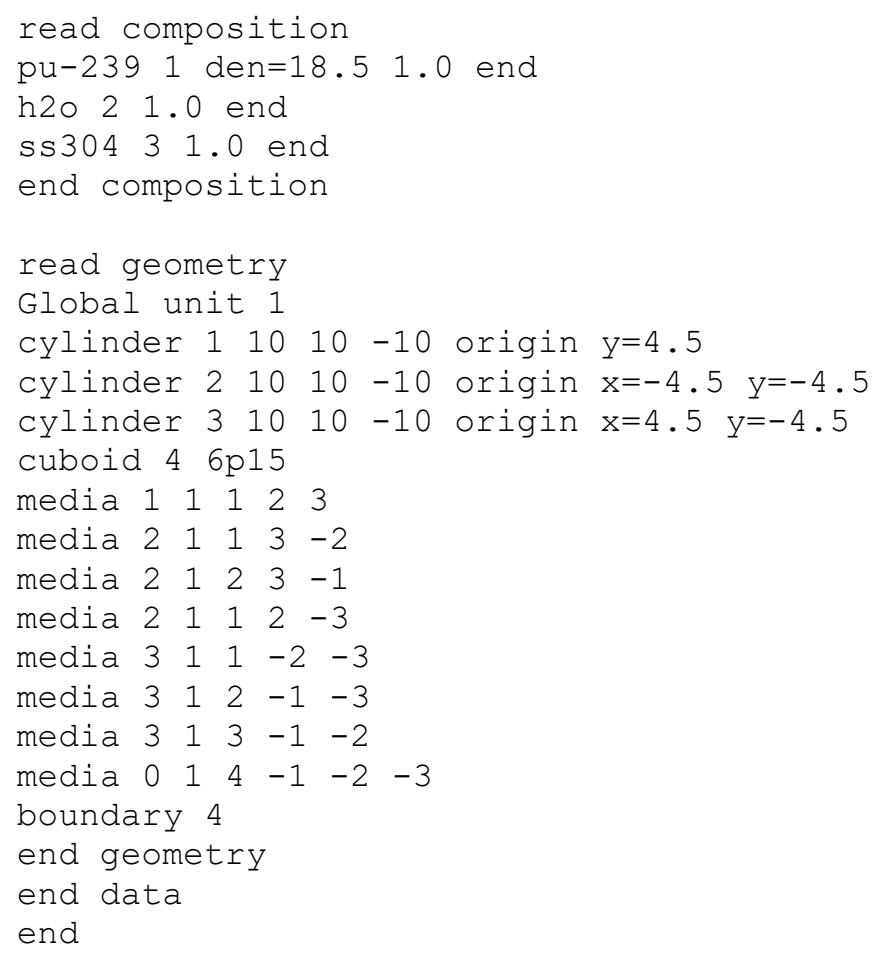

\section{INPUT FOR SECTION 5.4.4}

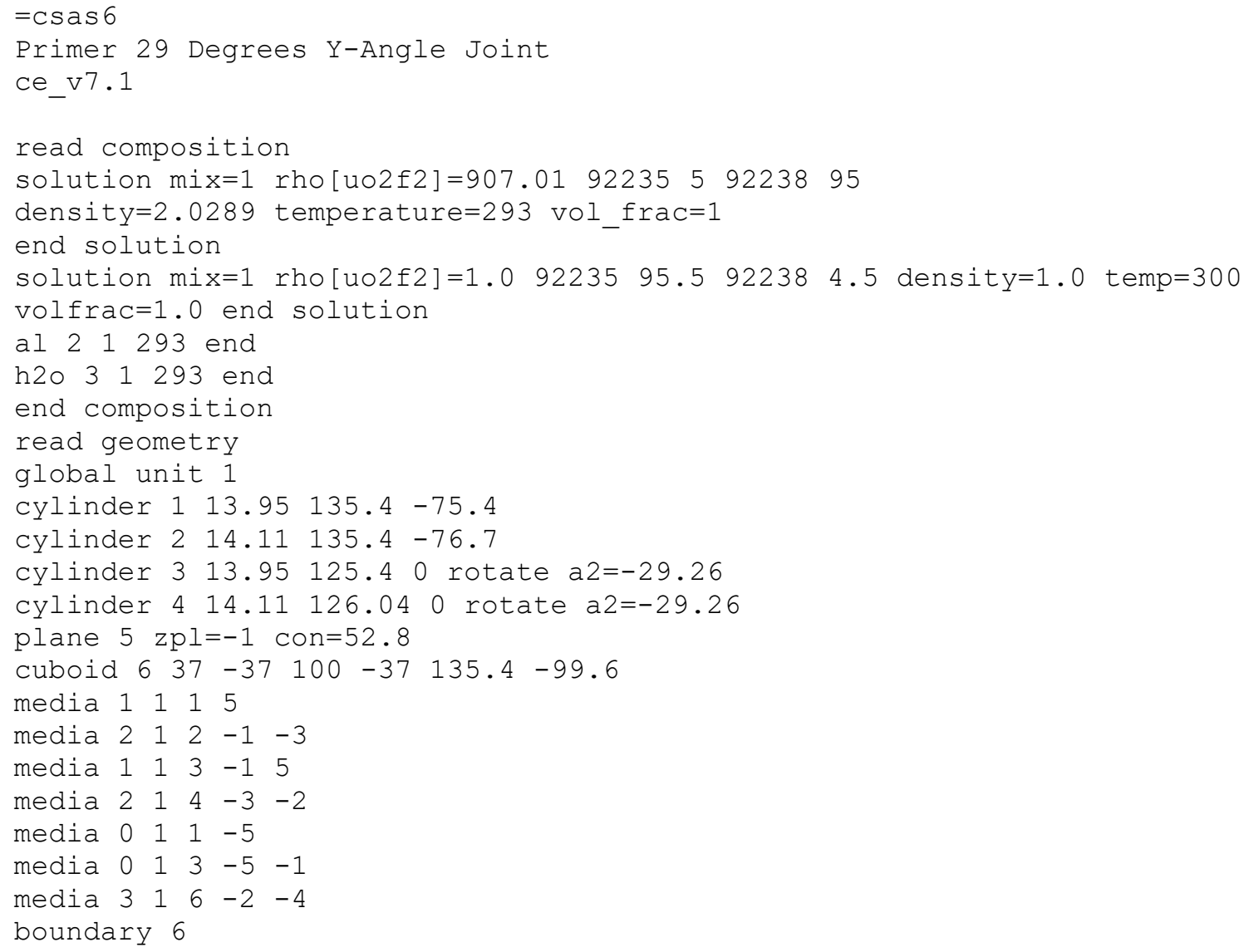


end geometry

end data

end

\section{INPUT FOR SECTION 5.5.2}

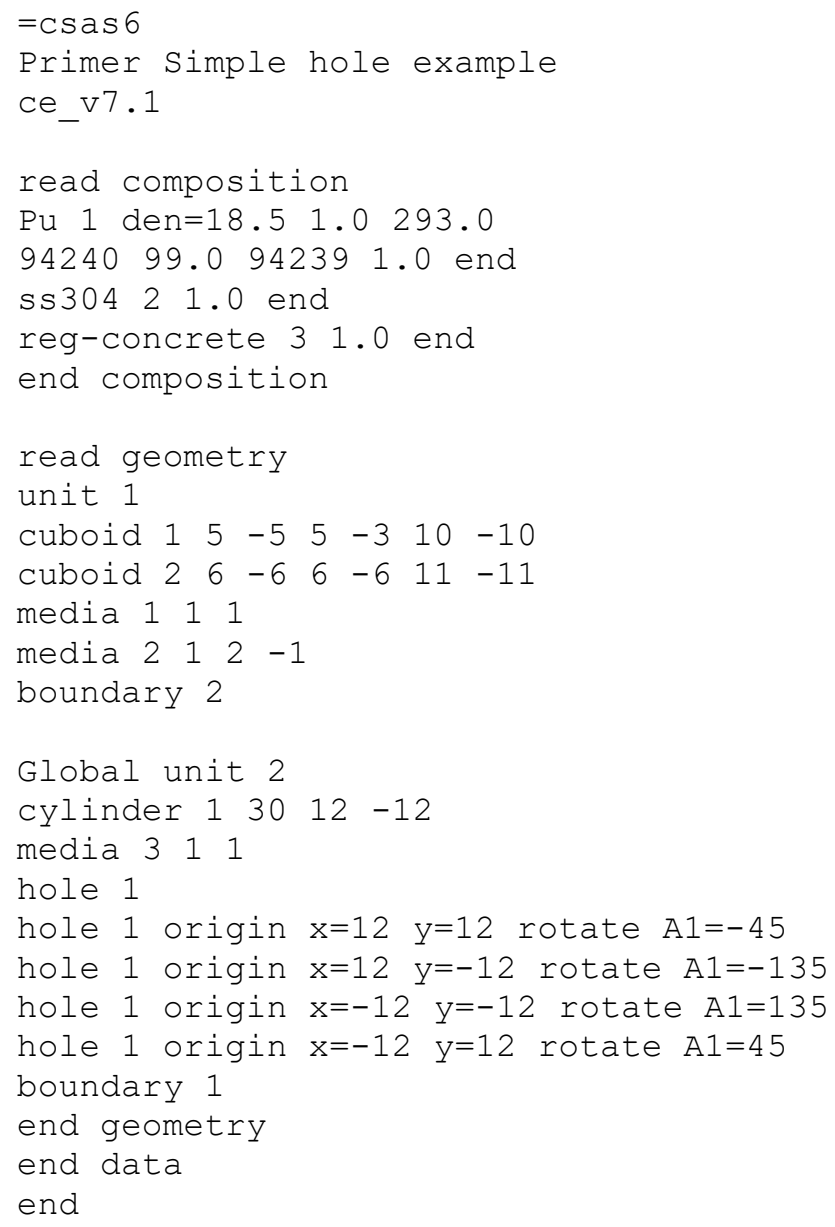

INPUT FOR SECTION 6.2

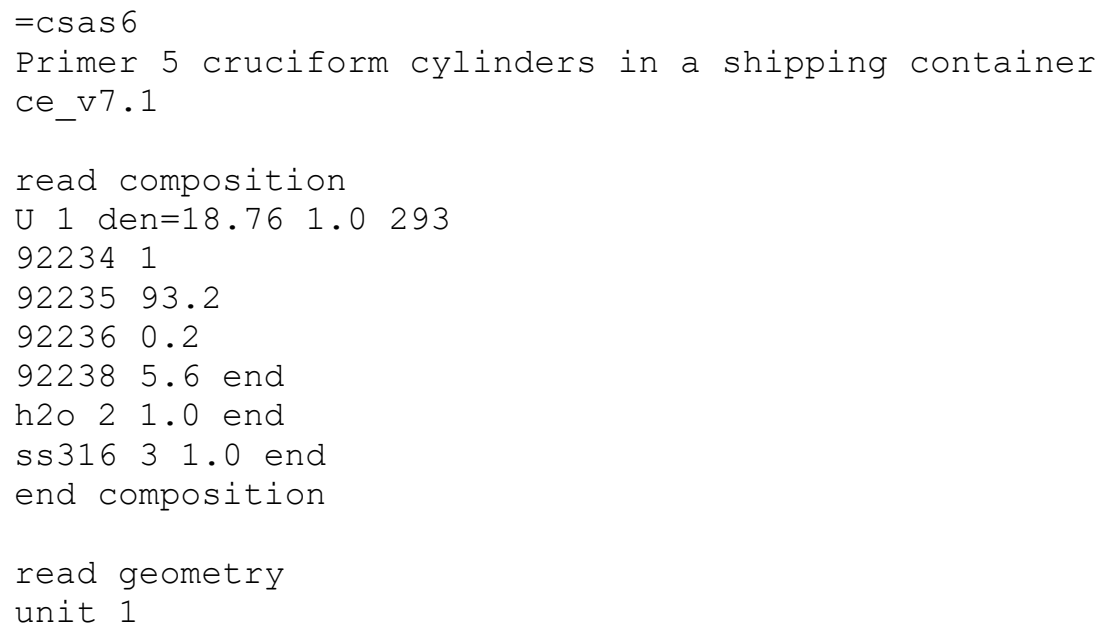




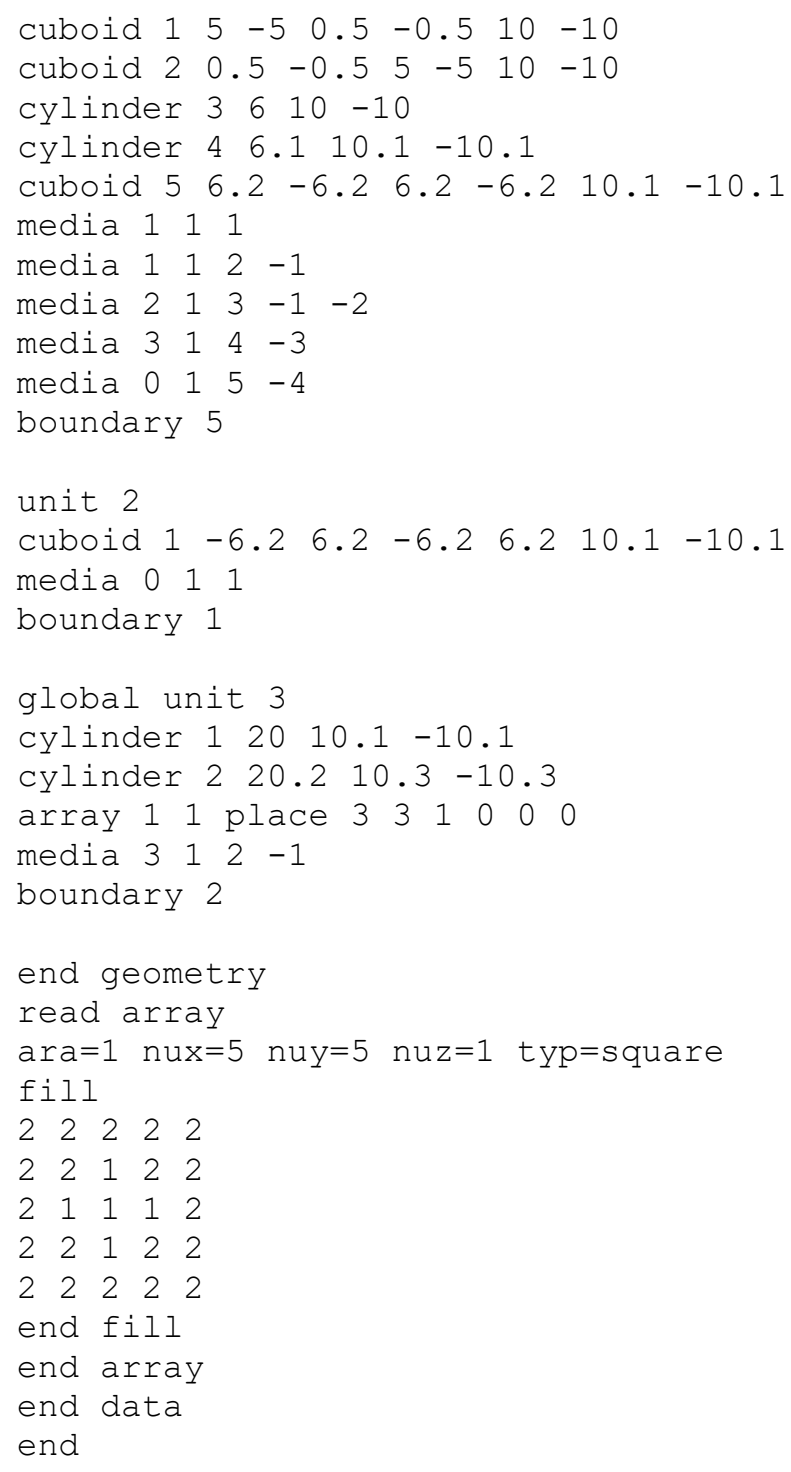

INPUT FOR SECTION 6.3

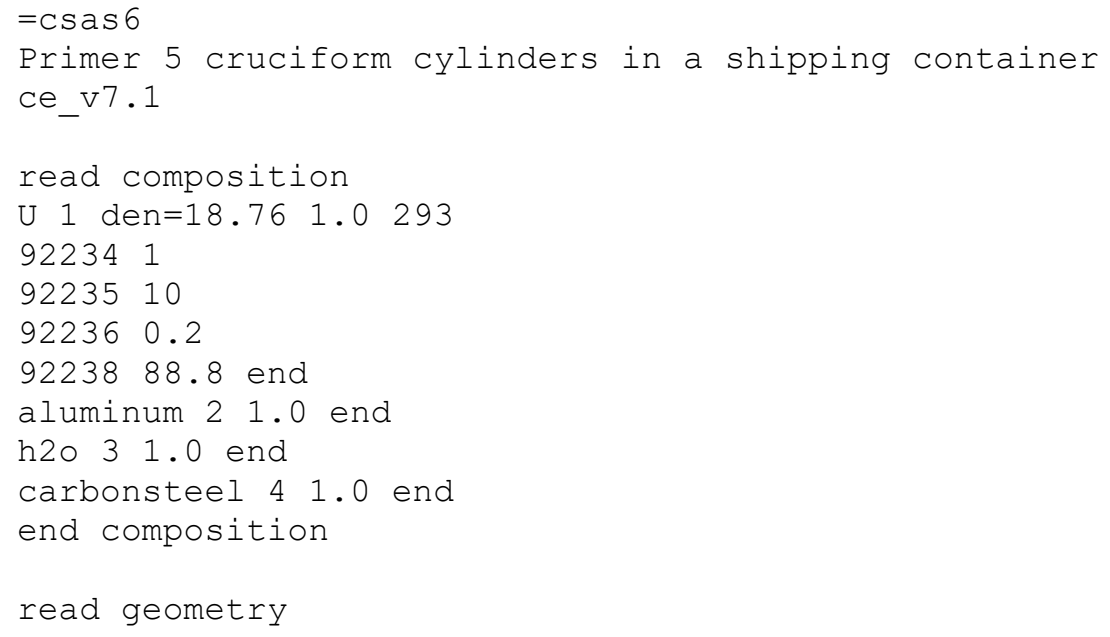




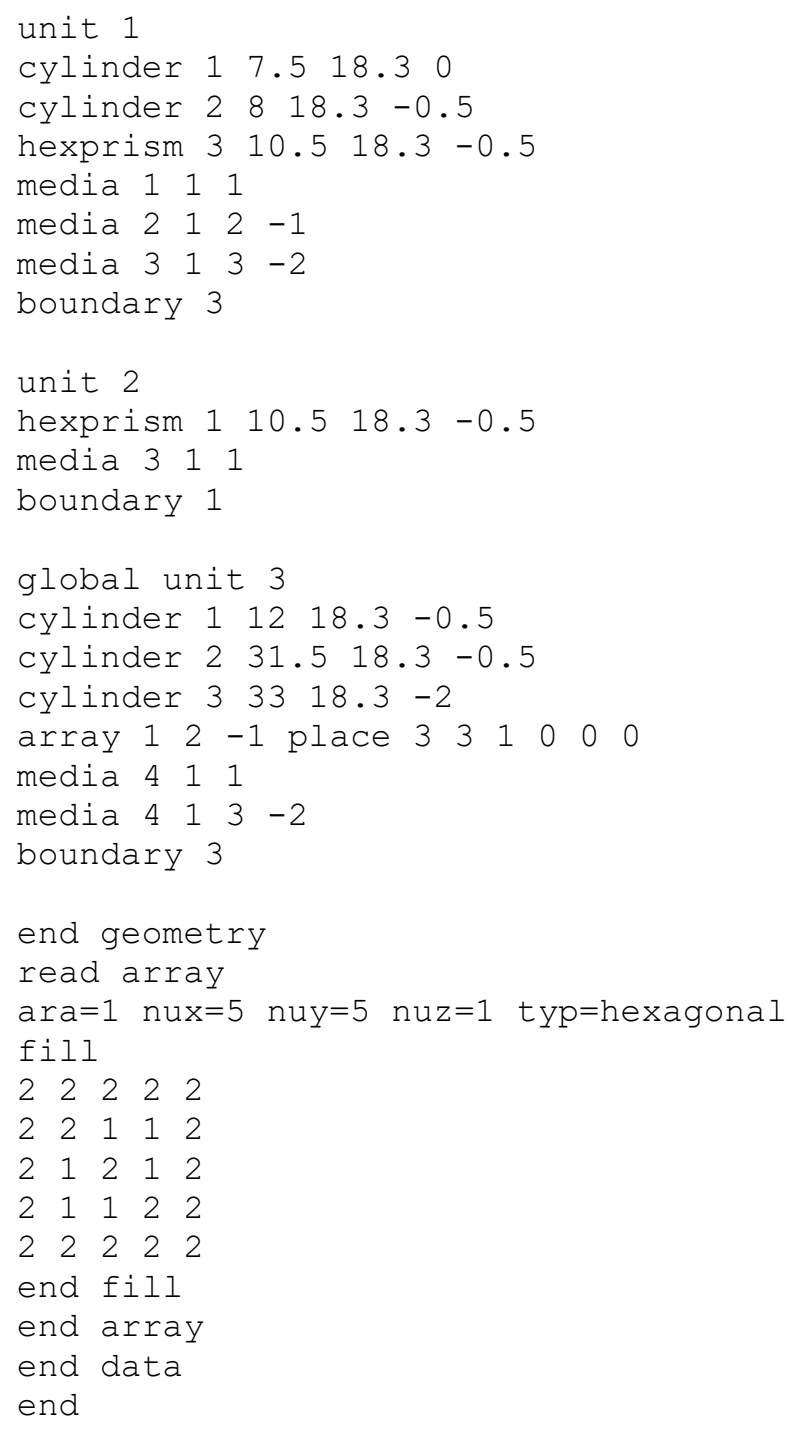

\section{INPUT FOR SECTION 7.2.1}

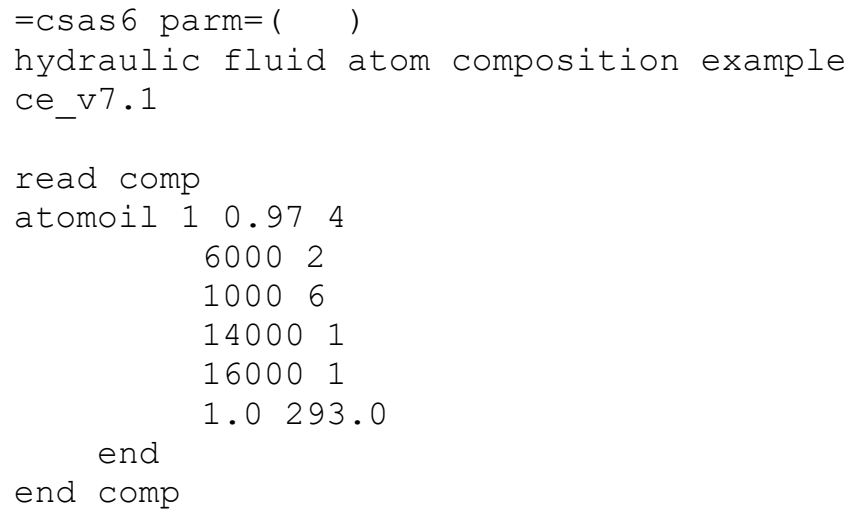

\section{INPUT FOR SECTION 7.2.2}

$=\operatorname{csas} 6 \operatorname{parm}=(\quad)$ 


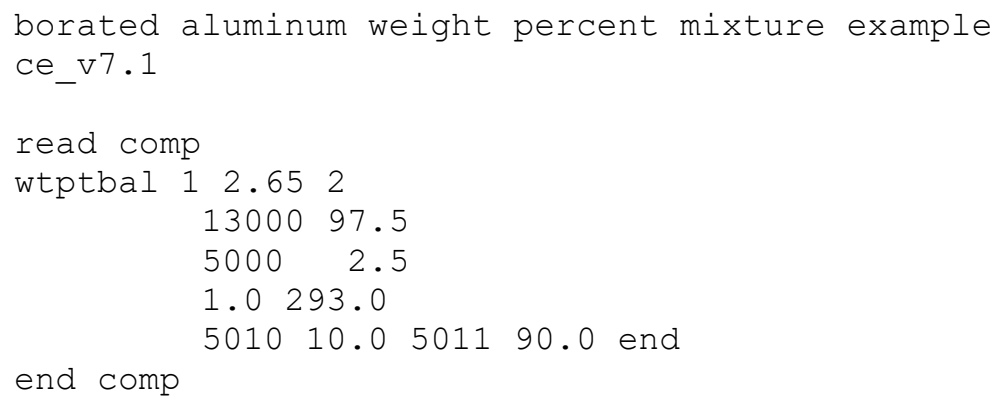

\section{INPUT FOR SECTION 7.2.3}

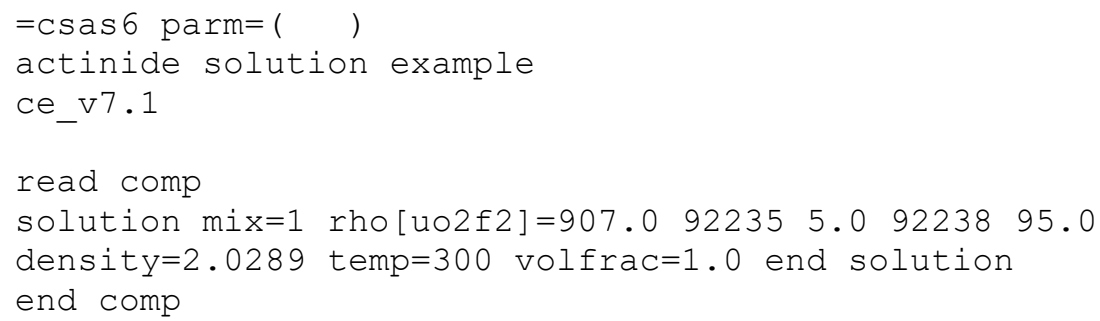

\section{INPUT FOR SECTION 8.5.1}

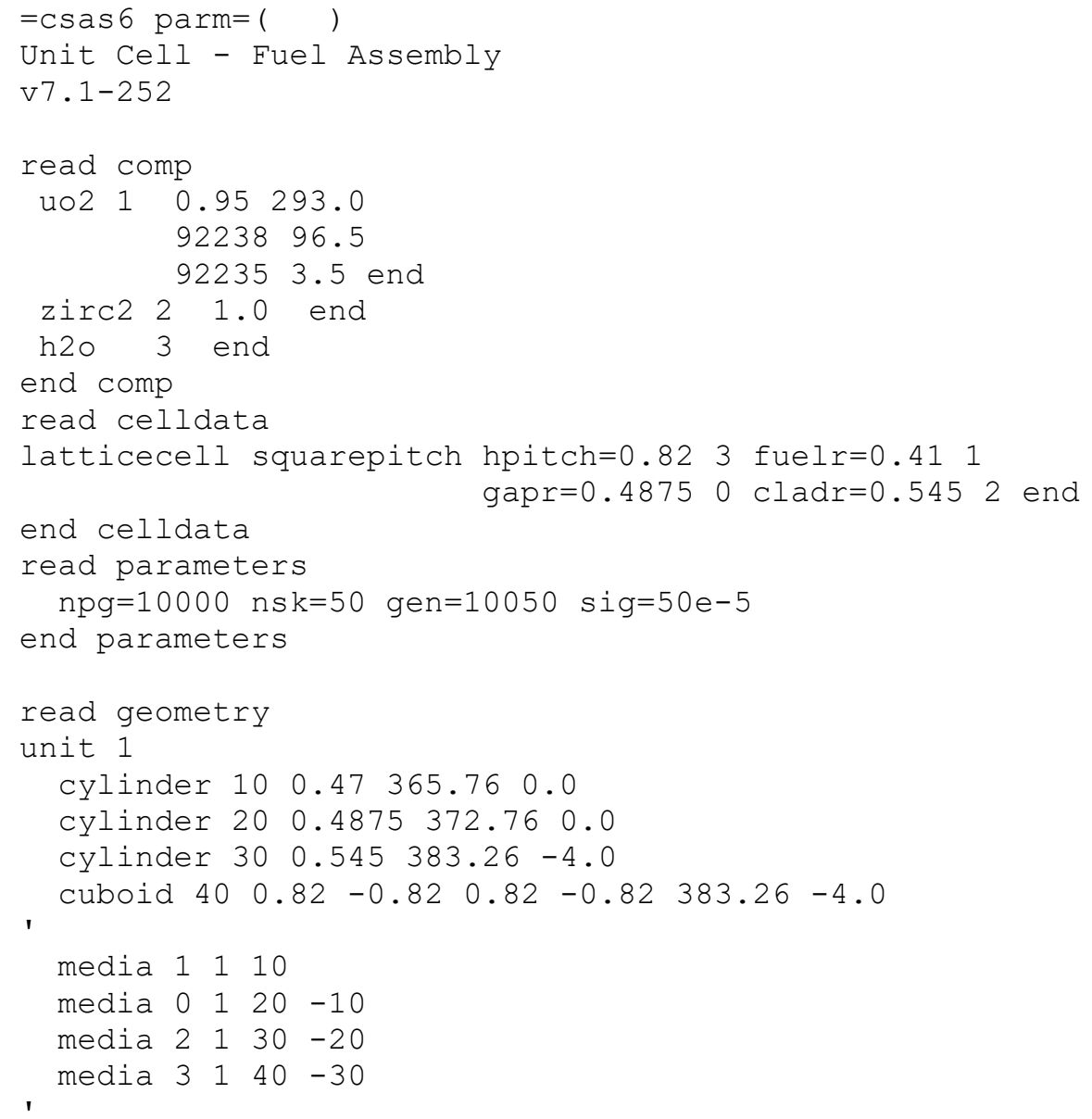




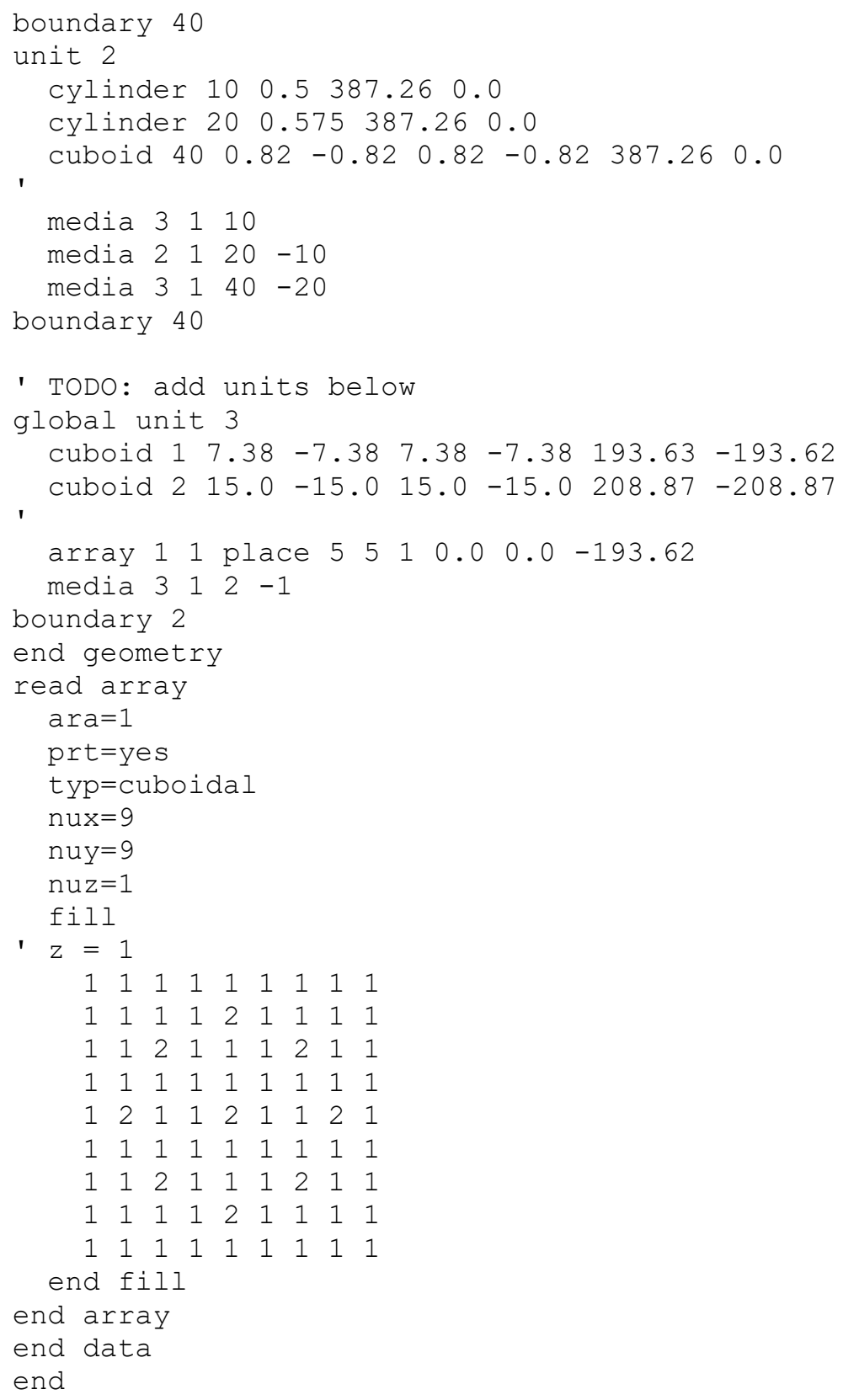

\section{INPUT FOR SECTION 8.5.2}

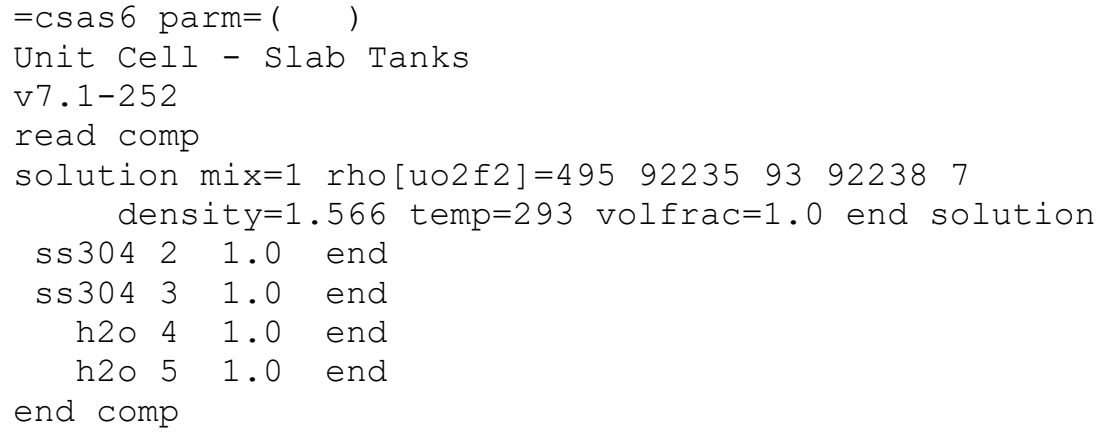




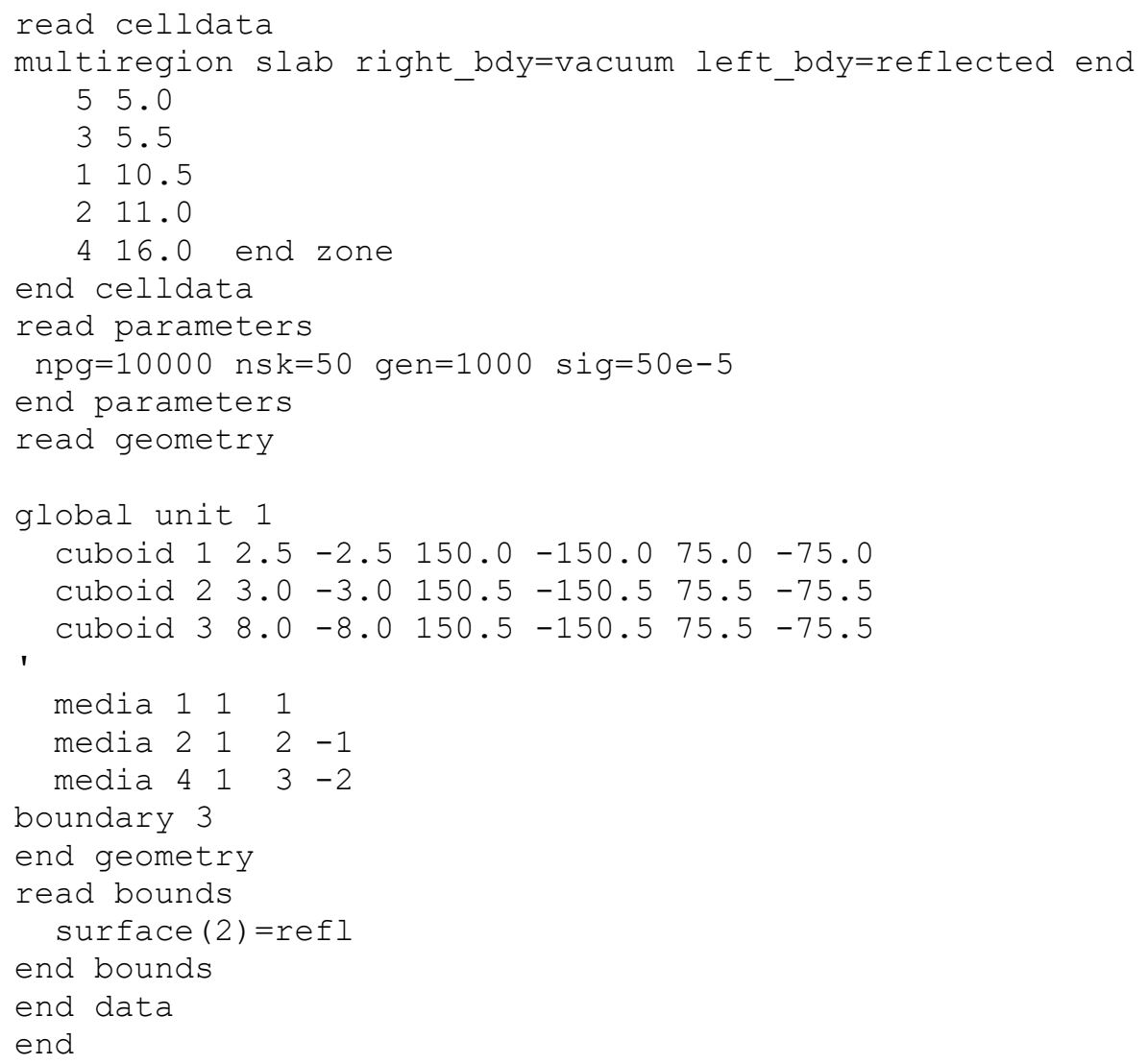

\section{INPUT FOR SECTION 8.5.3}

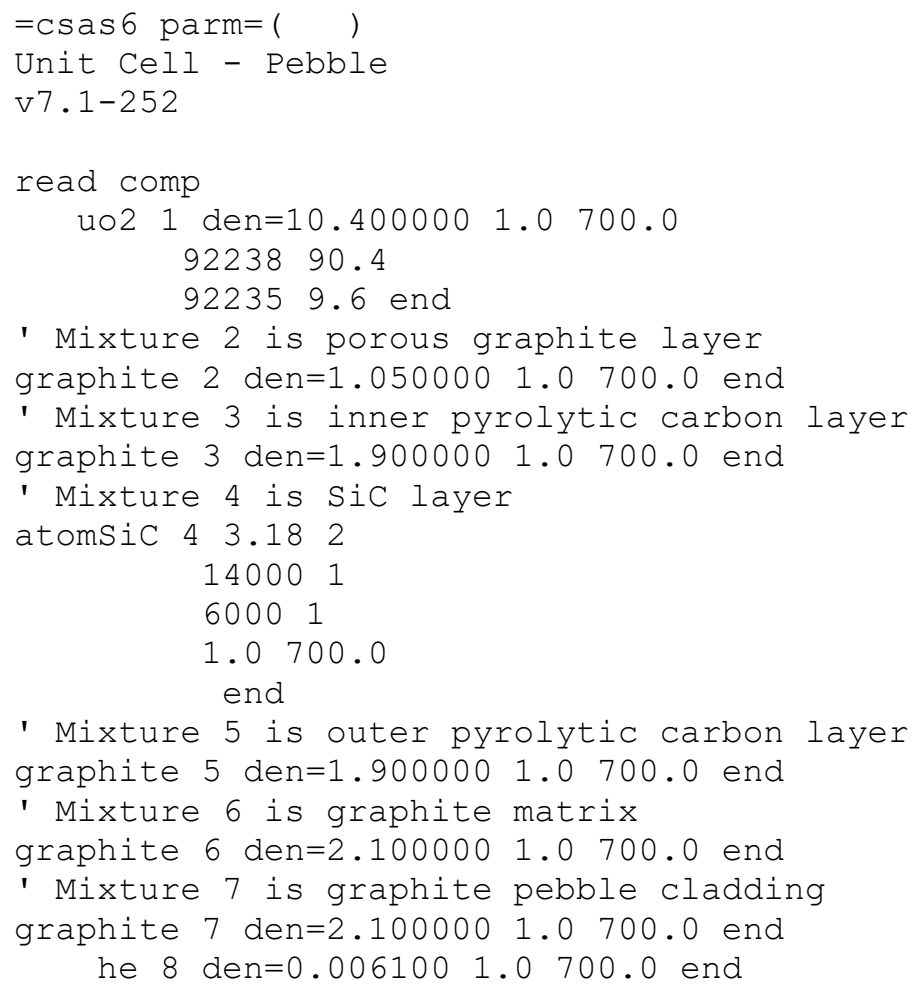




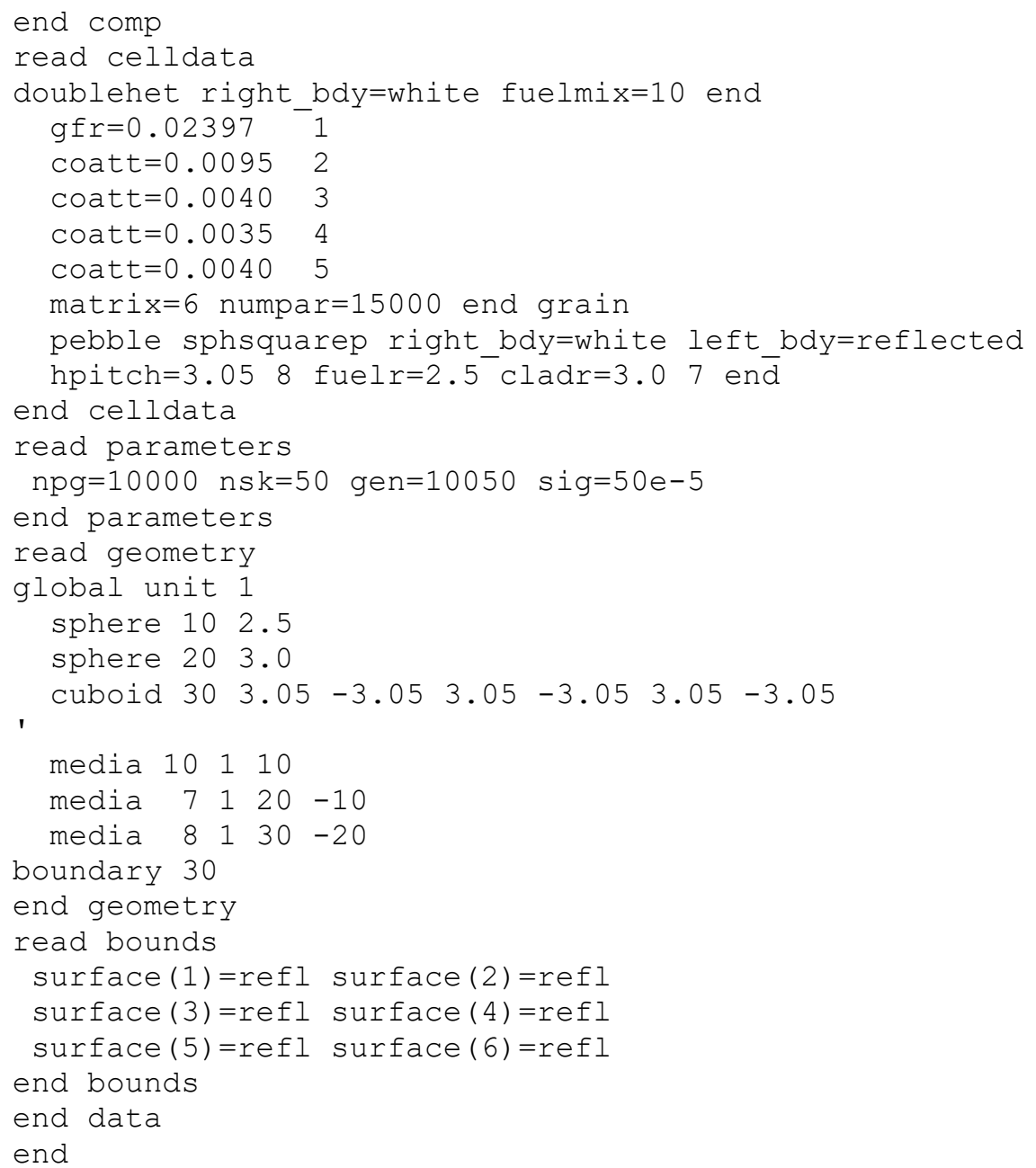

